\title{
Experimental Measurement of the 4-D Transverse Phase Space Map of a Heavy Ion Beam
}

H.S. Hopkins

(Ph.D.)

December 1997

U.S. Department of Energy

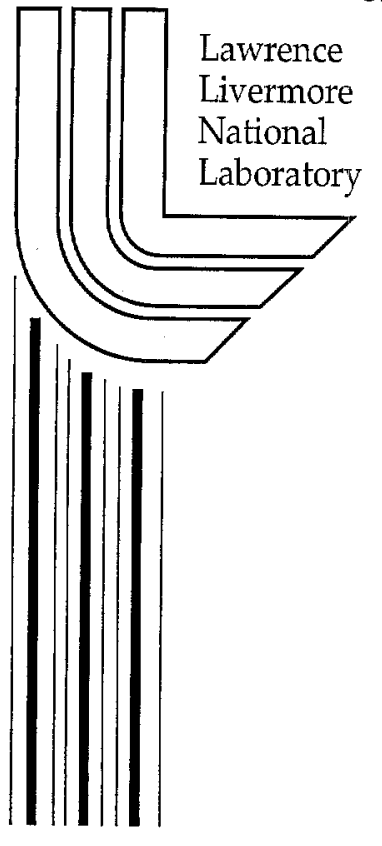




\section{DISCLAIMER}

This document was prepared as an account of work sponsored by an agency of the United States Government. Neither the United States Government nor the University of California nor any of their employees, makes any warranty, express or implied, or assumes any legal liability or responsibility for the accuracy, completeness, or usefulness of any information, apparatus, product, or process disclosed, or represents that its use would not infringe privately owned rights. Reference herein to any specific commercial product, process, or service by trade name, trademark, manufacturer, or otherwise, does not necessarily constitute or imply its endorsement, recommendation, or favoring by the United States Government or the University of California. The views and opinions of authors expressed herein do not necessarily state or reflect those of the United States Government or the University of California, and shall not be used for advertising or product endorsement purposes.

This work was performed under the auspices of the U. S. Department of Energy by the University of California, Lawrence Livermore National Laboratory under Contract No. W-7405-Eng-48.

This report has been reproduced directly from the best available copy.

Available electronically at http://www.doe.gov/bridge

Available for a processing fee to U.S. Department of Energy and its contractors in paper from

U.S. Department of Energy

Office of Scientific and Technical Information

P.O. Box 62

Oak Ridge, TN 37831-0062

Telephone: (865) 576-8401

Facsimile: (865) 576-5728

E-mail: reports@adonis.osti.gov

Available for the sale to the public from

U.S. Department of Commerce

National Technical Information Service

5285 Port Royal Road

Springfield, VA 22161

Telephone: (800) 553-6847

Facsimile: (703) 605-6900

E-mail: orders@ntis.fedworld.gov

Online ordering: http://www.ntis.gov/ordering.htm

OR

Lawrence Livermore National Laboratory

Technical Information Department's Digital Library

http://www.llnl.gov/tid/Library.html 


\title{
Experimental Measurement of the 4-D Transverse Phase Space Map of a Heavy Ion Beam
}

\author{
by
}

\author{
Harvey S. Hopkins
}

B.S. (United States Naval Academy) 1981

M.S. (University of California, Davis) 1987

A dissertation submitted in partial satisfaction of the requirements for the degree of

Doctor of Philosophy

in

Engineering-Nuclear Engineering

in the

GRADUATE DIVISION

of the

UNIVERSITY of CALIFORNIA at BERKELEY

Committee in charge:

Professor Edward C. Morse, Chair

Professor David L. Judd

Professor Jasmina L. Vujic

Fall 1997 
The dissertation of Harvey S. Hopkins is approved:

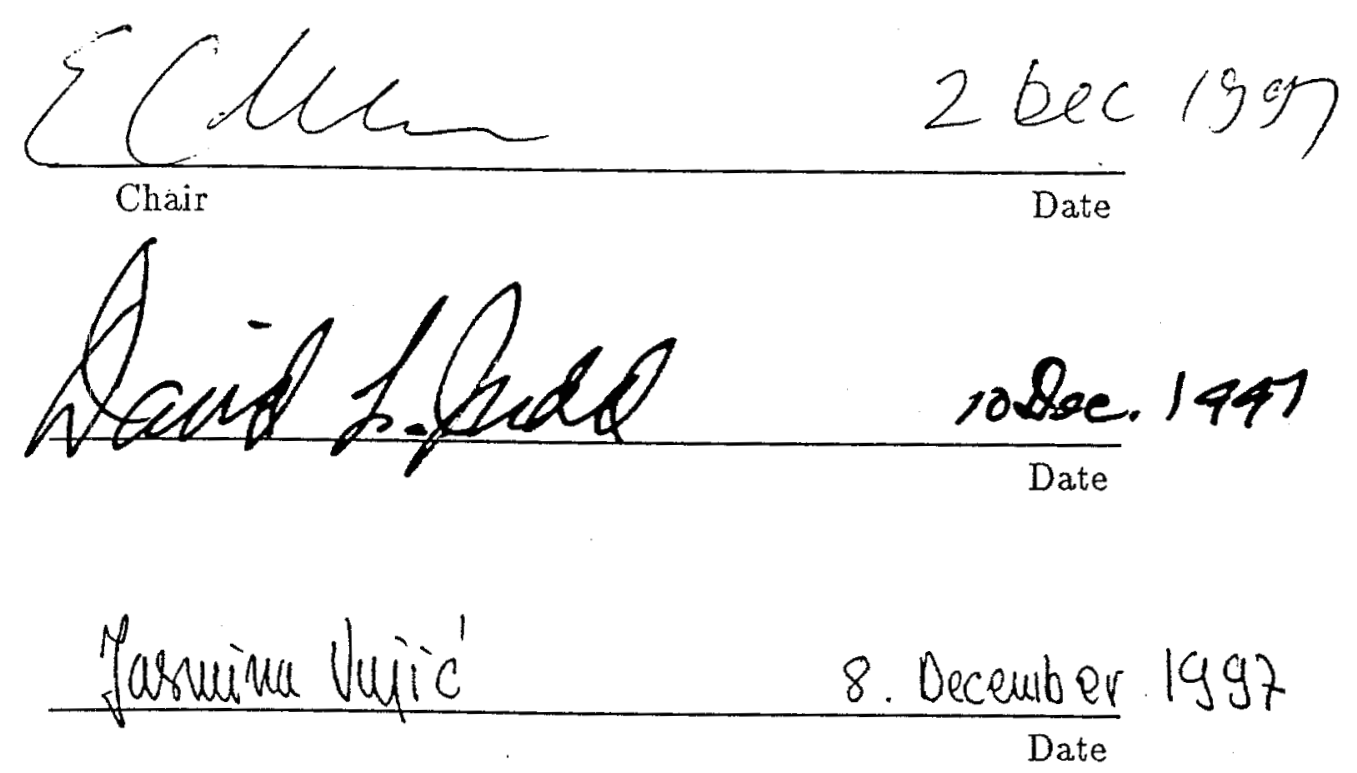

University of California at Berkeley

1997 


\begin{abstract}
Experimental Measurement of the 4-D Transverse Phase Space Map of a Heavy Ion Beam

by

Harvey S. Hopkins

Doctor of Philosophy in Engineering-Nuclear Engineering

University of California at Berkeley

Professor Edward C. Morse, Chair
\end{abstract}

The development and employment of a new diagnostic instrument for characterizing intense, heavy ion beams is reported on. This instrument, the "Gated Beam Imager" or "GBI" was designed for use on Lawrence Livermore National Laboratory Heavy Ion Fusion Project's "Small Recirculator", an integrated, scaled physics experiment and engineering development project for studying the transport and control of intense heavy ion beams as inertial fusion drivers in the production of electric power. The GBI allows rapid measurement and calculation of a heavy ion beam's characteristics to include all the first and second moments of the transverse phase space distribution, transverse emittance, envelope parameters and beam centroid. The GBI, with appropriate gating produces a time history of the beam resulting in a 4-D phase-space and time "map" of the beam. A unique capability of the GBI over existing diagnostic instruments is its ability to measure the "cross" moments between the two transverse orthogonal directions. Non-zero "cross" moments in the alternating gradient lattice of the Small Recirculator are indicative of focusing element rotational misalignments 
contributing to beam emittance growth. This emittance growth, while having the same effect on the ability to focus a beam as emittance growth caused by non-linear effects, is in principle removable by an appropriate number of focusing elements. The instrument uses the pepperpot method of introducing a plate with many pinholes into the beam and observing the images of the resulting beamlets as they interact with a detector after an appropriate drift distance. In order to produce adequate optical signal and repeatability, the detector was chosen to be a microchannel plate (MCP) with a phosphor readout screen. The heavy ions in the pepperpot beamlets are stopped in the MCP's thin front metal anode and the resulting secondary electron signal is amplified and proximity-focused onto the phosphor while maintaining the spatial and intensity characteristics of the heavy ion beamlets. The MCP used in this manner is a sensitive, accurate, and long-lasting detector, resistant against signal degradation experienced by previous methods of intense heavy ion beam detection and imaging. The performance of the GBI was benchmarked against existing mechanical emittance diagnostics and the results of sophisticated beam transport numerical simulation codes to demonstrate its usefulness as a diagnostic tool.

A method of beam correction to remove the effects of quadrupole focusing element rotational misalignments is proposed using data obtainable from a GBI. An optimizing code was written to determine the parameters of the correction system elements based on input from the GBI. The results of this code for the Small Recirculator beam are reported on.

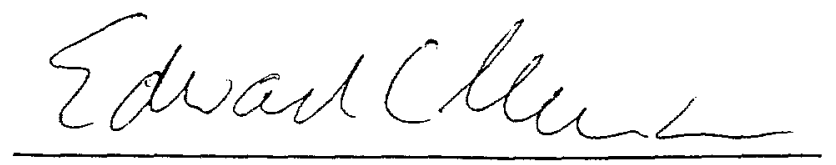

Professor Edward C. Morse

Dissertation Committee Chair 


\section{Contents}

List of Figures vi vi vis

List of Tables ix

1 Introduction 1

2 Small Recirculator Experiment Overview 5

2.1 Small Recirculator Description . . . . . . . . . . . . . 7

2.1.1 Full Ring Operation ................ 7

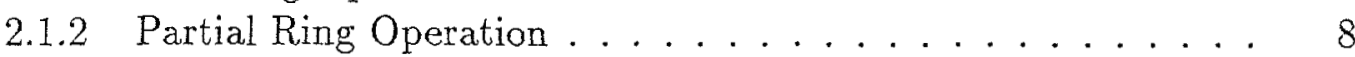

2.1.3 Potassium Ion Source . . . . . . . . . . . . . 10

2.1.4 Electrostatic Matching Section . . . . . . . . . . . . 12

2.1.5 Straight Magnetic Transport Section ............. 14

2.1.6 Electrostatic Bending Section .............. 15

2.1.7 Multi-Instrument Diagnostic Tank . . . . . . . . . 16

2.2 Beam Physics Scaling of the Small Recirculator . . . . . . . . . 18

2.2 .1 Physical Size Scaling . . . . . . . . . . . . . . 18

2.2.2 Maintaining Key Dimensionless Beam Physics Parameters . . 19

3 Heavy Ion Beam Transport 24

3.1 Origin of Beam Emittance . . . . . . . . . . . . . . . 25

3.2 Small Recirculator Beam Transport . . . . . . . . . . . . . . . 26

3.3 Moment Description of the Ion Beam . . . . . . . . . . . . 31

3.4 Definition of Emittance . . . . . . . . . . . . . . . . 34

4 Pepperpot Method and Image Data Analysis $\quad \mathbf{3 7}$

4.1 Capabilities of Existing Emittance Diagnostics . . . . . . . . . . 37

4.2 The Pepperpot Method . . . . . . . . . . . . . . . 38

4.3 Design of the Gated Beam Imager . . . . . . . . . . . . . . . . . 40

4.4 Pepperpot Method Data Analysis . . . . . . . . . . . . . . . . 42

4.5 Image Capture and Image Analysis Code . . . . . . . . . . . . . . 50

4.6 Sample Output from the Data Analysis Code . . . . . . . . . . 52

5 Detector Selection and Equipment Description 67 
5.1 Fast Plastic Scintillator as a Heavy Ion Detector . . . . . . . . . . . 67

5.2 Microchannel Plate Detector as a Heavy Ion Detector . . . . . . . . 71

5.3 Obtaining Time-Resolved Beam Data by Gating the MCP . . . . . 76

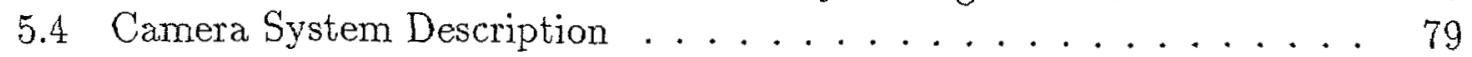

5.5 Operating the Gated Beam Imager . . . . . . . . . . . . . . . . 81

5.6 Applying Corrections to the GBI Images . . . . . . . . . . . . . . . . 83

5.6 .1 CCD Dark Charge Correction ............. 83

5.6.2 CCD Flat Field Response Correction . . . . . . . . . . . . 84

5.6.3 MCP Detector Flat Field Response Correction . . . . . . . . . 85

5.6.4 Preparing the Images for Data Analysis . . . . . . . . . . 88

6 A Beam Correction System 91

6.1 The Effect on the Beam of Rotationally Misaligned Quadrupoles . . . 91

6.2 Approximate Correction for a Single Misaligned Quadrupole . . . . . 95

6.3 Correction for Random Quadrupole Rotation Misalignments . . . . . 99

6.3.1 Applying GBI Data to a Beam Correction System . . . . . . 101

6.3.2 Determining the Optimum Correction System Parameters . . 104

6.3.3 Implementing the Optimizing Algorithm . . . . . . . . 106

7 Experimental Results 113

7.1 GBI Setup and Calibration . . . . . . . . . . . . . . 115

7.2 Observing the Effect of Axial Bearn Expansion . . . . . . . . . . . 115

7.3. Data Analysis and Results at the $45^{\circ}$ Bend Section . . . . . . . . . 119

7.3.1 Comparison of GBI and Slit Scanner Measurements . . . . . 120

7.3 .2 Error Analysis . . . . . . . . . . . . . . . . 122

7.3.3 Comparison of GBI Data with WARP Numerical Simulation Code Results . . . . . . . . . . . . . . . . . . . 124

7.4 Observing and Measuring the Effect of Magnetic Quadrupole Rotation Errors and Applying Approximate Corrections . . . . . . . . . . 126

7.5 Beam Transverse Intensity Distribution Measurement . . . . . . . 138

8 Summary and Conclusions $\quad 141$

$\begin{array}{ll}\text { A GBI Design Optimizing Spreadsheet } & 146\end{array}$

B Derivation of Beam Moment Evolution Equations $\quad 151$

C Explicit Solutions for $\Delta x^{2}$ and $\Delta y^{2} \quad \mathbf{1 5 6}$

D GBI Equipment Settings for Acquiring Gated Images 162

E Simulation and Data Analysis Codes $\mathbf{1 6 5}$

E.1 "FULLAG" ......................... 165 


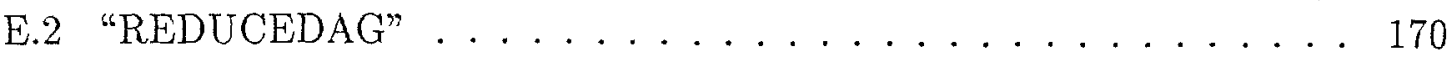

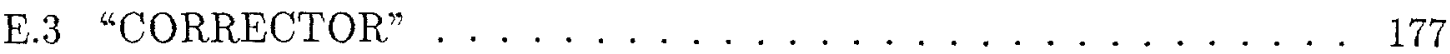

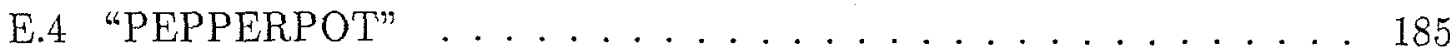




\section{List of Figures}

1.1 IFE Power Plant Conceptual View . . . . . . . . . . . . 2

2.1 Small Recirculator Plan View . . . . . . . . . . . . . . 6

2.2 Small Recirculator Schematic . . . . . . . . . . . . 7

2.3 Experimental Configuration of the Small Recirculator $\left(45^{\circ}\right) \ldots \ldots$

2.4 Experimental Configuration of the Small Recirculator $\left(90^{\circ}\right) \ldots . . .9$

2.5 Ion Source Assembly . . . . . . . . . . . . . . . . . . 11

2.6 Ion Source Pulser Power Supply Circuit. . . . . . . . . . . . . 12

2.7 Electrostatic Matching Section . . . . . . . . . . . . . . 13

2.8 Small Recirculator Straight Magnetic Transport Section . . . . . . . . 15

2.9 Small Recirculator $45^{\circ}$ Bend Section . . . . . . . . . . . 16

2.10 Multi-Instrument Diagnostic Chamber . . . . . . . . . . . . . 17

2.11 "C-design" Heavy Ion Driver Schematic . . . . . . . . . . . . 19

3.1 Beam 4-D Phase Space Volume ............... . . 27

3.2 2-D Phase Space Ellipse Coefficients . . . . . . . . . . . . . 29

3.3 Beam Sigma Matrix . . . . . . . . . . . . . 33

4.1 Nonlinear Evolution of Phase Space . . . . . . . . . . . . . . . . 39

4.2 Pepperpot Method Schematic . . . . . . . . . . . . . . . . 39

4.3 Simplified GBI Instrument Schematic . . . . . . . . . . . . . . . 40

4.4 Phase Space Ellipse Rotation Angle Determination . . . . . . . . . . 42

4.5 Beamlet Data Required for Moment Calculations . . . . . . . . . . . 43

4.6 Beam Phase Space Ellipse . . . . . . . . . . . . . . . 44

4.7 GBI Phase Space Plot . . . . . . . . . . . . . . . . . . 45

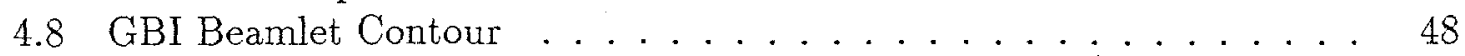

4.9 Typical GBI Data Image . . . . . . . . . . . . . . . . . . . 52

4.10 Identification of Beamlet Holes . . . . . . . . . . . . 53

4.11 Data Analysis Windows . . . . . . . . . . . . 54

4.12 Relationship Between Holes and Spots . . . . . . . . . . . . . 55

4.13 Contour Plot of the Beamlet Intensity . . . . . . . . . . . . . . 56

4.14 Beamlet Intensity Surface Plot . . . . . . . . . . . . . . . 57

4.15 Beamlet Intensity Surface Plot . . . . . . . . . . . . . 58

4.16 Smoothed Beamlet Surface Plot . . . . . . . . . . . . 58 
4.17 Beamlet Cross-section Plots . . . . . . . . . . . . . . . 59

4.18 Beamlet Profile Plot . . . . . . . . . . . . . . . . . 60

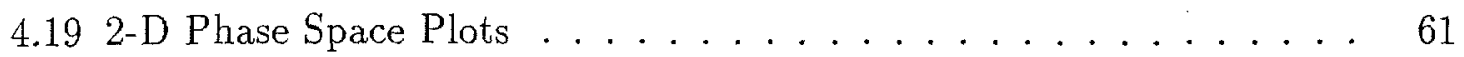

4.20 Pepperpot Rotation Angle Plot . . . . . . . . . . . . . . 62

4.21 Incorrect Rotation Angle . . . . . . . . . . . . . . . . 63

4.22 Cross Moment Plot $\left(\left\langle x^{\prime}-y^{\prime}\right\rangle,\langle x-y\rangle\right) \ldots \ldots \ldots$. . . . . . . . . . . . . . . 64

4.23 Cross Moment Plot $\left\langle x^{\prime}-y\right\rangle,\left\langle x-y^{\prime}\right\rangle \ldots \ldots$. . . . . . . . 65

4.24 Beam Statistics Plots . . . . . . . . . . . . . . 66

5.1 BC-412 Scintillator Light Output vs. Exposure . . . . . . . . . . . 69

5.2 Scintillator Exposure Image Sequence . . . . . . . . . . . . . . . . 71

5.3 Microchannel Plate Schematic . . . . . . . . . . . . . 73

5.4 Microchannel Plate Resolution . . . . . . . . . . . . . . 75

5.5 PINEX MCP Gate Circuit . . . . . . . . . . . . . . . . . 77

5.6 MCP Detector Gate Signals . . . . . . . . . . . . . . . . 78

5.7 GBI Equipment Interconnections . . . . . . . . . . . . . 79

5.8 GBI CCD Camera In Position . . . . . . . . . . . . . . 80

5.9 GBI Camera Control Equipment . . . . . . . . . . . . . . 82

5.10 GBI Image Capture Timing Diagram . . . . . . . . . . . . . . 83

5.11 Dark Charge Image Line Average Plot . . . . . . . . . . . . . . . 84

5.12 CCD Camera Flat Field Equipment Setup . . . . . . . . . . . 86

5.13 MCP X-ray Flat Field Equipment Setup . . . . . . . . . . . . . . 87

5.14 MCP X-ray Flat Field, Field-of-View . . . . . . . . . . . . 88

5.15 MCP Non-Uniform Response . . . . . . . . . . . . 89

6.1 Beam and Laboratory Coordinate System . . . . . . . . . . . . . 94

6.2 A Single Misaligned Quadrupole, Part A . . . . . . . . . . . . . . 96

6.3 A Single Misaligned Quadrupole, Part B . . . . . . . . . . . . . 97

6.4 Correction of a Known Quadrupole Rotation Error, Part A . . . . . . 98

6.5 Correction of a Known Quadrupole Rotation Error, Part B . . . . . . 99

6.6 Conceptual Beam Correction System . . . . . . . . . . . . . . 102

6.7 Verification of Simplified $\Delta x^{2}$ and $\Delta y^{2}$ solutions . . . . . . . . . 104

6.8 Beam Correction System Optimization Code Results Plots . . . . . . 109

6.9 Correction of Random Quadrupole Rotation Errors, Part A . . . . . . 111

6.10 Correction of Random Quadrupole Rotation Errors, Part B . . . . . . 112

7.1 Bending Dipole Voltage Scan . . . . . . . . . . . . 114

7.2 View of the GBI ....................... 116

7.3 Camera Field of View Calibration Image . . . . . . . . . . . . 117

7.4 Beam Axial Expansion Images . . . . . . . . . . . . . . . . 118

7.5 Source Pulser and Faraday Cup Waveforms . . . . . . . . . . . . . 119

7.6 Normalized Emittance vs. Time at the $45^{\circ}$ Bend . . . . . . . . . . . 120

7.7 Beam Ion Distribution Second Moments vs. Time . . . . . . . . . . 121 
7.8 Experimental Emittance Data Compared to "WARPxy" Simulation . 125

7.9 Experimental Envelope Data Compared with "WARPxy" Simulation 126

7.10 GBI Images with Quadrupole Rotation Errors . . . . . . . . . . . . . 128

7.11 Phase Space Emittance Plots with "Aligned" Quadrupoles . . . . . . 129

7.12 Cross Moment Plots with "Aligned" Quadrupoles . . . . . . . . . . . 130

7.13 Phase Space Emittance Plots with Quadrupole 8 rotated $5^{\circ}$ counterclockwise . . . . . . . . . . . . . . . . . 131

7.14 Cross Moment Plots with Quadrupole 8 rotated $5^{\circ}$ counter-clockwise 132

7.15 Phase Space Emittance Plots with Quarupole 13 rotated $5^{\circ}$ clockwise 133

7.16 Cross Moment Plot with Quarupole 13 rotated $5^{\circ}$ clockwise . . . . . . 134

7.17 Phase Space Emittance Plots with Quadrupoles 8 and 13 counter-rotated 135

7.18 Cross Moment Plot with Quadrupoles 8 and 13 counter-rotated . . . 136

7.19 Beam Intensity Surface Plot from the GBI . . . . . . . . . . . . . . 139

7.20 Beam Intensity Surface Plot from Cross Slits . . . . . . . . . . . . . . 139

7.21 GBI Image Pixel Row Average Plot . . . . . . . . . . . . . 140

B.1 Beam and laboratory coordinate system . . . . . . . . . 152 


\section{List of Tables}

2.1 Nominal Electrostatic Quadrupole Voltages for an $80 \mathrm{keV}$ Beam . . . 14

2.2 Small Recirculator Design Parameters . . . . . . . . . . . . . . 22

2.3 Comparison Between the Small Recirculator and C-design Driver . . 23

4.1 GBI Design Spreadsheet Results . . . . . . . . . . . . . . . 41

5.1 BC-412 Scintillator Properties . . . . . . . . . . . 68

5.2 Galileo Microchannel Plate Characteristics . . . . . . . . . . . . 73

5.3 TRIM Code Calculation Results . . . . . . . . . . . . 74

6.1 SPSA Optimization Results . . . . . . . . . . . . 110

7.1 Beam Ion Distribution Second Moments at the $45^{\circ}$ bend . . . . . . 122

7.2 Pepperpot Data Analysis Sensitivity to Errors . . . . . . . . . . . 123

7.3 Summary of Beam Parameters from Rotated Quadrupole Images . . . 137

D.1 GBI Equipment and Software Settings for GBI Measurements at $45^{\circ} .162$

D.2 GBI Measurement Parameters at $45^{\circ} \ldots \ldots \ldots$. . . . . . . . . . . . . . 163

D.3 $45^{\circ}$ Integrated image file information . . . . . . . . . . . . 163

D.4 $45^{\circ} 100$ ns gated image file information . . . . . . . . . . . 164 


\section{Acknowledgements}

I'd most like to thank my wife, Pam, for her steadfast support throughout my graduate education. Without her, I couldn't have done this work.

An overwhelming number of people provided assistance and information in support of this dissertation. Professor Ed Morse provided constructive criticism and direction when I needed it. Dr. Larry Nattrass of LLNL provided me the opportunity to work on LLNL's Heavy Ion Fusion Project. Dr. Alex Friedman provided financial support making it possible for me to work on the project and was always available with helpful suggestions. Through his efforts the Small Recirculator project was held together in a tough funding environment. Dr. Mike Cable suggested the Gated Beam Imager concept to me and Dr. Craig Sangster and Bo Nelson provided me with helpful suggestions with experimental methods. Dr. John Barnard and Dr. Steve Lund were always helpful in computational and theoretical aspects of heavy ion beam transport. I am indebted to Dr. Arthur Paul for his allowing me to use his Pepperpot data analysis code, instructing me in its use, and assisting me in modifying it.

There are many other people who have helped me and I thank them all. From the Heavy Ion Fusion Project, Dave Grote, George Craig, Larry Ahle, Gene Lauer, Bill Manning, Darryl Autrey, Art Molvik, Melissa Cisneroz, and Fred Dietrich. From Bechtel Nevada Corporation, Charlie Diamond, Ed Perreira, Dave Longinotti, Arte Debeling, Bill Fritz, and Chuck Ward. From LLNL Lasers Directorate, Dave Pendleton, Debbie Callaghan, Dean Lee, Roger Griffith, and Perry Bell. From Allied-Signal Corporation., Geoff Mant, and Rob Costa. From LBNL, Schmuel Eylon. 


\section{Chapter 1}

\section{Introduction}

The international search for a viable technology to employ in electric power generation from controlled fusion includes the United States' Inertial Confinement Fusion (ICF) Program. Large glass lasers including NOVA and the National Ignition Facility (NIF) at the Lawrence Livermore National Laboratory (LLNL) are being operated or built as research tools for achieving ignition and gain in a fusion target. ${ }^{1}$ Success in achieving ignition is a major hurdle to a practical, economical, and environmentally attractive source of electric power. The National Ignition Facility, using lasers as a driver, will demonstrate ignition and the response of target chamber materials to the fusion environment, as well as contributing to national security as part of the U.S. Department of Energy's Stockpile Stewardship Program.

A nominal 1000 MWe inertial fusion energy (IFE) power plant requires a high repetition rate and high driver efficiency for economic power production. ${ }^{2}$ In the U.S., several driver options are being studied to obtain the desired characteristics of high pulse rate, efficiency, reliability, and low cost. These options include the diodepumped solid state laser, light ion accelerator, and a heavy ion accelerator based on induction accelerator technology. ${ }^{3}$ The heavy ion driver research is centered at LLNL and Lawrence Berkeley National Laboratory. The major issues being studied at these sites, using theoretical and computational methods, and experiments, include ion source design and performance, beam transport, beam acceleration and control, final 


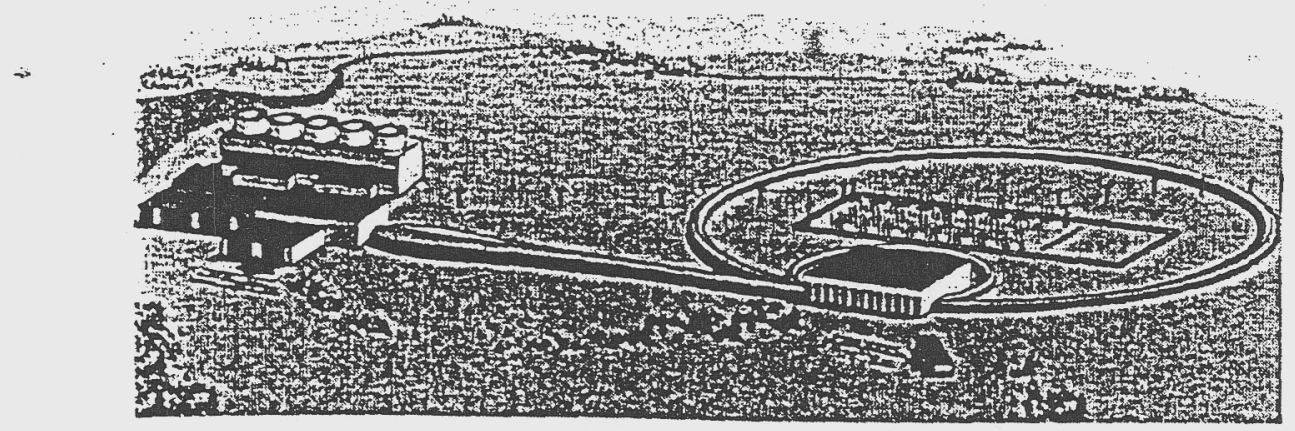

Figure 1.1: Conceptual view of an inertial fusion energy power plant. ${ }^{1}$

focusing element design, target chamber beam transport, and fusion target design and performance. Additionally integrated power plant systems studies done to model the plant and driver design, construction, and operation are being carried out to estimate the per kilowatt-hour cost of electricity from an inertial fusion energy plant.

Existing accelerators for high-energy physics research have had long lifetimes and high availability, and it is expected that IFE drivers could share these attributes. An induction accelerator in particular is efficient, can readily transport and amplify a high-current beam, and can easily meet the pulse rate requirement of $3-10 \mathrm{~Hz}$ needed for energy production. ${ }^{4}$ Conceptual designs for IFE power plants have been studied such as the artist's conception in Figure 1.1 and the HYLIFE II reactor chamber design. The HYLIFE II design addressed issues of how to convert the output of the target to a working fluid as well as alleviating material damage from intense neutron irradiation and other critical engineering issues. ${ }^{5}$

The requirements for the driver are imposed by the fusion target design and to a lesser extent the geometry of the target chamber. ${ }^{5-7}$ Current work at LLNL has focused on indirect drive targets in which a holraum with radiation converters encloses a deuterium-tritium pellet. The converters are illuminated by clusters of ion beams with a narrow cone angle. To achieve a gain of about 100 such a target is required to 
receive about $5 \mathrm{MJ}$ in incident ion beam energy in a suitably shaped pulse of about $10 \mathrm{~ns}$ in duration. ${ }^{8}$ For an ion mass of $100-200 \mathrm{amu}$ the required energy per ion is of order $5 \mathrm{GeV}$ based on radiation converter stopping range of $\mathrm{R}=.02-0.2 \mathrm{~g} / \mathrm{cm}^{2}{ }^{2}$ The use of heavy ions and their high individual energies allows a relatively low beam current (compared to light ion beams) and non-neutralized ballistic focusing of the beam across a 4-10 meter radius target chamber. The ability to focus to a nominal 3 millimeter spot is governed by four major factors: beam space charge, beam emittance, transverse focusing field misalignments, and transverse focusing field aberrations. Neglecting the effects of the other three factors an estimate of the maximum permissible beam emittance at the final focus lens can be made. Assuming 6 meters from the final focusing lens to the target and a 3 millimeter spot size on target, ions can be aimed with maximum convergence angle of 0.3 milliradians. This angle represents the ratio between ion transverse energy and beam directed energy. For a $5 \mathrm{GeV}$ beam, the transverse energy must remain less than $0.5 \mathrm{keV}$. The transverse emittance is the product of beam transverse dimension and velocity spread normalized to the beam's directed velocity. Uncorrected optics are limited to a focusing angle of about 15 milliradians and so for a 3 millimeter spot size, one obtains an emittance limit of $45 \mathrm{~mm}-\mathrm{mr}$.

Limiting the emittance growth from source to target of these heavy ion beams is a leading topic in the research and development of a heavy ion driver. Understanding the processes that cause emittance growth and developing the engineering to produce the necessary beam attributes in an economical way are of high priority. Theoretical, computational, and experimental work are in progress at LLNL with assistance from LBNL in the building and operating of the Small Recirculator, an integrated, scaled driver physics experiment and engineering technology development platform. ${ }^{9}$ Characterizing its beam of singly-charged potassium ions is crucial to verifying its design operating specifications and benchmarking sophisticated numerical beam simulation codes that will help design a full-scale power plant driver. For this purpose an improved emittance diagnostic, the Gated Beam Imager (GBI) was developed and fielded for this thesis to obtain information on the Small Recirculator beam not 
previously available.

A description of the Small Recirculator experiment and its technology is presented in Chapter 2. Chapter 3 presents a review of intense ion beam transport issues relevant to the design and operation of the GBI. Chapter 4 explains the design methodology used for the GBI and data analysis algorithm. Chapter 5 presents the work conducted to select the ion detector used in the GBI, a description of the instrument, and its operation. As an application of the unique information available from the GBI, a beam correction system concept is proposed in Chapter 6. This correction system uses inputs from the GBI to remove the effects of random quadrupole focusing magnet rotation errors from a heavy ion driver beam. Chapter 7 presents the results of GBI measurements on the Small Recirculator beam using the prototype instrument developed for this thesis and validation by comparison with existing diagnostic instrumentation and code results. Also, experimental results relevant to the beam correction system are presented. 


\section{Chapter 2}

\section{Small Recirculator Experiment Overview}

As achieving ignition and conducting an experimental program on the National Ignition Facility can address among many issues, fusion target physics, chamber materials issues, and give the necessary public awareness boost to inertial fusion energy research, LLNL is building a heavy ion recirculating induction accelerator (dubbed "Small Recirculator") to study in a scaled manner the physics and engineering of producing, accelerating, transporting, and controlling an intense, spacecharge-dominated heavy ion beam through multiple laps of a circular accelerator. ${ }^{9}$ The recirculating induction accelerator concept applied to a full-scale driver promises component construction cost reduction over a linear accelerator by re-using the same accelerating and focusing elements multiple times in a beam's travel from source to target. The long path length available in a multi-lap recirculator lessens the required accelerating gradient through each induction cell allowing smaller (and presumably cheaper) components than those of a shorter path length "once-through" linear accelerator. ${ }^{10}$

The Small Recirculator will be the first accelerator of its kind, and with multiple lap operation will explore the physics and engineering issues of beam control needed to economically build a driver-size machine. The repeated use of accelerator elements during beam acceleration and compression on multiple laps also requires new engineering development in areas of beam control, acceleration, and diagnostics. ${ }^{12}$ These issues are being addressed in the Small Recirculator project with the goal of an in- 
tegrated, scaled system to provide experience in all aspects that apply to building and operating a driver and to provide data to benchmark computer codes to aid in the design of a power plant size induction accelerator. To date, prior to multiple lap operation, the experiment has yielded valuable data on emittance growth, and beam control of intense heavy ion beams around bends. A summary of its design is in Table 2.2 at the end of this chapter on page 22 and drawings of its completed form are shown in Figures 2.1 and 2.2 .

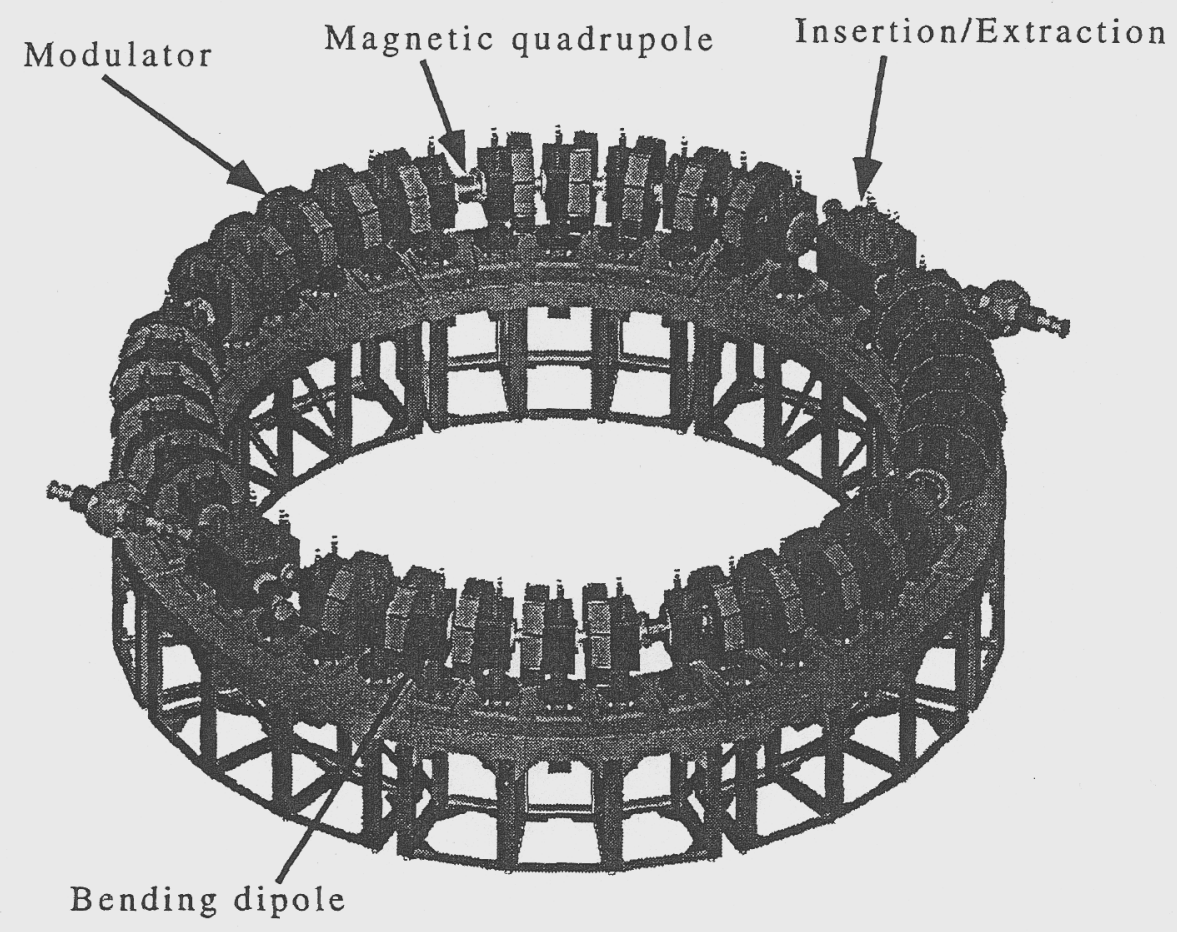

Figure 2.1: Final configuration of the Small Recirculator ring (source and matching section not pictured). 


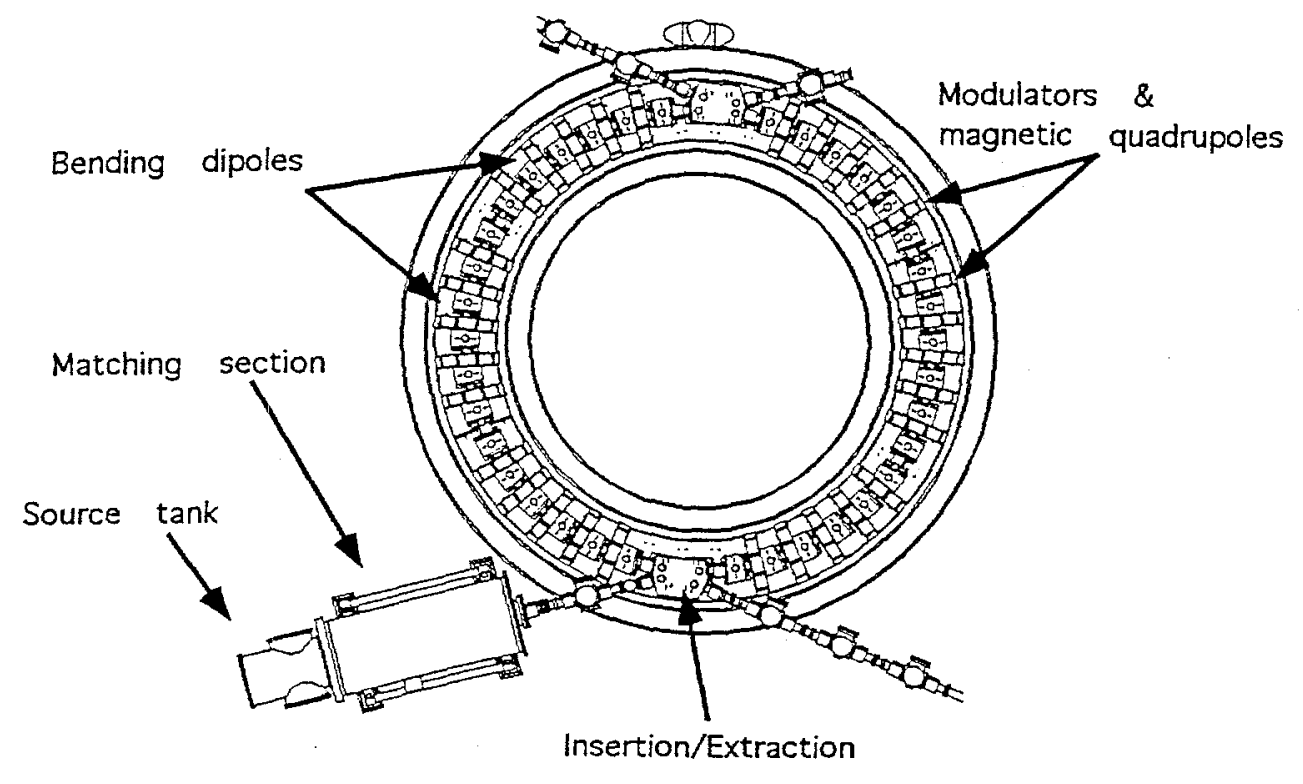

Figure 2.2: Final configuration schematic of the Small Recirculator ring

\subsection{Small Recirculator Description}

The Small Recirculator is being constructed on a "build and test" schedule allowing for component testing and beam experiments on that portion available at various milestones during construction. When completed, the machine will have a circumference of $14.4 \mathrm{~m}$, beam pipe radius of $3.5 \mathrm{~cm}$, two beam insertion/extraction sections, and an alternate-gradient focusing half-lattice period of $38 \mathrm{~cm}$, allowing 40 half-lattice periods (populated by induction acceleration modules, electrostatic bending dipoles, and permanent magnet transverse focusing quadrupoles). ${ }^{13}$

\subsubsection{Full Ring Operation}

Full ring operation is expected to include the study of intense beam dynamics over 15 laps equating to a path length of about 216 meters. Sophisticated numerical modeling of the beam using LLNL's WARP code predicts satisfactory beam trans- 
port through the 15 laps. ${ }^{14}$ Data from the full ring operation as well as partial ring operation during construction will be used to validate the code's initial beam conditions that are assumed regarding the ion distribution and gage the design operation of the accelerator system. To provide for the technology development needed for a full-scale recirculating ion driver in the areas of beam control, longitudinal confinement, induction modulator performance and energy recovery, and beam manipulation (insertion/extraction) with minimal emittance growth, the beam of singly-charged potassium ions will be accelerated from 80 to $320 \mathrm{keV}$ by 34 induction modules. The beam pulse, initially at a current of $2 \mathrm{~mA}, 4 \mu \mathrm{s}$ in length and $1.1 \mathrm{~cm}$ average radius will be accelerated and longitudinally compressed over 15 laps resulting in a beam pulse with a current of $8 \mathrm{~mA}, 1 \mu \mathrm{s}$ in length, and average radius of $1.3 \mathrm{~cm}$. The initial phase advance of the betatron motion of the ions is $\sigma_{0}=78^{\circ}$ in the absence of space-charge and $\sigma=16^{\circ}$ in the presence of space-charge. At final beam energy, the phase advances will decrease to $\sigma_{0}=45^{\circ}$ and $\sigma=12^{\circ}$ per lattice period.

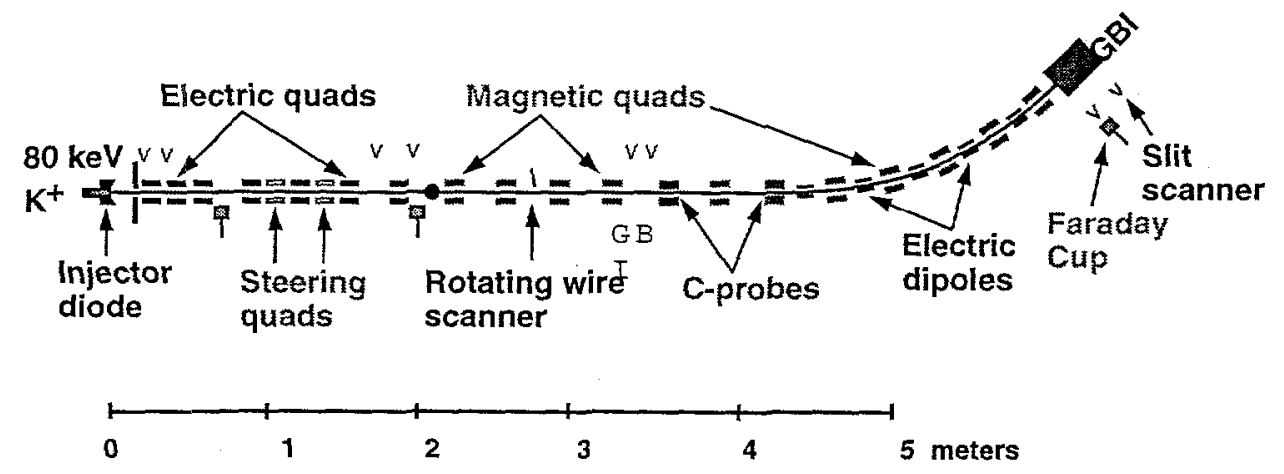

Figure 2.3: Experimental configuration of the Small Recirculator during the initial deployment of the Gated Beam Imager

\subsubsection{Partial Ring Operation}

During the experiments carried out in support of this thesis the configuration of the Small Recirculator was that shown in Figure 2.3 with a $45^{\circ}$ bend. Figure 2.4 


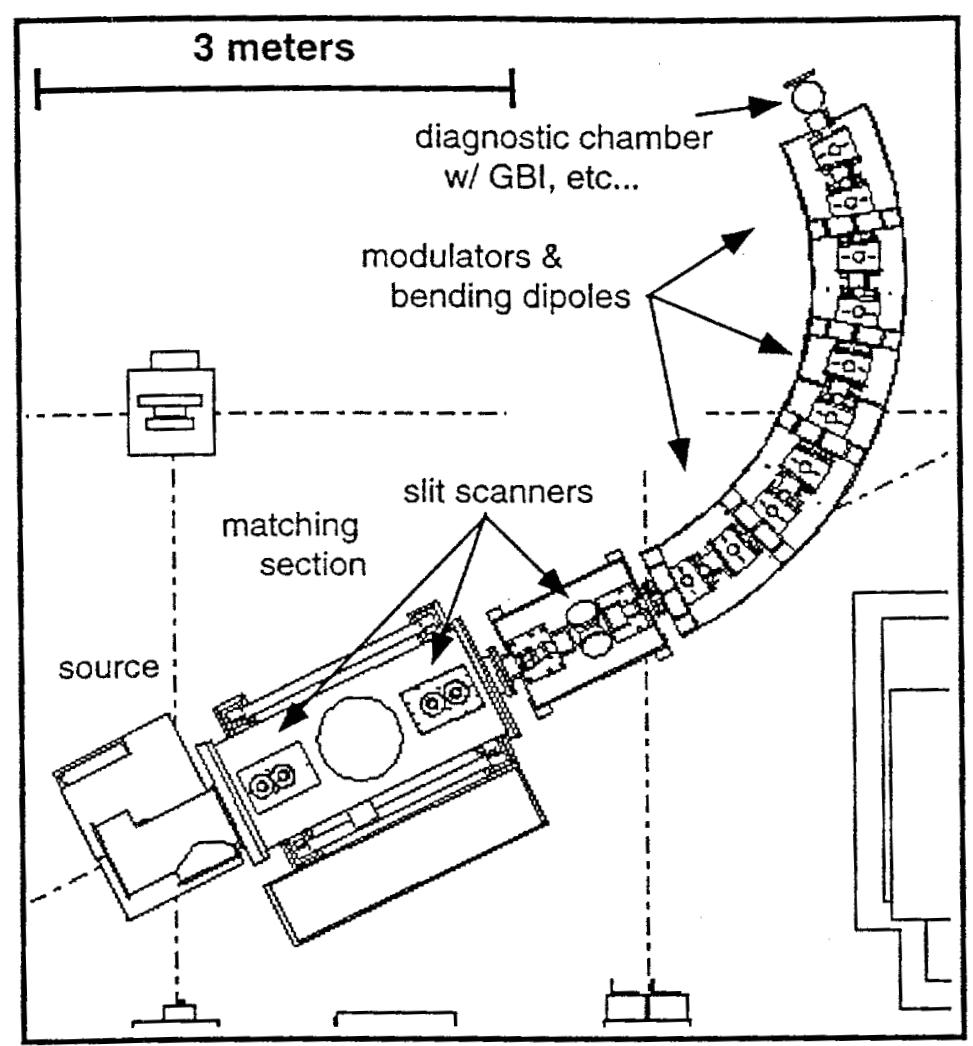

Figure 2.4: Experimental configuration of the Small Recirculator during the deployment of the Gated Beam Imager at the end of the $90^{\circ}$ bend.

shows the Small Recirculator with a $90^{\circ}$ bend installed. In this initial phase of construction and testing, several designs for the ion source pulser power supply were tested in order to obtain the desired beam ion extraction voltage waveform rising from 0 to $80 \mathrm{keV}$ in nominally $326 \mathrm{~ns}$ with a flattop, $4 \mu$ s long with less than $0.1 \%$ "ringing", and a sharp cut-off at pulse end. Testing of beam transport through an electrostatic quadrupole matching section into a straight magnetic transport section was completed prior to bending the beam initially around a $45^{\circ}$ bend in April 1997. Construction of a $90^{\circ}$ bend in started in August 1997. Bending such an intense spacecharge-dominated heavy ion beam around a curve while maintaining beam quality had never been accomplished before. Heavy ion beams are much more difficult to control than electron beams owing to their particle's large mass (a factor of $7 \times 10^{7}$ for 
potassium ions compared to electrons), requiring much larger applied fields and less room for error. Sophisticated particle-in-cell simulations, such as WARP, are required to accurately calculate the necessary fields and the shape of the elements that produce the fields such that higher order field components that produce emittance growth are minimized. ${ }^{15}$ The development of diagnostic instruments able to measure the subtle changes expected in the beam into and out of bends was a major effort at this stage of construction and testing. Development of the prototype GBI occurred over $2 \frac{1}{2}$ years using the heavy ion beam, first in the straight magnetic transport section and then at the end of the $45^{\circ}$ bend section. Its performance was compared with data from the mechanical 2-D emittance diagnostic known as "slit scanners" adapted from LBNL experiments to the Small Recirculator and to the moment and emittance calculations of the WARP code. For final testing, the Gated Beam Imager contained within a multi-instrument diagnostic chamber was placed at the end of the $45^{\circ}$ bend section around which the $80 \mathrm{keV}$ beam drifted without acceleration. These results are discussed in Chapter 7.

\subsubsection{Potassium Ion Source}

At the source end of the machine, ions are drawn from a diode source that delivers up to a $9 \mathrm{~mA}$ beam of singly-charged potassium ions $\left(\mathrm{K}^{+}\right)$at $80 \mathrm{keV}$. ${ }^{16}$ The ion source tank is mounted above the source pulser power supply housed in a tank filled with diala oil to provide cooling to power supply components and to prevent high voltage breakdown between components. ${ }^{17}$ This allows a compact physical arrangement of the pulser circuitry but does cause a considerable effort in tuning the circuit to account for stray impedances that are caused by circuit geometry which become evident at the high voltages and frequency necessary to produce the desired waveform.

The ion source is a one centimeter diameter zeolite cylinder coated with potassium. During operation the source is heated to approximately $1200^{\circ} \mathrm{F}$ by ohmic heating causing potassium ions to form a plasma just in front of its surface. The $80 \mathrm{keV}$ extraction voltage waveform that forms the beam is applied to a graphite Pierce 
electrode surrounding the source shown in Figure 2.5. The $80 \mathrm{keV}$ potential is dropped across a gap to a plate held at ground potential with a one centimeter round aperature centered on the beamline. The shape of the Pierce electrode provides a small focusing effect on the plasma and the aperature limits the current into the Small Recirculator to $2 \mathrm{~mA} .{ }^{16}$ The extraction voltage waveform has a design rise time of $326 \mathrm{~ns}$ which can be adjusted by an RC network in the power supply circuit shown in Figure 2.6. A nominal $4 \mu \mathrm{s}$ long pulse is generated with its length determined by the receipt of a second timing signal from the accelerator control system which causes a spark gap to crowbar the remaining pulser energy to ground with a fall time of $326 \mathrm{~ns}$.

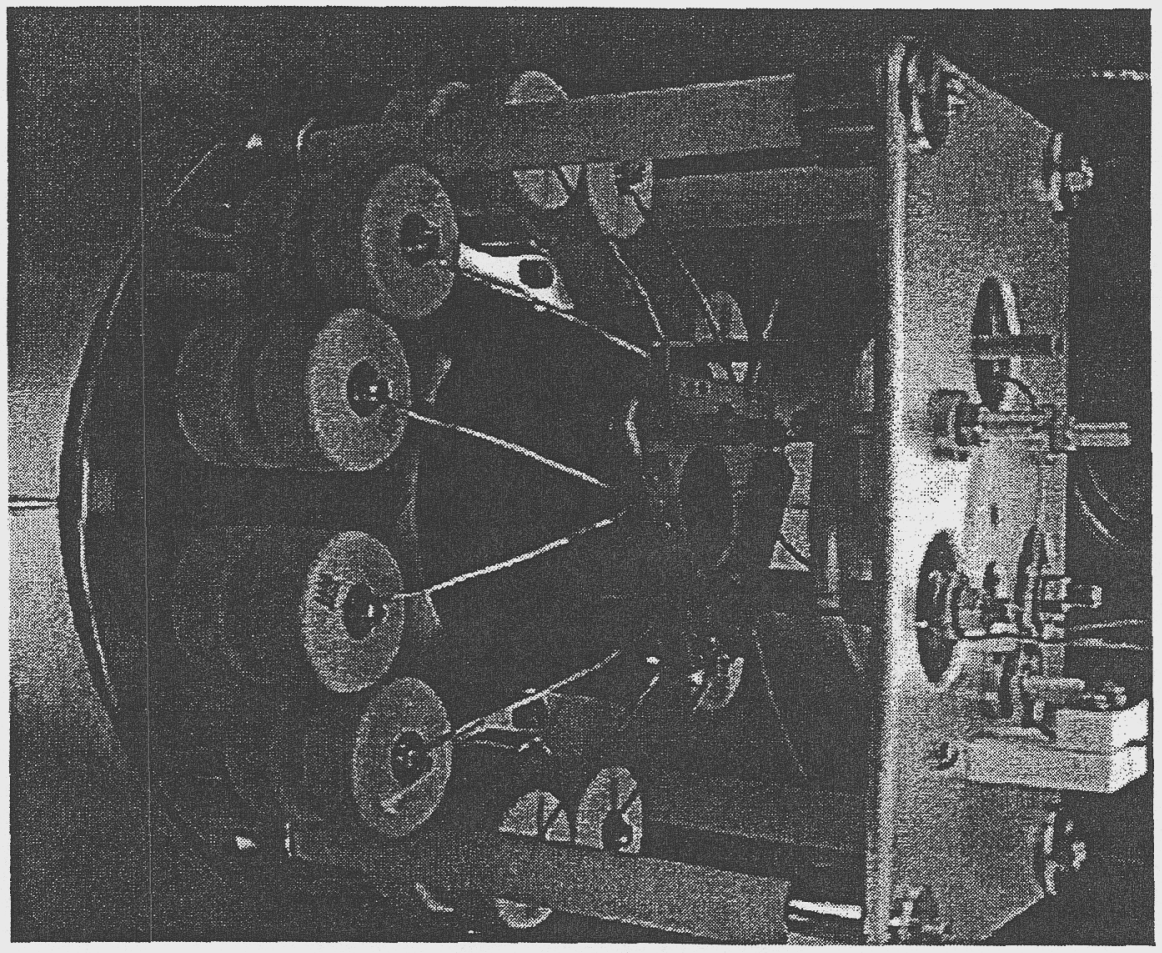

Figure 2.5: Pierce electrode (black) surrounding the potassium source (not visible) with power supply capacitors mounted on the periphery. The one centimeter diameter aperature to the matching section is to the left of the Pierce electrode 


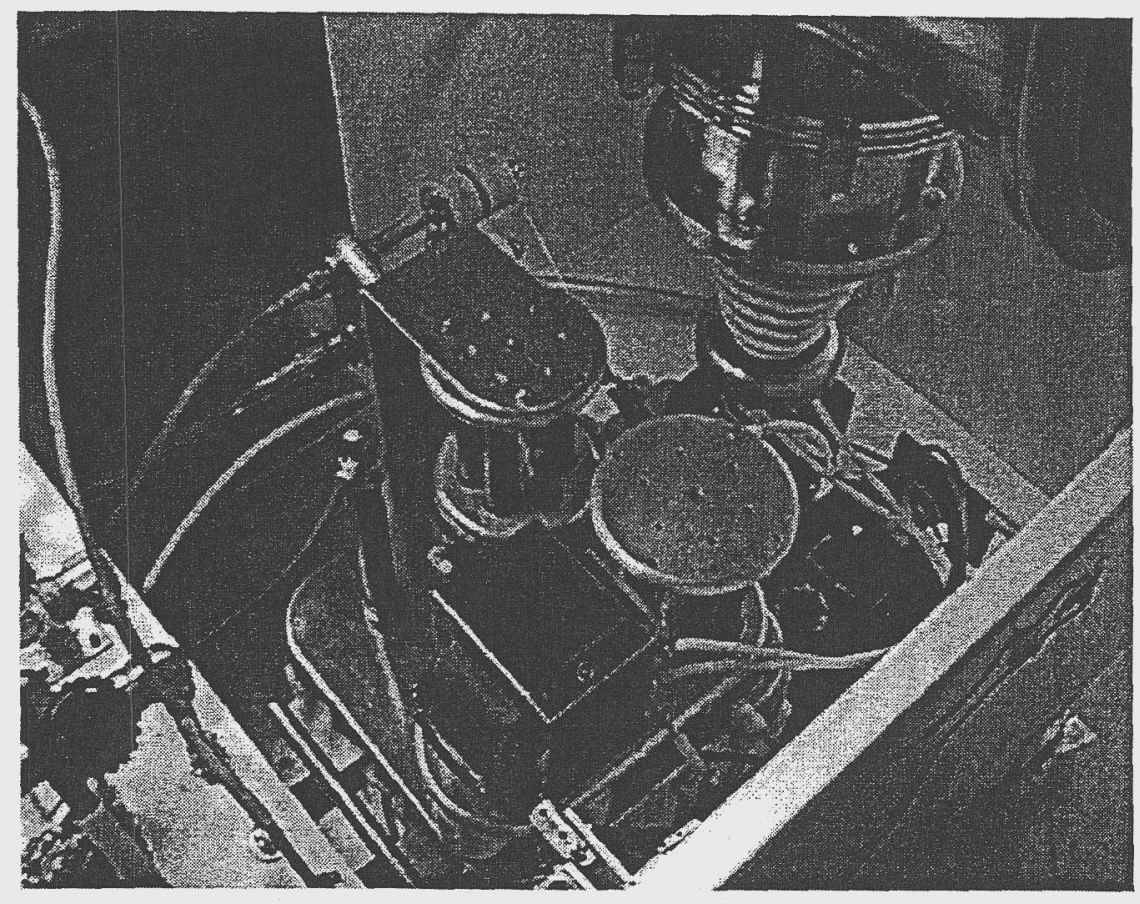

Figure 2.6: Source pulser power supply circuit in its oil tank below the ion source assembly. (Oil not present)

\subsubsection{Electrostatic Matching Section}

The one centimeter aperature passes the beam into the electrostatic matching section formerly used in LBNL's Single Beam Transport Experiment shown in Figure 2.7. The matching section contains seven electrostatic quadrupoles. High voltages whose values are calculated using the WARP code are applied to the quadrupoles which "matches" the beam by inducing the proper focusing in one transverse direction (e.g., $x$ or $y$, with $z$ being the axial direction) while defocusing in the orthogonal transverse direction. The correct combination of voltages and quadrupole polarity creates a beam envelope oscillation that matches the beam envelope to the orientation and spacing of the alternating gradient focusing elements of the magnetic transport section of the Small Recirculator. A correct match results in a beam envelope that remains less than the beam pipe's aperature dimension. Table 2.1 lists a typical set 
of electrostatic quadrupole voltages used during experiments to attain a matched 80 $\mathrm{keV}$ beam.

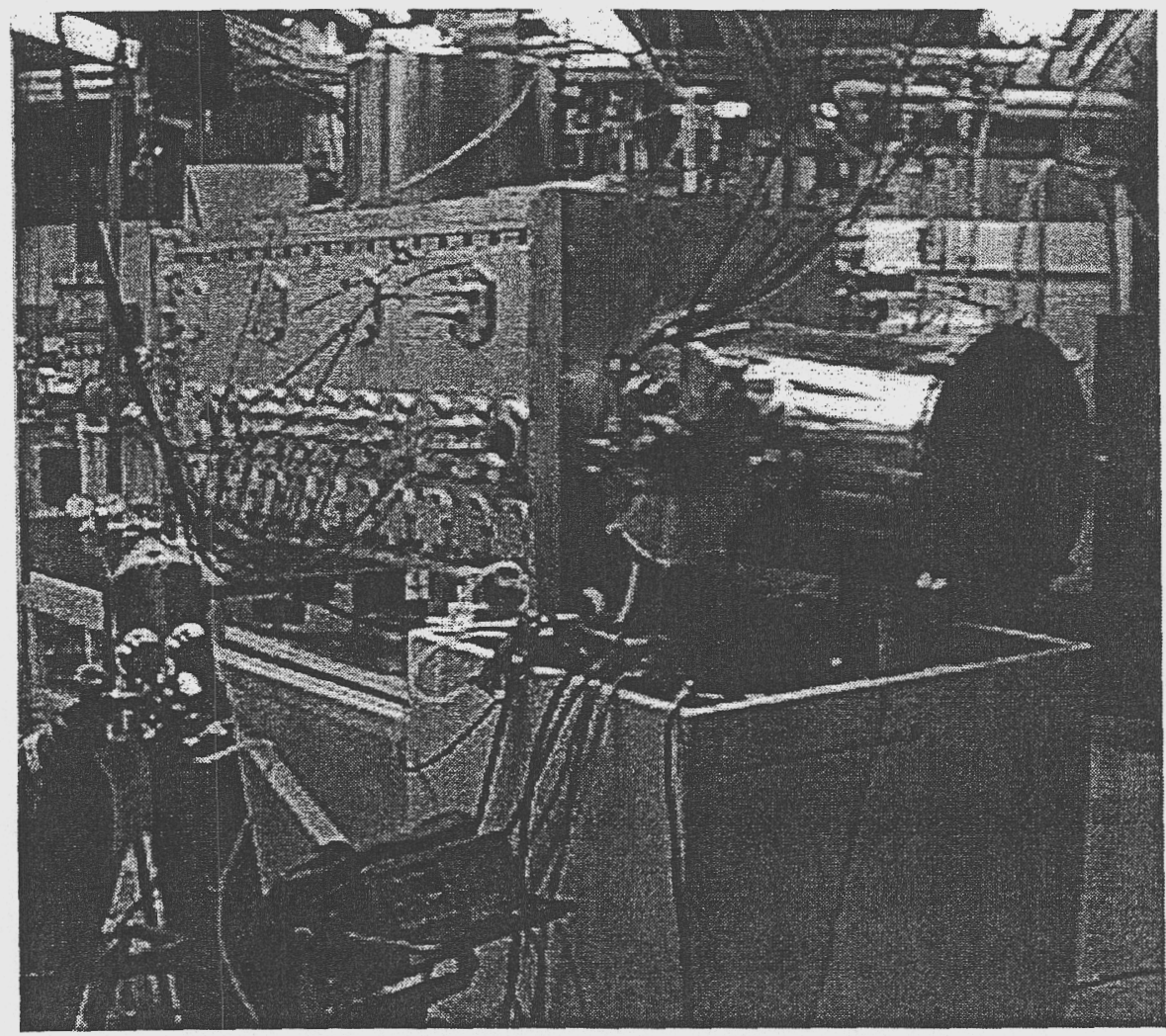

Figure 2.7: Electrostatic matching section. Cables supplying voltage to the electrostatic quadrupoles enter on the left side and the oil tank housing the ion source pulser circuit is shown below the ion source assembly tank to the right.

Faraday cup diagnostic instruments that measure total beam current as a function of time can be inserted into the matching section after electrostatic quadrupole numbers two and seven. Two sets of mechanical slit scanners, which can measure beam emittance in the $y$ (vertical) direction only, are located between quadrupoles two and three and between quadrupoles six and seven. A set of electrostatic steering quadrupoles for moving the beam a limited amount in the $\pm x, y$ direction are located after quadrupoles four and five. 
Table 2.1: Electrostatic quadrupole voltages used to "match" the Small Recirculator $80 \mathrm{keV}$ beam to the magnetic transport section lattice.

\begin{tabular}{cc}
\hline Quadrupole \# & Match Voltage (V) \\
1 & \pm 1975 \\
2 & \pm 3836 \\
3 & \pm 3085 \\
4 & \pm 1955 \\
5 & \pm 1840 \\
6 & \pm 1655 \\
7 & \pm 1665 \\
\hline
\end{tabular}

\subsubsection{Straight Magnetic Transport Section}

Following the matching section, during the straight magnetic transport experiments, were three magnetic transport sections, each $76.2 \mathrm{~cm}$ long, with two six-inch diagnostic or pumping ports and connections for capacitance probes (non-intercepting beam centroid diagnostics). ${ }^{18}$ The six-inch ports accepted a variety of diagnostic instruments including a LLNL-designed mechanical slit scanner, a rotating wire scanner for beam envelope measurements, and test assemblies used for GBI development. Small one-inch ports were used to bring out capacitance probe signals, and to accommodate GBI detector power supply leads. Each straight section consisted of two alternating gradient (or FODO, short for FOcusing/DefQcusing) half-lattice periods with two permanent magnetic quadrupole focusing elements with pole tip fields of 0.295 Tesla. Successive quadrupoles are aligned such that focusing in one transverse plane is followed by defocusing in the next quadrupole in the same plane. With a matched beam, the beam waist, (where both transverse radii of the beam are equal and the beam crossection is round), is at the center of the six-inch diagnostic ports. Figure 2.8 shows the three straight magnetic transport sections as configured during the experiments supporting this thesis. 


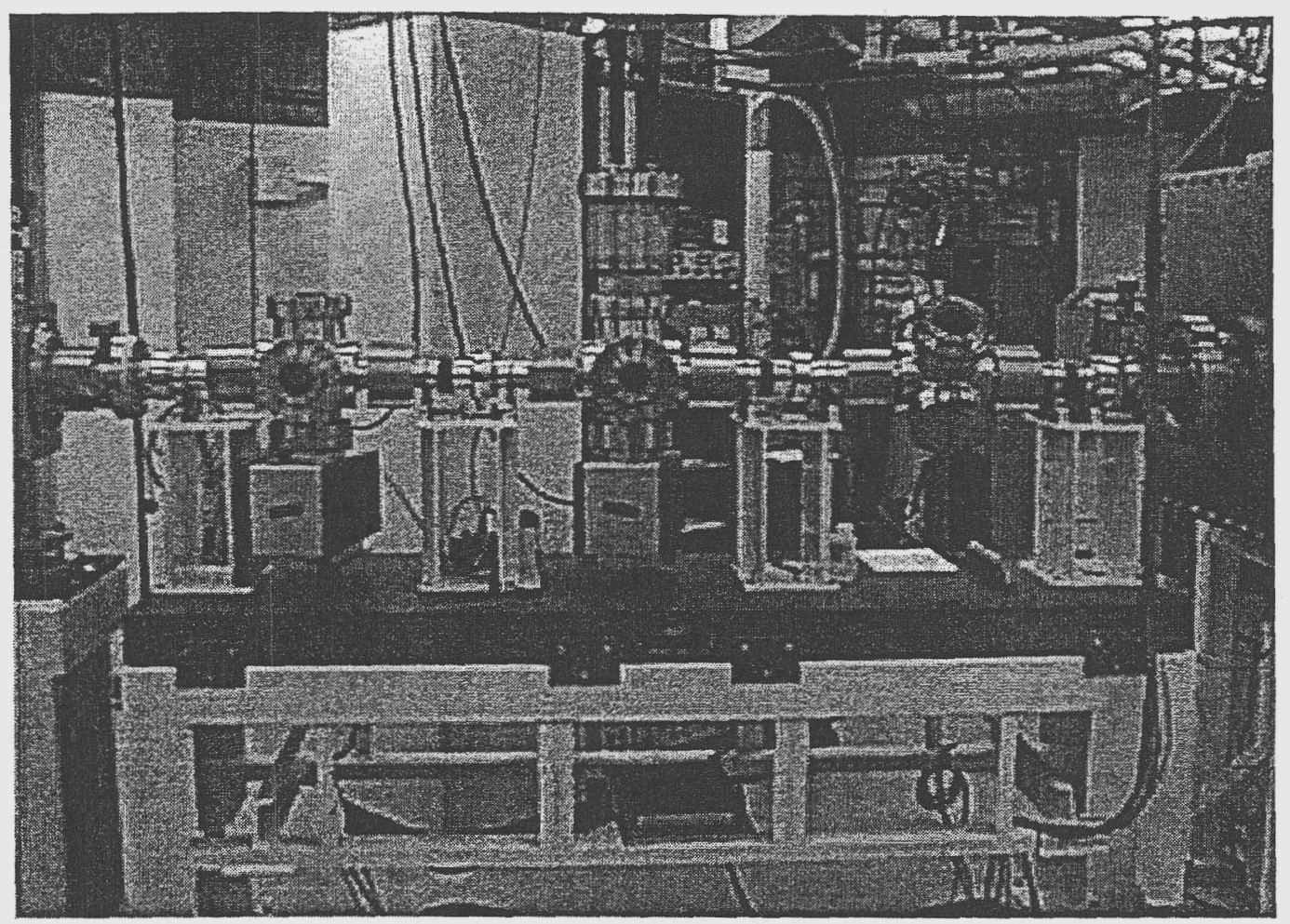

Figure 2.8: Small Recirculator straight magnetic transport section showing the 3 six-inch diagnostic ports.

\subsubsection{Electrostatic Bending Section}

Figure 2.9 shows the $45^{\circ}$ bend section that first accomplished the bending of an intense heavy ion beam with full beam transport. ${ }^{18}$ The FODO lattice of the straight transport continues in the bend with electrostatic dipole bending plates placed at the center point between successive magnetic quadrupoles. These plates are designed to bend the beam by $9^{\circ}$ in each half-lattice period requiring 5 dipoles to bend the beam by $45^{\circ}$. The nominal voltage to bend the beam $9^{\circ}$ at $80 \mathrm{keV}$ is $+6570 \mathrm{~V}$ placed on the outer plate and $-6570 \mathrm{~V}$ on the inner plate. The shape of the faces of the bending dipoles is slightly concave on the inner electrode and convex on the outer with "ridges" along the top and bottom edges. The shape, calculated using the WARP code was optimized to minimize the energy effect which shortens/lengthens the phase advance 
of particles not on the design orbit through the dipole and to minimize higher order field components. ${ }^{15}$

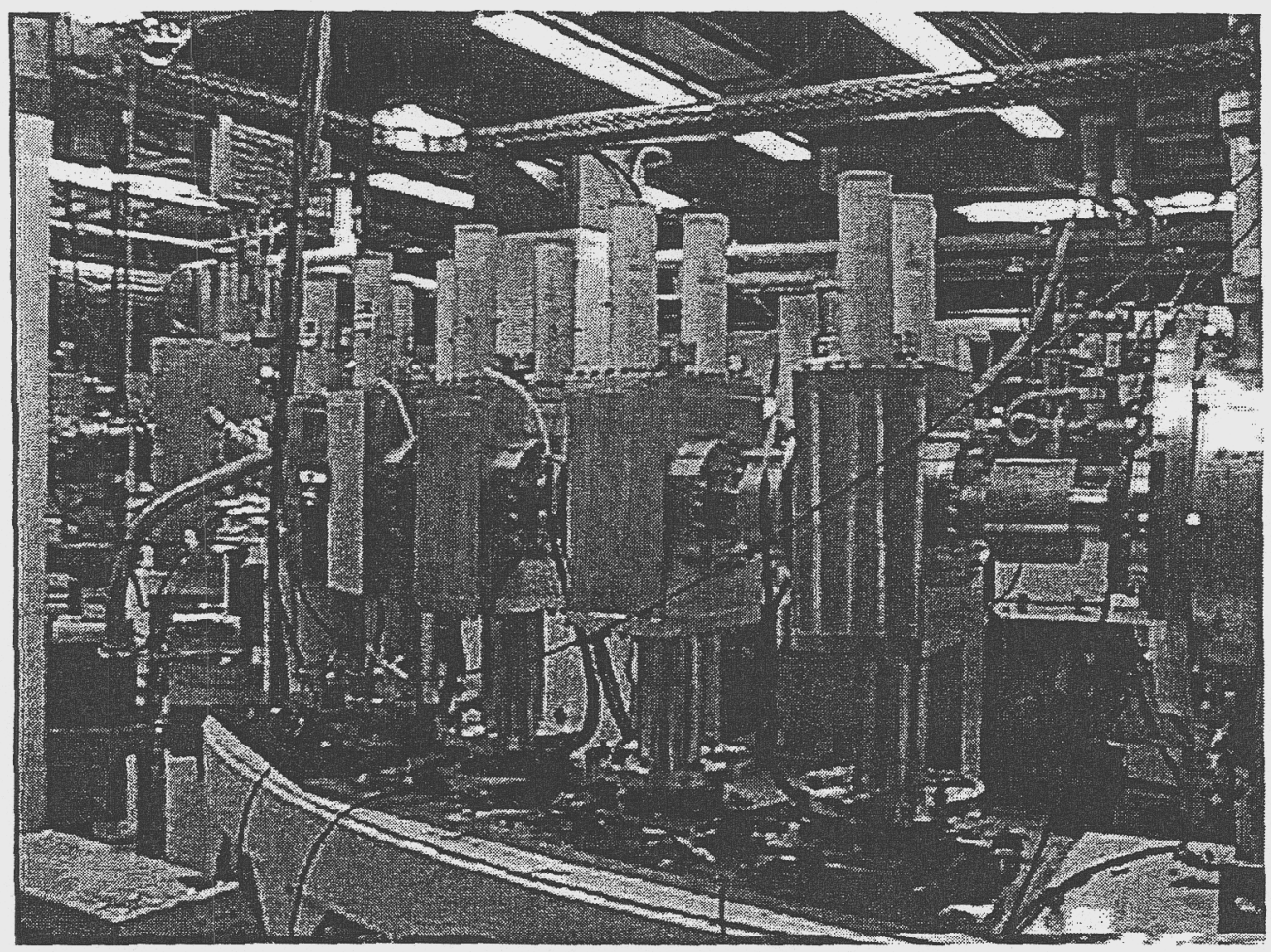

Figure 2.9: Small Recirculator $45^{\circ}$ bend section consisting of five half-lattice periods with electrostatic bending dipole plates.

\subsubsection{Multi-Instrument Diagnostic Tank}

No induction modulators were placed on the $45^{\circ}$ bend section at this point of the build and test schedule. They were being developed and bench tested during this time period. As such, no acceleration of the beam was accomplished during this phase of Small Recirculator operation and the beam drifted from extraction to the end of the beamline after the $45^{\circ}$ bend with no longitudinal confinement of beam ions. Measurement of the beam distribution's transverse moments was made at the beamline end in the multi-instrument diagnostic chamber I designed and built for acquiring 
data (Figure 2.10). The instruments contained in the chamber include a Faraday cup for measuring the total beam current, a mechanical slit scanner emittance diagnostic capable of 2-D phase space moment measurements, and the GBI diagnostic. This chamber will continue to be used at the end of the beamline as the Small Recirculator is built, eventually housing the final diagnostics following beam extraction after 15 lap operation.

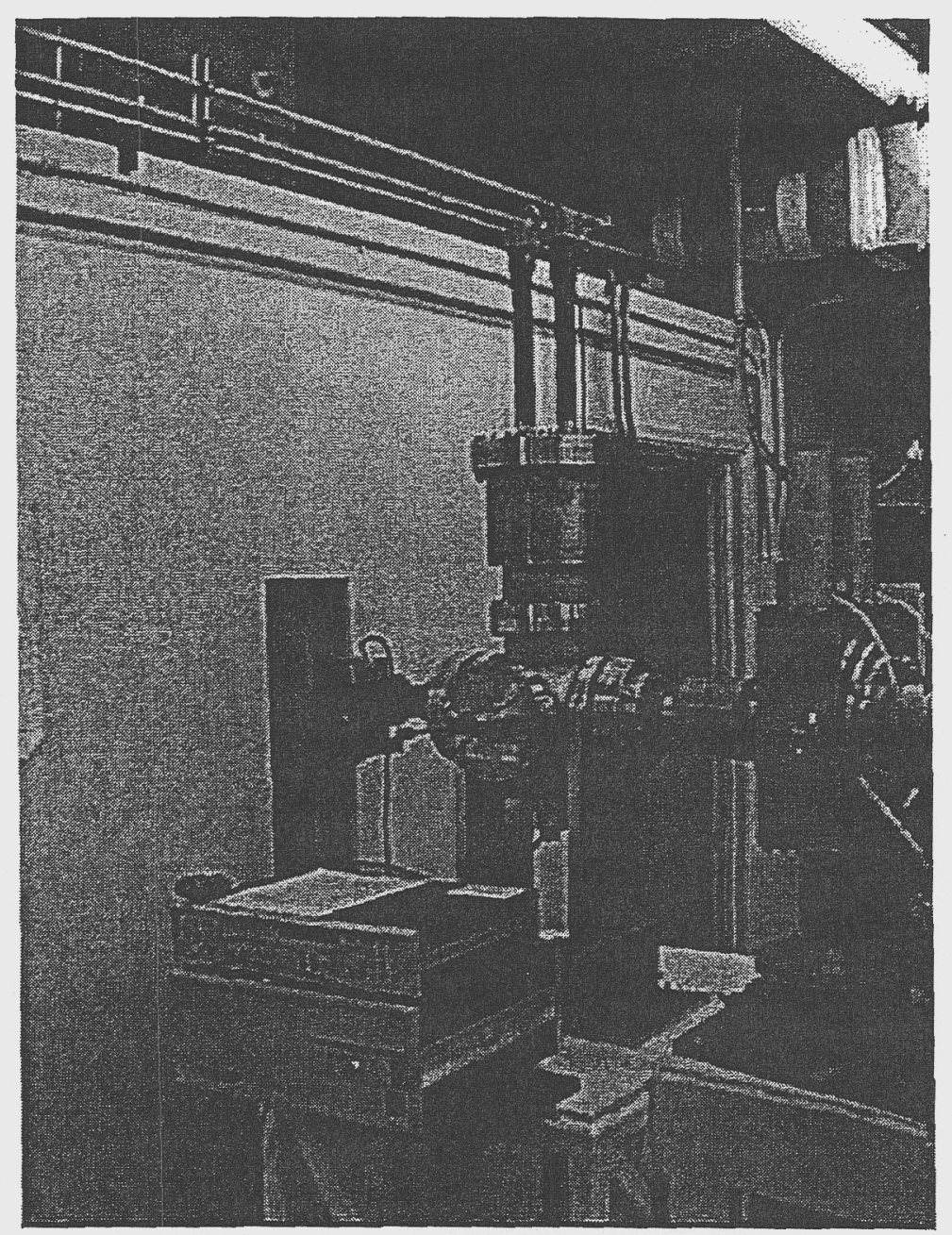

Figure 2.10: Multi-instrument diagnostic chamber at the beamline end showing the GBI CCD camera, the slit scanners mounted vertically for $y$-emittance measurements, and the Faraday Cup sliding manipulator on the horizontal port. The two boxes contain the controls for the GBI CCD camera. Slit scanner measurements of the $x$ emittance is accomplished by mounting the scanners horizontally on the chamber. 


\subsection{Beam Physics Scaling of the Small Recirculator}

That the Small Recirculator operate in the same beam physics regime as a fullscale driver is key to benchmarking the numerical simulation codes that will be used as aids to establish confidence in predicting the performance of a driver design. A LLNL design study conducted in 1991 proposed a "C-design" recirculator driver as an option for a full-scale IFE power plant. ${ }^{10}$ A schematic is shown in Figure 2.11. Appropriate scaling of key parameters from the Low Energy Ring of the C-design driver size machine to the Small Recirculator was done to ensure the data generated in the Small Recirculator experiment would apply to a scaled-up machine. ${ }^{14}$ A comparison of the small recirculator to the C-design Low Energy Ring is shown in Table 2.3 at the end of this chapter on page 23 .

\subsubsection{Physical Size Scaling}

Properly scaling the physical size of the Small Recirculator was the first consideration. The physical length of both the bending dipoles and focusing magnetic quadrupoles should be greater than 3 times the pipe radius or 1.5 times the aperture between the bending dipole plates. ${ }^{14}$ This condition ensures that the nonlinear fields associated with the finite length of the dipole and quadrupole are sufficiently small to minimize emittance growth. An additional length equal to the pipe radius is allotted on either end of each dipole and quadrupole yielding a ratio of $L / r_{p i p e}$ which is nearly identical to that of the C-design Low Energy Ring. A second scaling consideration is that the ratio of the beam pipe radius to the maximum beam radius be greater than 1.4. ${ }^{14}$ This rule of thumb gives adequate clearance to allow for beam motion within the beam pipe and ensures the image charge forces are unimportant. 


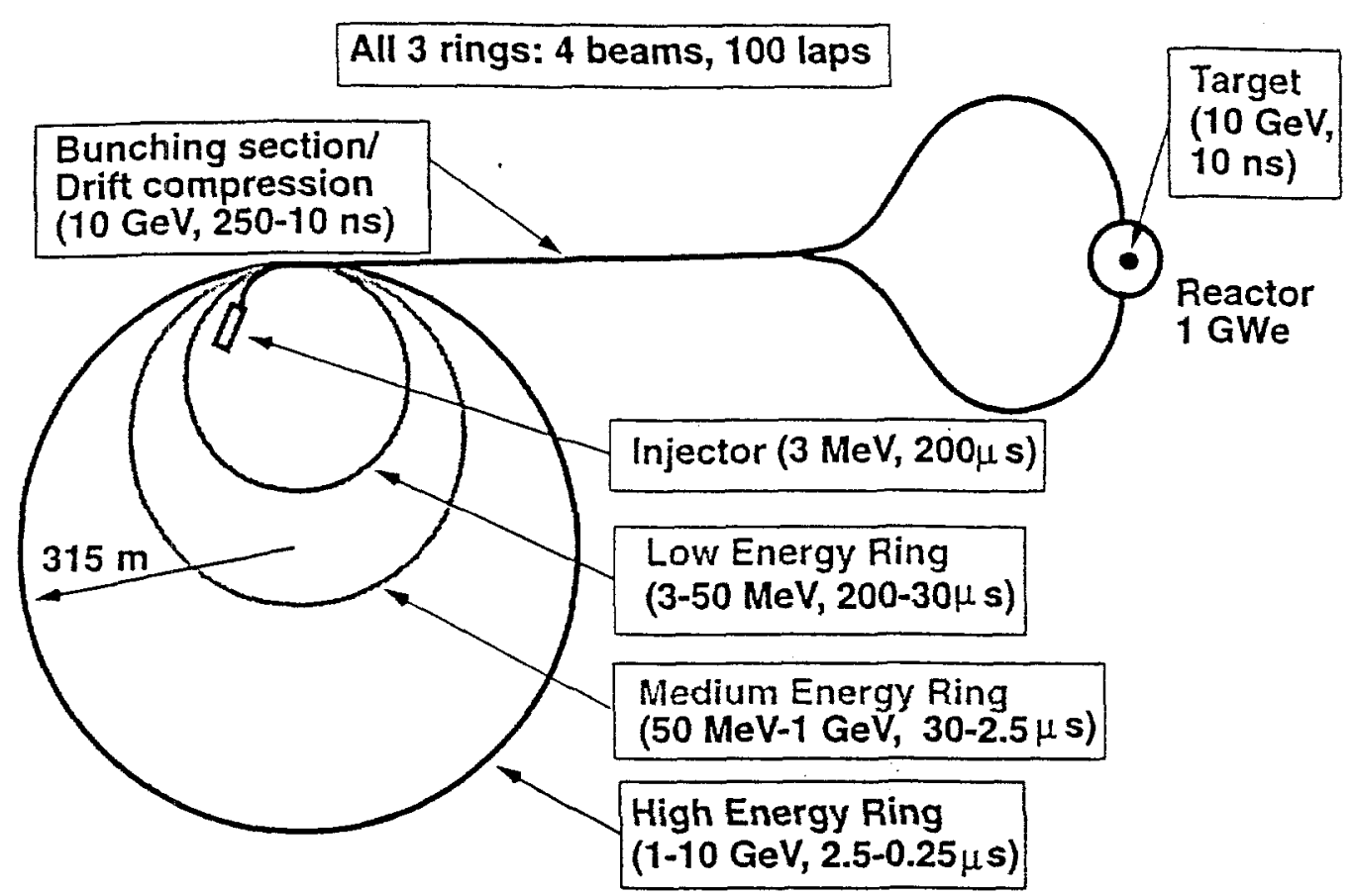

Figure 2.11: "C-design" heavy ion driver schematic

\subsubsection{Maintaining Key Dimensionless Beam Physics Pa- rameters}

Maintaining the values of three dimensionless parameters which characterize the focusing, space-charge, and inertial forces, which must balance to maintain beam equilibrium, ensures that the beam physics regime of the Small Recirculator is the same as that of a full scale driver. The first parameter, the phase advance per lattice 
period, $\sigma_{0}$ is a measure of the strength of the focusing forces relative to an ion's inertia. A resultant focusing of the beam occurs in an alternating gradient focusing system as follows. The beam is focused in the plane of the largest transverse beam envelope size and at the same time defocused in the orthogonal transverse plane of smallest envelope size. This is accomplished with quadrupole magnet fields aligned to the $x-y$ plane. Since the quadrupolar focusing force is linearly proportional to distance from the beam pipe axis, the result is net focusing through a series of magnets with focus/defocus axes rotated $90^{\circ}$ to one another. The ions at larger envelope dimension from the axis receive a greater inward focusing force than the outward defocusing force applied to the outer ions at smaller envelope dimension in the orthogonal plane. When the beam centroid is displaced from the axis it oscillates harmonically in the focusing field and the change in phase per lattice period is the phase advance, $\sigma_{0}$. Experimental studies on Lawrence Berkeley National Laboratory's Single Beam Transport Experiment (SBTE) showed that beam instabilities formed at $\sigma_{0}$ greater than $80^{\circ}$ which provides a constraint for both the driver and Small Recirculator. ${ }^{10}$

The beam perveance, $\kappa$ is the second dimensionless constant and is a measure of the space-charge forces relative to inertial forces. The expression for beam perveance is: $:^{11}$

$$
\kappa=\frac{q e I_{0}}{2 \pi \epsilon_{0} m_{0}(\beta \gamma c)^{3}}
$$

where $q$ is the charge state of beam ions, $e$ is the proton charge, $m_{0}$ is the beam ion mass in atomic mass units, $\gamma$ is the Lorentz factor, $\beta c$ is the ion velocity, $I_{0}$ is the beam current, and $\epsilon_{0}$ is he permittivity of free space. Scaling for the same perveance, $\left(\kappa=10^{-3}-10^{-4}\right)$ as that in the C-Design Low Energy Ring is accomplished in the Small Recirculator by lowering the current, ion mass, and ion velocity appropriately.

The third dimensionless parameter is the depressed phase advance, $\sigma$. In intense ion beams, the externally-applied focusing forces are countered by the outward force generated by space-charge and random ion thermal motion. The random ion thermal motion results in a thermal force and is characterized by the emittance. In space- 
charge-dominated ion beams of high line-charge density, the space-charge forces nearly equal the applied focusing forces. The emittance force makes up the difference. An off-axis beam particle performs quasi-harmonic oscillations in the combined external focusing and internal space-charge fields. The period of oscillation decreases as spacecharge forces approach the focusing forces. The ratio $\sigma / \sigma_{0}$ must be $\ll 1$ for a driver. ${ }^{14}$ The ratio for the Small Recirculator varies from 0.21 at $80 \mathrm{keV}$ to 0.27 at $320 \mathrm{keV}$.

Therefore, as the key dimensionless numbers are maintained in the scaling from a driver to the Small Recirculator, verification of the Small Recirculator's design performance will result in increased confidence in modeling a driver's performance. This along with the technology developed to successfully transport and control the beam through multiple laps will pave the way to scaling up the design to that required for a full-scale driver capable of economically delivering ion beams to a reactor chamber(s) many times a second. 
Table 2.2: Small Recirculator design parameters. ${ }^{13}$

\begin{tabular}{|c|c|c|}
\hline Ion mass (amu) & \multicolumn{2}{|l|}{39} \\
\hline Total flattop charge (C) & \multicolumn{2}{|l|}{$8 \times 10^{-9}$} \\
\hline Number of laps & \multicolumn{2}{|l|}{15} \\
\hline Physical quad occupancy fraction & \multicolumn{2}{|l|}{0.307} \\
\hline Physical dipole occupancy fraction & \multicolumn{2}{|l|}{0.291} \\
\hline Pipe radius/max beam radius $(x, y)$ & \multicolumn{2}{|l|}{$2.349,2.348$} \\
\hline Pipe radius $(\mathrm{cm})$ & \multicolumn{2}{|l|}{3.493} \\
\hline Circumference $(\mathrm{m})$ & \multicolumn{2}{|l|}{14.4} \\
\hline Half-lattice period $(\mathrm{cm})$ & \multicolumn{2}{|l|}{36} \\
\hline Half-lattice period/pipe radius & \multicolumn{2}{|l|}{10.308} \\
\hline Quad physical length/pipe radius & \multicolumn{2}{|l|}{3.164} \\
\hline Bend physical length/pipe radius & \multicolumn{2}{|l|}{2.996} \\
\hline Number of half-lattice periods & \multicolumn{2}{|l|}{40} \\
\hline \multirow[t]{2}{*}{ Residence time (ms) } & \multicolumn{2}{|l|}{0.229} \\
\hline & Initial & Final \\
\hline Beam energy (keV) & 80 & 320 \\
\hline Flattop pulse duration $(\mu \mathrm{s})$ & 4 & 1 \\
\hline Normalized emittance (mm-mr) & 0.1 & 0.2 \\
\hline Transverse temperature $(\mathrm{eV})$ & 0.739 & 1.478 \\
\hline Quadrupole field $(\mathrm{T})$ & 0.295 & 0.295 \\
\hline Dipole electrode voltage $( \pm \mathrm{kV})$ & 6.789 & 27.155 \\
\hline$\sigma_{0 x}\left({ }^{\circ}\right), \sigma_{0 y}\left({ }^{\circ}\right)$ & 78,78 & $44.554,44.554$ \\
\hline$\sigma_{0}$ (quadrupole only) $\left(^{\circ}\right)$ & 72 & 34.201 \\
\hline$\sigma_{x}, \sigma_{y}\left({ }^{\circ}\right)$ & $15.95,15.96$ & $11.55,11.55$ \\
\hline$\left(\sigma_{x} / \sigma_{0 x}\right),\left(\sigma_{y} / \sigma_{0 y}\right)$ & $0.205,0.205$ & $0.259,0.259$ \\
\hline Beam velocity/c $(\beta)$ & $2.1 \times 10^{-3}$ & $4.2 \times 10^{-3}$ \\
\hline Flattop bunch length $(\mathrm{m})$ & 2.517 & 1.258 \\
\hline Perveance & $3.57 \times 10^{-4}$ & $1.79 \times 10^{-4}$ \\
\hline Peak current $(\mathrm{mA})$ & 2 & 8 \\
\hline Line charge $(\mu \mathrm{C} / \mathrm{m})$ & $3.6 \times 10^{-3}$ & $7.21 \times 10^{-3}$ \\
\hline Modulator Repetition rate $(\mathrm{kHz})$ & 43.69 & 87.38 \\
\hline Beam radius in $x$ (mean,max) $(\mathrm{cm})$ & $1.11,1.422$ & $1.304,1.487$ \\
\hline Beam radius in $y($ mean, $\max )(\mathrm{cm})$ & $1.11,1.421$ & $1.305,1.488$ \\
\hline Number of betatron oscillations per lap in $x, y$ & $4.333,4.333$ & $2.475,2.475$ \\
\hline Ear rise or fall time/flattop time & 0.1 & 0.1 \\
\hline$\delta v / v$ from spatial compression & $1.89 \times 10^{-2}$ & $9.45 \times 10^{-3}$ \\
\hline$\delta v / v$ from ears & $6.73 \times 10^{-4}$ & $4.7 \times 10^{-4}$ \\
\hline Centroid offset from compression $(\mathrm{cm})$ & 0.231 & 0.354 \\
\hline Centroid offset from ears $(\mathrm{cm})$ & 0.008 & 0.018 \\
\hline
\end{tabular}


Table 2.3: Comparison between the Small Recirculator and C-design Low Energy Ring (LER) parameters. ${ }^{10}$

\begin{tabular}{|c|c|c|}
\hline & Small Recirculator & C-design LER \\
\hline \multicolumn{3}{|l|}{ General } \\
\hline Ion energy $(\mathrm{MeV})$ & $0.08-0.32$ & $3-50$ \\
\hline Pulse Duration $(\mu \mathrm{s})$ & $4-1$ & $200-30$ \\
\hline Circumference $(\mathrm{m})$ & 14 & 700 \\
\hline Current per beam (mA) & $2-8$ & $500-3300$ \\
\hline No. of Beams & 1 & 4 \\
\hline No. of laps & 15 & 100 \\
\hline Beampipe radius $(\mathrm{cm})$ & 3.5 & 7.8 \\
\hline Lattice half-period $(\mathrm{cm})$ & 36 & 85 \\
\hline Vacuum (torr) & $1 \times 10^{-8}$ & $5 \times 10^{-11}$ \\
\hline \multicolumn{3}{|l|}{ Induction modules } \\
\hline Inner radius $(\mathrm{m})$ & 0.163 & 0.313 \\
\hline Outer radius $(\mathrm{m})$ & 0.211 & 0.455 \\
\hline Length $(\mathrm{cm})$ & 8.5 & 40.3 \\
\hline No. of cores & 34 & 785 \\
\hline Cell voltage $(\mathrm{kV})$ & 0.50 & 0.60 \\
\hline \multicolumn{3}{|l|}{ Bends } \\
\hline Effective length (cm) & 13 & 15 \\
\hline No. of bends & 40 & 2680 \\
\hline Maximum field & $910 \mathrm{kv} \mathrm{m}^{-1}$ & $0.90 \mathrm{~T}$ \\
\hline \multicolumn{3}{|l|}{ Quadrupoles } \\
\hline Length (effective length) $(\mathrm{cm})$ & $10(10)$ & $47(23)$ \\
\hline No. of quads & 40 & 3139 \\
\hline Maximum quad field (T) & 0.3 & 2.0 \\
\hline \multicolumn{3}{|l|}{ Dimensionless parameters } \\
\hline Phase advance, $\sigma_{O}$ (degrees) & $78-44$ & $80-20$ \\
\hline$\sigma / \sigma_{0}$ & $0.20-0.27$ & $0.06-0.09$ \\
\hline Perveance, $\kappa$ & $(4.0-2.0) \times 10^{-4}$ & $(9.0-0.9) \times 10^{-4}$ \\
\hline Tune, $\nu$ & $4.3-2.5$ & $92-22$ \\
\hline
\end{tabular}




\section{Chapter 3}

\section{Heavy Ion Beam Transport}

The production, acceleration, and transport of high-quality intense heavy ion beams for use as drivers for inertial fusion energy is one of the key issues in determining the feasibility of the energy source. ${ }^{6}$ The specifications for the ion beams are determined by target physics considerations. Recent simulations using the code "LASNEX" have shown that to ignite one class of indirect-drive target tailored to heavy ions, the ions need to have an energy of about $4 \mathrm{GeV}$, a total beam energy of $5.8 \mathrm{MJ}$, and a spot size on target of about a few millimeters. ${ }^{8}$ The ions must be extracted from an ion source and manipulated within the driver in order to produce the desired beam spot size and energy deposition rate at the target. One key aspect of this process is the drift of the beam from the final focus lens across the target chamber to the target, a distance of about 4 meters. In a HYLIFE II-type IFE power plant the target chamber is necessarily large to provide a lower neutron fluence at the chamber first wall for materials damage limitations and to accommodate adequate liquid coolant/shielding within the reactor chamber. ${ }^{5}$ This large target chamber drift distance with no confining fields (except perhaps that of partial neutralization from the beam passing through chamber background gas) places a considerable constraint on the final focus lens. Among the detrimental processes during the chamber drift is the defocusing effect of the beam ions' random transverse thermal motion characterized by the beam transverse emittance. To account for this, a driver will have a transverse emittance specification at the final focus to ensure the required spot size 
on target is achieved. Measuring the emittance at the final focus and most likely at various locations along the beamline will be needed to ensure that this specification is met.

\subsection{Origin of Beam Emittance}

At the ion source, the extracted ions have an intrinsic thermal velocity spread which remains present in the beam from the source to target. This spread is present in both magnitude and direction of the extracted ions. During beam extraction, acceleration, and final focus, the velocity vector of each ion is changed under the influence of the applied electromagnetic fields of transverse focusing elements, the axial accelerating fields of induction modules, and by beam internal space-charge forces. In fact, the existence of a beam and not a bunch of randomly moving ions is owed to the induced directed motion of the ions along the accelerator swamping this random component. Despite focusing and acceleration of a beam, the intrinsic random ion velocity component remains and cannot be removed (except by cooling, generally deemed impractical in a driver). This is a consequence of Liouville's Theorem stating the volume of a particle ensemble in phase space cannot be reduced. The random component of an ion's velocity vector in a laboratory beam will vary considerably from the ideal thermal limit due to nonlinear focusing forces (unavoidable with practical focusing fields), and instabilities in the beam particle distribution (available free energy converted to random ion motion). Sophisticated modeling of the ion beam using the WARP code has shown that transitions between straight and curved sections of an accelerator are a major cause of emittance growth. ${ }^{50}$ Controlling this growth is dependent on the accelerator design and performance, and is a major goal of the Small Recirculator project. ${ }^{20}$

In a conceptual six-dimensional Euclidean phase space, combining configuration space $(x, y, z)$ and canonical momentum space $\left(p_{x}, p_{y}, p_{z}\right)$, a beam ion is represented by a point and all the points occupy a volume. This 6-D phase space can be reduced

to a 4-D transverse phase space of $\left(x, x^{\prime}, y, y^{\prime}\right)$ whose volume is conserved with the 
assumption of no coupling between the transverse $(x, y)$ and the axial $(z)$ motions of the particles as the beam travels through the accelerator. The canonical momenta are replaced by their corresponding transverse angles, $x^{\prime}=d x / d z$, and, $y^{\prime}=d y / d z$. Also, assuming a system of non-interacting particles, the movement of the beam ions is associated with the equivalent motion of points in phase space. Assuming no ions are lost from the beam, the number of points in phase space and the occupied volume is constant. The projections of the 4-D volume onto the two 2-D, $x$ and $y$ phase spaces results in two areas that are conserved if there is no coupling between the $x$ and $y$ motions of the ions. Figure 3.1 is a $2-\mathrm{D}$ visualization of the 4-D phase space and the two 2-D projections. The assumption of no coupling between the orthogonal motions of the ions in a laboratory beam is most likely a weak one. Experimental detection of the loss of directed energy from the $z$ direction to the $x-y$ direction by making measurements on the $z$ direction energy is unlikely. More feasible is the measurement of the longitudinal directed energy coupling by measuring the amount gained in the transverse directions. This coupling can be observed by detecting an increase in the emittance in the transverse directions. Direct detection of coupling between the transverse directions is more likely as the magnitude of any energy transfer would be a much greater relative percentage of the total transverse energy.

\subsection{Small Recirculator Beam Transport}

Nominally, the Small Recirculator beam consists of a distribution of $5 \times 10^{10}$ ions which are initially extracted from the ion source with an energy of $80 \mathrm{keV}$. Unless accelerated by induction modules this beam "drifts" through the lattice of focusing and bending elements of the machine. The Small Recirculator lattice is a periodic focusing system with two planes of symmetry $(x-z$ and $y-z)$. In the laboratory, $x$ is horizontal, $y$ is vertical, and $z$ is along the beamline which curves to the left around the bend. The paraxial motion of beam particles in the lattice (neglecting bending 


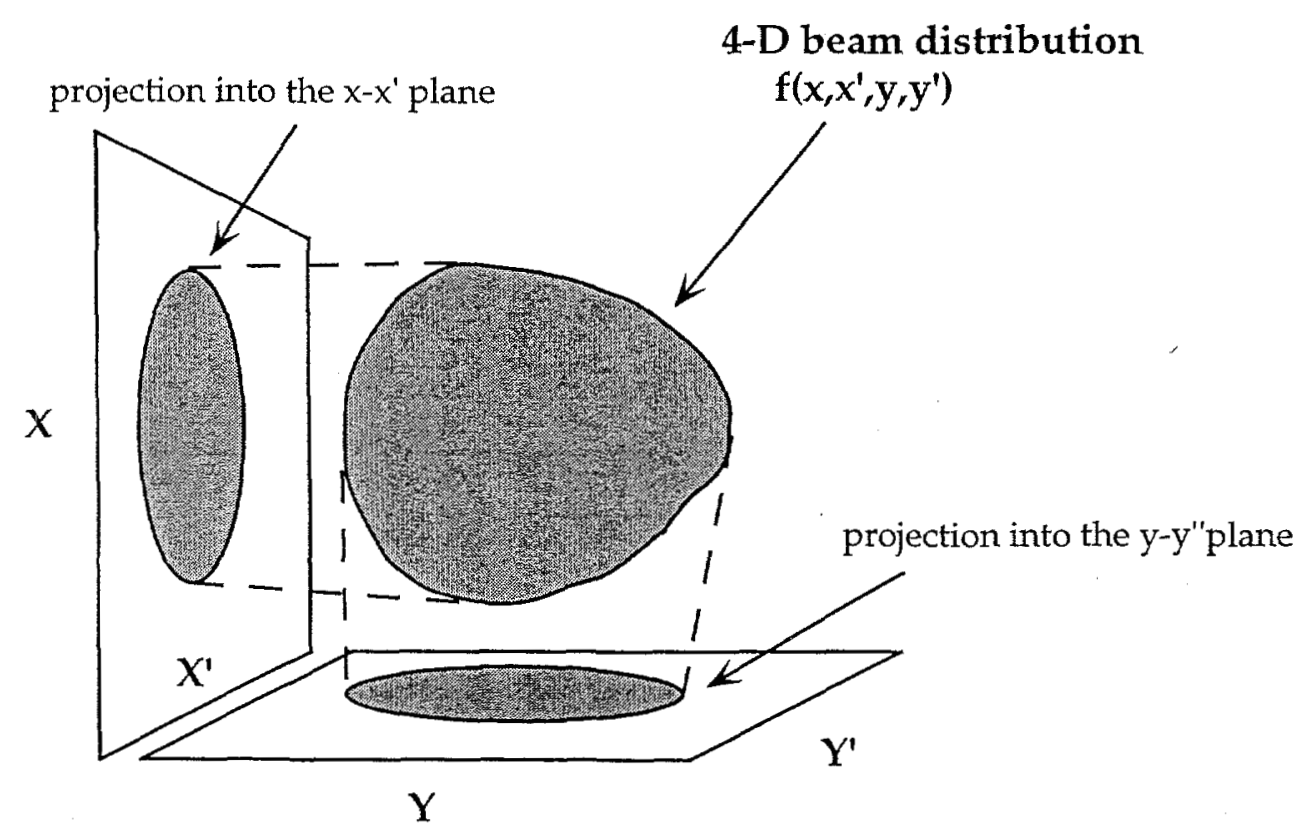

Figure 3.1: Representation of the beam's 4-D transverse phase space volume and the 2-D projections into the $x$ and $y$ planes.

and space-charge forces for now) is described by two equations:

$$
x^{\prime \prime}(z)+k_{x}(z) x=0 \quad y^{\prime \prime}(z)+k_{y}(z) y=0
$$

where $k_{x}(z)$ and $k_{y}(z)$ are the periodic focusing functions and satisfy the periodicity relation $k(z+L)=k(z)$ where $L$ is the length of one lattice period, $76.2 \mathrm{~cm}$.

Similarly, two sets of equations are necessary to describe the ion trajectories in the two planes of symmetry with space-charge taken into account now. These two sets of equations are coupled through the beam space-charge terms. Letting $X(z)$ denote the $x$-envelope and $Y(z)$ denote the $y$-envelope, the ellipse describing the transverse beam boundary obeys the equation

$$
\frac{x^{2}}{X^{2}}+\frac{y^{2}}{Y^{2}}=1
$$


and the charge density is defined by

$$
\rho(z)=\left\{\rho_{0} \text { for } \frac{x^{2}}{X^{2}}+\frac{y^{2}}{Y^{2}} \leq 1, \quad 0 \text { for } \quad \frac{x^{2}}{X^{2}}+\frac{y^{2}}{Y^{2}}>1\right.
$$

where

$$
\rho_{0}=\rho_{0}(z)=\frac{I}{\pi v X(z) Y(z)}
$$

is constant inside the beam at a given position $\mathrm{z}$, ( $\mathrm{x}, \mathrm{y}$ varying) but is a function of $\mathrm{z}$. The electric field for this charge distribution calculated from Poisson's Equation is:

$$
E_{x}=\frac{I}{\pi \epsilon_{0} v} \frac{x}{X(X+Y)} \quad E_{y}=\frac{I}{\pi \epsilon_{0} v} \frac{y}{Y(X+Y)}
$$

The ion trajectory equations including space-charge are:

$$
\begin{gathered}
x^{\prime \prime}(z)+k_{x}(z) x-\frac{2 \kappa}{X(X+Y)} x=0 \\
y^{\prime \prime}(z)+k_{y}(z) y-\frac{2 \kappa}{Y(X+Y)} y=0
\end{gathered}
$$

where $\kappa$ is the beam perveance defined in Equation 2.1. These equations are linear in $x$ and $y$, but are coupled through the space-charge terms which can be determined from the corresponding beam envelope equations. ${ }^{21}$ The trajectory equation solutions can be represented in phase-amplitude form:

$$
x(z)=A_{x} \omega_{x}(z) \cos \left[\psi_{x}(z)+\phi_{x}\right] \quad y(z)=A_{y} \omega_{y}(z) \cos \left[\psi_{y}(z)+\phi_{y}\right]
$$

where $A_{x}, A_{y}, \phi_{x}$, and $\phi_{y}$ depend on the initial conditions $\left(x_{0}, x_{0}^{\prime}\right)$ and $\left(y_{0}, y_{0}^{\prime}\right)$ and remain constant throughout the motion. The terms, $\omega_{x}(z)$, and $\omega_{y}(z)$ give the amplitude dependence on $z$ as the beam is transported through the alternate gradient focusing lattice. Differentiating $x(z)$ and $y(z)$ with respect to $z$ gives

$$
\begin{aligned}
& x^{\prime}=A_{x}\left[\omega_{x}^{\prime} \cos \left(\psi_{x}+\phi_{x}\right)-\omega_{x} \psi_{x}^{\prime} \sin \left(\psi_{x}+\phi_{x}\right)\right] \\
& y^{\prime}=A_{y}\left[\omega_{y}^{\prime} \cos \left(\psi_{y}+\phi_{y}\right)-\omega_{y} \psi_{y}^{\prime} \sin \left(\psi_{y}+\phi_{y}\right)\right]
\end{aligned}
$$


Eliminating $\psi$ and $\phi$ using the identity $\cos ^{2}(\psi+\phi)+\sin ^{2}(\psi+\phi)=1$ yields the equation of the 2-D phase space trajectories of an ion. ${ }^{22}$

$$
\hat{\gamma} x^{2}+2 \hat{\alpha} x x^{\prime}+\hat{\beta} x^{\prime 2}=A_{x}^{2} \quad \hat{\gamma} y^{2}+2 \hat{\alpha} y y^{\prime}+\hat{\beta} y^{\prime 2}=A_{y}{ }^{2}
$$

These are equations of ellipses whose shape and orientation are determined by the amplitude factor, $A$, and coefficients $\hat{\gamma}, \hat{\alpha}$, and $\hat{\beta}$ which are determined by $\omega(z)$, and $\omega^{\prime}(z)$. All ions in the beam with the same amplitude factor but differing $\phi$ lie on the same ellipse. Ions with different amplitude factors lie on other ellipses scaled in size but similar in shape. The relationship between the coefficients and the ellipse dimensions are shown in Figure 3.2. For the $x-x^{\prime}$ plane, and similarly for the $y-y^{\prime}$ plane, the area of the largest ellipse is $A_{0}=\pi \epsilon_{x}$ where $\epsilon_{x}$ is the beam emittance in the $x$ direction. Therefore, the equation of the largest ellipse can be written as

$$
\hat{\gamma} x^{2}+2 \hat{\alpha} x x^{\prime}+\hat{\beta} x^{2}=\epsilon_{x}^{2}
$$

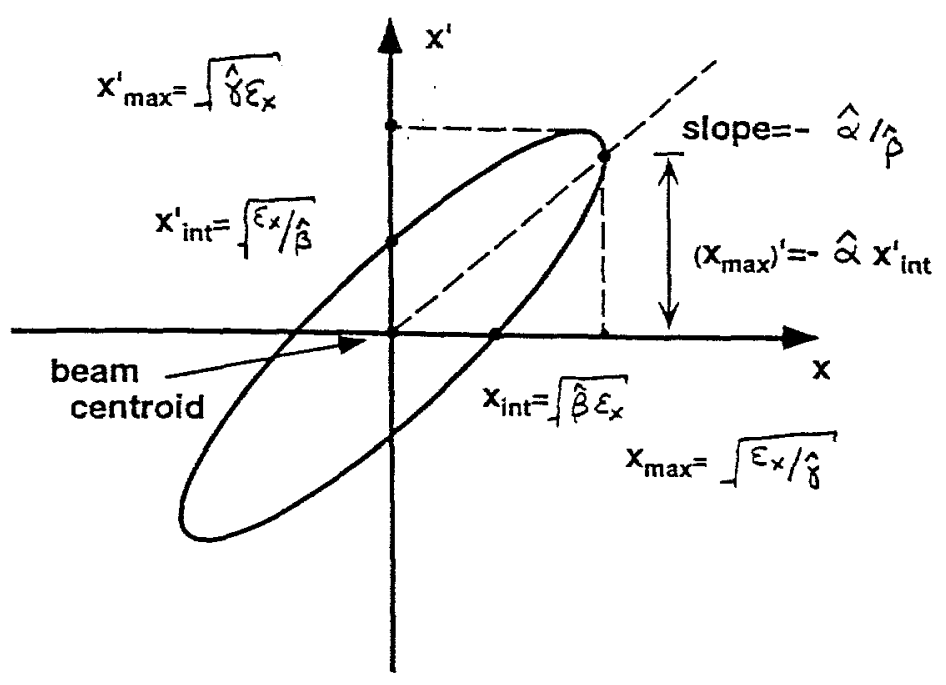

Figure 3.2: Relationship between phase space ellipse coefficients and the ellipse dimensions. 
As the beam drifts through the Small Recirculator lattice the emittance would be constant if the external focusing and bending fields and the space-charge fields were linear. ${ }^{23}$ With acceleration, the emittance decreases since $x^{\prime}=d x / d z$, but the normalized emittance remains constant as it includes factors which account for the increase in ion velocity. A discussion of the use of different emittance definitions follows later in this chapter. The only ion distribution that results in linear space-charge fields is the Kapchinsky-Vladimirski (K-V) distribution of the form $f\left(x, x^{\prime}, y, y^{\prime}\right)=F_{0} \delta\left(F-F_{0}\right){ }^{24}$ The point representation of this distribution is that all the points in a 4-D phase space representing ions lie on the surface of the 4-D hyperellipsoid of Figure 3.1. The projection of the hyperellipsoid onto the 2-D phase spaces results in ellipses with a uniform charge density.

A fundamental question of beam theory is to find equilibrium states where the ion distribution remains stationary (i.e., does not change with distance along the accelerator). Vlasov theory is a tool used in the absence of collisions to find equilibrium states. A stationary distribution represents a state of minimum total energy. A beam with higher total energy contains free energy which is available to be converted into random ion energy, thus increasing the emittance of the beam. This conversion is brought about by collisional processes, instabilities, and non-constant space-charge distributions and nonlinear focusing fields. ${ }^{25}$

Analytical techniques using the Vlasov equation to understand the stationary state or the evolution of a non-stationary state are useful only in axisymmetric cases and in the case of the $\mathrm{K}-\mathrm{V}$ distribution. ${ }^{26}$ Computational particle simulations must be used for other distributions such as the constant density with a semi-gaussian spread in ion velocities used to initialize WARP simulations. ${ }^{15}$ The initial magnitudes of the velocity spreads are, to date, only estimates in the code. A result of code simulations has been to show that the transverse emittance growth of a Small Recirculator beam as a result of the insertion/extraction into the ring is very sensitive to the magnitude of the axial velocity $\left(v_{z}\right)$ spread. Determining the proper spread by comparing experimental measurements to the code is of vital importance to code validation. 
The results of the analytical analysis of the K-V distribution can be used on unknown (laboratory) distributions, which are likely not K-V, using the concept of "equivalent beams". ${ }^{26,27}$ Treating the laboratory beam as an equivalent $\mathrm{K}-\mathrm{V}$ beam has yielded valuable tools for characterizing accelerator performance and as a tool for designing and predicting accelerator performance.

\subsection{Moment Description of the Ion Beam}

Sophisticated heavy ion beam simulation codes, such as LLNL's WARP, model the evolution of the beam distribution through a model recirculator using macroparticles, each representing a large number of ions. ${ }^{15}$ Choosing the appropriate parameters to use as code benchmarks involves surveying what can be measured in an experiment. The desirable (and reasonable) measurements include ion energy, beam envelope parameters, and transverse phase space first and second moments. These can be compared directly with code results or figures of merit such as the beam transverse emittance in the $x$ and $y$ directions. Relating the beam's representation in the conceptual 4-D phase space as a hyperellipsoid to its moments is seen with a matrix representation in Figure 3.1. ${ }^{28}$ In this context, $\sigma$ is not the beam phase advance, but the hyperellipsoid's sigma matrix. The difference between uses of the notation is apparent in context.

$$
\sigma=\left(\begin{array}{llll}
\sigma_{11} & \sigma_{12} & \sigma_{13} & \sigma_{14} \\
\sigma_{21} & \sigma_{22} & \sigma_{23} & \sigma_{24} \\
\sigma_{31} & \sigma_{32} & \sigma_{33} & \sigma_{34} \\
\sigma_{41} & \sigma_{42} & \sigma_{43} & \sigma_{44}
\end{array}\right)
$$

The equation of the hyperellipsoid is given by $\Gamma^{T} \sigma^{-1} \Gamma=1$ where the 4-component vector, $\Gamma=\left(x, x^{\prime}, y, y^{\prime}\right)$ and the dimensions of the hyperellipsoid are related to the 
matrix elements by:

$$
x_{\max }=\sqrt{\sigma_{11}} \quad x_{\max }^{\prime}=\sqrt{\sigma_{22}} \quad y_{\max }=\sqrt{\sigma_{33}} \quad y_{\max }^{\prime}=\sqrt{\sigma_{44}}
$$

Assuming a $\mathrm{K}-\mathrm{V}$ beam distribution, the relationship of the matrix elements to the second moments of the beam is:

$$
\begin{gathered}
\left.\sigma_{11}=4<x^{2}>\quad \sigma_{12}=4<x x^{\prime}\right\rangle \equiv 4<x^{\prime} x>=\sigma_{21} \quad \sigma_{22}=4<x^{\prime 2}> \\
\sigma_{13}=4<x y>\equiv 4<y x>=\sigma_{31} \quad \sigma_{23}=4<x^{\prime} y>\equiv 4<y x^{\prime}>=\sigma_{32} \\
\sigma_{14}=4<x y^{\prime}>\equiv 4<y^{\prime} x>=\sigma_{41} \quad \sigma_{24}=4<x^{\prime} y^{\prime}>\equiv 4<y^{\prime} x^{\prime}>=\sigma_{42} \\
\sigma_{33}=4<y^{2}>\quad \sigma_{34}=4<y y^{\prime}>\equiv 4<y^{\prime} y>=\sigma_{43} \quad \sigma_{44}=4<y^{\prime 2}>
\end{gathered}
$$

The $x$ and $y$ cross terms represent coupling between the motion of beam ions in the $x$ and $y$ planes. These elements are zero if no coupling is present or non-zero with coupling caused by, for example, a rotationally misaligned quadrupole focusing magnet. The second moments of the distribution are defined in the conventional manner, e.g.,

$$
<x^{2}>=\iiint \int x^{2} \rho\left(x, x^{\prime}, y, y^{\prime}\right) d x d x^{\prime} d y d y^{\prime}
$$

with $\rho\left(x, x^{\prime}, y, y^{\prime}\right) d x d x^{\prime} d y d y^{\prime}$ representing the beam ion distribution and having the normalization

$$
\iiint \int \rho\left(x, x^{\prime}, y, y^{\prime}\right) d x d x^{\prime} d y d y^{\prime}=1
$$

Without coupling between the ions' $x$ and $y$ motions, the projections of the hyperellipsoid into the $x-x^{\prime}$ and $y-y^{\prime}$ planes in Figure 3.1 are ellipses which contain the $x$ and $y$ motions of the beam particles. The applicability of Liouville's Theorem to the 4-D and to these 2-D phase spaces is predicated on no coupling between the orthogonal directions of motion. The separable nature of the $x$ and $y$ phase space ellipses is seen in the sigma matrix with no coupling, Equation 3.21. 


$$
\sigma=\left(\begin{array}{cccc}
\sigma_{11} & \sigma_{12} & 0 & 0 \\
\sigma_{21} & \sigma_{22} & 0 & 0 \\
0 & 0 & \sigma_{33} & \sigma_{34} \\
0 & 0 & \sigma_{43} & \sigma_{44}
\end{array}\right)
$$

Figure 3.3 shows the relationships between the sigma matrix elements and the 2-D phase space ellipse dimensions.

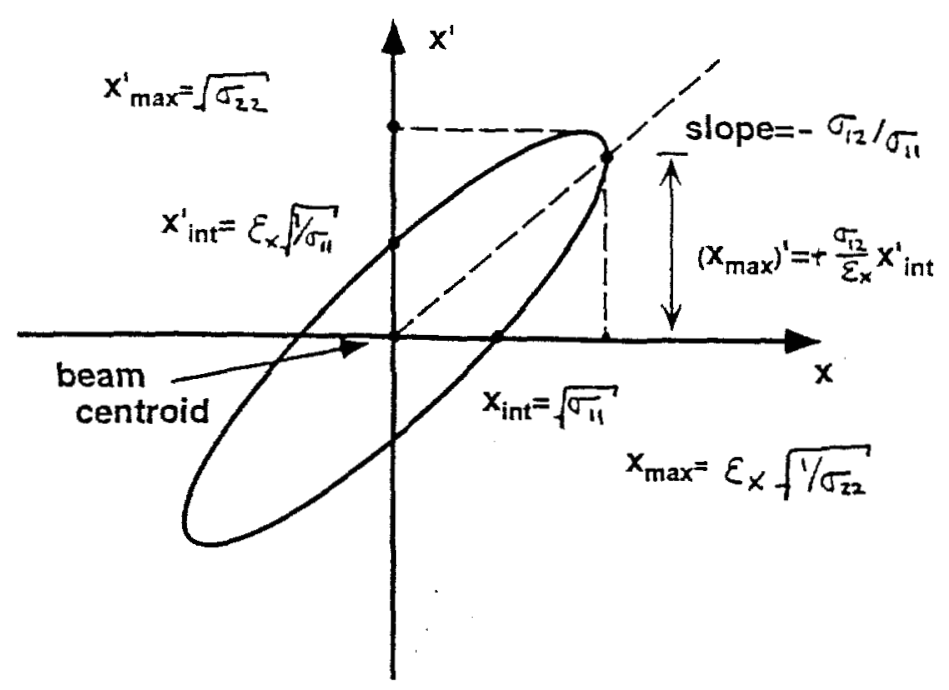

Figure 3.3: Relationship between the sigma matrix elements and the 2-D phase space ellipse dimensions.

A beam's phase space distribution can be described by its first and second moments. The actual beam distribution in a laboratory beam is not known. The usefulness of gross measurements such as the beam first and second moments is in the concept of equivalent beams. Two beams can be considered equivalent if they are composed of the same particle species, current, kinetic energy, and second moments. Detailed differences in their distributions are unimportant as long as the above constraints are met. This is fortunate as it allows comparison of a laboratory beam 
of unknown distribution with that of a known and probably different distribution used in a numerical simulation. The initial distribution for the WARP code that is most commonly used is one that is uniform in $x, y, z$, with a semi-gaussian spread in $v_{x}, v_{y}$, and $v_{z} \cdot{ }^{15}$ MOMENT, a simpler beam moment evolution code, uses a $\mathrm{K}-\mathrm{V}$ distribution. ${ }^{29}$ Therefore, a simulated beam and a laboratory beam with the same second moments and other quantities at the same time and location in the accelerator can be considered equivalent, and is one basis for comparing experimental data with numerical simulation results.

\subsection{Definition of Emittance}

The figure of merit most commonly used to describe beam quality is the transverse emittance of the beam. Beam "brightness" is another quantity used as a figure of merit when there is concern about the amount of beam current as well as the emittance. In the Small Recirculator, with full beam transport verified with Faraday cups, the emittance is the relevant quantity. It is closely related to the area of the two-dimensional projections of the 4-D phase space hyperellipsoid. Lapostalle and Sacherer introduced rms quantities in order to compare beams with differing distributions. ${ }^{26,27}$ The rms beam width is given by

$$
\tilde{x}=<x^{2}>^{\frac{1}{2}}
$$

The rms values of other quantities $\left(\tilde{x}^{\prime}, \tilde{y}, \tilde{y}^{\prime}\right)$ are similarly calculated. Statistically, the definition of the "rms transverse emittance" in the $x$ direction is

$$
\left.\tilde{\epsilon}_{x}=\left[<x^{2}><x^{\prime 2}>-<x x^{\prime}\right\rangle^{2}\right]^{\frac{1}{2}}
$$

where the term $\left\langle x x^{\prime}\right\rangle$ represents an $x-x^{\prime}$ correlated particle flow term that occurs when the beam is converging or diverging under the influence of external focusing forces. ${ }^{26,27}$ It represents an inward or outward flow term in the transverse kinetic energy. The difference between the total transverse kinetic energy, $\left\langle x^{2}\right\rangle\left\langle x^{\prime 2}\right\rangle$, and 
the flow energy represented by $\left\langle x x^{\prime}\right\rangle$ is the small random transverse kinetic energy from which the emittance rises. Correspondingly for the $y$ direction:

$$
\tilde{\epsilon}_{y}=\left[\left\langle y^{2}\right\rangle\left\langle y^{\prime 2}\right\rangle-\left\langle y y^{\prime}\right\rangle^{2}\right]^{\frac{1}{2}}
$$

Another common definition I will adopt for clarity is the "rms edge emittance" which is four times the rms emittance ${ }^{26}$ :

$$
\left.\epsilon_{x}=4 \tilde{\epsilon}_{x}=4\left[<x^{2}><x^{2}>-<x x^{\prime}\right\rangle^{2}\right]^{\frac{1}{2}}
$$

In terms of the hyperellipsoid sigma matrix:

$$
\epsilon_{x}=\left[\sigma_{11} \sigma_{22}-\sigma_{12}^{2}\right]^{\frac{1}{2}}
$$

As $x^{\prime}$ is defined as $d x / d z$, the instantaneous transverse angle with respect to motion along the accelerator, it is inversely proportional to the beam energy. A correction factor of $\beta \gamma$, the usual relativistic quantities, is applied to the emittance to give the normalized rms edge emittance which is constant with beam acceleration: ${ }^{30}$

$$
\epsilon_{n}=\beta \gamma \epsilon_{x}
$$

This is a figure of merit that can be easily used to compare the beam emittance if measurements are made at two points in an accelerator with acceleration in between.

There are ten independent transverse second moments. The existence of non-zero cross moments can be indicative of rotational misalignments of accelerator quadrupolar focusing components causing coupling between the $x$ and $y$ ion motions. The effects of misalignments are more fully discussed in Chapter 6 . These $x-y$ cross moments cannot be measured with a slit scanner diagnostic as this instrument averages beam information along the length of its slits. A useful quantity that becomes non-zero with $x-y$ coupling is indicative of the angular momentum developed by the 
beam about the $z$-axis. This quantity is defined by:

$$
l=\beta\left(<x^{\prime} y>-<x y^{\prime}>\right)
$$

Detection of beam rotation can indicate alignment problems in the accelerator which will have the ultimate effect of increasing the final spot size on target over that of a similar non-rotating beam.

The GBI measures these $x-y$ cross moments and extends the characterization of the Small Recirculator beam and understanding of possible causes of unpredicted beam behavior beyond that of previous instruments. 


\section{Chapter 4}

\section{Pepperpot Method and Image Data Analysis}

The motivation to design a new diagnostic instrument for measuring the characteristics of a heavy ion beam was fostered by the limitations of previous instruments. The new instrument developed in support of this thesis is the Gated Beam Imager (GBI) and is based on the pepperpot beam diagnostic method which has previously been used to diagnose electron beams. ${ }^{28}$ The diagnostics in predominant use to determine a heavy ion beam distribution's first and some of the second moments in transverse phase space have been capacitance probes and mechanical slit scanners.

\subsection{Capabilities of Existing Emittance Diagnostics}

Mechanical slit scanners, in which two thin slits are repositioned across the beam between successive shots are able to measure the second moments $\left(\left\langle x^{2}\right\rangle,\left\langle x x^{\prime}\right\rangle,\left\langle x^{\prime 2}\right\rangle\right.$ or, $\left.\left\langle y^{2}\right\rangle,\left\langle y y^{\prime}\right\rangle,\left\langle y^{\prime 2}\right\rangle\right)$ in one transverse plane at a time while averaging the beam information in the orthogonal plane. ${ }^{31}$ Thus, only the emittance in one plane at this particular place in the beam line can be obtained without disassembling the accelerator or having a second scanner at the same location, or some other extravagant solution. As a slit averages the beam in the direction of its length, a slit scanner cannot measure any of the cross moments, $\left(\langle x y\rangle,\left\langle x^{\prime} y\right\rangle,\left\langle x y^{\prime}\right\rangle,\left\langle x^{\prime} y^{\prime}\right\rangle\right)$ which are important to detecting a decrease in the accelerator performance due to a focusing field degradation, or the presence of a component physical misalignment such as 
a focusing quadrupole rotation error about the beam axis. These types of problems induce coupling between the $x$ and $y$ motions of the ions in an alternating gradient accelerator lattice causing an "effective" emittance growth in the beam. I call this "effective" emittance growth as the growth is genuine in its effect on the focusability of the ion beam. Because it is caused by linear forces coupled in the $x$ and $y$ directions, it can, in principle, be removed by the appropriate application of counteracting linear forces. Normally, emittance growth is associated with nonlinear forces acting on the beam distribution which entrains unoccupied phase space into the distribution making it for practical purposes impossible to sort out the actual distribution through diagnostic measurements. ${ }^{25}$ Figure 4.1 shows the entrainment of unoccupied phase space within the distribution as a result of nonlinear forces. The dotted line envelope shows the resolvable phase space ellipse.

The GBI is able to measure all the first and second phase space moments of the ion beam simultaneously, including the $x-y$ cross moments. With appropriate time gating it can produce the information for a 4-D and time history "map" of the beam using successive beam pulses. When installed after a bending dipole such as after the $45^{\circ}$ bend section of the Small Recirculator it was found useful as a qualitative energy analyzer to observe energy changes longitudinally along the beam pulse.

\subsection{The Pepperpot Method}

In the pepperpot method where a mask with small holes (the pepperpot) is introduced into the beam, the beam ions are stopped by the hole plate except where they pass through the holes forming small beamlets. These beamlets pass through a drift region where they freely expand from space-charge and emittance forces. The beamlets are intercepted by a detector which is excited and the spots are observed with a CCD camera, and digitized for analysis. Figure 4.2 is a schematic of the method.

Without the hole plate, the gross behavior of the beam in the drift space is similar 


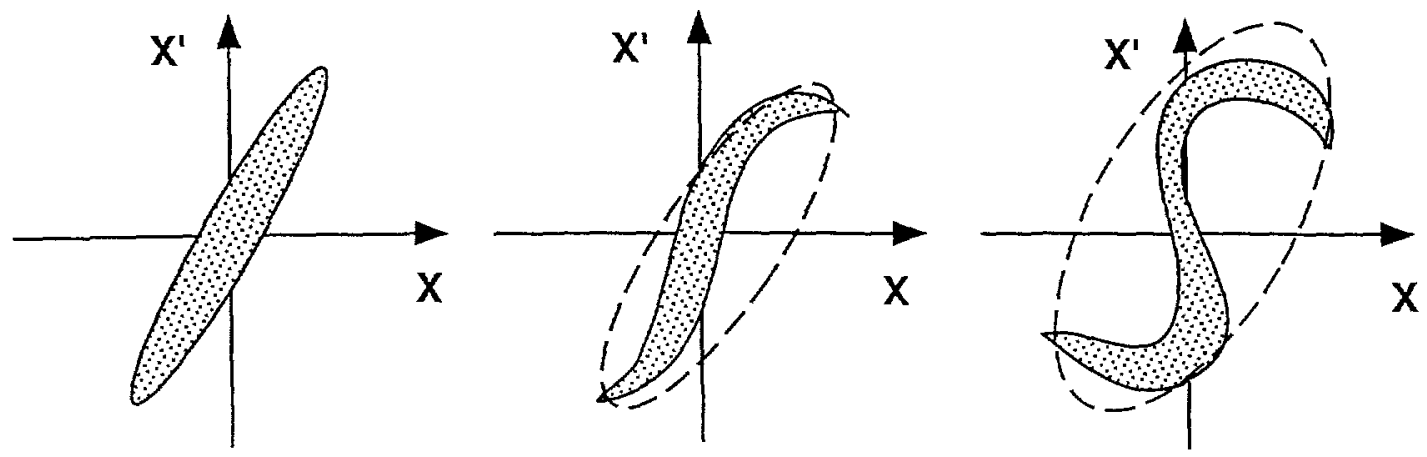

Figure 4.1: Entrainment of unoccupied space into the phase space area of the beam due to the action of nonlinear forces as the beam progresses through the accelerator. The dotted ellipses are fitted to shaded areas to illustrate how the resolvable emittance grows although the shaded areas are constant in area.

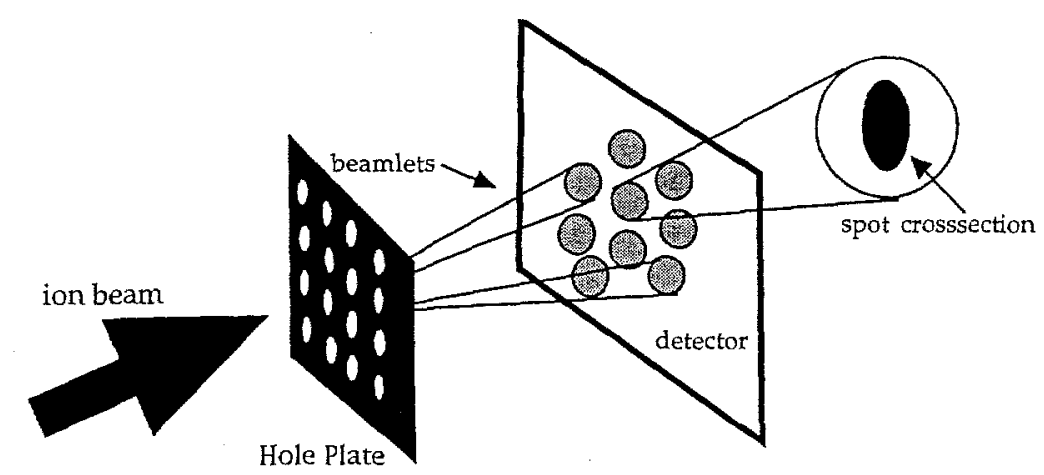

Figure 4.2: Schematic of the pepperpot method

to the beamlet but the small increase in beam dimension due to emittance forces is lost in the greater expansion due to space-charge. The small holes allow the emittancecaused transverse growth to dominate over space-charge expansion and thus be more easily measured as well as creating a known initial circular cross-section to compare to the beamlet cross-section at the detector. Creating the beamlets also allows information from the central regions of the beam to be used in determining its properties. Figure 4.3 is a simplified schematic of the Gated Beam Imager. 


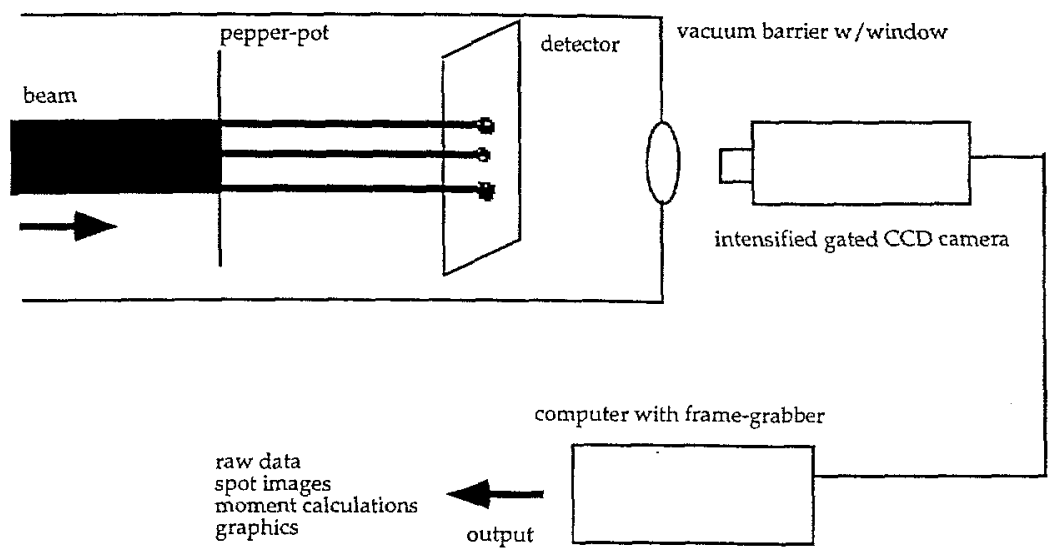

Figure 4.3: Simplified GBI instrument schematic

\subsection{Design of the Gated Beam Imager}

Several dimensions of the GBI such as pepperpot hole size, hole spacing, and beamlet drift length must be sized to achieve the goal of maximizing the beamlet's growth due to emittance while minimizing the growth from remaining space-charge forces. Additionally, adequate signal strength must be present at the CCD camera and the beamlet image spot size must be much greater than the camera system resolution so that statistical averages of the CCD pixels are valid. These requirements place constraints on the pepperpot hole size, hole spacing, and beamlet drift length. For instance, small beamlet size is desirable to limit space-charge forces but small beamlets reduce the detectable signal to the camera. Also, the hole spacing must be close enough to allow enough beamlets for sufficient statistical averaging but the spacing is limited by the requirement that the expanding beamlet image spots not overlap at the detector. The drift distance, as well, is limited by spot overlap due to the quadratic growth with distance of the beamlet from space-charge. Additionally, the drift distance must be sufficient to allow adequate beamlet spreading from emittance but prevent excessive space-charge spreading. An optimization of these competing parameters was done by developing a spread sheet to predict the performance of the GBI based on reasonable estimates of instrument dimensions and 
constraints imposed by the Small Recirculator design. The results used in the design of the GBI are shown in Table 4.1. The criteria used to define an acceptable design were that beamlet spread due to space-charge be less than $10 \%$ and that no beamlet spots could overlap due to expansion. The spreadsheet also makes estimates of the amount of optical signal available to the CCD camera based on the input design parameters. A copy of the spreadsheet is contained in Appendix A and details of the calculations are available in Reference 55.

Table 4.1: Results of the GBI design optimizing spreadsheet.

\begin{tabular}{|c|c|c|c|}
\hline \multicolumn{4}{|l|}{ Input Data } \\
\hline Beam current & $2 \mathrm{~mA}$ & Hole diameter & $100 \mu$ \\
\hline Beam radius & $0.5 \mathrm{~cm}$ & Hole spacing & $2.5 \mathrm{~mm}$ \\
\hline Beam energy & $80 \mathrm{keV}$ & Drift distance & $15 \mathrm{~cm}$ \\
\hline Beam length & $4 \mu \mathrm{s}$ & Beam emittance & $10 \mathrm{~mm}-\mathrm{mr}$ \\
\hline \multicolumn{4}{|c|}{ Beamlet Calculations } \\
\hline \multicolumn{3}{|c|}{ Max acceptance angle through hole } & $12.34^{\circ}$ \\
\hline \multicolumn{3}{|c|}{ Beamlet spread due to space charge } & $8.2 \% / 8.2 \mu$ \\
\hline \multicolumn{3}{|c|}{ Beamlet spread from emittance } & $214 \% / 214 \mu$ \\
\hline \multicolumn{3}{|c|}{ Beamlet diameter at detector } & $322.5 \mu$ \\
\hline \multicolumn{3}{|c|}{ Distance between beamlets } & $1.96 \mathrm{~mm}$ \\
\hline
\end{tabular}

The optimization calculations assume that the mean direction of the beam ions is parallel with the recirculator axis $(z)$. In general, the beam will be converging in one transverse plane while diverging in the other transverse plane through the FODO lattice. The random transverse thermal velocity of the ions will be superimposed on top of this directed transverse motion. While this directed transverse motion may not be overly significant to the design of the instrument, it is important to measure it in order to obtain an accurate calculation of the transverse emittance. 


\subsection{Pepperpot Method Data Analysis}

A diverging beam appears in phase space as a rotation of the major axis of the phase space ellipse containing the beam ion distribution into the first and third quadrant. Figure 4.4 shows a schematic of a diverging beam's phase space ellipse and the contribution to it of two beamlets created by a pepperpot.

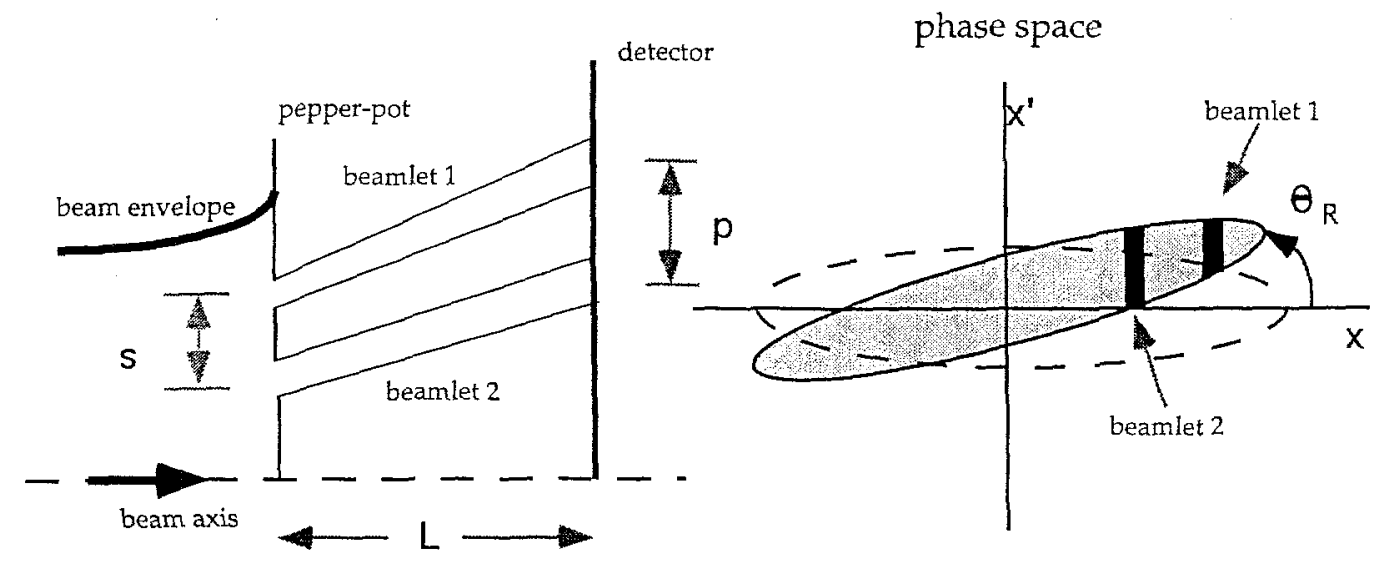

Figure 4.4: Determination of the phase space ellipse rotation angle.

Using some geometry and the assumption that the divergence angle is small yields the expression for the emittance ellipse rotation angle, " $\theta_{R}$ ":

$$
\theta_{R}=\arctan \left(\frac{p-s}{L}\right)
$$

The hole spacing, $s$, and drift distance, $L$, are known and the determination of the beamlet spot spacing, $p$, requires a calibration of the image pixel size so the image analysis software can measure the distance between spots. To improve the accuracy of the measurement the orthogonal distances between all the beamlet spots can be measured and $\theta_{R}$ calculated from the average image spot separation.

A schematic of a single beamlet is shown in Figure 4.5. The beamlet divergence angle is calculated based on the pepperpot hole size, spot size, and drift distance. For 
the $\mathrm{n}^{\text {th }}$ beamlet (from a pepperpot array of $\mathrm{N}$ holes) the divergence angle is:

$$
x_{n}^{\prime} \equiv \frac{d x_{n}}{d z}=\frac{1}{L}\left(x_{n}-r_{h}\right)
$$

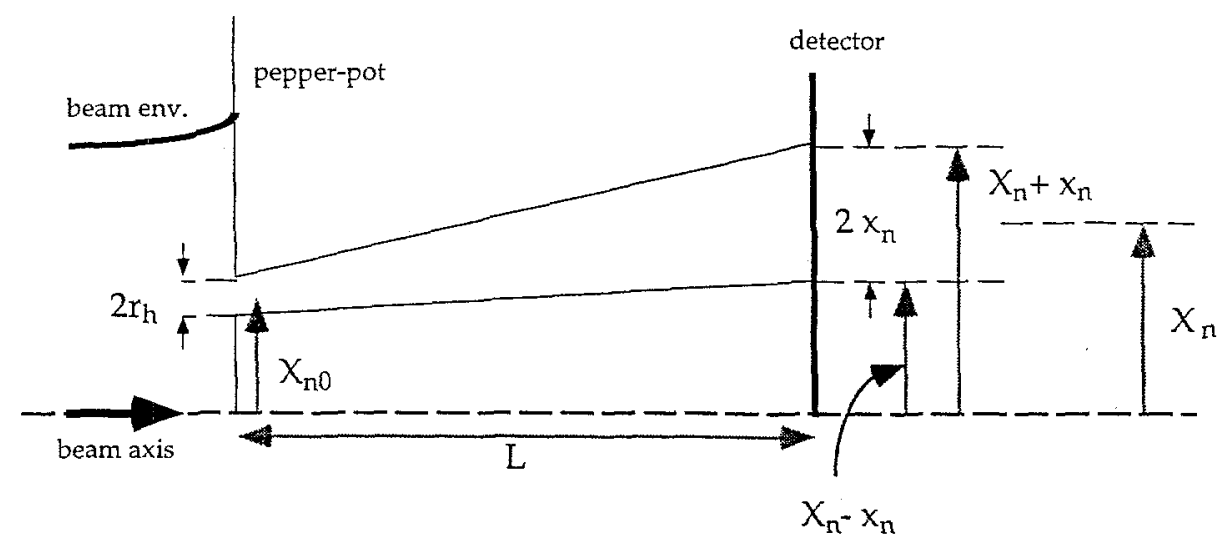

Figure 4.5: Beamlet data needed for beam moment and emittance calculations

As seen in Figure 4.6 each beamlet from the pepperpot contributes two points, $\left(X_{n 0}, X_{n 1}^{\prime}\right)$ and $\left(X_{n 0}, X_{n 2}^{\prime}\right)$ to defining the phase space ellipse. The pepperpot hole radius is assumed small compared to the beam radius. The maximum and minimum boundaries of the phase space ellipse at a given position is given by

$$
X_{n 2}^{\prime}=\frac{\left(X_{n}+x_{n}\right)-X_{n 0}}{L} \quad X_{n 1}^{\prime}=\frac{\left(X_{n}-x_{n}\right)-X_{n 0}}{L}
$$

The points from all beamlets will roughly define the phase space ellipse. Figure 4.7 shows data plotted from a GBI measurement on the Small Recirculator where each set of two points derived from one beamlet is plotted with a vertical line connecting 


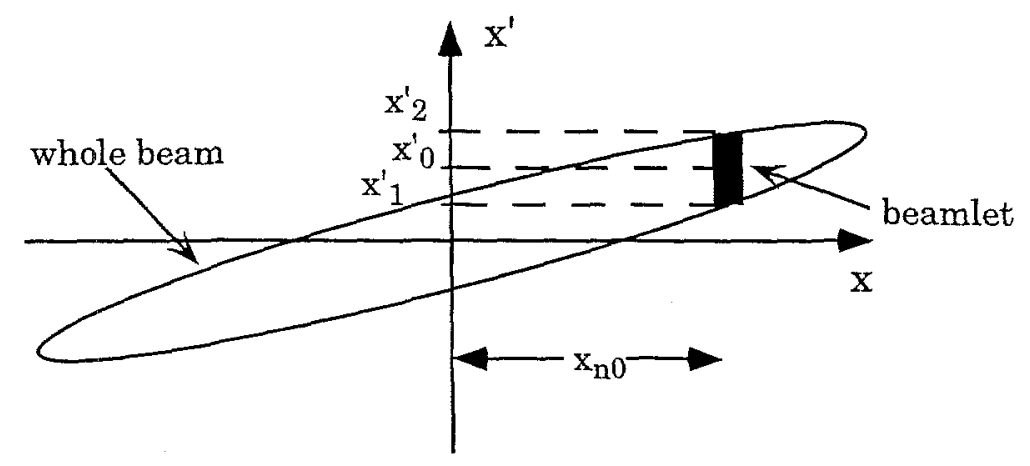

Figure 4.6: Beam phase space ellipse.

them. The area of the phase space ellipse is closely related to the beam emittance as discussed in Chapter 3.

The ellipse is generated using the first and second moments of the beam distribution. The moments of the entire beam are obtained by averaging the information obtained from each of the $\mathrm{N}$ total beamlets. For measurements made on the Small Recirculator beam, $N \approx 70$. The moments are calculated by discretizing the moment formula:

$$
<\phi>=\iiint \int \phi \rho\left(x, x^{\prime}, y, y^{\prime}\right) d x d x^{\prime} d y d y^{\prime}
$$

with the normalization

$$
\iint \rho\left(x, x^{\prime}, y, y^{\prime}\right) d x d x^{\prime} d y d y^{\prime}=1
$$

therefore the explicit forms for the first and second moments are obtained from the beamlet spots by the following calculations:

$$
<x>=\frac{\frac{1}{N} \sum_{n=1}^{N} I_{n} x_{n}}{I_{N}} \quad<x^{\prime}>=\frac{\frac{1}{N} \sum_{n=1}^{N} I_{n} x_{n}^{\prime}}{I_{N}}
$$




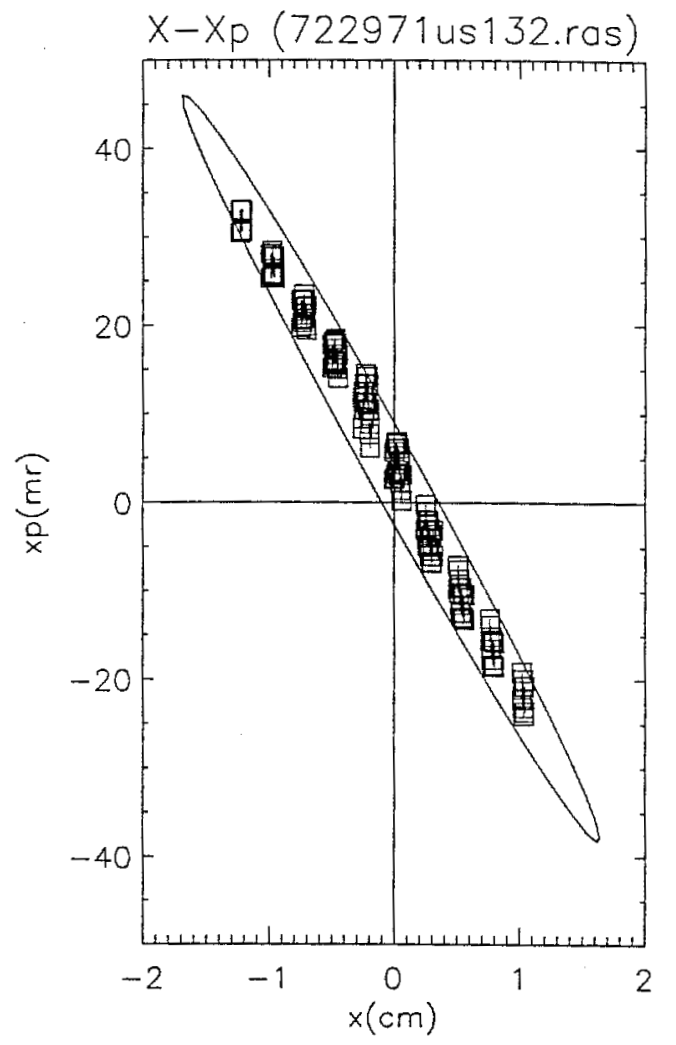

rms edge $x$ emittance $24.00 \mathrm{~mm}-\mathrm{mr}$
norm. $\times$ emittance $0.051 \mathrm{~mm}-\mathrm{mr}$
Level(percent) $=10$ at mask

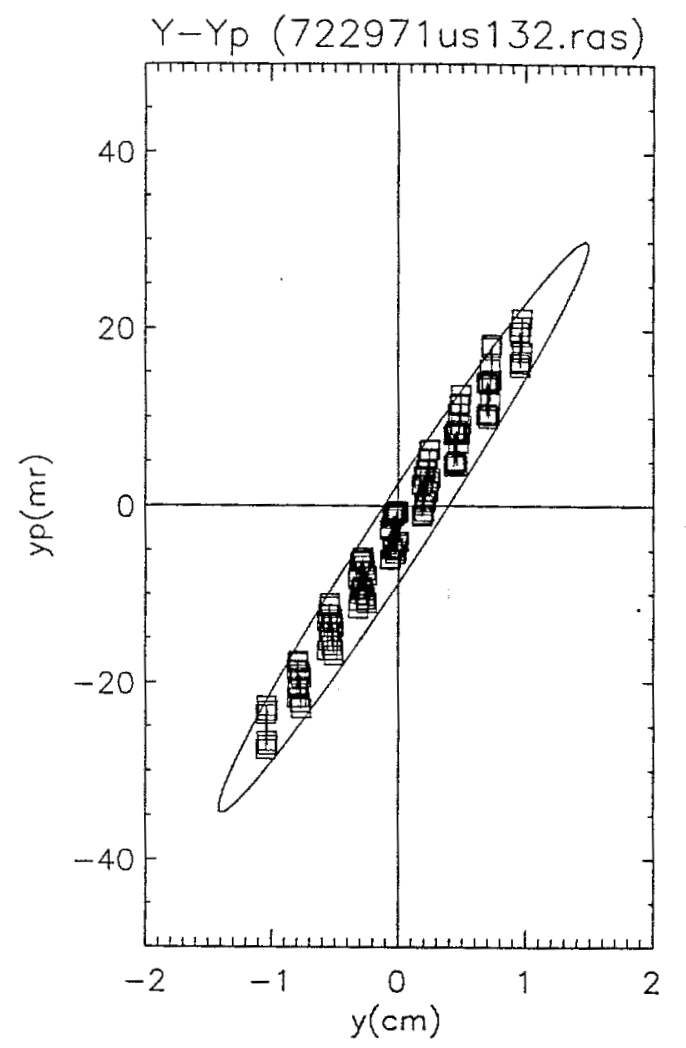

rms edge y emittance $20.67 \mathrm{~mm}-\mathrm{mr}$ norm. y emittonce $0.044 \mathrm{~mm}-\mathrm{mr}$ 67 beamlets Smoothing 0 Mask $-1.700 \mathrm{deg}$.

Figure 4.7: Phase space plot of a GBI measurement from the Small Recirculator. The ellipse rotation angles correspond to the FODO lattice focusing in the $x$-plane while defocusing in the $y$-plane at the GBI location.

$$
\begin{aligned}
<y>=\frac{\frac{1}{N} \sum_{n=1}^{N} I_{n} y_{n}}{I_{N}} & <y^{\prime}>=\frac{\frac{1}{N} \sum_{n=1}^{N} I_{n} y_{n}^{\prime}}{I_{N}} \\
<x^{2}>=\frac{\frac{1}{N} \sum_{n=1}^{N} I_{n} x_{n}^{2}}{I_{N}} & <y^{2}>=\frac{\frac{1}{N} \sum_{n=1}^{N} I_{n} y_{n}^{2}}{I_{N}} \\
<x x^{\prime}>=\frac{\frac{1}{N} \sum_{n=1}^{N} I_{n} x_{n} x_{n}^{\prime}}{I_{N}} & <y y^{\prime}>=\frac{\frac{1}{N} \sum_{n=1}^{N} I_{n} y_{n} y_{n}^{\prime}}{I_{N}} \\
<x^{\prime 2}>=\frac{\frac{1}{N} \sum_{n=1}^{N} I_{n} x_{n}^{\prime 2}}{I_{N}} & <y^{\prime 2}>=\frac{\frac{1}{N} \sum_{n=1}^{N} I_{n} y_{n}^{\prime 2}}{I_{N}}
\end{aligned}
$$




$$
\begin{aligned}
<x y>=\frac{\frac{1}{N} \sum_{n=1}^{N} I_{n} x_{n} y_{n}}{I_{N}} & <x^{\prime} y^{\prime}>=\frac{\frac{1}{N} \sum_{n=1}^{N} I_{n} x_{n}^{\prime} y_{n}^{\prime}}{I_{N}} \\
<x^{\prime} y>=\frac{\frac{1}{N} \sum_{n=1}^{N} I_{n} x_{n}^{\prime} y_{n}}{I_{N}} & <x y^{\prime}>=\frac{\frac{1}{N} \sum_{n=1}^{N} I_{n} x_{n} y_{n}^{\prime}}{I_{N}} \\
I_{n}=\frac{1}{P_{n}} \sum_{p=1}^{P_{n}} V_{p} & I_{N}=\frac{1}{N} \sum_{n=1}^{N} I_{n}
\end{aligned}
$$

where $N$ is the total number of beamlets, $n$ is the beamlet index, $P_{n}$ is the total number of pixels contained in the $n^{\text {th }}$ beamlet's digitized image, $V$ is the digitized pixel value, $p$ is the pixel index, $I_{n}$ is the average intensity of the $n^{\text {th }}$ beamlet, and $I_{N}$ is the average intensity of all $N$ beamlets. In the above formulas for the second moments the contribution from non-zero first moments (beam centroid not on the accelerator axis) is not removed. For a beam not on axis in phase space the following calculation is used to remove this contribution:

$$
\begin{gathered}
\Delta x^{2}=<x^{2}>-<x>^{2} \quad \Delta y^{2}=<y^{2}>-<y>^{2} \\
\Delta x^{\prime 2}=<x^{\prime 2}>-<x^{\prime}>^{2} \quad \Delta y^{\prime 2}=<y^{\prime 2}>-<y^{\prime}>^{2} \\
\Delta x x^{\prime}=<x x^{\prime}>-<x><x^{\prime}>\quad \Delta y y^{\prime}=<y y^{\prime}>-<y><y^{\prime}> \\
\Delta x y=<x y>-<x><y>\quad \Delta x^{\prime} y^{\prime}=<x^{\prime} y^{\prime}>-<x^{\prime}><y^{\prime}> \\
\Delta x^{\prime} y=<x^{\prime} y>-<x^{\prime}><y>\quad \Delta x y^{\prime}=<x y^{\prime}>-<x><y^{\prime}>
\end{gathered}
$$

where the $\Delta$ symbol introduced by Barnard will be used henceforth to indicate a second moment with the centroid contribution removed. ${ }^{29}$ The equation for the ellipse fitted to the pepperpot data is

$$
\gamma x^{2}+2 \alpha x x^{\prime}+\beta x^{\prime 2}=\epsilon_{x}
$$


where

$$
\gamma=\frac{\Delta x^{2}}{\epsilon_{x}} \quad \alpha=\frac{-\Delta x x^{\prime}}{\epsilon_{x}} \quad \beta=\frac{\Delta x^{\prime 2}}{\epsilon_{x}}
$$

The transverse rms edge emittance, $\epsilon_{x}$, is obtained from

$$
\epsilon_{x}=4\left(\Delta x^{2} \Delta x^{\prime 2}-\left(\Delta x x^{\prime}\right)^{2}\right)^{\frac{1}{2}}
$$

The previous discussion of measuring second moments by measuring the halfwidths of the beamlet spots assumed the spots were of regular shape (ellipses or circles) and the "edges" of the spots identifiable. The beamlets originate with round cross-section at the pepperpot but become elliptical due to different focusing in the $x$ and $y$ directions from the FODO lattice prior to the pepperpot, which distorts them into ellipse-like shapes at the detector.

The raw data available from the GBI consists of a two-dimensional array of digitized pixel values in the field of view of the CCD camera observing the detector. Pixel values range in value from 0 to 65535 (with 16-bit digitization of the CCD well). Generally, a contour of equal pixel value identified as the beamlet image edge will not be an ellipse. To make a spot size measurement, an ellipse is fitted to the CCD display intensity contour of interest and the measurement of major and minor axes based on this.

Practically this can be done by sampling the contour of interest (most likely the contour whose value is $10 \%$ of the spot's peak pixel value), fitting an ellipse and then rotating the ellipse to its upright position such that the major and minor axes lie on the $x$ and $y$ axes. This is done as follows: A thin band of $N$ points $\left(x_{j}, y_{j}\right)$ at the $10 \%$ contour level is chosen as in Figure 4.8 on page 48. The coefficients for an elliptical approximation to this band are calculated from:

$$
\overline{x^{2}}=\frac{2}{N} \sum_{n=1}^{N} x_{j}{ }^{2} \quad \overline{y^{2}}=\frac{2}{N} \sum_{n=1}^{N} y_{j}^{2} \quad \overline{x y}=\frac{2}{N} \sum_{n=1}^{N} x_{j} y_{j}
$$


The centroid $\left(x_{c}, y_{c}\right)$ of the ellipse is given by:

$$
\left(x_{c}, y_{c}\right)=\left(\frac{1}{N} \sum_{n=1}^{N} x_{j} \frac{1}{N} \sum_{n=1}^{N} y_{j}\right)
$$

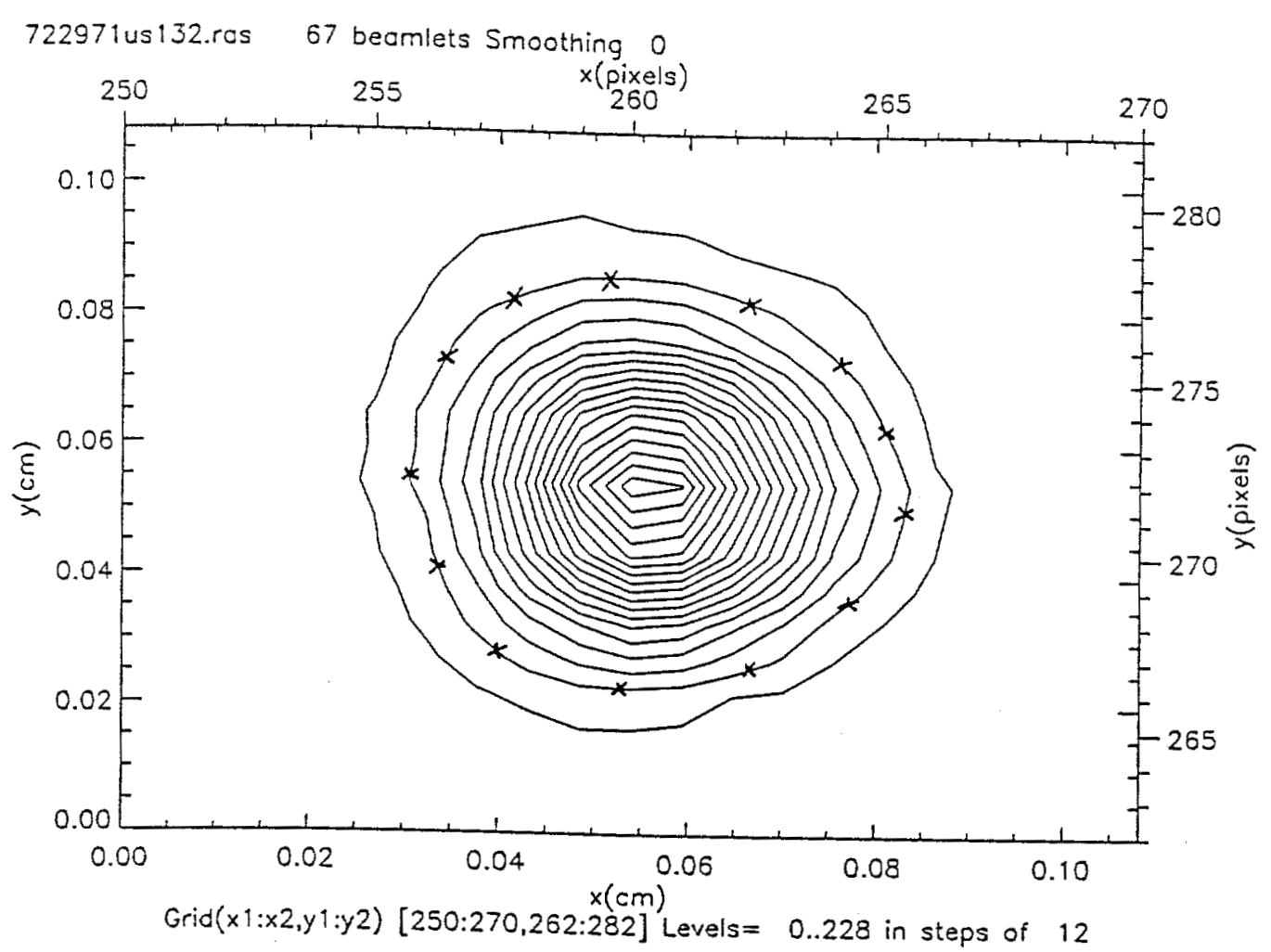

Figure 4.8: Contour of a single beamlet from a GBI measurement

These averages, $\left(\overline{x^{2}}, \overline{y^{2}}, \overline{x y}\right)$ can be equated to the elements of an ellipse's sigma matrix. The equation of the ellipse is:

$$
\gamma x^{2}+2 \alpha x y+\beta y^{2}=1
$$

and

$$
\sigma=\left[\begin{array}{ll}
\sigma_{11} & \sigma_{21} \\
\sigma_{21} & \sigma_{22}
\end{array}\right]=\left[\begin{array}{rr}
\beta & -\alpha \\
\alpha & \gamma
\end{array}\right]=\left[\begin{array}{cc}
\overline{x^{2}} & \overline{x y} \\
\overline{y x} & \overline{y^{2}}
\end{array}\right]
$$


thus,

$$
\sigma_{11}=\overline{x^{2}} \quad \sigma_{22}=\overline{y^{2}} \quad \sigma_{12}=\overline{x y}=\sigma_{21}=\overline{y x}
$$

The general rule for transforming the sigma matrix (e.g. a pure rotation) is

$$
\sigma^{*}=R \sigma R^{T}
$$

The transformation for a general rotation through angle, $\theta$ is

$$
\sigma^{*}=\left[\begin{array}{rr}
\cos \theta & \sin \theta \\
-\sin \theta & \cos \theta
\end{array}\right]\left[\begin{array}{ll}
\sigma_{11} & \sigma_{21} \\
\sigma_{21} & \sigma_{22}
\end{array}\right]\left[\begin{array}{rr}
\cos \theta & -\sin \theta \\
\sin \theta & \cos \theta
\end{array}\right]
$$

$$
\sigma^{*}=\left[\begin{array}{c}
\cos ^{2} \theta \sigma_{11}+2 \sin \theta \cos \theta \sigma_{12}+\sin ^{2} \theta \sigma_{22} \\
-\sin \theta \cos \theta \sigma_{11}+\left(\cos ^{2} \theta-\sin ^{2} \theta\right) \sigma_{12}+\sin \theta \cos \theta \sigma_{22} \\
-\sin \theta \cos \theta \sigma_{11}+\left(\cos ^{2} \theta-\sin ^{2} \theta\right) \sigma_{12}+\sin \theta \cos \theta \sigma_{22} \\
\sin ^{2} \theta \sigma_{11}+2 \sin \theta \cos \theta \sigma_{12}+\cos ^{2} \theta \sigma_{22}
\end{array}\right.
$$

To find the major and minor widths of the beamlet the required transformation is one that rotates the ellipse into its upright coordinate system. The correct rotation angle that puts the ellipse upright can be solved from:

$$
\sigma_{12}^{*}=\sigma_{21}^{*}=0 \quad \quad \quad>\quad \theta=\frac{1}{2} \arctan \left(\frac{2 \sigma_{12}}{\sigma_{11}-\sigma_{22}}\right)
$$

therefore the upright beamlet spot ellipse sigma matrix is:

$$
\sigma^{*}=\left[\begin{array}{rr}
A^{2} & 0 \\
0 & B^{2}
\end{array}\right]
$$


with major and minor axes of the fitted ellipse given by

$$
\begin{aligned}
& A=\left(\cos ^{2} \theta \sigma_{11}+2 \sin \theta \cos \theta \sigma_{12}+\sin ^{2} \theta \sigma_{22}\right)^{1 / 2} \\
& B=\left(\sin ^{2} \theta \sigma_{11}+2 \sin \theta \cos \theta \sigma_{12}+\cos ^{2} \theta \sigma_{22}\right)^{1 / 2}
\end{aligned}
$$

These values $(A, B)$ are the widths of the beamlet spots used in the calculation of the ion divergence angles and the ion distribution's first and second moments.

\subsection{Image Capture and Image Analysis Code}

To accomplish the immense task of data handling involved in capturing high resolution images, identifying and measuring beamlet spot sizes and locations, and performing the data analysis described above I used a combination of a commercial image capture software and a modified version of a pepperpot data analysis code developed at LLNL. ${ }^{28}$ The image capture software, IPLab Spectrum ${ }^{T M}$ by Signal Analytics Corporation, running on a Macintosh computer incorporates an extension allowing it to control the CCD camera and store images into the computer memory. ${ }^{32}$ It also allows other operations to be done such as focusing the camera and determining the field of view. Certain image manipulations were also accomplished with this software such as measuring distances between known points for pixel size calibration, animating a series of images of the beam into a "beam movie", an electronic version of a flip book of sequential images, and correcting images prior to data analyses. The mechanics of acquiring and correcting data images is discussed in Chapter 5 .

The pepperpot data analysis code, "PEPPERPOT" was written for analysis of pepperpot images generated by a pepperpot with holes arranged in concentric circles. It is written in Fortran-like commands of the Interactive Data Language (IDL) developed by Research Systems, Inc. ${ }^{33}$ I modified the source code to optimize its use with the GBI on the Small Recirculator. Changes included the ability to do calculations based on a rectangular array of beamlets. The rectangular array is more appropriate 
for diagnosing a beam with two orthogonal planes of motion produced by the FODO lattice. The original application was for solenoidal transport which produces radial symmetry, hence the circular pepperpot design. Other modifications I made included the ability to analyze different size data arrays to accommodate different CCD arrays, calculation of the $x-y$ cross moments, and I added in the algorithm for weighting each beamlet as prescribed earlier in this chapter. This last item was done to bring the calculation in line with the method by which the data from the slit scanner diagnostics is analyzed so that direct comparisons are possible. I also added a module to the source code that fits a gaussian to a cross-section of a beamlet so fitted beamlet size data could be used for moment calculations if desired. To gather time-sequenced data as a narrow gate is stepped through successive beam shots, moment and emittance data is written to files for later display using other graphics routines as shown in Chapter 7. The Pepperpot code allows interactive analysis of the GBI images with graphical and tabular output. A copy of the PEPPERPOT IDL source code is contained in Appendix E. 


\subsection{Sample Output from the Data Analysis Code}

The following figures are samples of the output of various analyses on an image.

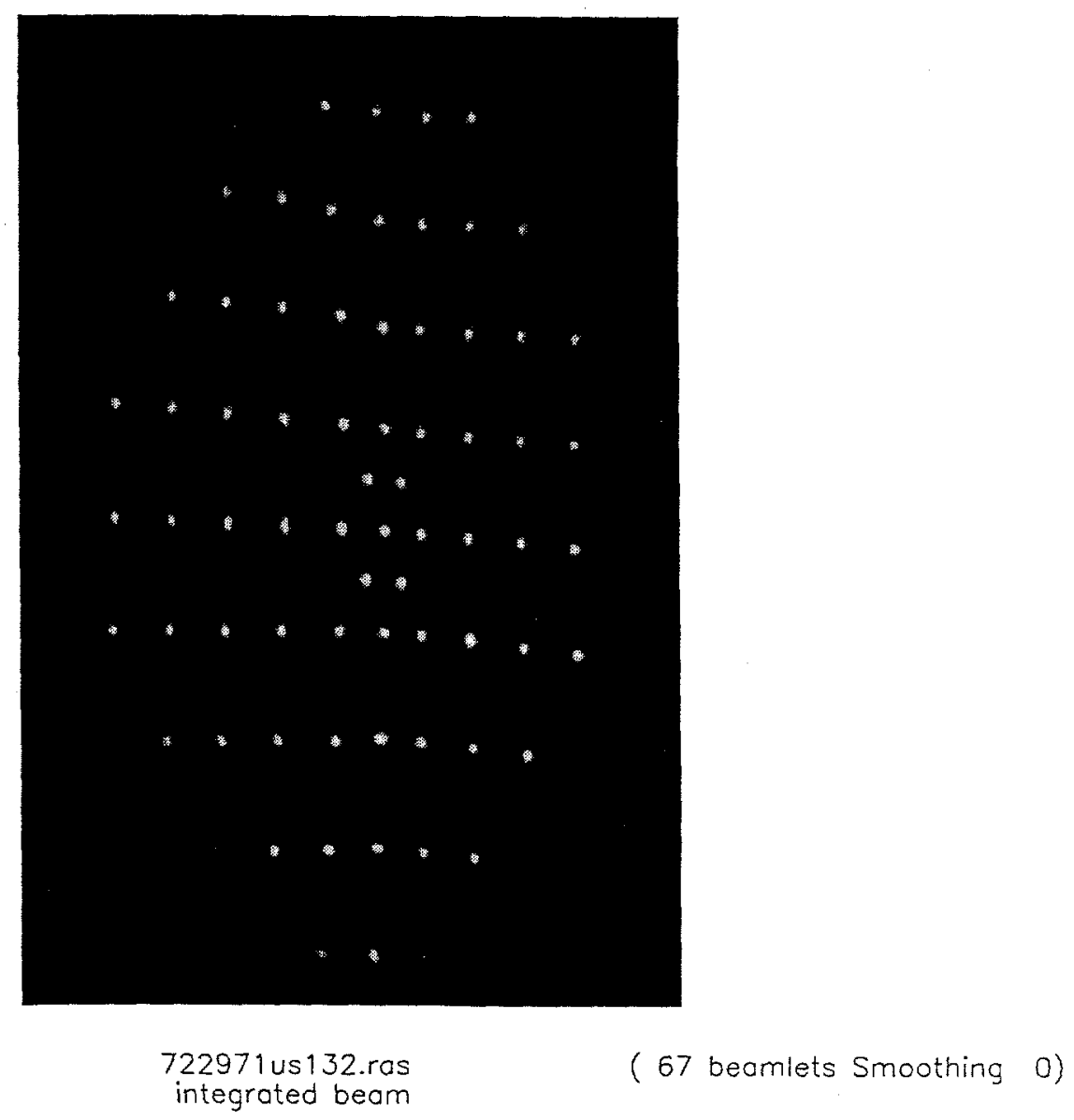

Figure 4.9: GBI image used for analysis of beam moments. The four spots between rows surrounding the center spot allow easy identification of the center hole as a reference and are not used in the data analysis. Each spot is approximately $0.5 \mathrm{~mm}$ in diameter and the field-of-view is 2.07 by $3.11 \mathrm{~cm}$. 


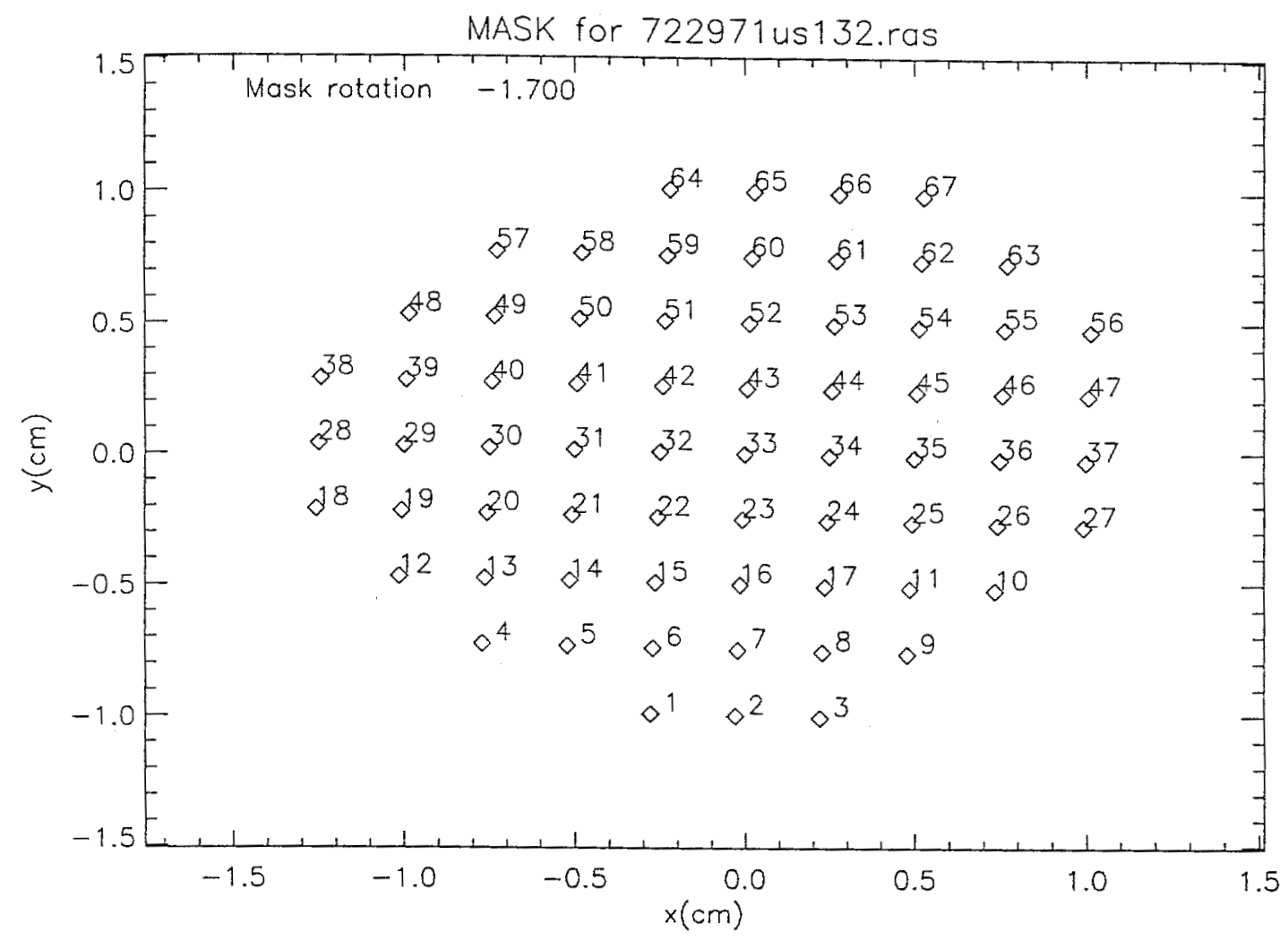

Figure 4.10: Identification of which holes the beamlets in Figure 4.9 originated from. 


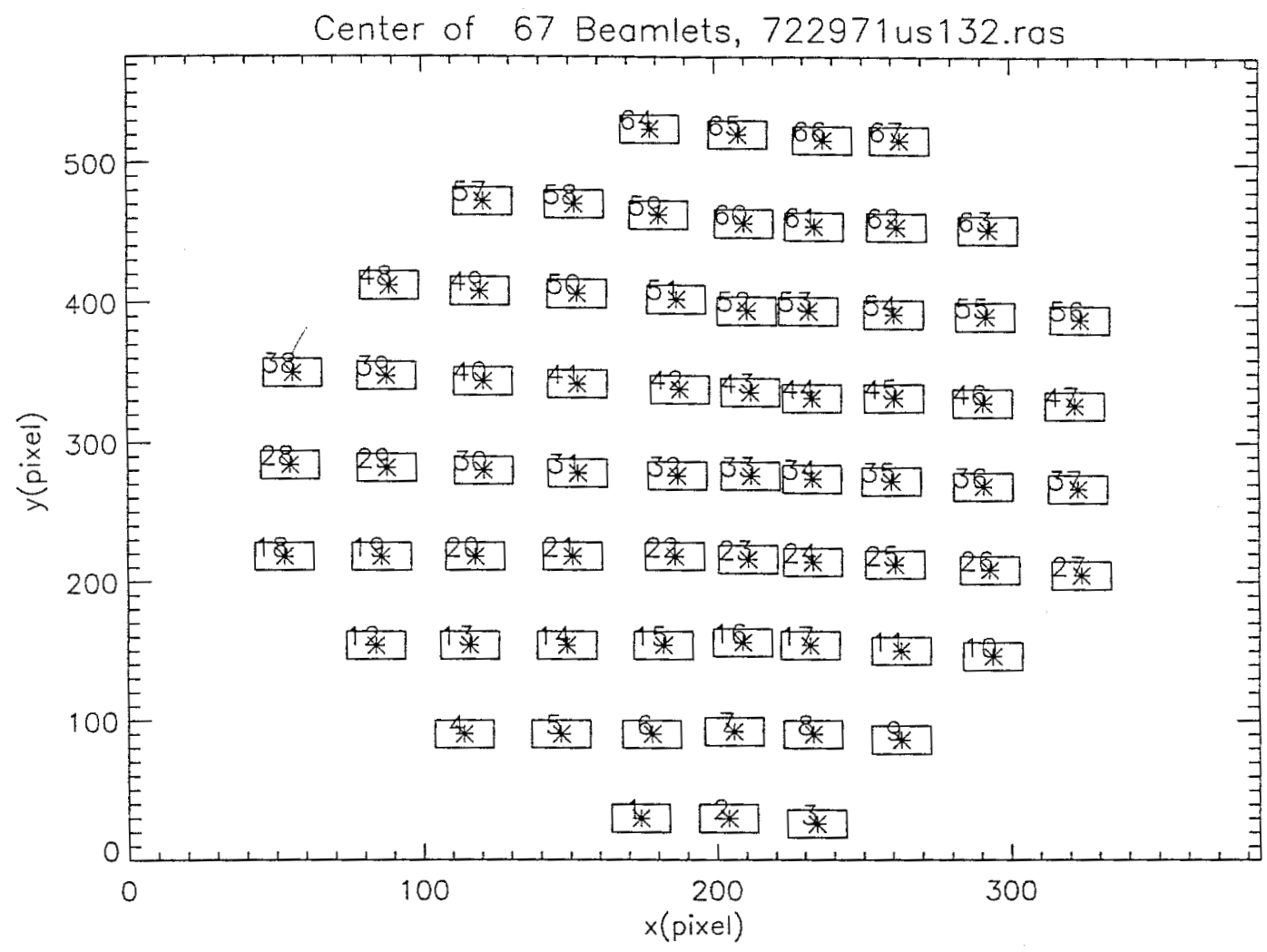

Figure 4.11: Plot of the data analysis windows surrounding the holes through which the beamlets passed. 


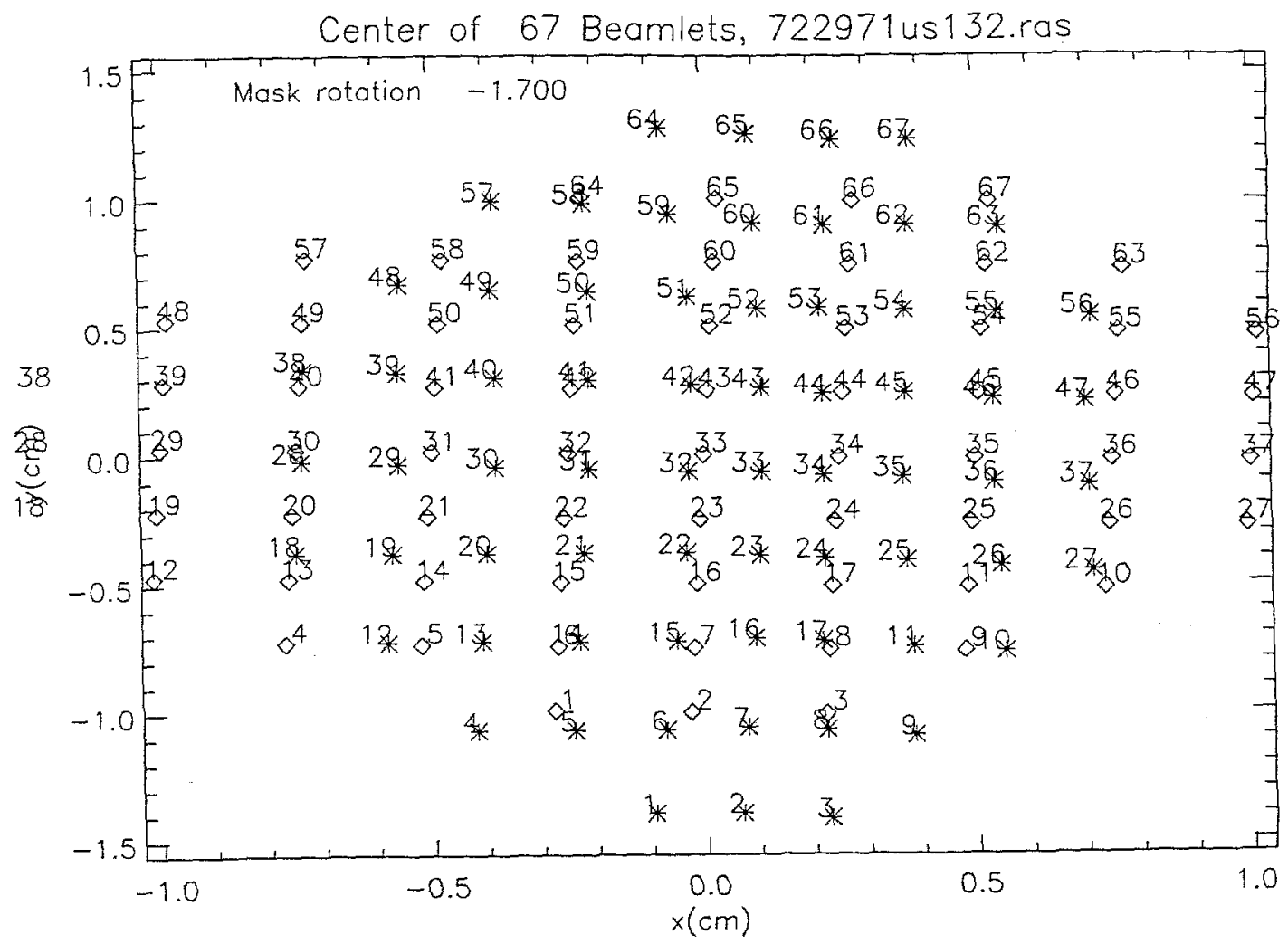

Figure 4.12: Plot superimposing the pepperpot holes (diamond symbols) and the centroids of the beamlet spots (asterisks) at the detector showing focusing in the $x$-plane and defocusing in the $y$-plane. 


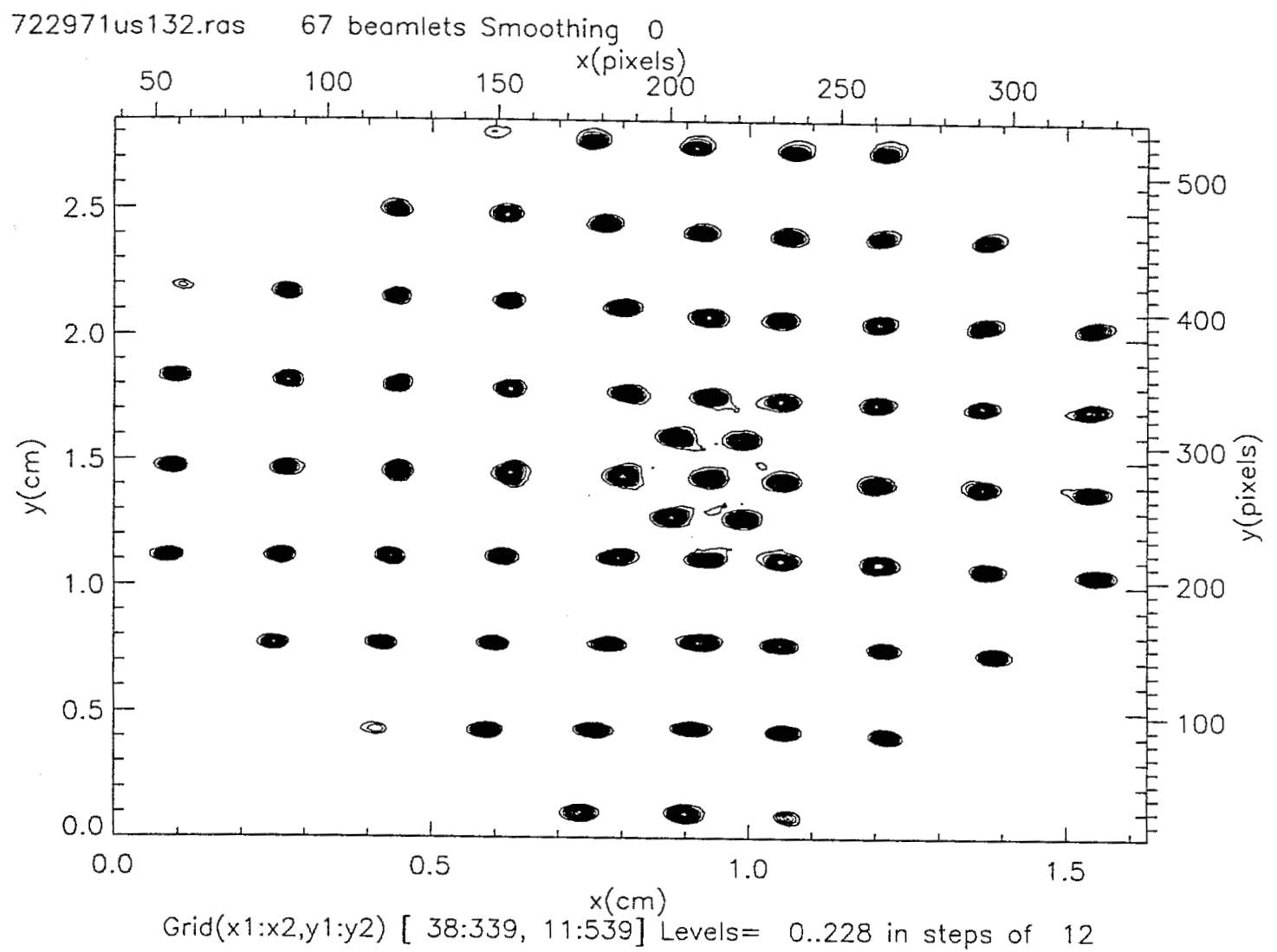

Figure 4.13: Contour plot of all data from the CCD camera field of view. 


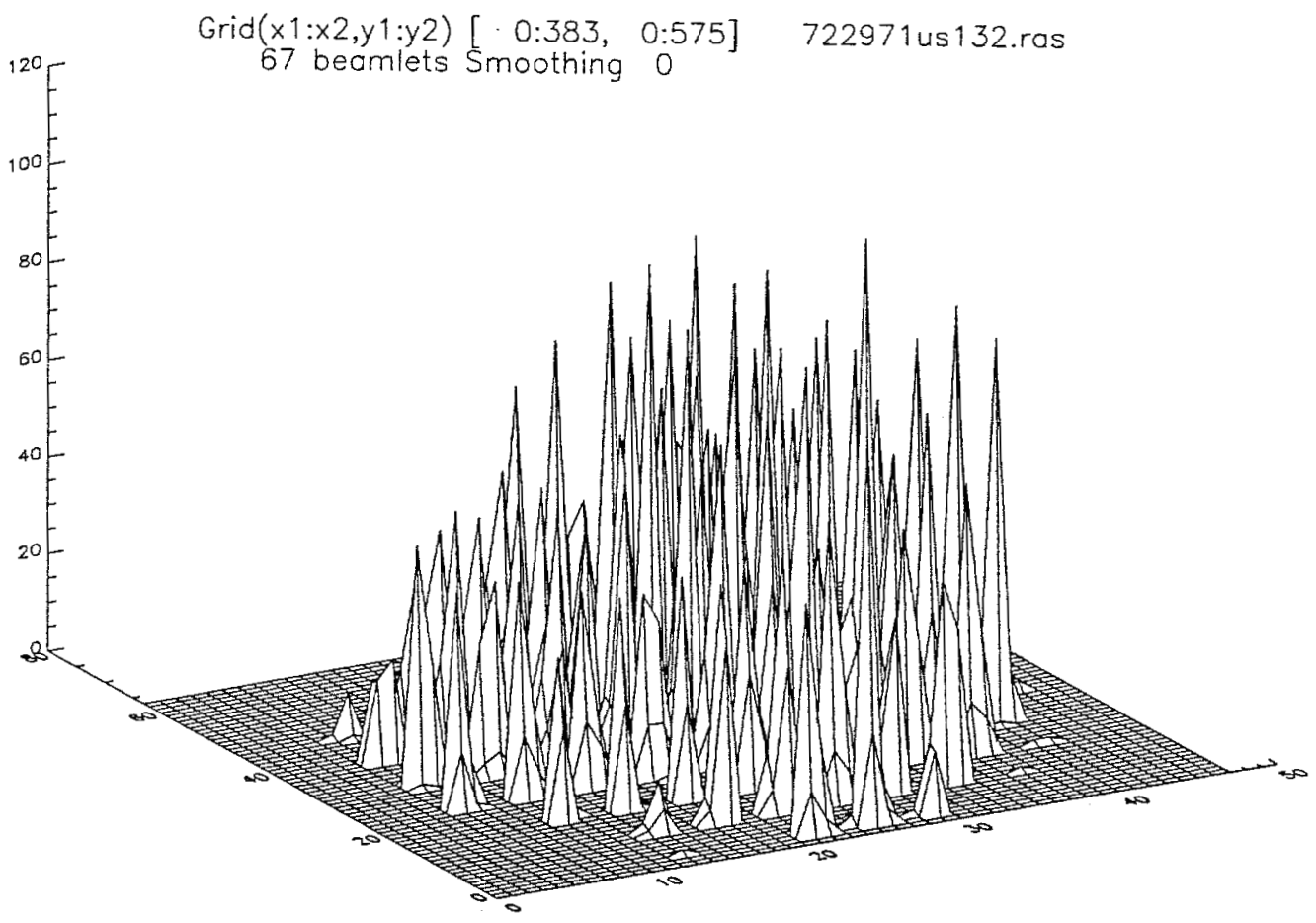

Figure 4.14: Surface plot of the intensity vs. position for the CCD camera field of view. 


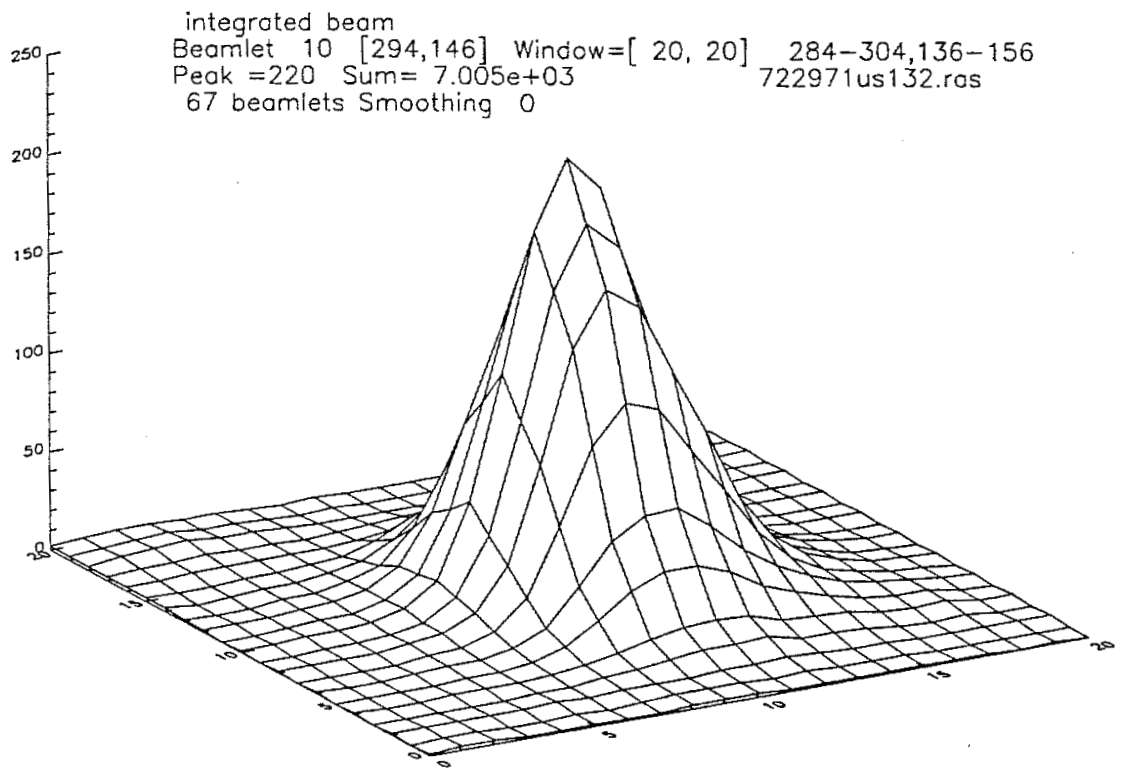

Figure 4.15: Surface plot of beamlet intensity vs. position limited to a 20 by 20 pixel data analysis window around one beamlet.

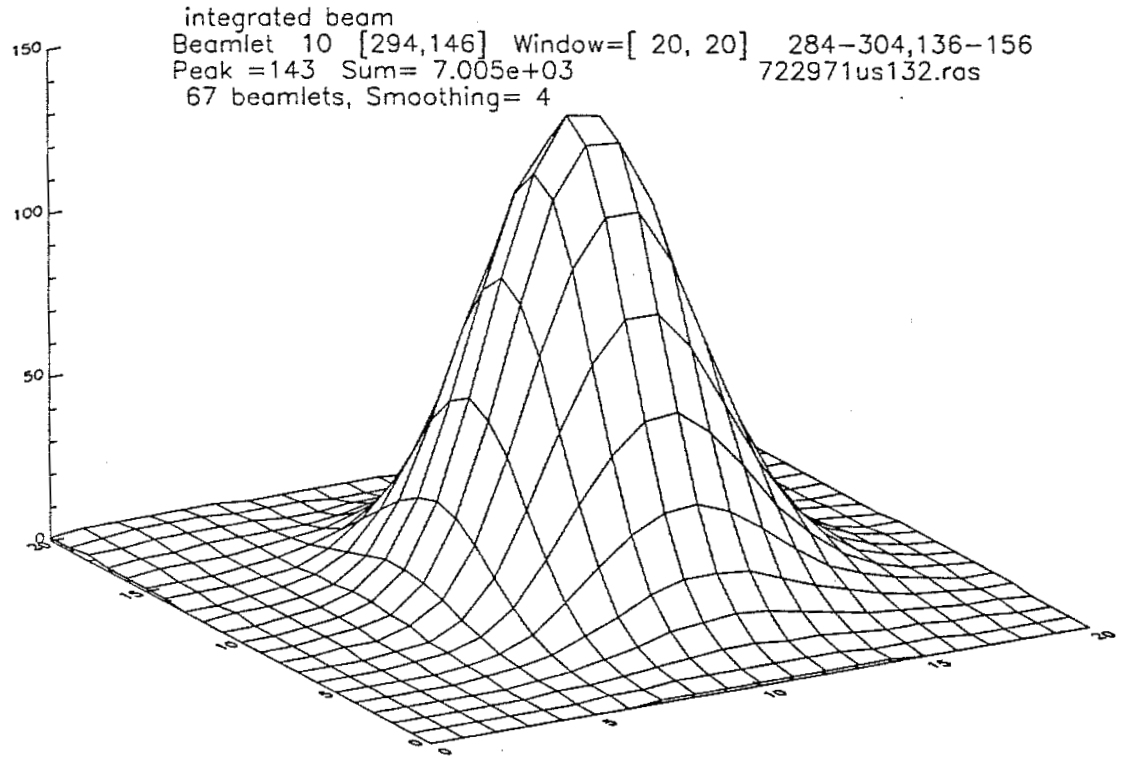

Figure 4.16: Surface plot of the beamlet of Figure 4.15 after a 4 by 4 pixel block averaging of the intensity values. 


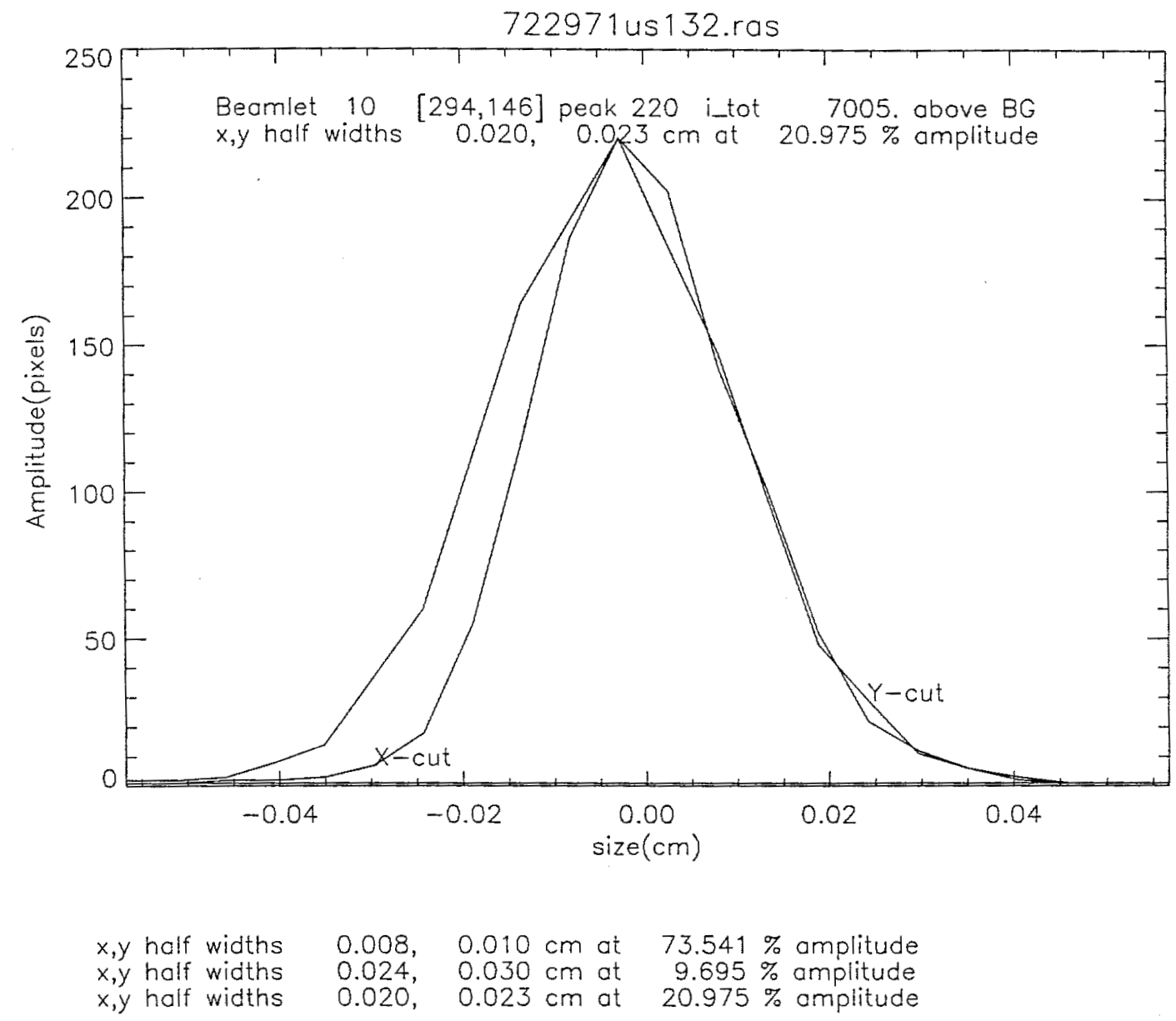

Figure 4.17: Beamlet cross-section plots of intensity vs. position. The width at different intensity levels can be determined by positioning a cursor. 


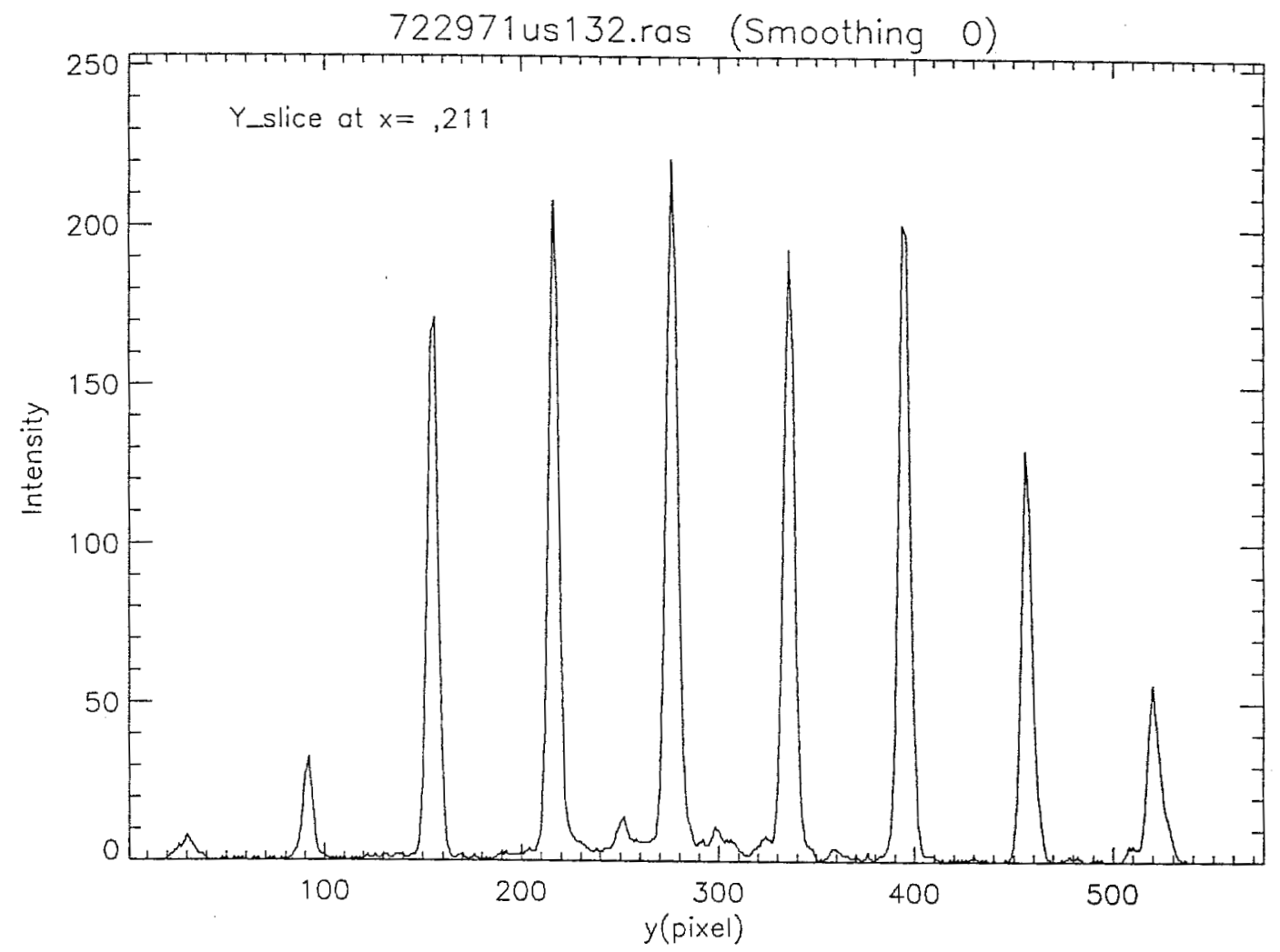

Figure 4.18: Plot of a cut through the image at a selected row or column. Here column 211 of Figure 4.9 is displayed. 

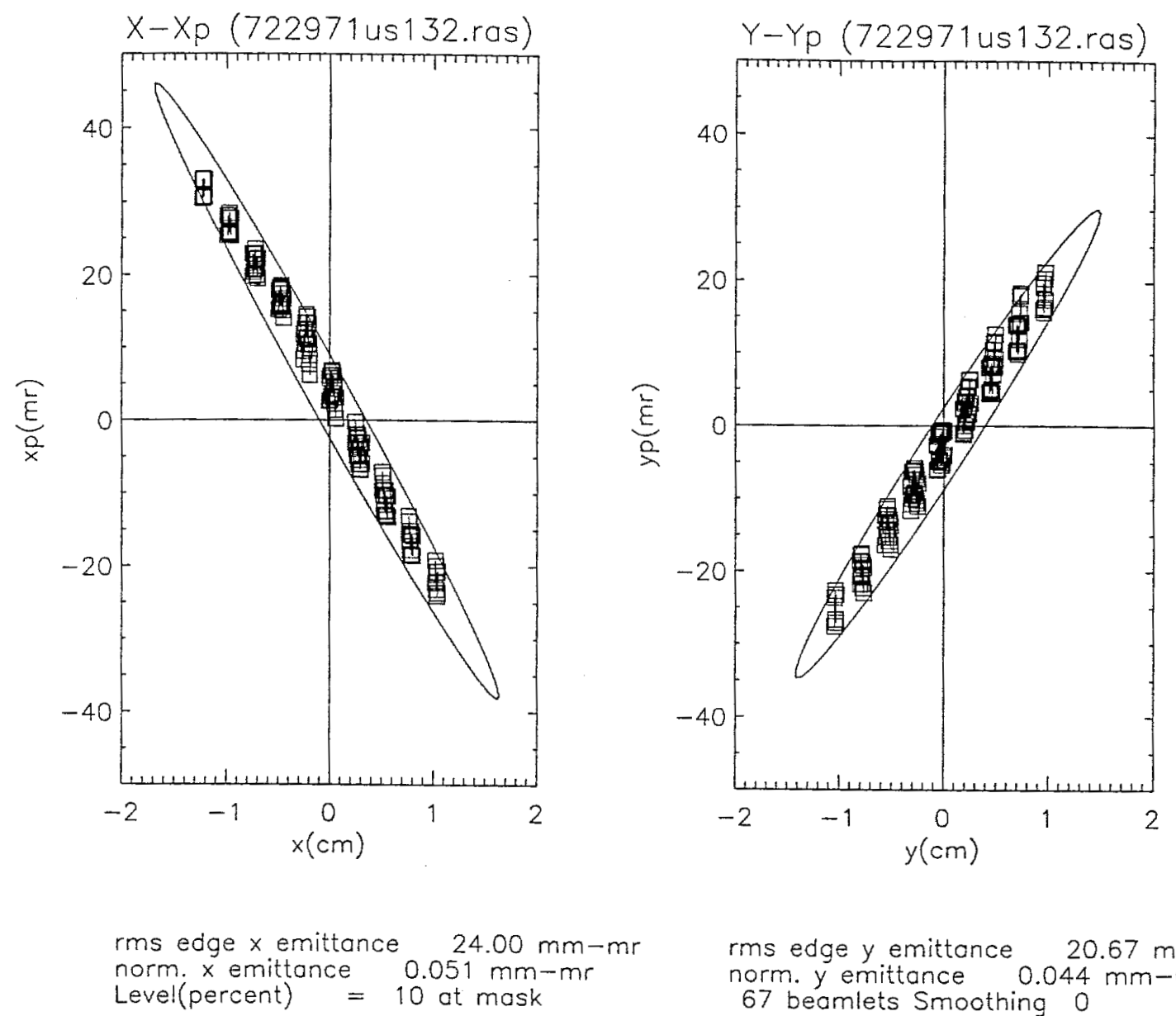

rms edge y emittance $20.67 \mathrm{~mm}-\mathrm{mr}$ norm. y emittance $0.044 \mathrm{~mm}-\mathrm{mr}$ 67 beomlets Smoothing 0 Mask $-1.700 \mathrm{deg}$.

Figure 4.19: 2-D phase space plots with individual beamlet data fitted with an ellipse and emittance calculations displayed. 


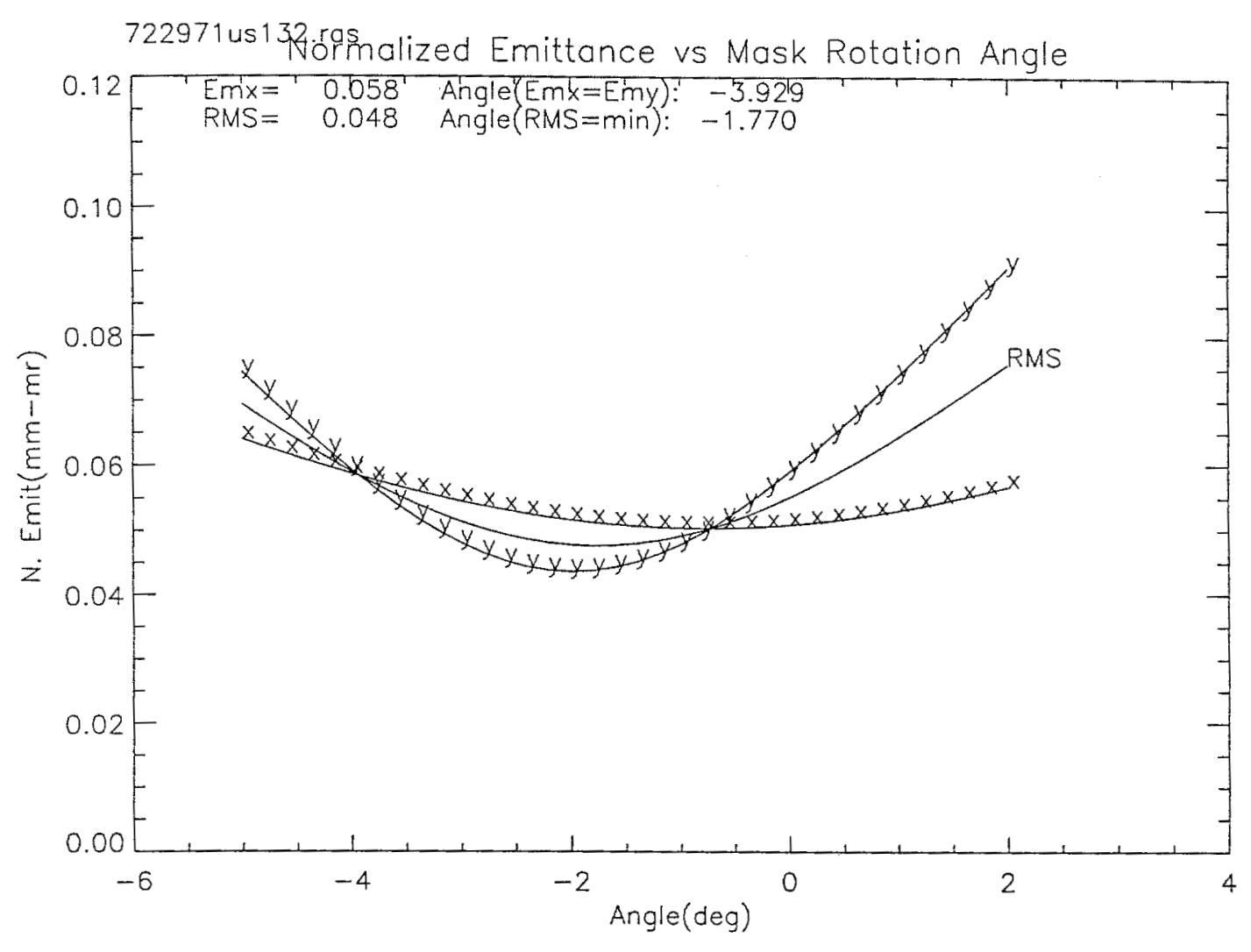

Figure 4.20: Plot of normalized emittance vs. pepperpot holeplate rotation angle. 


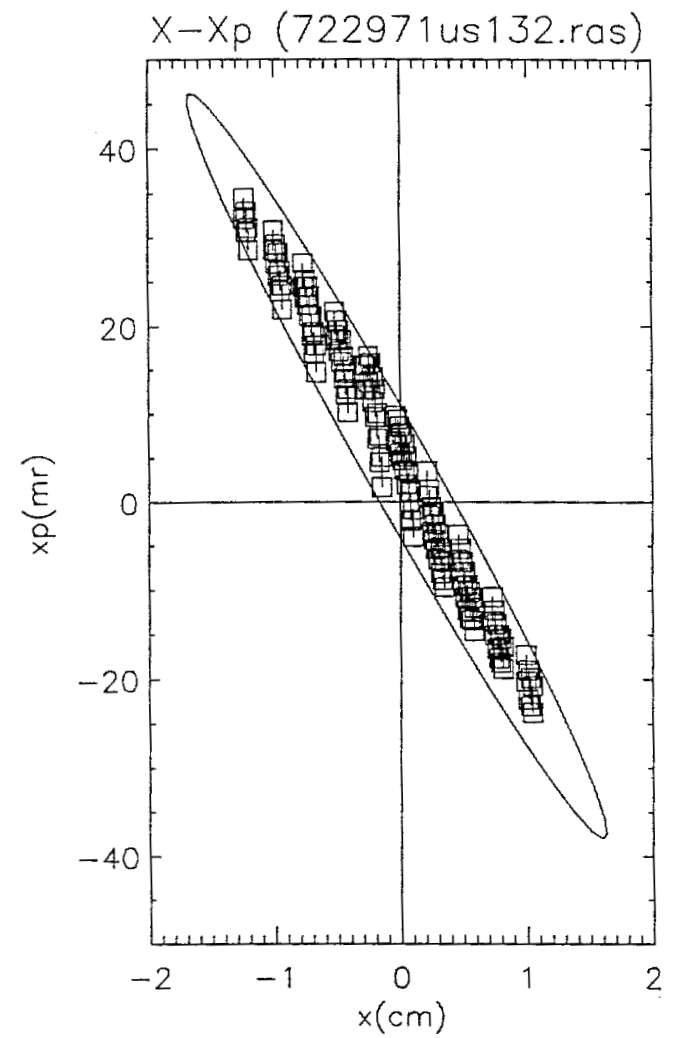

rms edge $x$ emittance $31.64 \mathrm{~mm}-\mathrm{mr}$
norm. x emittance $0.067 \mathrm{~mm}-\mathrm{mr}$
Level(percent) $=10$ at mask

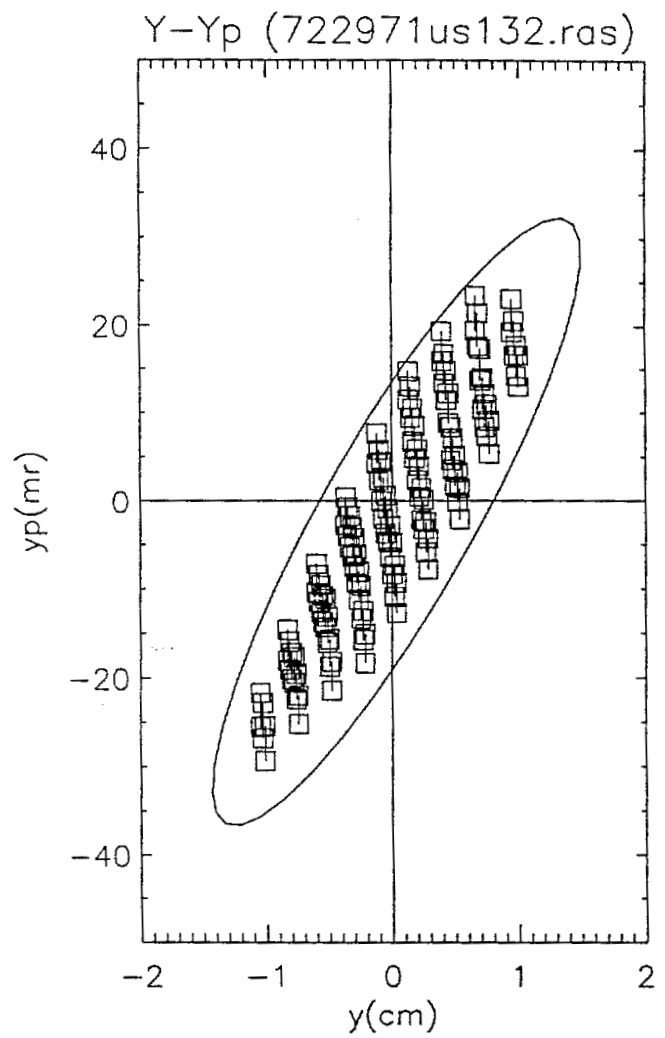

rms edge y emittance $59.65 \mathrm{~mm}-\mathrm{mr}$
norm. y emittance $0.127 \mathrm{~mm}-\mathrm{mr}$
67 beamlets Smoothing 0
Mask 4.000 deg.

Figure 4.21: The appearance of 2-D phase space plots when an incorrect holeplate rotation angle is used in calculating $x^{\prime}$ and $y^{\prime}$. Erroneous beamlet divergence angles are calculated resulting in a "vertical" separation of beamlet data and and a larger emittance value. 


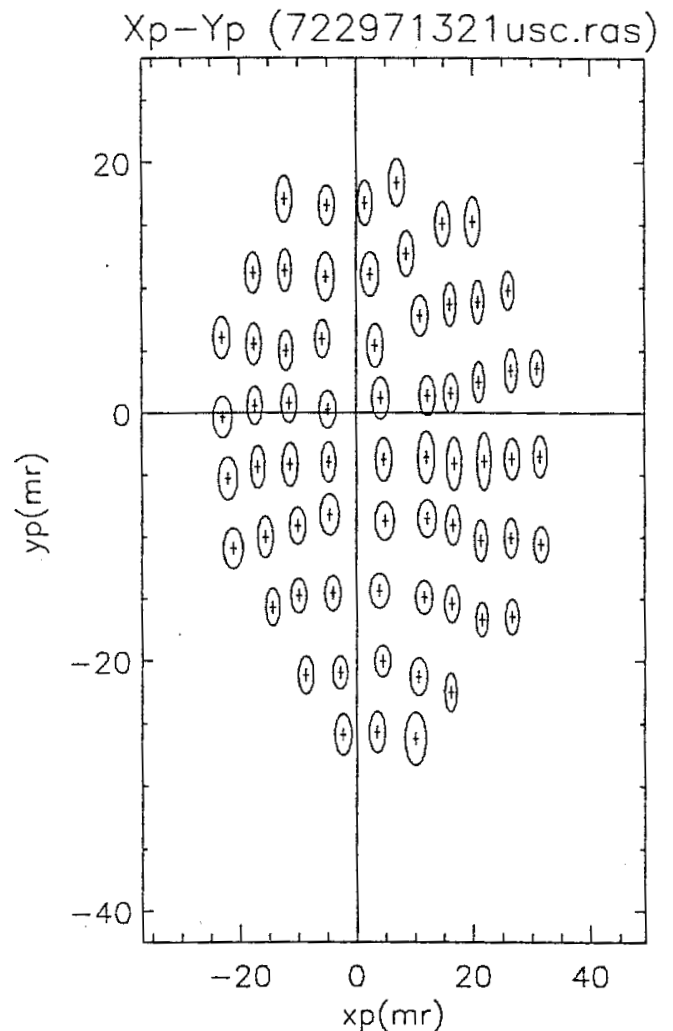

$-36.7649 .27-42.5228 .38$

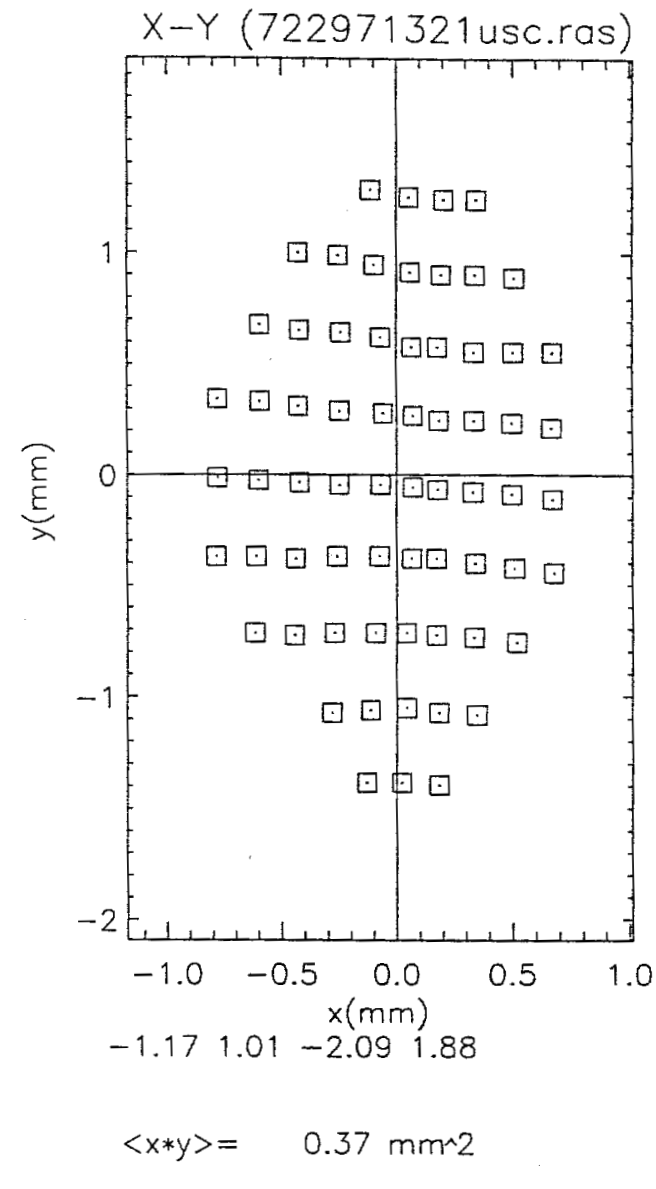

Figure 4.22: Plot of $x-y$ cross moments phase space with $\left\langle x^{\prime}-y^{\prime}\right\rangle$ and $\langle x-y\rangle$ shown. The magnitude of the axes of the $x^{\prime}-y^{\prime}$ ellipses are determined by the minimum and maximum divergence angles of each beamlet spot. 

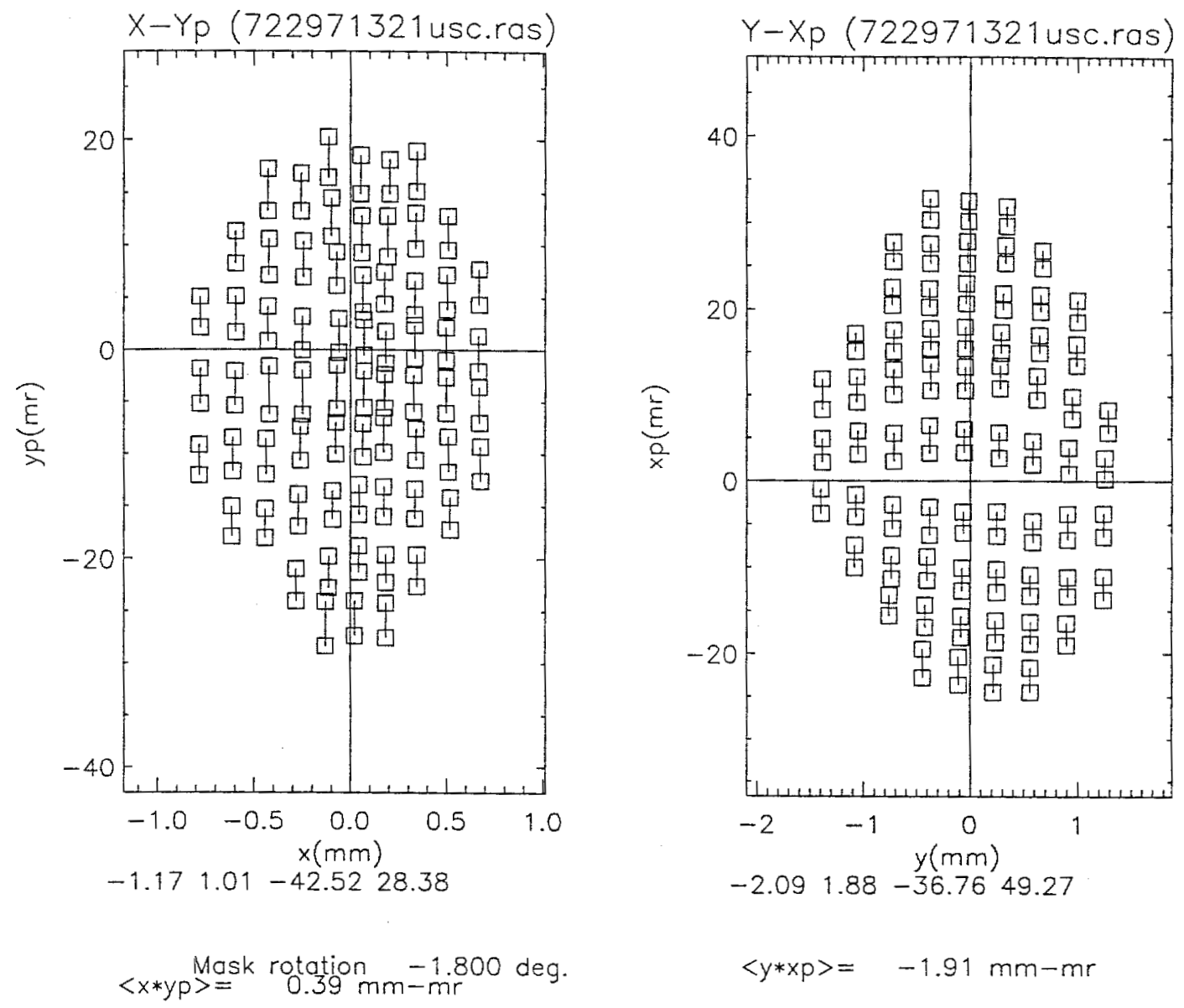

Figure 4.23: Plot of $x-y$ cross moments phase space with $\left\langle x^{\prime}-y\right\rangle$ and $\left\langle x-y^{\prime}\right\rangle$ shown. 

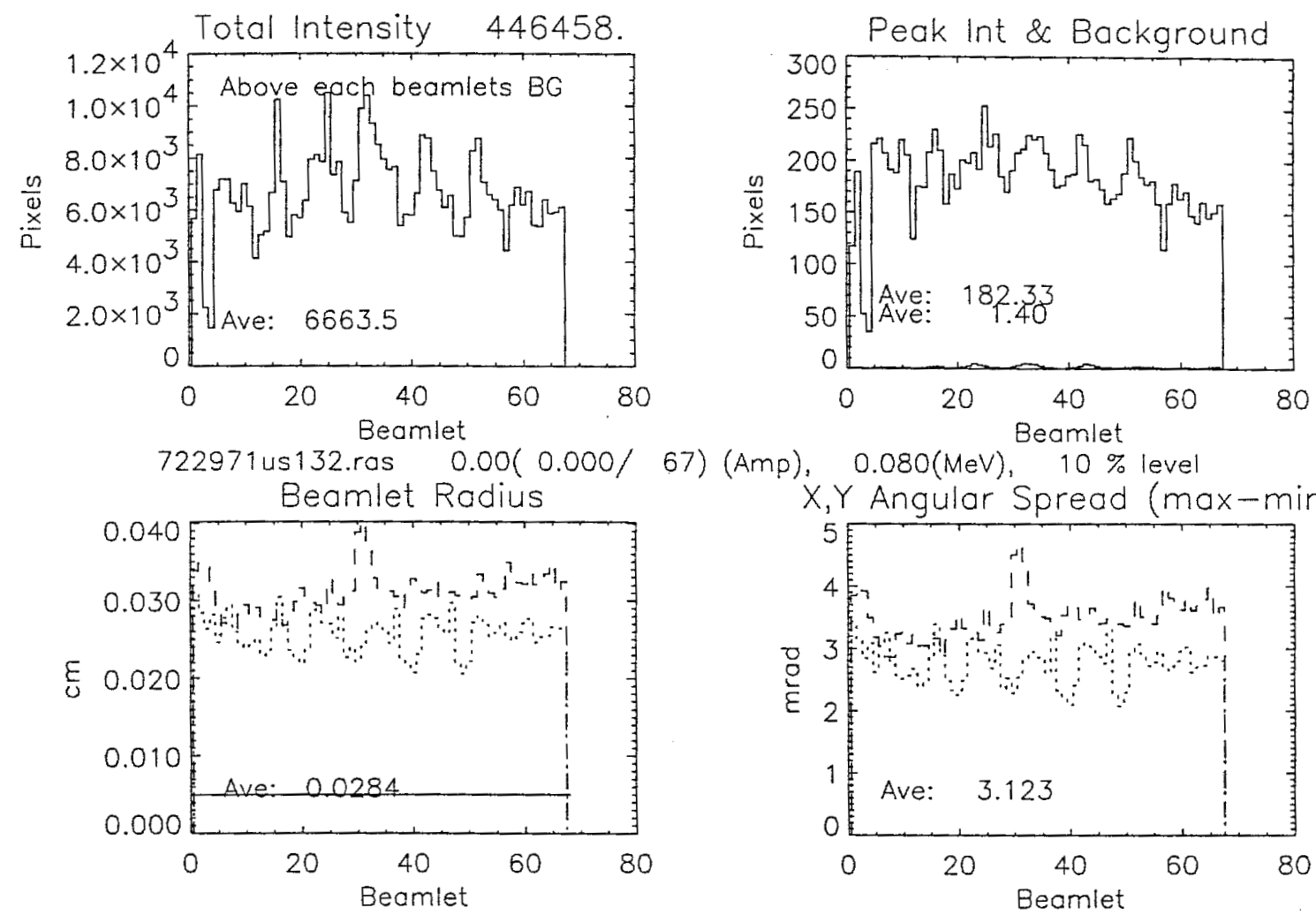

67) (Amp), $0.080(\mathrm{MeV}), \quad 10 \%$ level
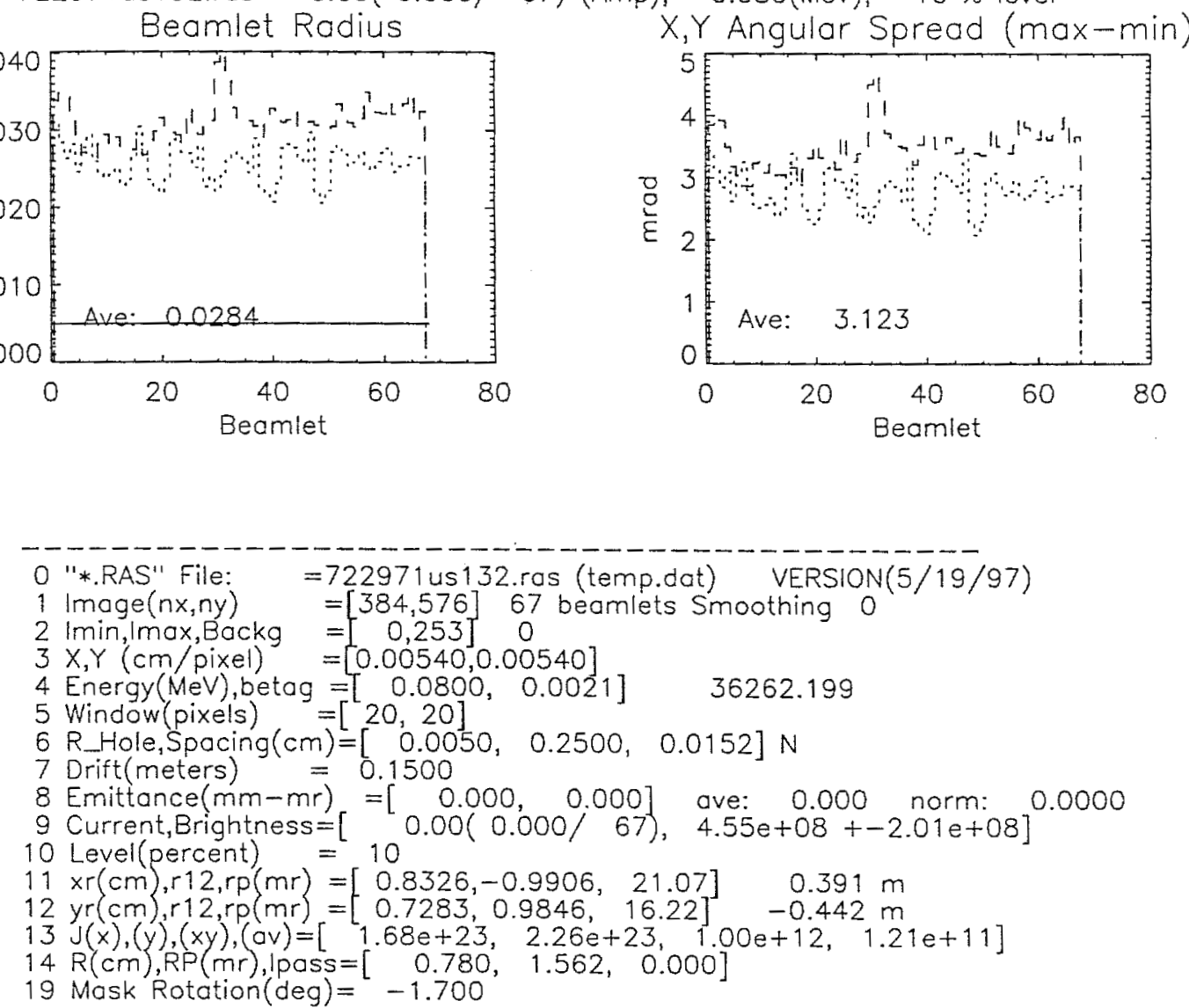

Figure 4.24: Plots of beamlet peak and total intensity statistics. 


\section{Chapter 5}

\section{Detector Selection and Equipment Description}

The selection of a specific material or device to serve as the beamlet detector in the GBI was done by considering the desired performance criteria. These criteria were:

1. Detector light output to be a linear function of incident beamlet intensity.

2. Shot-to-shot repeatability.

3. Fast detector response time to allow gating the camera.

4. Simplicity in construction.

\subsection{Fast Plastic Scintillator as a Heavy Ion Detector}

Due to the heavy ion's short range, its energy is deposited in a very small volume of material. ${ }^{34}$ Phosphors are known to damage easily necessitating their frequent replacement. They also have generally long decay constants on the order of $300 \mu \mathrm{s}$, making them unsuitable for gating the camera at the desired interval of $100 \mathrm{~ns}$ and thus were not considered. The initial promising material considered was commercially available fast plastic scintillator. I conducted experimental studies on fast plastic scintillator, specifically BICRON Corporation BC-412. Its important characteristics are summarized in Table 5.1.

BC-412 was chosen based on its short decay constant, and good mechanical properties. 
Table 5.1: BC-412 scintillator properties ${ }^{52}$

\begin{tabular}{|l|l|}
\hline Light Output \% Anthracene & 60 \\
\hline Wavelength of Maximum Emission & $434 \mathrm{~nm}$ \\
\hline Decay Constant & $3.3 \mathrm{~ns}$ \\
\hline Bulk Light Attenuation & $400 \mathrm{~cm}$ \\
\hline
\end{tabular}

It was unknown whether its light output would be sufficient for small, nanosecond gated signals or if its molecular structure responsible for scintillation would be rapidly damaged from intercepting the heavy ions. Initially its response to the $80 \mathrm{keV}$ potassium ion beam was characterized by placing it in the Small Recirculator beam and measuring its light output signal with a photomultiplier tube. The output signal was observed to be proportional to, and have the same features as the ion source pulser voltage waveform and Faraday cup traces. The light output closely tracked the signal produced by the ion current into the Faraday cup.

Encouraged by the initial photomultiplier tube results, I then conducted experiments to photograph the image of the beam on the scintillator with increasing ion exposure. I exposed the scintillator to repeated $4 \mu \mathrm{s}$ long, $80 \mathrm{keV}$ pulses ( 368 shots) and periodically examined the image of the beam on the scintillator with a CCD camera. The images showed a rapid degradation in image quality. A plot of the sum of the CCD camera pixel intensity values in the area of the beam image versus exposure is shown in Figure 5.1. It can be seen that the light output in the area of the beam exhibits a sharp drop in level then a slower degradation. The images are shown in Figures 5.2(a)-(d). The previous photomultiplier tube measurements had not shown such a decrease in light output. It appeared from the full CCD images that there had been a lot of light reflected off the interior of the chamber that the photomultiplier tube recorded although the image in the area of interest on the scintillator degraded significantly. This reflected light is not shown in the sequence of Figure 5.2. I conducted several tests to determine whether the surface of the scintillator was charging up and deflecting ions from their trajectories by attaching an electrically grounded, fine mesh screen with an ion transmittance of $90 \%$ to the scintillator surface. No 


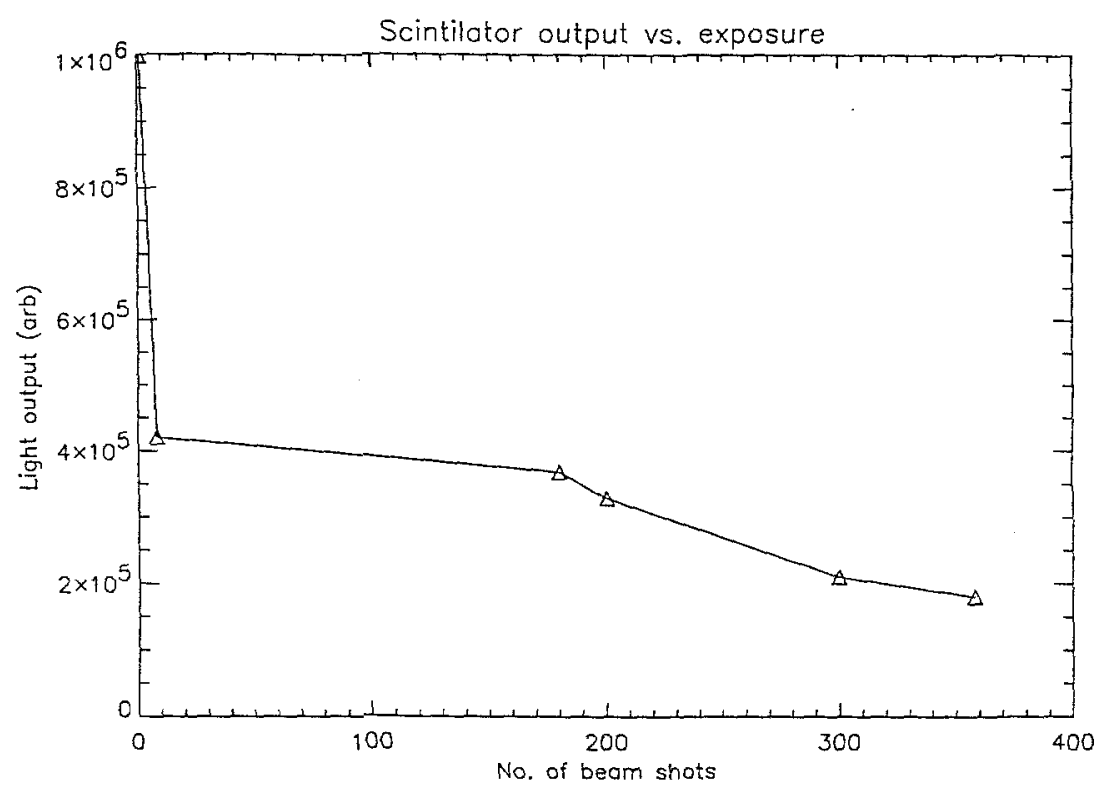

Figure 5.1: BC-412 scintillator light output vs. exposure.

significant effect on preventing image degradation was observed. Various techniques of scintillator surface preparation were also attempted to ensure a clean surface was exposed to the beam. Visual inspection of the scintillator after exposure showed no discernible changes such as clouding or yellowing of the material that would affect its light transmission. Scintillator once exposed also exhibited no recovery after not being exposed for up to a week afterwards and then tested again. Confirmation that damage was occurring in the localized area where beam ions interacted was tested by steering the beam to unexposed portions of the scintillator after at least 357 beam pulses. From Figure 5.2(d), it can be seen that the light output increases for that portion of the scintillator not previously exposed to the beam as the beam is steered to the right at the detector on shot 358 . Various schemes of introducing unexposed scintillator into the beam for each shot were considered such as a rotating wheel of scintillator or "window shade" roller assembly. Although this was a complication in the GBI design it appeared to be feasible.

The remaining question was whether sufficient optical signal would be present 
with a pepperpot introduced into the beam. This would cut the number of ions on the scintillator by roughly a factor of 40,000 and gating the CCD camera at a 100 ns interval would capture about $2.5 \%$ of that signal. I performed an experiment to image $4 \mu$ s long beamlets produced by a pepperpot with 400 micron diameter holes on unexposed scintillator. The images showed that not enough signal was produced to give confidence that accurate beamlet spot identification and analysis could be done. The ultimate pepperpot design was to have 100 micron diameter holes and to apply a $100 \mathrm{~ns}$ gate to the beam signal. As a result of the complications of scintillator damage and low light output in response to heavy ions, it was determined that fast plastic scintillator would be unsuitable as the detector in the GBI. Attention was now given to using a microchannel plate detector with the obvious sacrifice in simplicity and cost. 


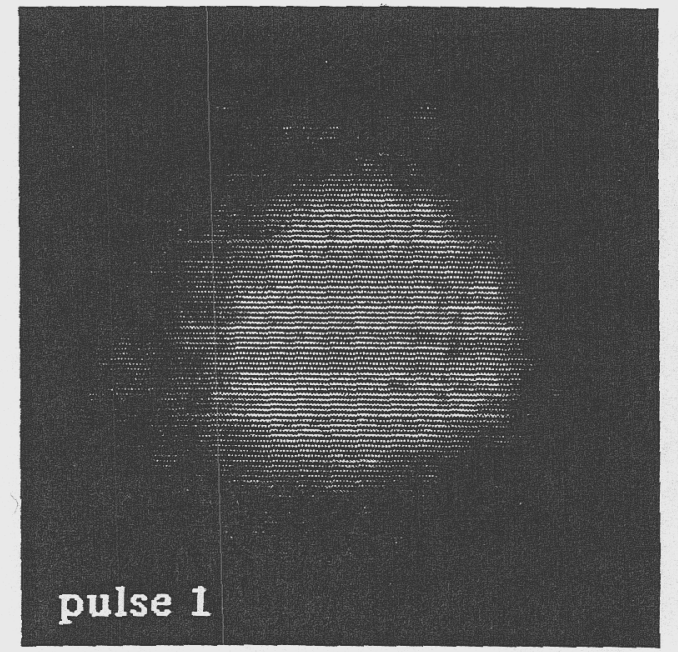

(a)

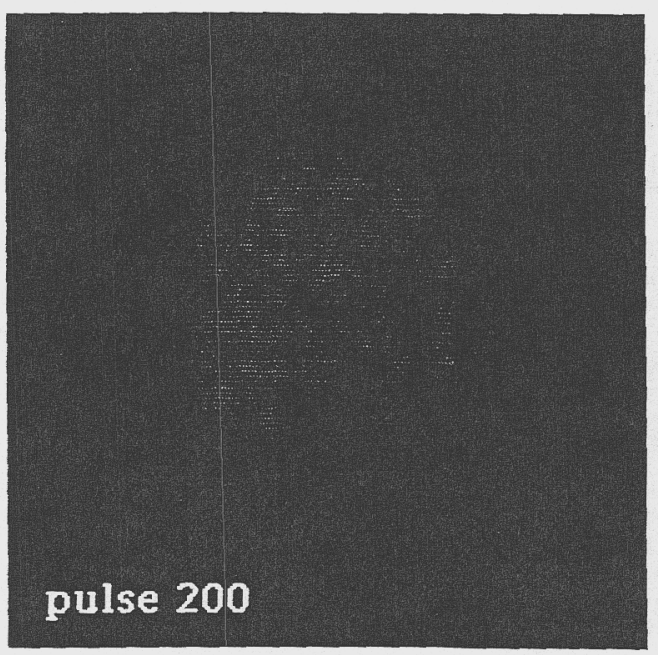

(c)

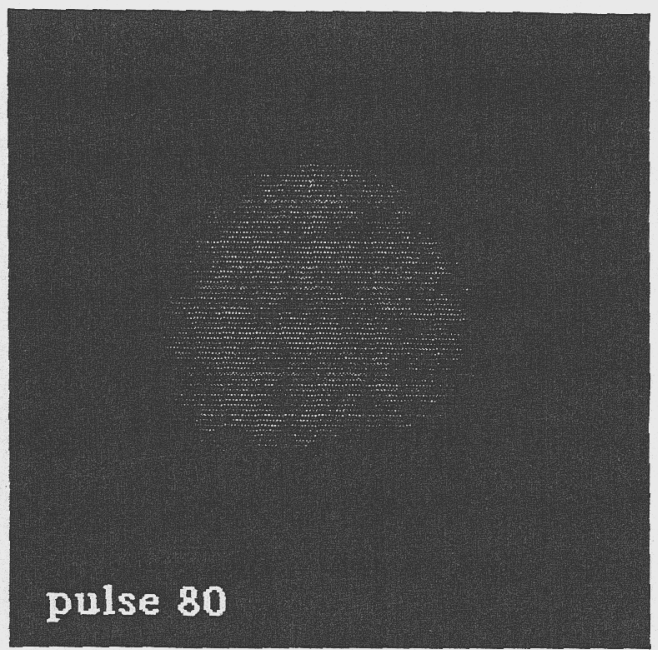

(b)

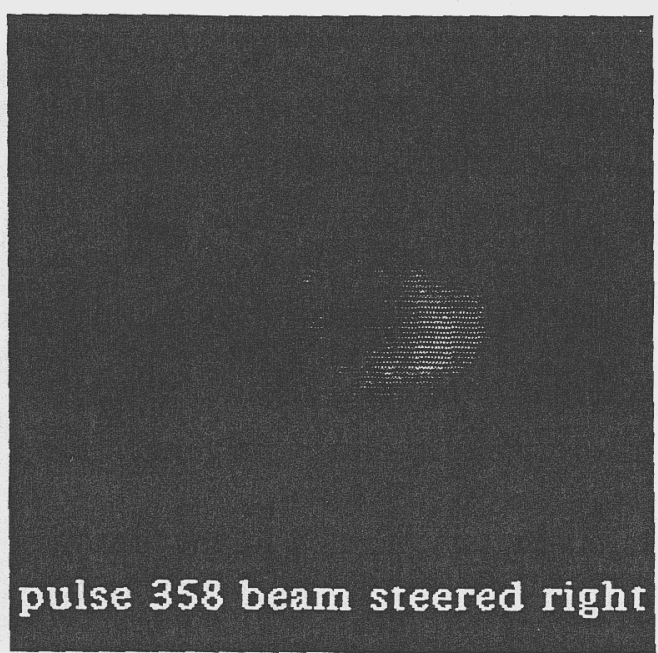

(d)

Figure 5.2: Sequence of whole beam images showing scintillator degradation with exposure. (a) First shot onto unexposed scintillator (b-c) Scintillator exposed to 80 and $2004 \mu$ s shots (d) Beam steered slightly right onto unexposed scintillator after 357 shots.

\subsection{Microchannel Plate Detector as a Heavy Ion Detector}

The response of phosphors and scintillators to intense electron beams is known to be satisfactory from the standpoint of using them as reliable and accurate particle 
detectors. ${ }^{35}$ The stopping power is much less for electrons than for heavy ions resulting in less energy deposited per unit volume and more interactions giving rise to photons in the visible spectrum. A device that converts the heavy ions in the pepperpot beamlets to an electron signal while maintaining the spatial orientation of original beamlets is the microchannel plate (MCP) consisting of many tiny parallel electron multiplying channels spaced tens of microns apart. Initial consideration on the use of a MCP as a detector revealed some useful advantages and some disadvantages that needed to be tested to determine whether they would limit the usefulness of the detector. The advantages which brought MCP's under initial consideration were:

1. The heavy ions could be converted to electrons which then interact with a detector material, thus side-stepping the problems seen with heavy ion damage.

2. MCP's could provide signal amplification once the heavy ions were converted to electrons while maintaining spatial resolution of the beamlet image.

Concerns regarding the MCP were:

1. Unknown damage response of the MCP due to intense exposure to heavy ions.

2. How to obtain fast gated images as commercially available MCP's use relatively slow phosphors as output screens.

A survey of commercially available devices showed that a detector with desirable characteristics could be purchased from several companies. A device purchased from Galileo Electro-Optics Corporation and used in these experiments is shown in Figure 5.3(a). A schematic of the device is shown in Figure 5.3(b) and its important characteristics shown in Table 5.2. An attractive feature of the Galileo MCP detector was the stainless steel front anode with minimum thickness of $1500-2000 \AA$ plated onto the input side of the MCP. Its primary purpose is to allow placing a bias voltage across the channels. It is also thick enough to stop all the incident potassium ions in the Small Recirculator beam at their incident energies of $80-320 \mathrm{keV}$, thus protecting the microchannels from any damage that would affect their performance. To model the ability of the front metal anode to stop the heavy ions, the computer code TRIM (Transport of Ions in Matter) was used to calculate the ion range and statistics of their interaction with the metal anode. ${ }^{36}$ Table 5.3 shows the calculated statistics of 
$320 \mathrm{keV}$ potassium ions stopping in the MCP front metal anode.
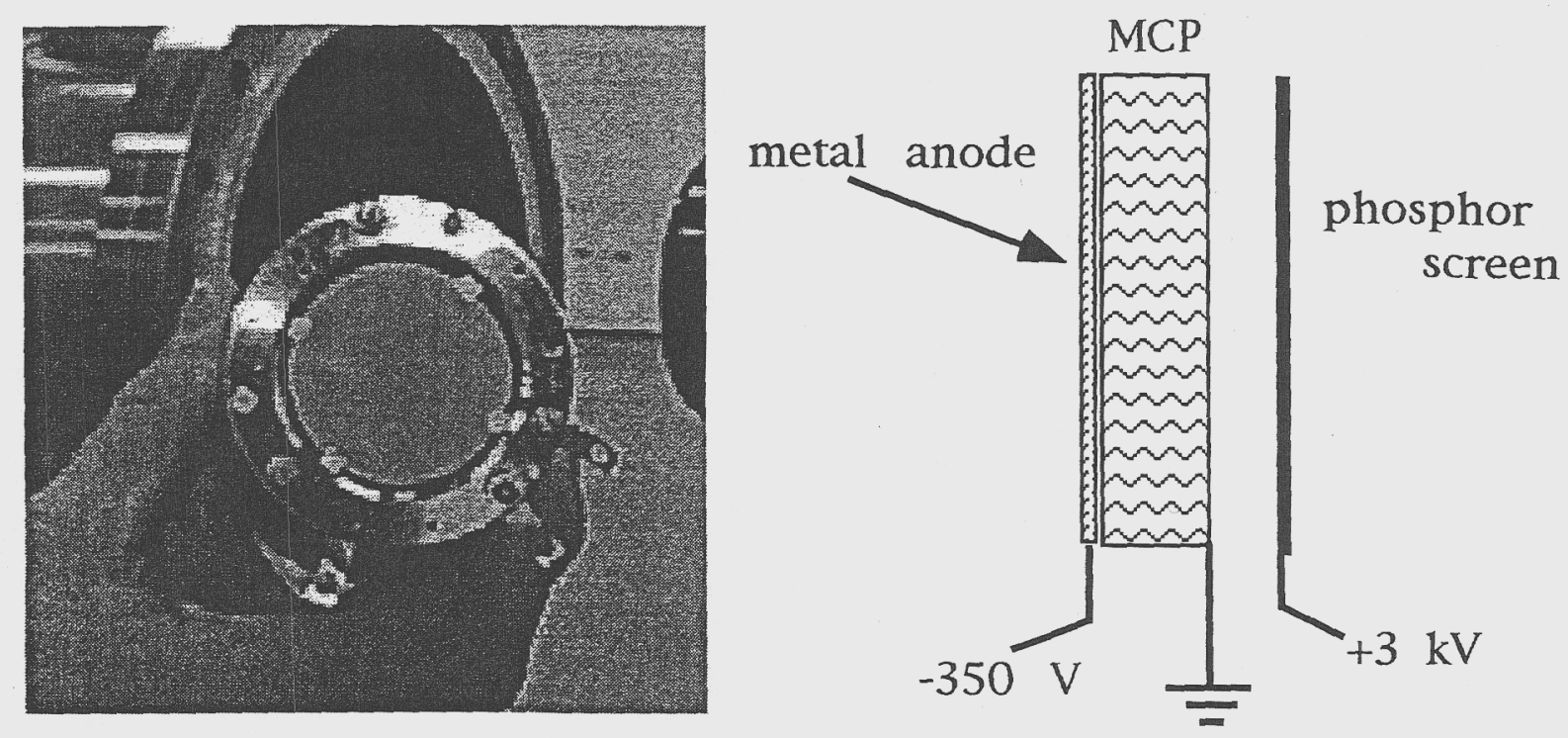

Figure 5.3: (a)Galileo Corp. microchannel plate and phosphor readout (b) MCP biasing scheme for detection of positive ions.

Table 5.2: Galileo microchannel plate characteristics ${ }^{37}$

\begin{tabular}{|l|l||l|l|}
\hline Diameter & $40 \mathrm{~mm}$ & Channel diameter & 10 microns \\
\hline Thickness & $0.46 \mathrm{~mm}$ & Channel spacing & 12 microns \\
\hline Anode thickness & $1500 \AA$ & Channel bias angle & $8^{\circ}$ \\
\hline Bias current & $8-40 \mu \mathrm{mps}$ & Channel coating & $\mathrm{MgO}$ \\
\hline Phosphor screen & $\mathrm{P} 20$ & Min. gain @1000V & 5000 \\
\hline
\end{tabular}

During the stopping of the ions in the metal anode, secondary electrons are produced. At $80 \mathrm{keV}$ it is estimated that 3-5 secondary electrons with initial energies of a few $\mathrm{eV}$ are produced based on the empirical data in Kaminsky. ${ }^{38}$ When the MCP is biased as shown in Figure 5.3(b) the electrons are accelerated into the MCP and amplified through interactions with the walls of the microchannels. A first order calculation of the number of secondary electrons produced in the anode from a $100 \mathrm{~ns}$ 
long, 100 micron diameter beamlet formed from the Small Recirculator beam pulse shows that about $9.4 \times 10^{4}$ electrons would be produced. The MCP has a minimum amplification of 5000 at $1 \mathrm{kV}$ bias so there is more than adequate electron signal from a single gated beamlet to produce an optical signal in the output phosphor. From the output of the MCP, the electrons are proximity focused across a thin gap by a strong positive bias of $3 \mathrm{kV}$ onto a P20 phosphor screen which produces the image of the beamlet spot. The adjustable gain of the MCP allows amplification of very fast gated signals which proved problematic in the testing of fast plastic scintillator as a detector material.

Table 5.3: Transport of Ions in Matter (TRIM code) ion stopping statistics for the front metal anode of the MCP detector

\begin{tabular}{lcc}
\hline & Range $(A)$ & Straggle $(\AA)$ \\
\cline { 2 - 3 } Longitudinal & 363 & 170 \\
Lateral & 124 & 71 \\
Radial & 194 & 108 \\
Vacancies/ion & 750.7 & \\
Energy loss (\%) & $\underline{\text { Ions }}$ & $\underline{\text { Recoils }}$ \\
Ionization & 22.67 & 18.39 \\
Vacancies & 0.14 & 1.73 \\
Phonons & 0.71 & 56.35 \\
\hline
\end{tabular}

The microchannel axes of the MCP are manufactured at an $8^{\circ}$ angle to the surface of the MCP. This improves the efficiency of the first interaction of incident electrons with the channel walls and prevents electrons from streaming through the channels without wall interactions. This $8^{\circ}$ angle results in a 46 micron position offset of the output of the channels from the input, thus all the beamlet images are equally shifted by this amount. The high voltage used in the proximity focusing and the short distance from the channel output to the phosphor reduces to a minimum additional offset across the output gap. This shift in the overall position of the beamlet images was considered negligible in the moment calculations discussed in Chapter 4.

The spatial resolution of the MCP is determined from the size and spacing of its 
microchannels. The Galileo MCP used in the GBI has channels 10 micron in diameter with a center-to-center spacing of 12 microns. A manufacturer's plot of resolution in microns versus channel spacing is shown in Figure 5.4. Given the channel spacing of 12 microns the uncertainty in detecting a "sharp" beamlet edge is 12 microns. This is an indication of the lower limit on the "graininess" of the beamlet images. However, it is not the limiting factor in system resolution as the pixel size on the camera CCD chip is 22.4 microns square, which is the limiting spatial factor in resolution. In fact, the optical resolution achieved in the GBI camera system during data runs was 54 microns per pixel, well within the MCP and CCD limit.

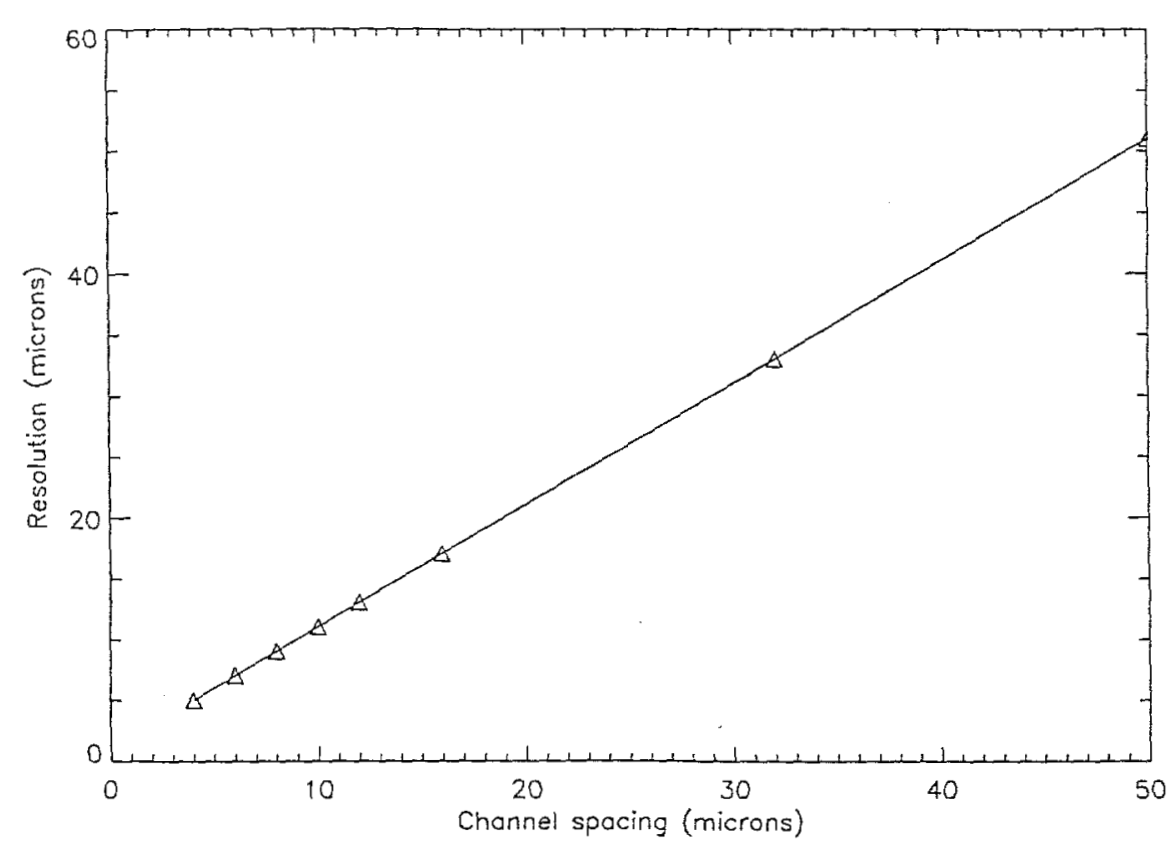

Figure 5.4: Microchannel plate resolution ${ }^{37}$ 


\subsection{Obtaining Time-Resolved Beam Data by Gating the $\mathrm{MCP}$}

Using a MCP detector with a slow phosphor output precluded gating the CCD camera at a gate much shorter than the phosphor decay constant. To do so would include the image produced from ions arriving at the detector prior to the desired time interval. Faster gating required a different method than camera gating to be able to resolve the time history of the beam. It was necessary to gate the MCP on and off such that it was "opaque" to ions outside the desired time interval.

The attractive feature of the $\mathrm{BC}-412$ fast plastic scintillator was its fast decay constant of 3.3 ns. This fast decay constant produced an image that contained the information from a 107.6 ns long slice of the beam when the camera was gated "ON" for 100 ns. The extra $7.6 \mathrm{~ns}$ of information results from light output corresponding to ions intercepted just prior to the camera gate. The fast decay constant easily allowed isolating narrow beam slices using the camera gate. On the other hand, the P20 phosphor of the MCP output has a decay constant of $200 \mu \mathrm{s}$, thus gating the camera for the desired $100 \mathrm{~ns}$ with the MCP operated in the DC mode "contaminates" the image obtained with the output of any previous beam impinging the detector prior to the gate interval. To overcome this difficulty a "Pinhole Neutron Experiment (PINEX)" MCP intensifier gate circuit and power supply (Figure 5.5) was obtained on loan from the Amador Valley Operations Office of Bechtel Nevada Corporation. The PINEX gate power supply is able to bias the MCP "ON" with a $-385 \mathrm{~V}$ pulse for $100 \mathrm{~ns}$ duration. An oscilloscope trace of a $100 \mathrm{~ns}$ and a $1 \mu \mathrm{s}$ gate signal is shown in Figure 5.6. The detector is effectively opaque to the secondary electrons generated by the heavy ions when not gated "ON", thus no optical output signal is produced. At these fast gates it was difficult to obtain a totally clean MCP bias signal with a rapid and smooth rise and fall. The trailing voltage spikes seen in the 100 ns trace of Figure 5.6(b) do not produce significant amplification of the beam signal due to the nonlinear response of the MCP to bias voltage, thus the gate signal is effectively 100 ns wide. Figure 5.7 is a schematic of the GBI equipment interconnections that were 
necessary to obtain gated images of the beam.

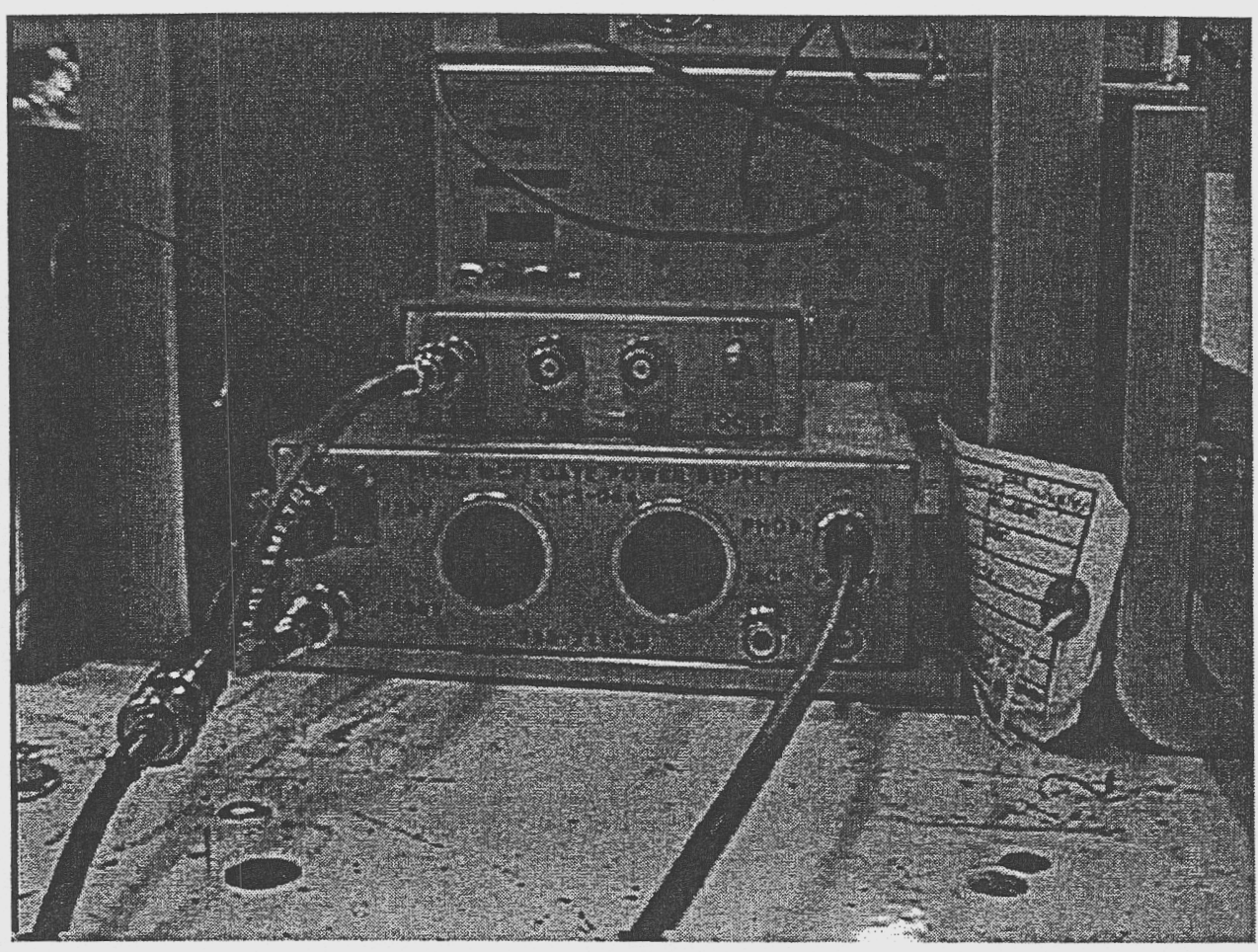

Figure 5.5: PINEX MCP gate circuit and power supply. 
(a) One us gate signai

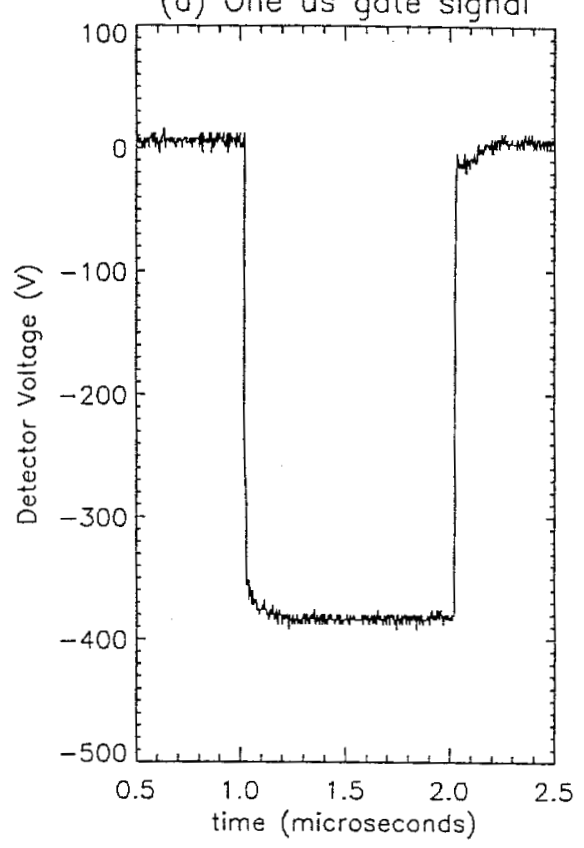

(b) $100 \mathrm{~ns}$ gate signa!

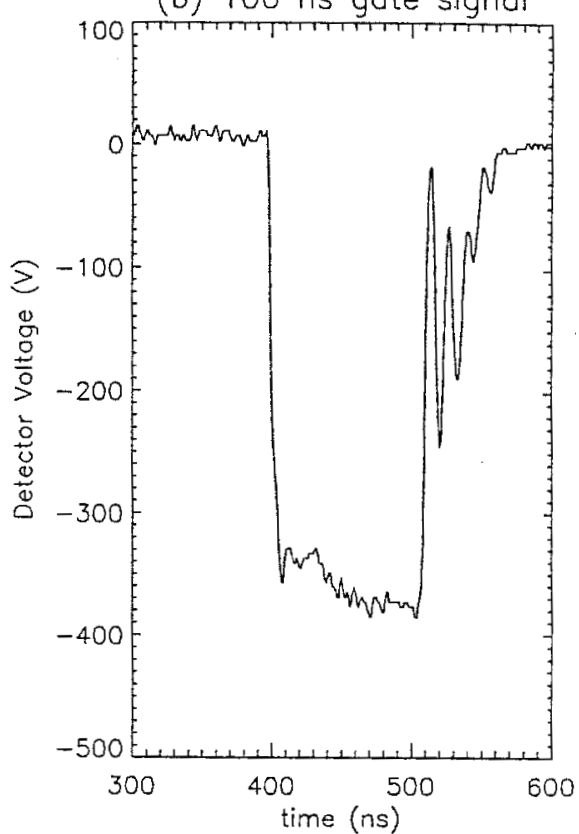

Figure 5.6: MCP detector gate signal (a) $1 \mu$ s gate used for image capture of a wide slice out of the beam flattop. (b) 100 ns gate used for the narrow slices to construct a time history of the beam. 


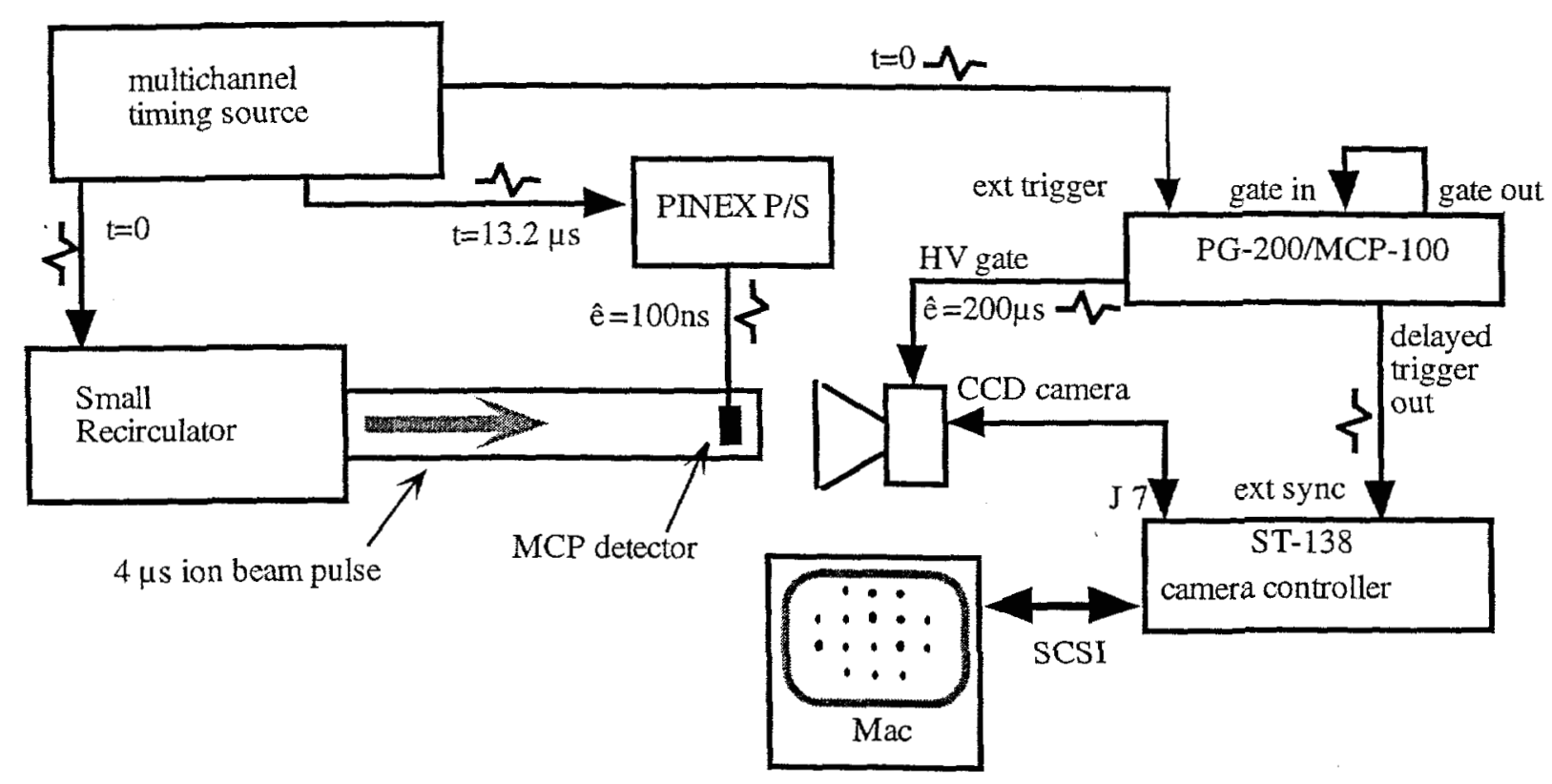

Figure 5.7: GBI equipment interconnections

\subsection{Camera System Description}

The Gated Beam Imager uses an intensified gated CCD camera to view the MCP detector output screen and capture the image of the beamlet on the detector. The camera is mounted outside the GBI diagnostic chamber in which the pepperpot and MCP detector are housed in the beamline as shown in Figure 5.8. The MCP phosphor screen is observed through a glass viewport either directly at the end of the beamline or with a mirror arrangement if placed in an intermediate diagnostic station. The camera system used was manufactured by Princeton Instruments, Inc. Inside the camera housing, a 384 by 576 pixel CCD of dimension 8.6 by $12.9 \mathrm{~mm}$ is fiber-optically coupled to an image intensifier allowing the observed image to be amplified and gated 
to approximately $5 \mathrm{~ns}$. To reduce dark charge buildup in the CCD during exposure and readout, it is seated on a cold finger which, in turn is seated on a thermoelectric (Peltier Effect) cooler. The cooler is mounted on a heat removal block cooled by air from cooling fans in the camera housing. To maximize the light transmission from the MCP phosphor screen to the camera CCD, I chose an $\mathrm{f} / 1.2$ Canon lens for its low f-stop and $85 \mathrm{~mm}$ focal length. In order to allow close-in focusing it was necessary to use a $32 \mathrm{~mm}$ lens extension between the camera and lens as shown in Figure 5.8. This allowed for a sharp focus to within 8 inches of the detector, and the appropriately sized field-of-view to maximize the spatial resolution of the data images.

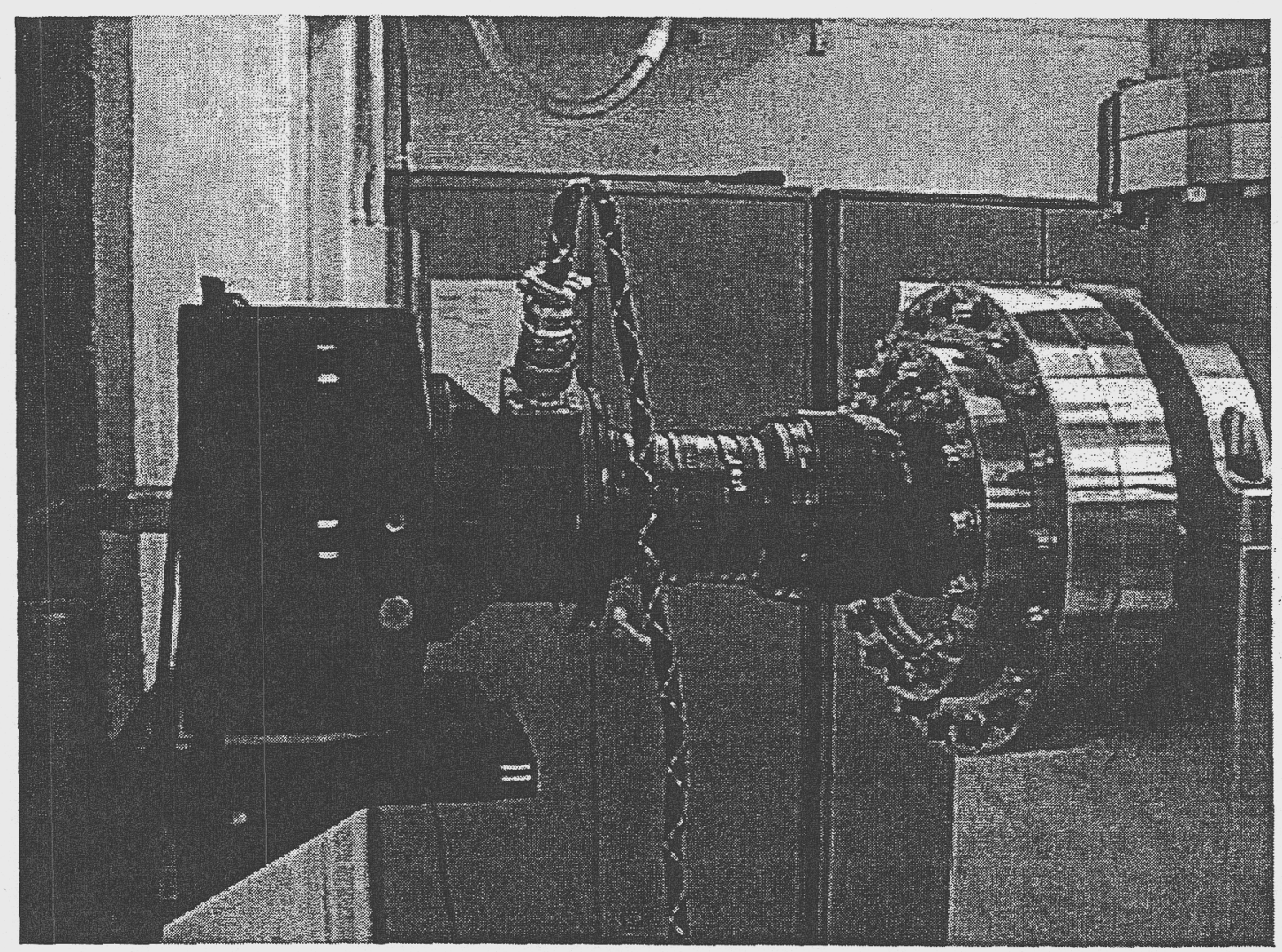

Figure 5.8: GBI CCD camera in position. Canon $\mathrm{f} / 1.2$ lens mounted on a $32 \mathrm{~mm}$ lens extension to provide close focusing to within 8 inches of the MCP detector. 
A Princeton Instruments ST-138 CCD camera controller controls the readout of the CCD and the operation of the CCD cooler. The readout of the CCD after exposure is acquired into memory of a Macintosh computer via a serial interface card connected to the ST-138. Gating the camera intensifier on and off is accomplished with a Princeton Instruments PG-200 Programmable Pulse Generator. Both the ST-138 and PG-200 are shown in Figure 5.9. Voltage ranging from 500-1000 V can be applied to the camera intensifier to amplify light passed to the camera CCD. The PG-200 also controls the timing of the voltage to the intensifier in response to the experiment timing reference. A commercial software, IPLab Spectrum ${ }^{T M}$ with Princeton Instruments-supplied plug-in software modules was used to both control the camera exposure and display the CCD readout of the beamlet images immediately after acquisition.

\subsection{Operating the Gated Beam Imager}

To acquire a gated image of the beam, a time-of-flight calculation for a given beam energy is made to estimate when ions will arrive at the detector after being transported along the Small Recirculator. The head of the beam is then searched for by adjusting the actual gate start time while observing the images. The proximity focusing voltage of $3 \mathrm{kV}$ from the microchannel output to the phosphor screen is applied continuously and the MCP is biased "ON" and "OFF" by the PINEX power supply. As mentioned before, the 100 ns gate on the MCP produces an image on the phosphor screen that has a $200 \mu \mathrm{s}$ decay time. In order to increase the signalto-noise ratio in the pixel voltages read out of the camera CCD it is desirable to gate the camera electronic shutter observing the MCP detector phosphor output. To accomplish this, the camera is gated on for $300 \mu$ s starting coincident with the MCP detector gate pulse. This gate proved to be optimum for excluding stray light in the GBI diagnostic chamber while allowing adequate signal for good beamlet spot resolution. A diagram of the timing sequence for acquiring a 100 ns gated image is shown in Figure 5.10. The long readout time $(4.4$ seconds) from the CCD to the 


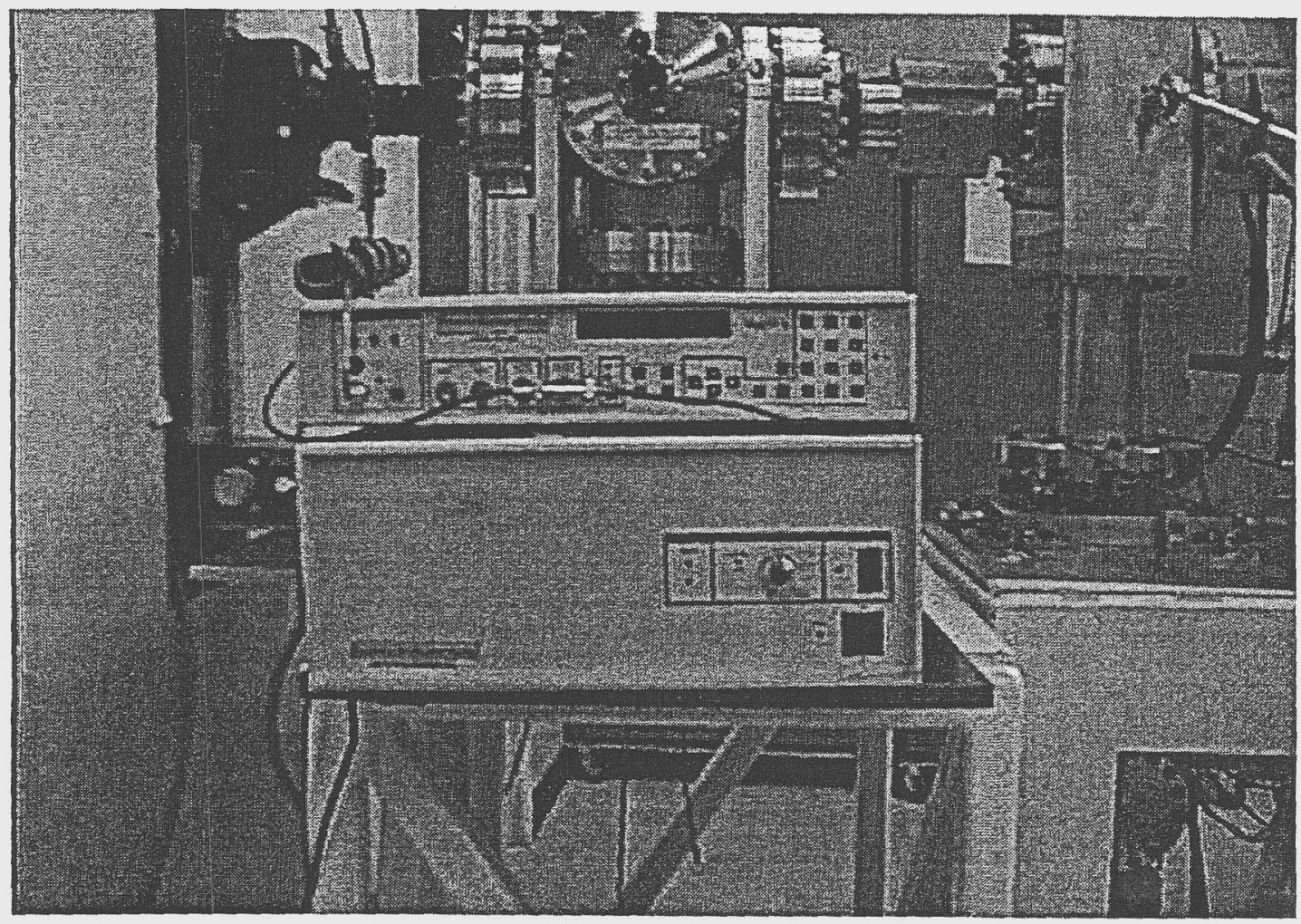

Figure 5.9: Princeton Instruments ST-138 CCD camera controller(lower) and PG-200 CCD intensifier power supply (upper). The wrapped coil on the left side of the PG200 adds inductance to the trigger input cable to prevent prematurely triggering on the rapid electrical transients picked up from the ion source pulser spark gap switches.

computer is a consequence of the camera's "slow scan mode" clock frequency of 50 $\mathrm{kHz}$. A slow readout of the camera CCD is important in limiting the readout noise in the image as readout noise is proportional to the readout clock frequency. ${ }^{39}$ Reading out images when not collecting data, such as focusing the camera was done at a $1 \mathrm{MHz}$ clock frequency resulting in a 0.22 second readout time allowing easier lens adjustment while viewing the image. After readout, the captured image is displayed and examined for quality prior to saving by the operator. For stepping through the beam, the trigger to the PINEX power supply is then delayed the appropriate interval (e.g., from $13.2 \mu$ s to $13.3 \mu$ s for 100 ns slices) and another image acquired on the next beam shot. 


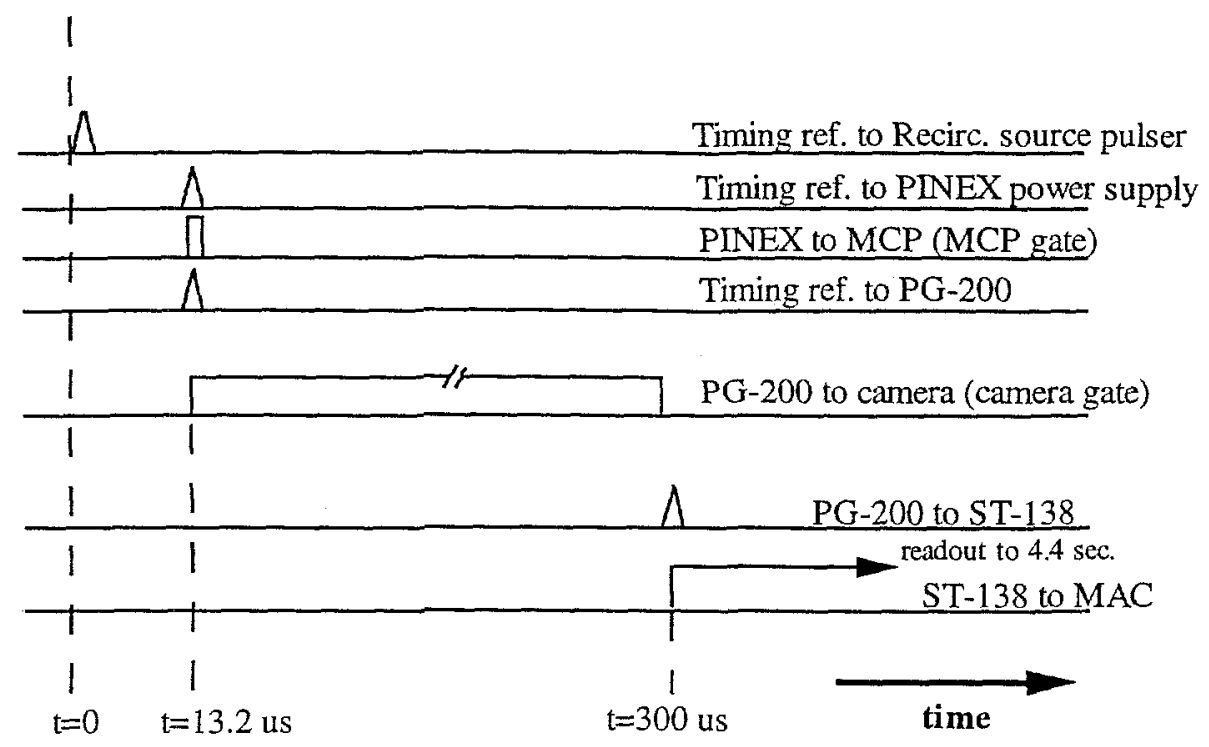

Figure 5.10: GBI image capture timing diagram.

\subsection{Applying Corrections to the GBI Images}

It was necessary to apply corrections to the data images in order to account for the response of the CCD to dark charge accumulation during exposure and readout and for differing pixel sensitivities that result from manufacturing tolerances from pixelto-pixel in the same CCD. Corrections were also made to account for the uneven response across the $\mathrm{MCP}$ detector to an even intensity input.

\subsubsection{CCD Dark Charge Correction}

Dark charge accumulation is a function of exposure and readout time, and the temperature of the CCD. ${ }^{40}$ Several dark charge measurements with the same exposure and readout times as data images were made during the data runs to account for any change due temperature drift of the camera cooling system, although changes are small due to the camera controller's ability to maintain CCD temperature within 
$\pm 0.05^{\circ} \mathrm{C} .{ }^{41}$ Figure 5.11 shows a typical dark charge result displayed by averaging the CCD pixel rows. The dark charge buildup is fully repeatable given a constant temperature and readout time between exposures and is corrected for by individual pixel value subtraction.

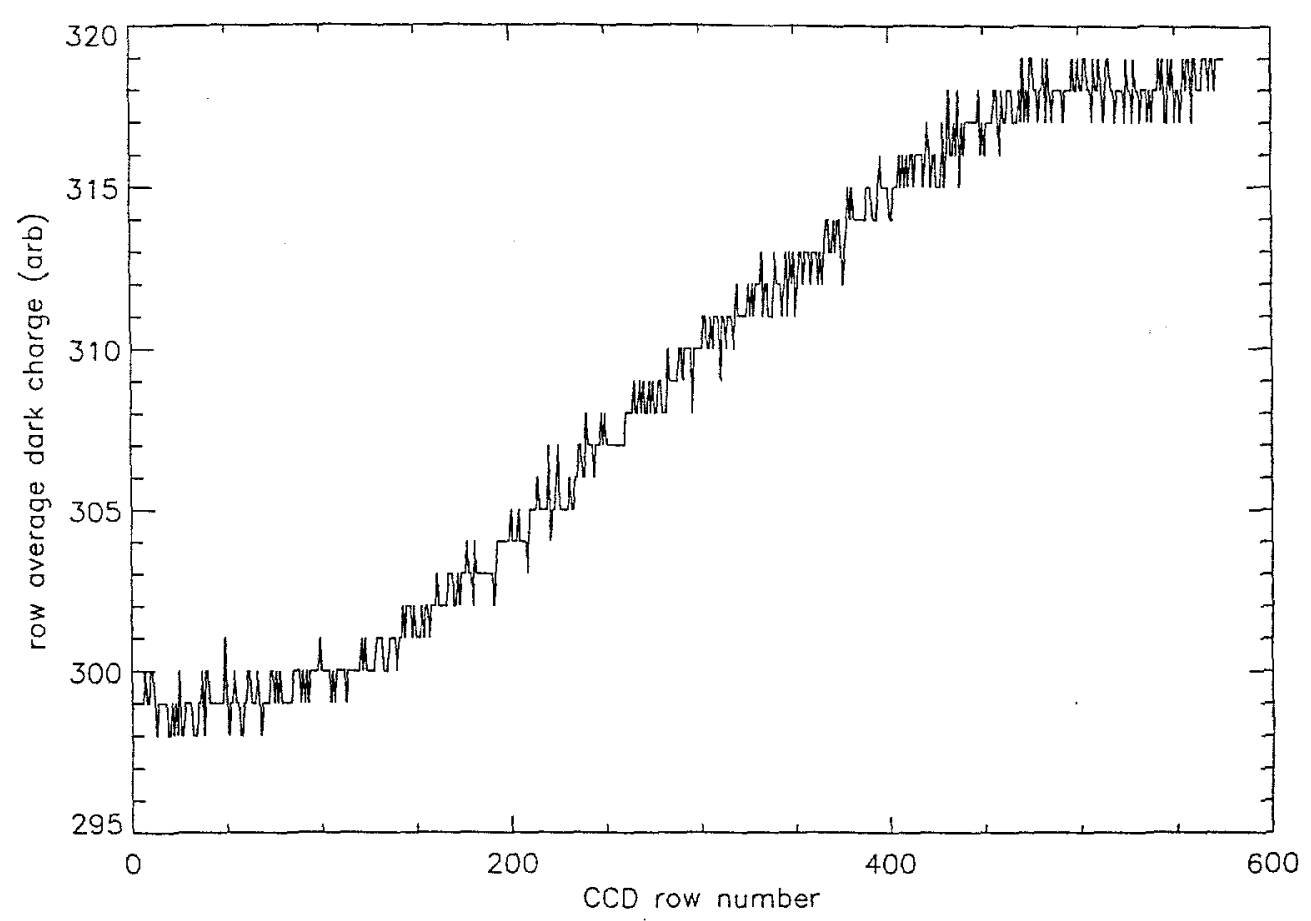

Figure 5.11: Image row average of camera CCD pixel values showing dark charge buildup during the finite time taken to read out the CCD by row from left to right.

\subsubsection{CCD Flat Field Response Correction}

Each CCD chip is unique in its pixel-to-pixel sensitivity due to slight variations in manufacturing. ${ }^{40}$ As well as being unique, the response to the same light level is fully repeatable from exposure to exposure. The difference in pixel sensitivity was determined with a "flat field" image which can be used to multiplicativly correct a data image. The "flat field" image was obtained using the "integrating light sphere" 
shown in Figure 5.12. The interior surface of the sphere is coated with a smooth white diffusing material which provides an evenly illuminated image from a light source in the side of the sphere. A flat field image was taken using a $300 \mu$ s exposure along with a nearly concurrent dark charge image to account for the CCD temperature at the exposure time. Along with these flat field and dark charge images, a data image of the GBI beamlet spots is manipulated within the IPLab Spectrum ${ }^{T M}$ software to correct for the dark charge buildup on the CCD during exposure and the uneven flat field response. The maximum dark charge level for a $300 \mu$ s exposure was about $1.2 \%$ of the peak spot intensity and the peak variation in the flat field measurement was $\pm 16 \%$. Pixel by pixel the correction is made using the formula ${ }^{32}$ :

$$
\text { Corrected Image }=\frac{\text { Mean }(\text { Flat }- \text { Dark } F)(\text { Image }- \text { DarkI })}{(\text { Flat }- \text { DarkF })}
$$

where "Flat" is a flat field image, "DarkF" is a dark charge image associated with the flat field image, "Image" is an image of the beamlet spots, and "Darkr" is a dark charge image associated with the beamlet spots image.

\subsubsection{MCP Detector Flat Field Response Correction}

The third correction made to all data images is a correction to account for the variation in response of the MCP microchannels to a flat field "illumination" ${ }^{37}$ Ideally all microchannels would give the same output for like input as would the phosphor screen. Lacking the ability to generate a detector-size flat field of heavy ions, I tested the MCP using a flat field x-ray source at LLNL. The machine shown in Figure 5.13 with the GBI attached to it generates $7.47 \mathrm{keV}$ x-rays from a nickel anode. Figure 5.14 shows the field-of-view of the CCD camera just prior to $x$-ray illumination and Figure 5.15 shows the flat field image obtained. The response of the MCP varied by as much as $70 \%$ from one edge to the other in a gradual and even manner. Extending this response to an experiment, equivalent beamlets would be weighted more or less in moment calculations depending on their position on the MCP. 


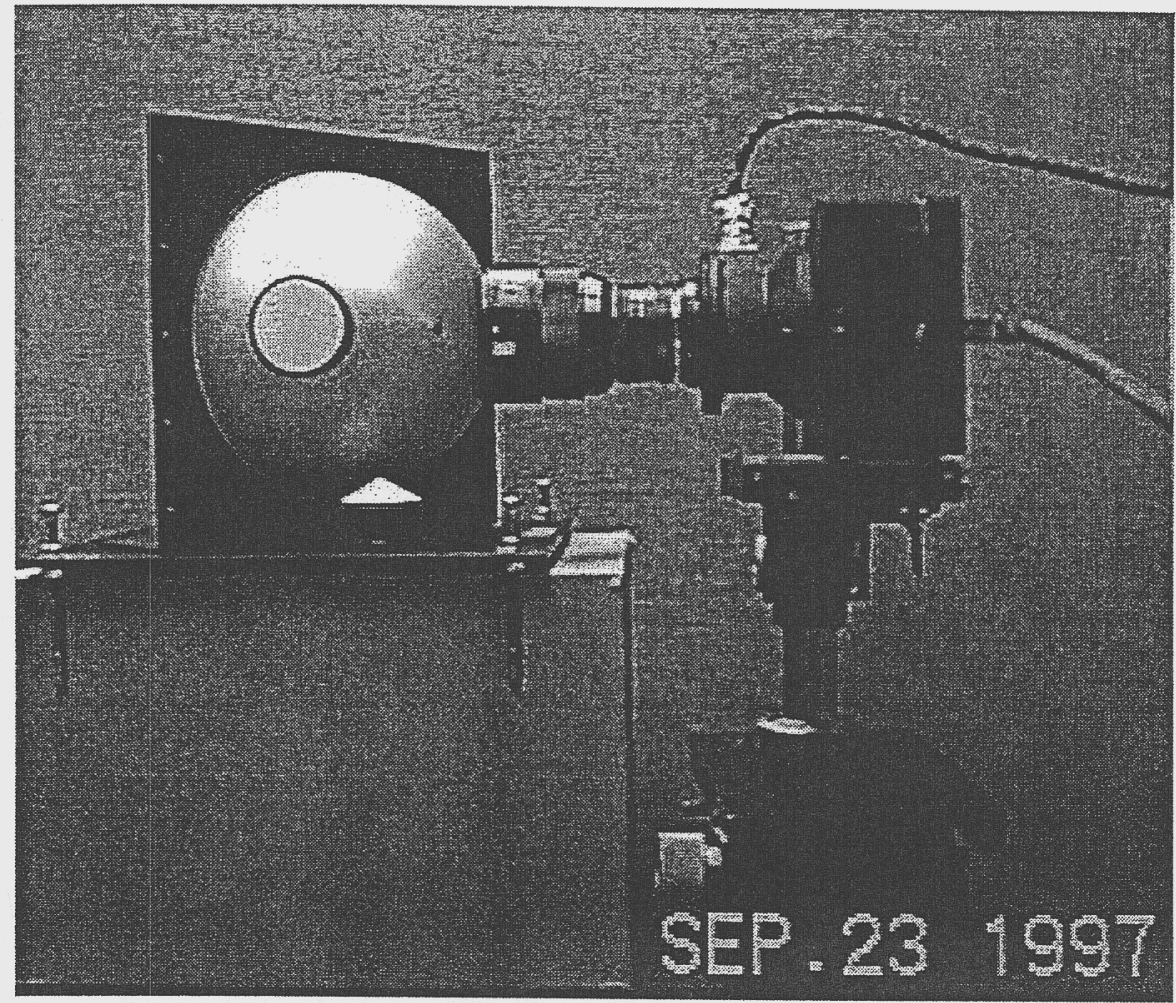

Figure 5.12: CCD camera and integrating light sphere setup for obtaining a flat field image. 


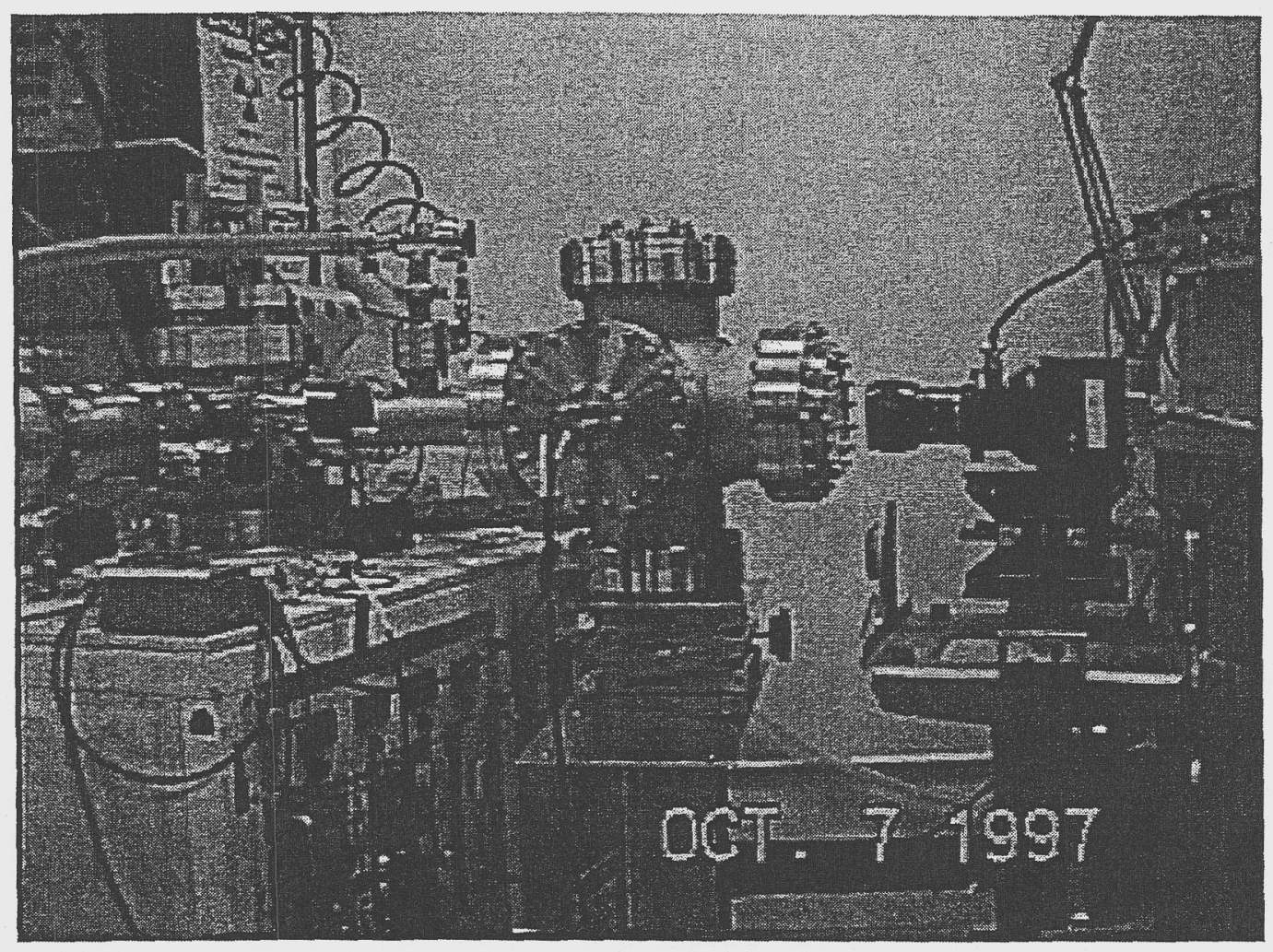

Figure 5.13: GBI attached to a LLNL x-ray source to measure the spatial response of the MCP to a uniform signal.

Once an evenly illuminated MCP image is obtained, the data images can be corrected. As the field of view of the CCD camera differs from the experiment setup to the MCP flat field setup, it is impossible to match up the exact position of the two images. This is not a severe limitation as the change in MCP response is gradual across the detector. The four fiducial marks shown in Figure 5.14 around the periphery of the detector allow the orientation of the flat field and data images to be matched. The larger field of view of the flat field image is measured and the image is extrapolated to the same size as the data image using the IPLab imaging software. The data image is then corrected for the non-uniform response of the MCP using the algorithm of Equation 5.1. 


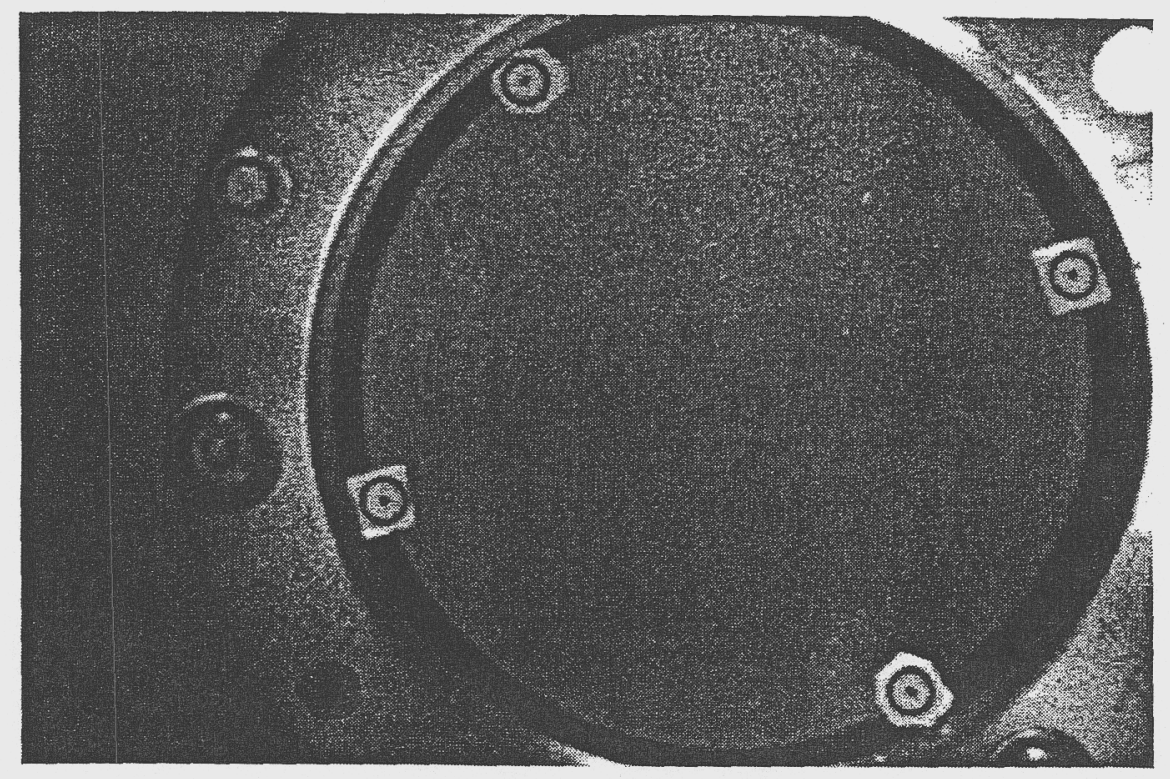

Figure 5.14: Field-of-view of the CCD camera observing the MCP detector for the MCP flat field x-ray measurements.

\subsubsection{Preparing the Images for Data Analysis}

Using a script of IPLab imaging software commands, the image corrections are applied in a matter of seconds. The corrected image in the form of a 2-D array (384 by 576 ) is then saved as a text file with each array address corresponding to a CCD pixel value representing the intensity of light which fell on the pixel. The array is then sent to the DEC Alpha workstation running the IDL pepperpot data analysis code. To conserve memory the array values are linearly scaled from 16 bit digitization ( 0 to 65535 ), to 8 bit digitization ( 0 to 256) and read into memory. Figure 5.16 shows a small part of a text array that includes a beamlet spot. Data analysis then can be carried out in accordance with the methods discussed in Chapter 4. 


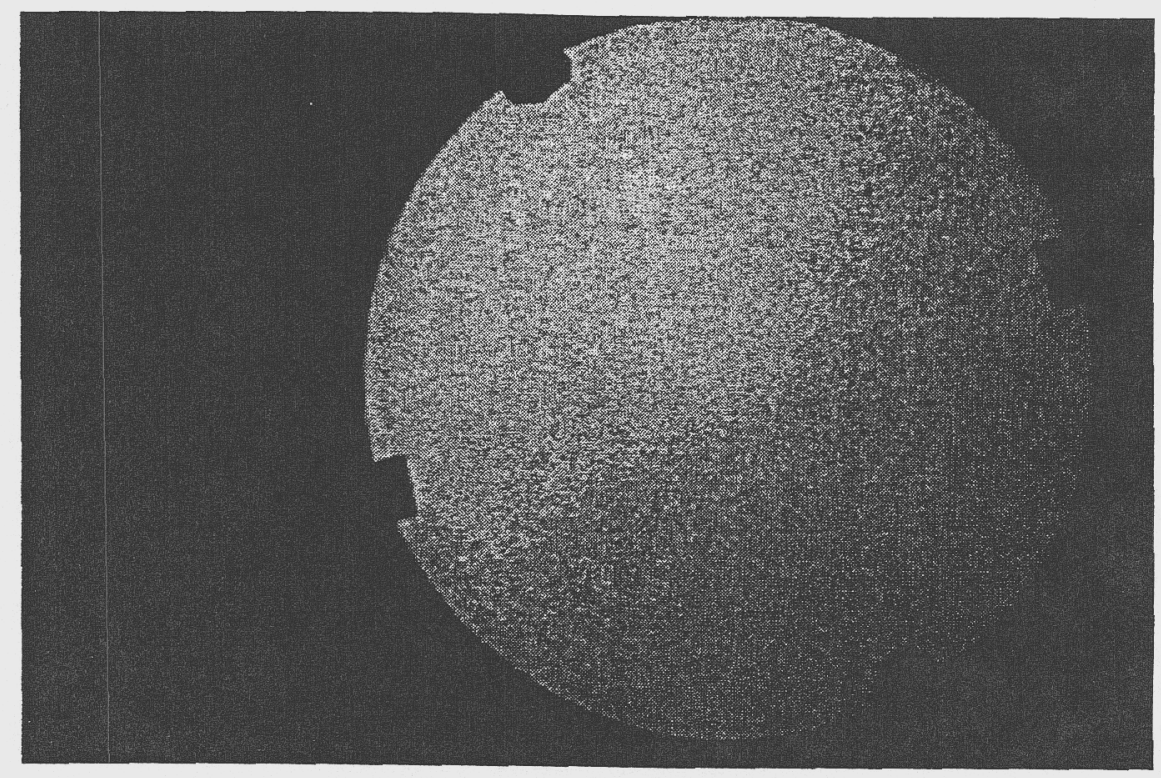

Figure 5.15: View of the non-uniform response of the MCP detector to a uniform field of $7.47 \mathrm{keV} x$-rays. The field of view that matches that of the experimental measurements is selected from this image and applied as a correction for the nonuniform response. 


\begin{tabular}{|cccccccccccccccccc|}
\hline 3 & 3 & 3 & 5 & 7 & 7 & 6 & 4 & 4 & 4 & 4 & 4 & 3 & 3 & 2 & 2 & 2 & 1 \\
3 & 3 & 2 & 3 & 5 & 5 & 5 & 4 & 4 & 5 & 5 & 5 & 4 & 3 & 3 & 2 & 3 & 1 \\
4 & 4 & 2 & 3 & 4 & 5 & 4 & 4 & 5 & 7 & 6 & 6 & 6 & 5 & 4 & 3 & 4 & 2 \\
3 & 3 & 2 & 2 & 4 & 4 & 5 & 7 & 9 & 12 & 12 & 11 & 9 & 7 & 5 & 3 & 4 & 2 \\
2 & 3 & 3 & 3 & 4 & 5 & 7 & 11 & 15 & 19 & 19 & 17 & 12 & 10 & 7 & 4 & 4 & 3 \\
2 & 2 & 2 & 3 & 4 & 5 & 10 & 18 & 29 & 39 & 46 & 42 & 31 & 22 & 12 & 7 & 4 & 2 \\
2 & 3 & 3 & 4 & 5 & 7 & 14 & 27 & 44 & 60 & 74 & 69 & 50 & 34 & 19 & 11 & 5 & 3 \\
2 & 2 & 3 & 4 & 5 & 8 & 17 & 35 & 66 & 98 & 122 & 116 & 88 & 58 & 32 & 18 & 8 & 4 \\
2 & 3 & 4 & 5 & 6 & 11 & 21 & 45 & 89 & 137 & 171 & 165 & 126 & 83 & 46 & 26 & 12 & 6 \\
2 & 3 & 3 & 4 & 6 & 12 & 24 & 50 & 100 & 160 & 197 & 190 & 143 & 95 & 53 & 30 & 13 & 7 \\
3 & 4 & 3 & 5 & 7 & 14 & 29 & 56 & 112 & 185 & 224 & 217 & 160 & 108 & 61 & 34 & 14 & 8 \\
2 & 4 & 3 & 4 & 6 & 13 & 27 & 52 & 100 & 159 & 191 & 184 & 140 & 91 & 51 & 28 & 11 & 7 \\
3 & 4 & 4 & 4 & 7 & 13 & 27 & 48 & 88 & 135 & 158 & 152 & 120 & 75 & 43 & 22 & 9 & 6 \\
2 & 3 & 3 & 3 & 5 & 10 & 21 & 39 & 66 & 97 & 111 & 106 & 83 & 53 & 31 & 17 & 7 & 5 \\
3 & 2 & 3 & 3 & 4 & 9 & 17 & 30 & 45 & 61 & 64 & 60 & 47 & 33 & 21 & 12 & 6 & 4 \\
2 & 2 & 2 & 2 & 4 & 7 & 13 & 22 & 30 & 41 & 43 & 40 & 30 & 22 & 15 & 10 & 5 & 3 \\
3 & 3 & 3 & 3 & 4 & 7 & 11 & 15 & 17 & 22 & 22 & 20 & 15 & 13 & 11 & 8 & 5 & 3 \\
3 & 3 & 2 & 3 & 4 & 6 & 9 & 12 & 13 & 16 & 14 & 13 & 10 & 8 & 7 & 6 & 3 & 2 \\
4 & 4 & 3 & 4 & 4 & 6 & 8 & 10 & 10 & 10 & 7 & 7 & 6 & 5 & 5 & 4 & 3 & 2 \\
3 & 3 & 2 & 3 & 4 & 5 & 7 & 8 & 8 & 7 & 4 & 5 & 5 & 4 & 3 & 3 & 2 & 2 \\
2 & 2 & 3 & 3 & 4 & 4 & 6 & 7 & 7 & 5 & 3 & 4 & 4 & 4 & 3 & 2 & 2 & 2 \\
\hline
\end{tabular}

Figure 5.16 A small sample of a beamlet spot data array file in text format encompassing one beamlet spot. 


\section{Chapter 6}

\section{A Beam Correction System}

The utility of measurements possible with the GBI in the design, construction, and operation of heavy ion accelerators can be realized in several ways. Experiments such as the LLNL Small Recirculator are meant to push the state of the art in induction accelerator design and in intense heavy ion beam control. More advanced measurements of more beam parameters are essential to evaluating its performance. The Small Recirculator is intended to produce data that will benchmark a numerical simulation code such as WARP..$^{15}$ One area that is ideally suited to being diagnosed by the GBI is the effect on the beam of rotationally misaligned quadrupole focusing magnets. The amount of misalignment that can be tolerated is an essential factor in quadrupole magnet design and accelerator construction costs. Measuring the effect of misalignments and removing the effect prior to final focus could increase the quadrupole magnet alignment tolerances and thus reduce accelerator construction and maintenance costs.

\subsection{The Effect on the Beam of Rotationally Misaligned Quadrupoles}

A magnetic quadrupole rotated about the beam axis will not cause a displacement of the beam centroid off-axis if it is initially on-axis. ${ }^{29}$ However, a rotated quadrupole will create a coupling between the previously uncoupled transverse $x$ and $y$ motions 
of beam ions. The beam control elements such as the quadrupoles, steering dipoles, insertion/extraction magnets, and beam final focus magnets are all assumed to be aligned to the laboratory frame. Barnard showed that in the presence of rotational misalignments, the beam 2-D transverse emittance is not conserved even under linear equations of motion, and the beam acquires a finite angular motion. ${ }^{29}$ The detrimental effect of this on the final focus element performance is indeed a real effect as any final focus lens will be designed to be aligned for an assumed orientation of the beam (most likely, upright in the laboratory frame). As the misaligned quadrupole still exerts a linear force on the beam it is possible to "correct" its effect using a suitable number of correction elements generating the proper combination of fields. Linear forces on the beam do not result in hopelessly entrained unoccupied phase space in the beam distribution as in Figure 4.1. A conceptual beam correction system is proposed in the following discussion (see Figure 6.6) that would use as input, the moment data obtained from the GBI.

Barnard derived beam first and second moment evolution equations for a beam transported and accelerated through an alternating gradient focusing, or FODO lattice. $^{29}$ An outline of the derivation appears in Appendix B. He assumed that:

1. The force on the ions comes from two sources; the external focusing (defocusing) from the quadrupolar field, and the internal defocusing from space charge of the beam.

2. The space charge is distributed in a uniform ellipse as in a $\mathrm{K}-\mathrm{V}$ distribution.

Stating the results obtained in Appendix B the two closed sets of coupled moment evolution equations for the first and second moments of the beam distribution are:

\section{First Moments}

$$
\begin{array}{ll}
\frac{d<x\rangle}{d z}=\left\langle x^{\prime}\right\rangle & \left.\frac{\left.d<x^{\prime}\right\rangle}{d z}=K_{q x x}<x>+K_{q x y}<y>-\left(d \ln \beta_{z} / d z\right)<x^{\prime}\right\rangle \\
\frac{d<y>}{d z}=\left\langle y^{\prime}\right\rangle & \left.\frac{\left.d<y^{\prime}\right\rangle}{d z}=K_{q y y}<y>+K_{q y x}<x>-\left(d \ln \beta_{z} / d z\right)<y^{\prime}\right\rangle
\end{array}
$$


Second Moments

$$
\begin{aligned}
& \frac{d \Delta x^{2}}{d z}=2 \Delta x x^{\prime} \\
& \frac{d \Delta x x^{\prime}}{d z}=\Delta x^{\prime 2}+K_{x x} \Delta x^{2}+K_{x y} \Delta x y-\left(d \ln \beta_{z} / d z\right) \Delta x x^{\prime} \\
& \frac{d \Delta x^{\prime 2}}{d z}=2 K_{x x} \Delta x x^{\prime}+2 K_{x y} \Delta x^{\prime} y-2\left(d \ln \beta_{z} / d z\right) \Delta x^{\prime 2} \\
& \frac{d \Delta y^{2}}{d z}=2 \Delta y y^{\prime} \\
& \frac{d \Delta y y^{\prime}}{d z}=\Delta y^{\prime 2}+K_{y y} \Delta y^{2}+K_{y x} \Delta x y-\left(d \ln \beta_{z} / d z\right) \Delta y y^{\prime} \\
& \frac{d \Delta y^{\prime 2}}{d z}=2 K_{y y} \Delta y y^{\prime}+2 K_{x y} \Delta x y^{\prime}-2\left(d \ln \beta_{z} / d z\right) \Delta y^{\prime 2} \\
& \frac{d \Delta x y}{d z}=\Delta x^{\prime} y+\Delta x y^{\prime} \\
& \frac{d \Delta x^{\prime} y}{d z}=\Delta x^{\prime} y^{\prime}+K_{x x} \Delta x y+K_{y x} \Delta y^{2}-\left(d l n \beta_{z} / d z\right) \Delta x^{\prime} y \\
& \frac{d \Delta x y^{\prime}}{d z}=\Delta x^{\prime} y^{\prime}+K_{y y} \Delta x y+K_{x y} \Delta x^{2}-\left(d \ln \beta_{z} / d z\right) \Delta x y^{\prime} \\
& \frac{d \Delta x^{\prime} y^{\prime}}{d z}=K_{x x} \Delta x y^{\prime}+K_{x y} \Delta y y^{\prime}+K_{y y} \Delta x^{\prime} y+K_{y x} \Delta x x^{\prime}-2\left(d \ln \beta_{z} / d z\right) \Delta x^{\prime} y^{\prime}
\end{aligned}
$$

where the notation $\Delta a b \equiv\left(<a^{2}>-<a>^{2}\right)\left(<b^{2}>-<b>^{2}\right)$ stands for a second moment with the contribution from a non-zero first moment removed. The coefficients in the evolution equations in terms of the external quadrupolar and internal spacecharge forces and angles defined in Figure 6.1 are:

$$
\begin{array}{lc}
K_{q x x}=-K_{q y y}=K_{q x 0} \cos 2 \theta \quad K_{q x y}=K_{q y x}=K_{q x 0} \sin 2 \theta \\
K_{s x x}=K_{s x b} \cos 2 \alpha+K_{s y b} \sin 2 \alpha & K_{s y y}=K_{s y b} \cos 2 \alpha+K_{s x b} \sin 2 \alpha \\
K_{s x y}=K_{s y x}=\left(K_{s x b}-K_{s y b}\right) \sin \alpha \cos \alpha & K_{q x 0}=-K_{q y 0}=\frac{B^{\prime}}{B \rho} \\
K_{s x b}=\frac{\kappa}{2\left(\Delta x_{b}^{2}+\left(\Delta x_{b}^{2} \Delta y_{b}^{2}\right)^{1 / 2}\right)} \quad K_{s y b}=\frac{K}{2\left(\Delta y_{b}^{2}+\left(\Delta x_{b}^{2} \Delta y_{b}^{2}\right)^{1 / 2}\right)}
\end{array}
$$

Barnard also found two independent constants of the motion of a beam in a FODO lattice with rotated quadrupoles inducing coupled motion between $x$ and $y .{ }^{29}$ As the 


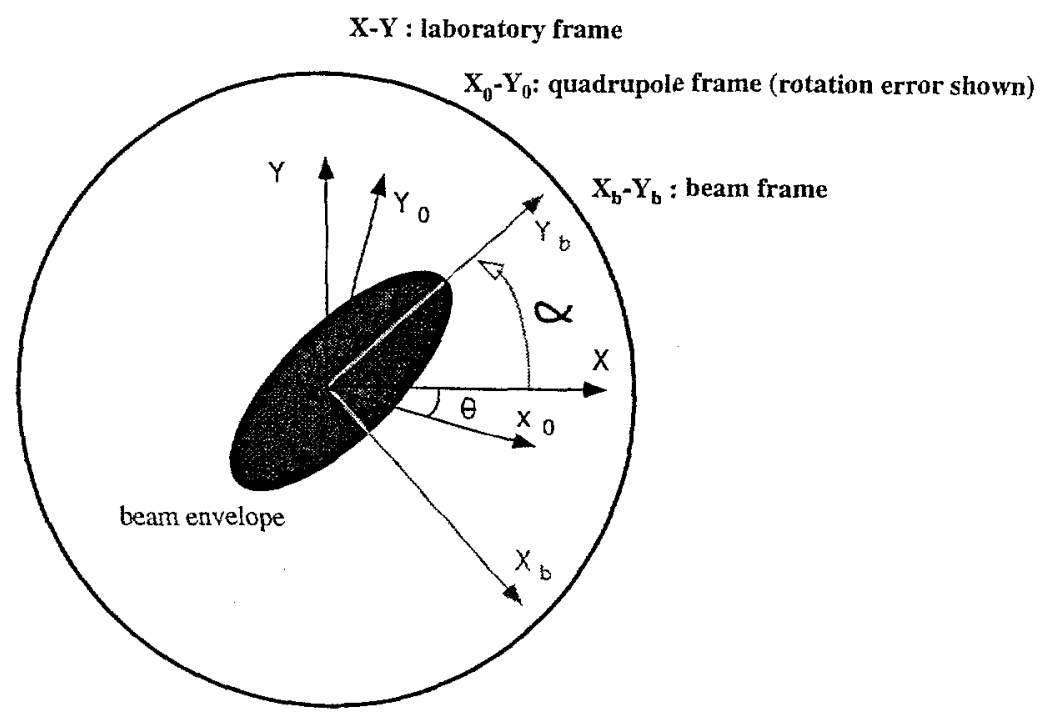

Figure 6.1: Beam and laboratory coordinate system.

emittance is a constant of the motion in an aligned FODO lattice, Barnard called these constants "generalized emittances". Their definitions are:

$$
\begin{aligned}
& \epsilon_{n g}=\left[\frac{1}{2} \epsilon_{n x}^{2}+\frac{1}{2} \epsilon_{n y}^{2}+(4 \beta)^{2}\left(\Delta x y \Delta x^{\prime} y^{\prime}-\Delta x y^{\prime} \Delta x^{\prime} y\right)\right]^{1 / 2} \\
& \epsilon_{n h}=\left[\left(\epsilon_{n x}^{2} \epsilon_{n y}^{2}+(4 \beta)^{4}\left(\left[\Delta x^{\prime} y^{\prime}\right]^{2}[\Delta x y]^{2}-2 \Delta x^{\prime} y \Delta x^{\prime} y^{\prime} \Delta x y \Delta x y^{\prime}+\left[\Delta x^{\prime} y\right]^{2}\right]^{1 / 2}(6\right.\right.
\end{aligned}
$$

where the terms $\epsilon_{n x}$ and $\epsilon_{n y}$ are the normalized transverse emittances defined in terms of the moments as:

$$
\begin{aligned}
\epsilon_{n x} & =4 \beta \gamma\left[\Delta x^{2} \Delta x^{\prime 2}-\left(\Delta x x^{\prime}\right)^{2}\right]^{1 / 2} \\
\epsilon_{n y} & =4 \beta \gamma\left[\Delta y^{2} \Delta y^{\prime 2}-\left(\Delta y y^{\prime}\right)^{2}\right]^{1 / 2}
\end{aligned}
$$

Any other combinations of second moments that yield constants can be reduced to these transverse and generalized emittances and in fact, these quantities reduce to linear combinations of the standard transverse emittances in an aligned FODO lattice 
which can be easily verified by setting the cross-moment terms to zero. ${ }^{42}$ To verify that the generalized emittances were indeed constants of the motion and to study the emittance growth caused by random quadrupole rotations Barnard wrote a computer code, "MOMENT" which numerically integrates the moment equations through a FODO lattice representing the C-design of a recirculator driver. ${ }^{29}$

\subsection{Approximate Correction for a Single Misaligned Quadrupole}

I modified the MOMENT code into several versions to support calculations to show that with a set of four adjustable magnetic quadrupoles, (variable rotation angle and field strength) and the information from the Gated Beam Imager (full set of beam moments), any effect of rotation errors could be removed from the beam and the transverse emittance growth caused by these errors removed from the beam.

The first modification (FULLAG in Appendix B) of the MOMENT code was to tailor its model parameters to match those of the Small Recirculator and to allow for the placing of known rotations on specific quadrupoles or to create random rotations on all quadrupoles. Figures 6.2 and 6.3 show the effect on the beam moments of a single misaligned quadrupole rotated $5^{\circ}$. counter-clockwise. A large error of $5^{\circ}$ was chosen to support experimental verification of the effect of rotation errors on the Small Recirculator. The evolution of the moments from the simulation is plotted with and without the error for comparison. There is only a slight effect on the $x$ and $y$ moments but the $x-y$ cross moments become non-zero indicating the the motion of the ions in the two orthogonal directions becomes coupled after the misalignment. The beam acquires a rapidly changing angular momentum as it passes through subsequent quadrupoles and the 2-D transverse emittance rises above its original value. An attempt to focus the beam to a "spot" at a point past the misalignment would not be as successful as had the misalignment not been present. Note that the generalized emittances in Figure 6.3 are unaffected by the rotation error as expected. 
(a) Delta $\times 2$ versus distance

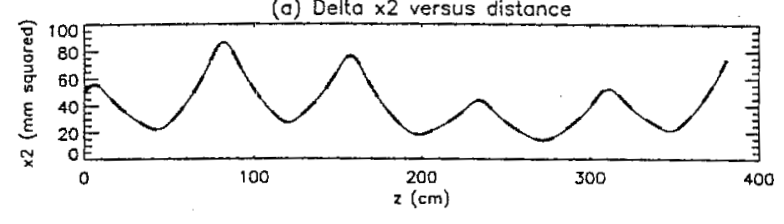

(c) Deltc xprime2 versus distonce

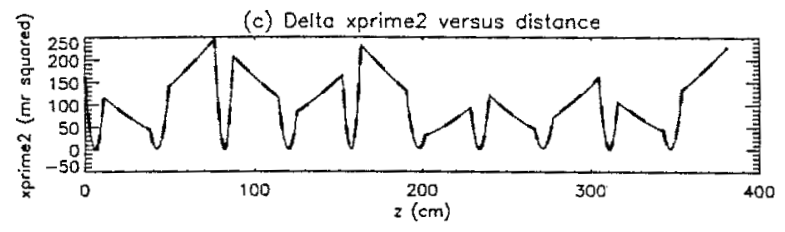

(e) Delto y-yprime versus distance

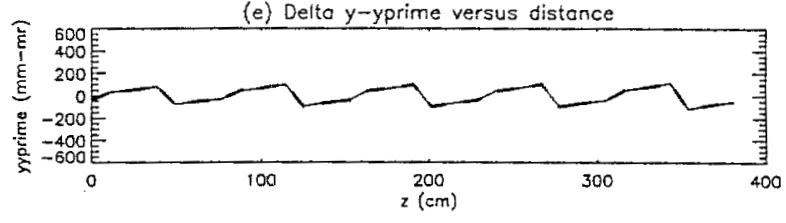

(g) Delta $x-y$ versus distance

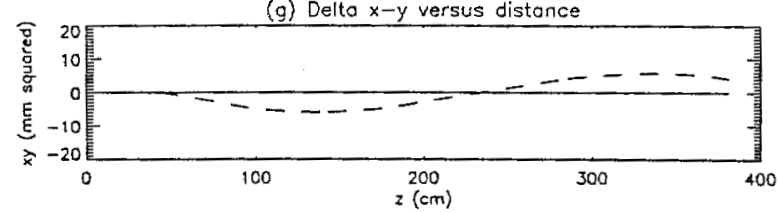

(i) Deltc $x$-yprime versus distence

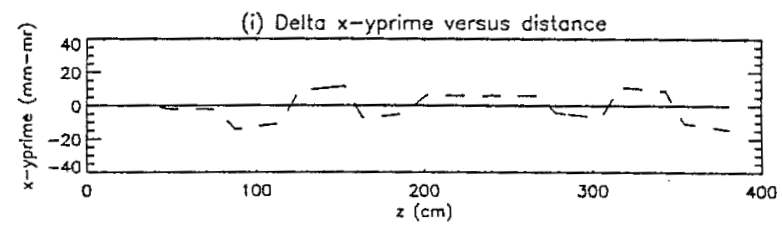

(b) Delto $x$-xprime versus distonce

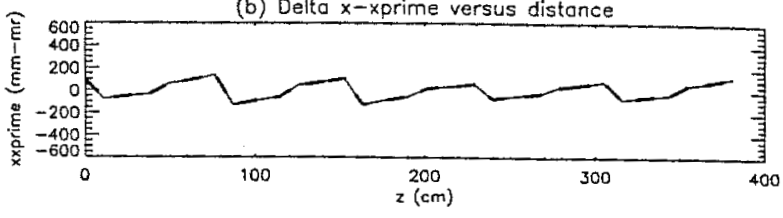

(d) Delta y 2 versus distonce

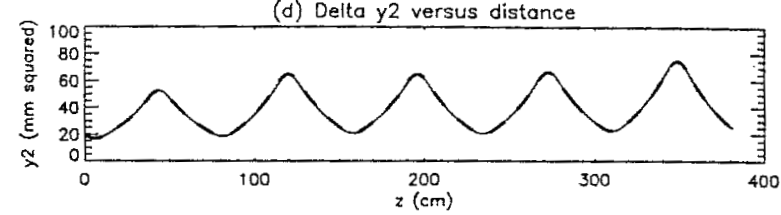

(f) Defta yprime2 versus distonce

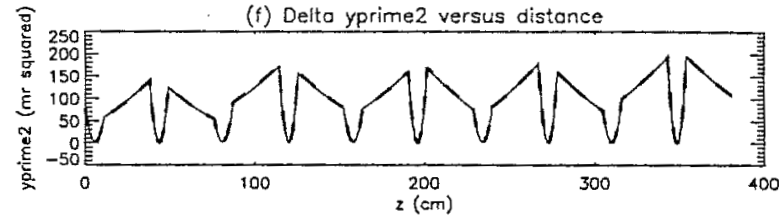

(h) Delto xprime-y versus distance

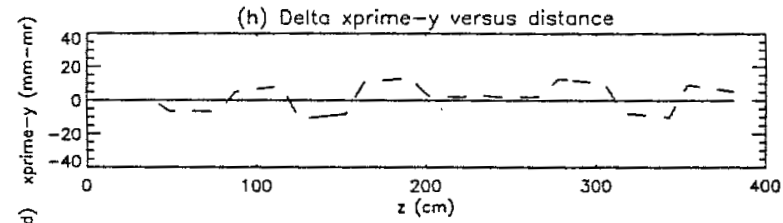

(j) Delto xprime-yprime versus distence

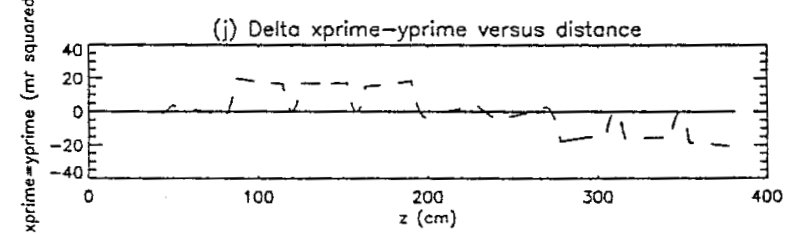

Figure 6.2: Effect of a misaligned quadrupole on the beam moments as a function of distance along the accelerator axis. Dashed lines denote the moments with a single error, quadrupole 2 at $z=38.1 \mathrm{~cm}$ rotated $5^{\circ}$ counter-clockwise. Solid lines are without a rotation error. Output from FULLAG. 

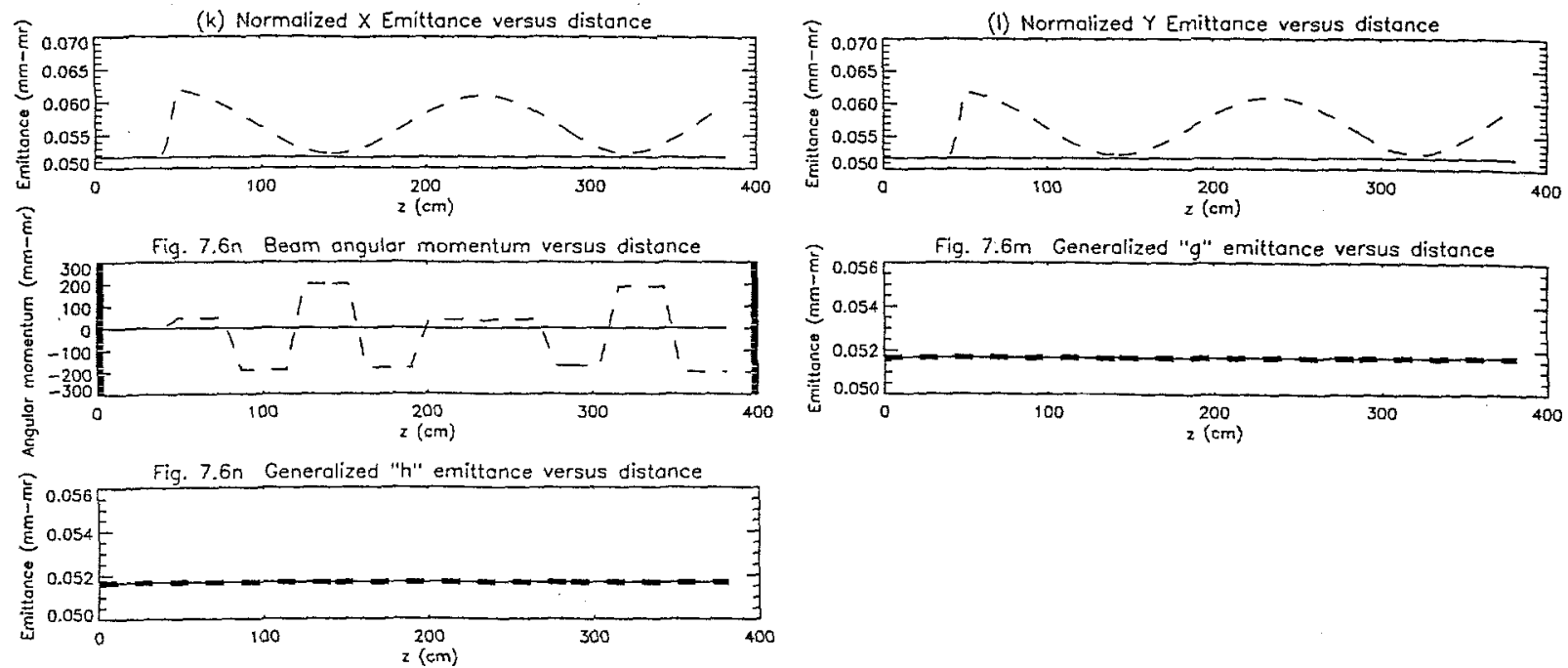

Figure 6.3: Effect of a misaligned quadrupole on the beam emittances and angular momentum. Dashed lines denote the quantities with a single error, quadrupole 2 at $z=38.1 \mathrm{~cm}$ rotated $5^{\circ}$ counter-clockwise. Solid lines are without a rotation error. Output of FULLAG.

A special case of an approximate beam correction method was noted while running the code with multiple known quadrupole rotations. With a known rotation error and beam phase advance (here $72^{\circ}$ ) that divides an integral number of times into $360^{\circ}$, the effect on the beam can be approximately removed by counter-rotating a quadrupole 5 half lattice periods subsequent to the misaligned quadrupole. This provided a rudimentary method of testing beam correction on the Small Recirculator. These tests are discussed in Chapter 7. Figures 6.4 and 6.5 show the results of a simulation run with quadrupole 2 rotated $5^{\circ}$ counter-clockwise, as above, and quadrupole 7 rotated $5^{\circ}$ clockwise. The motion between the $x$ and $y$ directions is mostly decoupled as shown in Figure 6.5 as the $x-y$ cross moments return to near zero. The 2-D transverse emittances return to their original values leading one to conclude that focusing the beam downstream of the quadrupoles would attain the almost the same same results as if the quadrupoles were perfectly aligned. Determining how to correct for any combination of upstream rotation errors would allow quadrupole alignment tolerances to increase, presumably simplifying the design and construction process of 
a driver size machine while maintaining the performance of an aligned lattice.

(a) Delta $\times 2$ versus distance

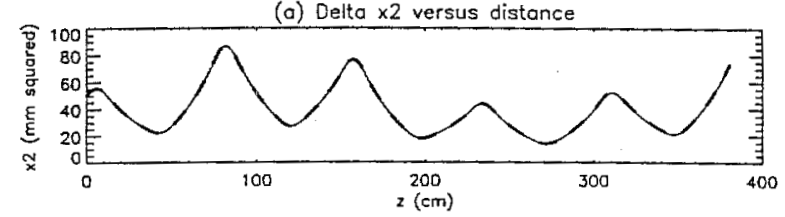

(c) Delta xprime2 versus distance

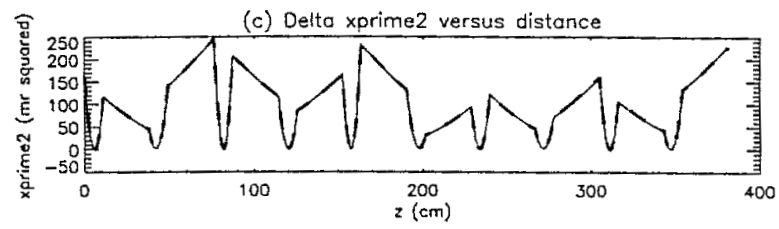

(e) Deita y-yprime versus distance

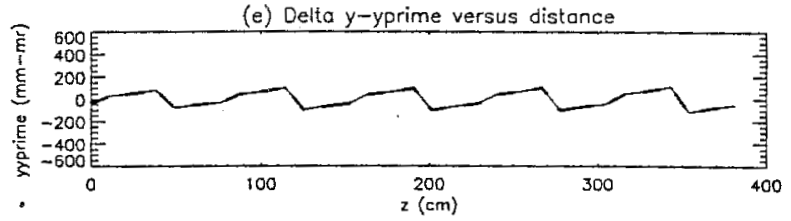

(g) Delta $x-y$ versus distance

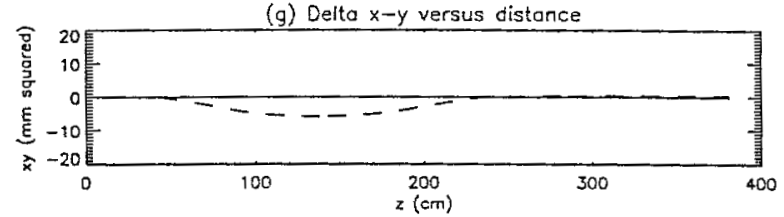

(i) Delto $x$-yprime versus distance

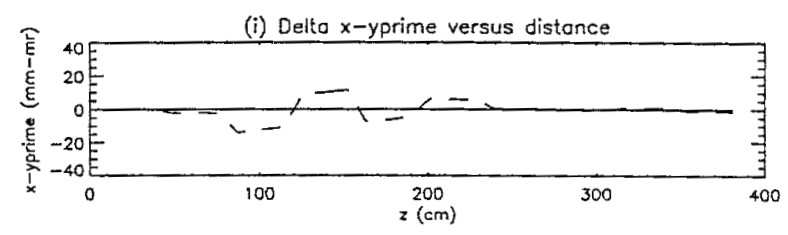

(b) Delto $x$-xprime versus distance

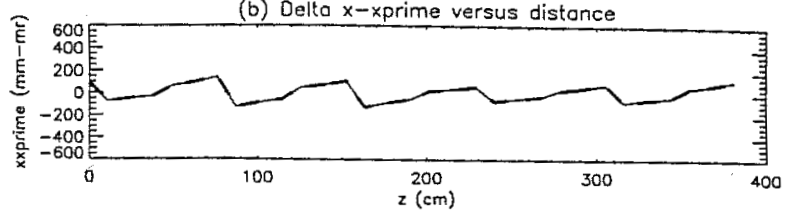

(d) Delta y2 versus distance

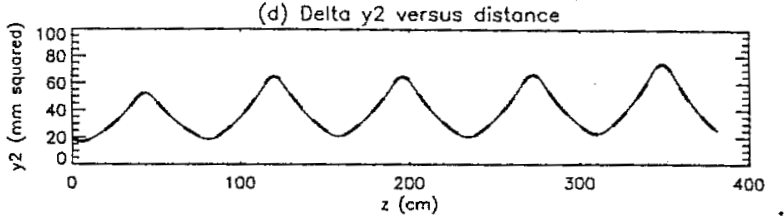

(f) Belto yprime2 versus distonce

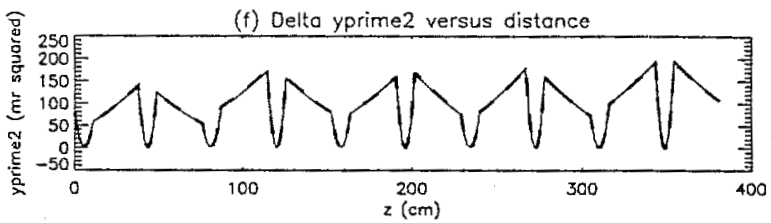

(h) Defto xprime-y versus distonce

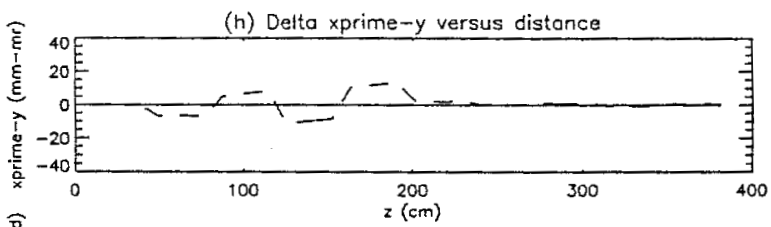

(i) Delto xprime-yprime versus distonce

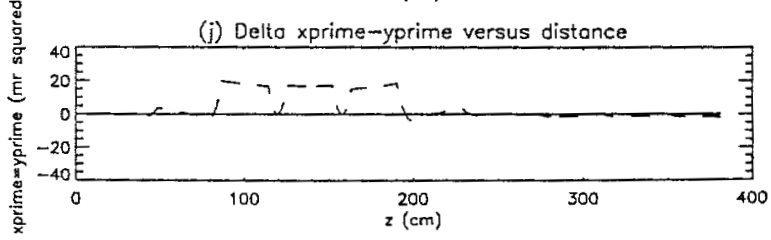

Figure 6.4: Correction of a known quadrupole rotation error. Quadrupole 2 at 38.1 $\mathrm{cm}$ rotated $5^{\circ}$ counter-clockwise and quadrupole 7 at $228.6 \mathrm{~cm}$ rotated $5^{\circ}$ clockwise. Dashed lines denote the moments with rotated quadrupoles. Solid lines are without quadrupole rotations. Output from FULLAG. 

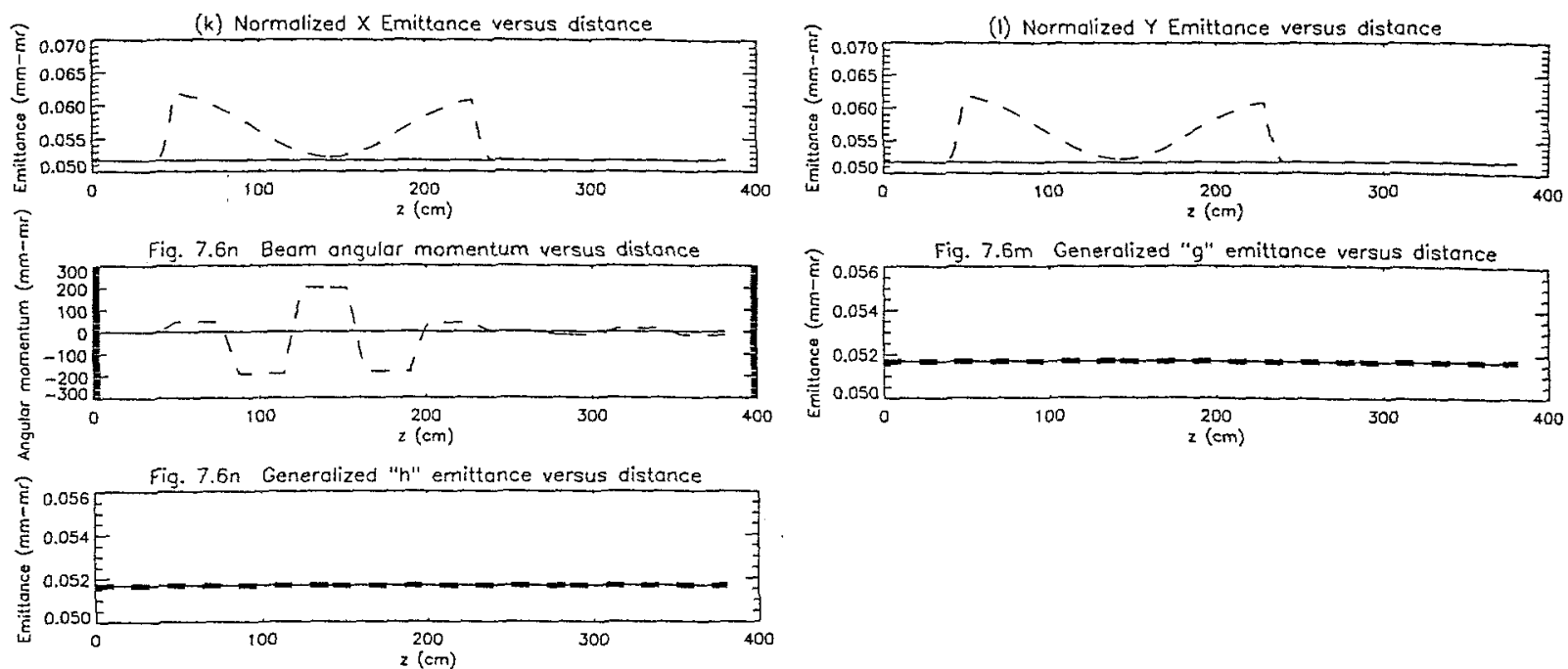

Figure 6.5: Correction of a known quadrupole rotation error. Quadrupole 2 at 38.1 $\mathrm{cm}$ rotated $5^{\circ}$ counter-clockwise and quadrupole 7 at $228.6 \mathrm{~cm}$ rotated $5^{\circ}$ clockwise. Dashed lines denote the quantities with rotated quadrupoles. Solid lines are without quadrupole rotations. Output from FULLAG.

\subsection{Correction for Random Quadrupole Rotation Misalign- ments}

For the more general case and one that exists in a real machine, the location and magnitude of quadrupole rotation errors is not known, only the moments of the beam can be measured at points along the beamline where a suitable diagnostic such as the GBI can be placed. Non-zero cross-moments would indicate the presence of quadrupole rotation errors causing coupling between $x$ and $y$ motions. Examining the second moment evolution equations one sees that they consist of a closed set of ten equations with ten independent parameters (the moments). A matrix representation of the equations at constant energy is: 
$\frac{d}{d z}\left(\begin{array}{c}\Delta x^{2} \\ \Delta x x^{\prime} \\ \Delta x^{\prime 2} \\ \Delta y^{2} \\ \Delta y y^{\prime} \\ \Delta y^{\prime 2} \\ \Delta x y \\ \Delta x^{\prime} y \\ \Delta x y^{\prime} \\ \Delta x^{\prime} y^{\prime}\end{array}\right)=\left(\begin{array}{cccccccccc}0 & 2 & 0 & 0 & 0 & 0 & 0 & 0 & 0 & 0 \\ K_{x x} & 0 & 1 & 0 & 0 & 0 & K_{x y} & 0 & 0 & 0 \\ 0 & 2 K_{x x} & 0 & 0 & 0 & 0 & 0 & 2 K_{x y} & 0 & 0 \\ 0 & 0 & 0 & 0 & 2 & 0 & 0 & 0 & 0 & 0 \\ 0 & 0 & 0 & K_{y y} & 0 & 1 & K_{y x} & 0 & 0 & 0 \\ 0 & 0 & 0 & 0 & 2 K_{y y} & 0 & 0 & 0 & 2 K_{x y} & 0 \\ 0 & 0 & 0 & 0 & 0 & 0 & 0 & 1 & 1 & 0 \\ 0 & 0 & 0 & K_{y x} & 0 & 0 & K_{x x} & 0 & 0 & 1 \\ K_{x y} & 0 & 0 & 0 & 0 & 0 & K_{y y} & 0 & 0 & 1 \\ 0 & K_{y x} & 0 & 0 & K_{x y} & 0 & 0 & K_{y y} & K_{x x} & 0\end{array}\right)\left(\begin{array}{c}\Delta x^{2} \\ \Delta x x^{\prime} \\ \Delta x^{\prime 2} \\ \Delta y^{2} \\ \Delta y y^{\prime} \\ \Delta y^{\prime 2} \\ \Delta x y \\ \Delta x^{\prime} y \\ \Delta x y^{\prime} \\ \Delta x^{\prime} y^{\prime}\end{array}\right)$

The beam can be manipulated to adjust these moments to the desired values. To simultaneously adjust the ten moments to desired values requires ten adjustable parameters.

A set of five quadrupole magnets with variable rotation angle and variable field strength would supply these manipulations. The generalized emittances provide relief in the engineering and cost of a beam correction system in that they provide constraints on the system of moment equations allowing the elimination of two parameters by solving explicitly for them in terms of the other parameters. Applying the two constraints and eliminating $\Delta x^{2}$ and $\Delta y^{2}$ by substituting in their explicit 
solutions yields:

$$
\frac{d}{d z}\left(\begin{array}{c}
\Delta x x^{\prime} \\
\Delta x^{\prime 2} \\
\Delta y y^{\prime} \\
\Delta y^{\prime 2} \\
\Delta x y \\
\Delta x^{\prime} y \\
\Delta x y^{\prime} \\
\Delta x^{\prime} y^{\prime}
\end{array}\right)=\left(\begin{array}{cccccccc}
K_{11} & K_{12} & K_{13} & K_{14} & K_{15} & K_{16} & K_{17} & K_{18} \\
2 K_{x x} & 0 & 0 & 0 & 0 & 2 K_{x y} & 0 & 0 \\
K_{31} & K_{32} & K_{33} & K_{34} & K_{35} & K_{36} & K_{37} & K_{38} \\
0 & 0 & 2 K_{y y} & 0 & 0 & 0 & 2 K_{x y} & 0 \\
0 & 0 & 0 & 0 & 0 & 1 & 1 & 0 \\
K_{61} & K_{62} & K_{63} & K_{64} & K_{65} & K_{66} & K_{67} & K_{68} \\
K_{71} & K_{72} & K_{73} & K_{74} & K_{75} & K_{76} & K_{77} & K_{78} \\
K_{y x} & 0 & K_{x y} & 0 & 0 & K_{y y} & K_{x x} & 0
\end{array}\right)\left(\begin{array}{c}
\Delta x x^{\prime} \\
\Delta x^{\prime 2} \\
\Delta y y^{\prime} \\
\Delta y^{\prime 2} \\
\Delta x y \\
\Delta x^{\prime} y \\
\Delta x y^{\prime} \\
\Delta x^{\prime} y^{\prime}
\end{array}\right)
$$

where the numbered coefficients are complicated functions of the second moments but are nevertheless calculable in a code, if not manually on paper. The resultant system of eight coupled equations and eight parameters allows beam correction to be accomplished by a system of four adjustable quadrupole magnets. A schematic of such a system is shown in Figure 6.6. Four adjustable magnetic quadrupoles replace four standard quadrupoles at some point in the beamline. A natural placement may be just before the final focus lens to ensure the beam enters in the best condition possible (upright, with zero angular momentum) for effective focusing. Other positions within the recirculator may be more effective in reducing the cumulative effect of quadrupole rotation errors. The difference between a regular quadrupole and these are in their ability to rotate the orientation of their magnetic fields and may have a greater degree of field strength adjustment. ${ }^{43}$

\subsubsection{Applying GBI Data to a Beam Correction System}

A GBI-type instrument would measure the moments of the beam at the input side of the correction system providing information on the condition of the beam as in Figure 6.6. A simulation code run would provide the desired (and attainable) moments at the output of the correction system. A benchmarked and calibrated code such as WARP would provide moments that included the unavoidable effects of the 


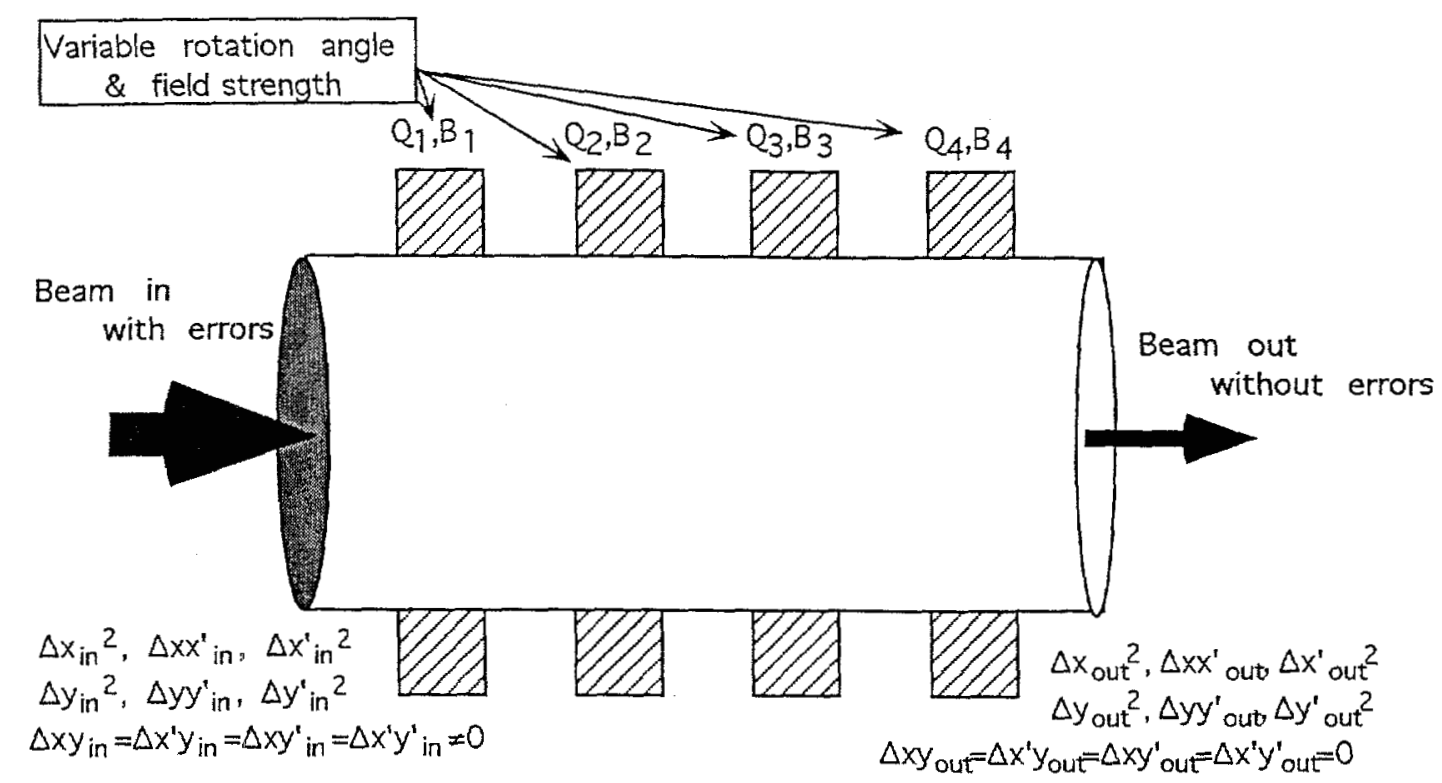

Figure 6.6: Conceptual beam correction system.

actual fields and resonances that result in nonlinear emittance growth even with a perfectly aligned model FODO lattice. This nonlinear effect on the moments cannot be removed through the application of linear fields so the desired moments calculated for the output of the correction system must be obtained from a sophisticated simulation that includes nonlinear effects. The simplest useful correction would be to correct the cross moments to zero which would deliver an upright and non-rotating beam ( $x$ and $y$ motions de-coupled) to the final focus. This may not be the best that can be done though which is why a sophisticated code which estimates nonlinear growth in an aligned lattice is desirable. Once the input moments are measured and the output moments are calculated, an optimization of the adjustable parameters in the correction quadrupoles is calculated and set until a decrease in beam transport element performance warrants another GBI measurement. A GBI measurement at the output of the correction system would be useful in directly verifying its performance.

I modified Barnard's MOMENT code into a third version to serve as an optimizer 
to calculate the field strengths and rotation angles of a correction system to remove the effect of quadrupole rotation errors. A copy of this code, "OPTIMIZE" is in Appendix C. Using the generalized emittance equations to explicitly solve for two moments proved quickly to be too difficult to do by hand. Using the simultaneous equation solving capabilities of Mathematica ${ }^{T M}$, a symbolic mathematics software system, allowed me to do this task and to find the simplest solution of the ten possibilities. ${ }^{49}$ Solving explicitly for $\Delta x^{2}$ and $\Delta y^{2}$ resulted in the 8 simplest moment evolution equations with 8 adjustable parameters. Even though these solutions were the simplest obtainable, the results consist of algebraic equations of over 100 terms each. The complete explicit solutions appear in Appendix B. While doing optimization calculations it was noticed that a large number of the terms in the solutions for $\Delta x^{2}$ and $\Delta y^{2}$ were much smaller than the rest (by 10 orders of magnitude) when using nominal Small Recirculator quantities. These terms can therefore be dropped without loss of accuracy and the simplified explicit expressions are shown here:

$$
\begin{aligned}
& \Delta x^{2}=\left(4 \beta^{4}\left(\Delta x^{\prime} y^{\prime}\right)^{2}\left(\Delta x x^{\prime}\right)^{2}-8 \beta^{4}\left(\Delta x^{\prime} y^{\prime}\right)^{3} \Delta x y+8 \beta^{4} \Delta x^{\prime} y\left(\Delta x^{\prime} y^{\prime}\right)^{2} \Delta x y^{\prime}\right. \\
& \left.+8 \beta^{4} \Delta x^{\prime} y^{\prime} \Delta x^{\prime 2} \Delta x x^{\prime} \Delta x y^{\prime}-4 \beta^{4}\left(\Delta x^{\prime 2}\right)^{2}\left(\Delta x y^{\prime}\right)^{2}-8 \beta^{4} \Delta x^{2}\left(\Delta x x^{\prime}\right)^{2} \Delta y 6^{2} .8\right) \\
& +8 \beta^{4} \Delta x^{\prime} y^{\prime} \Delta x^{\prime 2} \Delta x y \Delta y^{\prime 2}-8 \beta^{4} \Delta x^{\prime} y \Delta x^{\prime 2} \Delta x y^{\prime} \Delta y^{\prime 2}+4 \beta^{4}\left(\Delta x^{\prime} y\right)^{2}\left(\Delta y(\dot{b})^{3}\right) \\
& -8 \beta^{4} \Delta x^{\prime} y \Delta x^{\prime} y^{\prime} \Delta y^{\prime 2} \Delta y y^{\prime}+4 \beta^{4}\left(\Delta x^{\prime} y^{\prime}\right)^{2}\left(\Delta y y^{\prime 2}\right)+0.5 \beta^{2}\left(\Delta x^{\prime} y^{\prime}\right)^{2} \epsilon_{n g}^{2}(6.10) \\
& \left.-0.5 \beta^{2} \Delta x^{\prime 2} \Delta y^{\prime 2} \epsilon_{n g}^{2}\right) /\left(\beta^{4} \Delta x^{\prime 2}\left(8\left(\Delta x^{\prime} y^{\prime}\right)^{2}-8 \Delta x^{\prime 2} \Delta y^{\prime 2}\right)\right) \\
& \Delta y^{2}=0.5\left(-\beta^{2}\left(256 \beta^{2}\left(\Delta x^{\prime} y^{\prime}\right)^{2}\left(\Delta x x^{\prime}\right)^{2}-512 \beta^{2}\left(\Delta x^{\prime} y^{\prime}\right)^{3} \Delta x y+512 \beta^{2} \Delta x^{\prime} y\left(\Delta x^{\prime} y^{\prime}\right)^{2} \Delta 6 x y^{2}\right)\right. \\
& -512 \beta^{2} \Delta x^{\prime} y^{\prime} \Delta x^{\prime 2} \Delta x x^{\prime} \Delta x y^{\prime}+256 \beta^{2}\left(\Delta x^{\prime 2}\right)^{2}\left(\Delta x y^{\prime}\right)^{2}+512 \beta^{2} \Delta x^{\prime} y^{\prime} \Delta x^{\prime 2} \Delta x\left(6 \Delta 13 f^{2}\right) \\
& -512 \beta^{2} \Delta x^{\prime} y \Delta x^{\prime 2} \Delta x y^{\prime} \Delta y^{\prime 2}-256 \beta^{2}\left(\Delta x^{\prime} y\right)^{2}\left(\Delta y^{\prime 2}\right)^{2}+512 \beta^{2} \Delta x^{\prime} y \Delta x^{\prime} y^{\prime} \Delta y^{\prime 2}(\Delta y) \\
& +256 \beta^{2}\left(\Delta x^{\prime} y^{\prime}\right)^{2}\left(\Delta y y^{\prime 2}\right)-512 \beta^{2} \Delta x^{\prime 2} \Delta y^{\prime 2}\left(\Delta y y^{\prime 2}\right)+32\left(\Delta x^{\prime} y^{\prime}\right)^{2} \epsilon_{n g}^{2} \\
& \left.\left.-32 \Delta x^{\prime 2} \Delta y^{\prime 2} \epsilon_{n g}^{2}\right)\right) /\left(\beta^{4} \Delta y^{\prime 2}\left(-256\left(\Delta x^{\prime} y^{\prime}\right)^{2}+256 \Delta x^{\prime 2} \Delta y^{\prime 2}\right)\right)
\end{aligned}
$$

To verify the accuracy of these expressions the reduced set of eight moment evolution equations were used to simulate the Small Recirculator beam drifting through a 
lattice of perfectly aligned magnetic quadrupoles. Two calculations were performed. The first calculation integrated the full set of ten moment equations. The second calculation integrated the reduced set of eight moment with the simplified forms of $\Delta x^{2}$ and $\Delta y^{2}$ shown above. Figure 6.7 shows the difference between these calculations and that the maximum error is $<0.1 \%$.

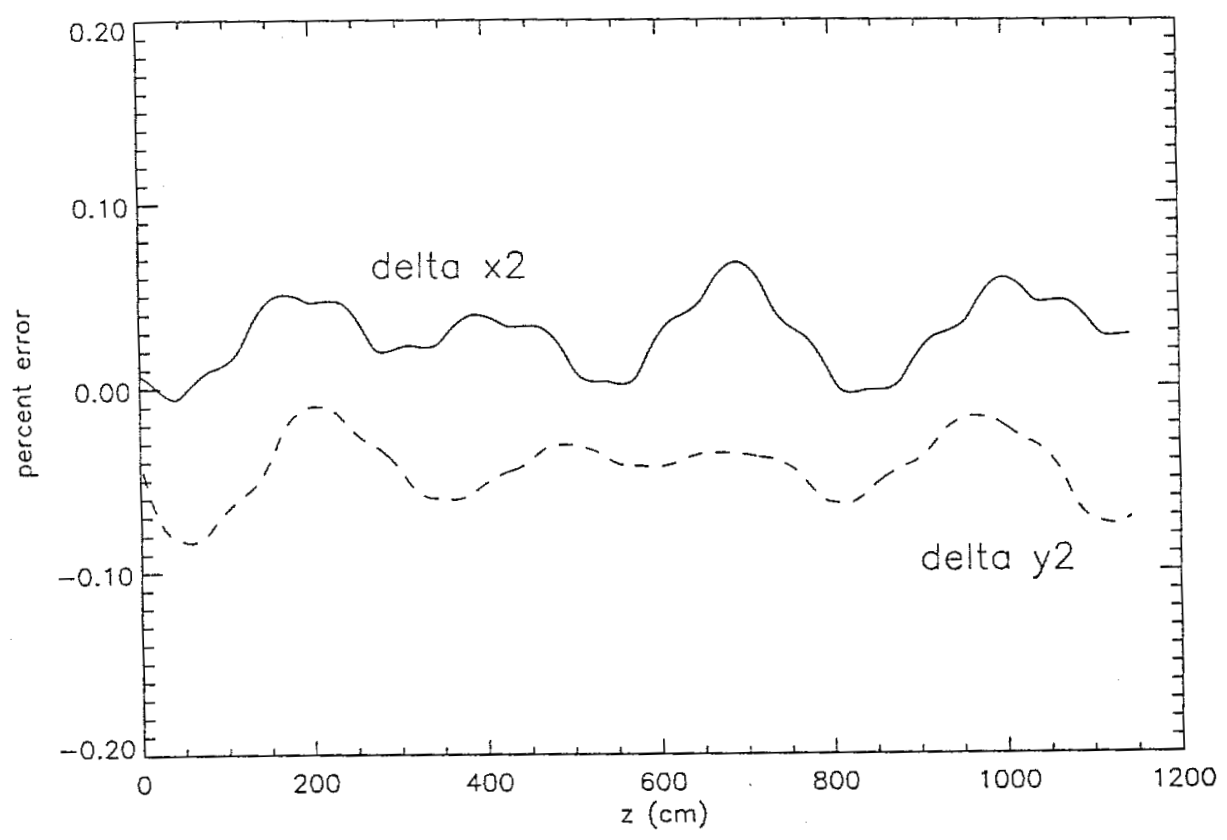

Figure 6.7: Verification of simplified $\Delta x^{2}$ and $\Delta y^{2}$ solutions. Each line is the percent difference between the calculated moments with the full set of 10 moment evolution equations and with the reduced set of 8 equations incorporating the simplified explicit solutions for $\Delta x^{2}$ and $\Delta y^{2}$.

\subsubsection{Determining the Optimum Correction System Param- eters}

Modifying Barnard's MOMENT code to calculate the optimized values of the correction quadrupole rotation angle and field strength presented a challenge due to the moment evolution equations' nonlinear dependence on the two quantities (angle 
and field strength) to be optimized. An elegant and efficient solution was found by adapting Spall's "Simultaneous Perturbation Stochastic Approximation" (SPSA) algorithm to the problem. ${ }^{44}$

The SPSA algorithm has been applied successfully to many problems where the equations governing the system are unknown and therefore obtaining the gradient of the objective function with respect to the parameters being optimized is not possible. ${ }^{45,46}$ These so-called model-free applications are treatable with SPSA as it is based on a highly efficient gradient approximation requiring only two objective function measurements regardless of the dimensionality of the gradient vector. In the beam correction system the parameters to be optimized are the rotation angle and magnetic field strengths of the four quadrupole magnets with the aim of adjusting the ten second moments to remove the effect of previous rotation errors. The objective function I have defined is the root sum square of the difference between the moments of the beam emerging from the correction system and the desired moments:

$$
\begin{aligned}
& L(\underline{M})= \\
& \left(\left(\Delta x_{c}^{2}-\Delta x_{d}^{2}\right)^{2}+\left(\Delta x x_{c}^{\prime}-\Delta x x_{d}^{\prime}\right)^{2}+\left(\Delta x_{c}^{\prime 2}-\Delta x_{d}^{\prime 2}\right)^{2}\right. \\
& +\left(\Delta y_{c}^{2}-\Delta y_{d}^{2}\right)^{2}+\left(\Delta y y_{c}^{\prime}-\Delta y y_{d}^{\prime}\right)^{2}+\left(\Delta y_{c}^{\prime 2}-\Delta y_{d}^{\prime 2}\right)^{2} \\
& \left.+\left(\Delta x y_{c}-\Delta x y_{d}\right)^{2}+\left(\Delta x^{\prime} y_{c}-\Delta x^{\prime} y_{d}\right)^{2}+\left(\Delta x y_{c}^{\prime}-\Delta x y_{d}^{\prime}\right)^{2}+\left(\Delta x^{\prime} y_{c}^{\prime}-\Delta x^{\prime} y_{d}^{\prime}\right)^{2}\right)^{\frac{1}{2}} \\
& \quad \underline{M}=\left[\Delta x^{2}, \Delta x x^{\prime}, \Delta x^{\prime 2}, \Delta y^{2}, \Delta y y^{\prime}, \Delta y^{\prime 2}, \Delta x y, \Delta x^{\prime} y, \Delta x y^{\prime}, \Delta x^{\prime} y^{\prime}\right]
\end{aligned}
$$

where, for example, $\Delta x_{c}^{2}$ is the calculated moment through the beam correction system using the moment evolution equations, and $\Delta x_{d}^{2}$ is the desired moment at the correction system output based on a calculation with a perfectly aligned transport lattice. Although in the set of moment evolution equations, the gradients of the objective function, $L(\underline{M})$ with respect to rotation angle and field strength could be found and applied to a standard finite difference scheme, the nonlinearity of the equations makes this difficult. The SPSA algorithm allows one to sidestep this issue with what has appeared to be no loss of accuracy and a definite improvement in computational efficiency. SPSA requires only two measurements of the objective function rather 
than the 16 of the standard finite differencing scheme. ${ }^{44}$ This is quite a saving as each measurement requires integrating the moment evolution equations through the beam correction system which is quite demanding using the reduced set of equations required for a four magnet system. The goal is to minimize the objective function resulting in the best least squares fit of the desired moment vector $\underline{M}$. The SPSA algorithm works by iterating from an initial guess of the optimal $\underline{M}$, where the iteration process depends on the "simultaneous perturbation" approximation to the gradient

$$
g(\underline{M})=\frac{\partial L(\underline{M})}{\partial \underline{\theta}}
$$

where $\underline{\theta}$ is the 8 -dimensional vector of quadrupole rotation angles and field strengths. It is assumed that $L(\underline{M})$ is a differentiable function of $\underline{\theta}$ and the minimum point $\underline{\theta}^{*}$ corresponds to the zero point of the gradient, i.e., $\underline{\theta}^{*}$ is such that

$$
g\left(\underline{\theta}^{*}\right)=\left.\frac{\partial L(\underline{M})}{\partial \underline{\theta}}\right|_{\underline{\theta}=\underline{\theta}^{*}}=0 .
$$

As a consequence of the basic recursive form of the algorithm there is generally not a risk of converging to a maximum or a saddle point. Nevertheless, one is able to check for convergence to a local minimum by examining the behavior of the moments in the lattice past the correction system, particularly whether the $x-y$ cross moments remain near zero.

\subsubsection{Implementing the Optimizing Algorithm}

The SPSA algorithm iterates through the following steps:

1. Pick an initial guess for $\underline{\theta}$ which for lack of any better information and the desire for the least adjustment for engineering considerations is zero quadrupole rotation angle and nominal magnetic field strength. 
2. Set the counter index, $k=0$ and compute the SPSA gain sequences

$$
a_{k}=a(A+k+1)^{-\alpha} \quad c_{k}=c(k+1)^{-\gamma}
$$

Spall states that $\alpha=0.602$ and $\gamma=0.101$ are effective and theoretically valid and also gives guidelines for the selection of $a, A$, and $c$ [footnote].

3. Generate by Monte Carlo method a p-dimensional random vector, $\Delta_{k}$ ( $\mathrm{p}$ being the number of adjustable parameters, here, 8) where each component is independently generated from a zero-mean probability distribution satisfying the conditions of Spall. ${ }^{44}$ The Bernoulli \pm 1 distribution is a valid choice with probability $\frac{1}{2}$ for each \pm 1 .

4. Obtain two measurements of the loss function, $L(\underline{M})$ based on a simultaneous perturbation around the current $\underline{\theta}_{k}, y\left(\underline{\theta}_{k}+c_{k} \Delta_{k}\right)$ and $y\left(\underline{\theta}_{k}-c_{k} \Delta_{k}\right)$. These measurements are computed by first adjusting the quadrupole parameters by $+c_{k} \Delta_{k}$ and integrating the input moments through the beam correction system and computing the root sum square of the difference between the output moments and the desired moments. This process is repeated with the beginning quadrupole values adjusted by $-c_{k} \Delta_{k}$. This is where the advantage of needing only two measurements rather 16 is realized as integration of the reduced set of moment evolution equations is computationally demanding.

5. Using the two measurements of the objective function obtained in the last step generate the simultaneous perturbation approximation to the 8-dimensional gradient,

$$
g_{k}\left(\underline{\theta}_{k}\right)=\left[\begin{array}{c}
\frac{L\left(\underline{\theta}_{k}+c_{k} \Delta_{k}\right)-L\left(\underline{\theta}_{k}-c_{k} \Delta_{k}\right)}{2 c_{k} \Delta_{k 1}} \\
\vdots \\
\frac{L\left(\underline{\theta}_{k}+c_{k} \Delta_{k}\right)-L\left(\underline{\theta}_{k}-c_{k} \underline{\Delta}_{k}\right)}{2 c_{k} \Delta_{k 8}}
\end{array}\right]
$$

where $\Delta_{k i}$ is the $i^{t h}$ component, $( \pm 1)$, of the $\Delta_{k}$ vector. Note the denominators change (stochastically) while the numerators are all the same, making possible only the two measurements to produce gradient approximations. 
6. Update the adjustable quadrupole parameters using the standard stochastic approximation form

$$
\underline{\theta}_{k+1}=\underline{\theta}_{k}-a_{k} g_{k}\left(\underline{\theta}_{k}\right)
$$

7. The algorithm is terminated if there is little change in several successive iterations. If this condition is not met, the iteration counter is updated by one and the iteration is repeated from step 2. The last iterate, $\underline{\theta}_{k}$ is the estimate of the optimum $\underline{\theta}^{*}$ which corrects the beam for previous quadrupole rotation errors such that the beam is upright with zero angular momentum.

I modified Barnard's Moment code to include optimization iteration loops implementing the SPSA algorithm. This code CORRECTOR (see Appendix C) models the beam correction system previously mentioned and shown in Figure 6.6. The results of an optimization run are shown in Figures 6.8 and Table 6.1. The input moments to the beam correction system resulted from a calculation of a recirculator transport through 562 half lattice periods with maximum rotation angle error of $0.5^{\circ}$. The desired output moments were generated by the REDUCEDAG code, a modification of the "MOMENT" code modeling a perfectly aligned lattice which can be seen by the zero values of the $x-y$ cross moments in Table 6.1. Although the SPSA algorithm remained noisy, the desirable settings were those that produced the smallest difference between the calculated and desired beam moments. From Figure 6.8 one can see that the best parameters were not always obtained on the last iteration. 

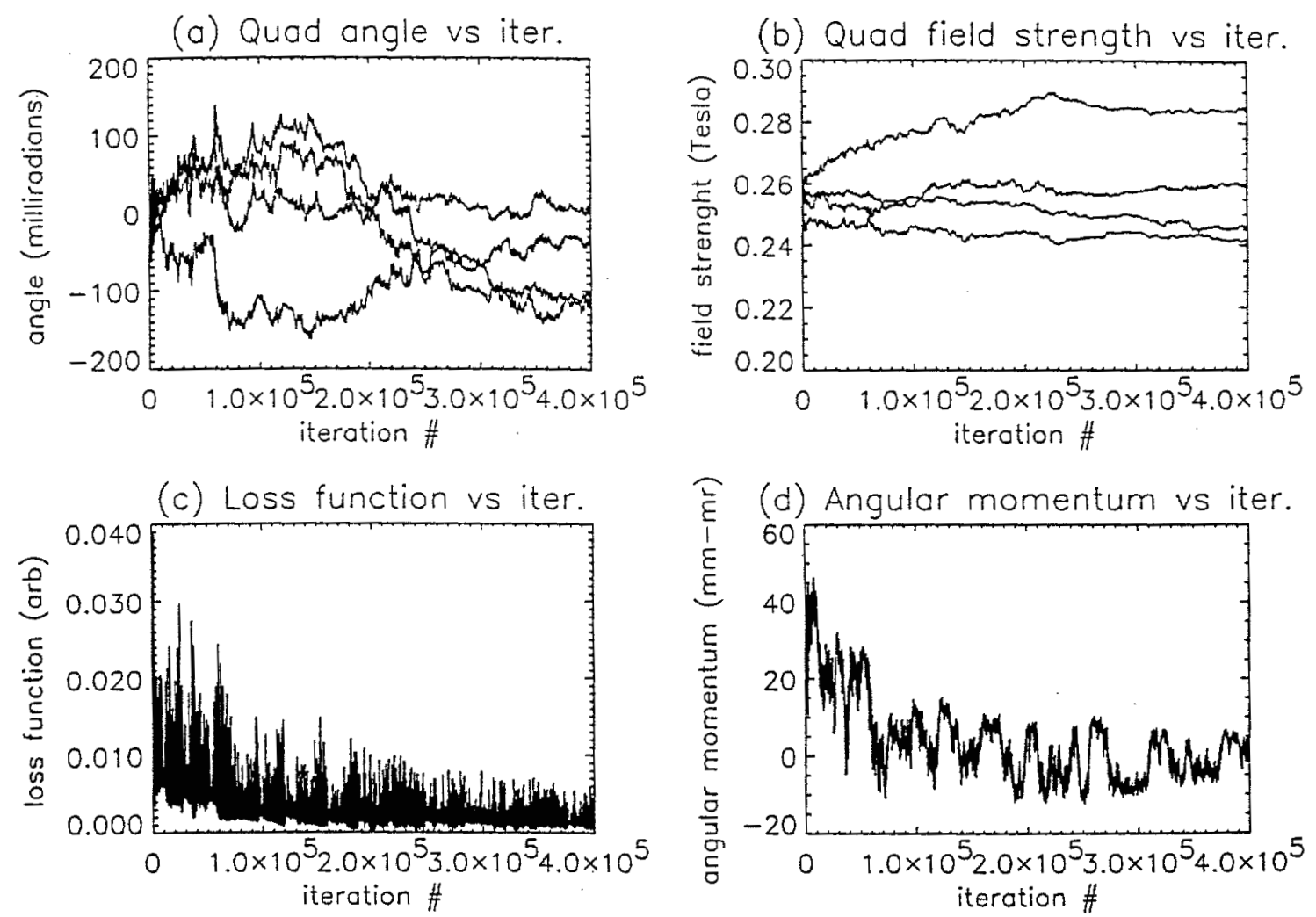

Figure 6.8: Beam correction system optimization code results plots. The best parameters occurred on iteration number 245,753 of 400,000 . 
Table 6.1: Results of the SPSA optimization run to determine beam correction system settings

\begin{tabular}{|c|c|c|c|}
\hline \multicolumn{2}{|c|}{ Least Loss Function Calculated } & \multicolumn{2}{|l|}{$1.629 \times 10^{-4}$} \\
\hline \multicolumn{2}{|l|}{ Initial Loss Function } & \multicolumn{2}{|l|}{0.1562} \\
\hline \multicolumn{2}{|l|}{ Angular Momentum } & \multicolumn{2}{|l|}{$3.740 \times 10^{-4}$} \\
\hline \multicolumn{2}{|l|}{ Initial Angular Momentum } & \multicolumn{2}{|l|}{0.126} \\
\hline Nominal Quad Field (T) & 0.25258 & Nominal Quad Angle (०) & 0 \\
\hline Quad Field 1 (T) & 0.25692 & Quad Angle $1\left(^{\circ}\right)$ & -3.956 \\
\hline Quad Field 2 (T) & 0.24212 & Quad Angle $2\left(^{\circ}\right)$ & 0.621 \\
\hline Quad Field 3 (T) & 0.25033 & Quad Angle $3\left(^{\circ}\right)$ & -2.112 \\
\hline Quad Field 4(T) & 0.28685 & Quad Angle $4\left(^{\circ}\right)$ & -3.651 \\
\hline \multicolumn{2}{|c|}{ Calculated Moments } & \multicolumn{2}{|l|}{ Desired Moments } \\
\hline$\Delta x^{2}\left(m^{2}\right)$ & 54.54 & $\Delta x^{2}\left(\mathrm{~mm}^{2}\right)$ & 54.54 \\
\hline$\Delta x x^{\prime}(\mathrm{mm}-\mathrm{mr})$ & 102.28 & $\Delta x x^{\prime}(\mathrm{mm}-\mathrm{mr})$ & 101.66 \\
\hline$\Delta x^{\prime 2}\left(m r^{2}\right)$ & 179.35 & $\Delta x^{\prime 2}\left(\mathrm{mr}^{2}\right)$ & 177.19 \\
\hline$\Delta y^{2}\left(\mathrm{~mm}^{2}\right)$ & 15.96 & $\Delta y^{2}\left(\mathrm{~mm}^{2}\right)$ & 15.96 \\
\hline$\Delta y y^{\prime}(\mathrm{mm}-\mathrm{mr})$ & -31.92 & $\Delta y y^{\prime}(\mathrm{mm}-\mathrm{mr})$ & -30.51 \\
\hline$\Delta y^{\prime 2}\left(\mathrm{mr}^{2}\right)$ & 66.27 & $\Delta y^{\prime 2}\left(\mathrm{mr}^{2}\right)$ & 60.78 \\
\hline$\Delta x y\left(\mathrm{~mm}^{2}\right)$ & $-4.13 \times 10^{-3}$ & $\Delta x y\left(\mathrm{~mm}^{2}\right)$ & 0 \\
\hline$\Delta x^{\prime} y(\mathrm{~mm}-\mathrm{mr})$ & $4.84 \times 10^{-4}$ & $\Delta x^{\prime} y(\mathrm{~mm}-\mathrm{mr})$ & 0 \\
\hline$\Delta x y^{\prime}(\mathrm{mm}-\mathrm{mr})$ & $1.92 \times 10^{-2}$ & $\Delta x y^{\prime}(\mathrm{mm}-\mathrm{mr})$ & 0 \\
\hline$\Delta x^{\prime} y^{\prime}\left(\mathrm{mr}^{2}\right)$ & $2.02 \times 10^{-2}$ & $\Delta x^{\prime} y^{\prime}\left(m r^{2}\right)$ & 0 \\
\hline \multicolumn{4}{|c|}{ SPSA Parameters } \\
\hline Maximum iterations & $4 \times 10^{5}$ & Gain coefficient, $\mathrm{A}$ & $2 \times 10^{4}$ \\
\hline Gain coefficient, a & 0.06 & Gain coefficient, b & 0.0033 \\
\hline Gain coefficient, $c$ & $5 \times 10^{-6}$ & Gain coefficient, $\mathrm{d}$ & $5 \times 10^{-6}$ \\
\hline
\end{tabular}

The optimized values of the four rotation angles and field strengths are used in the simulation shown in Figures 6.9-6.10. A system of 4 quadrupoles modeling the beam correction system accepts a beam whose input moments are obtained from a lattice of 562 quadrupoles with random errors up to $\pm 0.5^{\circ}$. With the optimized settings of Table 6.1 the four quadrupoles deliver a beam that is "upright" and not rotating and whose other moments are nearly returned to the desired values. 
(a) Delto $\times 2$ versus distance

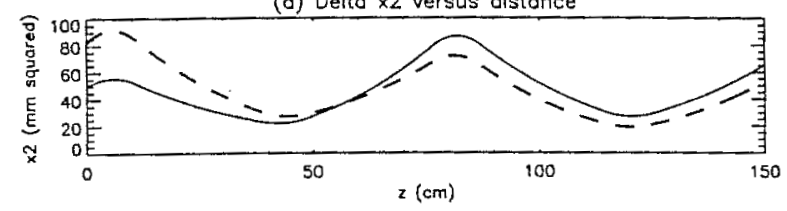

(b) Delta $x$-xprime versus distance

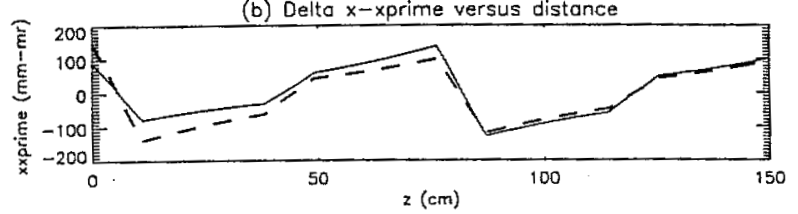

(c) Delto xprime2 versus distance

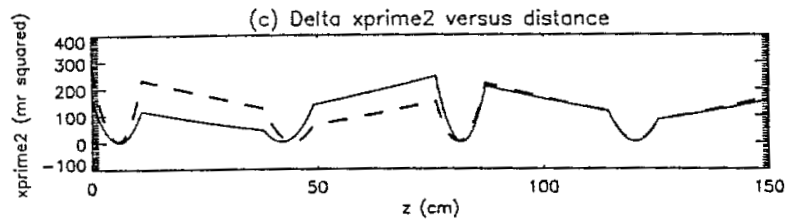

(g) Deito $x-y$ versus distance

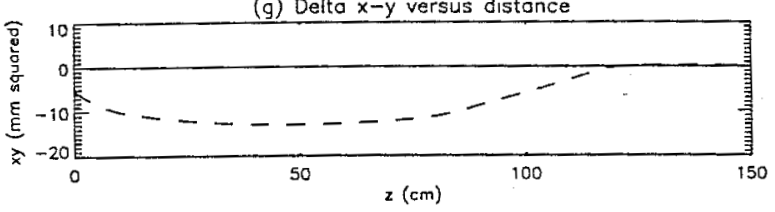

(h) Deito xprime-y versus distance

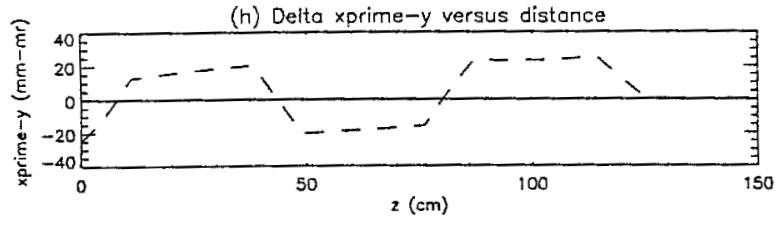

(d) Delto y 2 versus distance

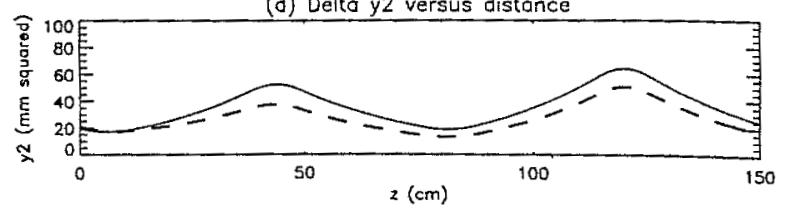

(e) Delta y-yprime versus distance

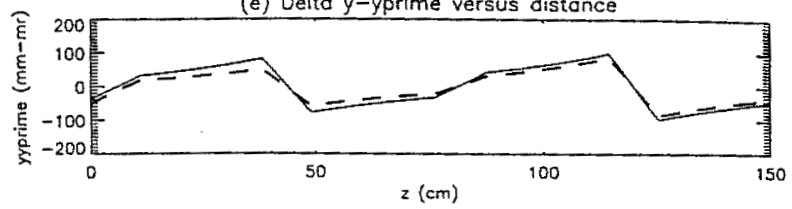

(f) Delta yprime2 versus oistance

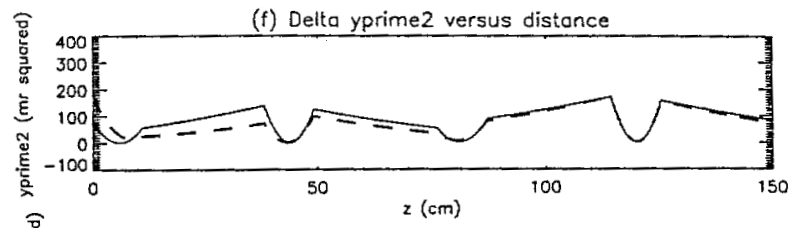

(j) Delto xprime-yprime versus distonce
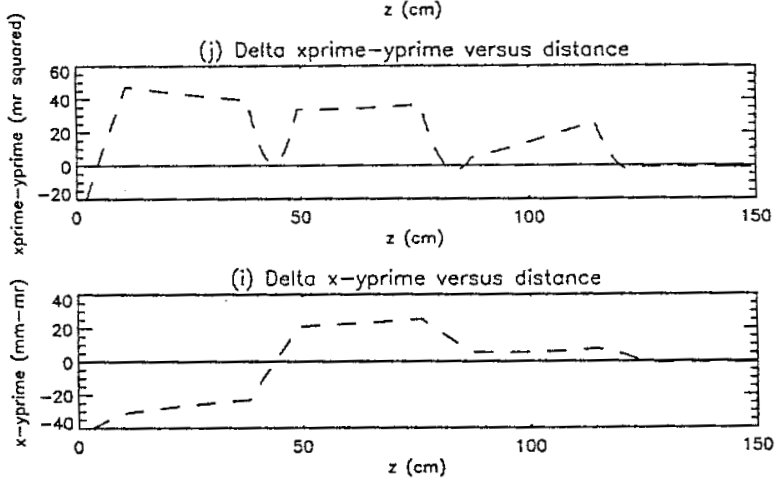

Figure 6.9: Correction of randorn quadrupole rotation errors of up to $\pm 0.5^{\circ}$ from 562 half lattice periods of a Small Recirculator lattice. The beam correction system is placed between $\mathrm{z}=0$ and $152 \mathrm{~cm}$. The dashed lines show the moments as the beam is in the correction system being "corrected" to the desired values. The solid lines are the moments from an aligned lattice and are shown to depict the convergence of the misaligned beam to the desired moment values by the end of the correction system at $\mathrm{z}=152 \mathrm{~cm}$. 
Thus, looking at these results it can be concluded that such a correction system
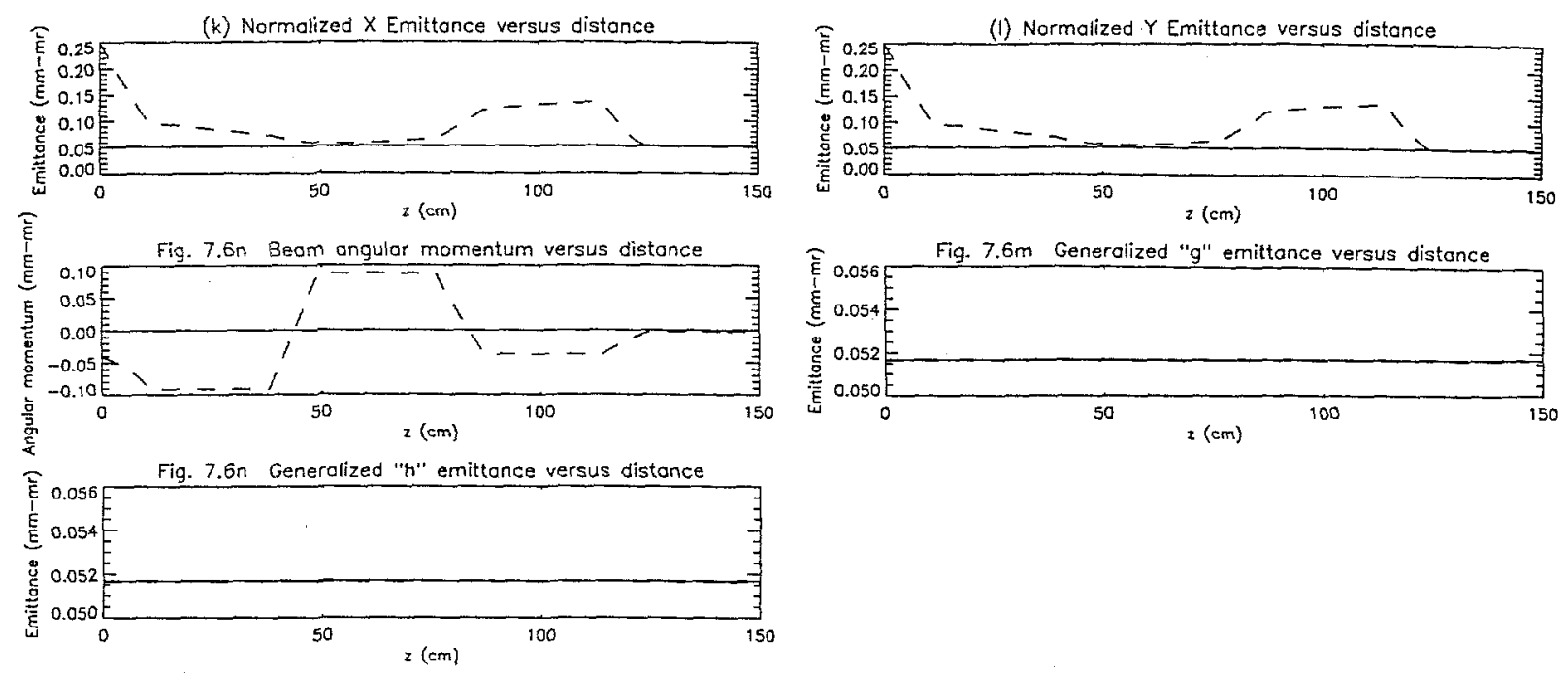

Figure 6.10: Correction of random quadrupole rotation errors of up to $\pm 0.5^{\circ}$ from 562 half lattice periods of a Small Recirculator lattice. The beam correction system is placed between $z=0$ and $152 \mathrm{~cm}$. The dashed lines show the moments as the beam is in the correction system being "corrected" to the desired values. The solid lines are the moments from an aligned lattice and are shown to depict the convergence of the misaligned beam to the desired moment values by the end of the correction system at $z=152 \mathrm{~cm}$.

placed prior to the final focusing element can have a positive impact on lessening the rotational alignment tolerance on a driver's quadrupole focusing elements. Whether or not the degree of precision required for the field strength and rotation angle of the four correction quadrupoles can be attained is not determined here. Nevertheless, for any kind of system that will correct for rotation errors, the $x-y$ cross moments will have to be measured. The GBI is well suited to do this. A test of rudimentary beam correction was carried out on the Small Recirculator using single quadrupoles and the results of these tests as measured by the GBI are discussed in Chapter 7 . 


\section{Chapter 7}

\section{Experimental Results}

A complete set of measurements were made on the Small Recirculator beam at the end of the $45^{\circ}$ bend section with the Gated Beam Imager on July 22, 1997. Good results were obtained and show the effectiveness of the GBI as a diagnostic tool when combined with appropriate image analysis software. During the measurements, the beam energy was $80 \mathrm{keV}$ with an initial $4 \mu \mathrm{s}$ long pulse length which lengthened as the beam traveled through the accelerator since no axial confinement was possible without the modulators installed. The data run consisted of gathering $100 \mathrm{~ns}$ long gated images stepped through successive beam shots to time-resolve the beam into approximately 40 slices. Additionally, $1 \mu$ s long gated images were obtained during the flattop of the beam. Images with upstream magnetic quadrupole rotation errors were taken to study the effect on the beam emittance as discussed in Chapter 6 . The rudimentary beam correction system of counter-rotating one quadrupole at the appropriate distance from a known quadrupole rotation error was also studied to determine the effect on the beam. Appendix $D$ lists the important equipment and software settings used to obtain the data images and information about each image.

As the images were obtained during the initial testing phase of the Small Recirculator $45^{\circ}$ bend section it was necessary to determine the optimum voltage settings for the bending dipole plates in each half lattice period for a drifting $80 \mathrm{keV}$ beam such that the beam centroid would be on centerline when intercepted by the GBI. This was 


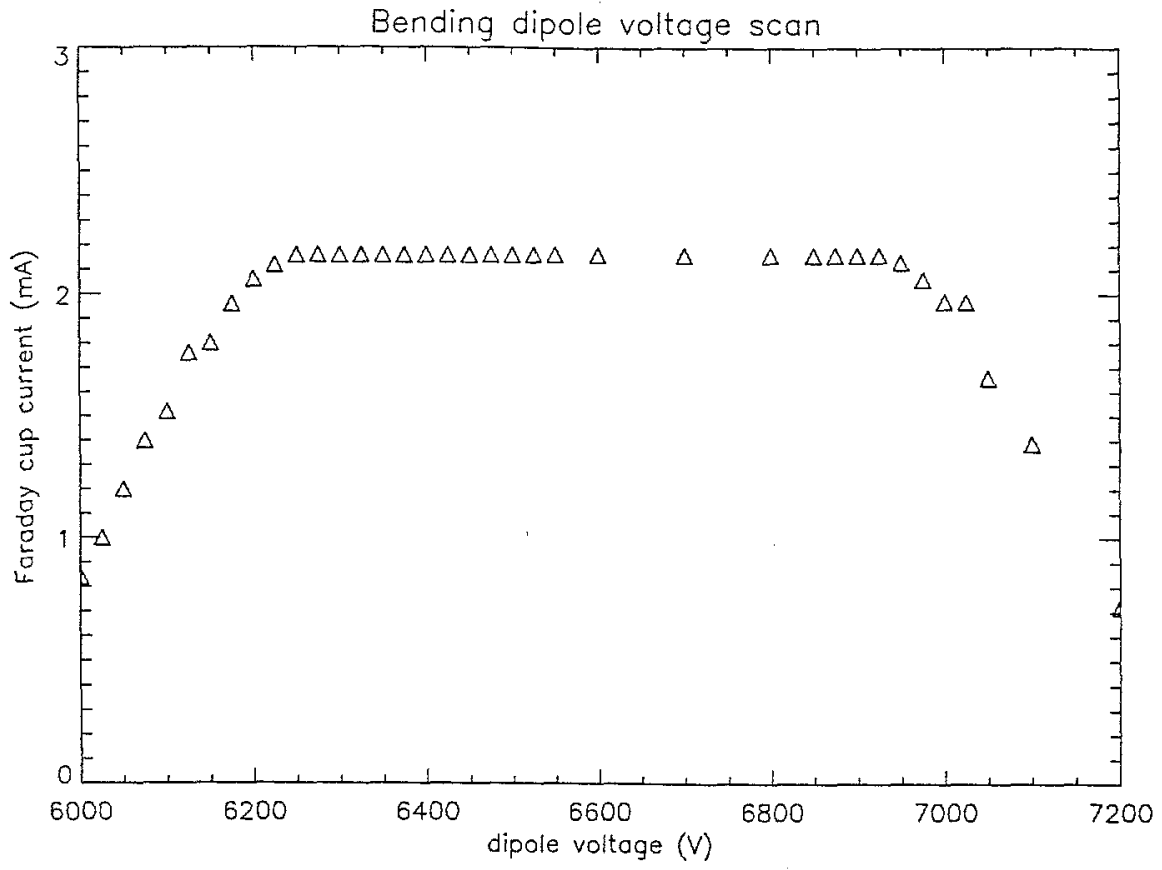

Figure 7.1: Faraday cup current vs. bending dipole voltage scan for determining the optimum dipole voltage to transport the $80 \mathrm{keV}$ around the $45^{\circ}$ bend.

done prior to installing the GBI by inserting a Faraday cup in the multi-diagnostic chamber such that it was centered on the beamline at the outlet of the bend. The beam current into the cup was measured while changing the bending dipole plate voltage in the half lattice periods and then plotted in Figure 7.1. The voltage values of the flattop corresponded to a beam current of $2.06 \mathrm{~mA}$ indicating full beam transport around the $45^{\circ}$ bend. The $4 \mathrm{~cm}$ diameter of the Faraday cup is larger than the nominal diameter $(2.6 \mathrm{~cm})$ of the ion beam resulting in the wide plateau in the curve indicating that the cup intercepted all of the beam over a wide dipole voltage range. A first order estimate of the voltage that put the the beam on the centerline was made by taking the midpoint between the "shoulders" of the curve. This voltage of $6570 \mathrm{~V}$ was applied to the bending dipoles in the subsequent GBI measurements and verified as the proper bending voltage by the images obtained. 


\subsection{GBI Setup and Calibration}

After determining the bending dipole voltage setting, the Faraday cup was withdrawn and the pepperpot and MCP detector of the GBI inserted in their centering mount within the multi-diagnostic chamber and the electrical connections supplying the detector intensifier and proximity focusing voltages made up. The camera was then positioned and focused to give a sharp image of the fiducial marks and scale on the face of the detector. Figure 7.2 shows the camera in position observing the detector through a window in the diagnostic chamber. A calibration image (Figure 7.3) of a scale mounted on the detector was then taken with the camera in place. Analysis of the calibration image with the measurement tools of the IPLAB imaging software determined the spatial resolution of the GBI to be 54 microns per CCD pixel and the field of view to be 2.07 by $3.11 \mathrm{~cm}$. This resolution was used as an input to the IDL Pepperpot analysis code to determine beamlet spot sizes in the subsequent data images and as an input for estimating error bars on the analysis.

\subsection{Observing the Effect of Axial Beam Expansion}

Measurements at the end of the $45^{\circ}$ bend captured a total of 56 images ranging from $4 \mu \mathrm{s}$ long images to gated $100 \mathrm{~ns}$ long images. A time-of-flight calculation predicted the beam head would arrive at the detector at $11.9 \mu \mathrm{s}$ after the source pulser was triggered. The beam head was measured arriving at the detector after $11.7 \mu \mathrm{s}$ with beam tail arriving after $16.4 \mu \mathrm{s}$. Thus, the initial beam length of $4 \mu \mathrm{s}$ had expanded to $4.7 \mu \mathrm{s}$ long. This axial expansion of the beam was not unexpected as no axial confinement fields were applied. In fact, the time-resolved beam images can be viewed as a qualitative measure of the difference in ion energy axially along the beam as seen in the 3 images of Figure 7.4. These images also confirm that the timing signals gating the MCP "ON" and "OFF" are correct and discrete beam slices are being imaged by the GBI. The detector intercepts the head of the beam as it swings wide around the bend as the head is accelerated by space-charge forces within 


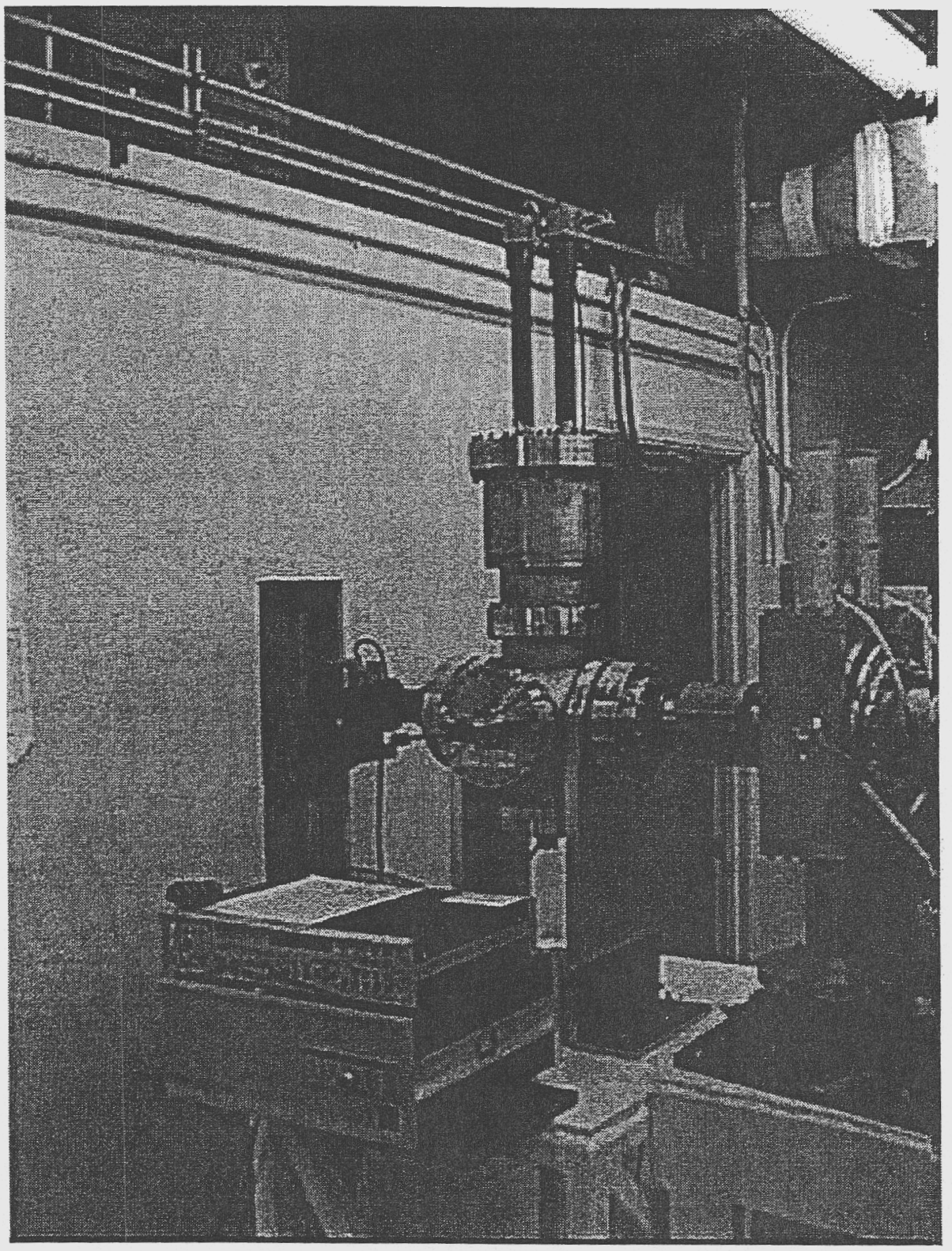

Figure 7.2: Gated Beam Imager in position for data collection at the end of the $45^{\circ}$ bend section 


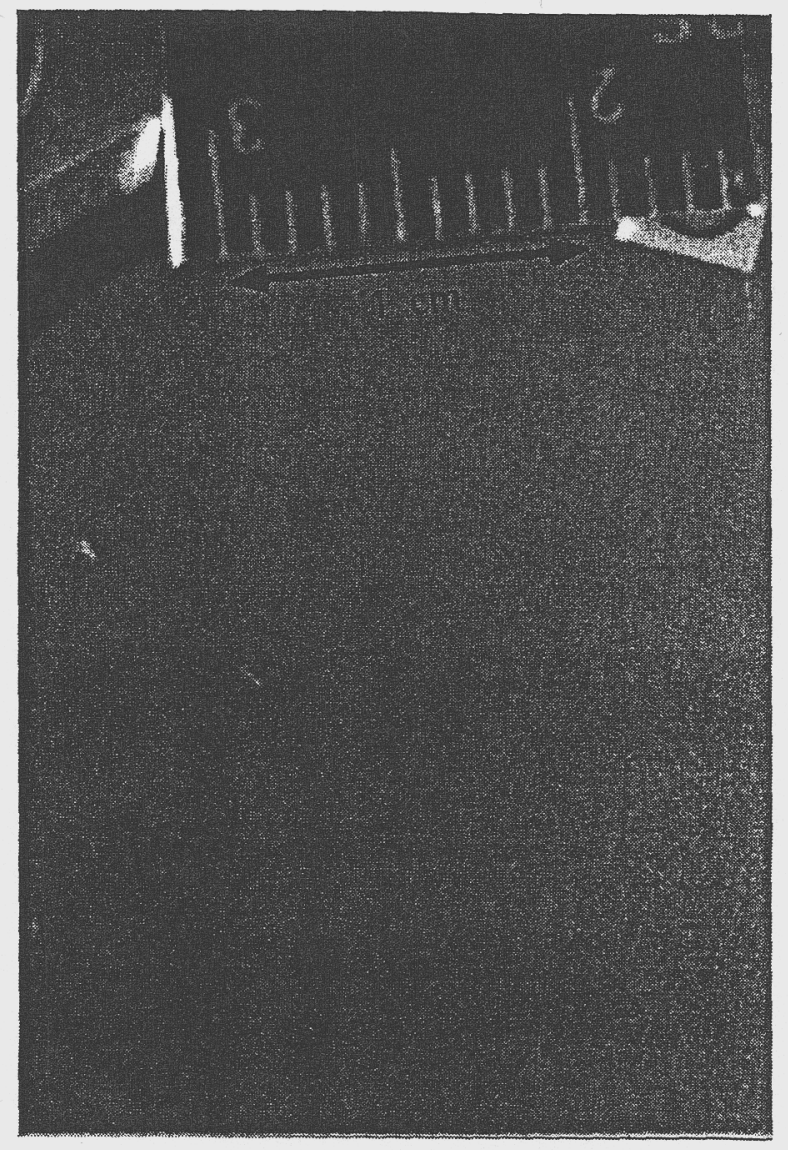

Figure 7.3: Calibration image taken to determine the CCD camera pixel size

the beam (Figure 7.4a). The center image (Figure 7.4b), at $14.0 \mu$ s representing the beam flattop is intercepted at the center of the beamline as it experiences little net longitudinal space-charge force. In the third image (Figure 7.4c), at $15.6 \mu \mathrm{s}$, the ions have been retarded and the beam swings to the inside in the bending dipole field. A "beam movie" was made of the series of 100 ns slices that graphically illustrates the acceleration of the beam head, retardation of the beam tail and the stability of the flattop region of the beam. 


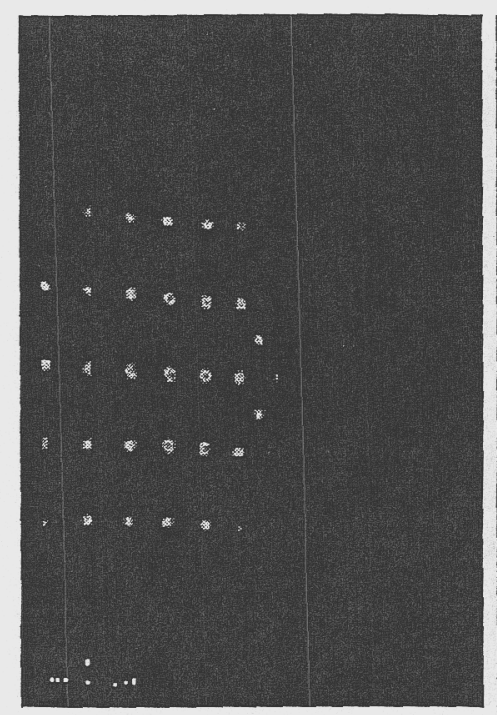

(a)

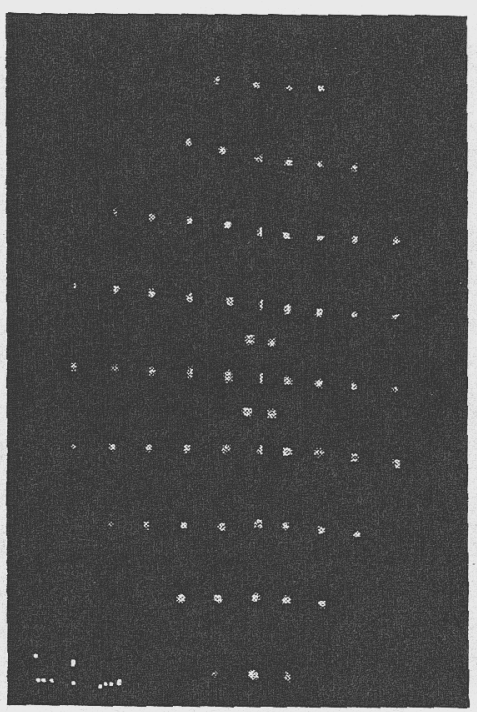

(b)

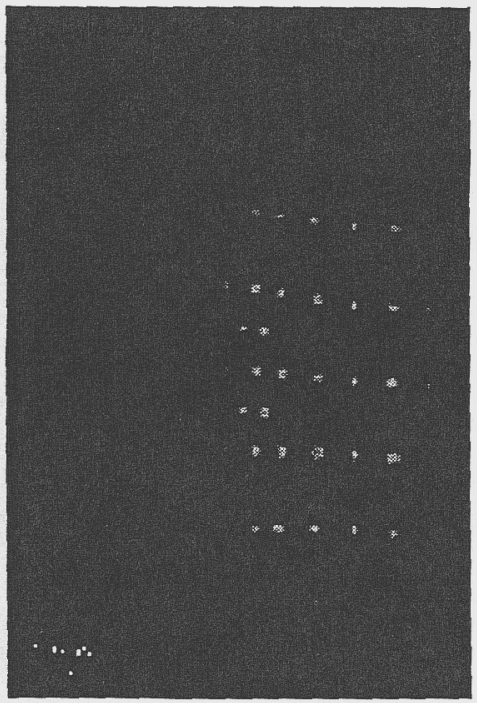

(c)

Figure 7.4: $100 \mathrm{~ns}$ images at the end of $45^{\circ}$ without beam axial confinement showing: a. the beam head swinging wide due to space-charge acceleration, $b$. the beam flattop on center, $\mathrm{c}$. the beam tail swinging inside the bend due to space-charge retardation. 


\subsection{Data Analysis and Results at the $45^{\circ}$ Bend Section}

A total of 48, $100 \mathrm{~ns}$ long gated images were obtained each delayed by $100 \mathrm{~ns}$ from the previous image giving a time history of the beam. Additionally, three $1 \mu \mathrm{s}$ long gated images from the flattop of the beam were captured. These images were taken after times-of-flight of $12.2,13.2$ and $14.2 \mu$ s to characterize the center of the beam shown as the flattop in the oscilloscope trace of the beam into the Faraday cup (Figure 7.5). Each data image was modified prior to analysis as described in Chapter 5 to account for dark charge accumulation and non-uniform response of the camera CCD and the MCP detector.

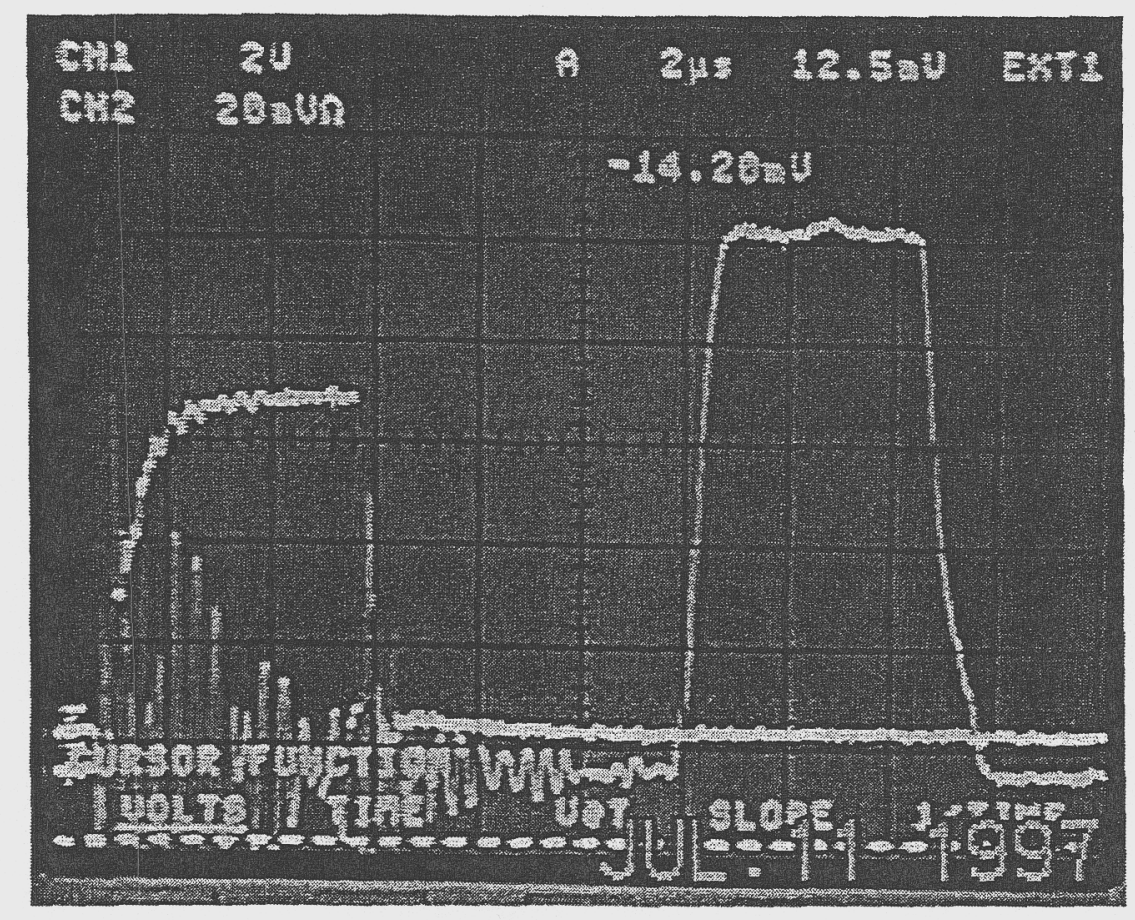

Figure 7.5: Oscilloscope traces of the source pulser (left) and the Faraday cup signal of the beam at the end of the $45^{\circ}$ bend section. The time scale is $2 \mu$ s per division. 


\subsubsection{Comparison of GBI and Slit Scanner Measurements}

Using the IDL Pepperpot analysis code, each image of the beam was analyzed. The emittance and second moments of the beam during each time slice was calculated in order to obtain a time history of the beam. The calculation was carried out as described in Chapter 4. The normalized emittance versus time along the beam with the time origin referenced to the beam head is shown in Figure 7.6. The value of the emittance in the $y$-direction obtained with a slit scanner is also indicated on Figure 7.6 with the triangle symbol. There is essential agreement between the two methods. Figure 7.7 shows the time history of the ten second moments of the beam. These figures represent a graphical distillation of the 4-D and time phase space map of the beam. The flattop numerical values characterizing the beam are shown in Table 7.1 , where the symbol " $\Delta$ " is the second moment minus the contribution of a non-zero first moment, following the convention of Chapter 4 .

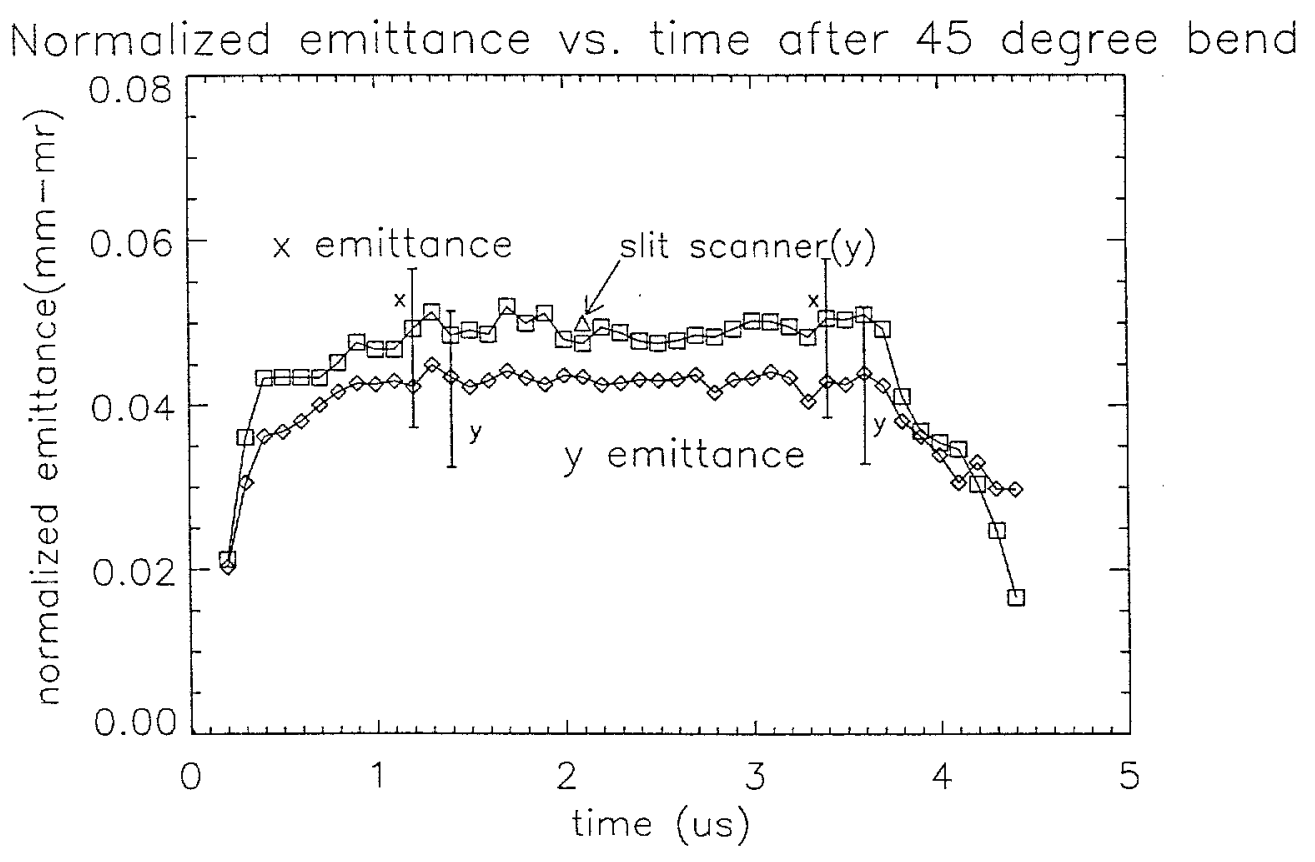

Figure 7.6: Normalized emittance vs. time from the GBI. A single data point representing the slit scanner flattop emittance measurement in the $y$-direction is shown with the triangle symbol. 
(a) $\langle x 2\rangle$ versus time

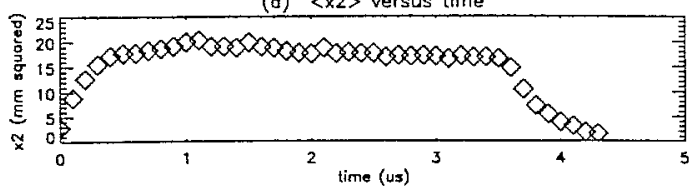

(c) <x-xprime $>$ versus time

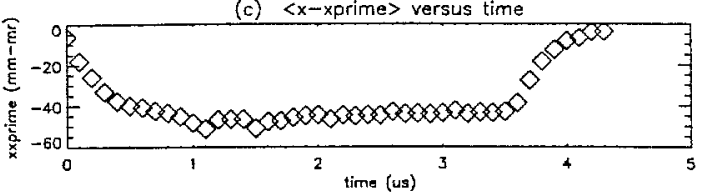

(e) <xprime2> versus time

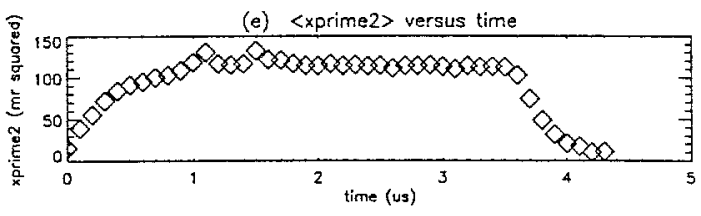

(a) $\langle x-y\rangle$ versus time

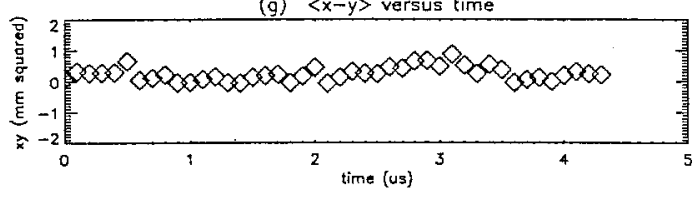

(i) <xprime-y> versus time

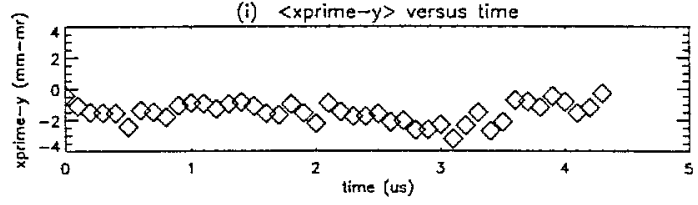

(b) $\langle y 2\rangle$ versus time

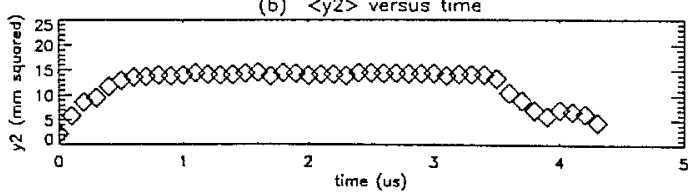

(d) $\langle y$-yprime $>$ versus time

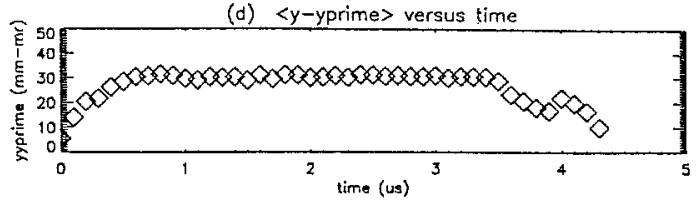

(f) <yprime2> versus time

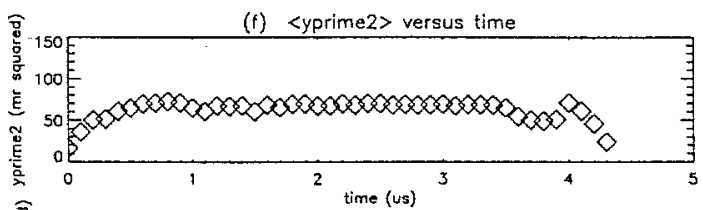

(h) <xprime-yprime> versus time

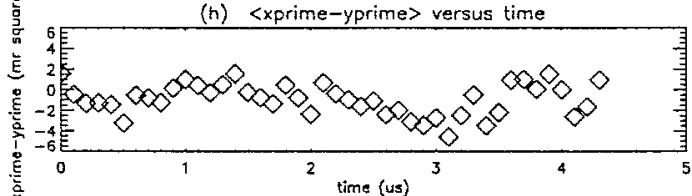

(j) <x-yprime> versus time

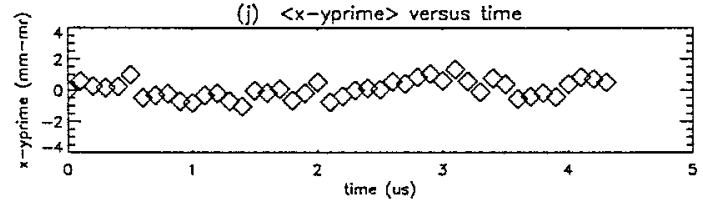

Figure 7.7: Beam ion distribution second moments vs. time 
Table 7.1: Characterization of the Small Recirculator beam after the $45^{\circ}$ bend section using the GBI.

\begin{tabular}{|l|c|c|c|}
\hline$\langle x\rangle(\mathrm{mm})$ & 0.67 & $\langle y\rangle(\mathrm{mm})$ & 0.39 \\
\hline$\left\langle x^{\prime}\right\rangle(\mathrm{mr})$ & 1.06 & $\left\langle y^{\prime}\right\rangle(\mathrm{mr})$ & -3.35 \\
\hline$\Delta x^{2}\left(\mathrm{~mm}^{2}\right)$ & 34.6 & $\Delta y^{2}\left(\mathrm{~mm}^{2}\right)$ & 27.4 \\
\hline$\Delta x x^{\prime}(\mathrm{mm}-\mathrm{mr})$ & -87.3 & $\Delta y y^{\prime}(\mathrm{mm}-\mathrm{mr})$ & 60.0 \\
\hline$\Delta x^{\prime 2}\left(\mathrm{mr}^{2}\right)$ & 223.8 & $\Delta y^{\prime 2}\left(\mathrm{mr}^{2}\right)$ & 135.3 \\
\hline$\Delta x y\left(\mathrm{~mm}^{2}\right)$ & 0.736 & $\left.\Delta x^{\prime} y^{\prime}(\mathrm{mr})^{2}\right)$ & -3.881 \\
\hline$\Delta x^{\prime} y(\mathrm{~mm}-\mathrm{mr})$ & -3.814 & $\Delta x y^{\prime}(\mathrm{mm}-\mathrm{mr})$ & 0.773 \\
\hline$\epsilon_{x}$ (rms edge) $(\mathrm{mm}-\mathrm{mr})$ & 22.73 & $\epsilon_{n x}(\mathrm{~mm}-\mathrm{mr})$ & 0.0484 \\
\hline$\epsilon_{y}$ (rms edge) $(\mathrm{mm}-\mathrm{mr})$ & 20.06 & $\epsilon_{n y}(\mathrm{~mm}-\mathrm{mr})$ & 0.0427 \\
\hline x envelope (mm) & 11.8 & y envelope $(\mathrm{mm})$ & 10.5 \\
\hline x' envelope $(\mathrm{mr})$ & -29.7 & y' envelope $(\mathrm{mr})$ & 22.9 \\
\hline angular momentum (mm-mr) & 4.587 \\
\hline
\end{tabular}

\subsubsection{Error Analysis}

The error bars on the emittance data points in Figure 7.6 were calculated based on known or estimated tolerances in the physical size of GBI dimensions and measurement errors that have a substantial effect on the moment calculations. Each worst case tolerance value was input into the Pepperpot analysis code. The effect on the calculation was assumed independent of the effect of the other tolerances. Like sign effects were summed to generate the high and low limits of the error bars. The tolerances that were accounted for and their contributions to the emittance are summarized in Table 7.2. Additionally the Peppperpot analysis code was run with all of the like effect tolerances applied together to calculate the result when they are allowed to interact.

Proper interpretation of the error bars is important to drawing a conclusion as to the relative magnitudes of the $x$ and $y$ emittances. Those errors that tend to cause the emittance to be greater or smaller than the calculated value (where the 
data point falls) track each other in both the $x$ and $y$ directions. The conclusion drawn from this is that indeed the $x$ emittance which is "in-plane" with the bend will remain larger than the $y$ emittance and both values will track together up or down their respective error bars. As can be seen in Table 7.2 the calculation of the emittance is most sensitive to errors in the alignment about the $z$-axis of the grid of holes in the pepperpot. A misalignment of the pepperpot results in an error in the calculation of the angle from the position of the hole to the position of the beamlet spot. Additionally this error is not constant but increases with the distance from the beam axis. Empirically, it was seen with the images obtained at the $45^{\circ}$ bend, the error introduced did not contribute equally to the error bar in both the $x$ and $y$ directions. An error resulting from the misalignment of the pepperpot is however applied equally to all calculations from image to image within a series where the GBI has not been moved.

Table 7.2: Pepperpot data analysis sensitivity to measurement errors, and instrument manufacturing and alignment tolerances. (Emittance units are mm-mr)

\begin{tabular}{|l|c|c|c|c|}
\hline Maximum Error $\downarrow$ & $\epsilon_{n x}$ & $\Delta \epsilon_{n x}$ & $\epsilon_{n y}$ & $\Delta \epsilon_{n y}$ \\
\hline \hline Nominal Emittances & 0.04838 & 0 & 0.04269 & 0 \\
\hline Hole Size $+5 \%$ & 0.0409 & -0.00029 & 0.04237 & -0.00032 \\
Hole Size $-5 \%$ & 0.0487 & 0.00031 & 0.04301 & 0.00032 \\
\hline mask rot/cam. angle $+0.5^{\circ}$ & 0.04768 & -0.00070 & 0.04461 & 0.00195 \\
mask rot/cam. angle - $0.5^{\circ}$ & 0.04952 & 0.00114 & 0.04312 & 0.00043 \\
\hline w/o intensity weighting & 0.05042 & 0.00204 & 0.04474 & 0.00206 \\
\hline$+5 \%$ pixel calibration error & 0.05089 & 0.00251 & 0.04496 & 0.00227 \\
\hline$-5 \%$ pixel calibration error & 0.04587 & -0.00251 & 0.04042 & -0.00227 \\
\hline spot radius meas. error $(+27 \mu)$ & 0.05160 & 0.00322 & 0.04619 & 0.00350 \\
\hline spot radius meas. error $(-27 \mu)$ & 0.04538 & -0.00300 & 0.03928 & -0.00341 \\
\hline change beam energy $+1 \%$ & 0.04799 & -0.00039 & 0.04234 & -0.00035 \\
change beam energy - $1 \%$ & 0.04751 & -0.00087 & 0.04192 & -0.00077 \\
\hline space charge + 8.22\% spot size & 0.04363 & -0.00475 & 0.03838 & -0.00431 \\
\hline \hline \multicolumn{4}{|c|}{0.00719} \\
\hline + Error bar x emittance & -0.01212 \\
\hline - Error bar x emittance & -0.00802 \\
\hline + Error bar y emittance \\
\hline - Error bar y emittance
\end{tabular}




\subsubsection{Comparison of GBI Data with WARP Numerical Sim- ulation Code Results}

Members of the LLNL HIF project staff have been developing methods to apply the numerical simulation code WARP as a tool both the theorist, and experimentalist can use to resolve questions regarding the performance of the Small Recirculator. Although this effort is in its infancy and the experimental results are meant to verify the correct values of variable initial parameters in the WARP code, it is nevertheless useful as a confidence building measure in the utility of the GBI to compare its measurements to the code results using reasonable values of the input parameters. The 6-D phase space distribution of the beam initially loaded into the WARP code is a constant density particle distribution in physical space $(x, y, z)$ with a semi-gaussian spread in the velocities of the ions in the transverse $\left(v_{x}, v_{y}\right)$ and axial $\left(v_{z}\right)$ directions. It is the width of the semi-gaussian velocity spread in the axial direction that most influences the growth in transverse emittance caused by the transition from the straight transport section to the bend section. In the code results, the emittance growth occurs most in the "in-plane" direction $(x)$ of the bend and to a lesser extent in the $y$ direction until the perturbation thermalizes spreading the emittance across both directions. Figure 7.8 shows a graphical output from the WARP code with the data points from the GBI placed at their measured values. It can be seen from the sensitivity in the emittance growth that determining the proper initial value of the axial velocity spread is important. The data from the GBI and slit scanners indicates that a narrower spread is appropriate as the emittance in both the $x$ and $y$ directions does not grow as much as predicted using the initial velocity spreads $(0.250 .11$ The envelope of the beam and its slope at the pepperpot location can be easily calculated from the moments measured by the GBI. A module of the WARP code (WARPenv) calculates the beam envelope parameters. Figure 7.9 shows data points calculated from GBI moments for the envelope and envelope slope along with code simulation 


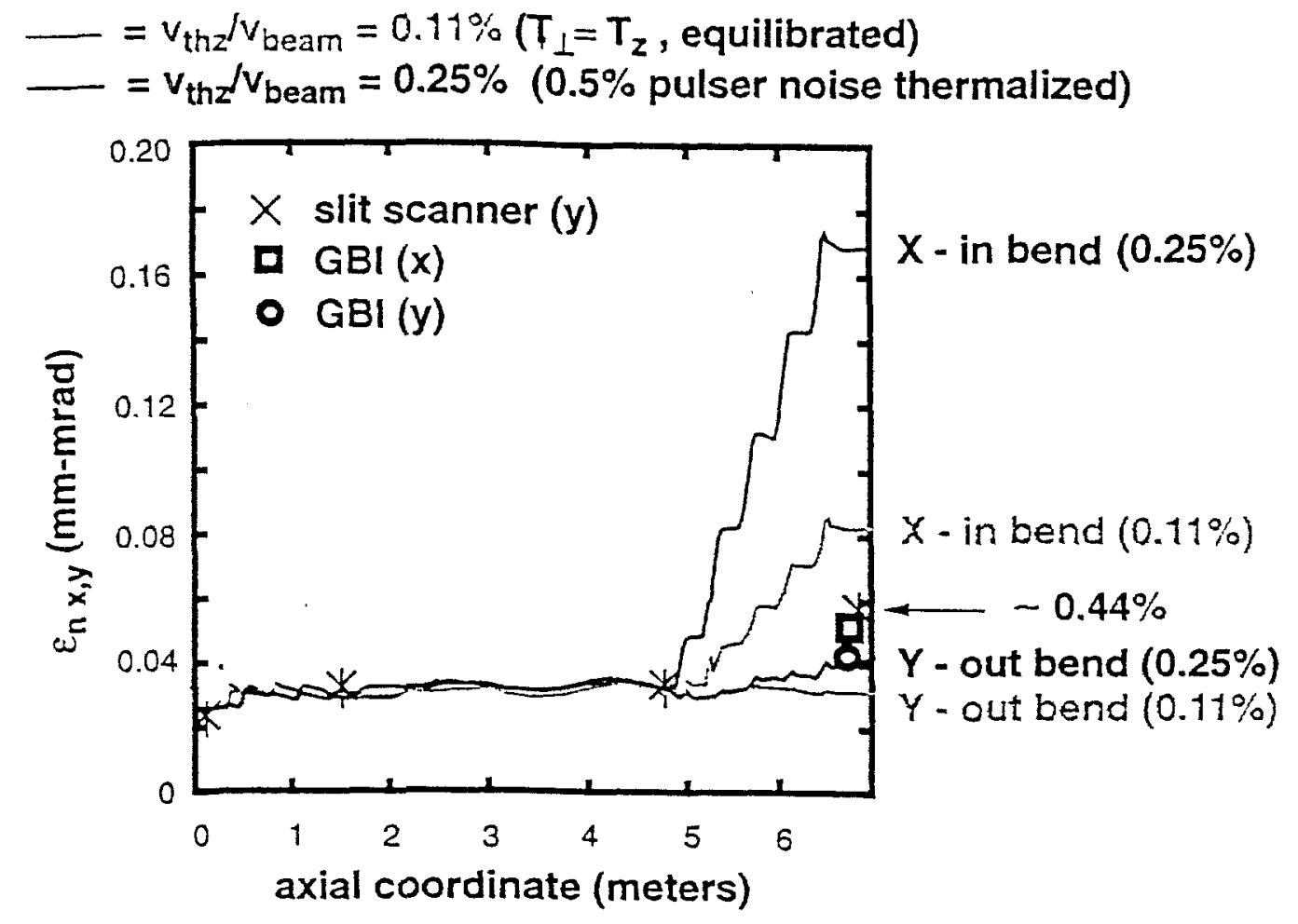

Figure 7.8: Experimental emittance data compared to "WARPxy" simulation. ${ }^{47}$

output. This shows a favorable comparison between the code envelope calculations and the experimental measurements. The envelope dimensions $(a, b)$, and envelope slopes $\left(a^{\prime}, b^{\prime}\right)$ were calculated from the second moments using:

$$
a=2 \sqrt{\left\langle(x-\langle x\rangle)^{2}\right\rangle} \quad b=2 \sqrt{\left.<(y-<y\rangle)^{2}\right\rangle}
$$

and

$$
a^{\prime}=2 \frac{(x-<x>)\left(x^{\prime}-<x^{\prime}>\right)}{\sqrt{\left.<(x-<x>)^{2}\right\rangle}} \quad b^{\prime}=2 \frac{(y-<y>)\left(y^{\prime}-<y^{\prime}>\right)}{\sqrt{\left.<(y-<y>)^{2}\right\rangle}}
$$

The slope equations having been obtained by taking the derivative with respect to $z$ of the envelope equations. 

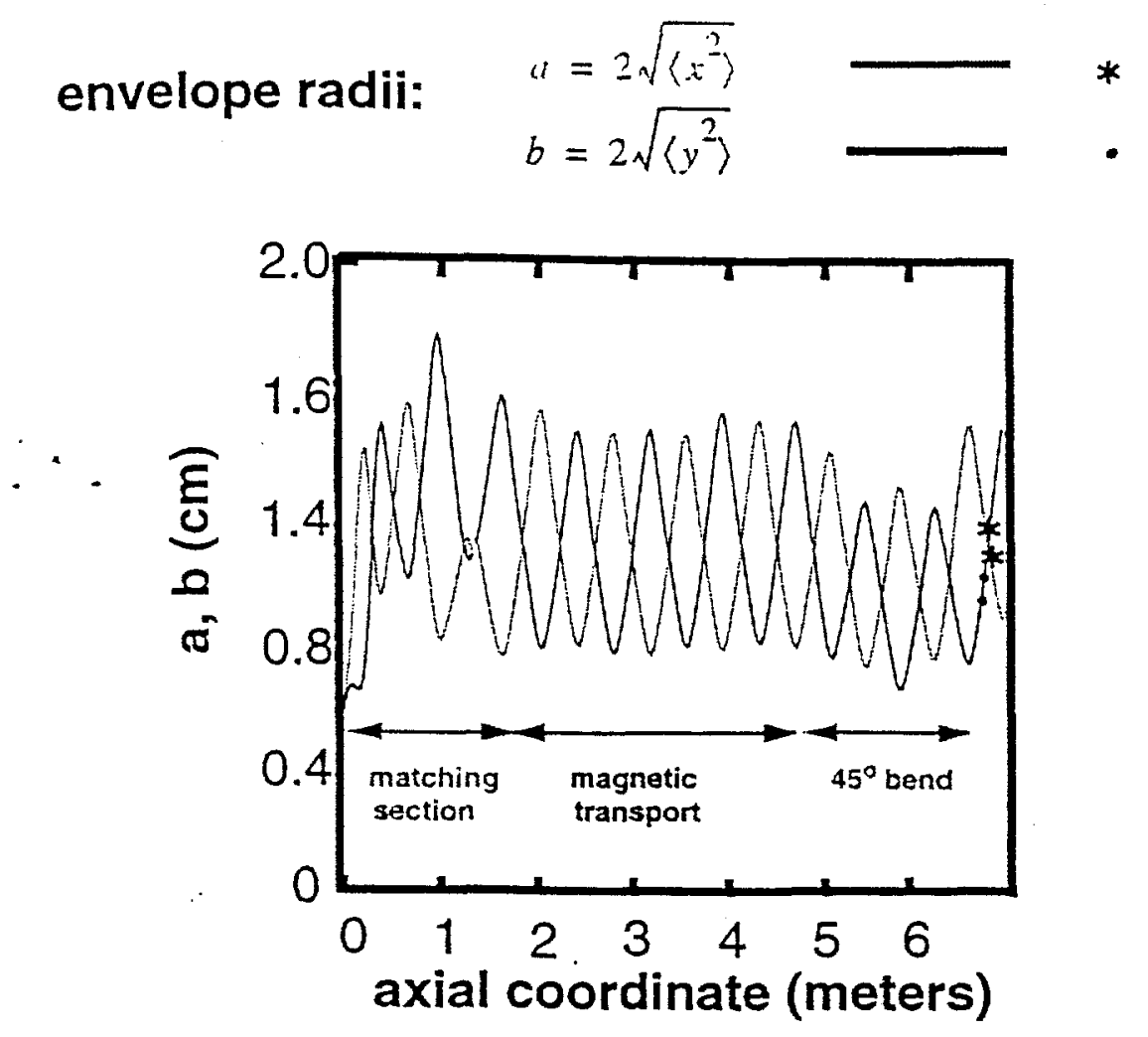

Figure 7.9: Experimental beam envelope data compared with "WARPxy" numerical simulation simulation. The two asterisks show the envelope detected by the GBI at the pepperpot and the detector in the $x$-direction. Similarly the two dots are the data in the $y$-direction. ${ }^{47}$

\subsection{Observing and Measuring the Effect of Magnetic Quadrupole Rotation Errors and Applying Approximate Correc- tions}

The effect on the beam of magnetic quadrupole rotation errors discussed in Chapter 6 was tested by imaging the beam with known quadrupole rotation errors applied to the Small Recirculator. The rudimentary correction of the beam by counterrotation of a single quadrupole of the same field strength at the appropriate position was also tested. The proposed beam correction system of four adjustable quadrupoles 
was not tested on the Small Recirculator and the construction of such a system is not yet included in project plans. Nevertheless, the results of the rudimentary tests conducted point to the utility of the more complicated system.

From Chapter 6, empirical simulation studies showed that the effects of a known single rotation error can be largely removed by counter-rotating a single, downstream quadrupole. The proper number of half-lattice periods between the adjusted quadrupoles is given by $360 / \sigma_{0}$ when this quotient is an integer. As the GBI was positioned at the output of magnetic quadrupole 13, testing was accomplished with quadrupole 8 and 13 with $5^{\circ}$ rotations. The limitations to the tests conducted included the fact that the actual phase advance of the Small Recirculator is $78^{\circ}$ when the effect of the bending dipoles is added to the $72^{\circ}$ phase advance of the magnetic quadrupoles alone. Because $360^{\circ}$ divided by the actual phase advance is not an integer, but is 4.61 , rotating the $8^{\text {th }}$ and $13^{\text {th }}$ quadrupole is a close approximation. The other limitation was the existence of unknown random quadrupole rotation errors that exist in any machine. These unknown errors are most likely a contributor to the non-zero $x-y$ cross moments obtained in the images without any intentional rotations. Nevertheless, inducing known quadrupole errors was instructive in confirming their effect on the beam which exploring the concepts of rotation error tolerances and beam correction. Figure 7.10 shows a series of images taken and analyzed for beam moment characterization. They are all $1 \mu \mathrm{s}$ long gated images of the flattop of the beam starting at a time-of-flight of $13.2 \mu \mathrm{s}$, about $1 \mu \mathrm{s}$ back from the beam head.

Figure 7.10(a) shows an image of the GBI beamlet spots with only the small existing random quadrupole rotation errors causing coupling between the $x$ and $y$ directions. The calculations of the second moments results in non-zero $x-y$ cross moments as listed in Table 7.3. Looking at the image, what appears like a tilt in the rows and columns of the spots, is more likely a combination of the random rotation errors and a $1.7^{\circ}$ rotation of the pepperpot from the "laboratory frame". The tilt in the pattern can also be identified as not solely due to the pepperpot rotation as the angle of the rows with the $x$-axis is greater than the angle of the columns with the $y$ - 


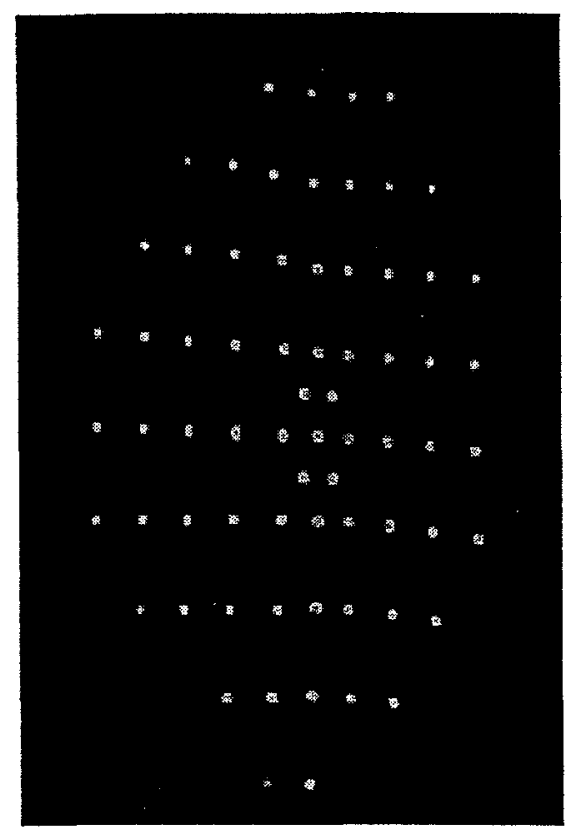

(a)

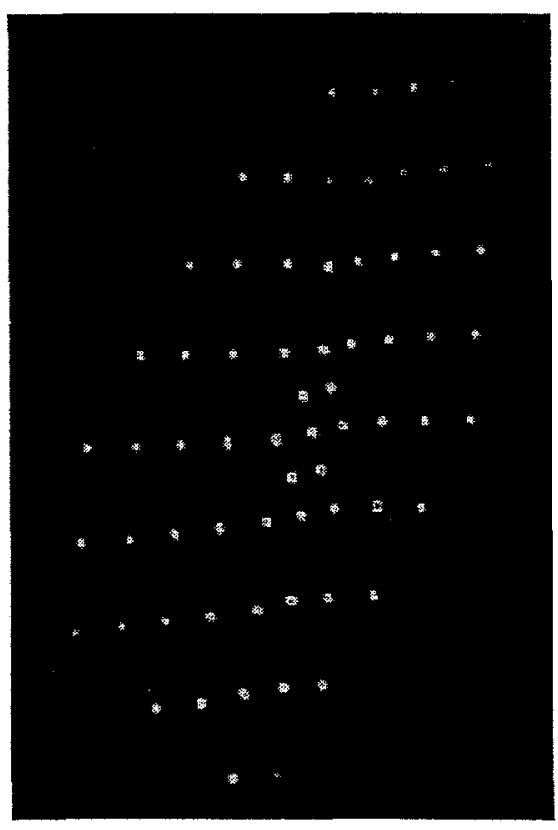

(c)

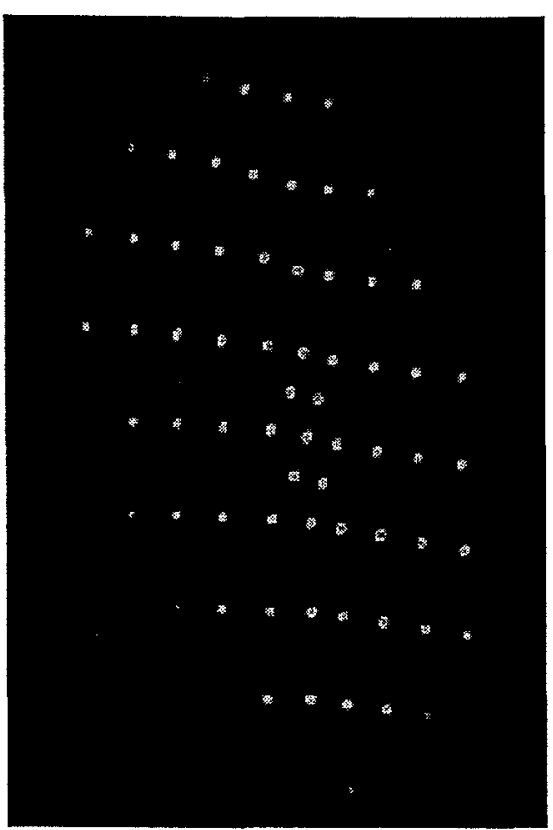

(b)

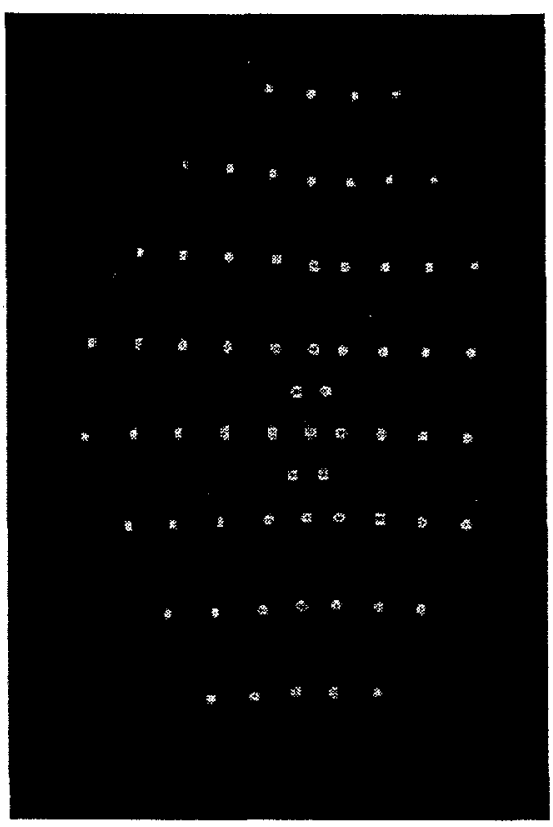

(d)

Figure 7.10: GBI images with quadrupole rotation errors. All images are $1 \mu \mathrm{s}$ long from the beam flattop at $13.2 \mu \mathrm{s}$. a. Existing experimental rotation errors and other effects b. Quad 8 rotated $5^{\circ}$ counter-clockwise only c. Quad 13 rotated $5^{\circ}$ clockwise only d. "Corrected" beam with quad 8 at $5^{\circ} \mathrm{CCW}$ and quad 13 at $5^{\circ} \mathrm{CW}$. 
axis. With the pepperpot rotation angle removed during the calculation of moments, $x-y$ cross moments remained non-zero, albeit much smaller than when coupling is intentionally induced. Figures 7.11 , and 7.12 show the emittance ellipses and cross moment data plots for this case.

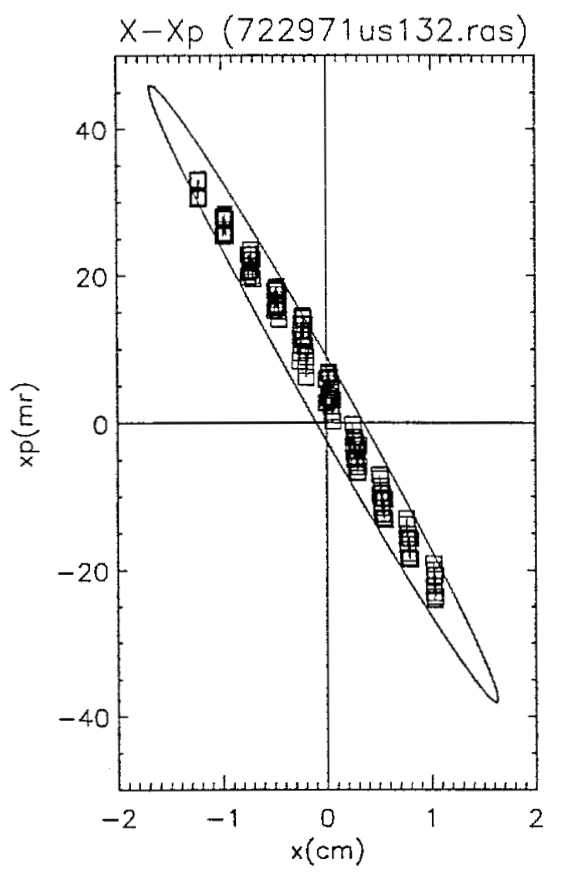

rms edge $\times$ emittance $24.00 \mathrm{~mm}-\mathrm{mr}$
norm. $x$ emittance $0.051 \mathrm{~mm}-\mathrm{mr}$ norm. $x$ emittance
Level(percent) $=10$ at mosk

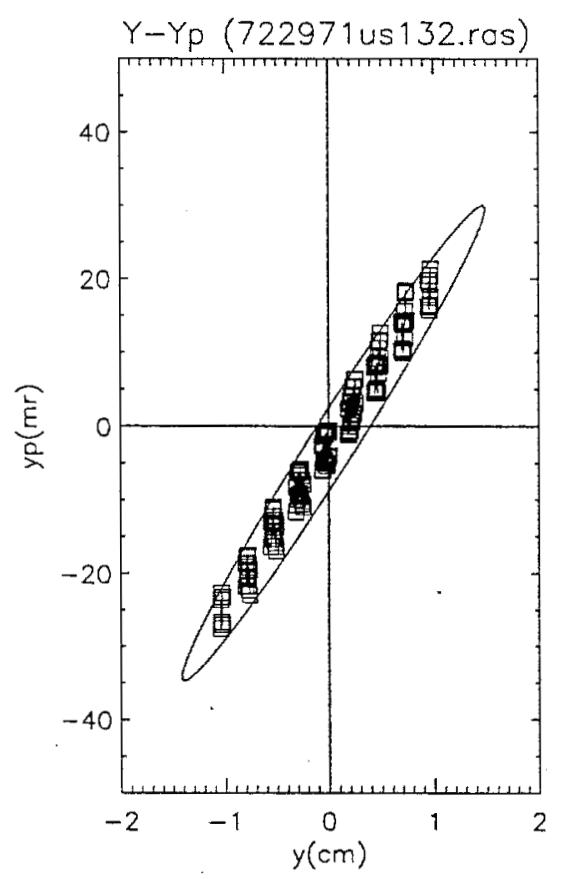

rms edge y emittance $20.67 \mathrm{~mm}-\mathrm{mr}$
norm. y emittonce $0.044 \mathrm{~mm}-\mathrm{mr}$
67 beomlets Smoothing 0
Mask -1.700 deg.

Figure 7.11: Phase space emittance plots with "aligned" quadrupoles. 

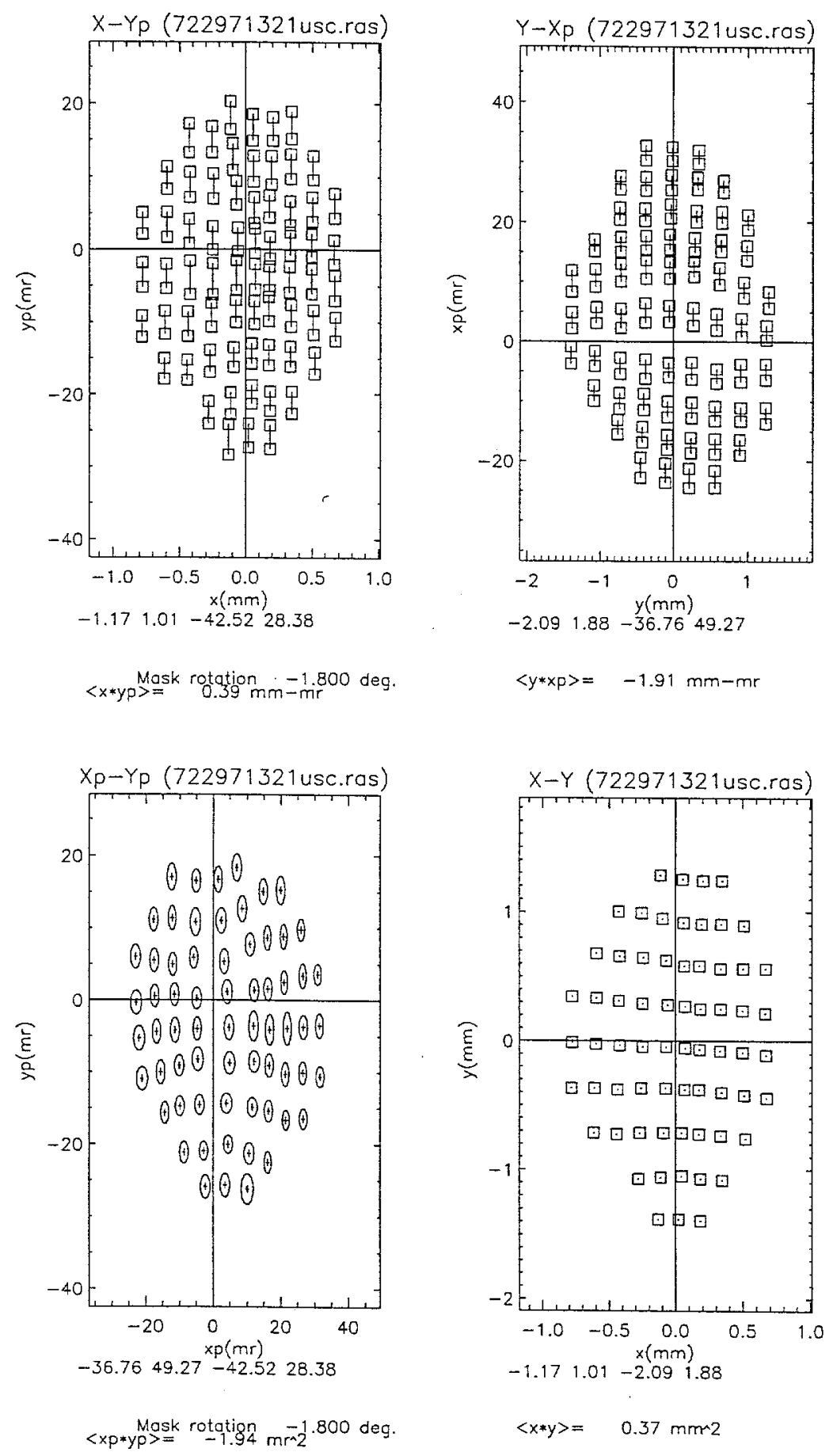

Figure 7.12: Cross moment plots with "aligned" quadrupoles. 
Figure 7.10(b) shows the beamlet spot images when quadrupole 8 was rotated $5^{\circ}$ counterclockwise. Its moment values are listed in Table 7.2 and emittance ellipses and cross moments data plots are shown in Figures 7.13, and 7.14. Not unexpectantly, the emittance in the $x$ and $y$ directions increases indicating the decrease in focusability of the beam due to their (more) non-zero angular momentum. This image along with Figure 7.10(c) illustrate the ease with which one can observe using the GBI that quadrupole rotation errors have caused an increase in the emittance. A slit scanner will detect the same emittance increase from Figure 7.10(a) to $7.10(\mathrm{~b})$ but with the data available from the measurement the analysis would be hard-pressed to definitively indicate that the cause. As the slit scanner averages the beam information parallel to its slits, detection of the slight envelope changes even for a gross rotation error as I induced here is not likely.
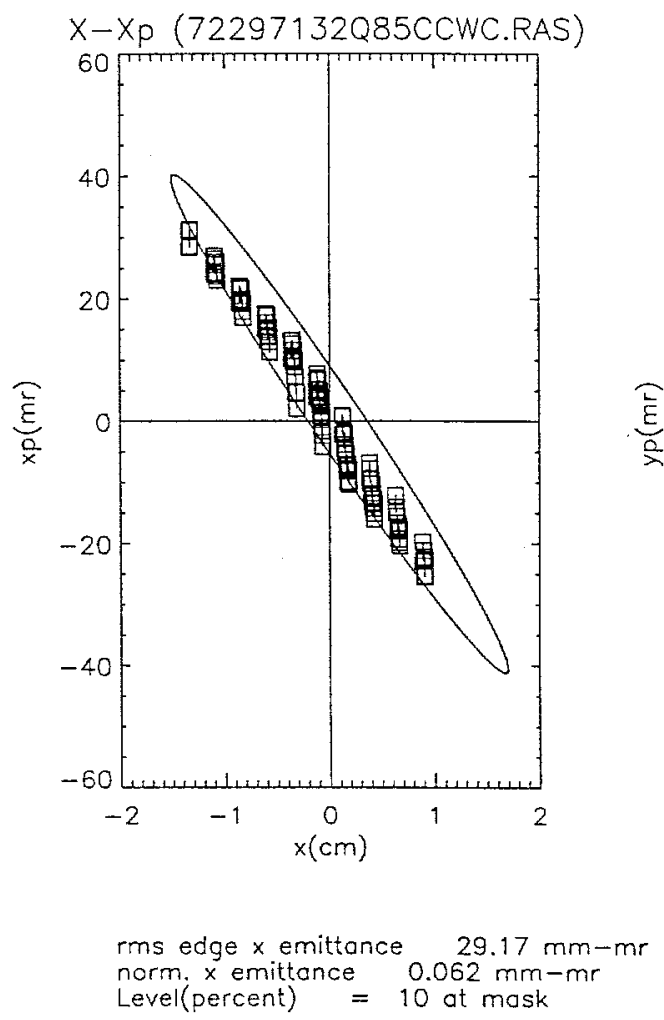
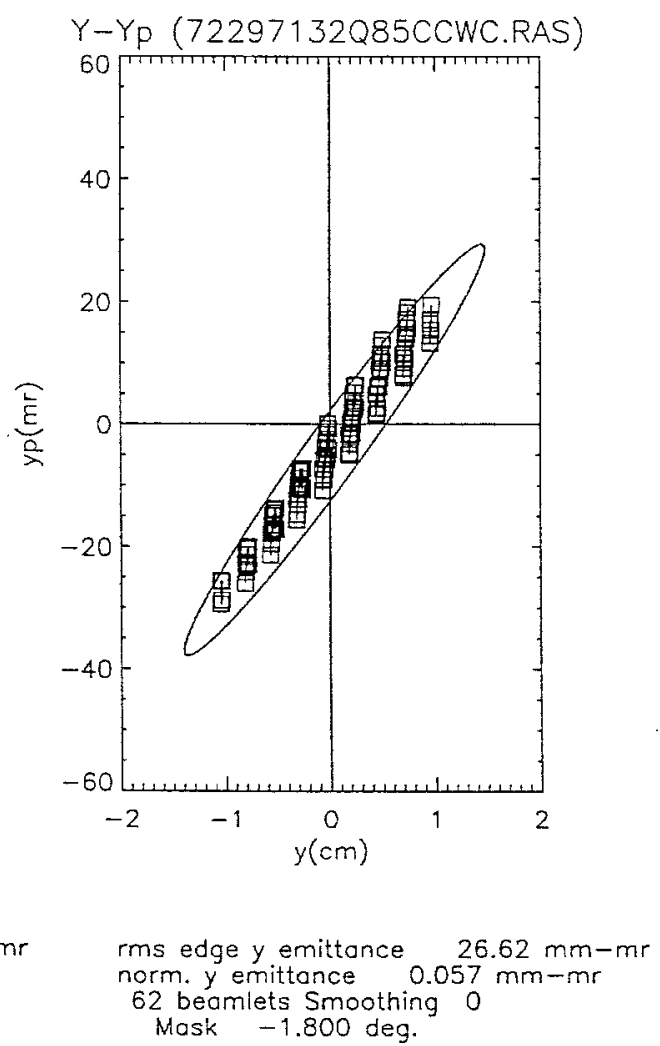

Figure 7.13: Phase space emittance plots with quadrupole 8 rotated $5^{\circ}$ counterclockwise. 

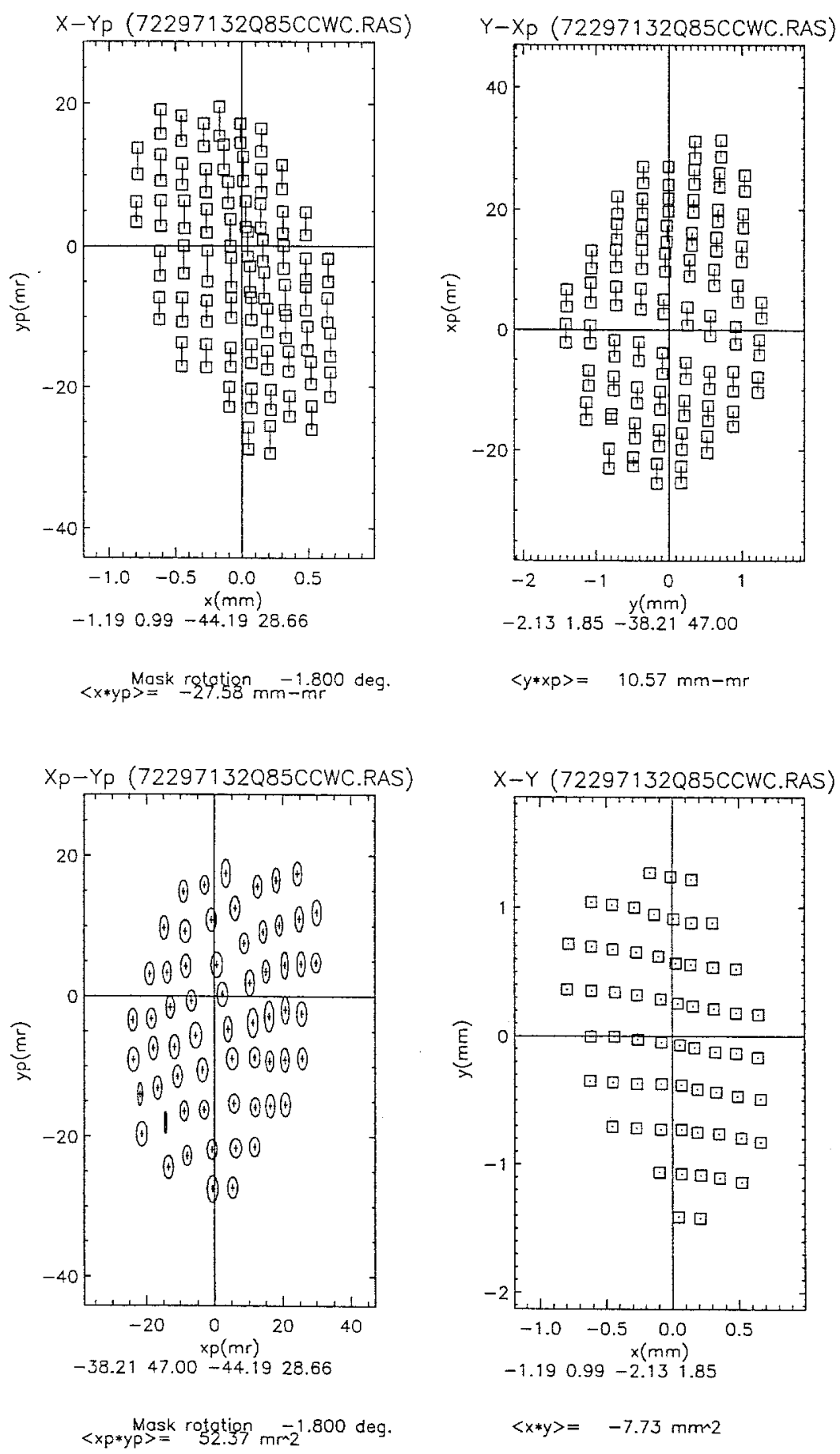

Figure 7.14: Cross moment plots with quadrupole 8 rotated $5^{\circ}$ counter-clockwise. 
Figure 7.10(c) shows the image obtained when quadrupole 8 is returned to alignment and a $5^{\circ}$ clockwise rotation is placed on quadrupole 13 . Similar to the rotation of quadrupole 8, the beam's $x$ and $y$ emittances increase along with the cross moments because of the increased coupling. Figures 7.15, 7.4, and 7.16 show the emittance ellipse and cross moment data plots.
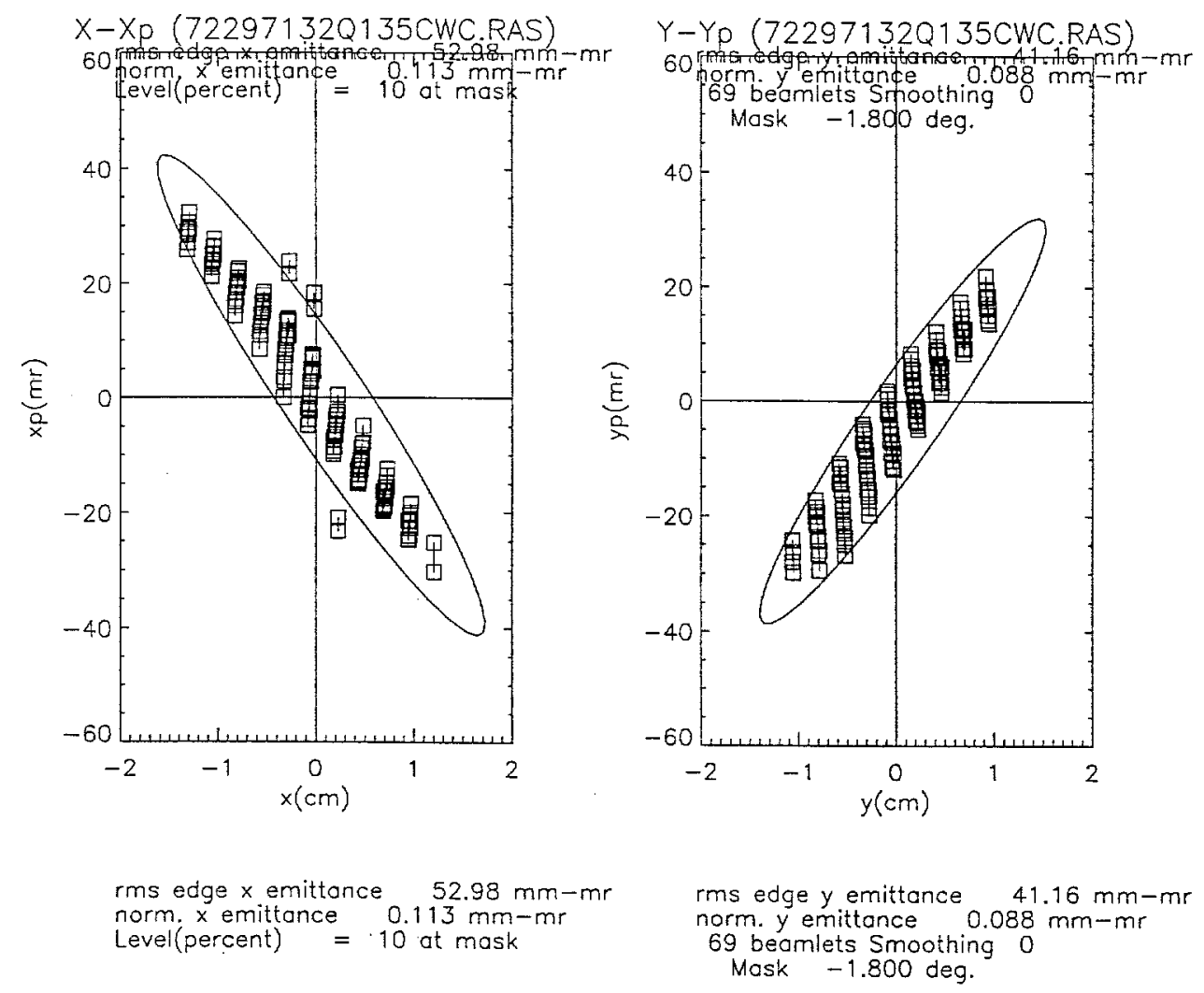

Figure 7.15: Phase space emittance plots with quadrupole 13 rotated $5^{\circ}$ clockwise. 

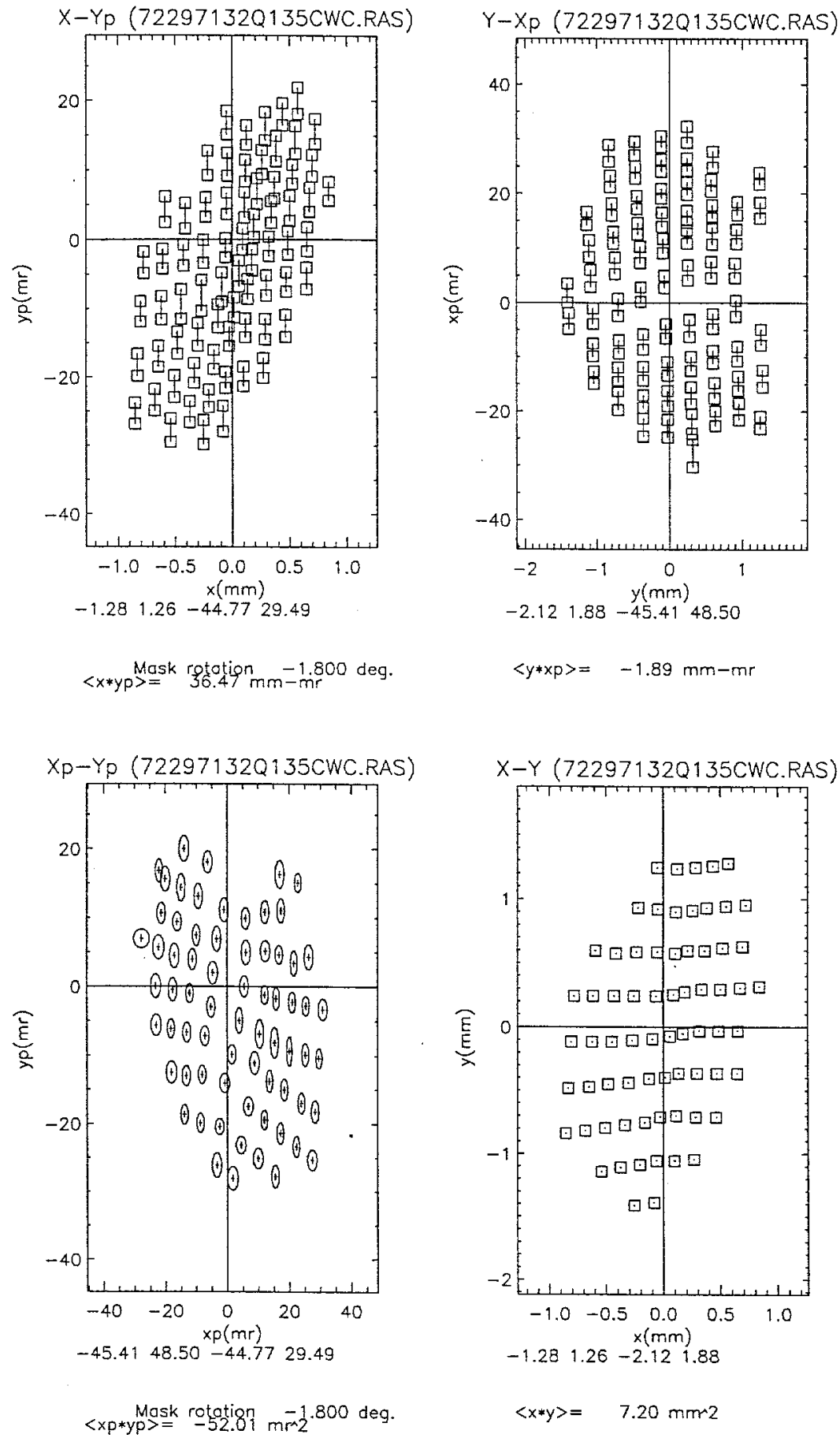

Figure 7.16: Cross moment plot with quadrupole 13 rotated $5^{\circ}$ clockwise. 
Figure 7.10(d) shows the results when quadrupole 8 and 13 are counter-rotated by $5^{\circ}$ approximating the rudimantary beam correction for a known quadrupole rotation error. In this case the error in quadrupole 8 being corrected by quadrupole 13. Again it is an approximation to the special case simulation results shown in Figure 6.4 due to the physical limitations previously discussed. The image itself is upright and the rows and columns are parallel to the $x$ and $y$ axes. As all the other images indicated the $1.7^{\circ}$ rotation of the pepperpot, this parallelism indicates that the "correction" is less than optimal as expected. The calculated moments and emittances do however substantially show values much improved over those with only one quadrupole rotated. Figures 7.17, 7.4, and 7.18 show the emittance ellipse and cross moment data plots.

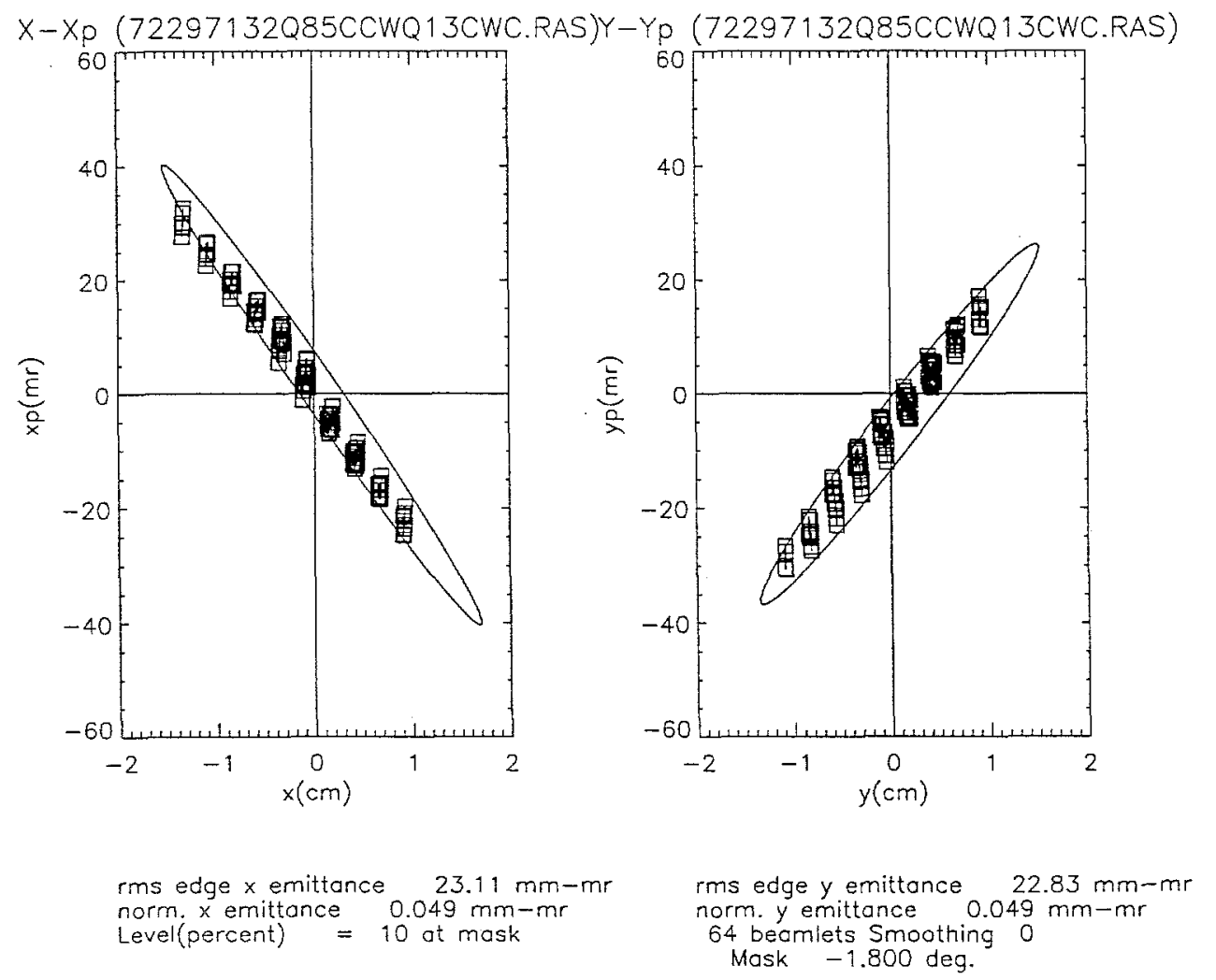

Figure 7.17: Phase space emittance plots with quadrupoles 8 and 13 counter-rotated by $5^{\circ}$. 

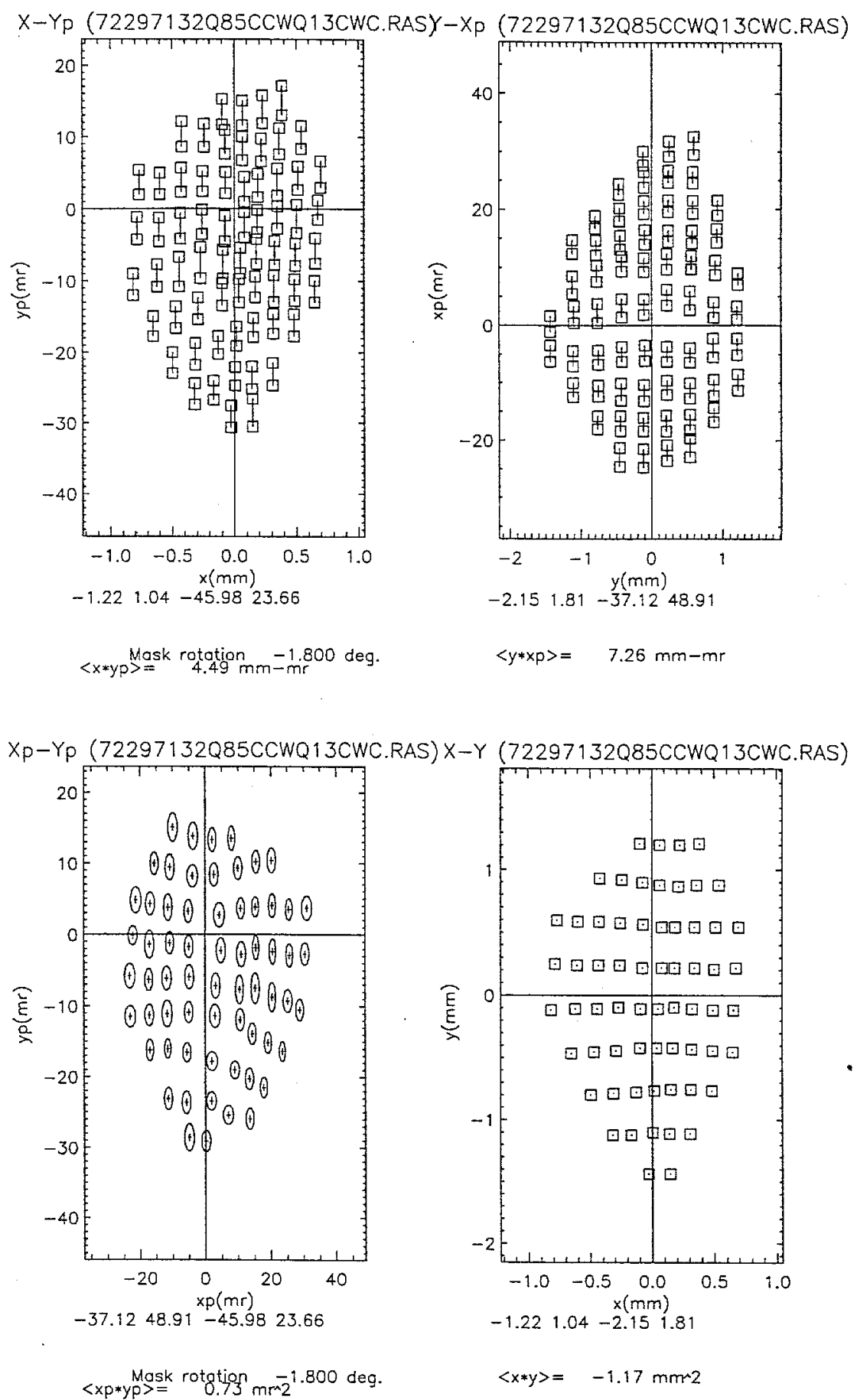

Figure 7.18: Cross moment plot with quadrupoles 8 and 13 counter-rotated by $5^{\circ}$. 
Table 7.3: Summary of beam parameters with known quadrupole rotation errors. a. Quadrupole 8 rotated $5^{\circ}$ counter-clockwise only b. Quadrupole 13 rotated $5^{\circ}$ clockwise only $\mathrm{c}$. Rudimentary beam correction applied by counter-rotation of quadrupoles 8 and $13 \mathrm{~d}$. Parameters from a beam with no rotation errors.

\begin{tabular}{|l|c|c|c|c|}
\hline Beam parameter & Q8 CCW & Q13 CW & "Corrected" & No Rotation \\
\hline \hline$\langle x\rangle(\mathrm{mm})$ & 0.96 & -0.52 & 0.084 & 0.67 \\
\hline$\left\langle x^{\prime}\right\rangle(\mathrm{mr})$ & -0.5 & 0.6 & 0 & 1.1 \\
\hline$\langle y\rangle(\mathrm{mm})$ & 0.40 & 0.63 & 0.83 & 0.39 \\
\hline$\left\langle y^{\prime}\right\rangle(\mathrm{mr})$ & -4.3 & -3.3 & -5.2 & -3.3 \\
\hline$\Delta x^{2}\left(\mathrm{~mm}^{2}\right)$ & 32.3 & 35.1 & 32.9 & 34.6 \\
\hline$\Delta x x^{\prime}\left(\mathrm{mm}^{2} \mathrm{mr}\right)$ & -80.7 & -83.4 & -81.2 & -87.3 \\
\hline$\Delta x^{\prime 2}\left(\mathrm{mr}^{2}\right)$ & 208.2 & 218.3 & 204.1 & 223.8 \\
\hline$\Delta y^{2}\left(\mathrm{~mm}^{2}\right)$ & 26.0 & 26.8 & 25.9 & 27.4 \\
\hline$\Delta y y^{\prime}\left(\mathrm{mm}^{2}-\mathrm{mr}\right)$ & 59.3 & 61.5 & 55.8 & 60.0 \\
\hline$\Delta y^{\prime 2}\left(\mathrm{mr}^{2}\right)$ & 142.0 & 157.0 & 125.0 & 135.3 \\
\hline$\Delta x y\left(\mathrm{~mm}^{2}\right)$ & -7.7 & 7.2 & -1.2 & 0.7 \\
\hline$\Delta \dot{x}^{\prime} y(\mathrm{~mm}-\mathrm{mr})$ & 10.6 & -1.9 & 7.3 & -3.8 \\
\hline$\Delta x y^{\prime}(\mathrm{mm}-\mathrm{mr})$ & -27.6 & 36.5 & 4.5 & 0.8 \\
\hline$\Delta x^{\prime} y^{\prime}\left(\mathrm{mr}{ }^{2}\right)$ & 52.4 & -52.0 & 0.7 & -3.9 \\
\hline$\epsilon_{x}(\mathrm{rms})(\mathrm{mm}-\mathrm{mr})$ & 29.2 & 53.0 & 23.1 & 22.7 \\
\hline$\epsilon_{y}$ (rms)(mm-mr) & 26.6 & 41.2 & 22.8 & 20.1 \\
\hline$\epsilon_{n x}(\mathrm{~mm}-\mathrm{mr})$ & 0.062 & 0.11 & 0.049 & 0.048 \\
\hline$\epsilon_{n y}(\mathrm{~mm}-\mathrm{mr})$ & 0.057 & 0.09 & 0.049 & 0.043 \\
\hline $\mathrm{x}$ envelope $(\mathrm{mm})$ & 11.3 & 11.8 & 11.4 & 11.7 \\
\hline $\mathrm{x}^{\prime}$ envelope $(\mathrm{mr})$ & -28.4 & -28.1 & -28.2 & -29.6 \\
\hline $\mathrm{y}$ envelope (mm) & 10.2 & 10.3 & 10.1 & 10.4 \\
\hline $\mathrm{y}^{\prime}$ envelope $(\mathrm{mr})$ & 23.3 & 23.7 & 21.9 & 22.9 \\
\hline angular momentum (mm-mr) & -38.2 & 38.4 & -2.8 & 4.6 \\
\hline
\end{tabular}




\subsection{Beam Transverse Intensity Distribution Measurement}

An examination of the intensity distribution in the $x-y$ plane is also of great interest in determining the performance of the ion source. The calculations of the emittance yields a value for an equivalent $\mathrm{K}-\mathrm{V}$ beam (uniform distribution in phase space) but the actual distribution may be quite different. A surface plot of the beamlet total intensity vs. $\mathrm{x}-\mathrm{y}$ position with interpolation between spots is shown in Figure 7.19. As one can see the Small Recirculator beam at the end of $45^{\circ}$ was quite different from a uniform distribution. A surface plot of this kind is very useful as the difference in intensity level is less evident looking at the image or a 1-D cut along a line of beamlet spots shown in Figure 7.21. The resulting conclusion that the intensity distribution is far from uniform across the beam was not evident (nor easily observable without a lot of effort) from previous slit scanner measurements. Using the slit scanner data the degradation of the ion source had not been detected, although a re-examination of the data shows correlation between it and the GBI intensity data. A scan of the source near its output aperature using a pair of $1 \mathrm{~mm}$ cross slits and a Faraday cup was performed on August 2, 1997. This scan shown in Figure 7.20 also showed a large variation in source intensity prior to beam transport through the electric and magnetic quadrupole lattice. Subsequent physical analysis of the ion source at Lawrence Berkeley Laboratory showed that the source was largely depleted of potassium. ${ }^{54}$ The attributes of the GBI that allow it to quickly measure the 2$D$ intensity profile of the beam make it most useful in gaging source performance degradation once calibrated against a known good source and any anomalies in beam transport are eliminated in contributing to a changed intensity profile. 


\section{Beam Intensity After 45 Deg Bend}

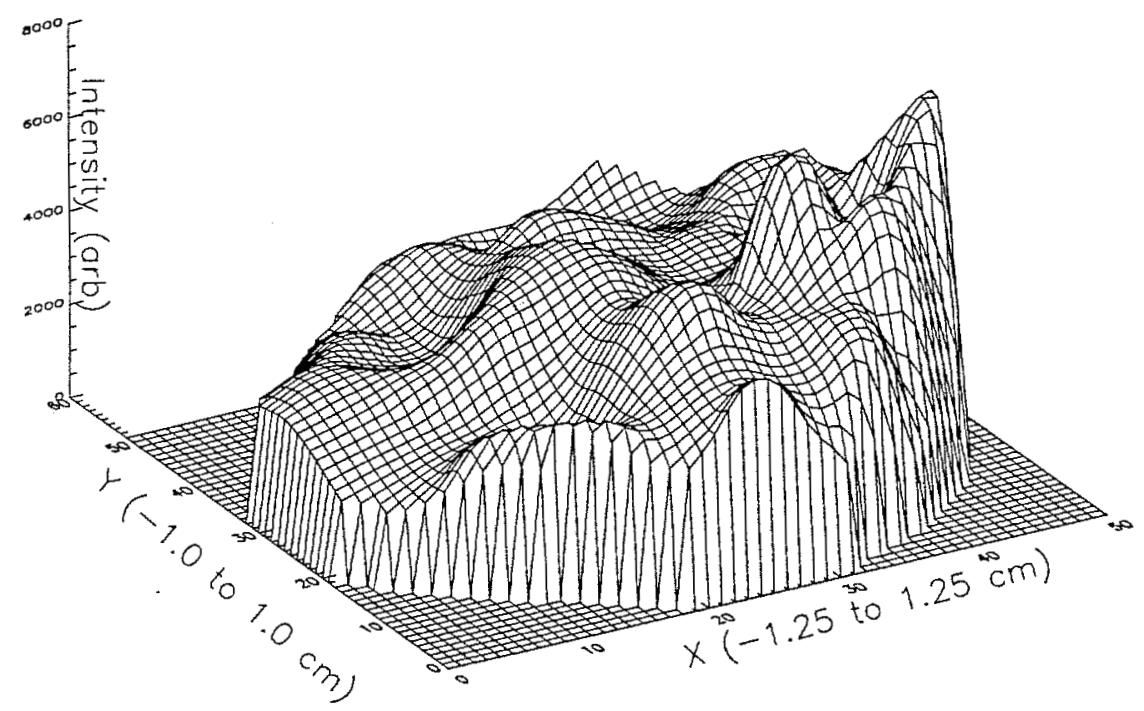

Figure 7.19: Small Recirculator beamlet intensity surface plot after the $45^{\circ}$ bend showing the uneven emission of the potassium ion source.

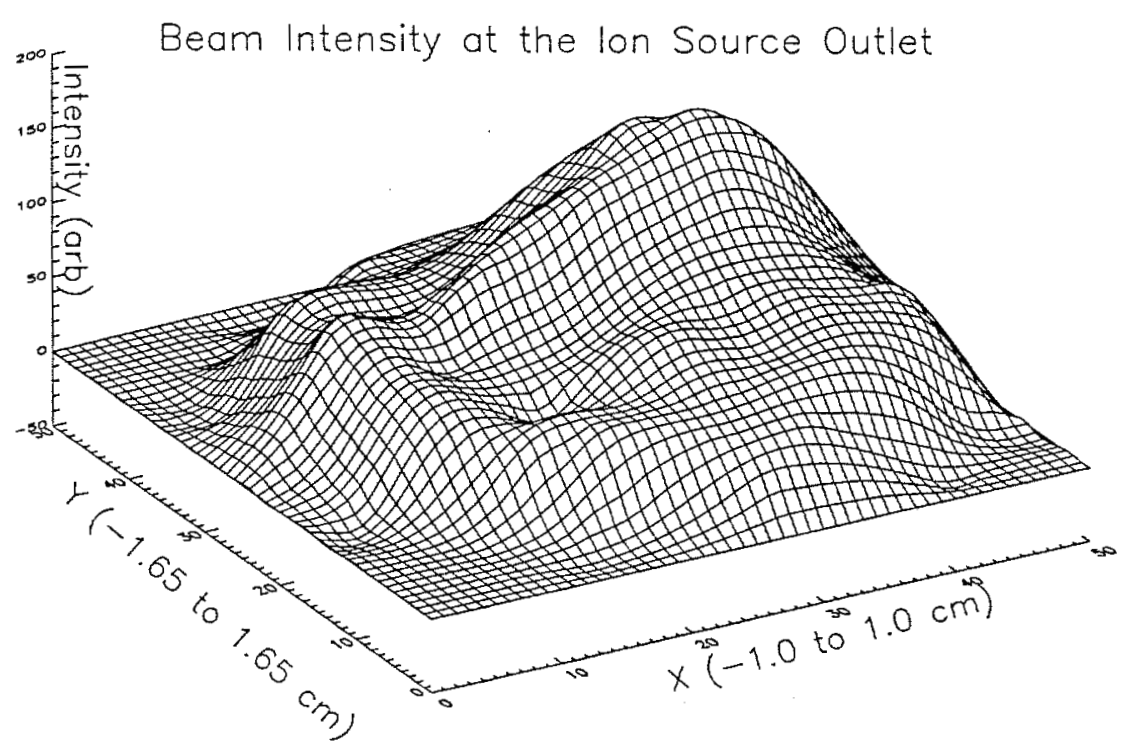

Figure 7.20: Small Recirculator ion source scan at the source indicating nonuniformity of emission. 


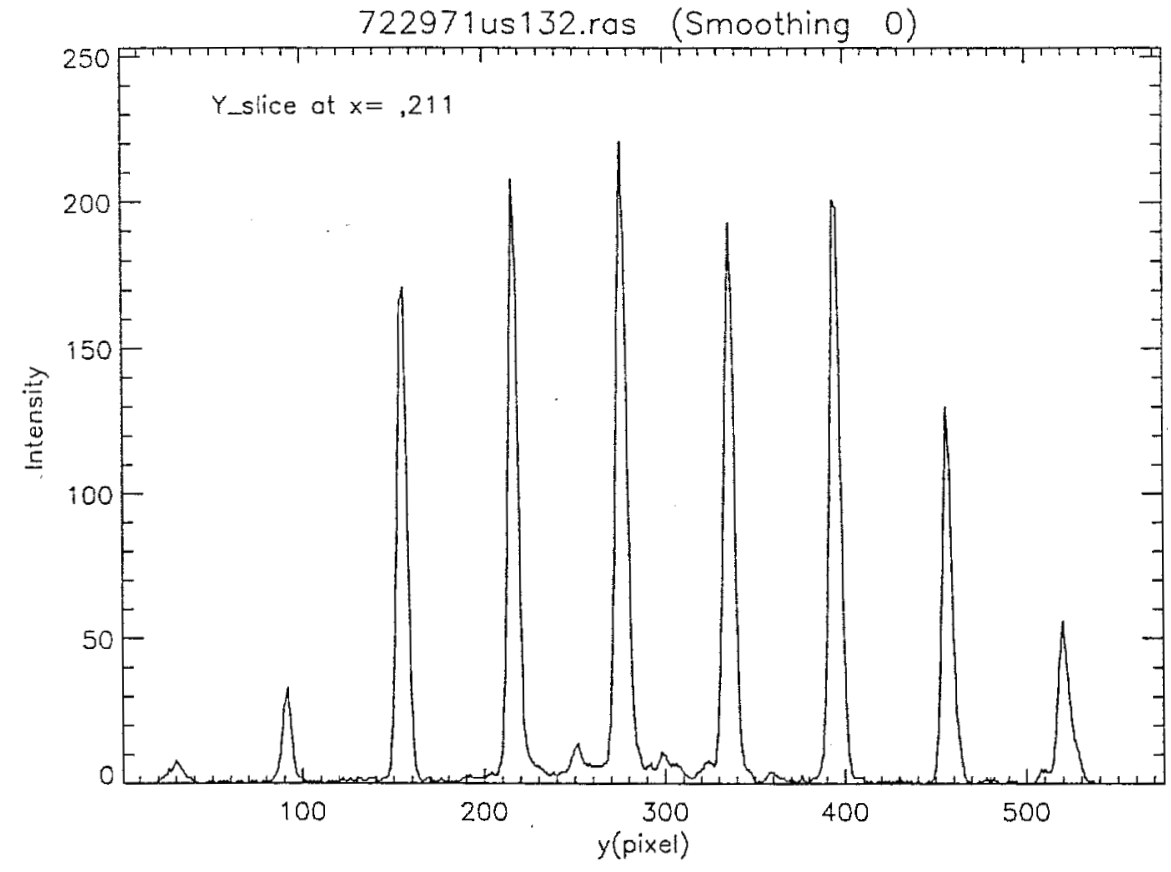

Figure 7.21: GBI image pixel row average plot for a column of beamlets. 


\section{Chapter 8}

\section{Summary and Conclusions}

The principle goal of this dissertation was to design, build, and test a new diagnostic instrument capable of rapidly, and durably measuring the four-dimensional transverse phase space characteristics of an intense space- charge-dominated heavy ion beam. The Gated Beam Imager was developed and uses the pepperpot method of analysis to statistically extract the beam ion's positions and corresponding transverse velocities. The GBI provides durable, and repeatable measurements by employing a microchannel plate as the ion beamlet detector. The GBI was successfully deployed on LLNL's Small Recirculator experiment and measured the characteristics of a $2 \mathrm{~mA}$ potassium ion beam. In the construction and testing of this instrument, a few of the more challenging issues included the selection of a material or device to detect the beamlets reliably with no loss of sensitivity with high exposure to the ion beam, optimizing the design of the instrument and the camera optical system, and developing software analysis tools to analyze the images and provide a convenient user interface.

A secondary goal of this work was to show the utility of the additional information available from the GBI, but not from existing diagnostic instruments. This was done by developing a conceptual design for a beam correction system addressing how to correct for rotational misalignments of quadrupoles in an accelerator's alternating gradient focusing system. A demonstration of a computational method, using the information available from a GBI, for determining the settings of such a correction system was performed. A practical use for this correction system would be to increase 
the focusing quadrupole alignment tolerances during the construction of a driver-scale accelerator which would presumably lead to a decrease in construction and operating costs.

The predominant phase space diagnostic in use on heavy ion beams is the mechanical slit scanner. This diagnostic uses two parallel, narrow slits moved through the beam on successive beam shots to measure the first and second transverse moments of the beam distribution in the direction perpendicular to the slits. Averaging beam intensity information occurs in the direction parallel to the slits resulting in the measurement containing a contribuiton from any coupled motion between the orthogonal transverse directions ( $x$ and $y$ ). The time to scan the beam is about 15 minutes and requires about 400 beam shots. To obtain the moments in the orthogonal direction the slit scanners are rotated $90^{\circ}$ or a second set of scanners is installed at the same position. With the GBI, one is able to obtain in a single beam shot, the first and second moments of the beam in both transverse directions and the four cross-moments between the $x$ and $y$ directions which are indicative of coupling between motion in the $x$ and $y$ directions. A cause of this coupling in an alternate gradient focusing lattice are quadrupole rotational misalignments. The transverse cross-moments cannot be measured with the existing slit scanners due to the averaging of beam information in the direction parallel to the slits. The moments are intensity-weighted as the GBI preserves the intensity characteristics of each beamlet as it is transformed to an optical signal and digitized by the CCD camera. A time history of the beam is obtainable by gating the GBI detector on for a short time and moving the gate time through the beam on successive shots. Thus, a transverse phase space time-dependent map of the beam is obtained which can be compared with detailed numerical simulations or used as input for a beam correction system.

The unique design of the GBI includes the use of a microchannel plate to detect the ion beamlets produced by the pepperpot mask, and convert this signal to an visible optical ouput that preserves the intensity distribution of the beamlets. The spatial orientation of the beamlets is also preserved allowing the hole in the mask, from which the beamlet originated, to be identified for moment calculations. In the 
work to develop the GBI, fast plastic scintillator material was first investigated as a possible beamlet detector. Plastic scintillator was investigated based on its rapid scintillation decay constant which would allow obtaining narrow time slices from a beam by gating the observing camera rapidly "on" and "off". This would allow the construction of an inexpensive detector and take advantage of existing camera capabilities. During tests, plastic scintillator proved to be rapidly de-sensitized under repeated exposure to the ion beam. Additionally, its initial light output was too low in response to the ions to obtain accurate images of the beamlet spots. Presumably, the short range of the heavy ions precluded sufficient interaction with the scintillator to produce adequate visible output.

The microchannel plate detector eventually incorporated into the GBI has several features that allow it to produce accurate, and repeatable detection of the beamlets and produce an optical signal strong enough to be useful for transverse phase space moment analysis. To avoid the damaging effects of the heavy ions, the microchannel plate selected has a thin stainless steel coating on the front face of the microchannels. The primary purpose of this coating is to provide an anode to allow placing a voltage across the microchannels to produce amplification of an incoming signal. The anode is thick enough to stop heavy ions in the energy range of the Small Recirculator. The secondary electrons produced during ion stopping enter the microchannel plate and are amplified and detected with a phosphor screen at the output of the microchannel plate. This "shielding" of the microchannel plate from the heavy ions side steps any damage issues. Additionally the gain of the microchannel plate is linear allowing optical signal amplification while maintaining the intensity characteristics of the beamlets. The spatial resolution of the position and size of the beamlets is not significantly affected by the "discretizing" of the beamlets into channels of finite size and spacing as the resolution of CCD camera systems is less than the microchannel spacing. Obtaining narrow time slices of the beam proved more difficult with a microchannel plate detector as it was necessary to gate the bias voltage to the detector rather than use the built-in gate on a CCD camera. This was a consequence of commercially-available microchannel plates using phosphor screens which produce 
an output with a long response time. Confirmed during testing, the microchannel plate detector produced accurate, repeatable, and flexible images well suited to data analysis.

GBI measurements on the Small Recirculator beam showed good agreement with mechanical slit scanner emittance data obtained in the $y$-direction. Similar magnitude error bars as those calculated for the GBI are applicable to the slit scanner data. Comparison of the GBI moment data with the output of the WARP code, a sophisticated particle-in-cell numerical beam simulation, shows that the assumed initial velocity spread of the ions in the axial direction should be adjusted. WARP calculation of the beam emittance at the position of the GBI shows much more growth in emittance in the $x$ (in-plane with the bend) direction than measured. The simulation results are much greater than the data and well outside experimental error bars. It is known that the emittance growth is very sensitive to the assumed initial width of the semi-gaussian spread in the ion axial velocity. Beam moments from the GBI data, analyzed to yield the beam envelope and envelope convergence angle, agree well with the WARP calculations. These envelope calculations are insensitive to the assumed ion axial velocity spread and lead to the conclusion that the code axial velocity spread assumptions should be re-examined. These results are a measure of the validity of the Small Recirculator experimental program of which a main goal is to benchmark sophisticated codes such as WARP. This requires that those quantities not obtainable through first principles or direct measurement be empirically determined.

The usefulness of a 4-D phase space diagnostic such as the GBI is demonstrated in the conceptual beam correction system addressing quadrupole rotation errors proposed in this work. The ability to measure the $x-y$ cross-moments makes such a system possible. Extension of Barnard's work that found constants of the motion in the presence of rotation errors was necessary to demonstrate efficient design of the correction system. That such a system will decrease the construction and operating costs of a driver-scale accelerator is realizable in the ability to increase alignment tolerances of focusing components and to be able to withstand any component aging effects resulting in rotational misalignments. The "model-free" attributes of the Si- 
multaneous Perturbation Stochastic Approximation (SPSA) algorithm for optimizing correction system parameters were successful due the nonlinear dependence of the beam moments on the optimized parameters. SPSA requires only two calculations of the parameter gradients regardless of the dimensionality of the system. This was very efficient, although it was noted that the difficulty in tuning the algorithm to converge quickly increased with the increasing dimensionality of the problem.

The GBI combined with user-friendly analysis software has been shown to rapidly, and efficiently provide accurate, and repeatable characterization of heavy ion beams. It provides additional information beyond that of other diagnostics. This information is useful in diagnosing the cause of beam emittance growth and allows the concept of "correcting" a beam to be further studied. The GBI developed for this work is a prototype and showed that reliable detection of an intense beam heavy ions could be accomplished in a manner allowing characterization of the beam's 4-D transverse phase space. The further development of the basic components of this instrument will continue allowing it to be a more fexible, and capable instrument in meeting the need to supply data on the operation of heavy ion accelerators. 


\section{Appendix A}

\section{GBI Design Optimizing Spreadsheet}

The following is a printout of the spreadsheet incorporating the design equations of the GBI. It allows the user to vary the beam current, average radius, energy, length, emittance, ion mass, pepperpot mask thickness, hole diameter and spacing, drift distance, and the gate interval. The effect on beamlet spread due to emittance and space charge as well as beamlet spot spacing at the detector is calculated and checked against a minimum criteria. Additionally, estimates of the signal from the detector is estimated. 


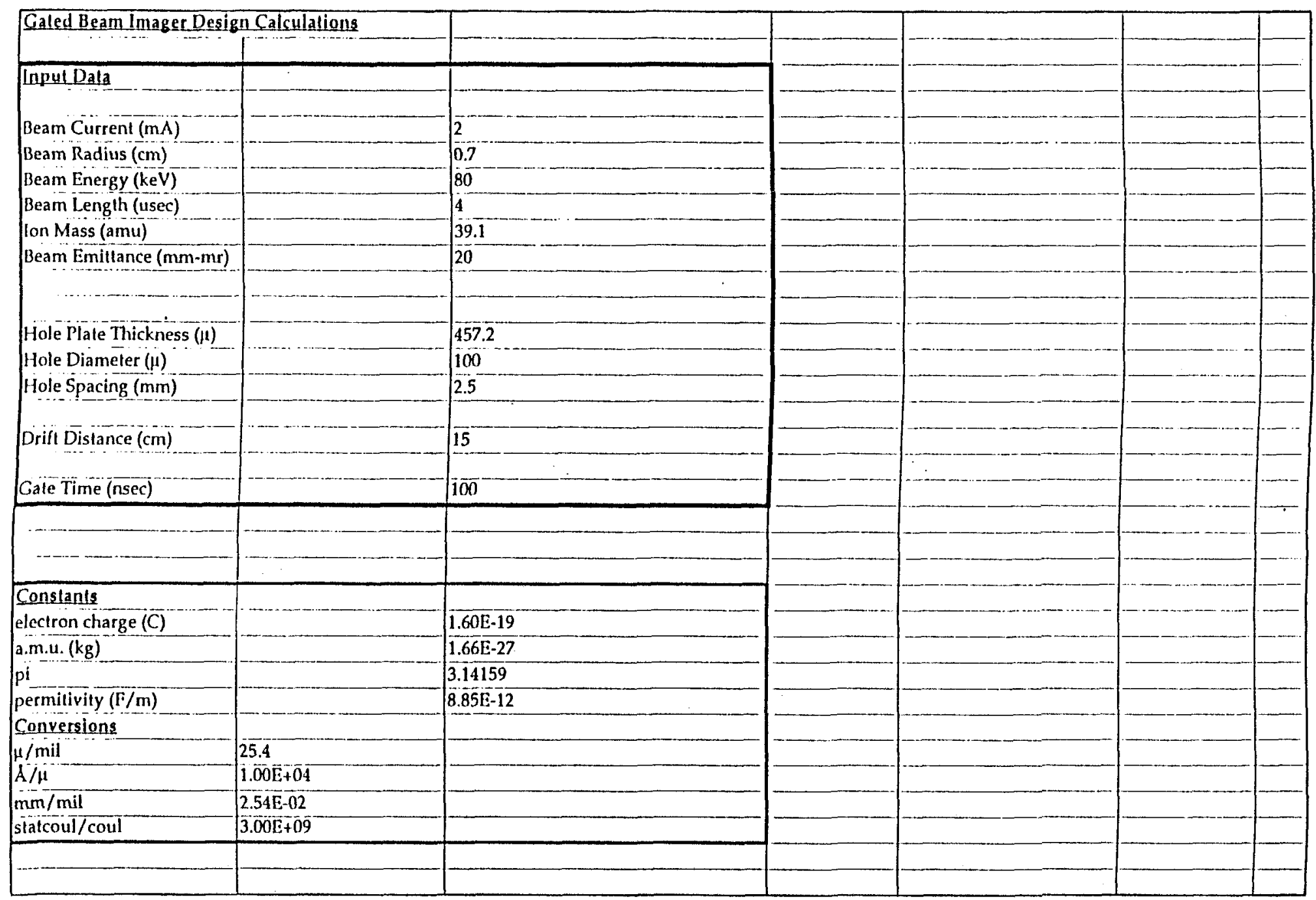




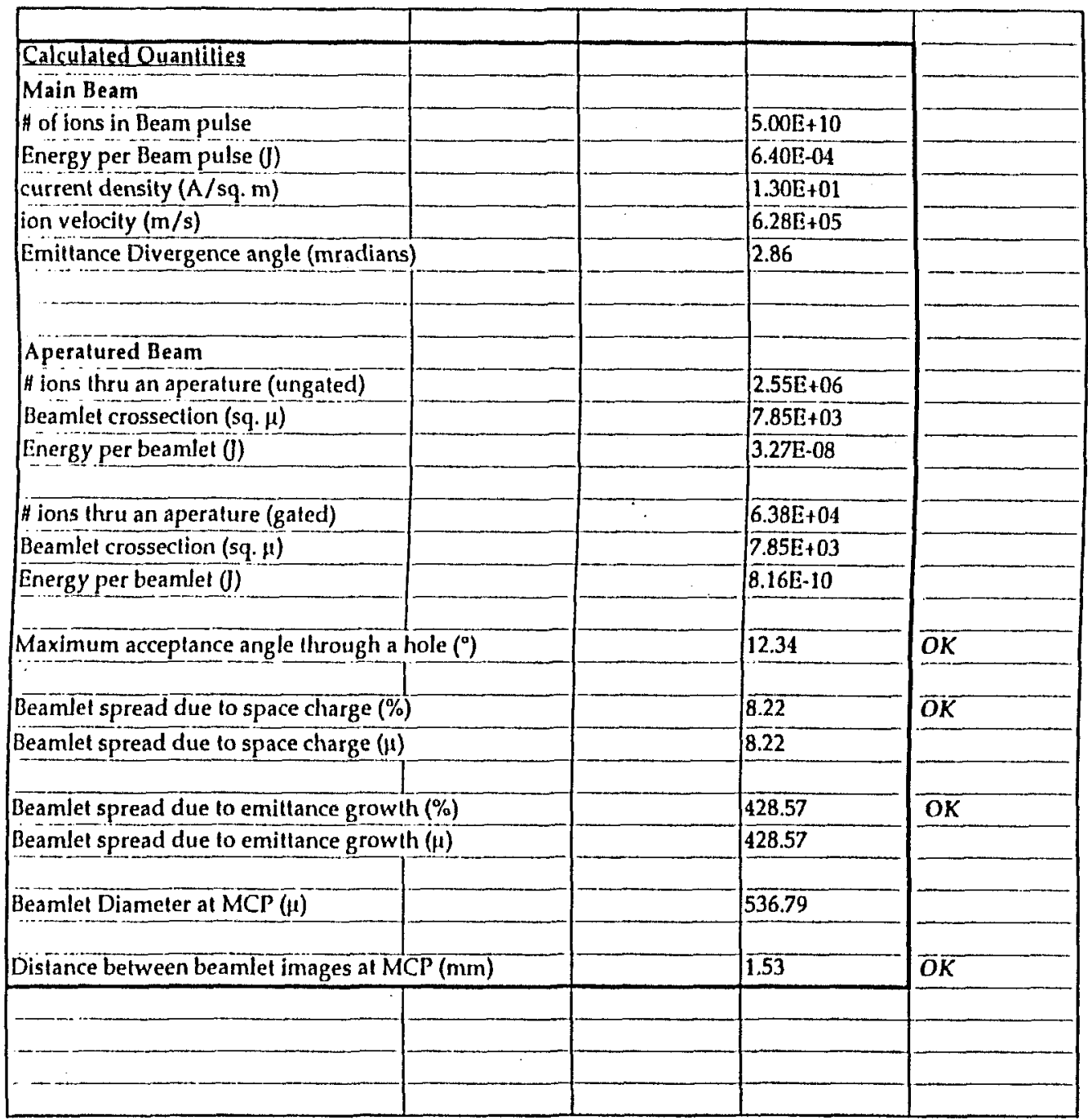




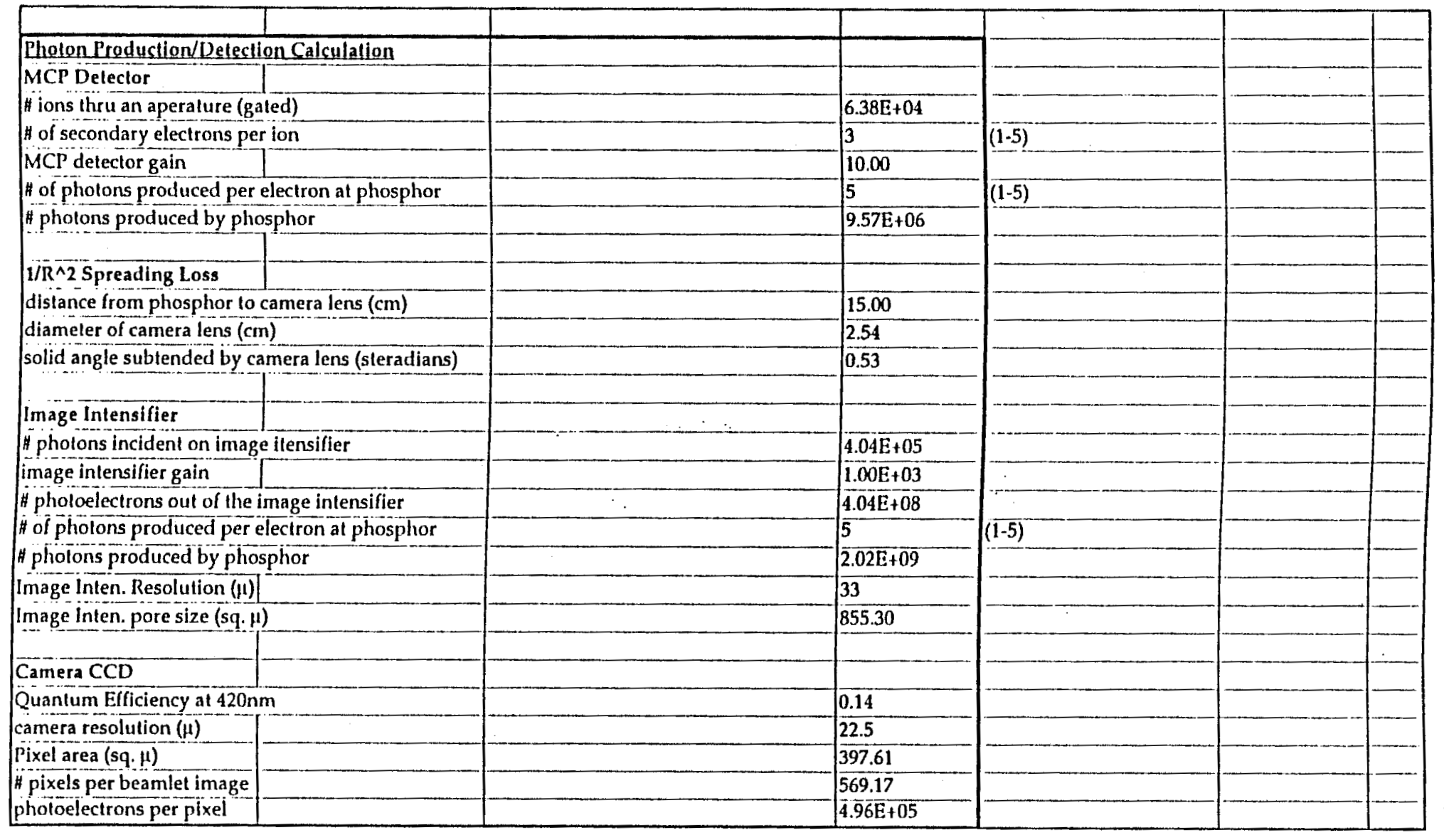




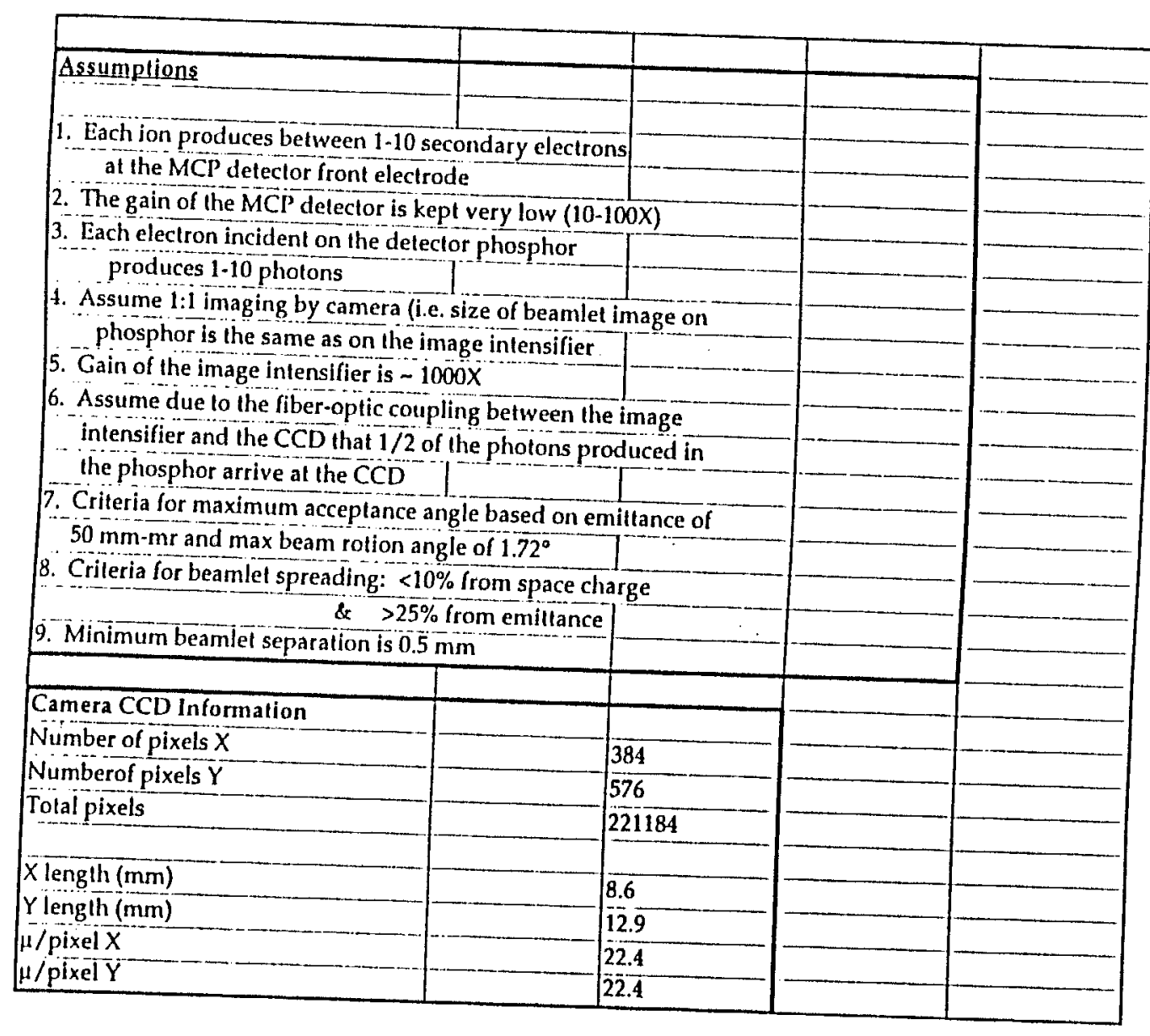




\section{Appendix B}

\section{Derivation of Beam Moment Evolution Equations}

This appendix follows the work of Barnard in deriving the moment evolution equations of Chapter 6 [footnote]. Barnard assumed that the force on an ion comes from two sources only, the external focusing force from a purely quadrupolar field, and an internal force from the space charge of the beam. The calculation assumes the space charge is evenly distributed in a uniform density ellipse but the semi-axes and rotation angle of the ellipse is allowed to evolve as a function of the axial coordinate, z. Quadrupoles can be rotated by an angle, $\theta$ from the $x$-axis, and the beam can be rotated by an angle, $\alpha$ from the $\mathrm{x}$-axis as seen in Figure reffig:coordsyst

The relation between the coordinate in the quadrupole frame (subscript 0 ) and the lab frame (no subscript) are given by:

$$
x=x_{0} \cos \theta-y_{0} \sin \theta \quad y=y_{0} \cos \theta+x_{0} \sin \theta
$$

and similarly for the beam frame:

$$
x-<x>=x_{b} \cos \alpha-y_{b} \sin \alpha \quad y-<y>=y_{b} \cos \alpha+x_{b} \sin \alpha
$$

The " $<>$ " indicates a statistical average over the beam ion distribution For a non-relativistic beam, with velocity ratio $\beta$, moving along the z-axis the paraxial 


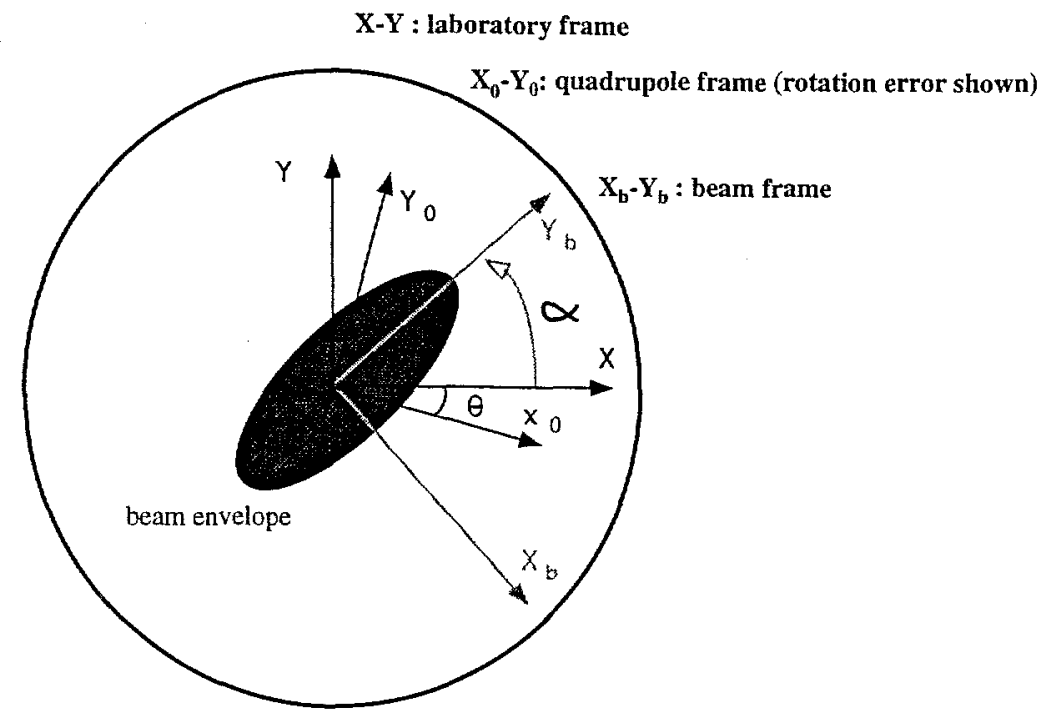

Figure B.1: Beam and laboratory coordinate system

equations of motion can then be written as:

$$
\begin{aligned}
& x^{\prime \prime}=\frac{d^{2} x}{d z^{2}}=K_{q x x} x+K_{q x y} y+K_{s x x}(x-<x>)+K_{s x y}(y-<y>) \\
& y^{\prime \prime}=\frac{d^{2} y}{d z^{2}}=\underbrace{K_{q y y} y+K_{q y x} x}_{\text {externalforces }}+\underbrace{K_{s y y}(y-<y>)+K_{s y x}(x-<x>)}_{\text {internalforces }} \\
K_{q x x} \equiv & K_{q x 0} \cos ^{2} \theta+K_{q y 0} \sin ^{2} \theta=K_{q x 0} \cos 2 \theta \\
K_{q y y} \equiv & K_{q y 0} \cos ^{2} \theta+K_{q x 0} \sin ^{2} \theta=-K_{q x 0} \cos 2 \theta \\
K_{q x y} \equiv & K_{q y x}=\left(K_{q x 0}-K_{q y 0}\right)(\sin \theta \cos \theta)=K_{q x 0} \sin 2 \theta \\
K_{q y y} \equiv & K_{q y 0} \cos ^{2} \theta+K_{q x 0} \sin { }^{2} \theta=-K_{q x 0} \cos 2 \theta \\
K_{s x x}= & K_{s x b} \cos 2 \alpha+K_{s y b} \sin 2 \alpha \\
K_{s x y}= & K_{s y x}=\left(K_{s x b}-K_{s y b}\right) \sin \alpha \cos \alpha \\
K_{q x 0}= & -K_{q y 0}=\frac{B^{\prime}}{B \rho}
\end{aligned}
$$


For instance, $K_{q x x}$ is the proportional constant for the quadrupolar force on an ion in the $x$-direction caused by a dispacement in the $x$-direction from the center of the magnetic quadrupole which corresponds with the center of the beamline. $K_{s x y}$ is the proportional constant for the space-charge force on an ion in the $x$-direction caused by a dispacement in the $y$-direction from the centroid of the beam. In the rotated beam frame:

$$
K_{s x b}=\frac{\kappa}{2\left(\Delta x_{b}^{2}+\left(\Delta x_{b}^{2} \Delta y_{b}^{2}\right)^{1 / 2}\right)} \quad K_{s y b}=\frac{\kappa}{2\left(\Delta y_{b}^{2}+\left(\Delta x_{b}^{2} \Delta y_{b}^{2}\right)^{1 / 2}\right)}
$$

and $\Delta x_{b}$ and $\Delta y_{b}$ are the moments in the rotated beam frame:

$$
\begin{aligned}
& \Delta x_{b}^{2}=\Delta x^{2} \cos 2 \alpha+\Delta y^{2} \sin 2 \alpha+2 \Delta x y \cos \alpha \sin \alpha \\
& \Delta y_{b}^{2}=\Delta y^{2} \cos 2 \alpha+\Delta x^{2} \sin 2 \alpha-2 \Delta x y \cos \alpha \sin \alpha
\end{aligned}
$$

Here $\kappa$ is the beam perveance and is a measure of the space charge forces relative to inertial forces.

$$
\kappa=\frac{q e I_{0}}{2 \pi \epsilon_{0} m_{0}(\beta \gamma c)^{3}}
$$

where $q$ is the charge state of beam ions, $e$ is the proton charge, $m_{0}$ is the beam ion mass in atomic mass units, $\gamma$ is the Lorentz factor, $\beta c$ is the ion velocity, $I_{0}$ is the beam current, and $\epsilon_{0}$ is he permittivity of free space. The symbol, $\Delta$ is shorthand for the second moment of the distribution with the contribution from non-zero first moments removed, (i.e., $\Delta a b \equiv\langle a b\rangle-<a\rangle<b>$ ).

The beam rotation angle may be expressed in terms of the second order moments by

$$
\tan 2 \alpha=\frac{2 \Delta x y}{\Delta x^{2}-\Delta y^{2}}
$$

Let the beam distribution function $f$, be the number of particles $d N$ per unit transverse phase space volume:

$$
f\left(x, x^{\prime}, y, y^{\prime}\right)=\frac{d N}{d x d x^{\prime} d y d y^{\prime}}
$$

Since the beam can be described as a collision-less non-neutral plasma the evolution 
of $\mathrm{f}$ can be described by the Vlasov/Collisionless Boltzman Equation

$$
\frac{\partial f}{\partial z}+x^{\prime} \frac{\partial f}{\partial x}+x^{\prime \prime} \frac{\partial f}{\partial x^{\prime}}+y^{\prime} \frac{\partial \dot{f}}{\partial y}+y^{\prime \prime} \frac{\partial f}{\partial y^{\prime}}+\left(\frac{\partial x^{\prime \prime}}{\partial x^{\prime}}+\frac{\partial y^{\prime \prime}}{\partial y^{\prime}}\right) f=0
$$

where $x^{\prime \prime}$ and $y^{\prime \prime}$ are determined by the equations of motion. The average of a variable over a continuous distribution is given by:

$$
<\phi>(z) \equiv \frac{1}{N} \iiint \int \phi f\left(x, x^{\prime}, y, y^{\prime}\right) d x d x^{\prime} d y d y^{\prime}
$$

substituting the moments into the above equation and integrating by parts yields the moment evolution equations:

$$
\begin{aligned}
& \frac{d \Delta x^{2}}{d z}=2 \Delta x x^{\prime} \\
& \frac{d \Delta x x^{\prime}}{d z}=\Delta x^{\prime 2}+K_{x x} \Delta x^{2}+K_{x y} \Delta x y-\left(d \ln \beta_{z} / d z\right) \Delta x x^{\prime} \\
& \frac{d \Delta x^{\prime 2}}{d z}=2 K_{x x} \Delta x x^{\prime}+2 K_{x y} \Delta x^{\prime} y-2\left(d \ln \beta_{z} / d z\right) \Delta x^{\prime 2} \\
& \frac{d \Delta y^{2}}{d z}=2 \Delta y y^{\prime} \\
& \frac{d \Delta y y^{\prime}}{d z}=\Delta y^{\prime 2}+K_{y y} \Delta y^{2}+K_{y x} \Delta x y-\left(d \ln \beta_{z} / d z\right) \Delta y y^{\prime} \\
& \frac{d \Delta y^{\prime 2}}{d z}=2 K_{y y} \Delta y y^{\prime}+2 K_{x y} \Delta x y^{\prime}-2\left(d \ln \beta_{z} / d z\right) \Delta y^{\prime 2} \\
& \frac{d \Delta x y}{d z}=\Delta x^{\prime} y+\Delta x y^{\prime} \\
& \frac{d \Delta x^{\prime} y}{d z}=\Delta x^{\prime} y^{\prime}+K_{x x} \Delta x y+K_{y x} \Delta y^{2}-\left(d \ln \beta_{z} / d z\right) \Delta x^{\prime} y \\
& \frac{d \Delta x y^{\prime}}{d z}=\Delta x^{\prime} y^{\prime}+K_{y y} \Delta x y+K_{x y} \Delta x^{2}-\left(d \ln \beta_{z} / d z\right) \Delta x y^{\prime} \\
& \frac{d \Delta x^{\prime} y^{\prime}}{d z}=K_{x x} \Delta x y^{\prime}+K_{x y} \Delta y y^{\prime}+K_{y y} \Delta x^{\prime} y+K_{y x} \Delta x x^{\prime}-2\left(d \ln \beta_{z} / d z\right) \Delta x^{\prime} y^{\prime}
\end{aligned}
$$

where

$$
\begin{aligned}
& K_{x x} \equiv K_{q x x}+K_{s x x} \\
& K_{y y} \equiv K_{q y y}+K_{s y y} \\
& K_{x y} \equiv K_{q x y}+K_{s x y}=K_{y x}
\end{aligned}
$$


and similarly the first moment evolution equations are given by

$$
\begin{array}{ll}
\left.\frac{d<x\rangle}{d z}=<x^{\prime}\right\rangle & \left.\frac{\left.d<x^{\prime}\right\rangle}{d z}=K_{q x x}<x>+K_{q x y}<y>-\left(d \ln \beta_{z} / d z\right)<x^{\prime}\right\rangle \\
\left.\frac{d<y>}{d z}=<y^{\prime}\right\rangle & \frac{\left.d<y^{\prime}\right\rangle}{d z}=K_{q y y}<y>+K_{q y x}<x>-\left(d \ln \beta_{z} / d z\right)<y^{\prime}>
\end{array}
$$




\section{Appendix $\mathrm{C}$}

\section{Explicit Solutions for $\Delta x^{2}$ and $\Delta y^{2}$}

From an engineering and cost point of view it is desirable to reduce the number of independent adjustable parameters necessary to correct the beam for random quadrupole rotatational misalignments. Using Barnard's generalized emittances the matrix of ten moment evolution equations may be reduced to eight by explicitly solving the expressions for the generalized emittances for two of the moments in terms of the remaining eight moments. It was found helpful to use the capabiltiies of Mathematica to do this and it was found that solving for $\Delta x^{2}$ and $\Delta y^{2}$ yielded the "simplest" result. The Mathematica command for doing this was:

$$
\text { Solve }[<<\text { constraints.m }>>,\{d x 2, d y 2\}]
$$

where the file "constraints.m" contained the generalized emittance expressions and "dx2,dy2" are $\Delta x^{2}, \Delta y^{2}$ in a form readable by Mathematica. The explicit solutions are very long and as discussed in Chapter 6 they were able to be simplified when the numerical values for the beam moments corresponding to the Small Recirculator were used in the formulas. The terms that were dropped from the following explicit solutions to obtain the results of Equations 6.11 and 6.16 appear in a lighter shade.

The numerical value of the terms that were dropped was about 20 orders of magnitude smaller than those terms kept. These solutions are then substituted anywhere $\Delta x^{2}, \Delta y^{2}$ appear in the ten moment equations and the eight independent moment evolution equations of the reduced matrix of Equation 6.6 are the result. All the 
complications are contained in the numbered coefficients.

$$
\begin{aligned}
& \Delta \mathrm{x}^{2}=\left[4 \beta^{4} \Delta \mathrm{x}^{\prime} \mathrm{y}^{\prime 2} \Delta \mathrm{xx}^{\prime 2}-8 \beta^{4} \Delta \mathrm{x}^{\prime} \mathrm{y}^{\prime 3} \Delta \mathrm{xy}+8 \beta^{4} \Delta \mathrm{x}^{\prime} \mathrm{y} \Delta \mathrm{x}^{\prime} \mathrm{y}^{\prime 2} \Delta \mathrm{xy}^{\prime}+\right. \\
& 8 \beta^{4} \Delta \mathrm{x}^{\prime} \mathrm{y}^{\prime}\left(\Delta \mathrm{x}^{\prime 2}\right) \Delta \mathrm{xx}^{\prime} \Delta \mathrm{xy}^{\prime}-4 \beta^{4}\left(\Delta \mathrm{x}^{\prime 2}\right)^{2} \Delta \mathrm{xy}^{\prime 2} \\
& 8 \beta^{4}\left(\Delta \mathrm{x}^{\prime 2}\right) \Delta \mathrm{xx}^{\prime 2}\left(\Delta \mathrm{y}^{\prime 2}\right)+8 \beta^{4} \Delta \mathrm{x}^{\prime} \mathrm{y}^{\prime}\left(\Delta \mathrm{x}^{\prime 2}\right) \Delta \mathrm{xy}\left(\Delta \mathrm{y}^{\prime 2}\right) \\
& 8 \beta^{4} \Delta \mathrm{x}^{\prime} \mathrm{y}\left(\Delta \mathrm{x}^{\prime 2}\right) \Delta \mathrm{xy}^{\prime}\left(\Delta \mathrm{y}^{\prime 2}\right)+4 \beta^{4} \Delta \mathrm{x}^{\prime} \mathrm{y}^{2}\left(\Delta \mathrm{y}^{\prime 2}\right)^{2}- \\
& 8 \beta^{4} \Delta \mathrm{x}^{\prime} \mathrm{y} \Delta \mathrm{x}^{\prime} \mathrm{y}^{\prime}\left(\Delta \mathrm{y}^{\prime 2}\right) \Delta \mathrm{yy}^{\prime}+4 \beta^{4} \Delta \mathrm{x}^{\prime} \mathrm{y}^{\prime 2} \Delta \mathrm{yy}^{\prime 2}+ \\
& 0.5 \beta^{2} \Delta \mathrm{x}^{\prime} \mathrm{y}^{\prime 2} \epsilon_{\mathrm{g}}{ }^{2}-0.5 \beta^{2}\left(\Delta \mathrm{x}^{\prime 2}\right)\left(\Delta \mathrm{y}^{\prime 2}\right) \epsilon_{\mathrm{g}}{ }^{2}- \\
& 0.015625\left[65536 \beta^{8} \Delta x^{\prime} y^{\prime 4} \Delta x x^{4}-262144 \beta^{8} \Delta x^{\prime} y^{\prime 5} \Delta x x^{\prime 2} \Delta x y+\right. \\
& 262144 \beta^{8} \Delta x^{\prime} y^{\prime 6} \Delta x y^{2}+ \\
& 262144 \beta^{8} \Delta x^{\prime} y \Delta x^{\prime} y^{\prime 4} \Delta x x^{\prime 2} \Delta x y^{\prime}- \\
& 262144 \beta^{8} \Delta x^{\prime} y^{\prime 3}\left(\Delta x^{\prime 2}\right) \Delta x x^{\prime 3} \Delta x y^{\prime}- \\
& 524288 \beta^{8} \Delta x^{\prime} y \Delta x^{\prime} y^{\prime 5} \Delta x y \Delta x y^{\prime}+524288 \beta^{8} \Delta x^{\prime} y^{\prime 4}\left(\Delta x^{\prime 2}\right) \Delta x x^{\prime} \Delta x y \Delta x y^{\prime}+ \\
& 262144 \beta^{8} \Delta x^{\prime} y^{2} \Delta x^{\prime} y^{\prime 4} \Delta x y^{\prime 2}-524288 \beta^{8} \Delta x^{\prime} y \Delta x^{\prime} y^{\prime 3}\left(\Delta x^{\prime 2}\right) \Delta x x^{\prime} \Delta x y^{\prime 2}+ \\
& 393216 \beta^{8} \Delta x^{\prime} y^{\prime 2}\left(\Delta x^{\prime 2}\right)^{2} \Delta x x^{\prime 2} \Delta x y^{\prime 2}- \\
& 262144 \beta^{8} \Delta x^{\prime} y^{\prime 3}\left(\Delta x^{\prime 2}\right)^{2} \Delta x y \Delta x y^{\prime 2}+ \\
& 262144 \beta^{8} \Delta x^{\prime} y \Delta x^{\prime} y^{\prime 2}\left(\Delta x^{\prime 2}\right)^{2} \Delta x y^{\prime 3}- \\
& 262144 \beta^{8} \Delta x^{\prime} y^{\prime}\left(\Delta x^{\prime 2}\right)^{3} \Delta x x^{\prime} \Delta x y^{\prime^{3}}+65536 \beta^{8}\left(\Delta x^{\prime 2}\right)^{4} \Delta x y^{\prime 4}+ \\
& 262144 \beta^{8} \Delta x^{\prime} y^{\prime 3}\left(\Delta x^{\prime 2}\right) \Delta x x^{\prime 2} \Delta x y\left(\Delta y^{\prime 2}\right)- \\
& 786432 \beta^{8} \Delta x^{\prime} y^{\prime 4}\left(\Delta x^{\prime 2}\right) \Delta x y^{2}\left(\Delta y^{\prime 2}\right)- \\
& 262144 \beta^{8} \Delta x^{\prime} y \Delta x^{\prime} y^{\prime 2}\left(\Delta x^{\prime 2}\right) \Delta x x^{\prime 2} \Delta x y^{\prime}\left(\Delta y^{\prime 2}\right)+ \\
& 1.5728610^{6} \beta^{8} \Delta x^{\prime} y \Delta x^{\prime} y^{\prime 3}\left(\Delta x^{\prime 2}\right) \Delta x y \Delta x y^{\prime}\left(\Delta y^{\prime 2}\right)- \\
& 524288 \beta^{8} \Delta x^{\prime} y^{\prime 2}\left(\Delta x^{\prime 2}\right)^{2} \Delta x x^{\prime} \Delta x y \Delta x y^{\prime}\left(\Delta y^{\prime 2}\right)- \\
& 786432 \beta^{8} \Delta x^{\prime} y^{2} \Delta x^{\prime} y^{\prime 2}\left(\Delta x^{\prime 2}\right) \Delta x y^{\prime 2}\left(\Delta y^{\prime 2}\right)+ \\
& 524288 \beta^{8} \Delta x^{\prime} y \Delta x^{\prime} y^{\prime}\left(\Delta x^{\prime 2}\right)^{2} \Delta x x^{\prime} \Delta x y^{\prime 2}\left(\Delta y^{\prime 2}\right)+ \\
& 262144 \beta^{8} \Delta x^{\prime} y^{\prime}\left(\Delta x^{\prime 2}\right)^{3} \Delta x y \Delta x y^{\prime 2}\left(\Delta y^{\prime 2}\right)-
\end{aligned}
$$




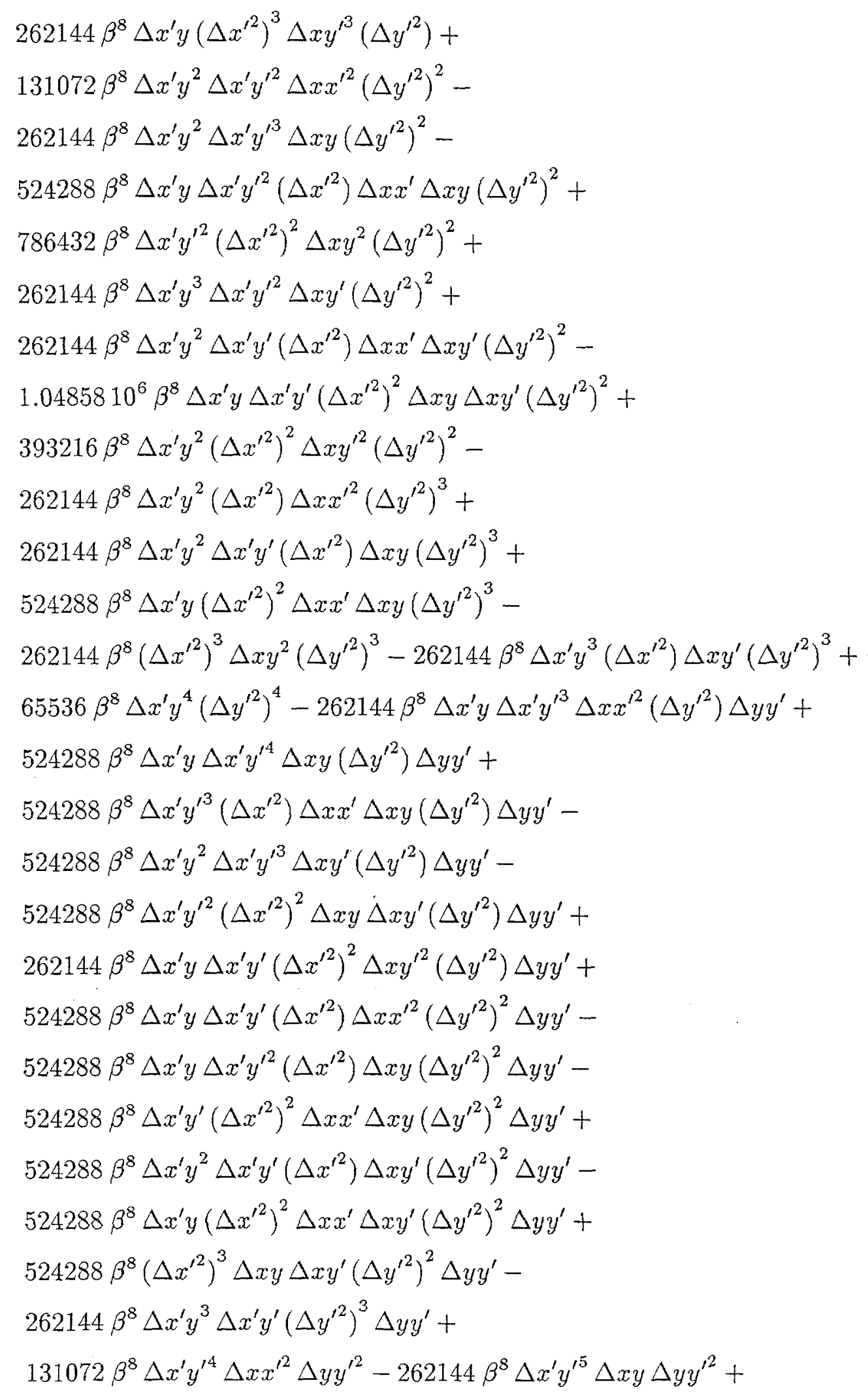




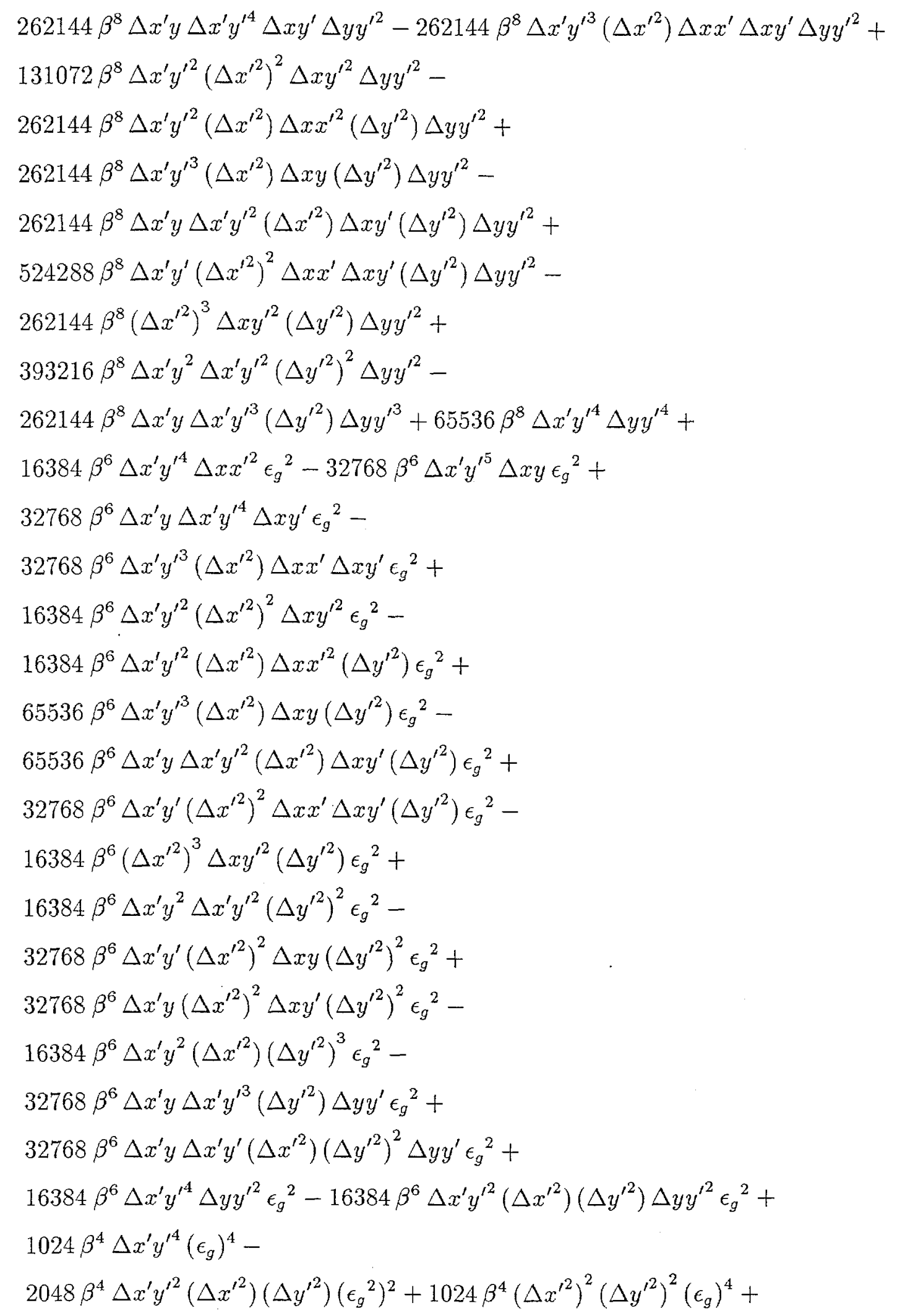




$$
\begin{aligned}
& \left.\left.1024 \beta^{4} \Delta x^{\prime} y^{\prime 2}\left(\Delta x^{\prime 2}\right)\left(\Delta y^{\prime 2}\right)\left(\epsilon_{h}\right)^{4}-1024 \beta^{4}\left(\Delta x^{\prime 2}\right)^{2}\left(\Delta y^{\prime 2}\right)^{2}\left(\epsilon_{h}\right)^{4}\right]^{0.5}\right] / \\
& {\left[\beta^{4} \Delta \mathbf{x}^{\prime 2}\left(8 \Delta \mathbf{x}^{\prime} \mathbf{y}^{\prime 2}-8 \Delta \mathbf{x}^{\prime 2} \Delta \mathbf{y}^{\prime 2}\right)\right]}
\end{aligned}
$$

$\Delta y^{2}=0.5\left[\beta^{2}\left[256 \beta^{2} \Delta \mathrm{x}^{\prime} \mathrm{y}^{\prime 2} \Delta \mathrm{xx}^{\prime 2}-\right.\right.$

$512 \beta^{2} \Delta \mathrm{x}^{\prime} \mathrm{y}^{\prime 3} \Delta \mathrm{xy}+$

$512 \beta^{2} \Delta \mathrm{x}^{\prime} \mathrm{y} \Delta \mathrm{x}^{\prime} \mathrm{y}^{\prime 2} \Delta \mathrm{xy}^{\prime}-512 \beta^{2} \Delta \mathrm{x}^{\prime} \mathrm{y}^{\prime} \Delta \mathrm{x}^{\prime 2} \Delta \mathrm{xx}^{\prime} \Delta \mathrm{xy}^{\prime}+$

$256 \beta^{2}\left(\Delta \mathbf{x}^{\prime 2}\right)^{2} \Delta \mathrm{xy}^{\prime 2}+$

$512 \beta^{2} \Delta \mathrm{x}^{\prime} \mathrm{y}^{\prime} \Delta \mathrm{x}^{\prime 2} \Delta \mathrm{xy} \Delta \mathrm{y}^{\prime 2}-512 \beta^{2} \Delta \mathrm{x}^{\prime} \mathrm{y} \Delta \mathrm{x}^{\prime 2} \Delta \mathrm{xy}^{\prime} \Delta \mathrm{y}^{\prime 2}-$

$256 \beta^{2} \Delta \mathrm{x}^{\prime} \mathrm{y}^{2}\left(\Delta \mathrm{y}^{\prime 2}\right)^{2}+512 \beta^{2} \Delta \mathrm{x}^{\prime} \mathrm{y} \Delta \mathrm{x}^{\prime} \mathrm{y}^{\prime} \Delta \mathrm{y}^{\prime 2} \Delta \mathrm{yy}^{\prime}+$

$256 \beta^{2} \Delta \mathrm{x}^{\prime} \mathrm{y}^{\prime 2} \Delta \mathrm{yy}^{\prime 2}-512 \beta^{2} \Delta \mathrm{x}^{\prime 2} \Delta \mathrm{y}^{\prime 2} \Delta \mathrm{yy}^{\prime 2}+$

$\left.32 \Delta x^{\prime} y^{\prime 2} \epsilon_{\mathrm{g}}^{2}-32 \Delta \mathrm{x}^{\prime 2} \Delta \mathrm{y}^{\prime 2} \epsilon_{\mathrm{g}}{ }^{2}\right]-$

$\left[\beta^{4}\left[256 \beta^{2} \Delta x^{\prime} y^{\prime 2} \Delta x x^{\prime 2}-512 \beta^{2} \Delta x^{\prime} y^{\prime 3} \Delta x y+512 \beta^{2} \Delta x^{\prime} y \Delta x^{\prime} y^{\prime 2} \Delta x y^{\prime}-\right.\right.$

$512 \beta^{2} \Delta x^{\prime} y^{\prime} \Delta x^{\prime 2} \Delta x x^{\prime} \Delta x y^{\prime}+$

$256 \beta^{2}\left(\Delta x^{\prime 2}\right)^{2} \Delta x y^{\prime 2}+512 \beta^{2} \Delta x^{\prime} y^{\prime} \Delta x^{\prime 2} \Delta x y \Delta y^{\prime 2}-512 \beta^{2} \Delta x^{\prime} y \Delta x^{\prime 2} \Delta x y^{\prime} \Delta y^{\prime 2}-$

$256 \beta^{2} \Delta x^{\prime} y^{2}\left(\Delta y^{\prime 2}\right)^{2}+512 \beta^{2} \Delta x^{\prime} y \Delta x^{\prime} y^{\prime} \Delta y^{\prime 2} \Delta y y^{\prime}+$

$\left.256 \beta^{2} \Delta x^{\prime} y^{\prime 2} \Delta y y^{\prime 2}-512 \beta^{2} \Delta x^{\prime 2} \Delta y^{\prime 2} \Delta y y^{\prime 2}+32 \Delta x^{\prime} y^{\prime 2} \epsilon_{g}{ }^{2}-32 \Delta{x^{\prime}}^{2} \Delta{y^{\prime}}^{2} \epsilon_{g}\right]^{2}-$ 


$$
\begin{aligned}
& 4 \beta^{4} \Delta y^{\prime 2}\left[-256 \Delta x^{\prime} y^{\prime 2}+256 \Delta{x^{\prime}}^{2} \Delta y^{\prime 2}\right]\left[-256 \beta^{4} \Delta x^{\prime} y^{\prime 2} \Delta x^{\prime 2} \Delta x y^{2}+\right. \\
& 512 \beta^{4} \Delta x^{\prime} y \Delta x^{\prime} y^{\prime} \Delta x^{\prime 2} \Delta x y \Delta x y^{\prime}-256 \beta^{4} \Delta x^{\prime} y^{2} \Delta x^{\prime 2} \Delta x y^{\prime 2}+ \\
& 256 \beta^{4} \Delta x^{\prime} y^{2} \Delta x x^{\prime 2} \Delta y^{\prime 2}-512 \beta^{4} \Delta x^{\prime} y^{2} \Delta x^{\prime} y^{\prime} \Delta x y \Delta y^{\prime 2}- \\
& 512 \beta^{4} \Delta x^{\prime} y \Delta x^{\prime 2} \Delta x x^{\prime} \Delta x y \Delta y^{\prime 2}+256 \beta^{4}\left(\Delta x^{\prime 2}\right)^{2} \Delta x y^{2} \Delta y^{\prime 2}+ \\
& 512 \beta^{4} \Delta x^{\prime} y^{3} \Delta x y^{\prime} \Delta y^{\prime 2}- \\
& 512 \beta^{4} \Delta x^{\prime} y \Delta x^{\prime} y^{\prime} \Delta x x^{\prime 2} \Delta y y^{\prime}+1024 \beta^{4} \Delta x^{\prime} y \Delta x^{\prime} y^{\prime 2} \Delta x y \Delta y y^{\prime}+ \\
& 512 \beta^{4} \Delta x^{\prime} y^{\prime} \Delta x^{\prime 2} \Delta x x^{\prime} \Delta x y \Delta y y^{\prime}- \\
& 1024 \beta^{4} \Delta x^{\prime} y^{2} \Delta x^{\prime} y^{\prime} \Delta x y^{\prime} \Delta y y^{\prime}+512 \beta^{4} \Delta x^{\prime} y \Delta x^{\prime 2} \Delta x x^{\prime} \Delta x y^{\prime} \Delta y y^{\prime}- \\
& 512 \beta^{4}\left(\Delta x^{\prime 2}\right)^{2} \Delta x y \Delta x y^{\prime} \Delta y y^{\prime}-512 \beta^{4} \Delta x^{\prime} y^{\prime} \Delta x^{\prime 2} \Delta x y \Delta y y^{\prime 2}+ \\
& 512 \beta^{4} \Delta x^{\prime} y \Delta x^{\prime 2} \Delta x y^{\prime} \Delta y y^{\prime 2}+ \\
& 256 \beta^{4} \Delta x^{\prime} y^{2} \Delta y^{\prime 2} \Delta y y^{\prime 2}-512 \beta^{4} \Delta x^{\prime} y \Delta x^{\prime} y^{\prime} \Delta y y^{\prime 3}+ \\
& 256 \beta^{4} \Delta x^{\prime 2} \Delta y y^{4}+ \\
& 32 \beta^{2} \Delta x^{\prime} y^{2} \Delta y^{\prime 2} \epsilon_{g}^{2}-64 \beta^{2} \Delta x^{\prime} y \Delta x^{\prime} y^{\prime} \Delta y y^{\prime} \epsilon_{g}{ }^{2}+ \\
& \left.\left.32 \beta^{2} \Delta{x^{\prime}}^{2} \Delta y y^{\prime 2} \epsilon_{g}{ }^{2}+\Delta x^{2} \epsilon_{h}{ }^{4}\right]^{0.5}\right] / \\
& \left(\beta^{4} \Delta \mathrm{y}^{\prime 2}\left[-256 \Delta \mathrm{x}^{\prime} \mathrm{y}^{\prime 2}+256 \Delta \mathrm{x}^{\prime 2} \Delta \mathrm{y}^{\prime 2}\right]\right)
\end{aligned}
$$




\section{Appendix D}

\section{GBI Equipment Settings for Acquiring Gated Images}

Table D.1: GBI Equipment and Software Settings for GBI Measurements at $45^{\circ}$

\begin{tabular}{|l|l|c|}
\hline CCD camera settings & Lens aperature & $\mathrm{f} / 1.4$ \\
\hline \hline ST-138 Controller & CCD temperature & $-30^{\circ} \mathrm{C}$ \\
\hline \hline PG-200 Voltage P/S & Gate width & $200 \mu \mathrm{s}$ \\
\hline & Voltage for $100 \mathrm{~ns} / 1 \mu \mathrm{s}$ gates & $900 / 700 \mathrm{~V}$ \\
\hline & Mode & "Gate" \\
\hline & Brightness Control & "Off" \\
\hline \hline GBI Settings & Gate width & $100 \mathrm{~ns} / 1 \mu \mathrm{s}$ \\
\hline & Int. Voltage & $-350 \mathrm{~V}$ \\
\hline & Proximity focusing voltage & $3000 \mathrm{~V}$ \\
\hline \hline IPLAB Settings & Mode & "Synchronous" \\
\hline & Exposure & $200 \mu \mathrm{s}$ \\
\hline & Timing & "Ext. Sync" \\
\hline & Cleans & "Continuous" \\
\hline & Region & $384 \times 576$ pixels \\
\hline & A/D Converter & "16 bit $50 \mathrm{kHz}$ readout" \\
\hline & CCD Selected & "Electronic 3 ph" \\
\hline & Bending dipole volt. & $\pm 6570 \mathrm{~V}$ \\
\hline \hline Small Recirculator & Vacuum & $4 \mathrm{E}-7$ torr \\
\hline & & \\
\hline & &
\end{tabular}


Table D.2: GBI Measurement Parameters at $45^{\circ}$

\begin{tabular}{|l|c|}
\hline Date of Measurement & $7 / 22 / 97$ \\
\hline GBI Position (descriptive) & after $45^{\circ}$ bend \\
\hline GBI Postion (cm along beamline) & $670 \mathrm{~cm}$ \\
\hline Measured after Quad No. & 13 \\
\hline Beamlet drift distance & $15 \mathrm{~cm}$ \\
\hline Camera field of view & 2.07 by $3.11 \mathrm{~cm}$ \\
\hline Lens Extension needed & $32 \mathrm{~mm}$ \\
\hline
\end{tabular}

Table D.3: $45^{\circ}$ Integrated image file information

\begin{tabular}{|l|l|c|}
\hline Image File name & $\begin{array}{l}\text { Gate } \\
\text { start time } \\
(\mu \mathrm{s})\end{array}$ & \\
\hline "72297.ras" & no gate & integrated image \\
\hline "722971us132.ras" & 13.2 & flattop image \\
\hline "722971us142.ras" & 14.2 & flattop image \\
\hline "722971us152.ras" & 15.2 & flattop image \\
\hline "Q8CCWQ13CW132.ras" & 13.2 & rotated quadrupole error \\
\hline "Q13CW132.ras" & 13.2 & rotated quadrupole error \\
\hline "Q13CCW132.ras" & 13.2 & rotated quadrupole error \\
\hline "Q8CCW132.ras" & 13.2 & rotated quadrupole error \\
\hline
\end{tabular}


Table D.4: $45^{\circ} 100$ ns gated image file information

\begin{tabular}{|l|c|l|l|l|l|}
\hline $\begin{array}{l}\text { Image } \\
\text { Filename }\end{array}$ & $\begin{array}{l}\text { Gate } \\
\text { start } \\
(\mu \mathrm{s})\end{array}$ & $\begin{array}{l}\text { Image } \\
\text { Filename }\end{array}$ & $\begin{array}{l}\text { Gate } \\
\text { start } \\
(\mu \mathrm{s})\end{array}$ & $\begin{array}{l}\text { Image } \\
\text { Filename } \\
(\mu \mathrm{s})\end{array}$ & $\begin{array}{l}\text { Gate } \\
\text { start }\end{array}$ \\
\hline "72297117.ras" & 11.7 & "72297133.ras" & 13.3 & "72297149.ras" & 14.9 \\
\hline "72297118.ras" & 11.8 & "72297134.ras" & 13.4 & "72297150.ras" & 15.0 \\
\hline "72297119.ras" & 11.9 & "72297135.ras" & 13.5 & "72297151.ras" & 15.1 \\
\hline "72297120.ras" & 12.0 & "72297136.ras" & 13.6 & "72297152.ras" & 15.2 \\
\hline "72297121.ras" & 12.1 & "72297137.ras" & 13.7 & "72297153.ras" & 15.3 \\
\hline "72297122.ras" & 12.2 & "72297138.ras" & 13.8 & "72297154.ras" & 15.4 \\
\hline "72297123.ras" & 12.3 & "72297139.ras" & 13.9 & "72297155.ras" & 15.5 \\
\hline "72297124.ras" & 12.4 & "72297140.ras" & 14.0 & "72297156.ras" & 15.6 \\
\hline "72297125.ras" & 12.5 & "72297141.ras" & 14.1 & "72297157.ras" & 15.7 \\
\hline "72297126.ras" & 12.6 & "72297142.ras" & 14.2 & "72297158.ras" & 15.8 \\
\hline "72297127.ras" & 12.7 & "72297143.ras" & 14.3 & "72297159.ras" & 15.9 \\
\hline "72297128.ras" & 12.8 & "72297144.ras" & 14.4 & "72297160.ras" & 16.0 \\
\hline "72297129.ras" & 12.9 & "72297145.ras" & 14.5 & "72297161.ras" & 16.1 \\
\hline "72297130.ras" & 13.0 & "72297146.ras" & 14.6 & "72297162.ras" & 16.2 \\
\hline "72297131.ras" & 13.1 & "72297147.ras" & 14.7 & "72297163.ras" & 16.3 \\
\hline "72297132.ras" & 13.2 & "72297148.ras" & 14.8 & "72297164.ras" & 16.4 \\
\hline
\end{tabular}




\section{Appendix E}

\section{Simulation and Data Analysis Codes}

The following are the codes used in the course of this work, to simulate the Small Recirculator beam using the moment evolution equations, to find the optimized values of the strength and rotation angle of the quadrupoles in the beam correction system, and to perform the GBI image analysis.

\section{E.1 "FULLAG"}

"FULLAG" is a FORTRAN 77 code modified from J. Barnard's MOMENT code. Its algorithm incorporates the full set of 10 second moment evolution equations and allows the setting of either random quadrupole rotation errors or discrete errors of the user's choice. It was used to produce moment data to compare against that produced by the reduced set of 8 second moment evolution equations in their approximate form. This comparison is shown in Figure 6.7. 


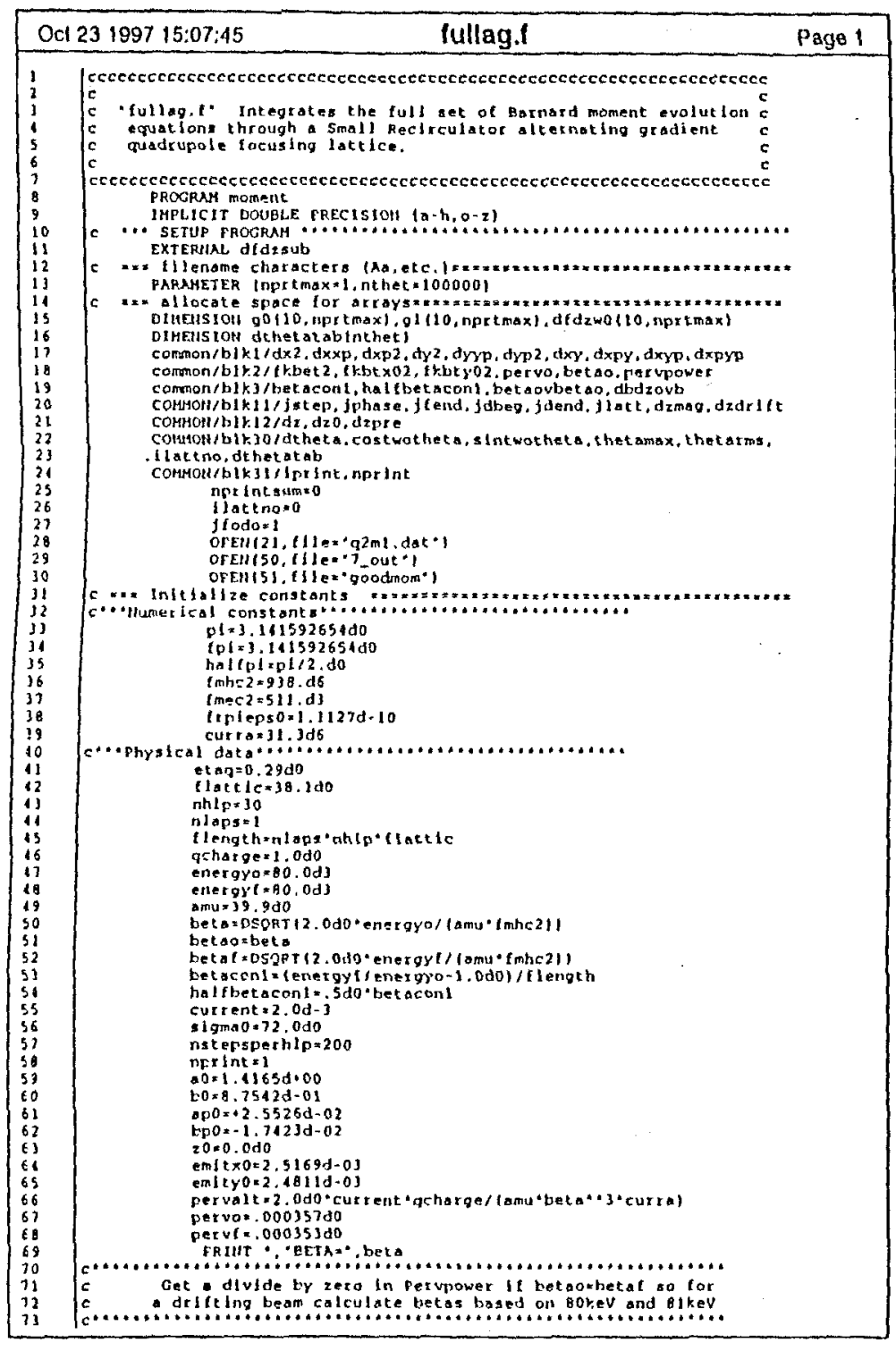

\begin{tabular}{|c|c|c|}
\hline 0,12 & $23199715: 07: 45$ & Page 2 \\
\hline 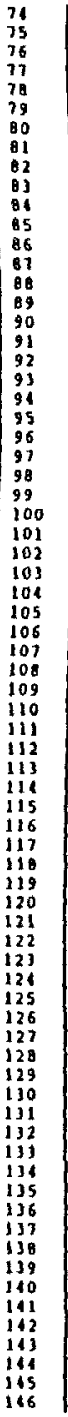 & 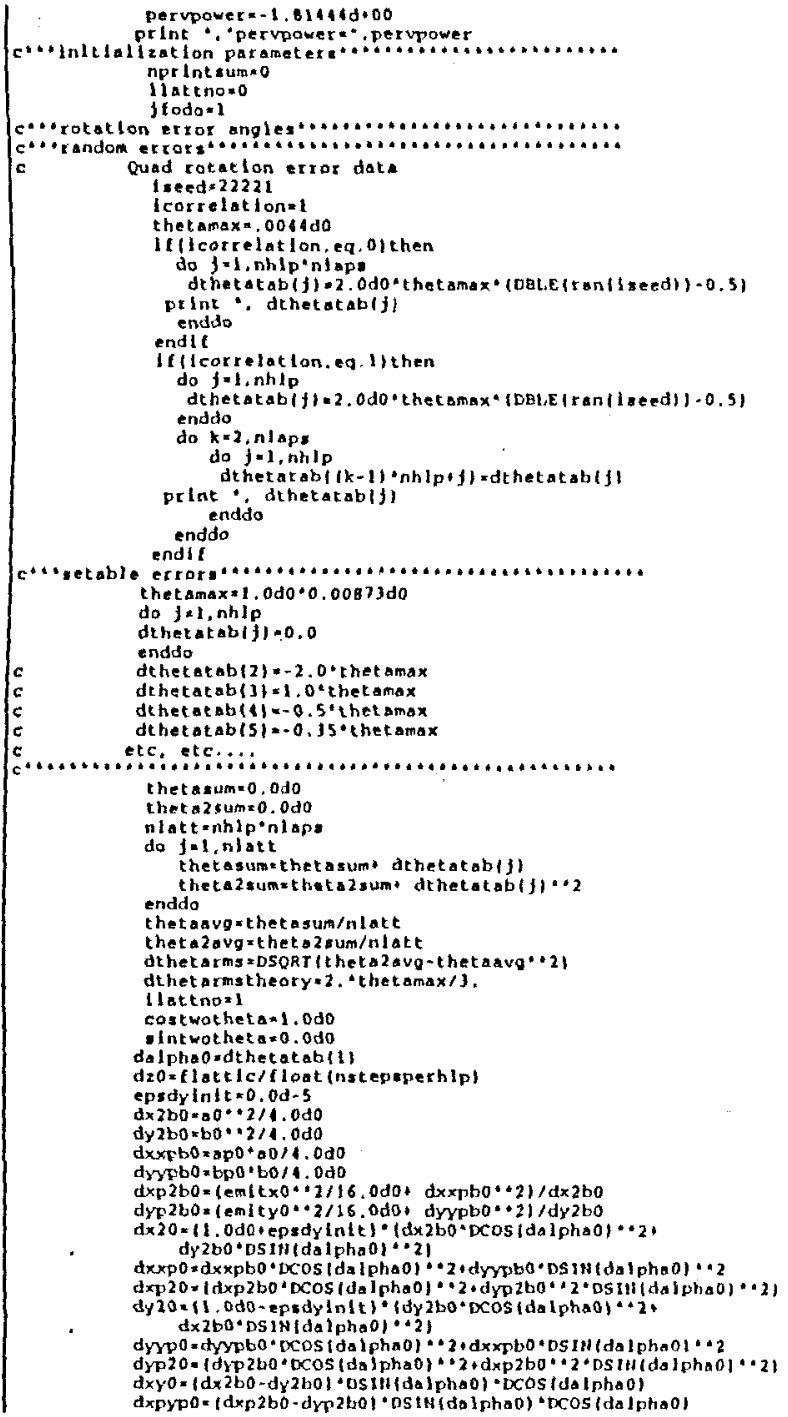 & \\
\hline
\end{tabular}




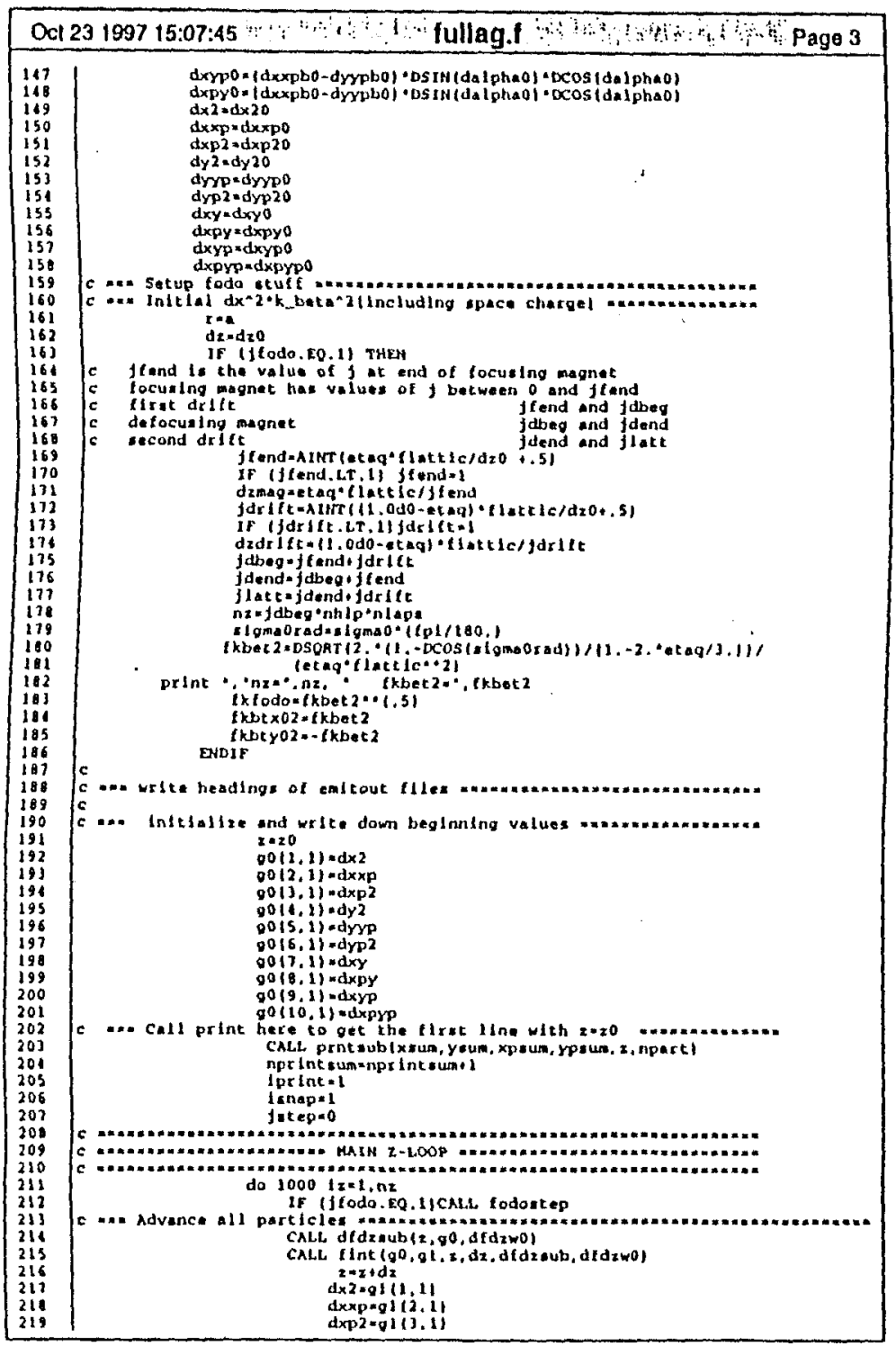

\begin{tabular}{|c|c|c|c|}
\hline \multicolumn{4}{|c|}{ Oct 231997 15:07:45 $\quad \cdots \quad$ fullag. $\quad$ Page 4} \\
\hline 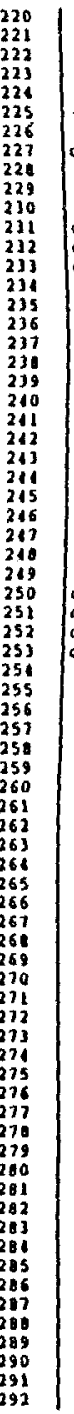 & 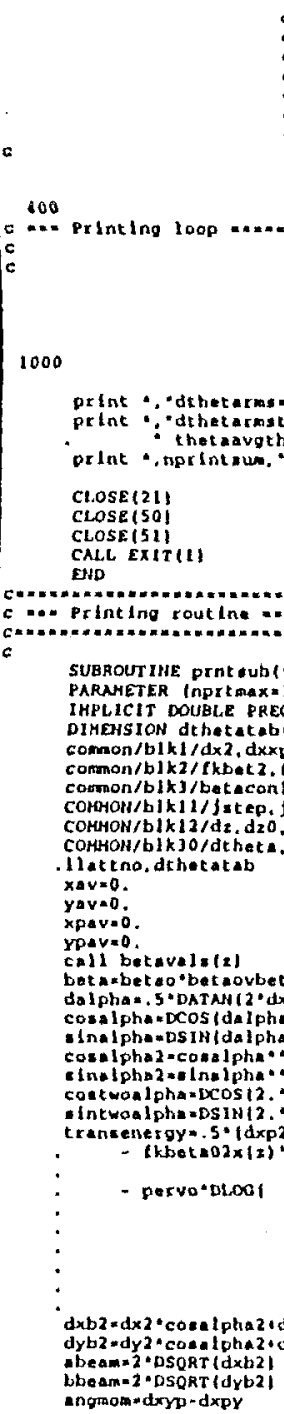 & 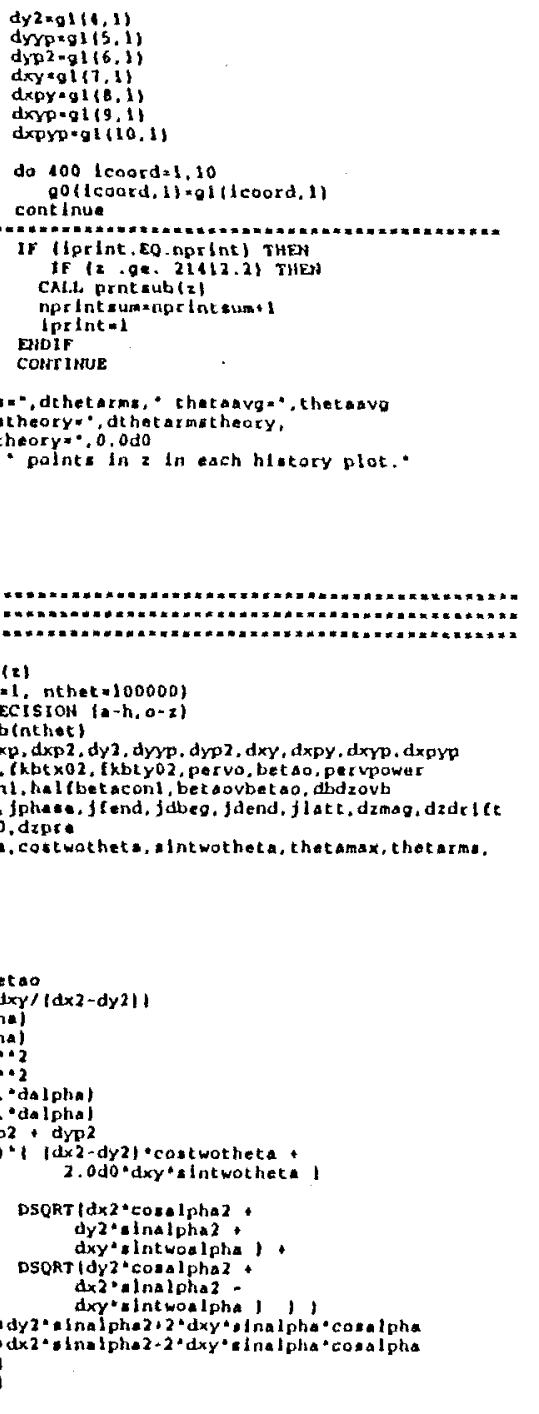 & \\
\hline
\end{tabular}



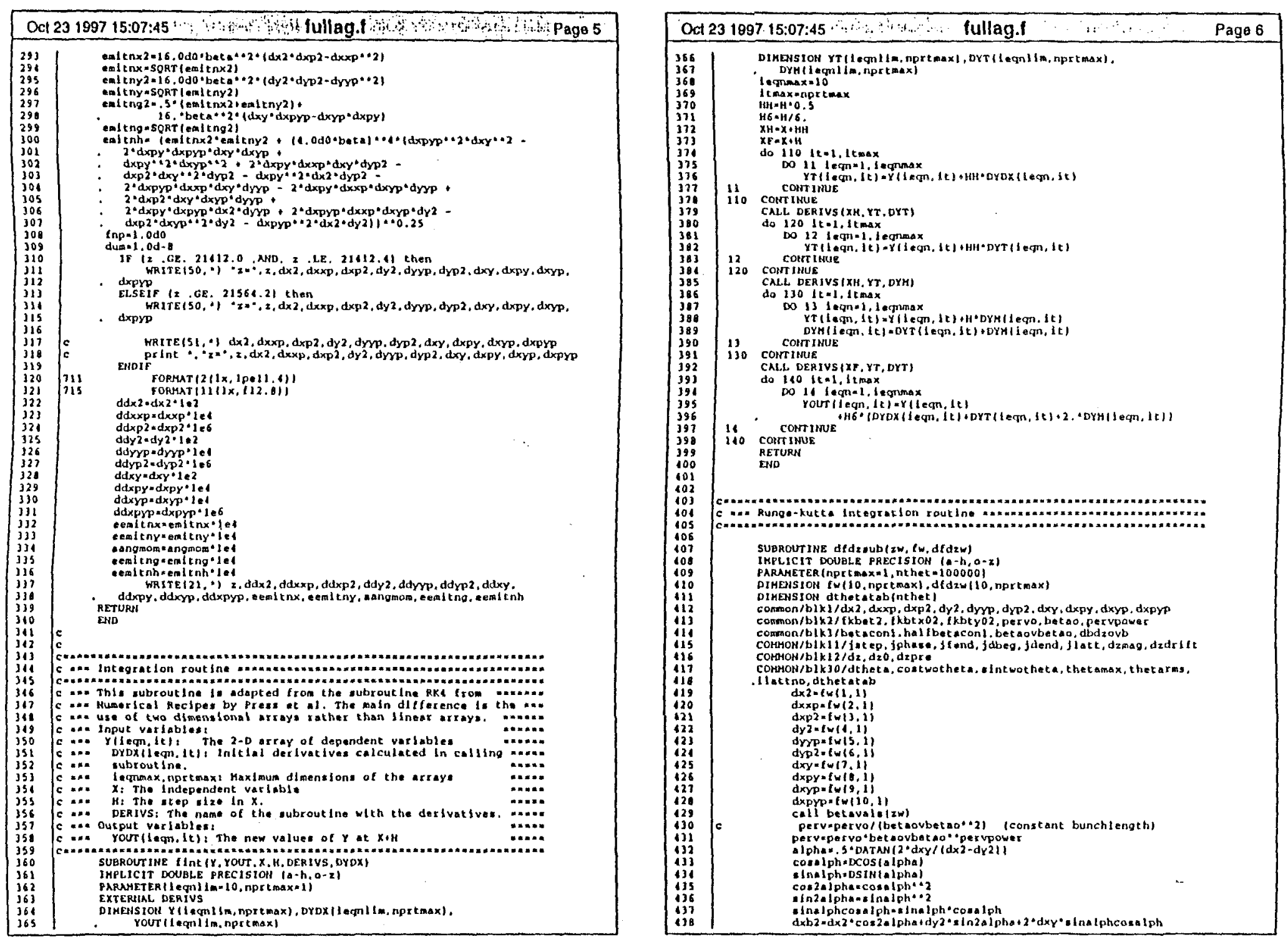


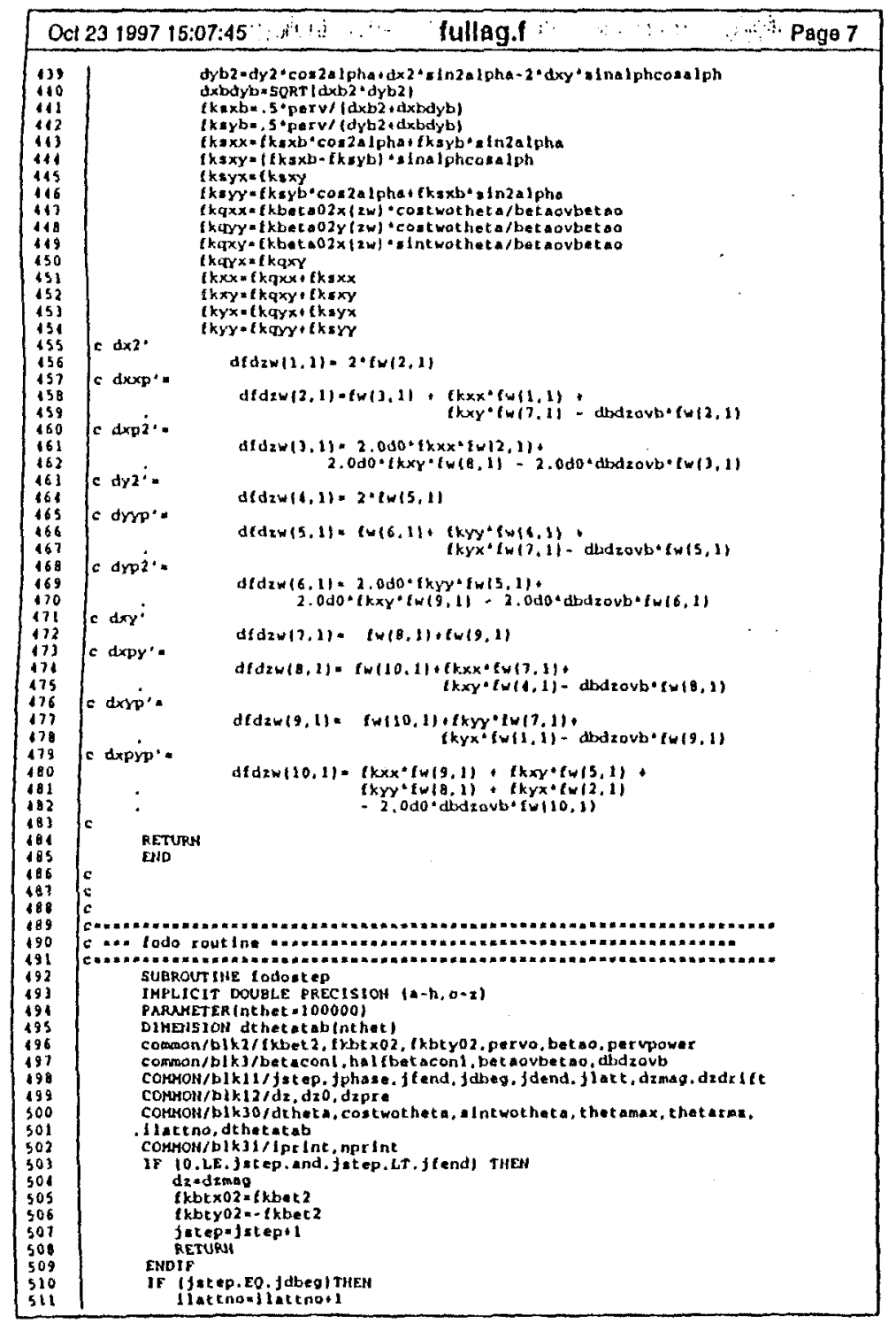

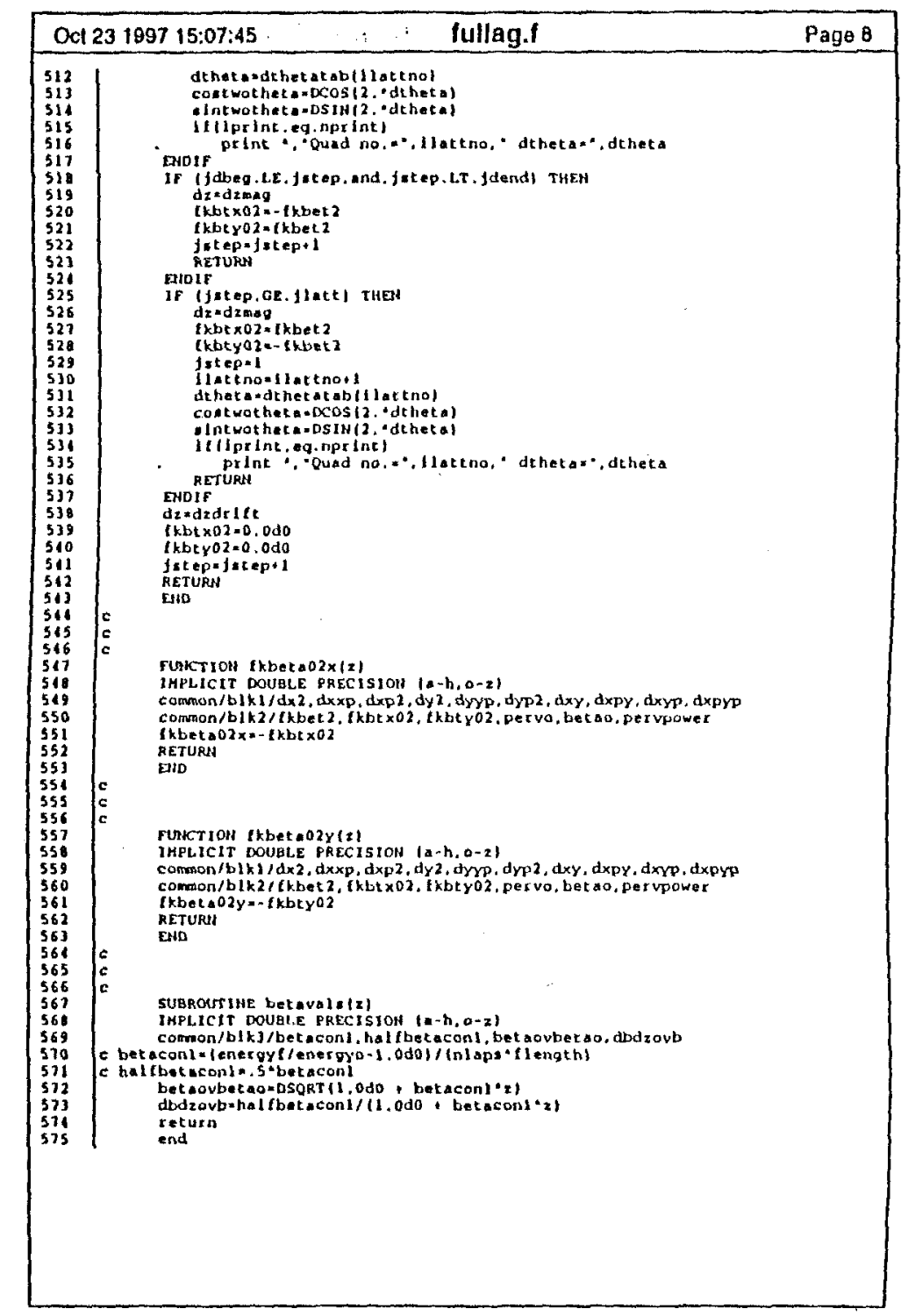




\section{E.2 "REDUCEDAG"}

"REDUCEDAG" is a FORTRAN 77 code modified from J. Barnard's MOMENT code. Its algorithm incorporates the reduced set of 8 second moment evolution equations by substituting in the approximate explicit solutions for $\Delta x^{2}$ and $\Delta y^{2}$ (Equations 6.11 and 6.16 ) wherever they occur in the moment evolution equations. The code allows the setting of either random quadrupole rotation errors or discrete errors of the user's choice. It was used to produce moment data to compare against that produced by the set of 10 second moment evolution equations of "FULLAG". This comparison is shown in Figure 6.7. 


\begin{tabular}{|c|c|}
\hline Ocl 231997 15:27:17 & Page 1 \\
\hline 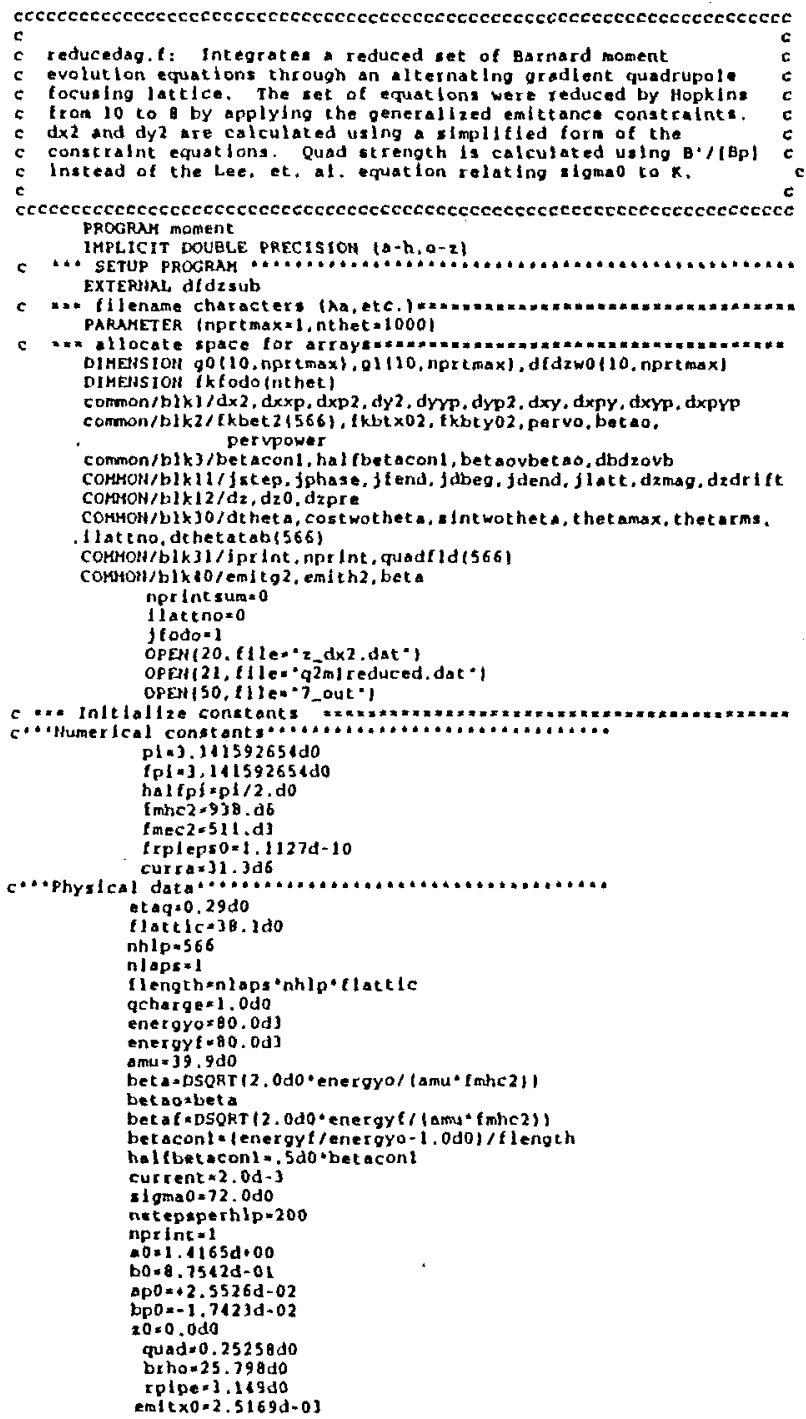 & \\
\hline
\end{tabular}

\begin{tabular}{|c|c|}
\hline Oct 231997 15:27:17 & Page 2 \\
\hline 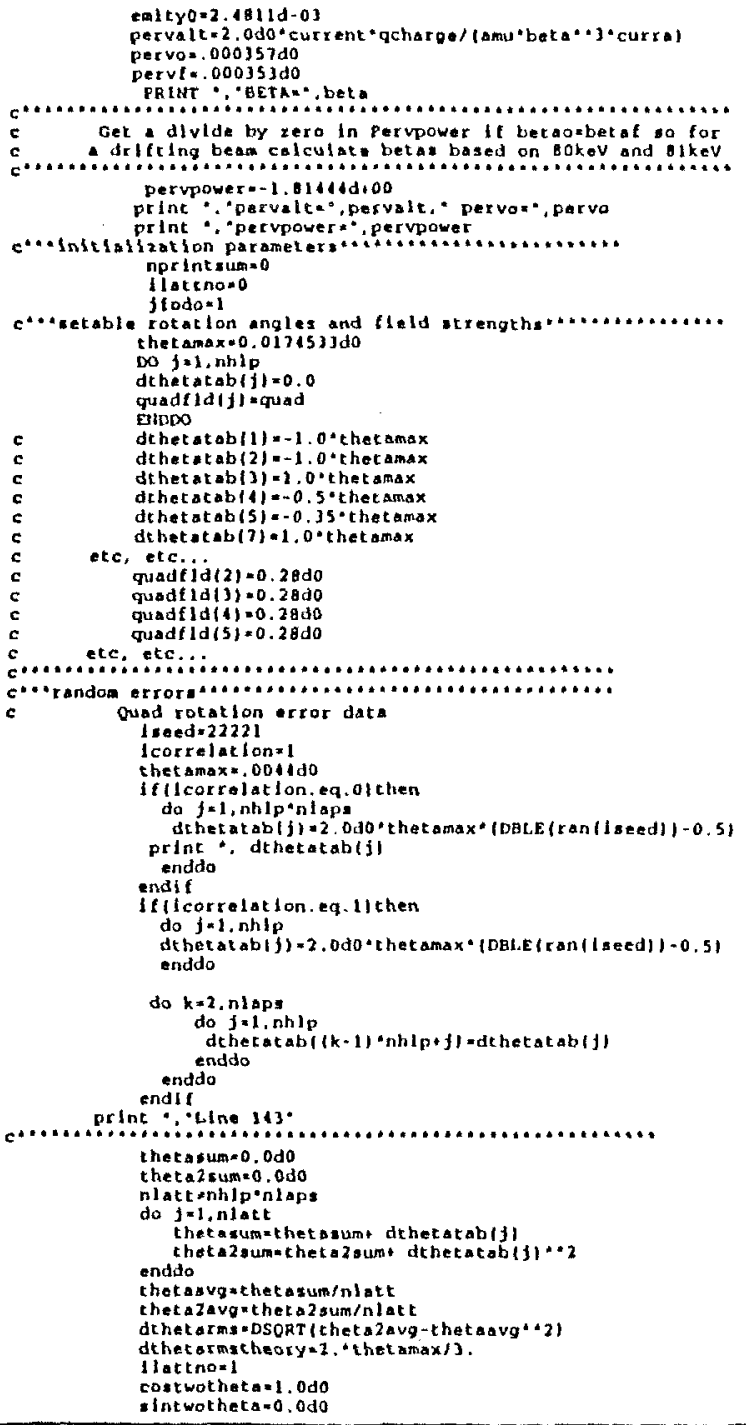 & \\
\hline
\end{tabular}




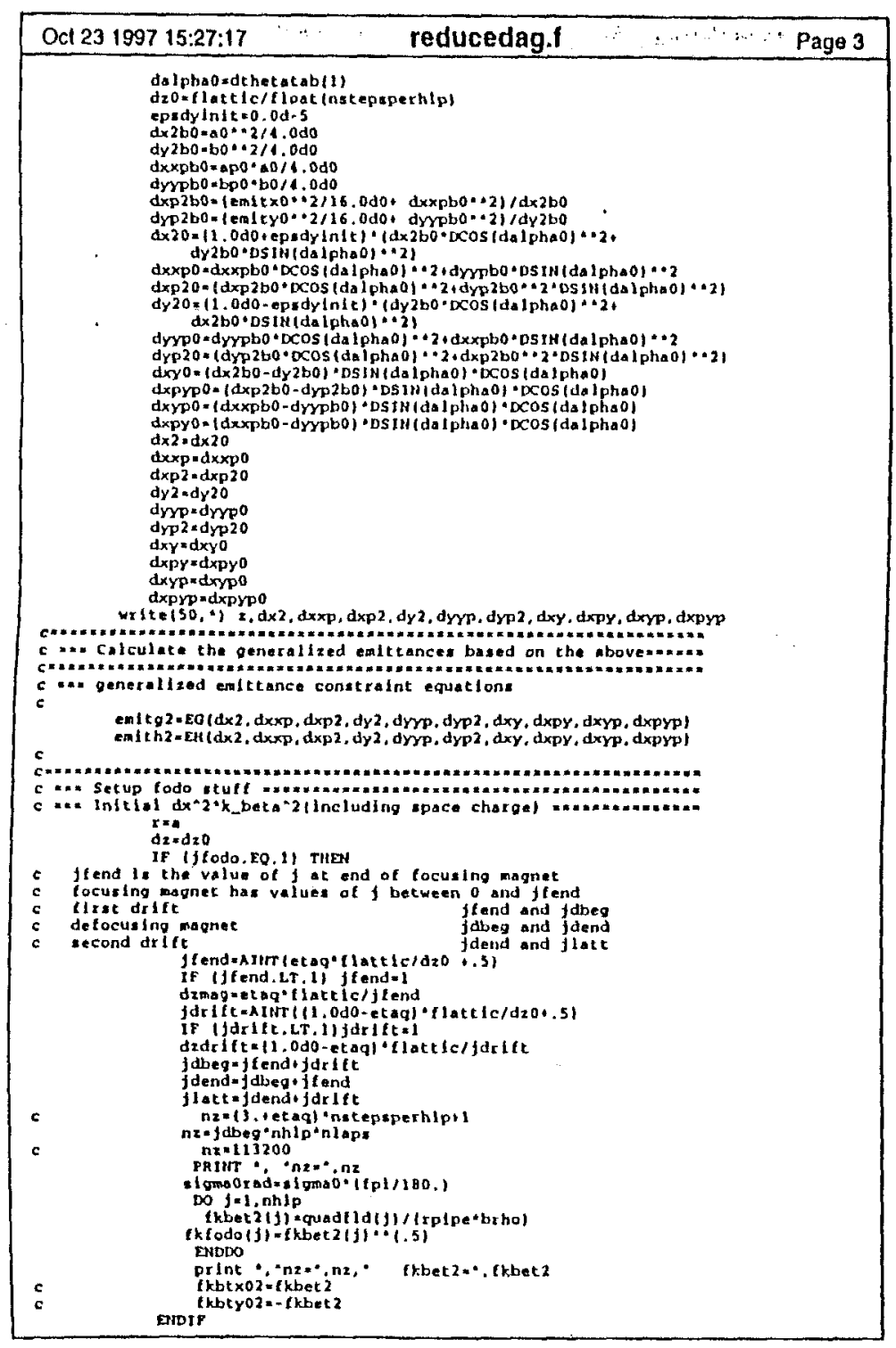

\begin{tabular}{|c|c|}
\hline Oct 231997 15:27:17 & Page 4 \\
\hline 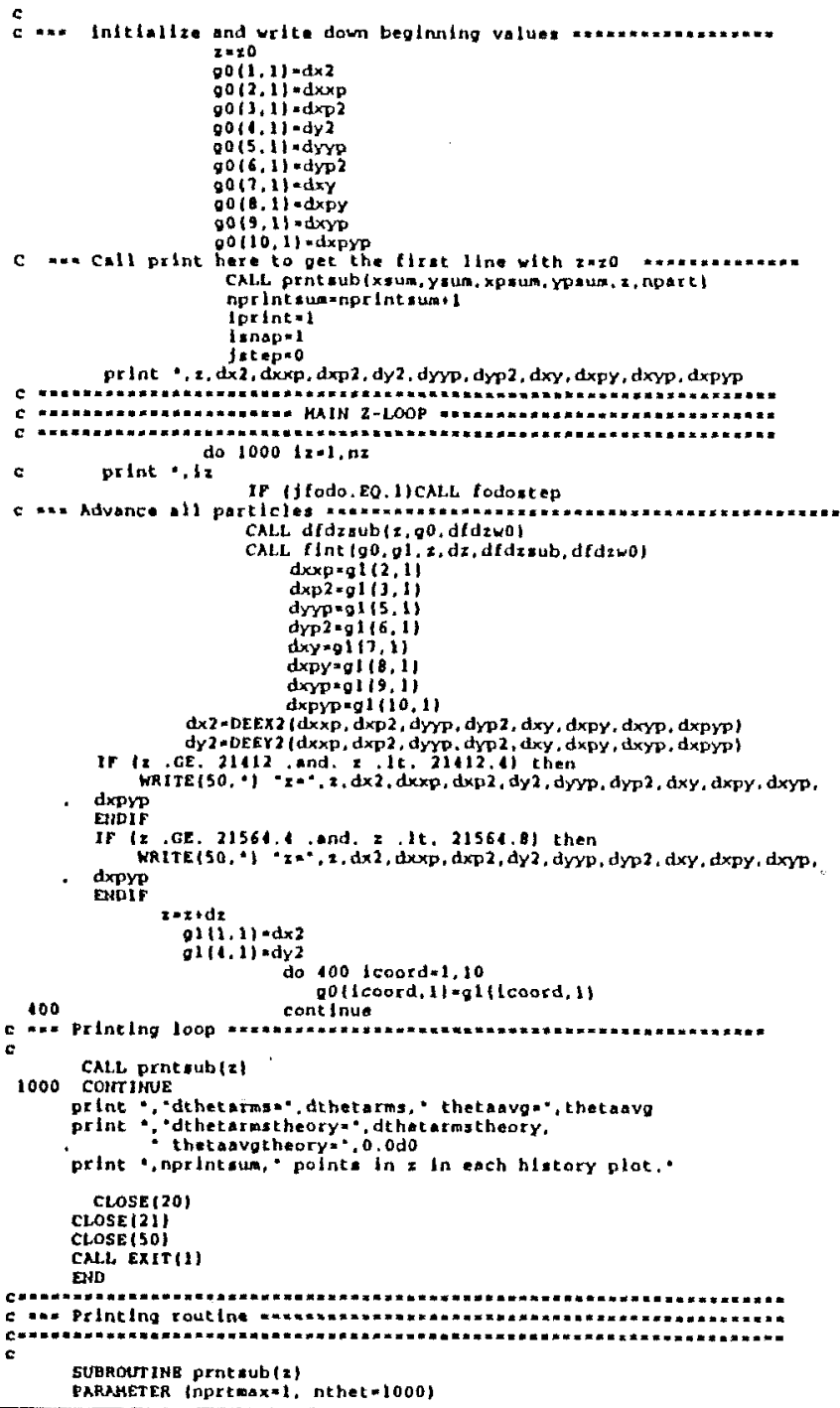 & $=$ \\
\hline
\end{tabular}




\begin{tabular}{|c|c|c|}
\hline Oct & reducedag.f & Page 5 \\
\hline $\begin{array}{l}c \\
c \\
c \\
c \\
c \\
i 11 \\
710 \\
c \cdot T h e\end{array}$ & 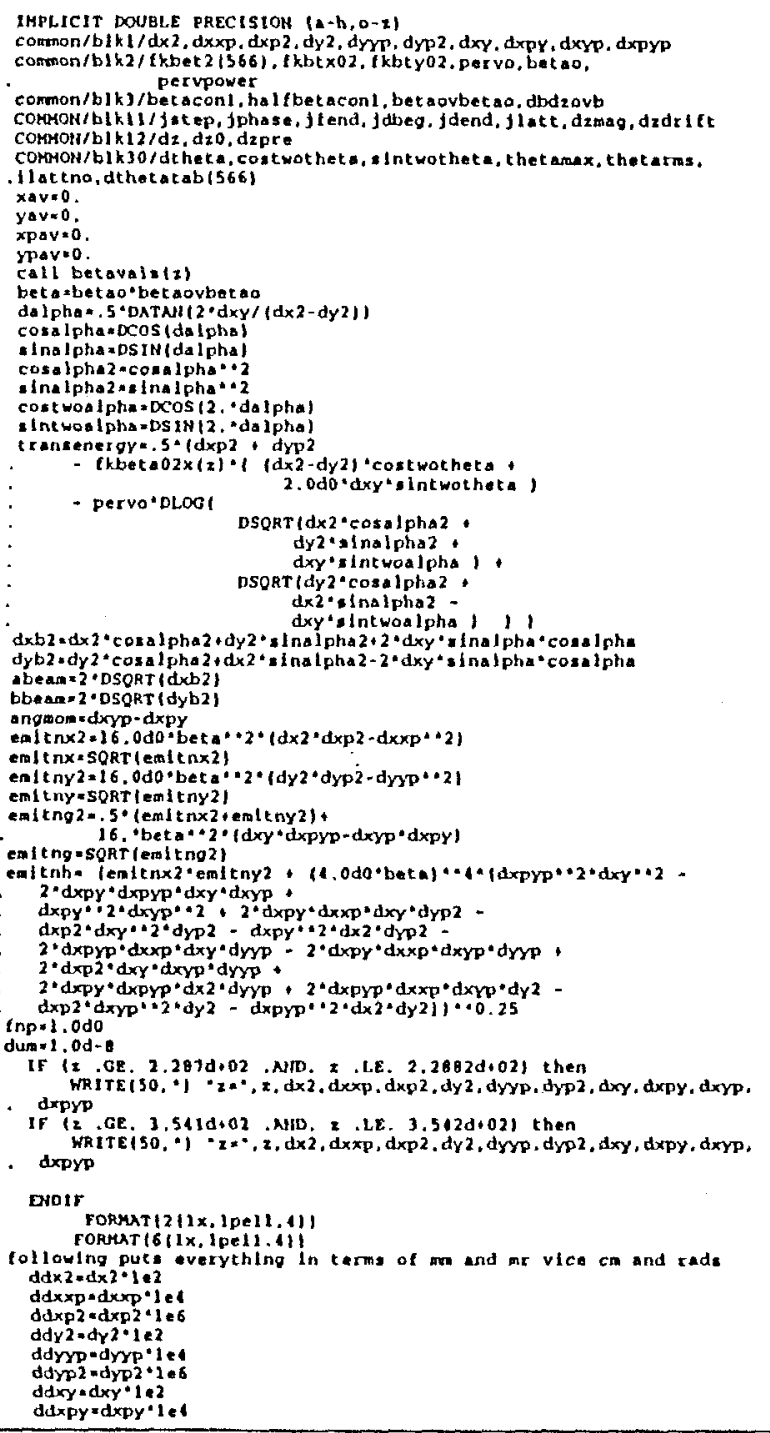 & . \\
\hline
\end{tabular}

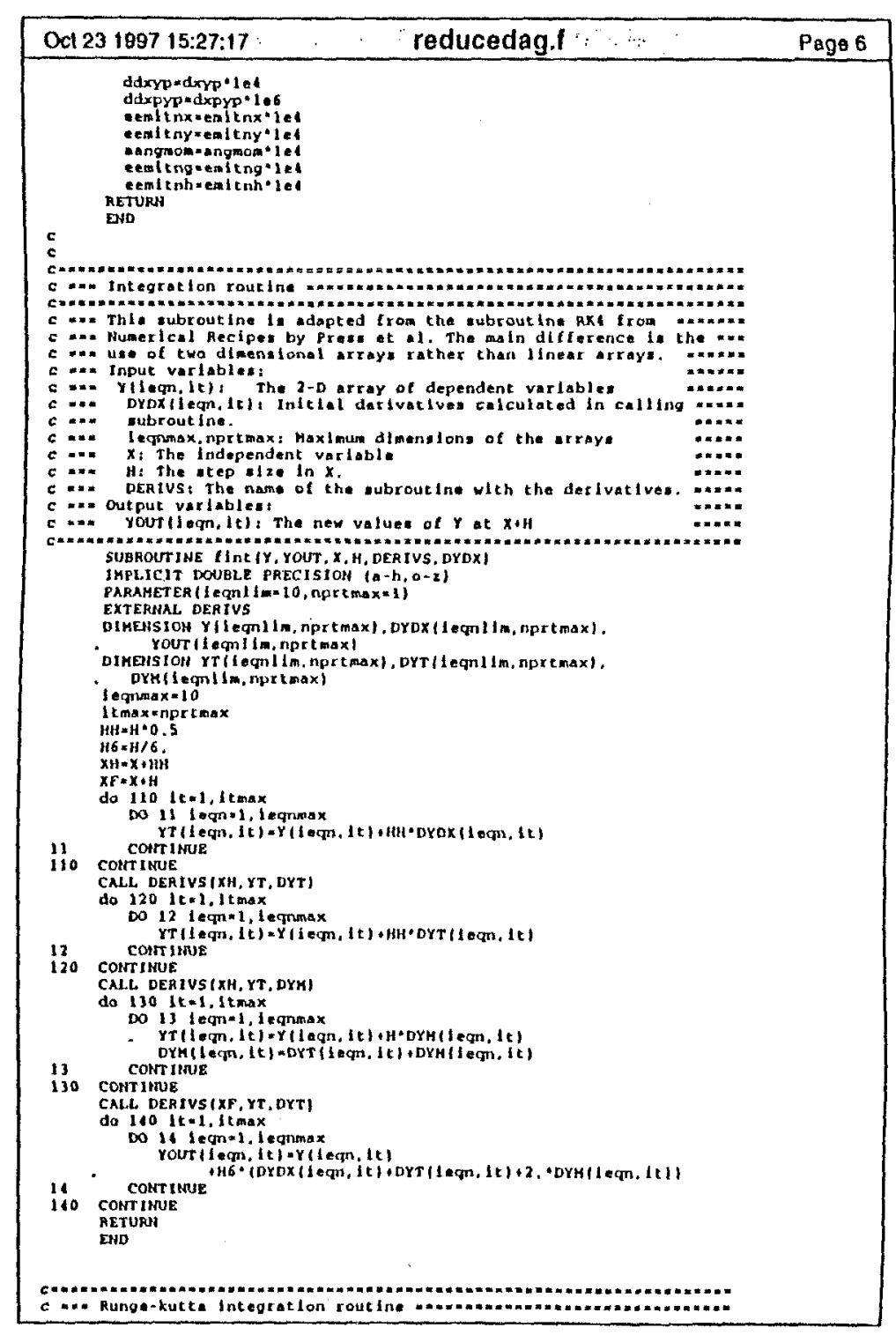




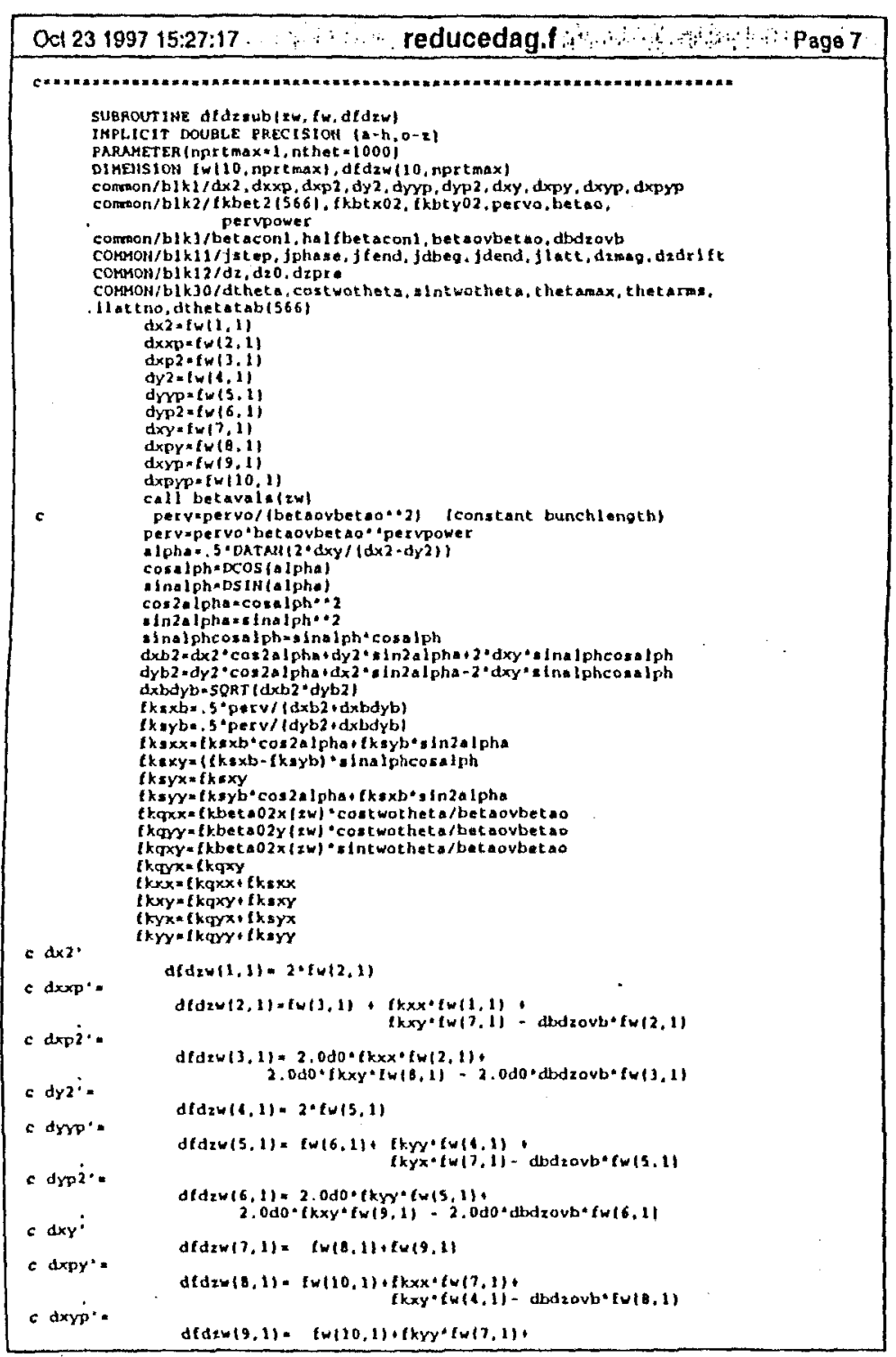

\begin{tabular}{|c|c|}
\hline OCt 231997 15:27:17 $\quad: \cdots$ reducedag. $f$. is & Page 8 \\
\hline 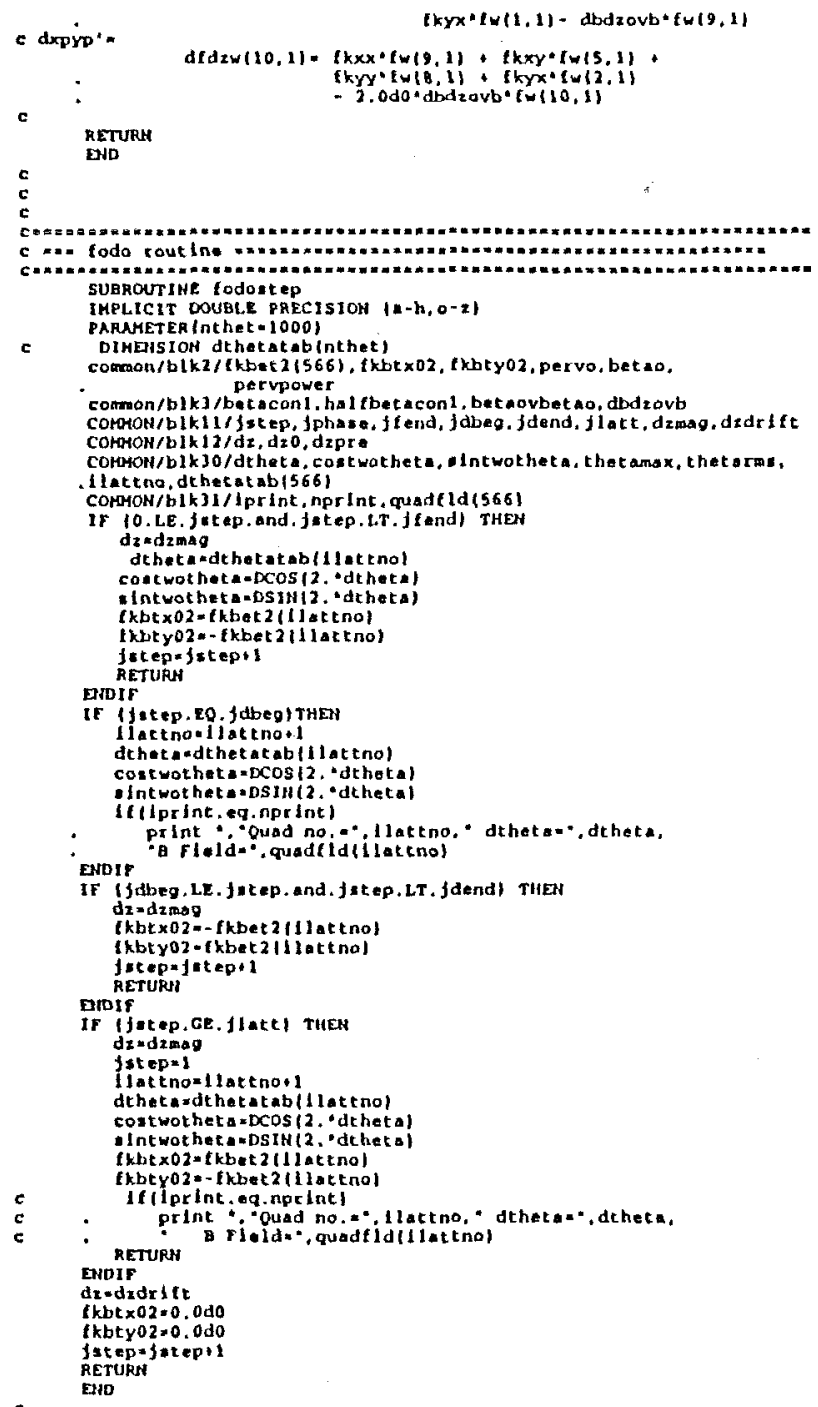 & . \\
\hline
\end{tabular}




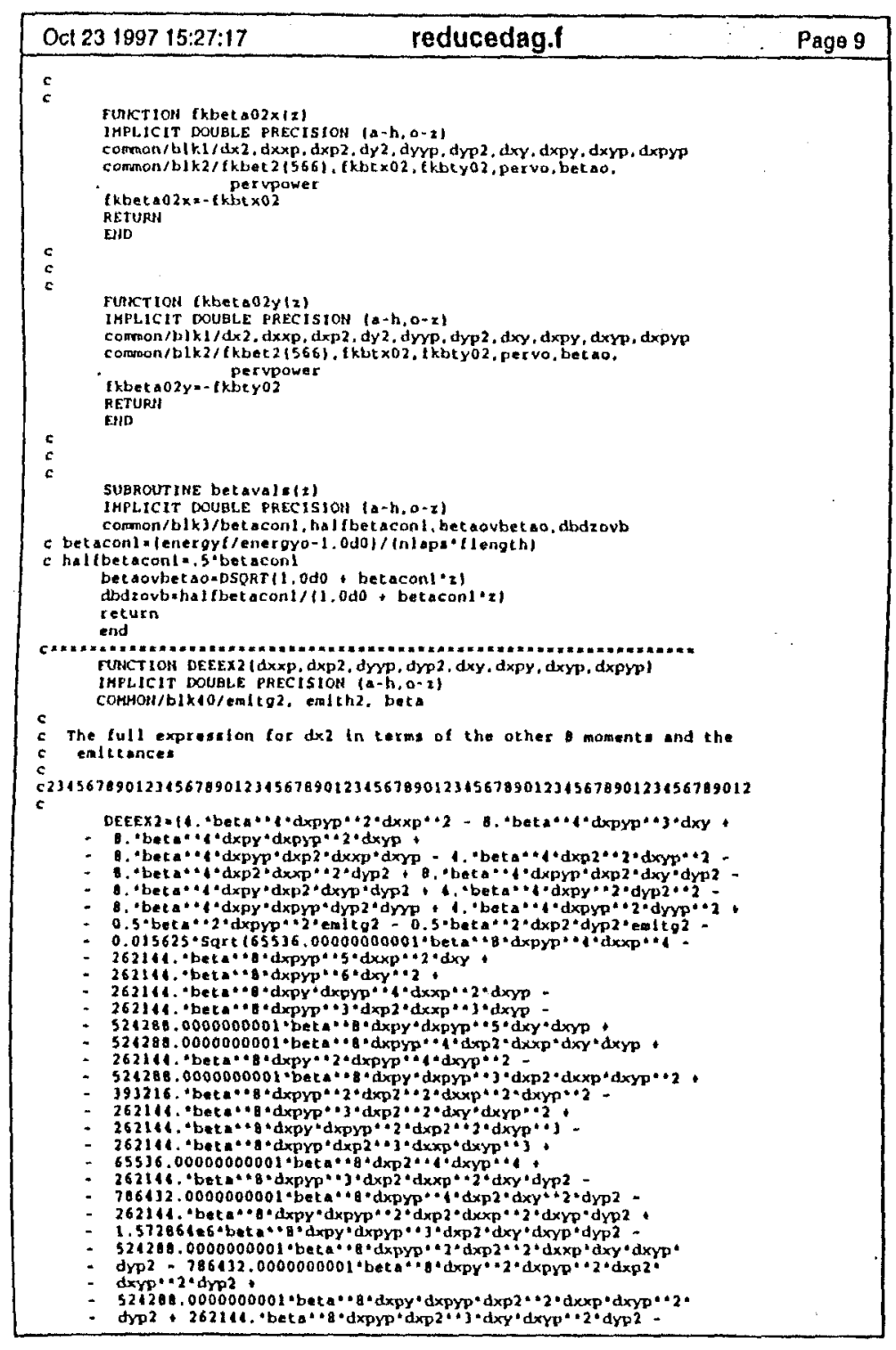

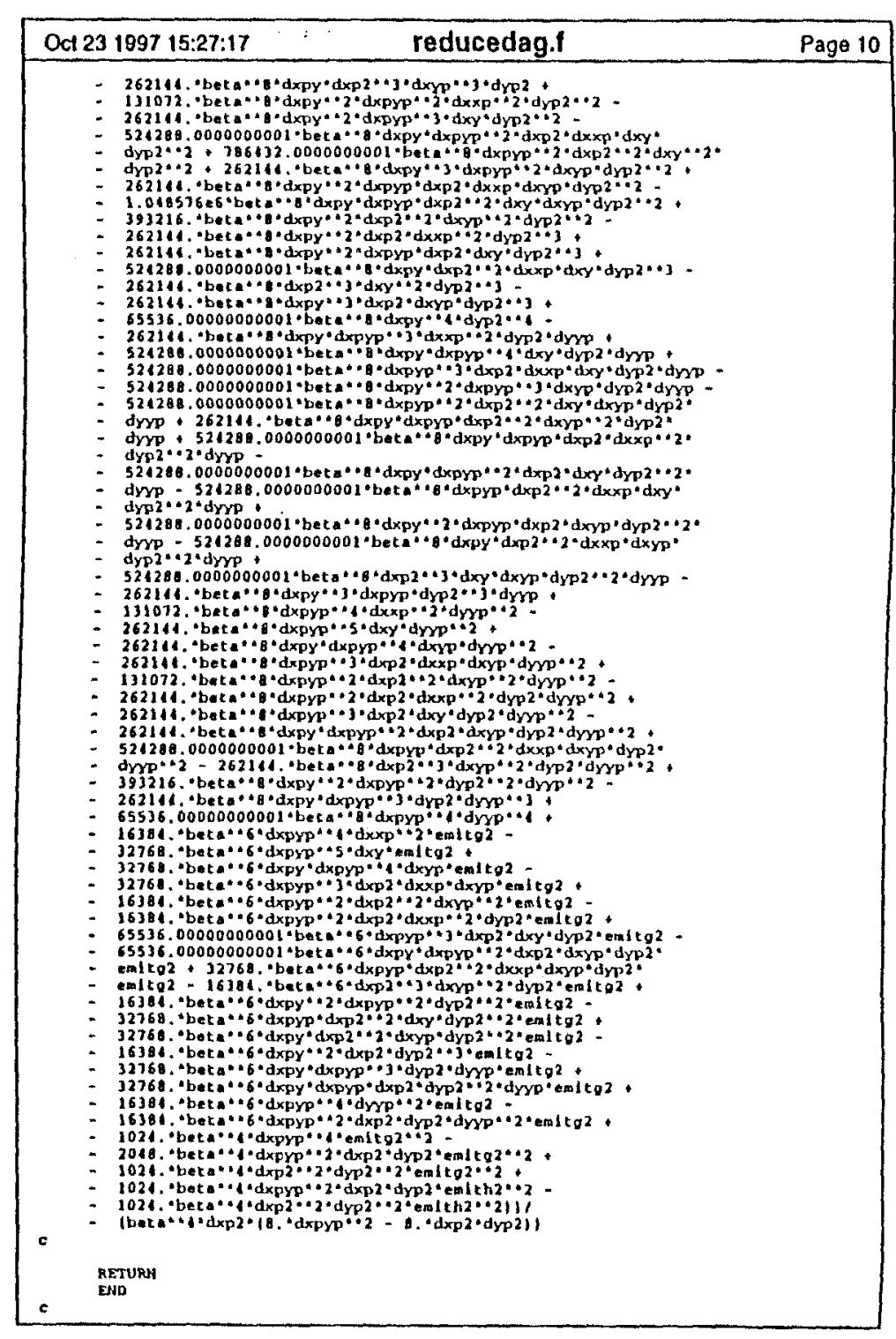




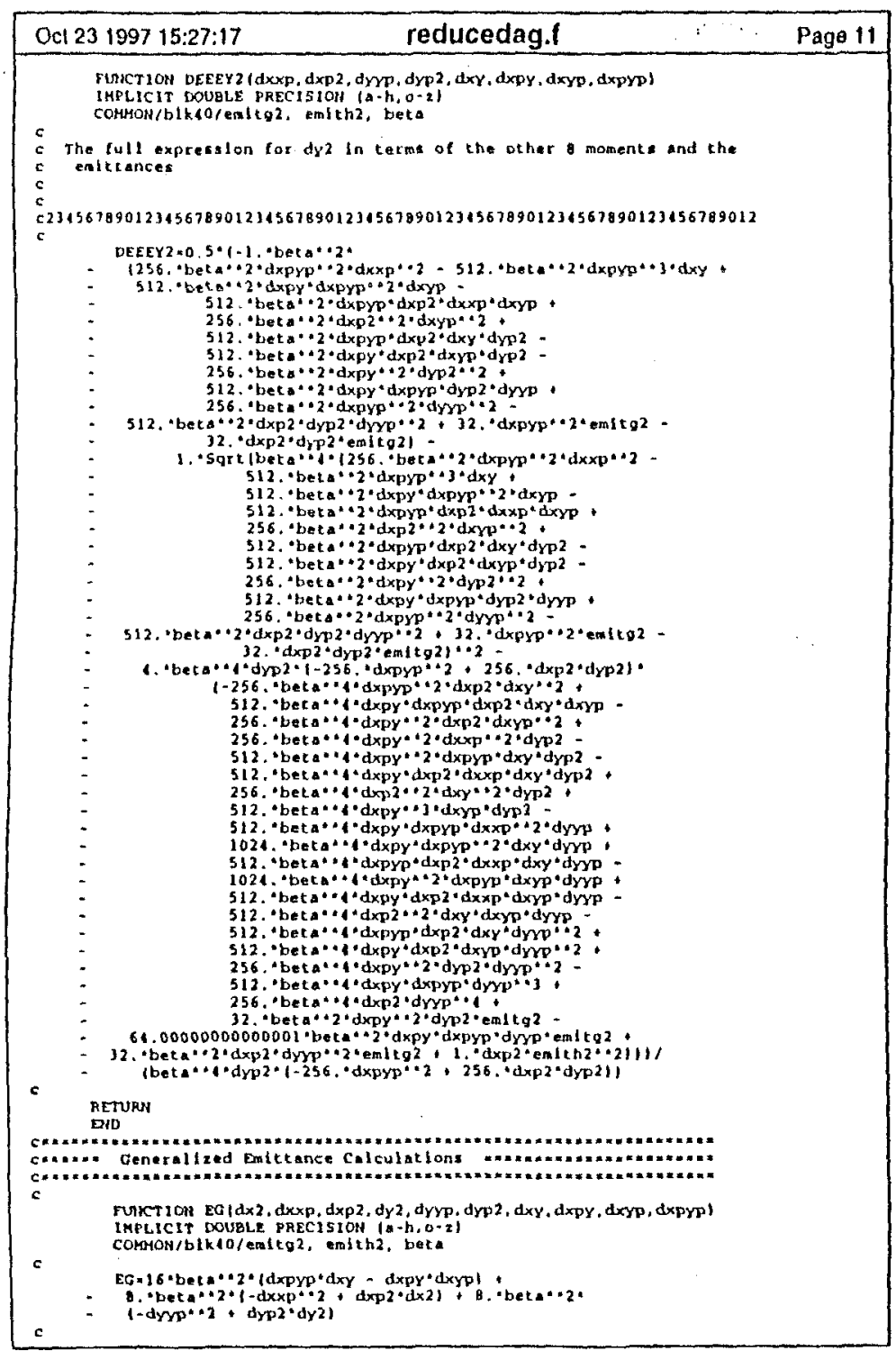

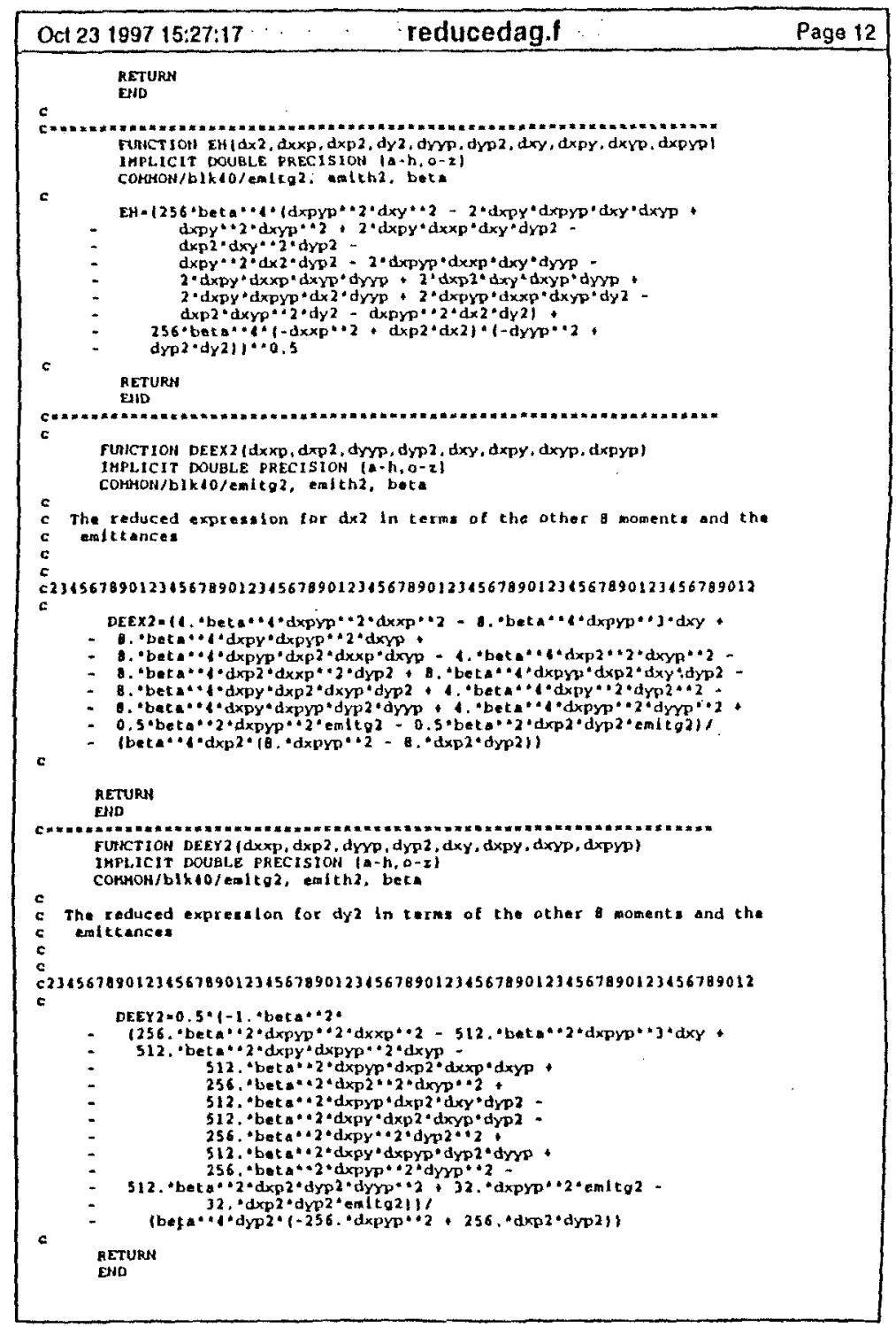




\section{E.3 "CORRECTOR"}

"CORRECTOR" is a FORTRAN 77 code modified from J. Barnard's MOMENT code. It implements the SPSA algorithm to optimize the settings of the adjustable magnetic quadrupoles of the beam correction system based on a desired set of beam second moments. The code allows the user to determine the initial settings of the quadrupole field strengths and rotation angles. "CORRECTOR's" algorithm incorporates the reduced set of 8 second moment evolution equations by substituting in the approximate explicit solutions for $\Delta x^{2}$ and $\Delta y^{2}$ (Equations 6.11 and 6.16) wherever they occur in the moment evolution equations. 


\begin{tabular}{|c|c|}
\hline Ocl 231997 14:47:28 & Page 1 \\
\hline 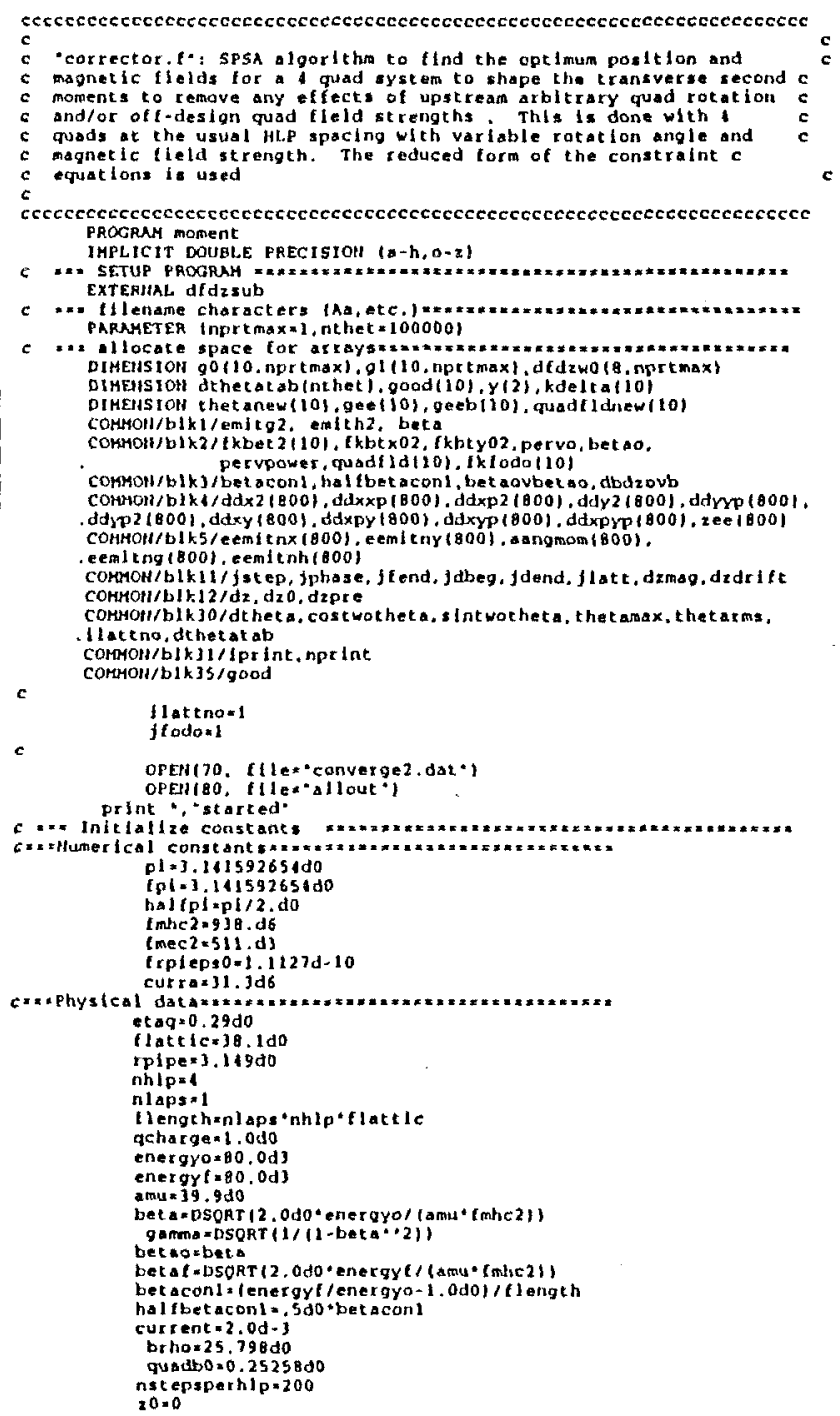 & c \\
\hline
\end{tabular}

\begin{tabular}{|c|c|}
\hline Oct $23 \quad 1997$ 14:47:28 & Page 2 \\
\hline 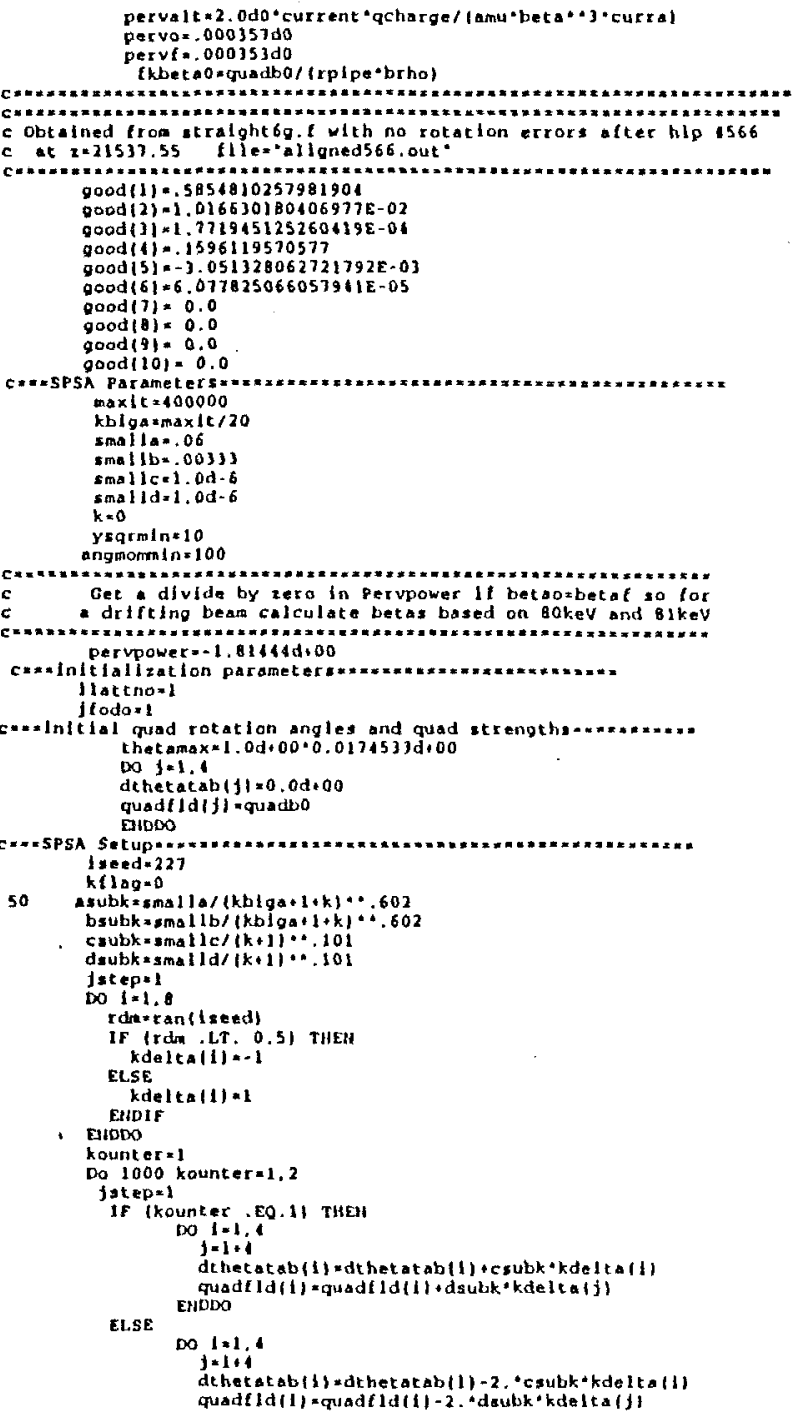 & \\
\hline
\end{tabular}




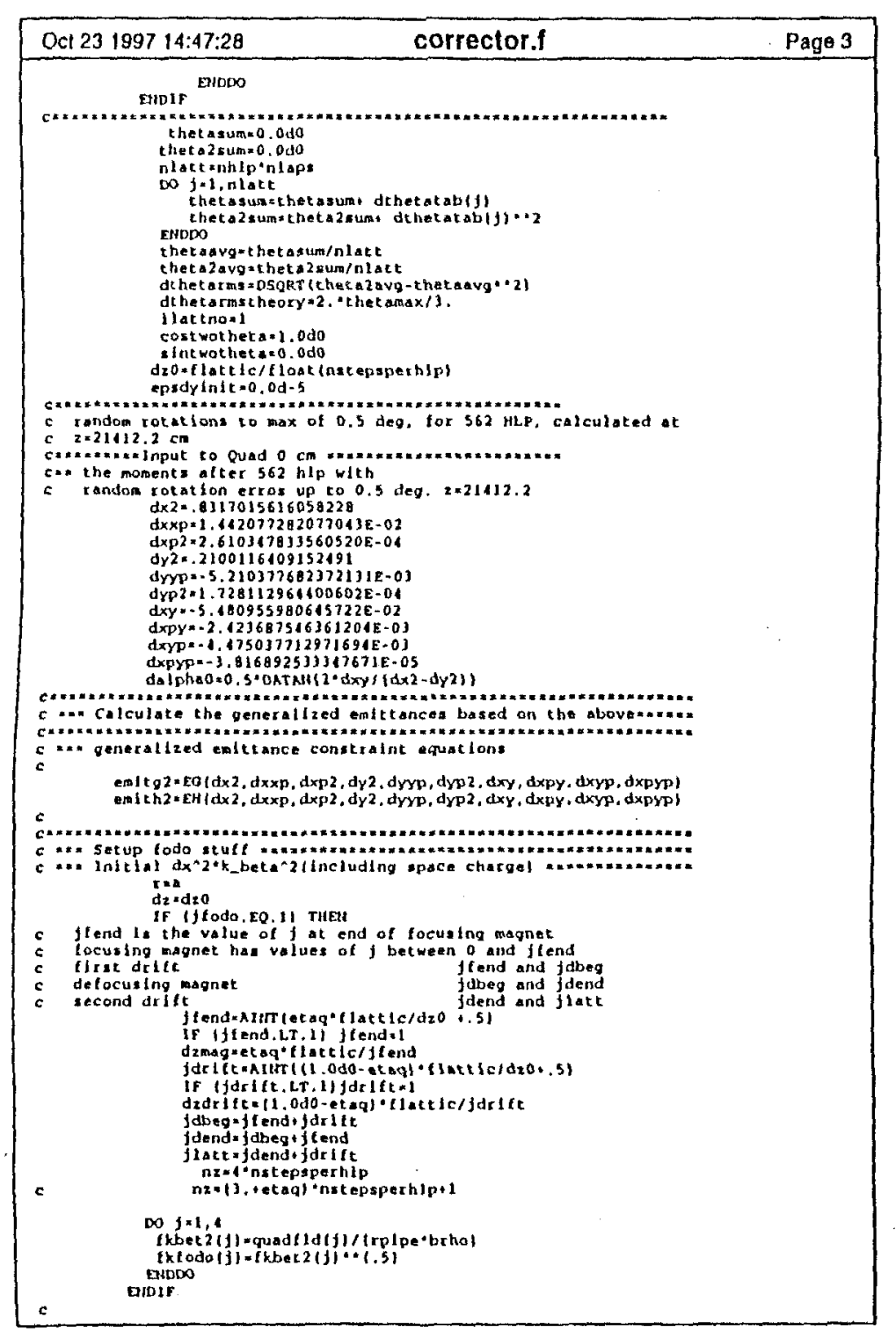

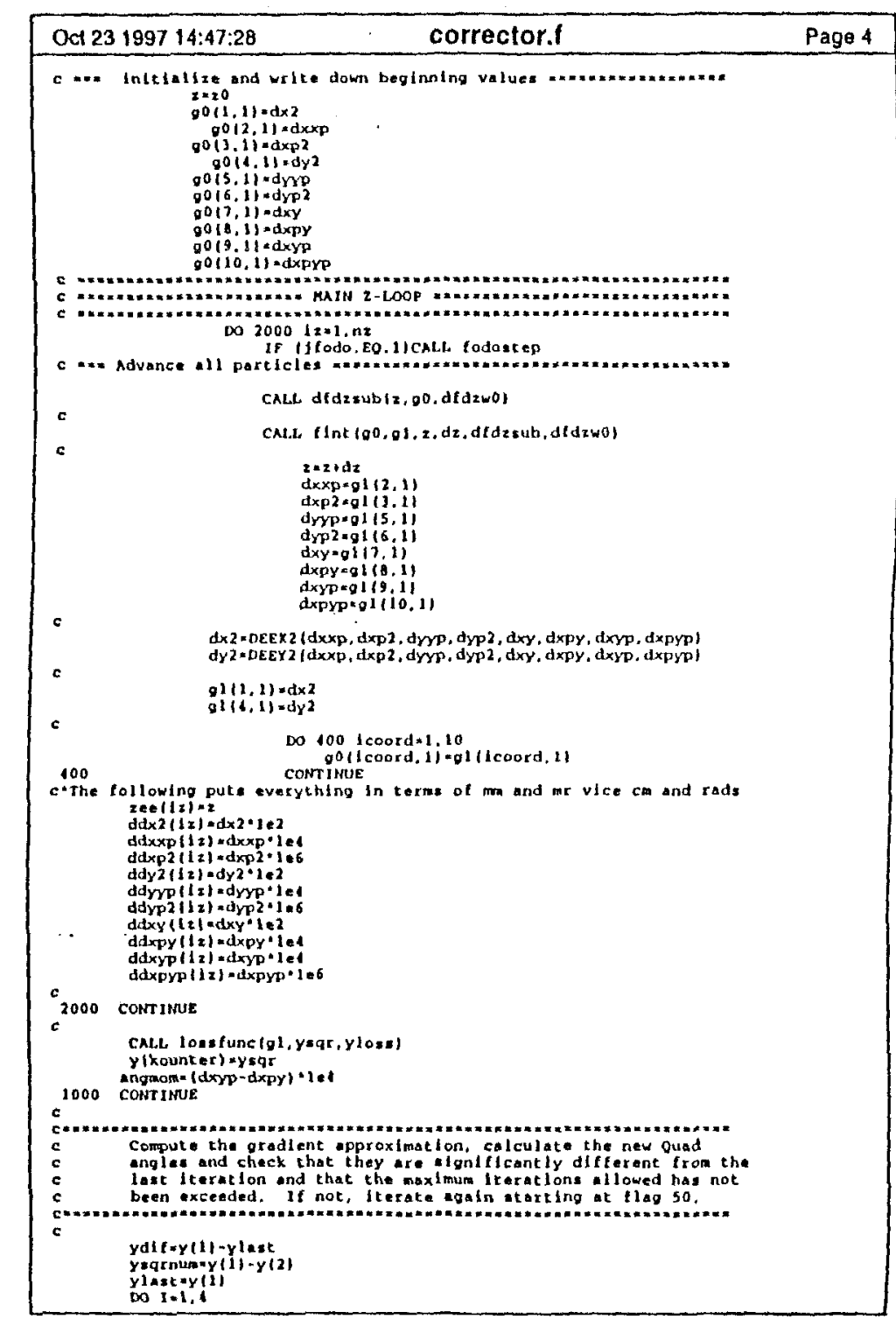




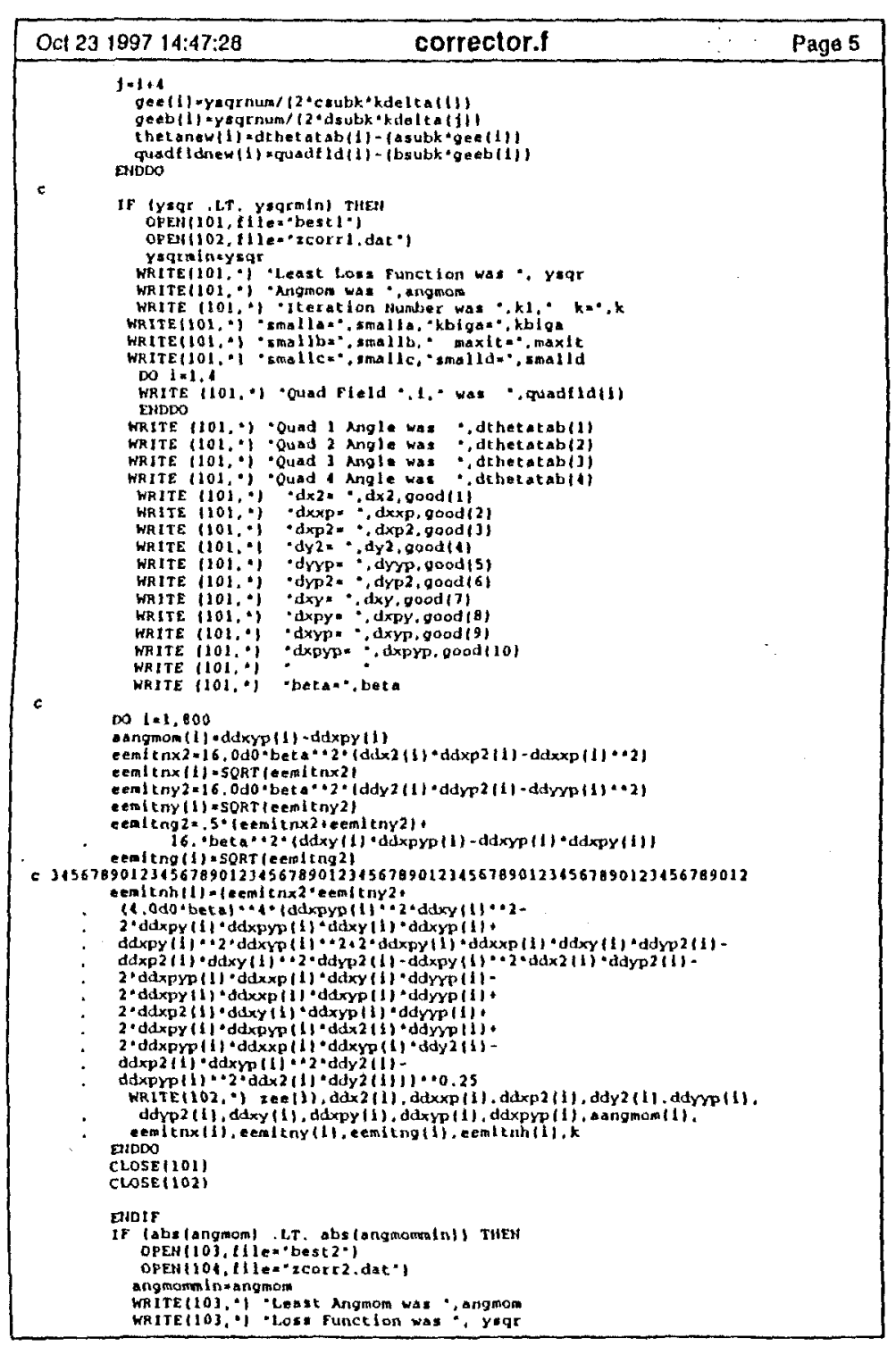

\begin{tabular}{|c|c|}
\hline Oct 231997 14:47:28 & Paga 6 \\
\hline 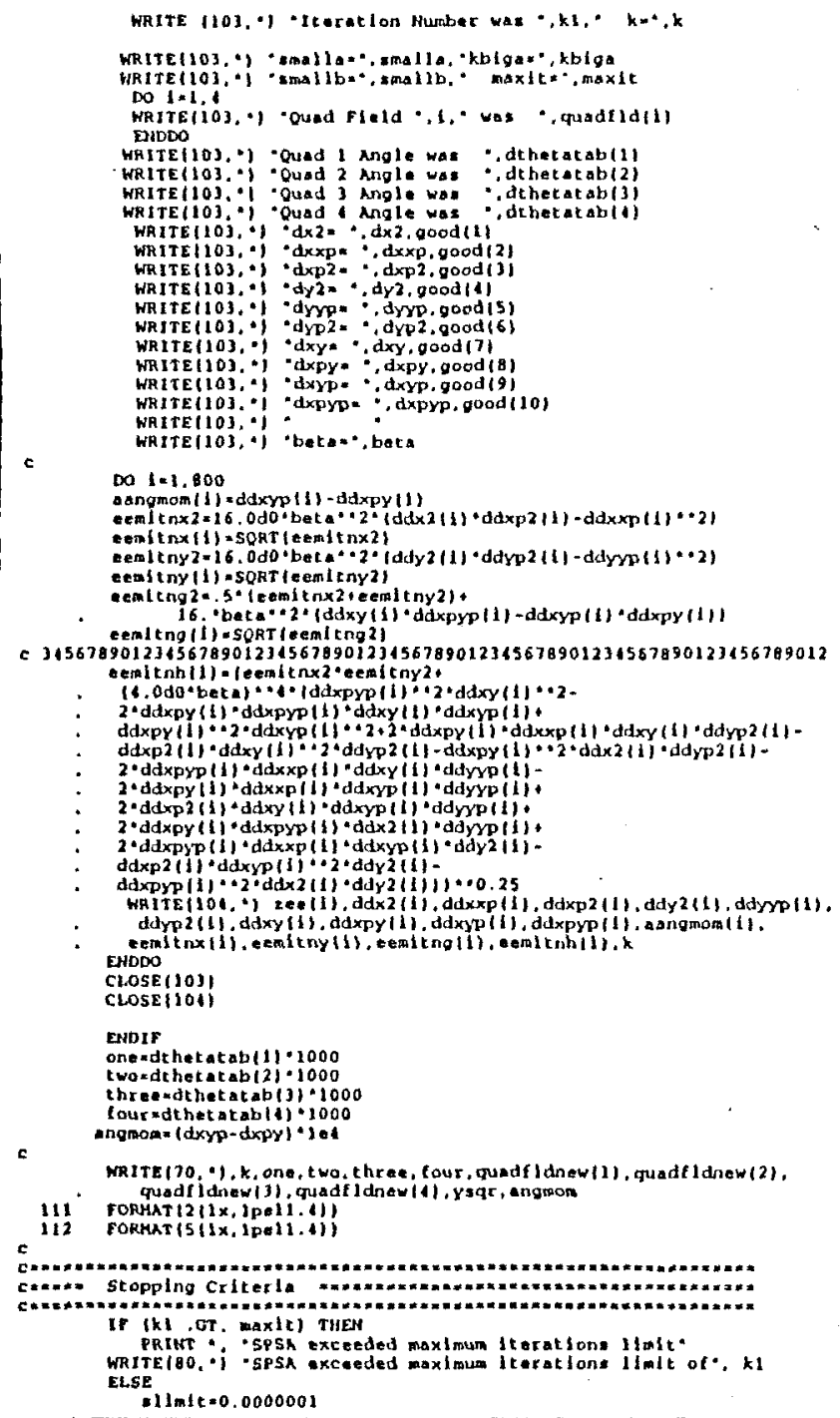 & \\
\hline
\end{tabular}




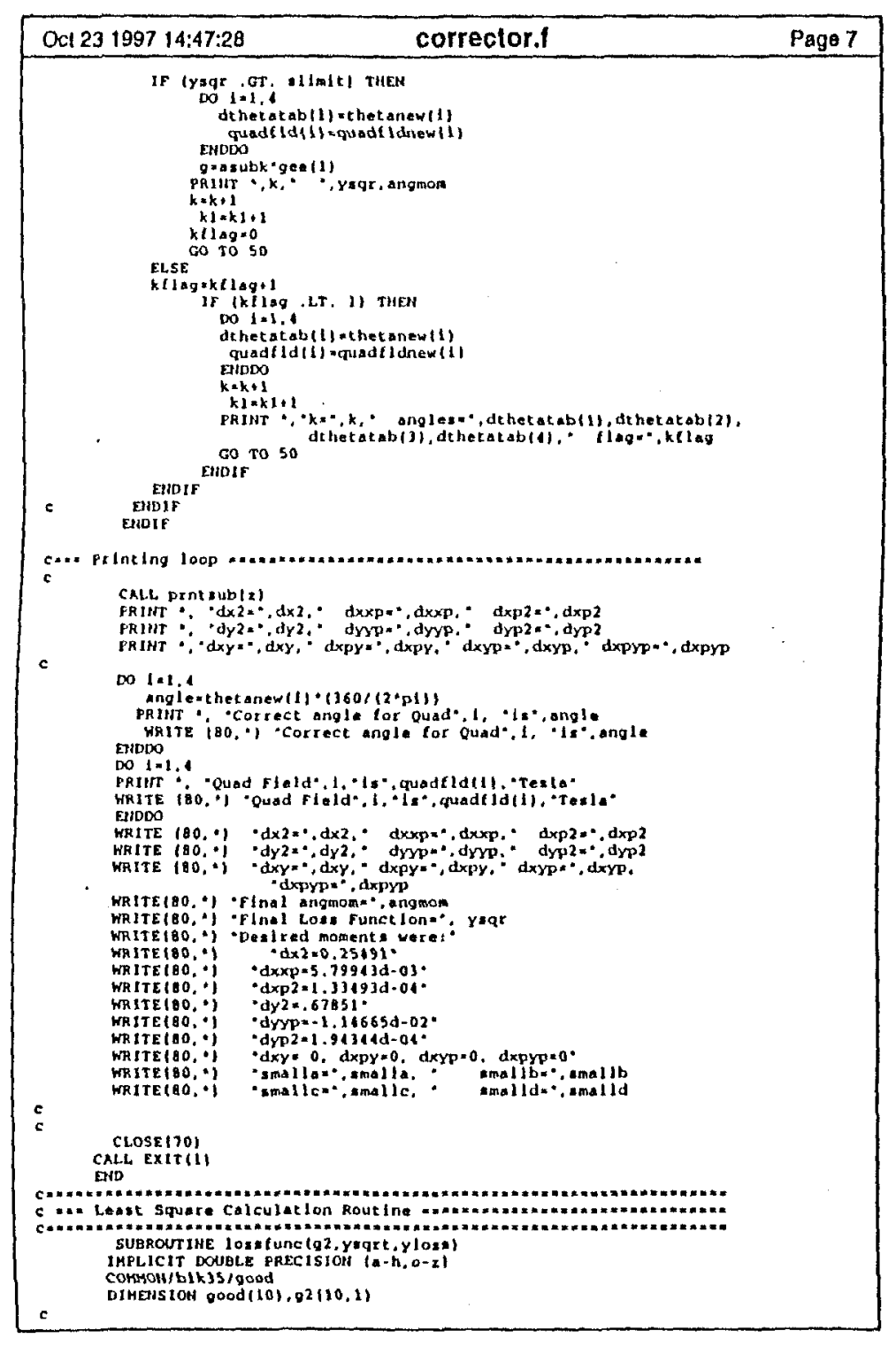

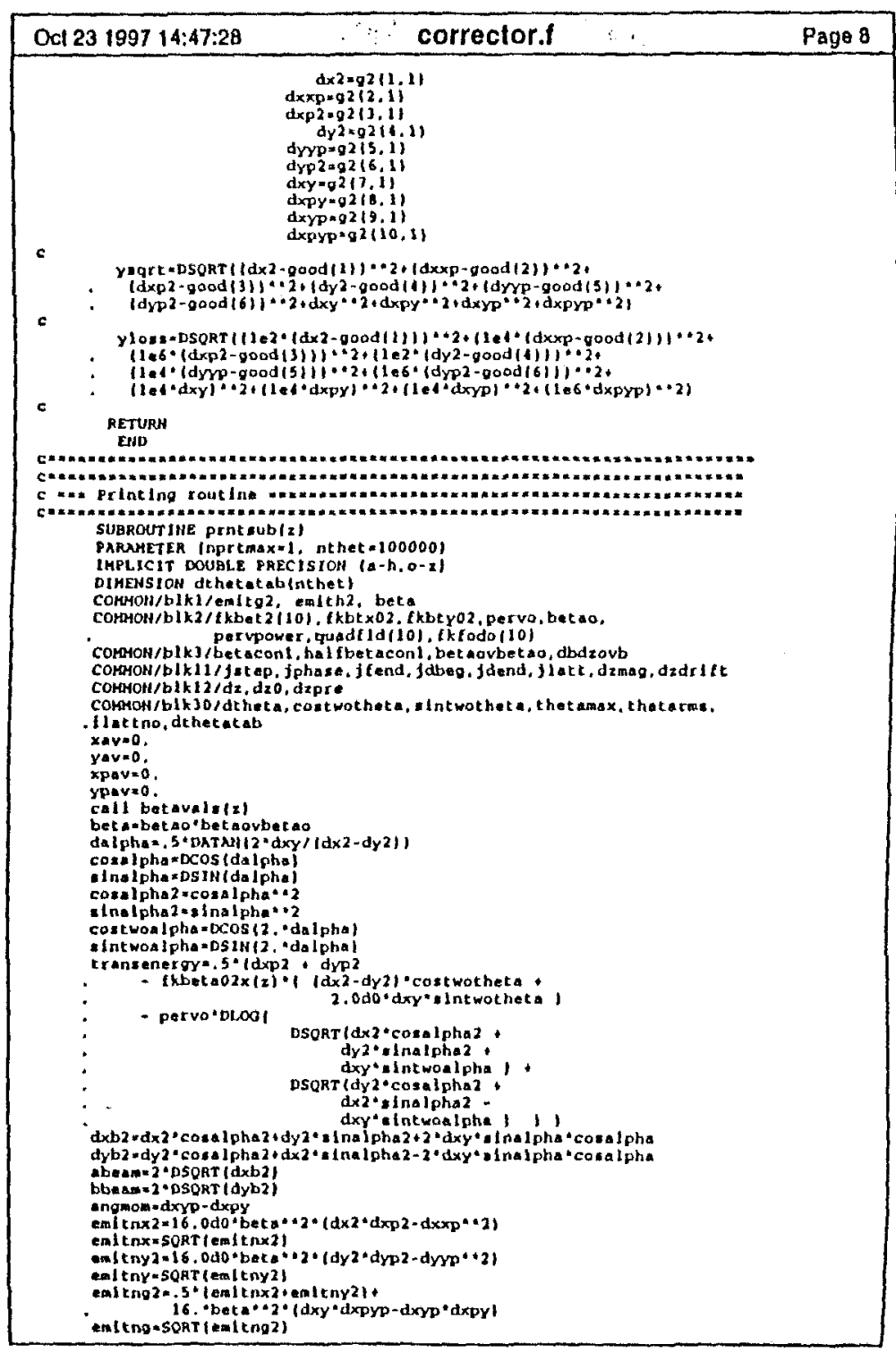




\begin{tabular}{|c|c|}
\hline Oct 23 & 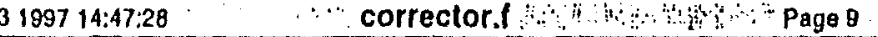 \\
\hline 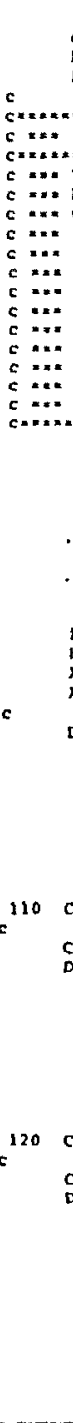 & 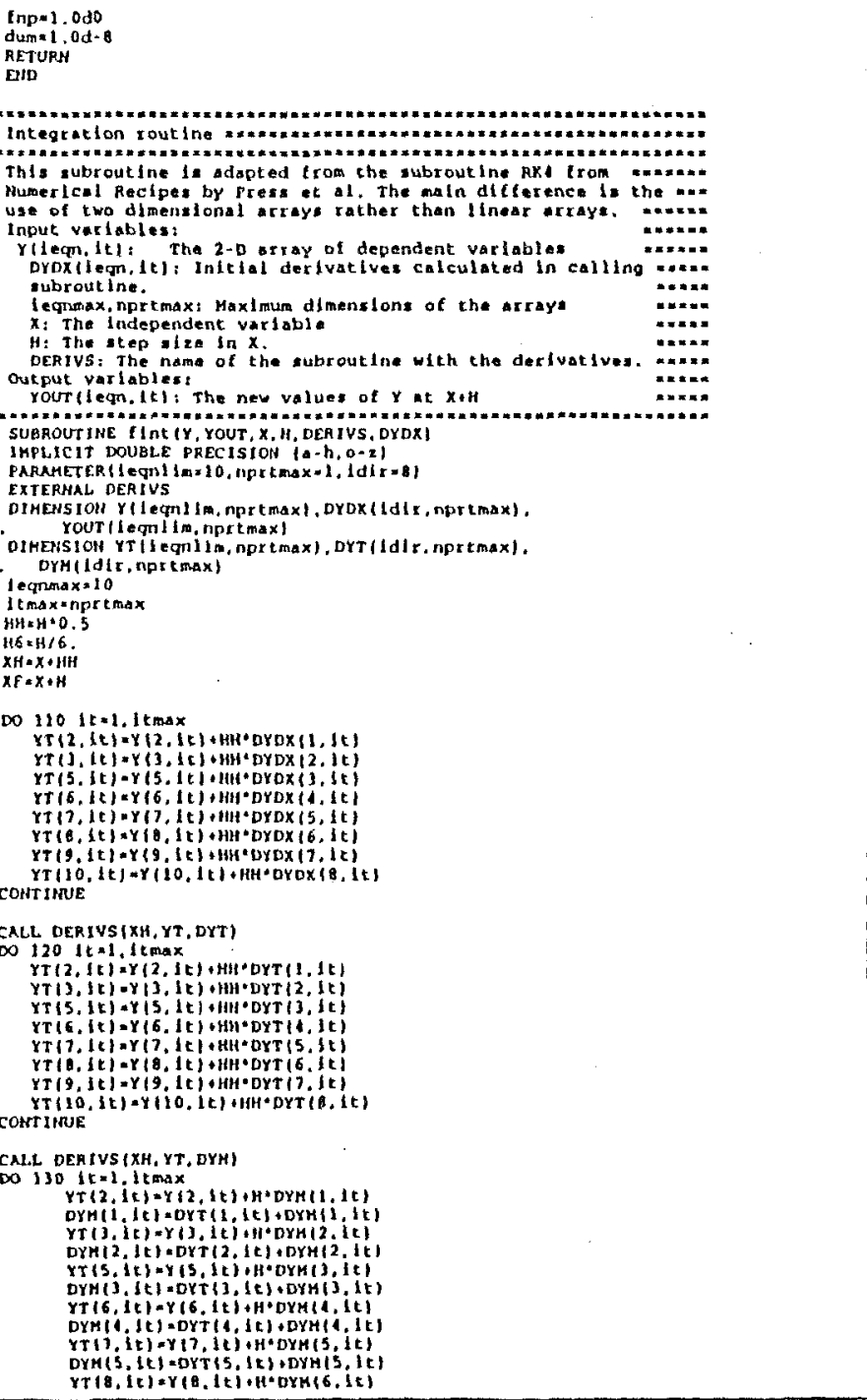 \\
\hline
\end{tabular}

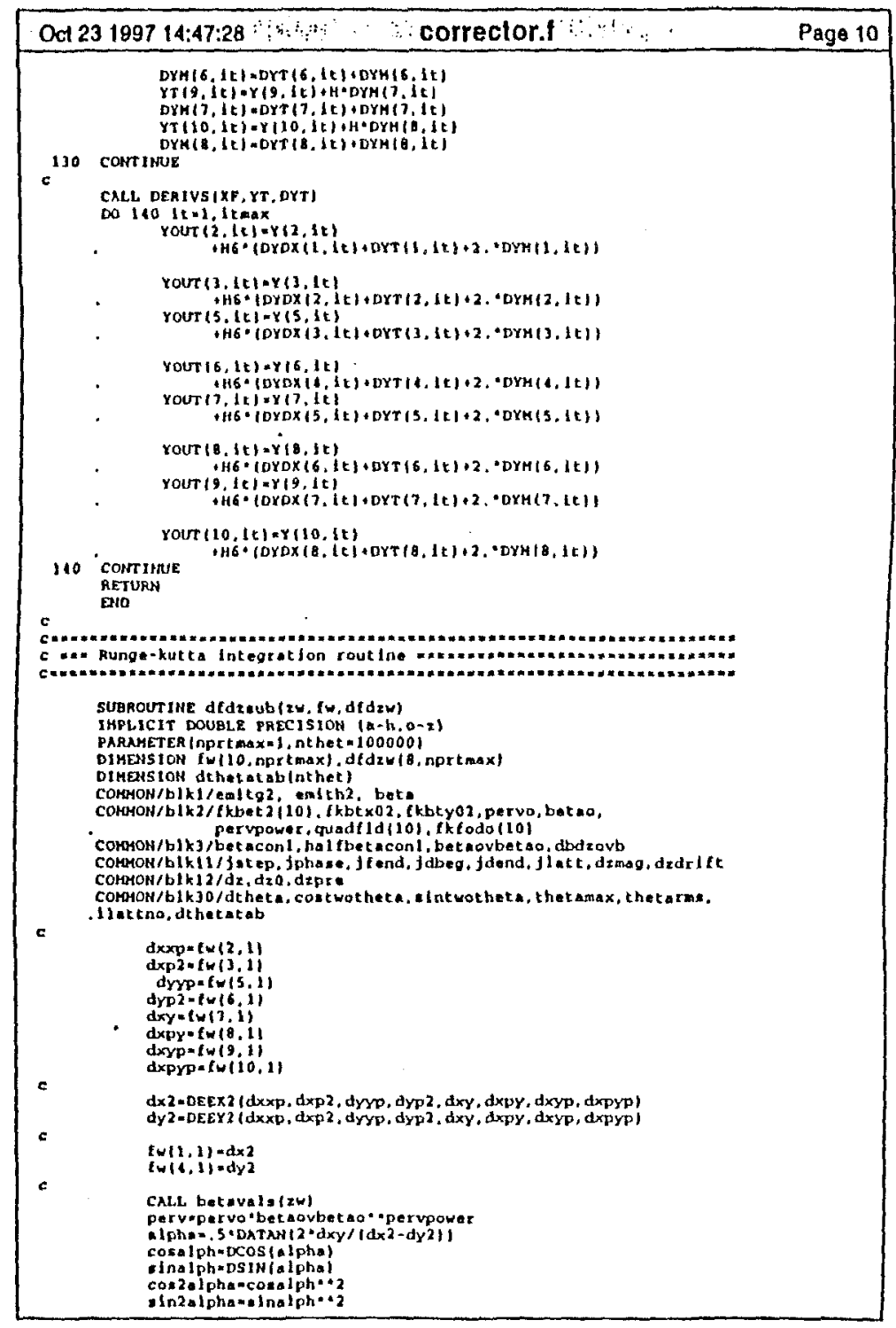




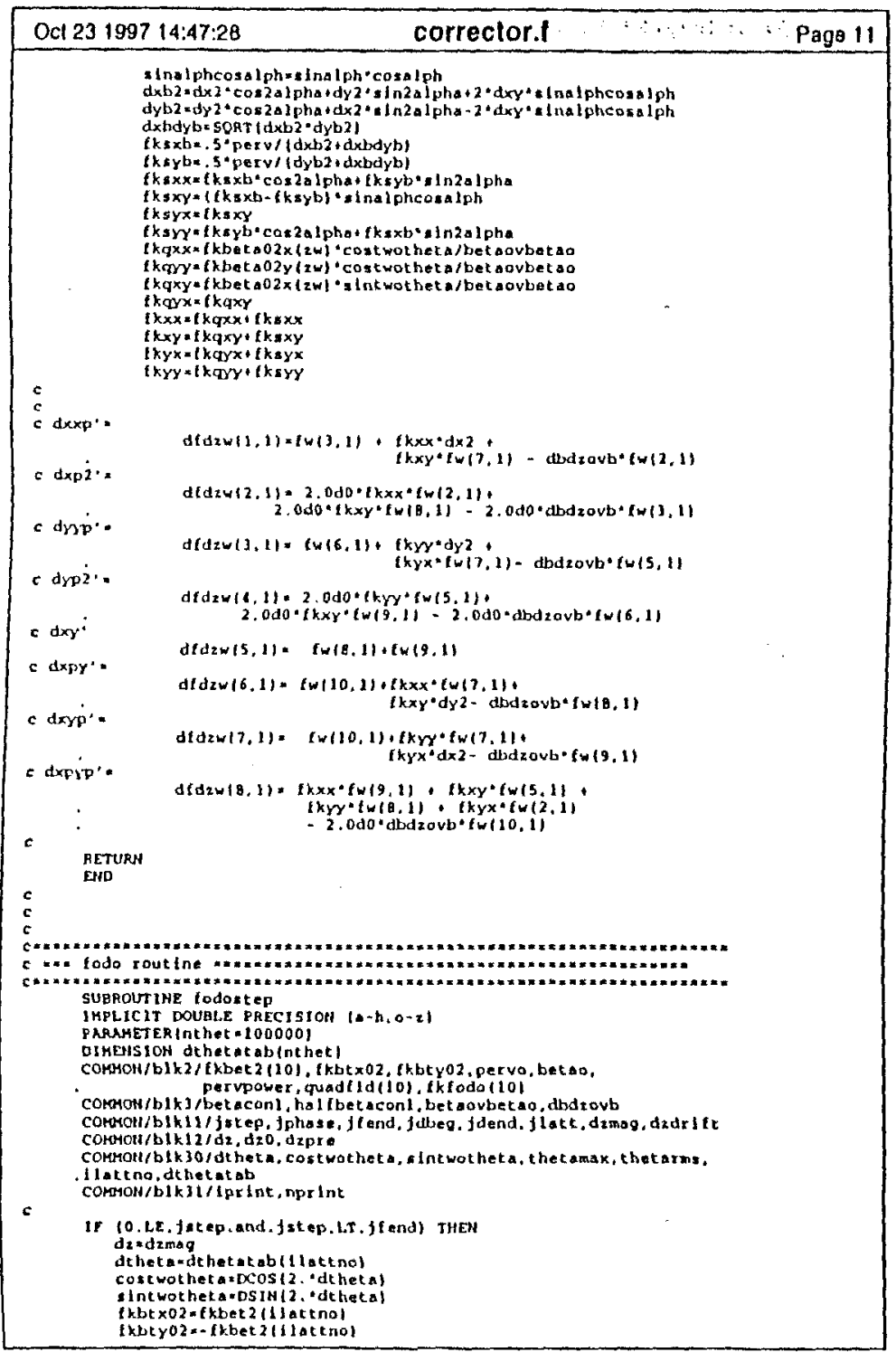

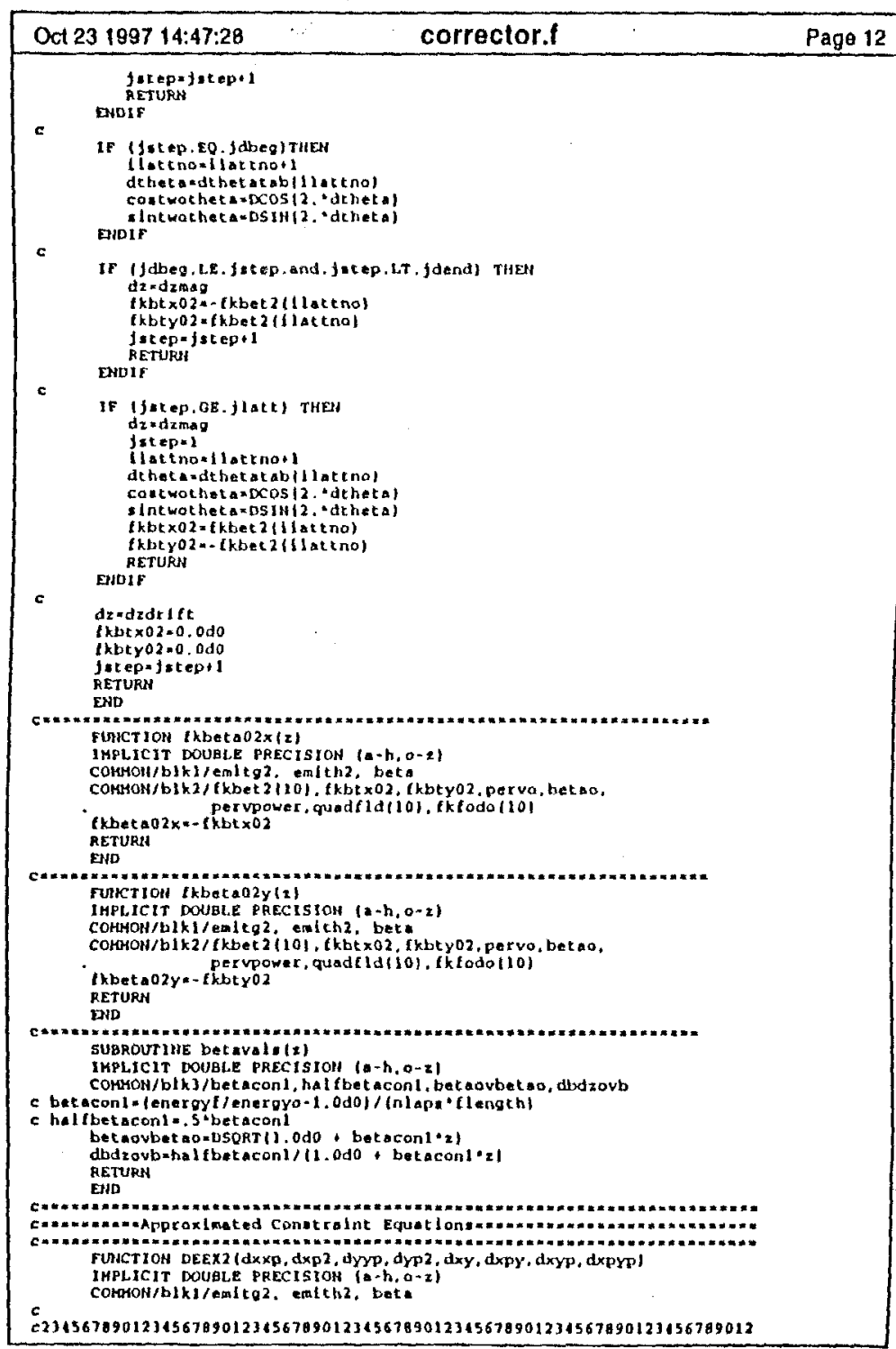




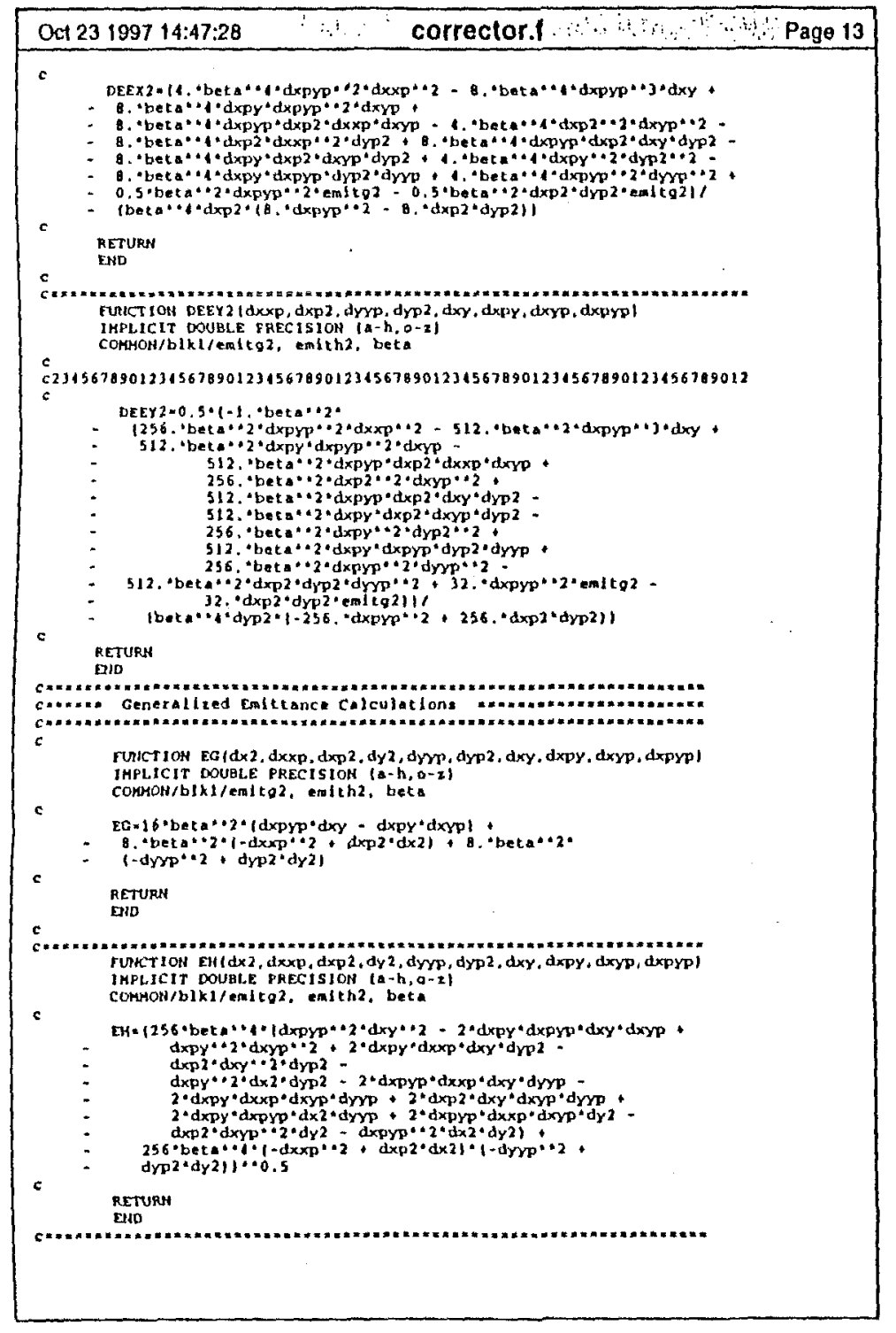




\section{E.4 "PEPPERPOT"}

"PEPPERPOT" is a code written in IDL. It was modified from A. Paul's IDL pepperpot image analysis code used with images of electron beams from the ETAII accelerator. It was modified to analyze ion beams passed through a rectangular pattern pepperpot mask more suitable to the analysis of a beam produced by an alternating gradient focusing lattice. A modification was made to calculate the transverse cross-moments and display these correlations. The ability to fit a gaussian curve to beamlet spot cross-sections was also added. 


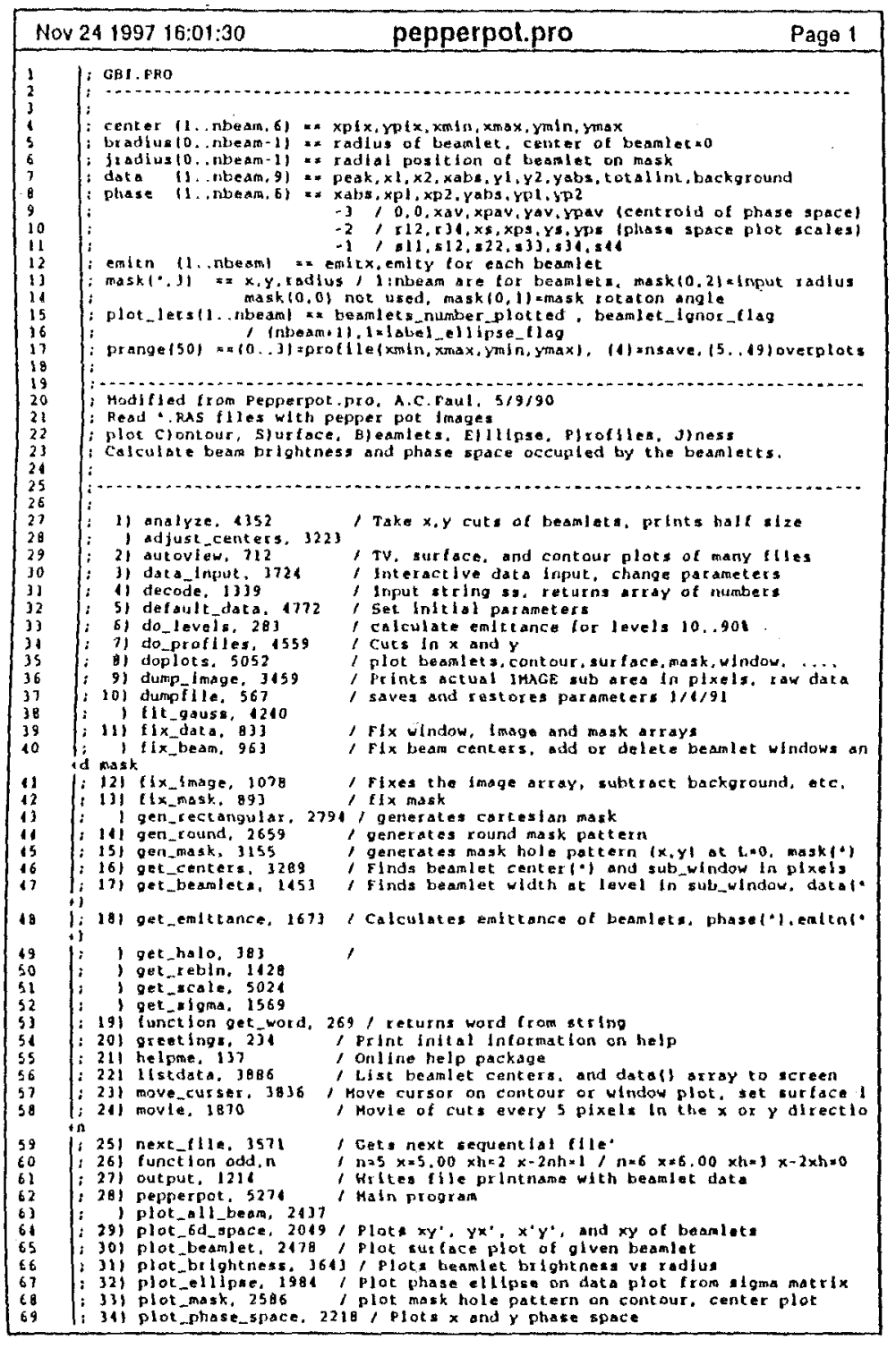

\begin{tabular}{|c|c|c|}
\hline \multicolumn{2}{|c|}{ Nov 241997 16:01:30 } & pepperpot.pro \\
\hline 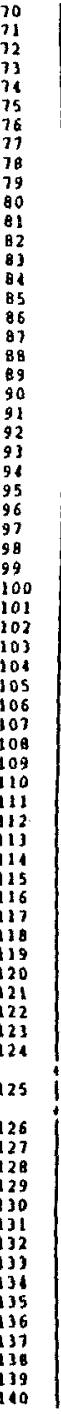 & 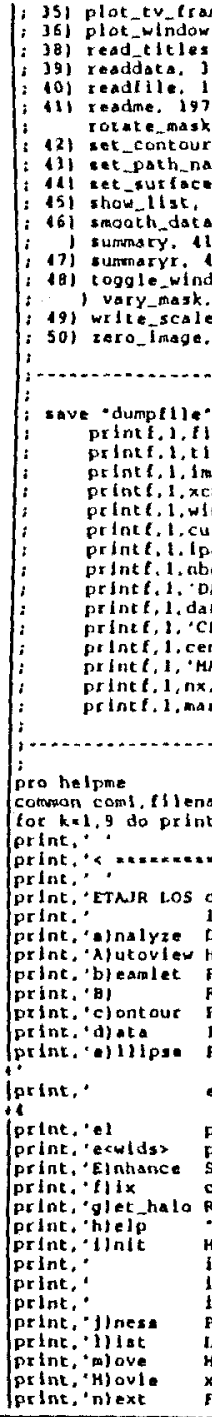 & 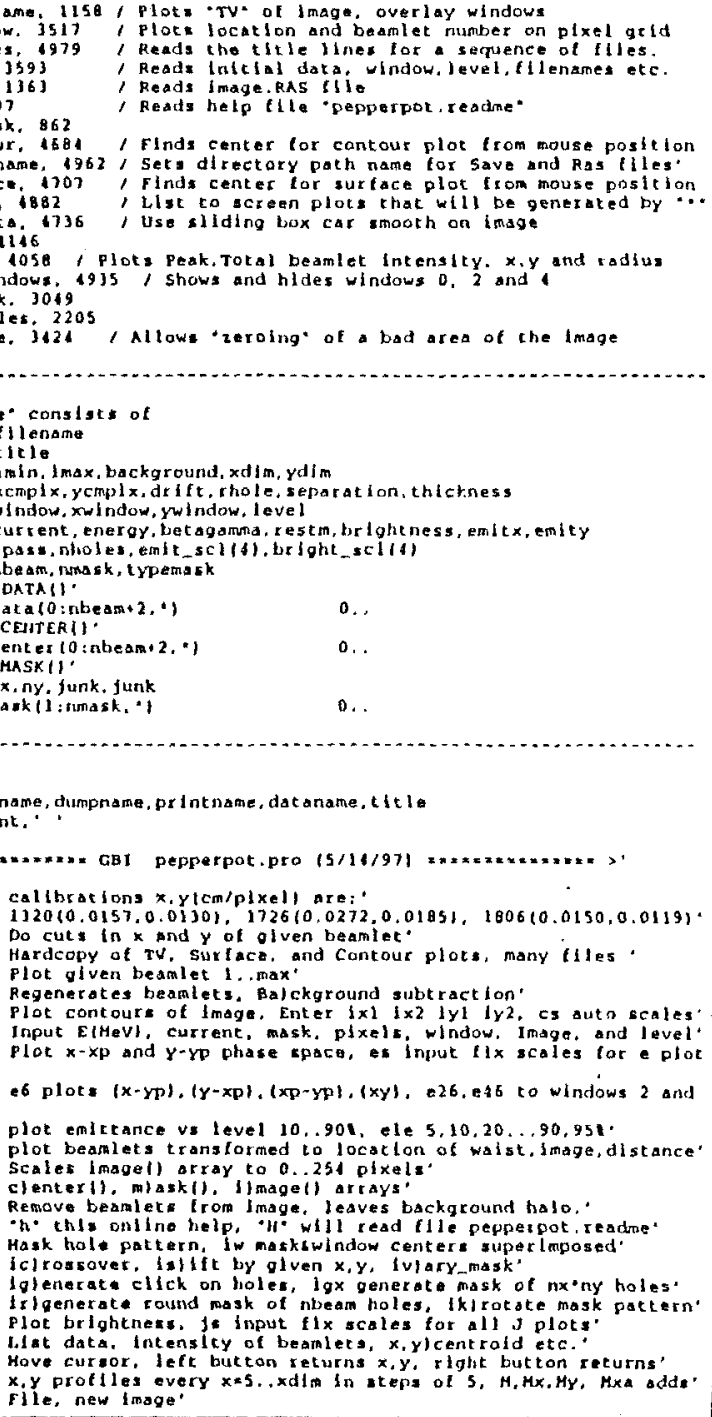 \\
\hline
\end{tabular}



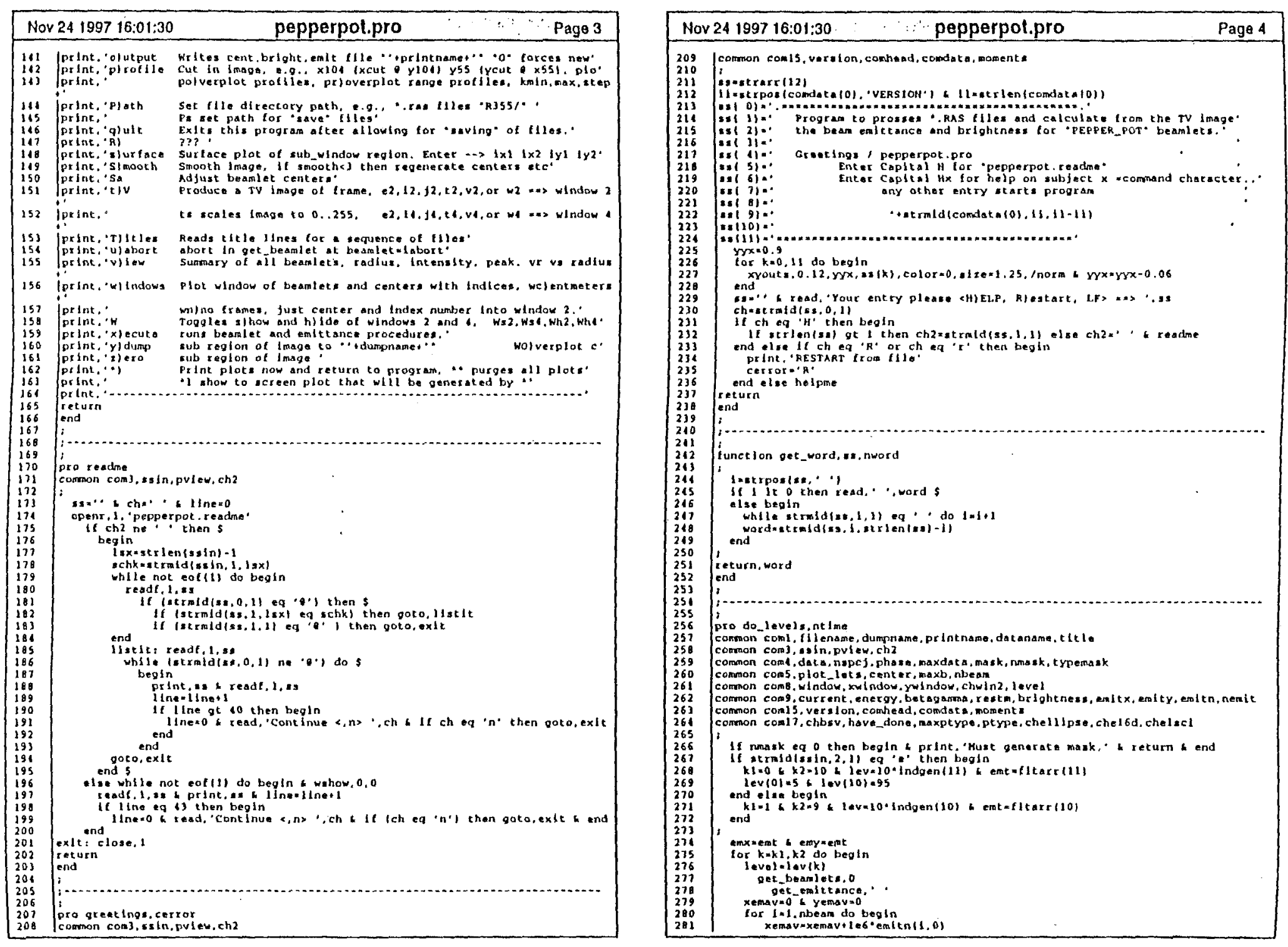

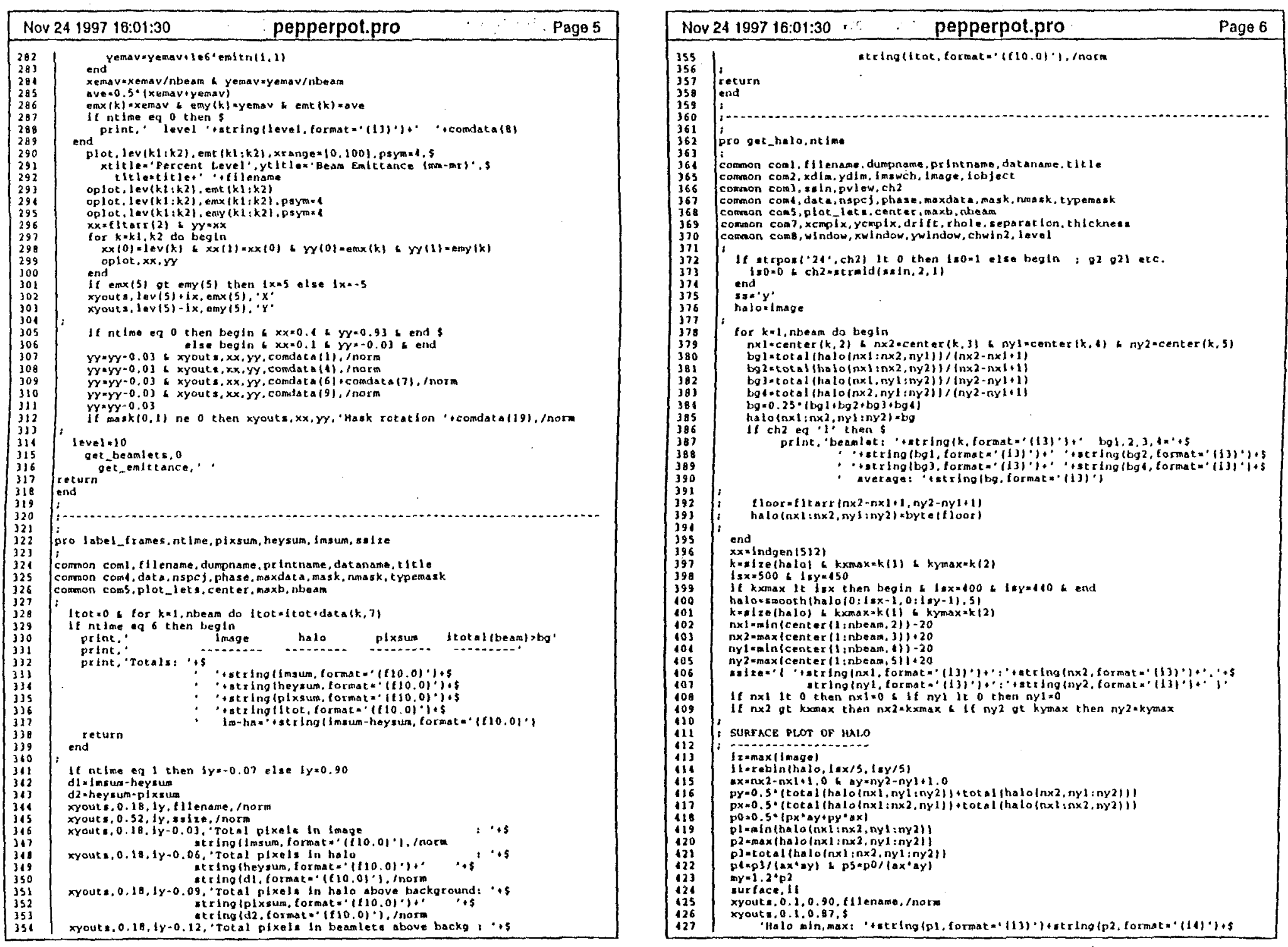

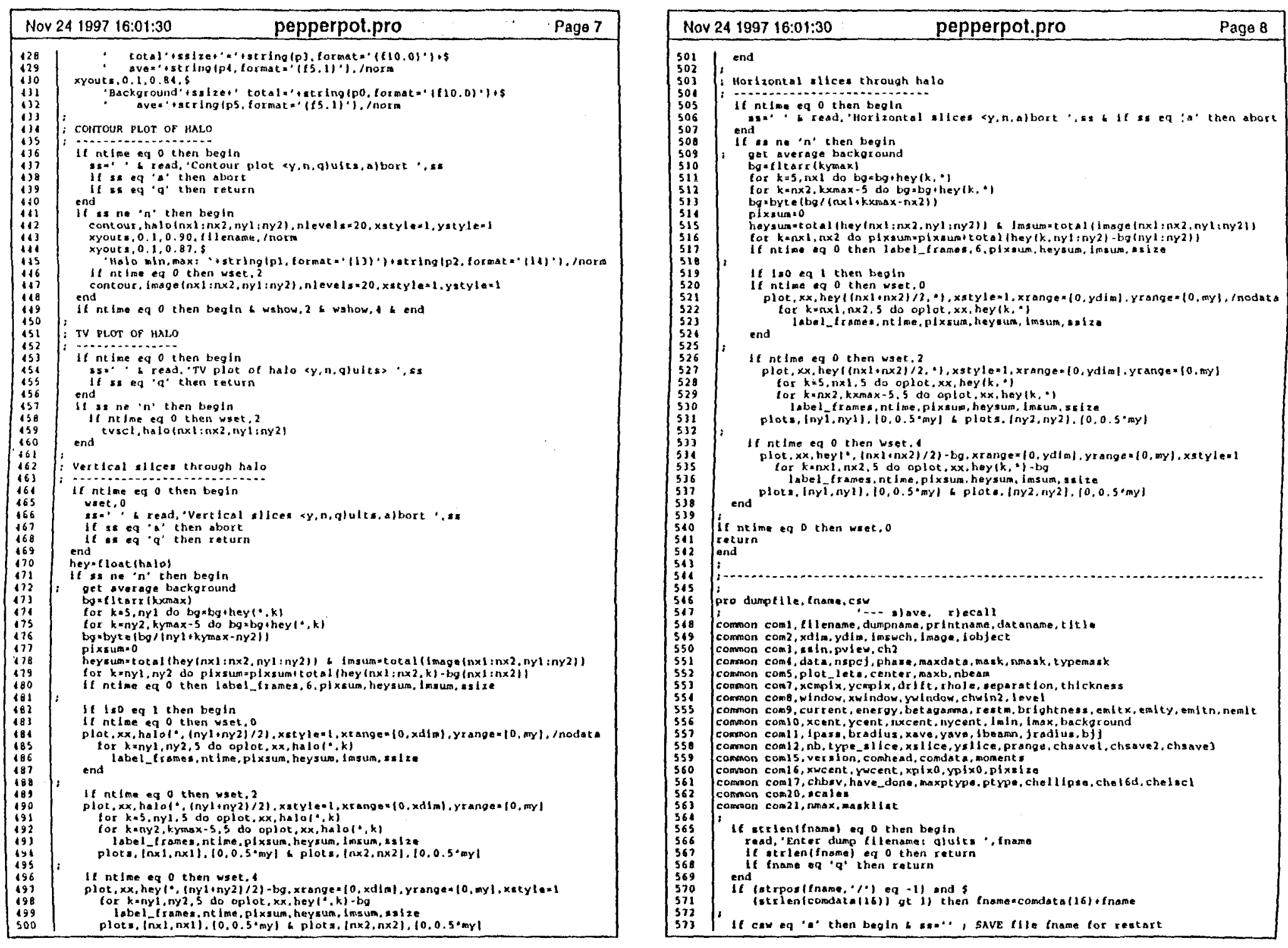

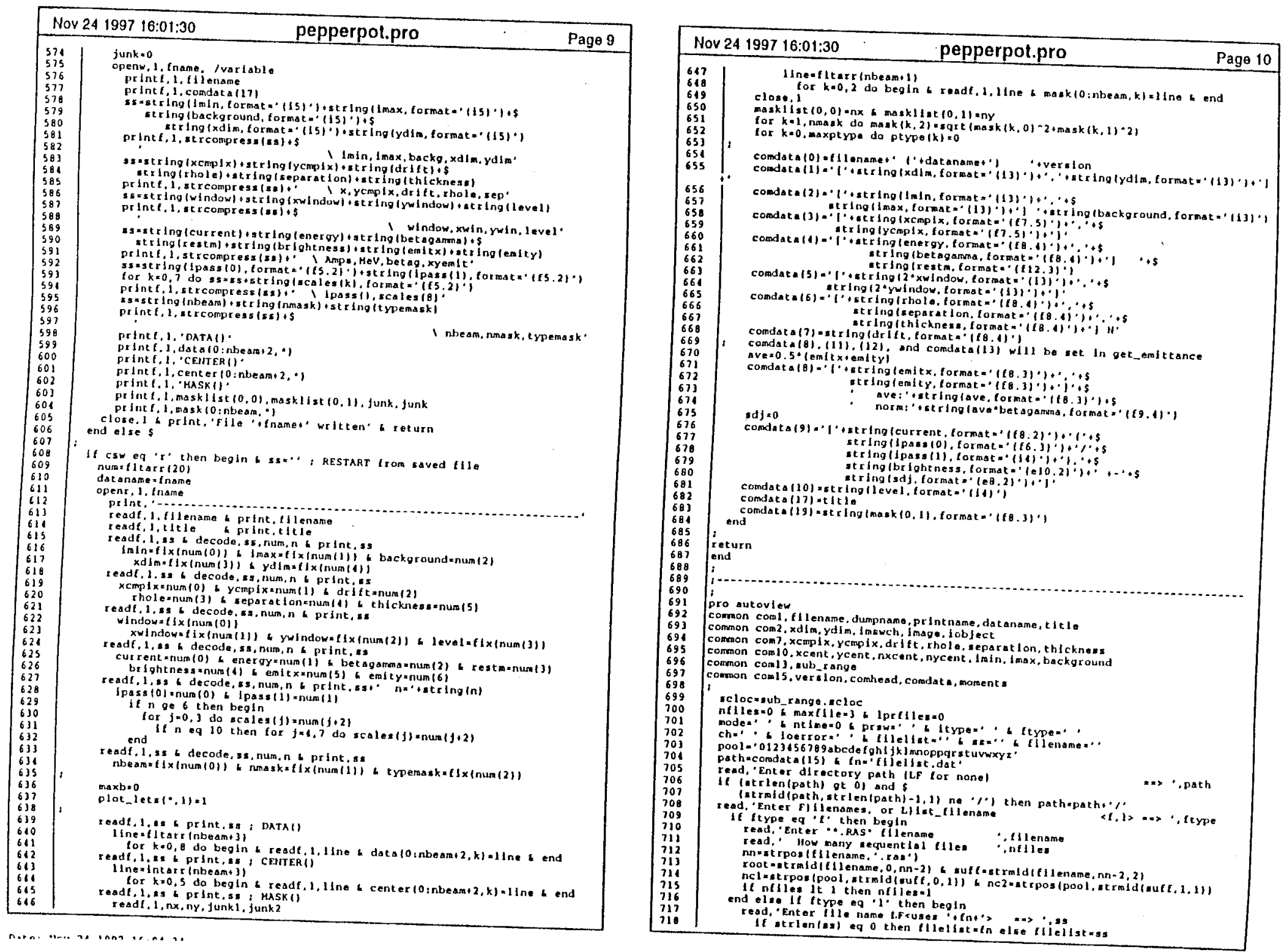


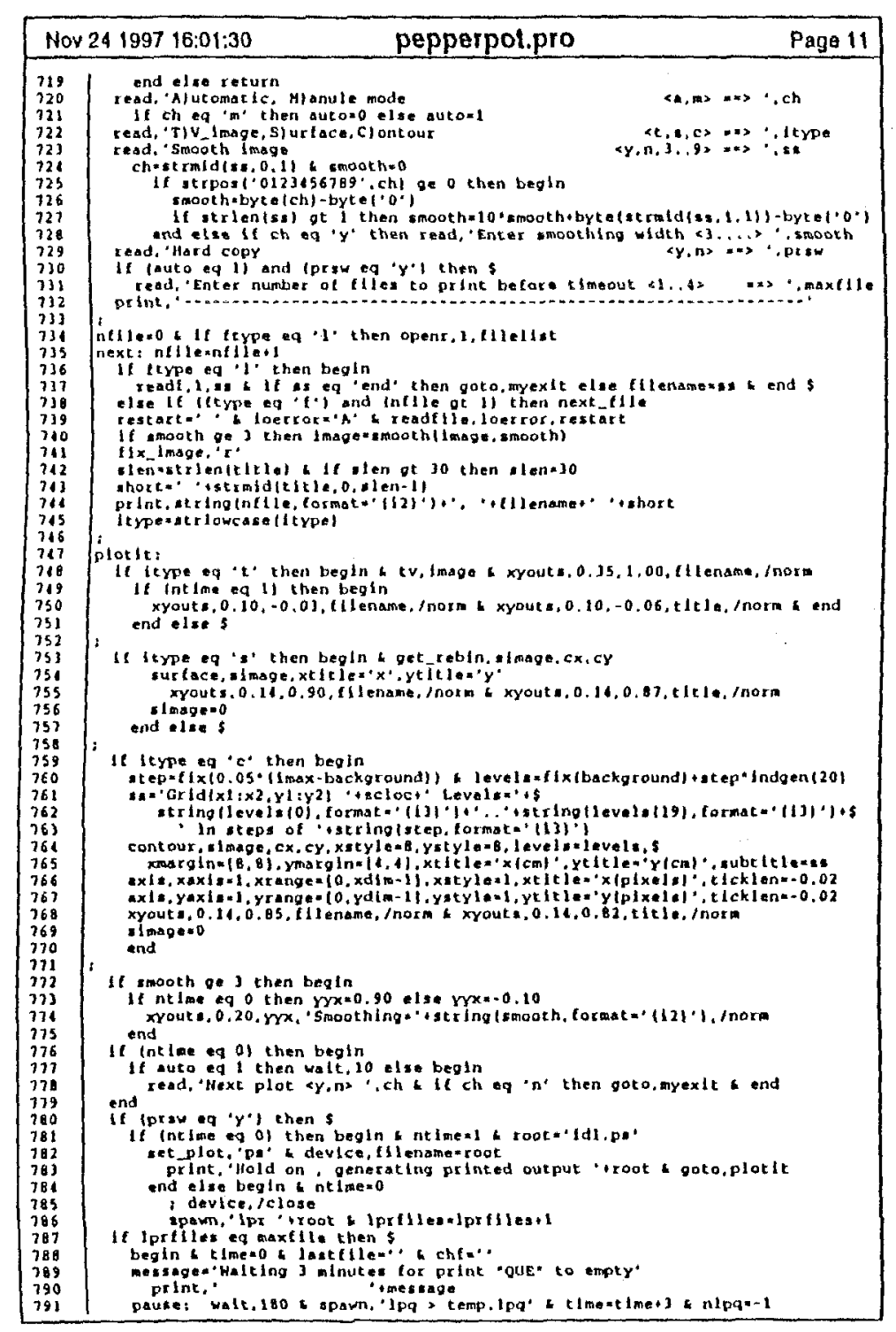

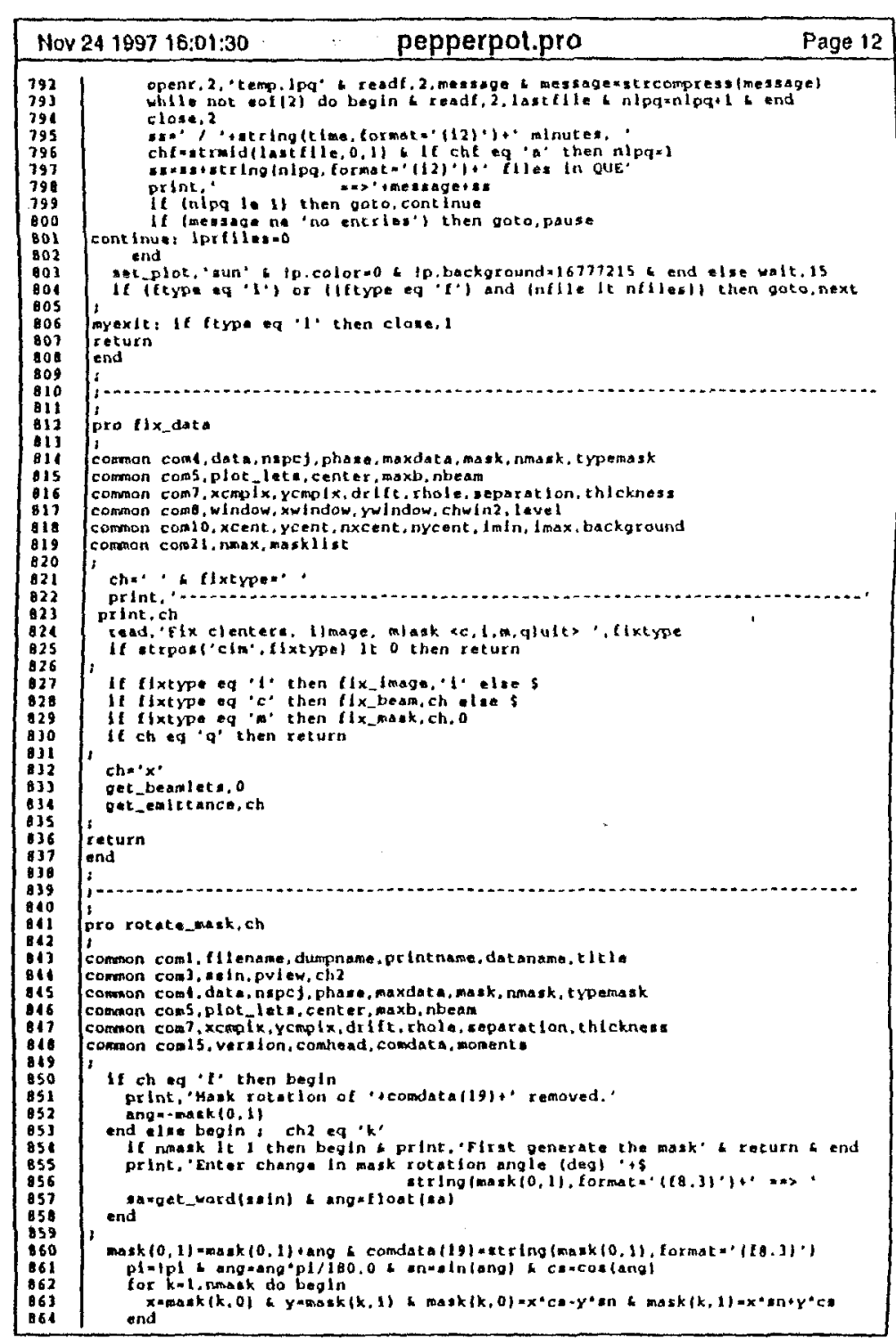




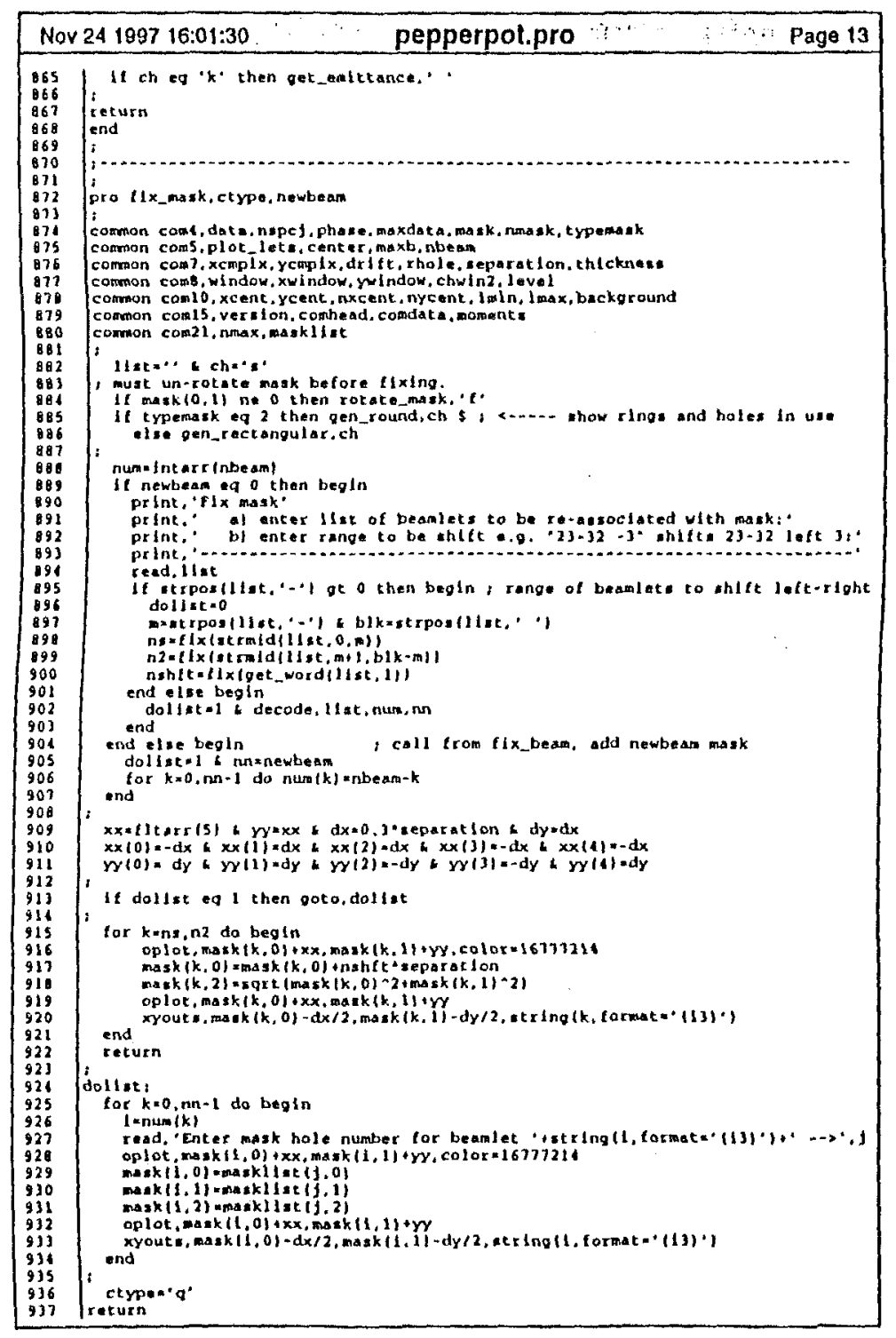

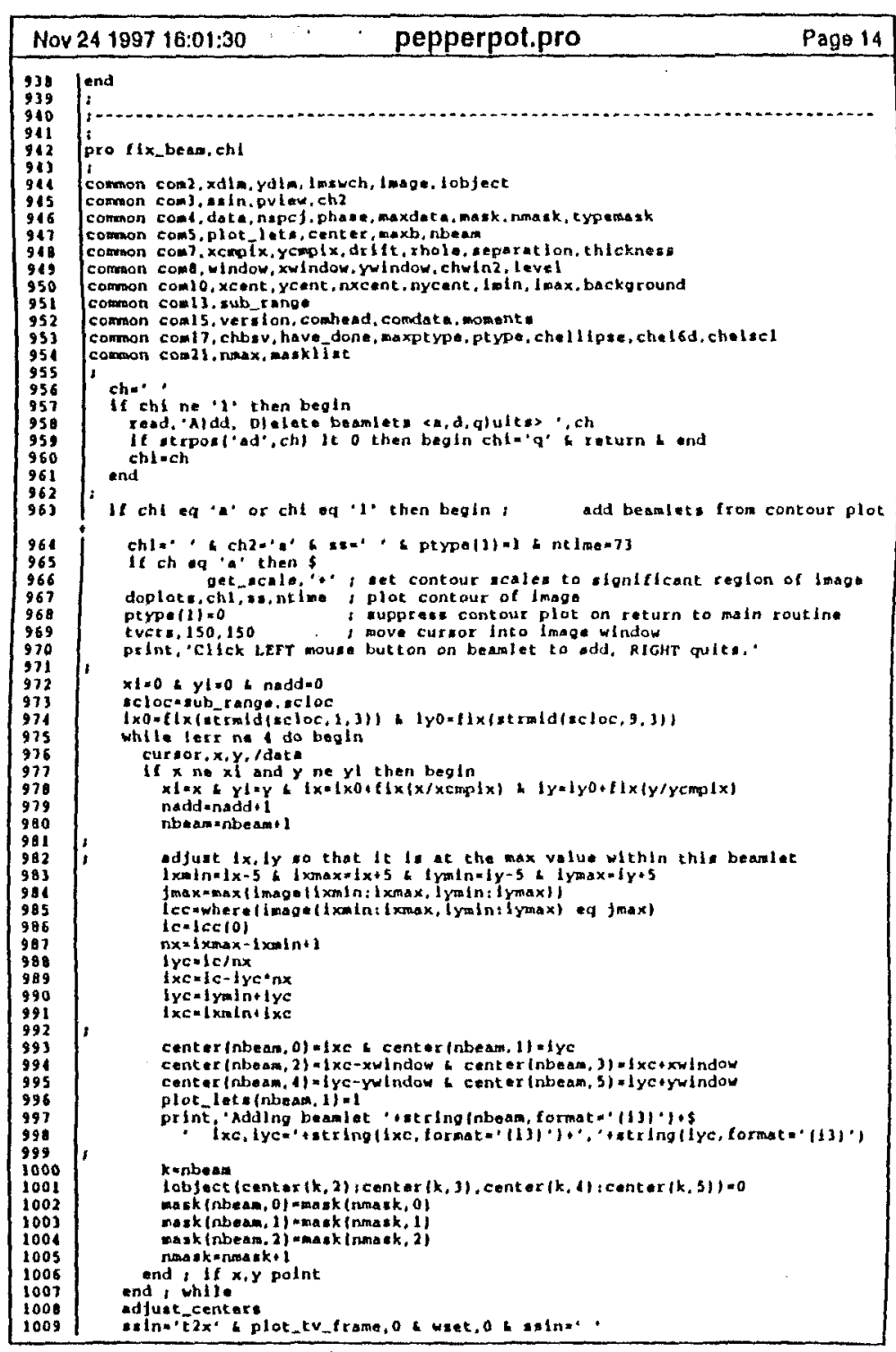



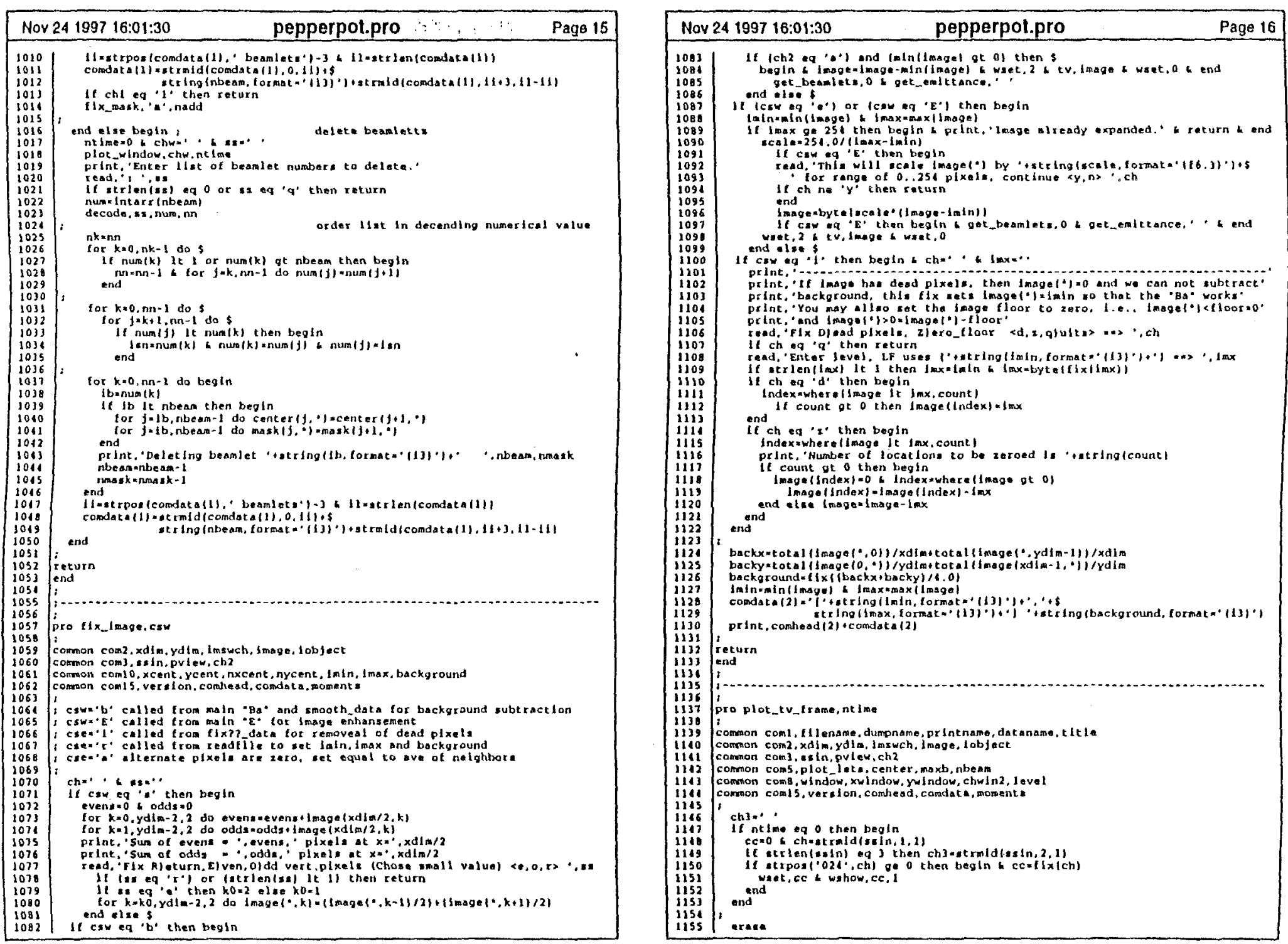

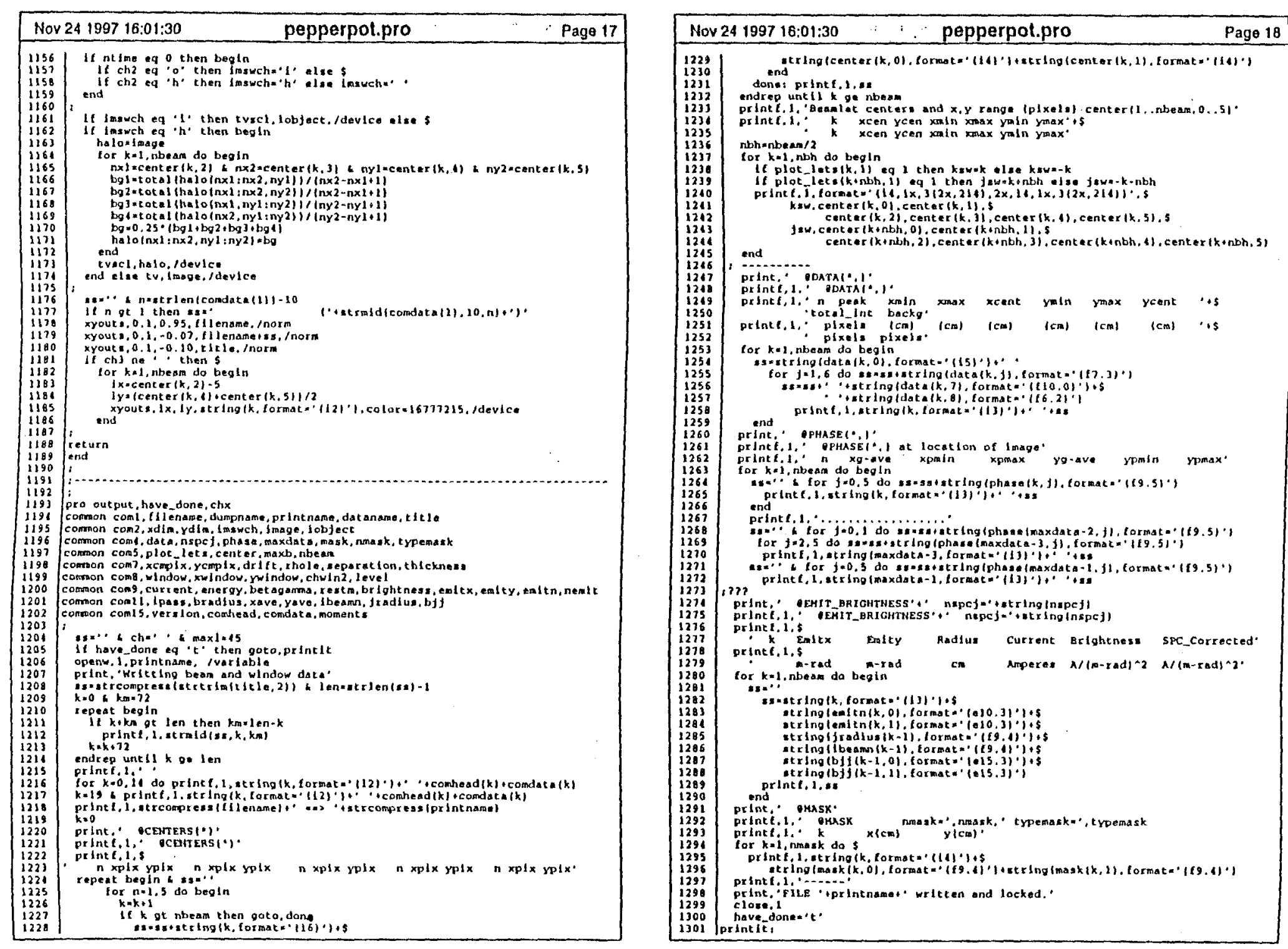

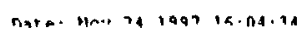




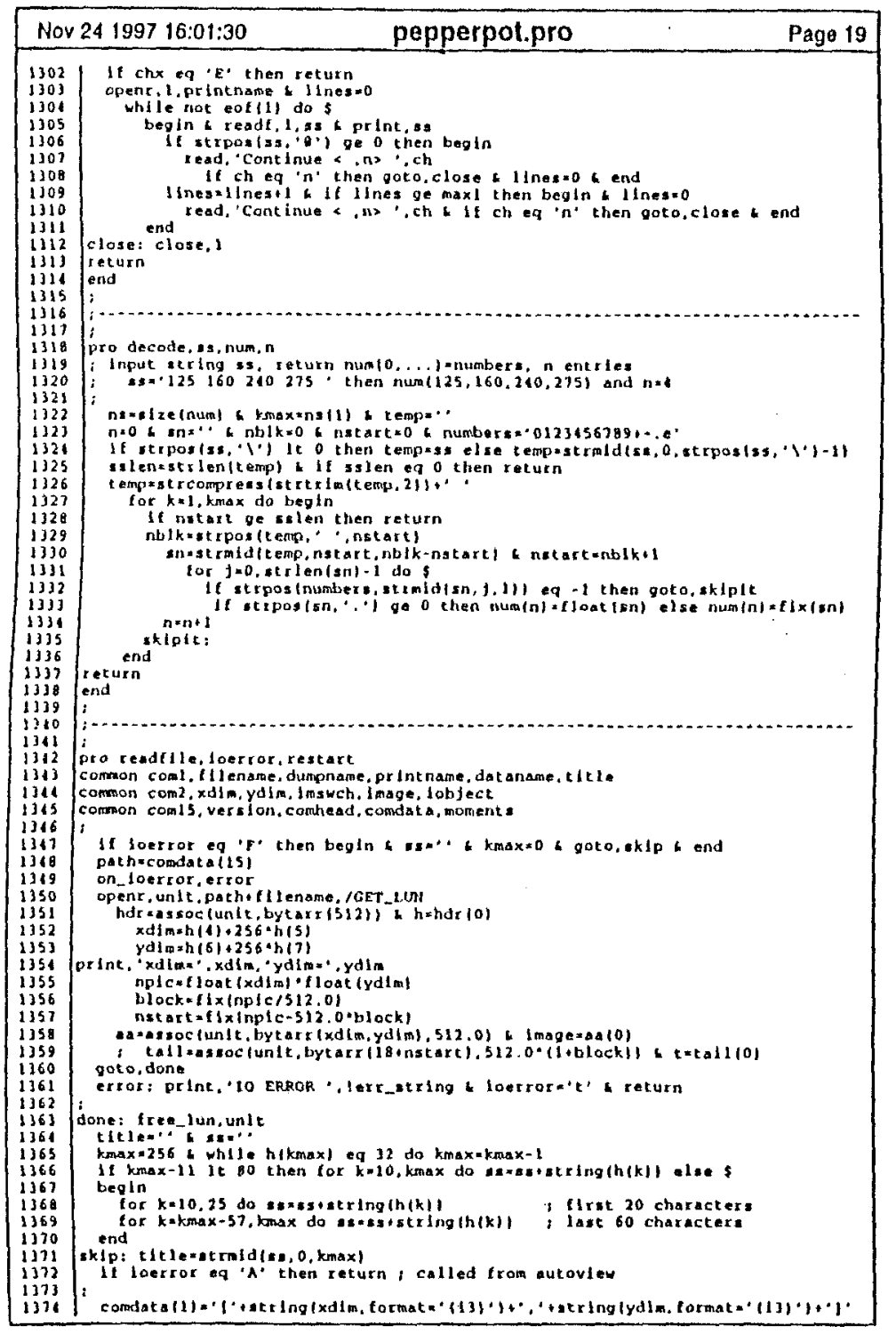

\begin{tabular}{|c|c|}
\hline Nov 2 & pepperpot.pro \\
\hline 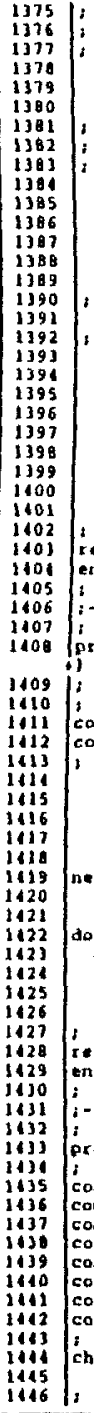 & 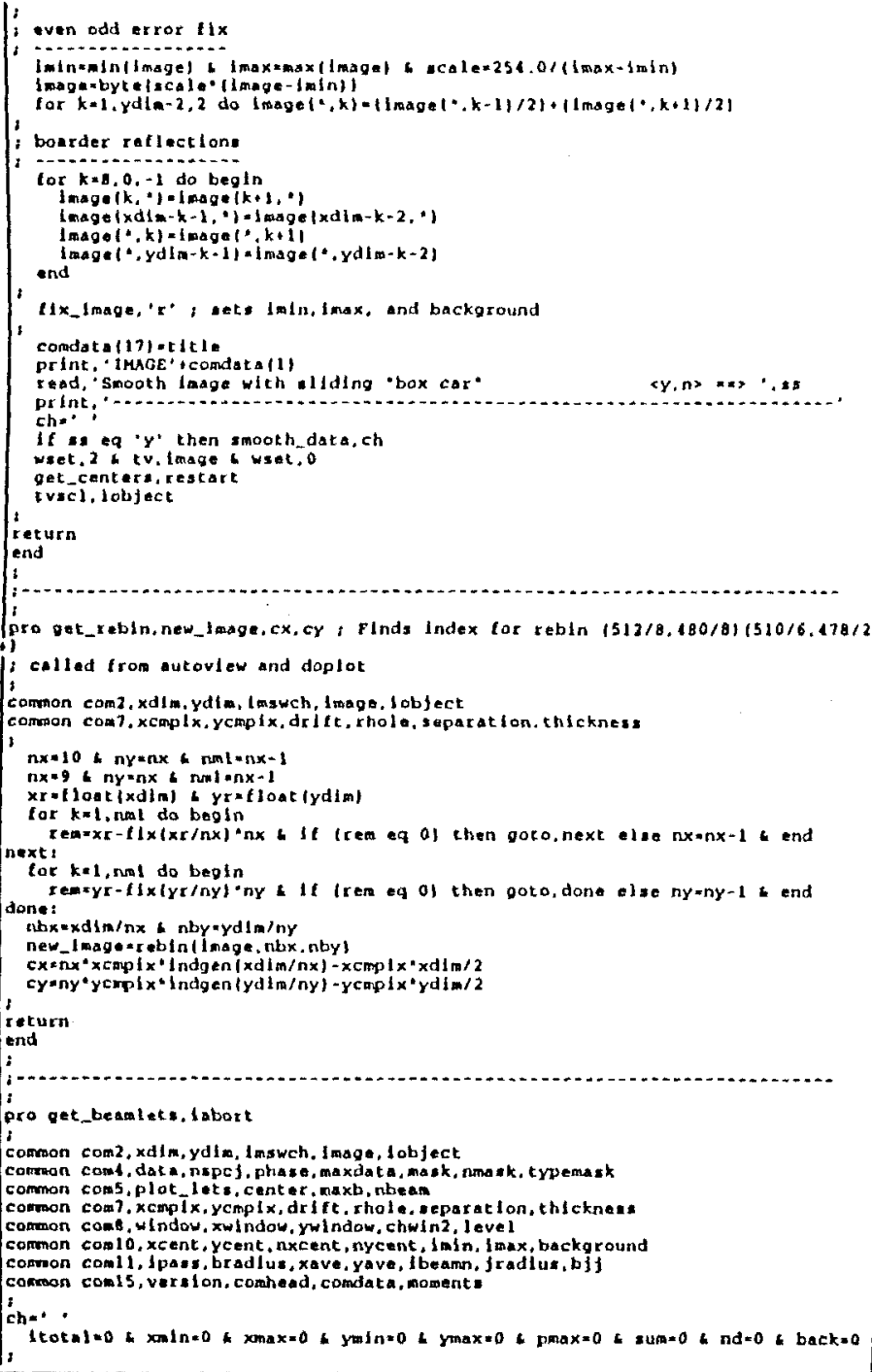 \\
\hline
\end{tabular}




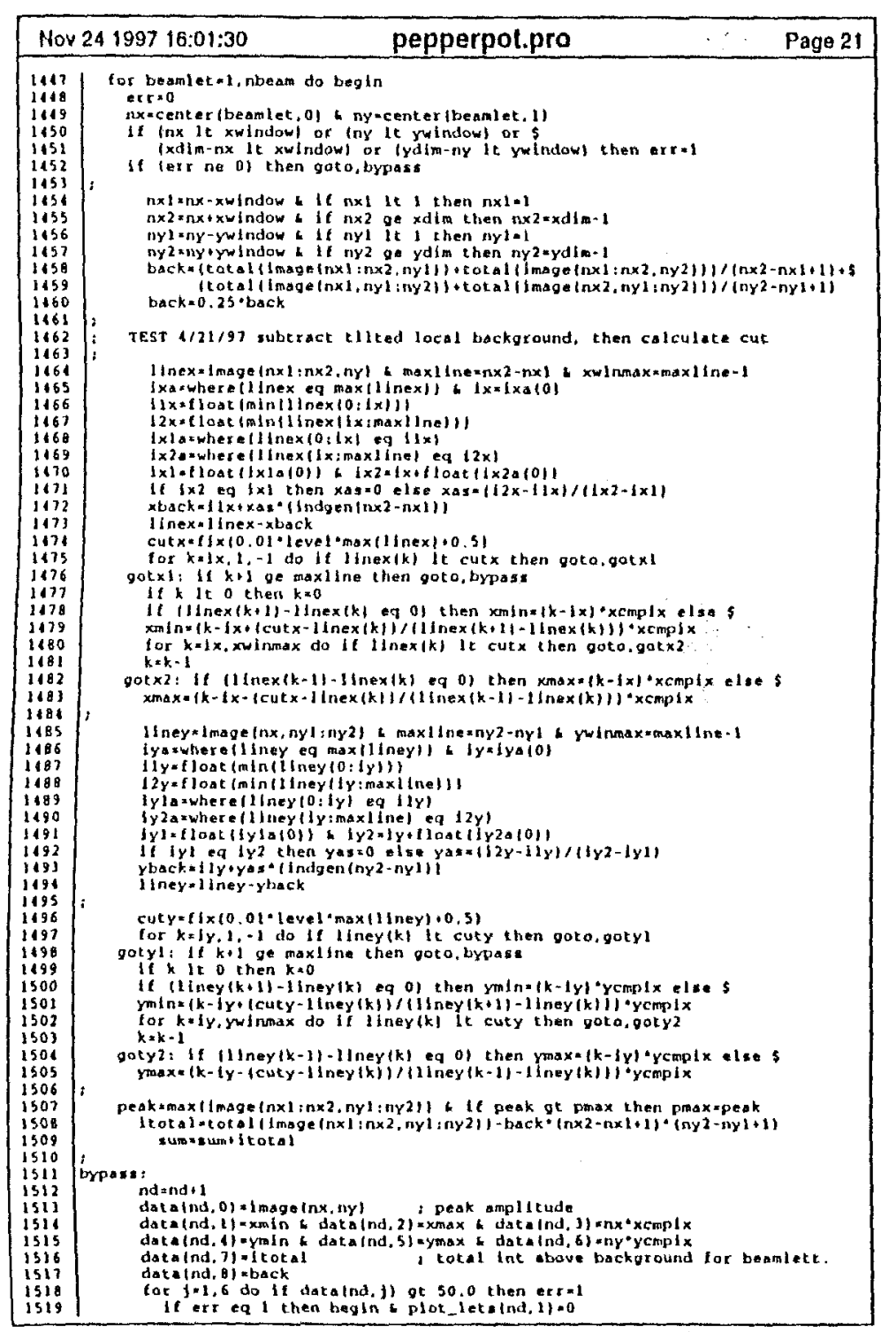

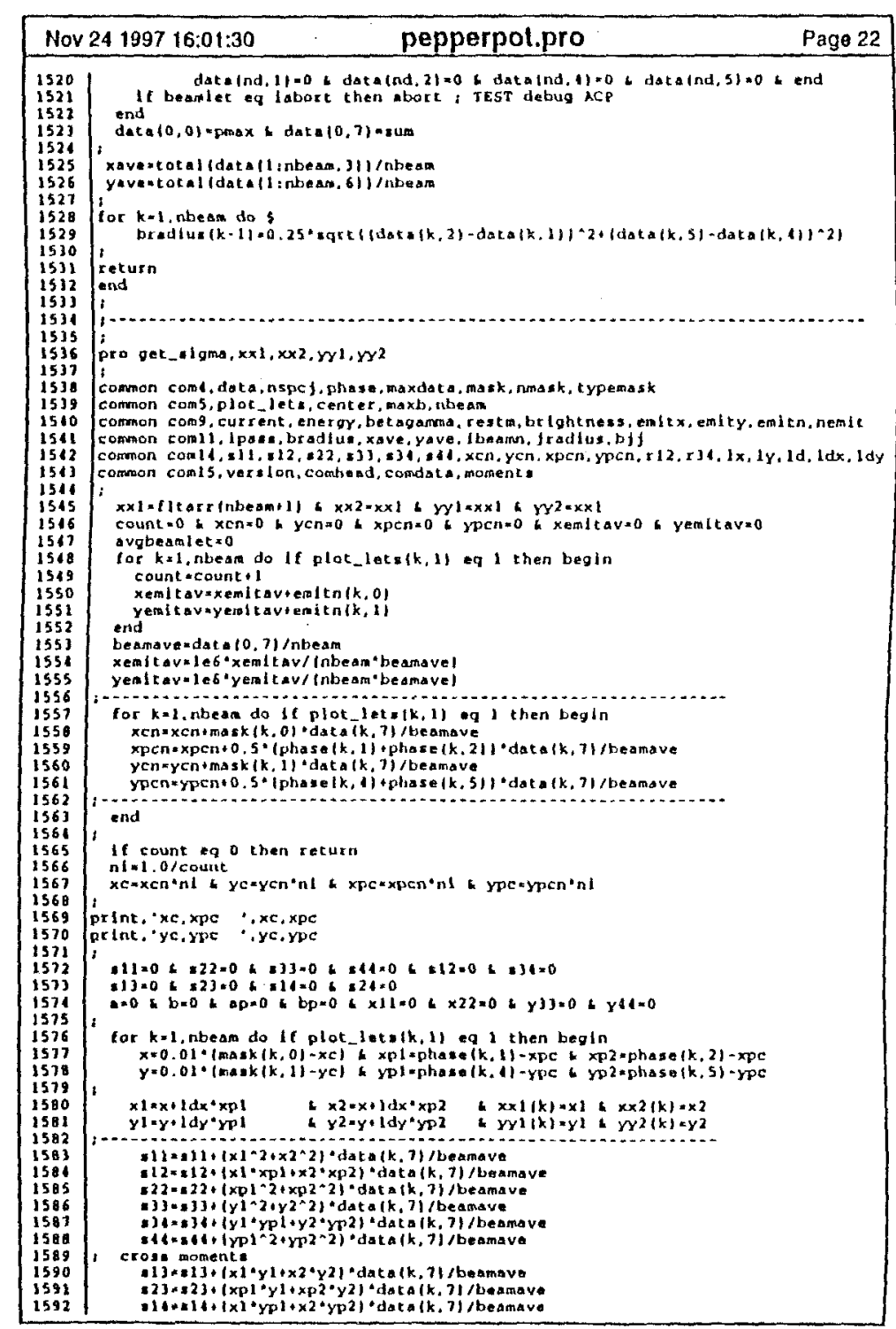



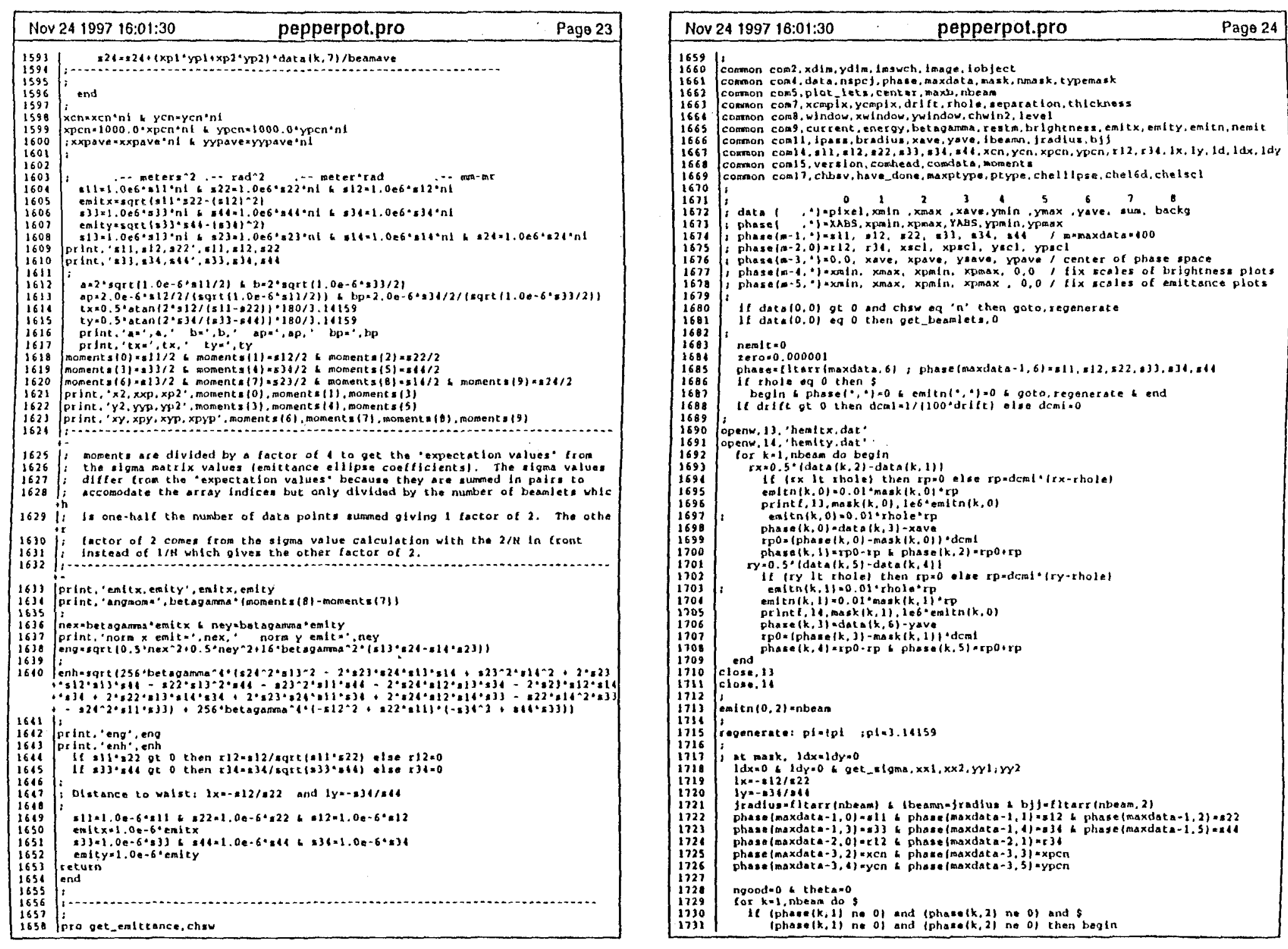


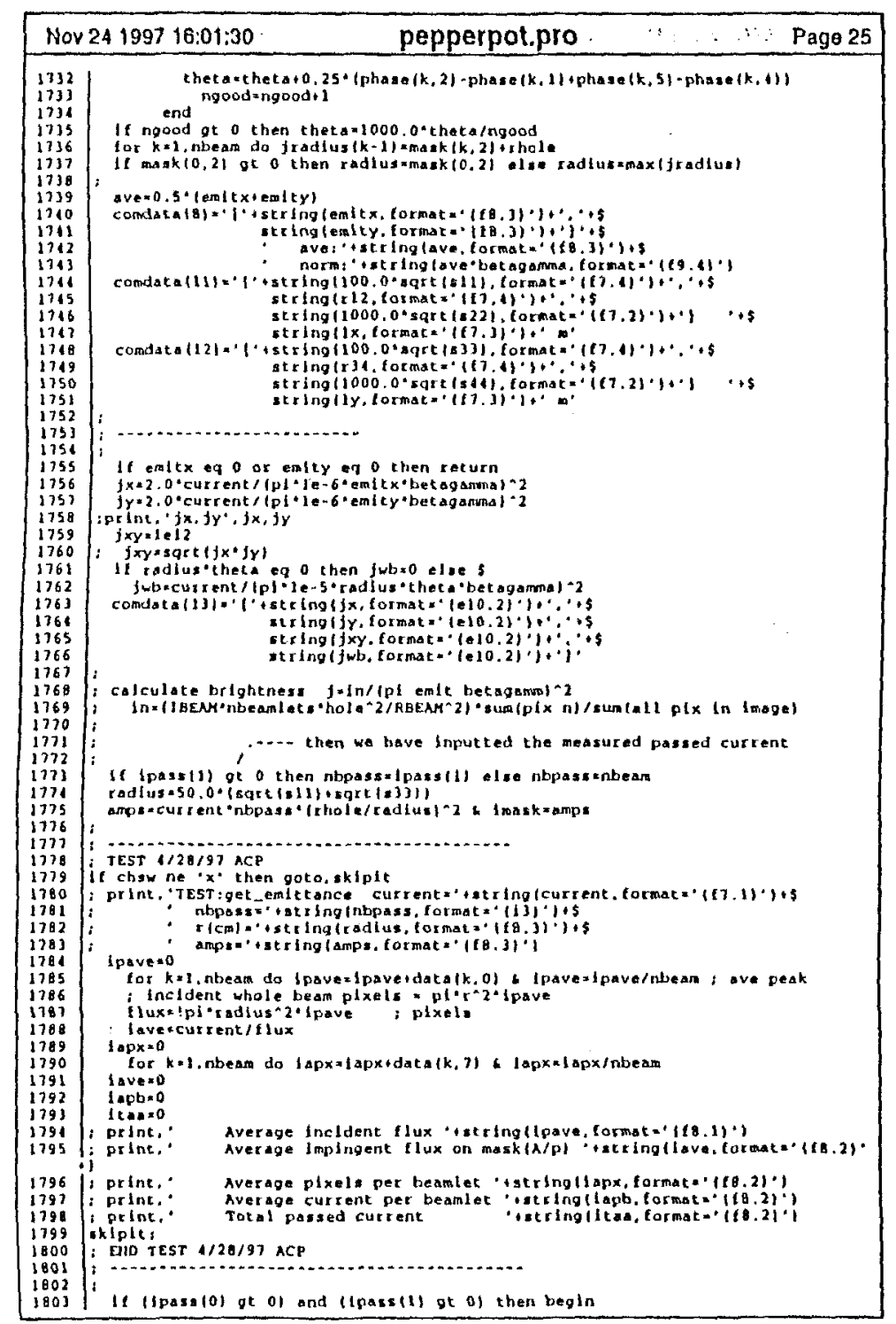

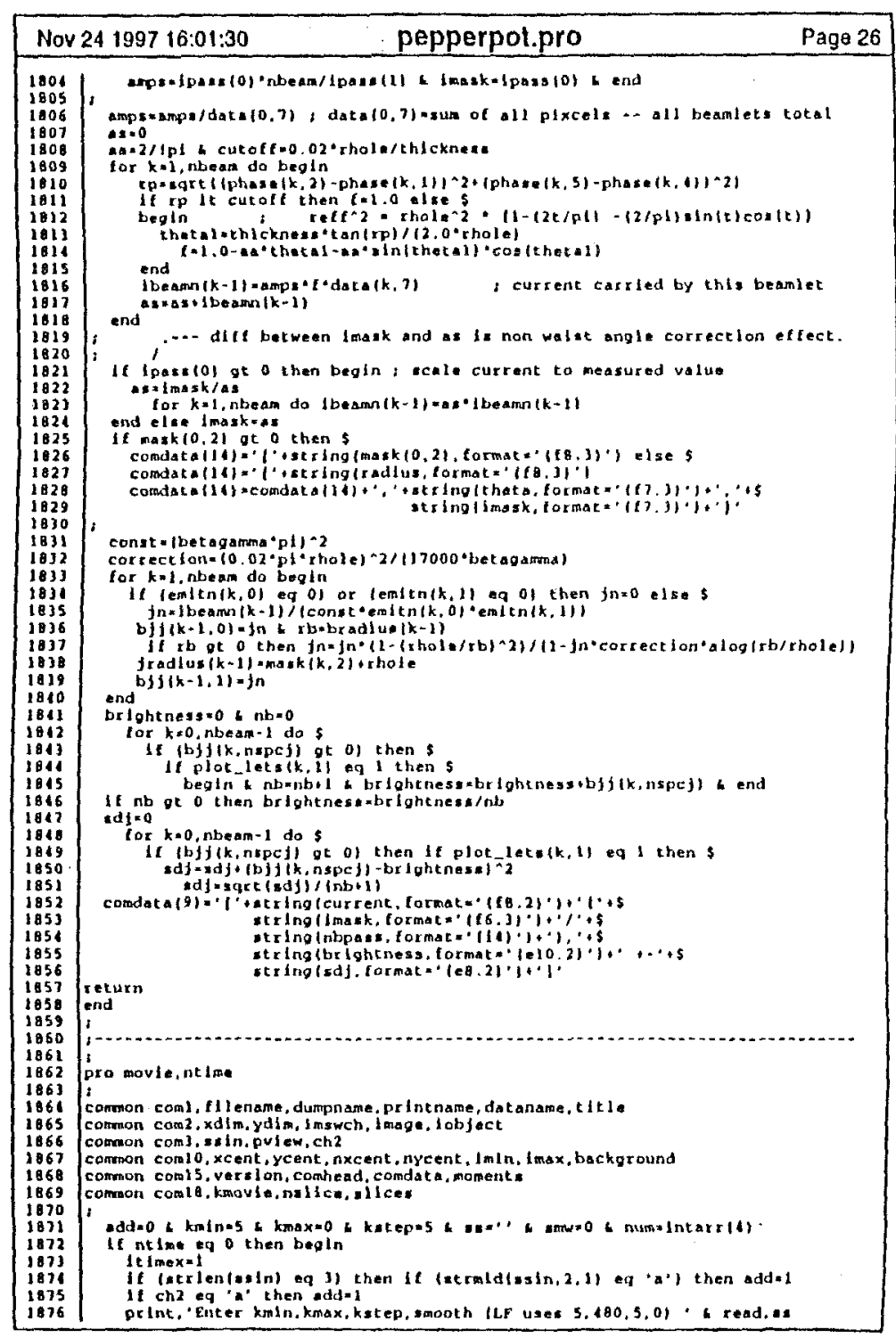



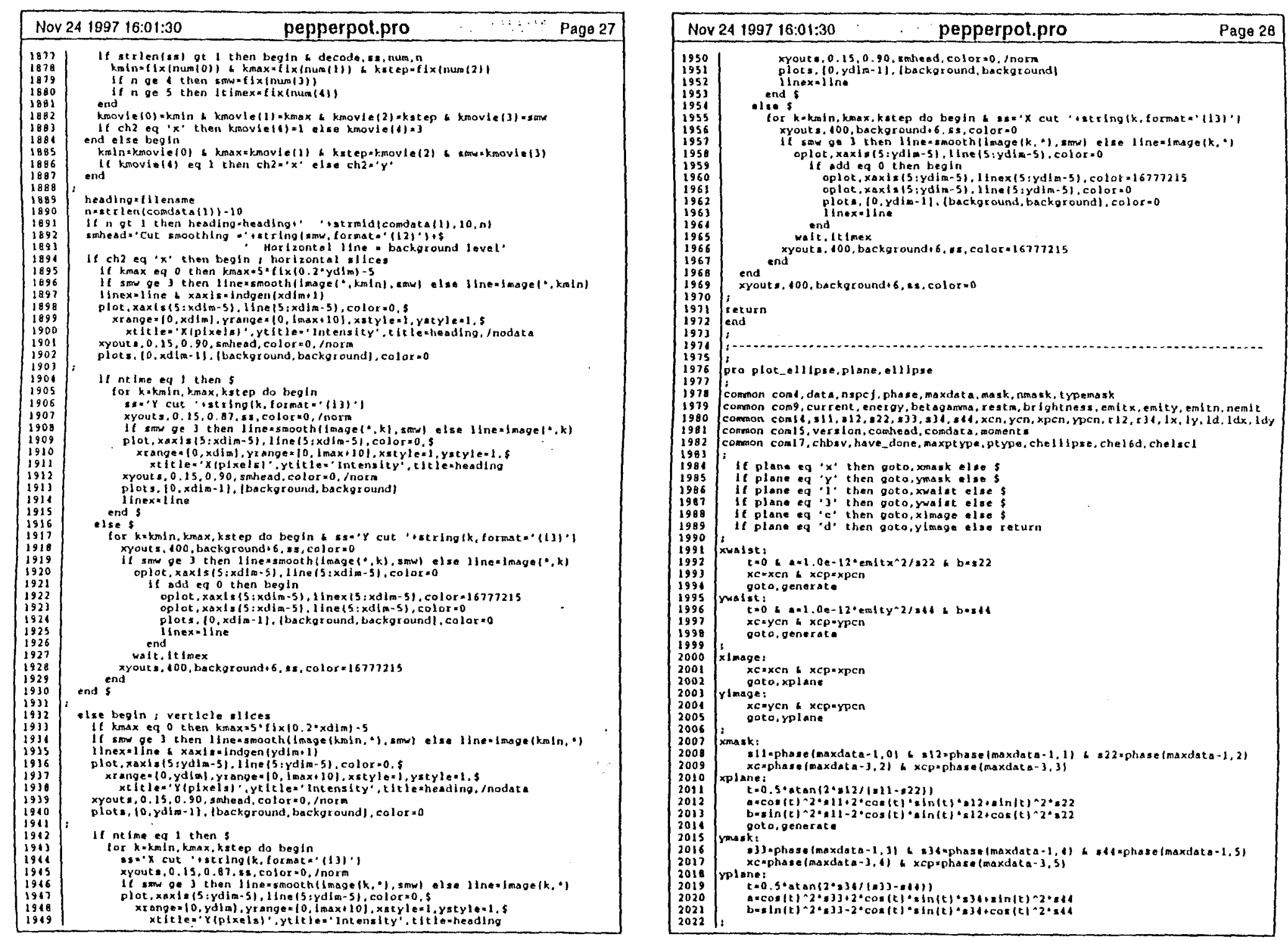


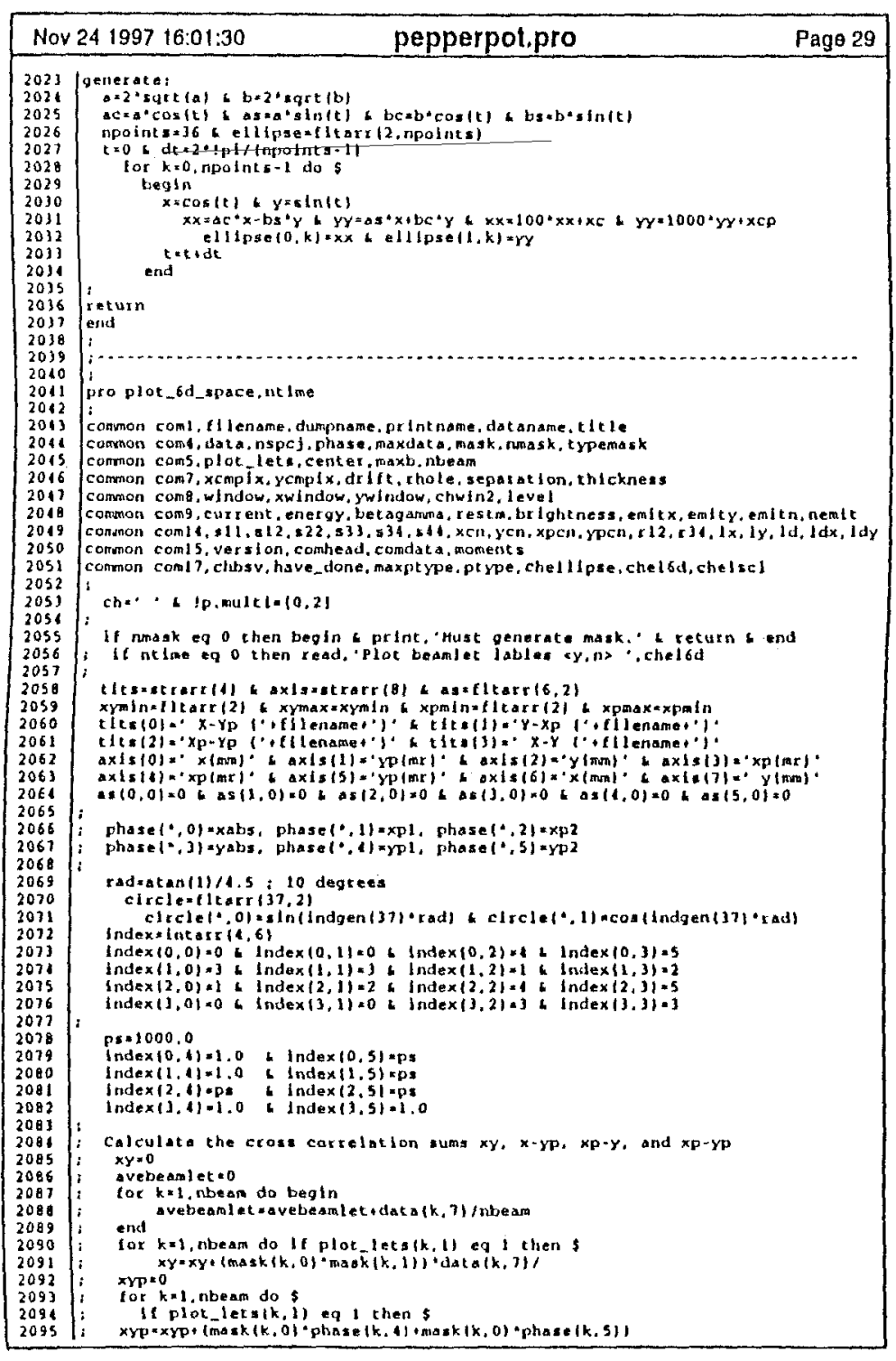

\begin{tabular}{|c|c|c|}
\hline \multicolumn{3}{|c|}{ Nov 241997 16:01:30 } \\
\hline 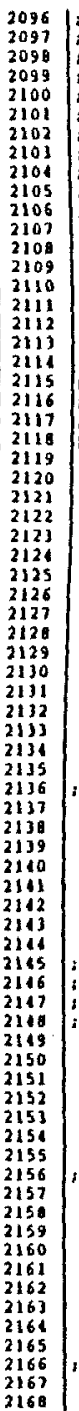 & 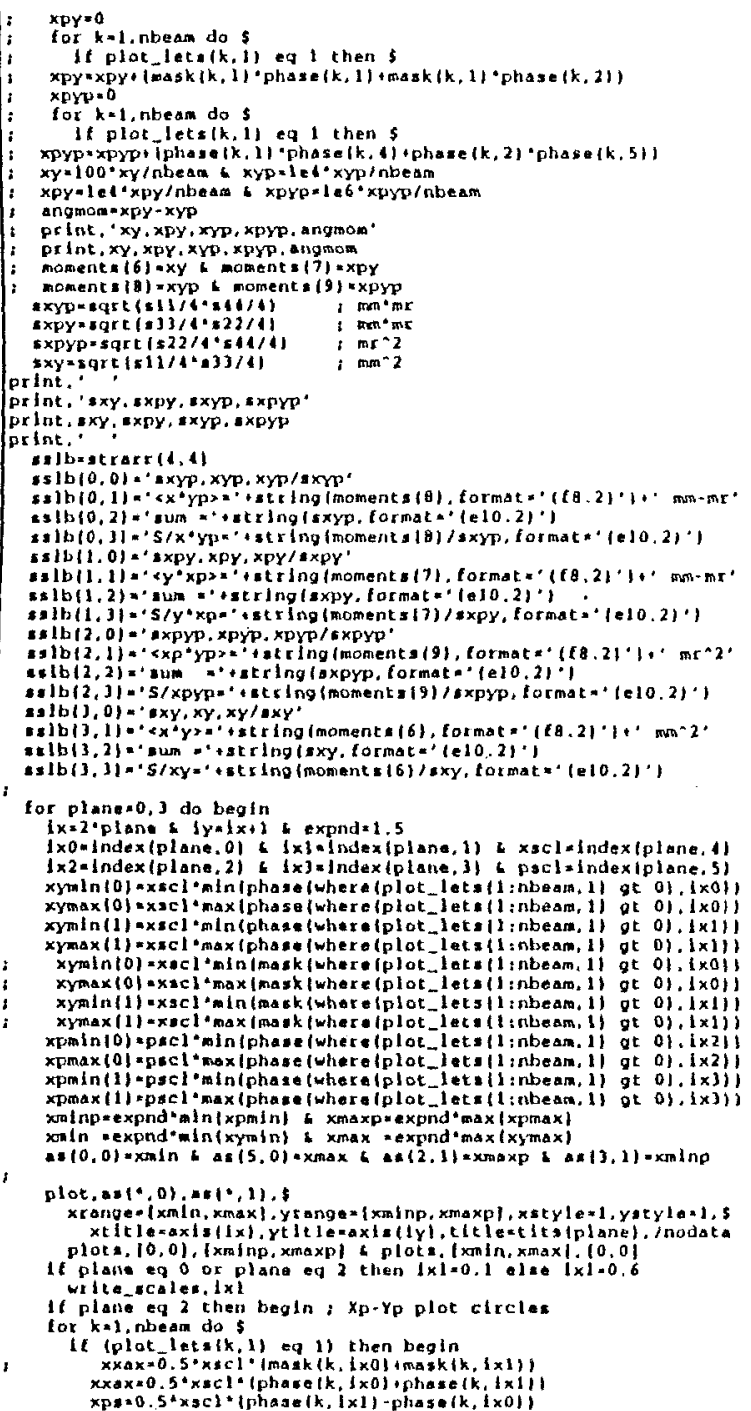 & \\
\hline
\end{tabular}



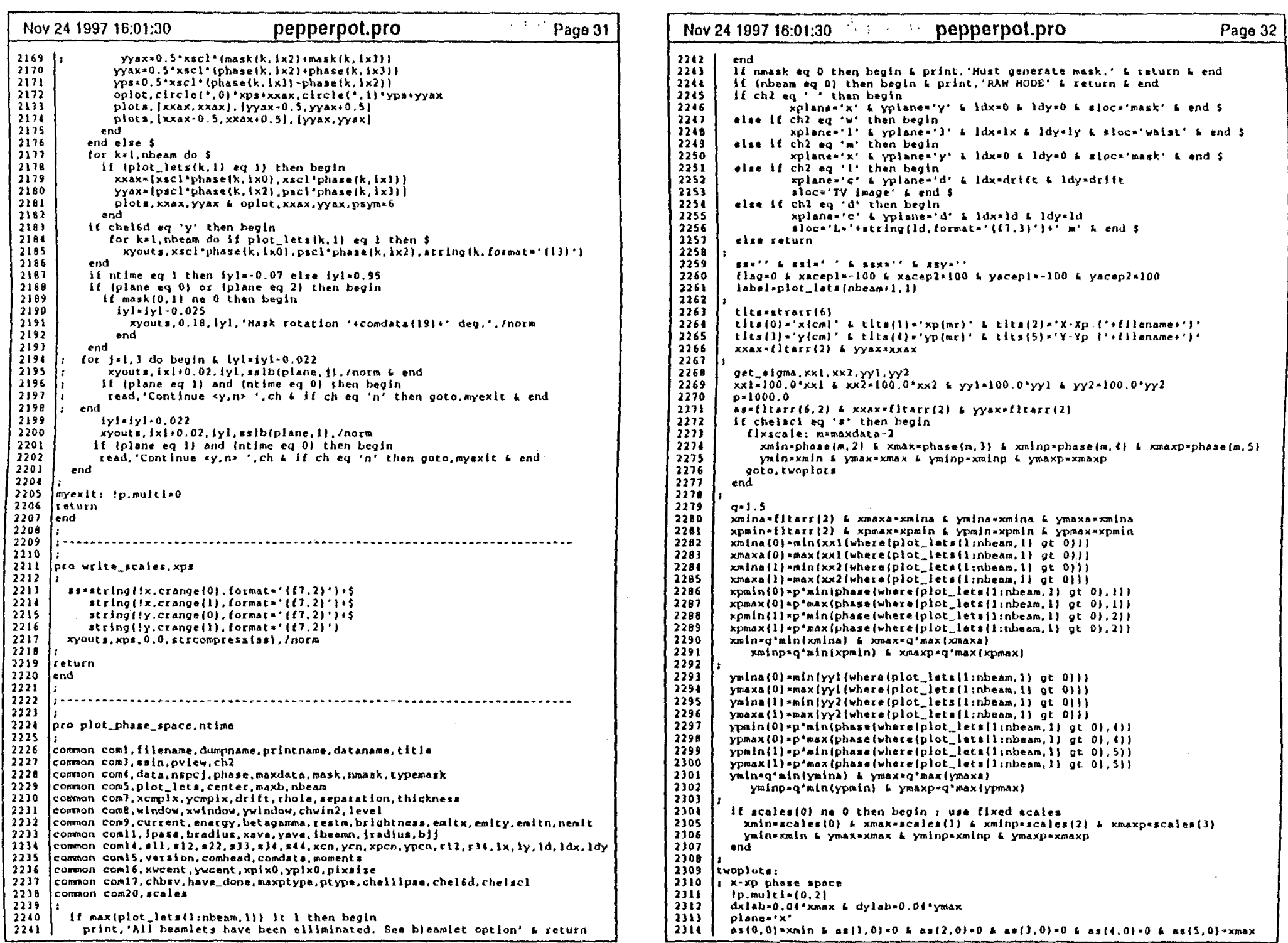


\begin{tabular}{|c|c|}
\hline \multicolumn{2}{|c|}{ Nov 24 1997 16:01:30 } \\
\hline $\begin{array}{l}2315 \\
2316\end{array}$ & 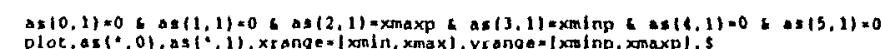 \\
\hline $\begin{array}{l}2316 \\
2317\end{array}$ & 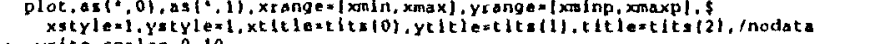 \\
\hline 2118 & 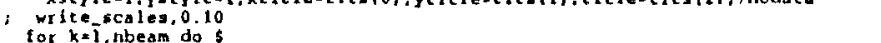 \\
\hline $\begin{array}{l}23320 \\
2320\end{array}$ & 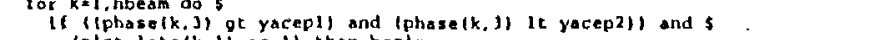 \\
\hline $\begin{array}{l}321 \\
2322\end{array}$ & Iflot - letsik. 11 eq 11 then beg la \\
\hline 2321 & 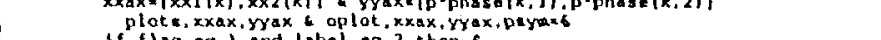 \\
\hline $\begin{array}{l}2124 \\
2125\end{array}$ & 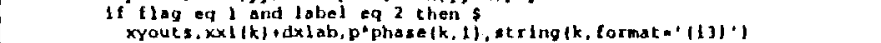 \\
\hline 2326 & end \\
\hline 2328 & 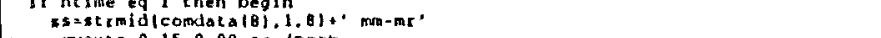 \\
\hline $\begin{array}{l}2329 \\
2330\end{array}$ & 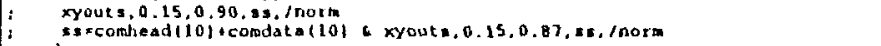 \\
\hline $\begin{array}{l}2331 \\
2312\end{array}$ & 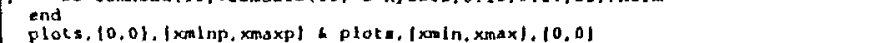 \\
\hline $\begin{array}{l}2313 \\
2134\end{array}$ & 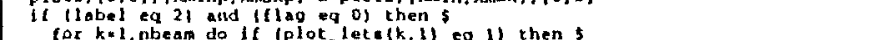 \\
\hline $\begin{array}{cccc}2135 \\
235 \\
3116\end{array}$ & 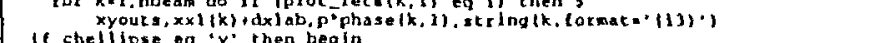 \\
\hline 2337 & 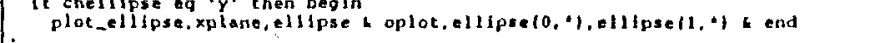 \\
\hline 2339 & y-yp phase apace \\
\hline $\begin{array}{l}2340 \\
2301\end{array}$ & 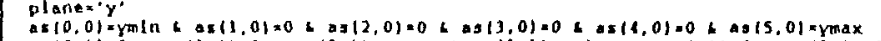 \\
\hline $\begin{array}{l}2142 \\
230\end{array}$ & 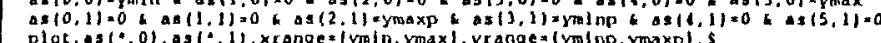 \\
\hline 2116 & 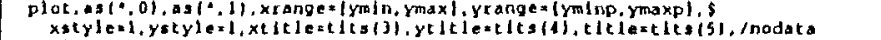 \\
\hline $\begin{array}{l}2345 \\
2316\end{array}$ & wrlé.xales. 0.60 \\
\hline 2317 & 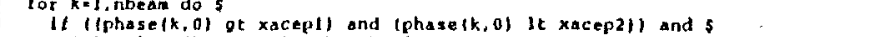 \\
\hline $\begin{array}{l}2348 \\
2369\end{array}$ & 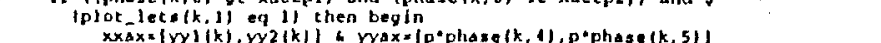 \\
\hline $\begin{array}{l}2350 \\
2351\end{array}$ & 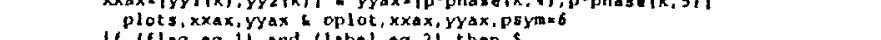 \\
\hline $\begin{array}{l}2351 \\
2352\end{array}$ & 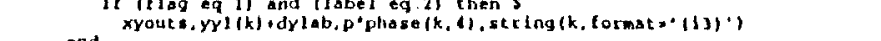 \\
\hline $\begin{array}{l}2353 \\
2351\end{array}$ & 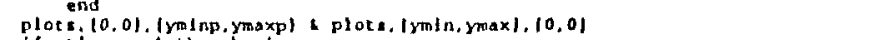 \\
\hline $\begin{array}{l}2355 \\
2356\end{array}$ & $\begin{array}{l}\text { if nelme eq i then beqin } \\
\text { ssestrmidicomdata }(8), 10,81, \cdots \text { mo-mr. }\end{array}$ \\
\hline $\begin{array}{l}2357 \\
2358\end{array}$ & end $x$ xout $x, 0.6$. \\
\hline $\begin{array}{l}2359 \\
2360\end{array}$ & 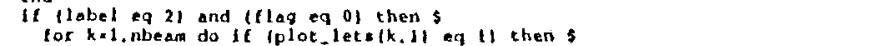 \\
\hline $\begin{array}{lll}2161 \\
2362\end{array}$ & 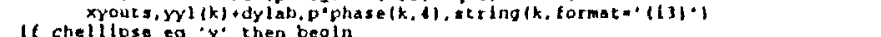 \\
\hline 2363 3163 & 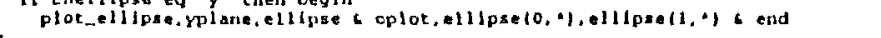 \\
\hline $\begin{array}{llll}2366 \\
2365\end{array}$ & plot labels \\
\hline $\begin{array}{l}2366 \\
2367\end{array}$ & if ntime eq o eth \\
\hline $\begin{array}{lll}2168 \\
2369\end{array}$ & 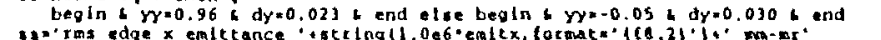 \\
\hline 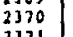 & 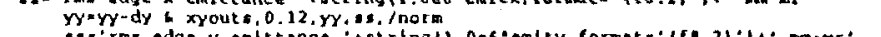 \\
\hline $\begin{array}{l}2313 \\
2372\end{array}$ & 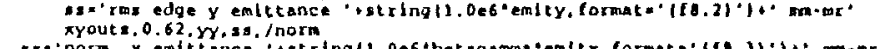 \\
\hline 2373 & $.31 \% 1, \ldots \operatorname{mom}$ \\
\hline $\begin{array}{l}2374 \\
2375\end{array}$ & 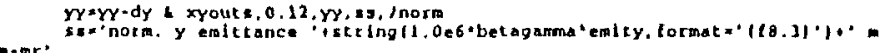 \\
\hline 2376 & 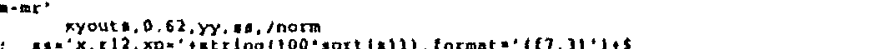 \\
\hline 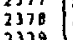 & 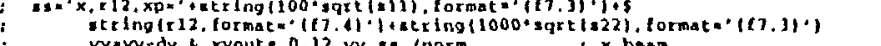 \\
\hline $\begin{array}{l}2379 \\
2380\end{array}$ & 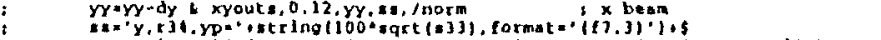 \\
\hline $\begin{array}{l}2301 \\
2282\end{array}$ & 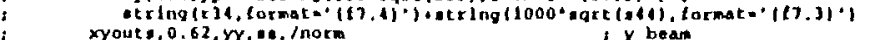 \\
\hline $\begin{array}{l}2389 \\
2395\end{array}$ & 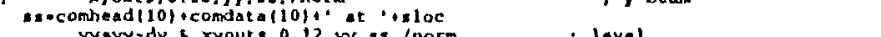 \\
\hline $\begin{array}{l}3384 \\
2385\end{array}$ & 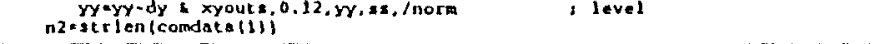 \\
\hline
\end{tabular}

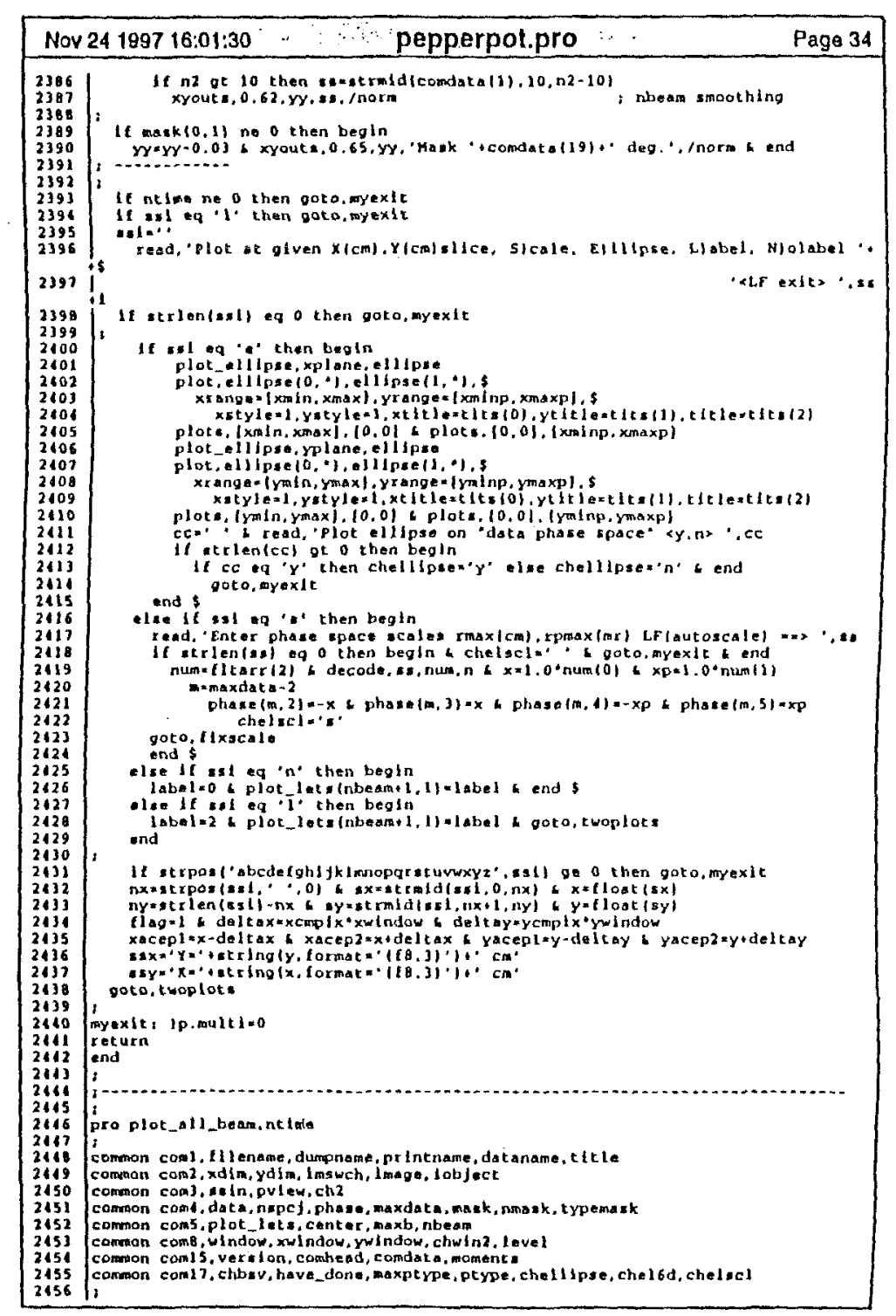



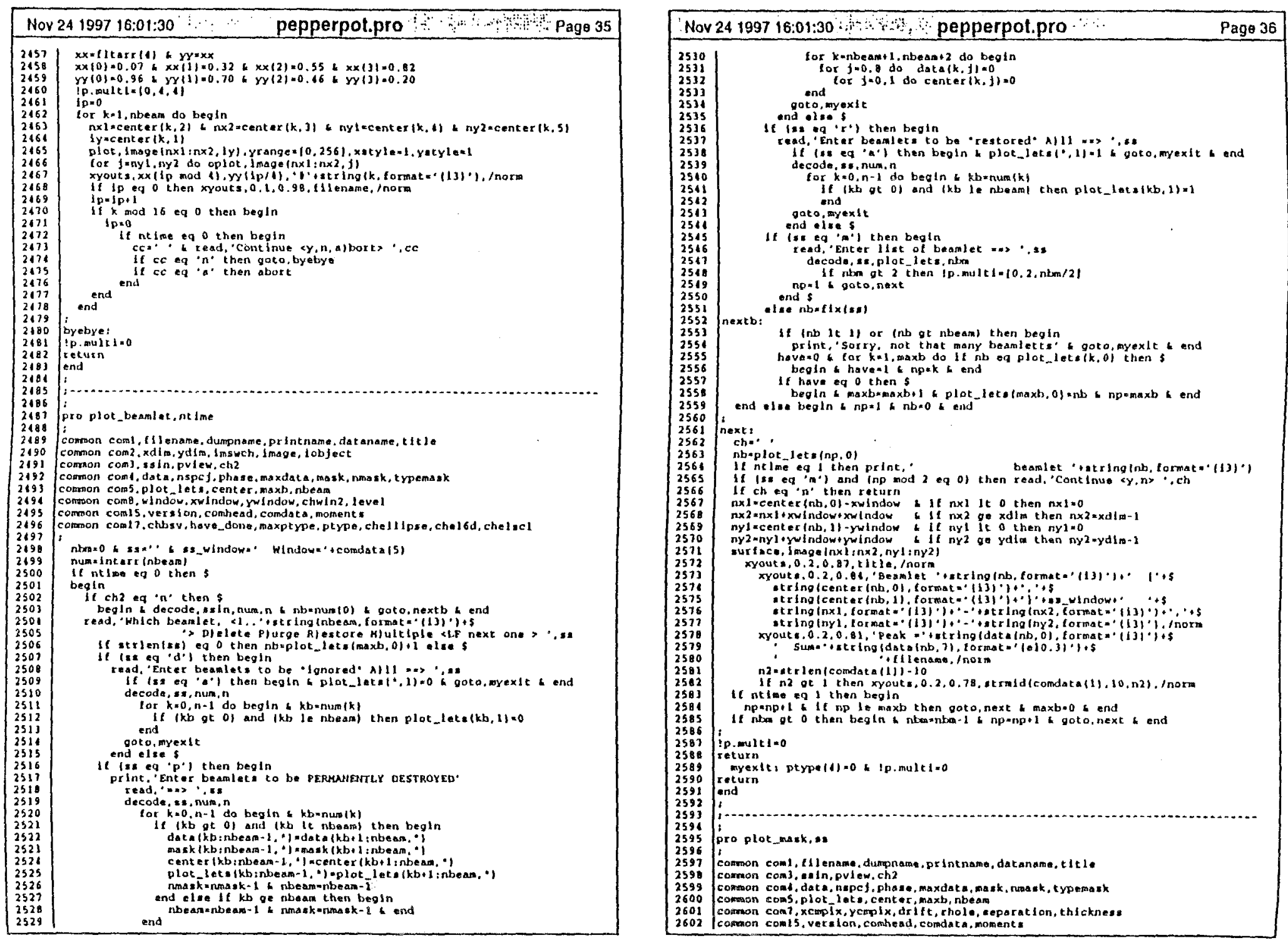

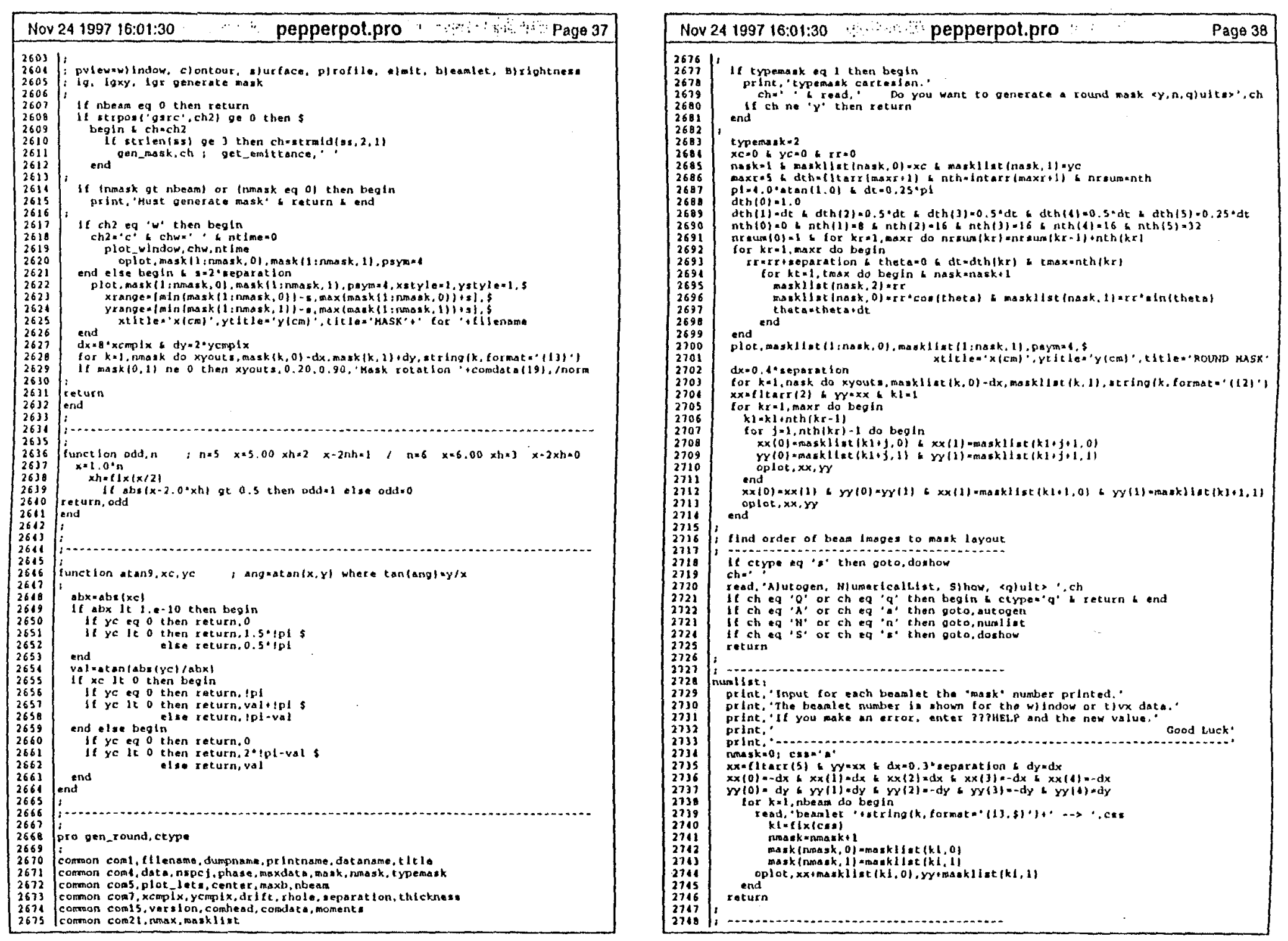

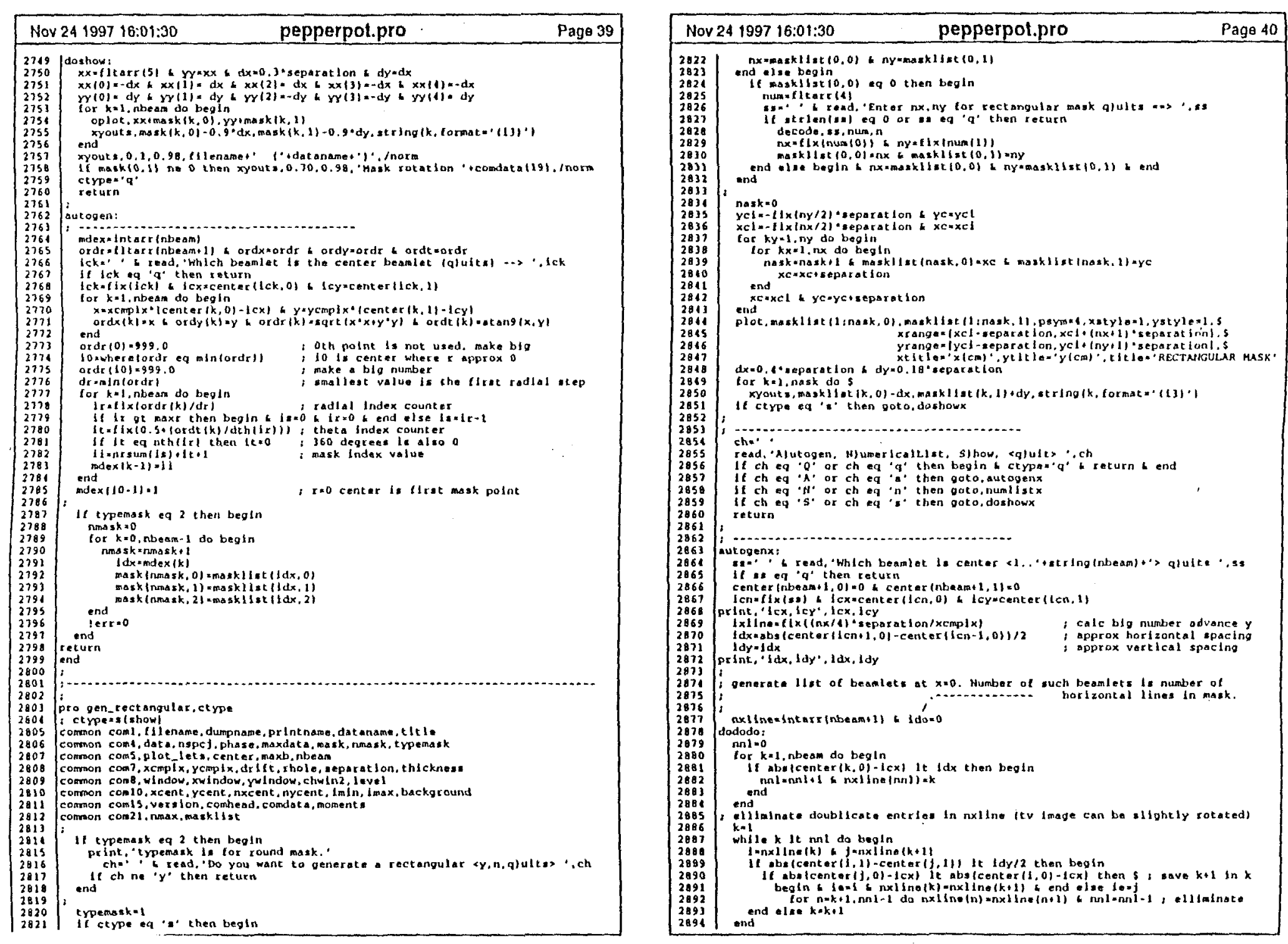

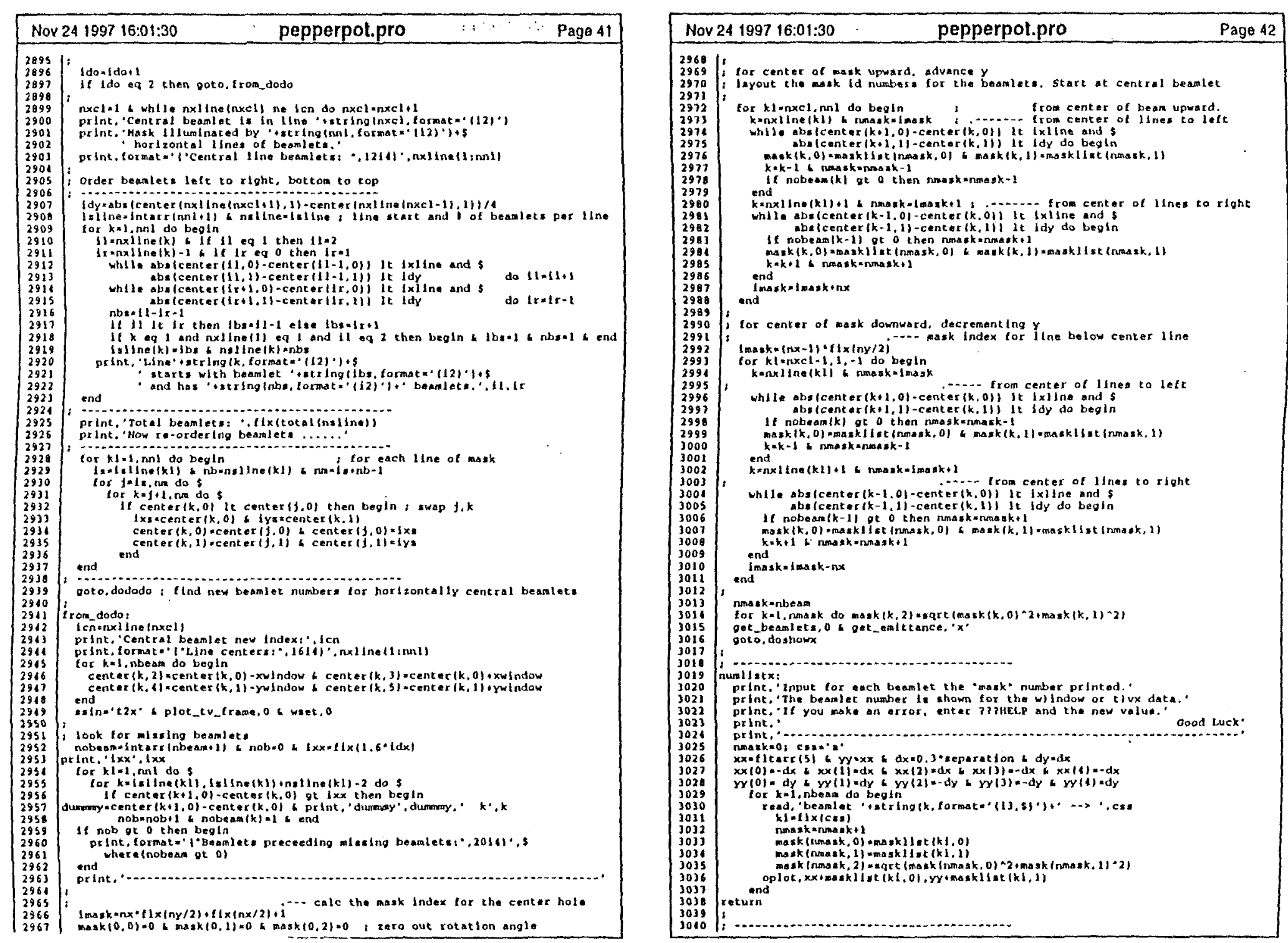


\begin{tabular}{|c|c|c|c|}
\hline \multicolumn{2}{|c|}{ Nov 24 1997 16:01:30 } & pepperpot.pro & Pago 43 \\
\hline & doshowx: & & \\
\hline $\begin{array}{l}3012 \\
3043\end{array}$ & 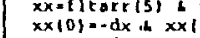 & 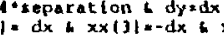 & \\
\hline 3044 & 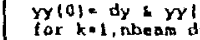 & $i=-d y \leq x y(3) \cdot-d y=$ & \\
\hline $\begin{array}{l}3006 \\
1047\end{array}$ & $\begin{array}{l}\text { oplot ix masask } \\
\text { xyouts, mask (k }\end{array}$ & 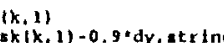 & \\
\hline $\begin{array}{l}3048 \\
3019\end{array}$ & end $\begin{array}{l}\text { end } \\
\text { xyout , } 0,0,98\end{array}$ & 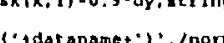 & \\
\hline $\begin{array}{l}3019 \\
3050\end{array}$ & $\begin{array}{l}\text { xyouta } 0,1,0.98 \\
\text { II maskio, } 11 \text { ne }\end{array}$ & 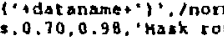 & 1./norm \\
\hline $\begin{array}{l}3051 \\
1052\end{array}$ & $\begin{array}{l}\text { ctypes: } \\
\text { return }\end{array}$ & & \\
\hline $\begin{array}{l}3053 \\
3054\end{array}$ & :ind & & \\
\hline $\begin{array}{l}3055 \\
3056\end{array}$ & $: \ldots$ & & \\
\hline $\begin{array}{l}3057 \\
3058\end{array}$ & & & \\
\hline $\begin{array}{l}3058 \\
3059\end{array}$ & 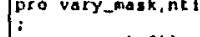 & & \\
\hline $\begin{array}{l}1060 \\
1061 \\
3065\end{array}$ & $\begin{array}{l}\text { conmon com1, fllen } \\
\text { cormman cont, dats. }\end{array}$ & $\begin{array}{l}\text { printrname, dataname, } \\
\text { axdat a, mask, nma sk, }\end{array}$ & \\
\hline $\begin{array}{l}3062 \\
3063 \\
3064 \\
3065\end{array}$ & $\begin{array}{l}\text { comman coms.plot } \\
\text { couman coms curre } \\
\text { common com15, ver } \\
\text { common com } 21 \text {, rumax }\end{array}$ & $\begin{array}{l}\text { axb, mbeam } \\
\text { agamman, restm, bri lohtn } \\
\text { omdaca, moments }\end{array}$ & itn, nemit \\
\hline $\begin{array}{l}3066 \\
3067\end{array}$ & I It namask it it & rlat, First generate & and end \\
\hline $\begin{array}{l}3060 \\
3069\end{array}$ & 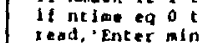 & and step Cmask rot & amn sanx da da \\
\hline 3070 & nomaxe nmax & 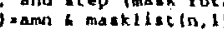 & 2) $=\mathrm{da} a$ \\
\hline $\begin{array}{l}3071 \\
3072 \\
3073\end{array}$ & narmax truax & $(n, 0)$ amxamaskilat & $(n .2)$ \\
\hline 3073 & 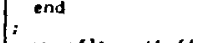 & . & \\
\hline $\begin{array}{l}3075 \\
3076 \\
307\end{array}$ & 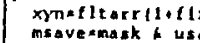 & ab.1) Exray to sav & \\
\hline & 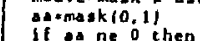 & $\quad: \ln 1 \mathrm{c}$ & ingle \\
\hline $\begin{array}{l}3078 \\
3080 \\
1080\end{array}$ & 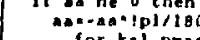 & al a cencostaxit & ation \\
\hline $\begin{array}{l}1000 \\
3001 \\
3092\end{array} \mid$ & 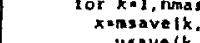 & $k, 1$ & \\
\hline $\begin{array}{l}3002 \\
3003 \\
3082\end{array}$ & end usover $1 \mathrm{k}$. & save $(k, 1)=x^{\circ} \operatorname{snt} y^{0}$ & \\
\hline $\begin{array}{l}3081 \\
3005\end{array}$ & $\begin{array}{l}\text { end } \\
\text { eng a amn }\end{array}$ & & \\
\hline $\begin{array}{l}3086 \\
3087\end{array}$ & 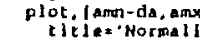 & 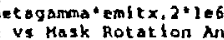 & nodata.s \\
\hline $\begin{array}{l}3088 \\
3089\end{array}$ & 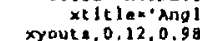 & 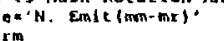 & \\
\hline $\begin{array}{l}3090 \\
3090\end{array}$ & 3 anco & & \\
\hline $\begin{array}{l}3091 \\
3092 \\
3007\end{array}$ & $\begin{array}{l}\text { Lanno } \\
\text { while ang } 1\end{array}$ & & \\
\hline $\begin{array}{l}3093 \\
3094\end{array}$ & 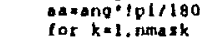 & (a) $1 \cos * \cos (\operatorname{as})$ & \\
\hline 3095 & $\underset{\substack{x=\text { usave } \\
\text { end }}}{x}, 0 \mid$ & $11=\operatorname{mask}(k, 0)=x^{\circ} \cdot c s-$ & sney+cs \\
\hline 3097 & get_enlt tance. & ,0) ang $\&$ xyn $(1 \operatorname{an}, 1)$ & $t x \leqslant x y n 11$ \\
\hline 3098 & $\begin{array}{l}\text { Non } \\
\text { xyouts ang }\end{array}$ & $-x_{0} \cdot x^{\prime}$ & \\
\hline $\begin{array}{l}3099 \\
1100\end{array}$ & $\tan x y$ & $\gamma \cdot \gamma$ & \\
\hline 3101 & & & \\
\hline 3102 & oplot, xyn $1 \cdot 01, x$ & $.0 t, x y n(1,01, x y n(+, 2)$ & \\
\hline 1104 & $\because$ locate where eml & there Ras emitrance & \\
\hline $\begin{array}{l}3106 \\
1106\end{array}$ & rmsesgrtixymik & $11-21 / \operatorname{sqxt}(2) \& \mathrm{xym} i \mathrm{x}$ & \\
\hline 3100 & aplot.xym 1,0 & & \\
\hline $\begin{array}{l}3109 \\
3110\end{array}$ & xyauta ixmilan & RMS & \\
\hline $\begin{array}{l}311 \\
3112 \\
3112\end{array}$ & $1 t \operatorname{xyn} 10.11$ at & 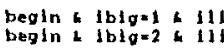 & \\
\hline
\end{tabular}

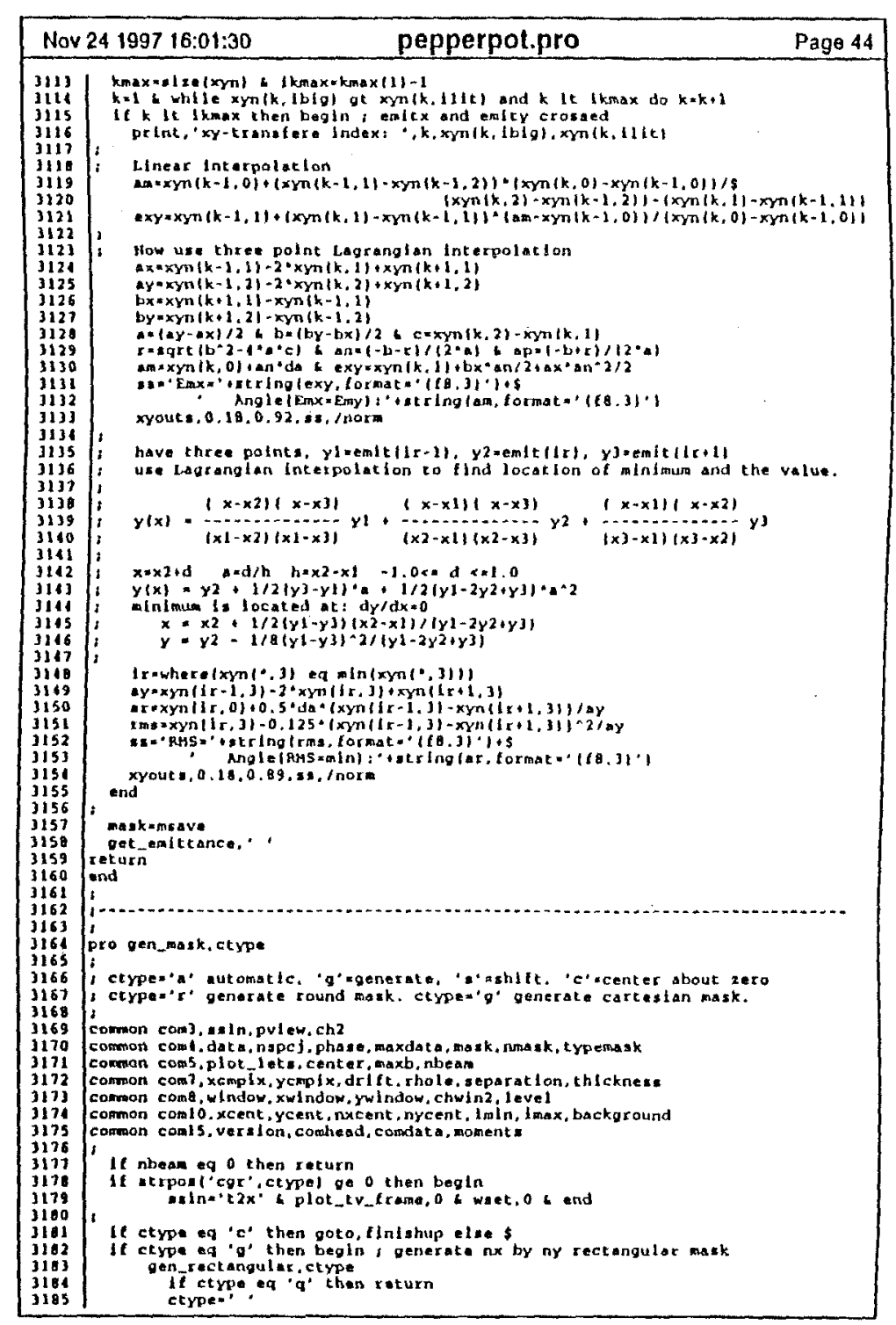



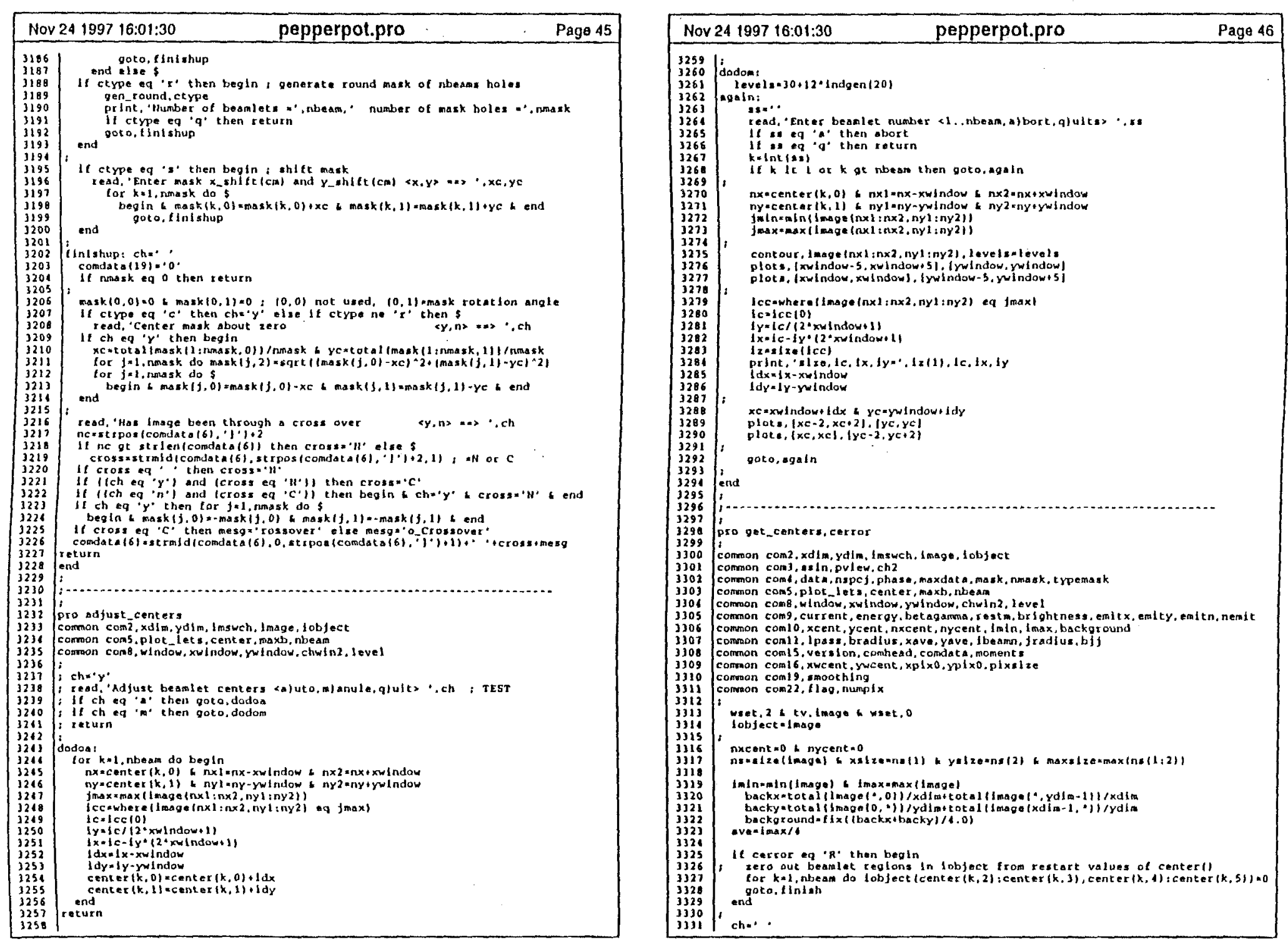


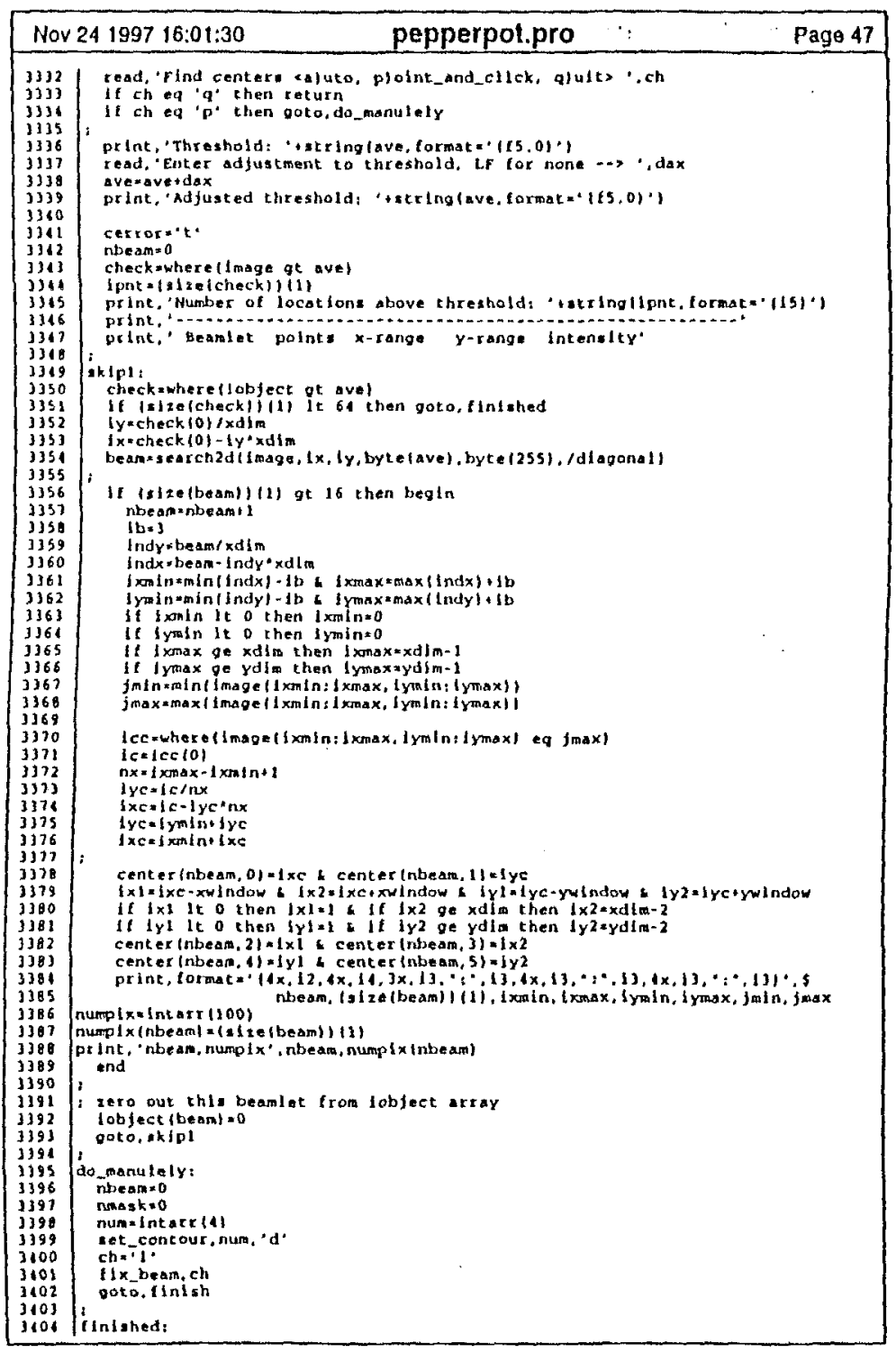

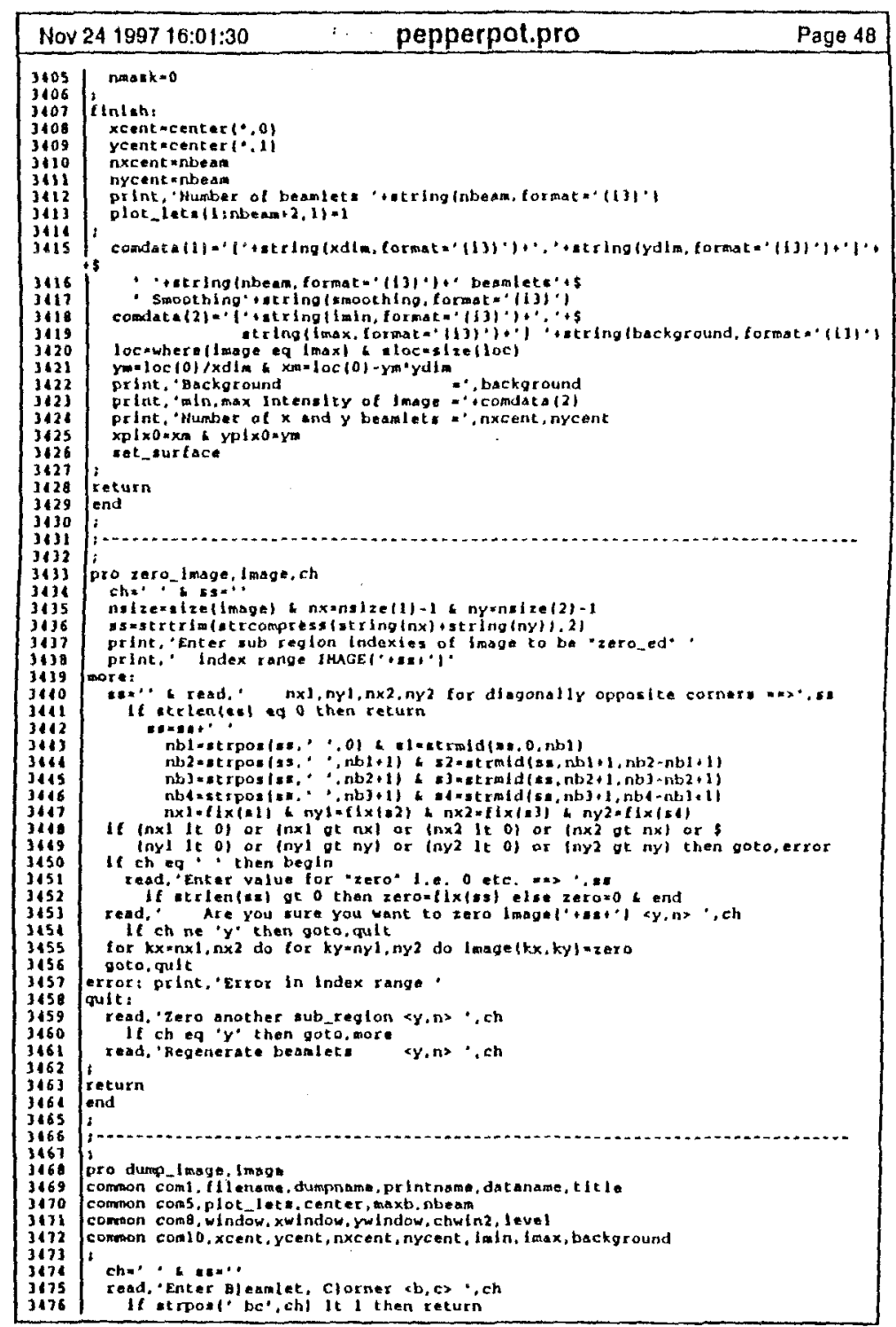




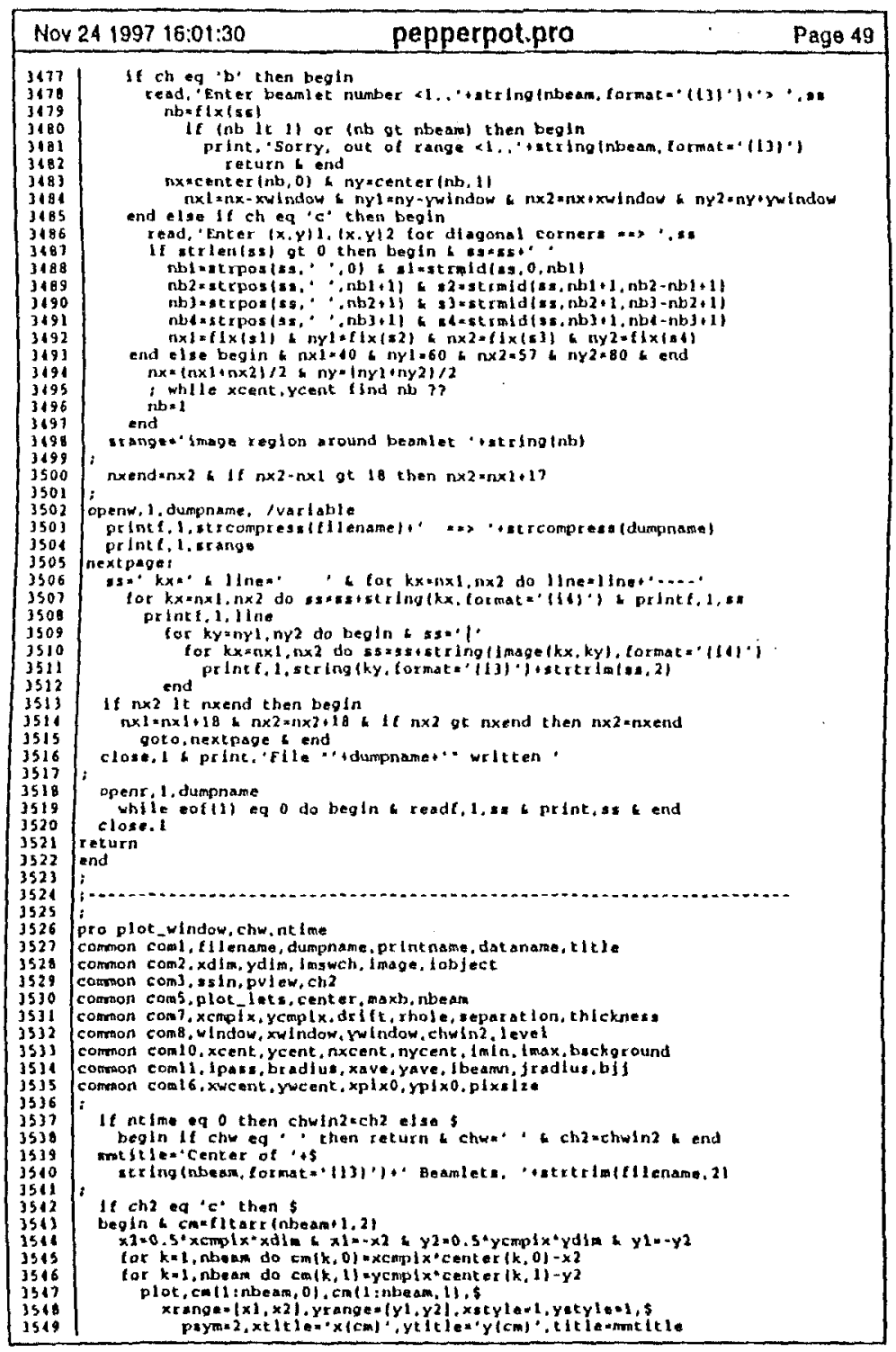

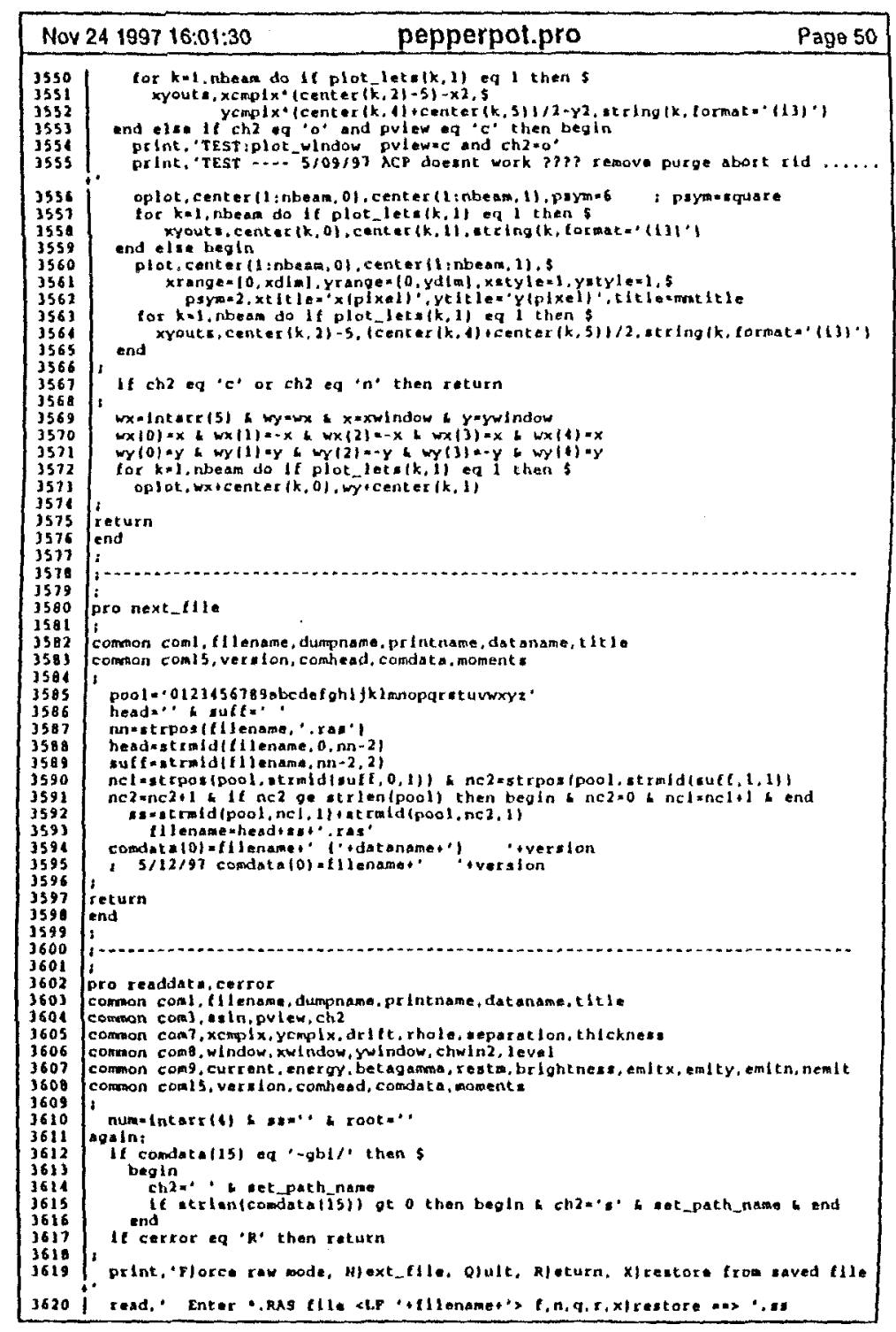



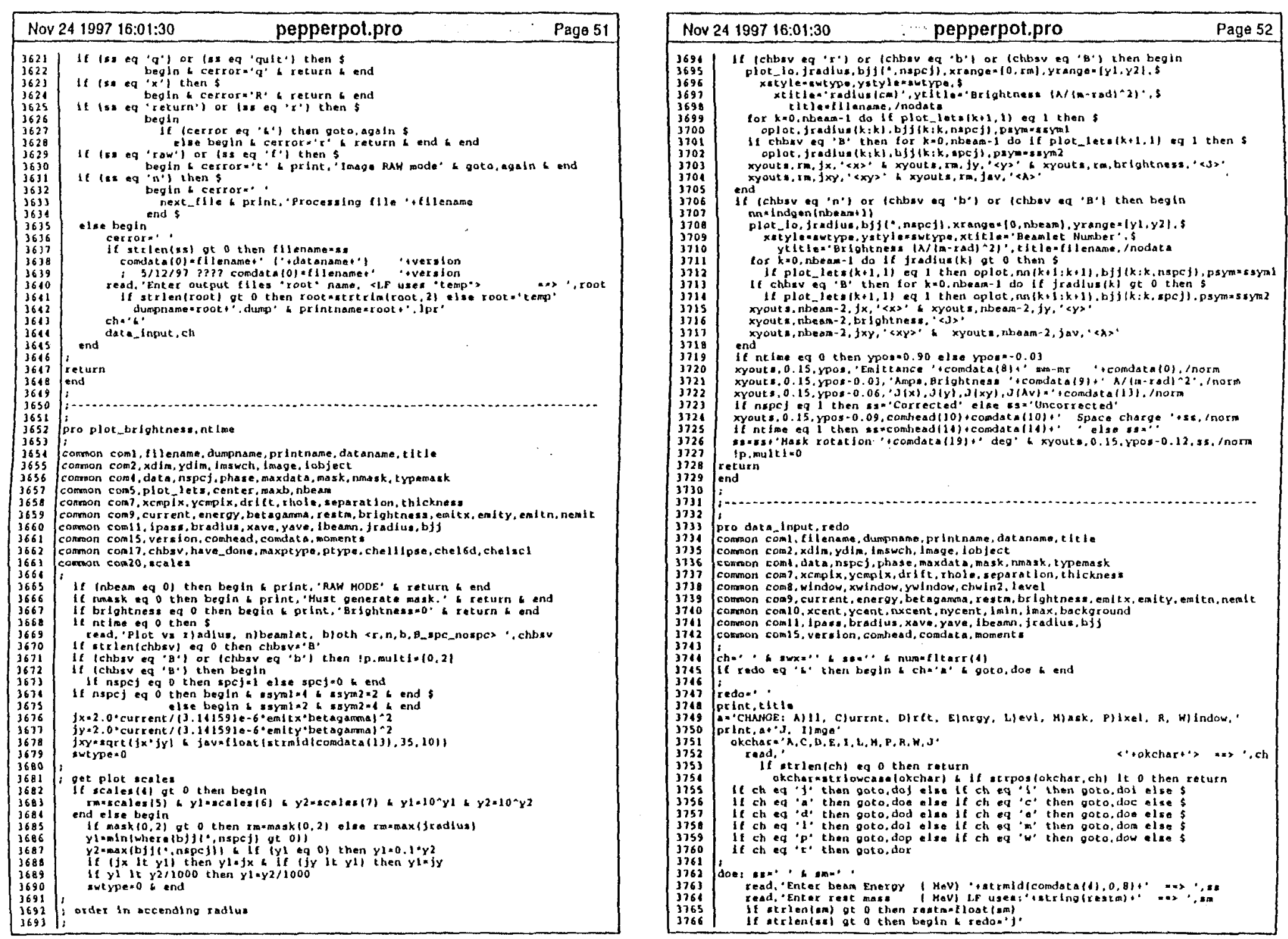

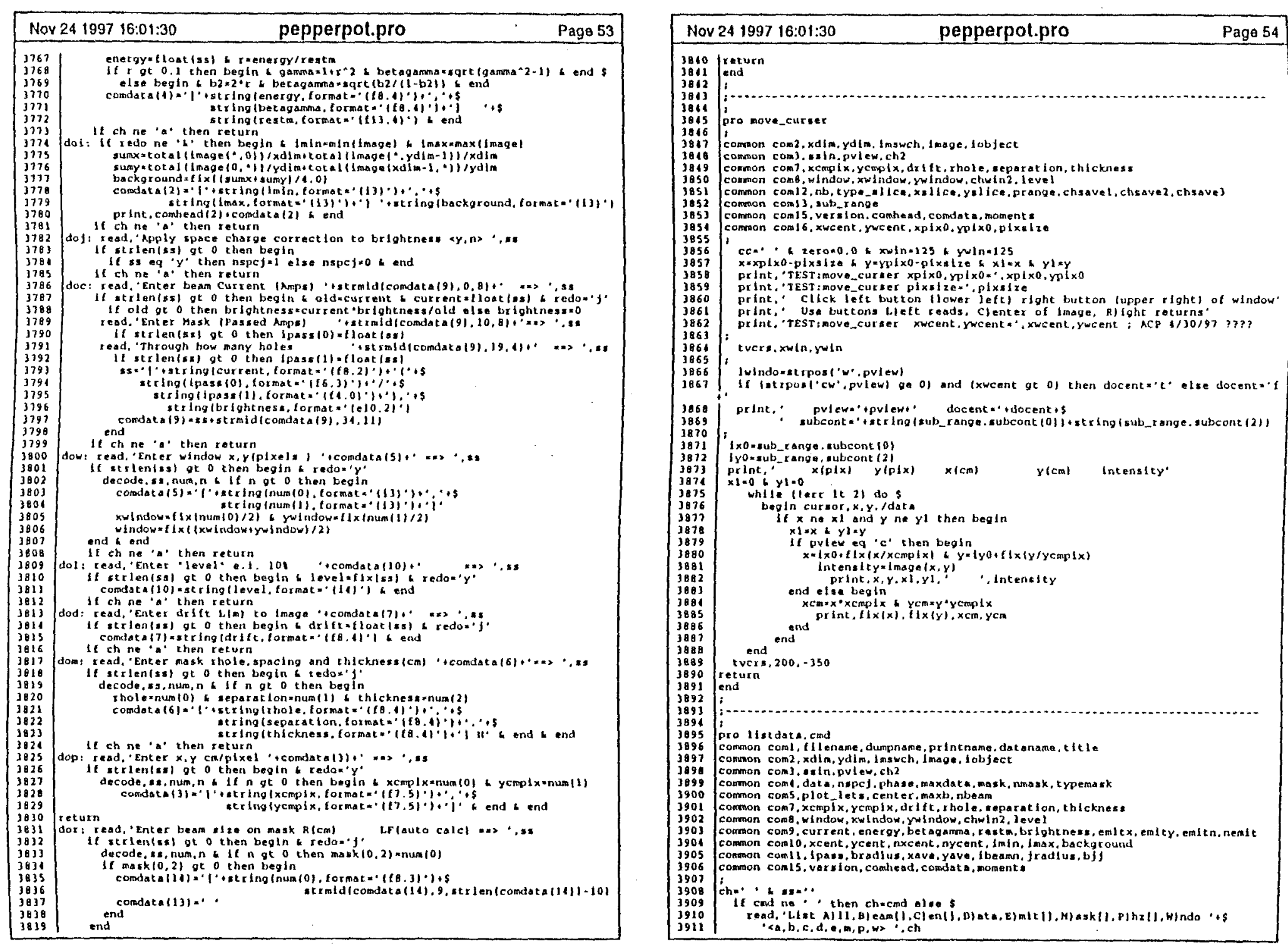

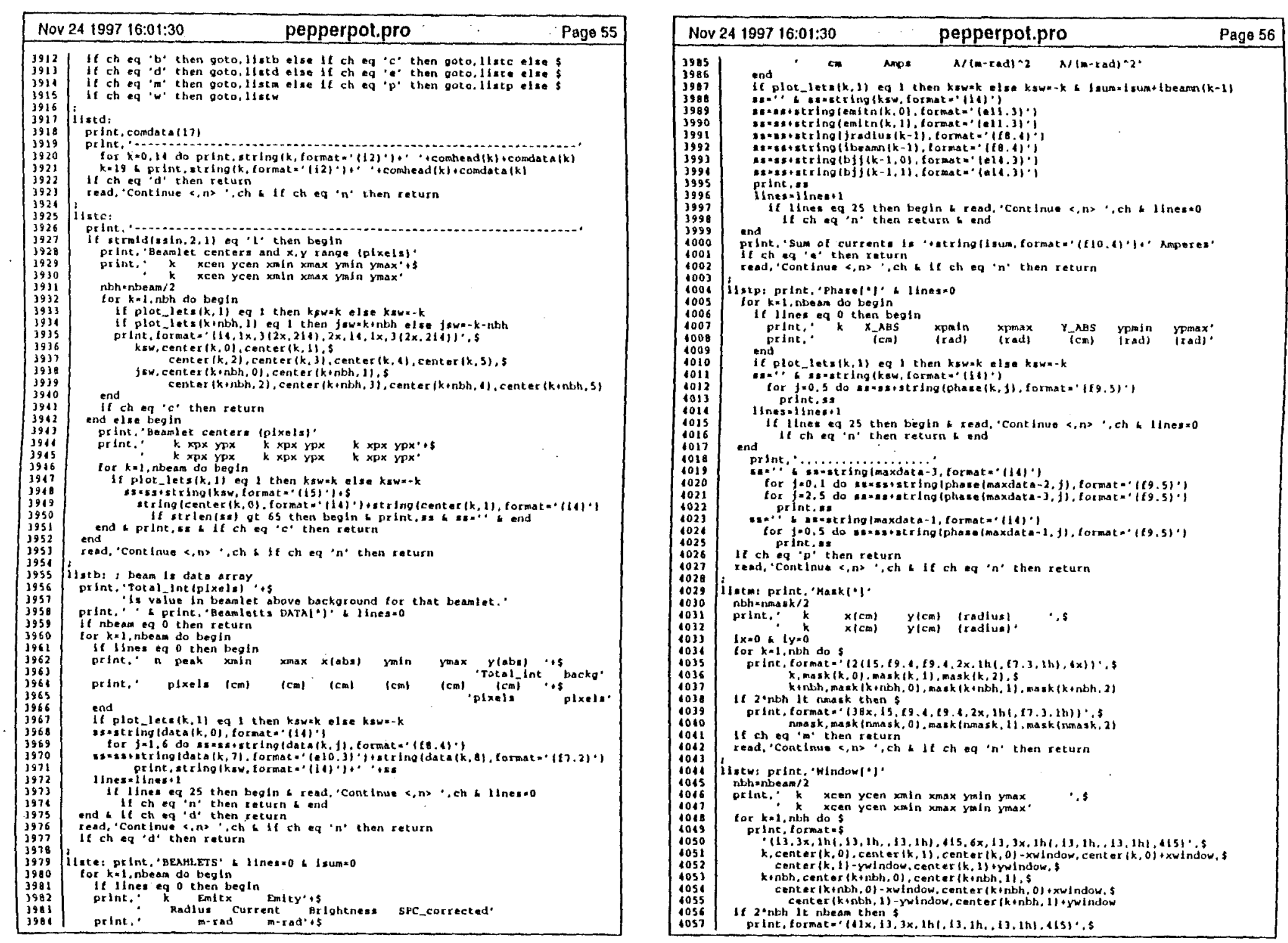


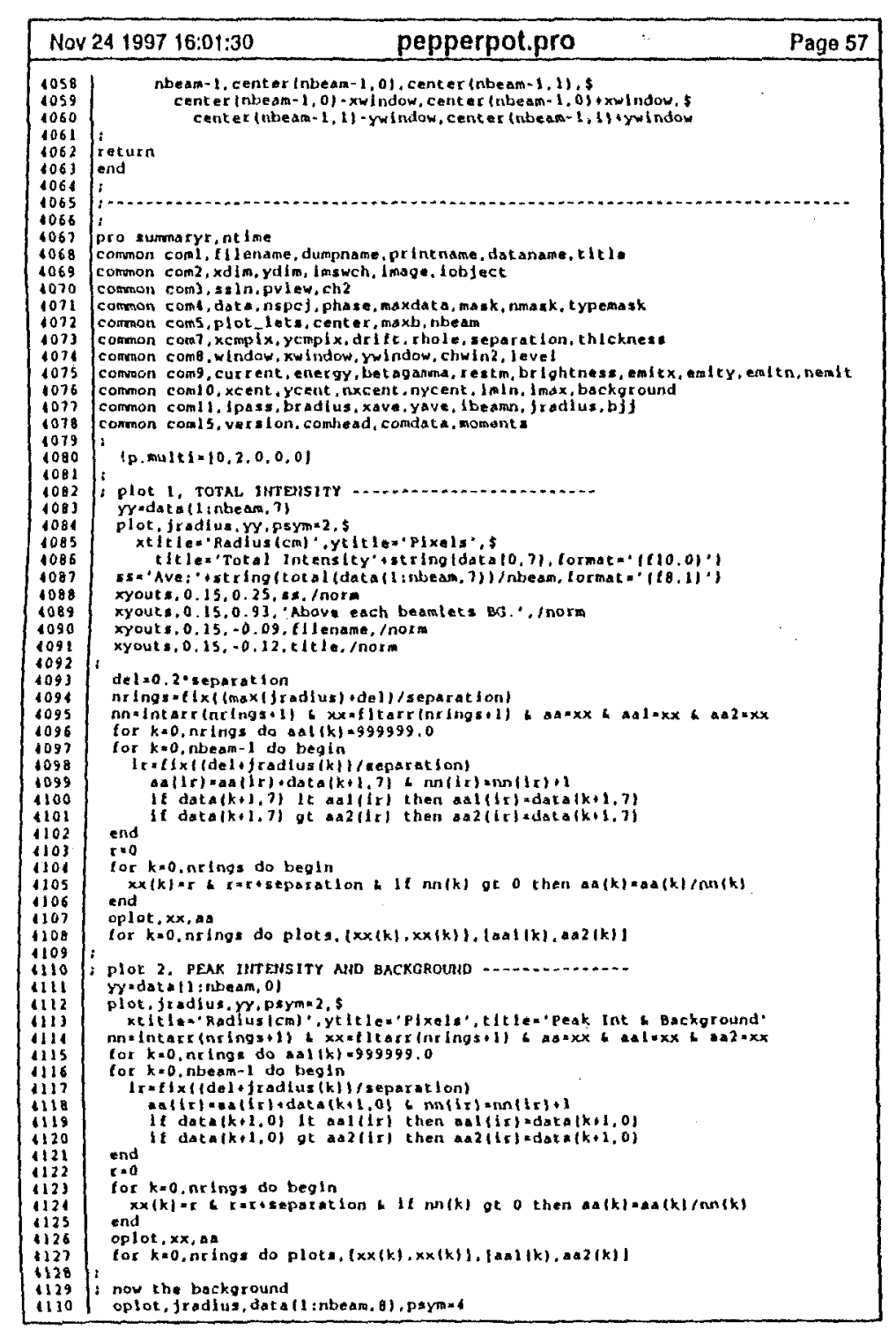

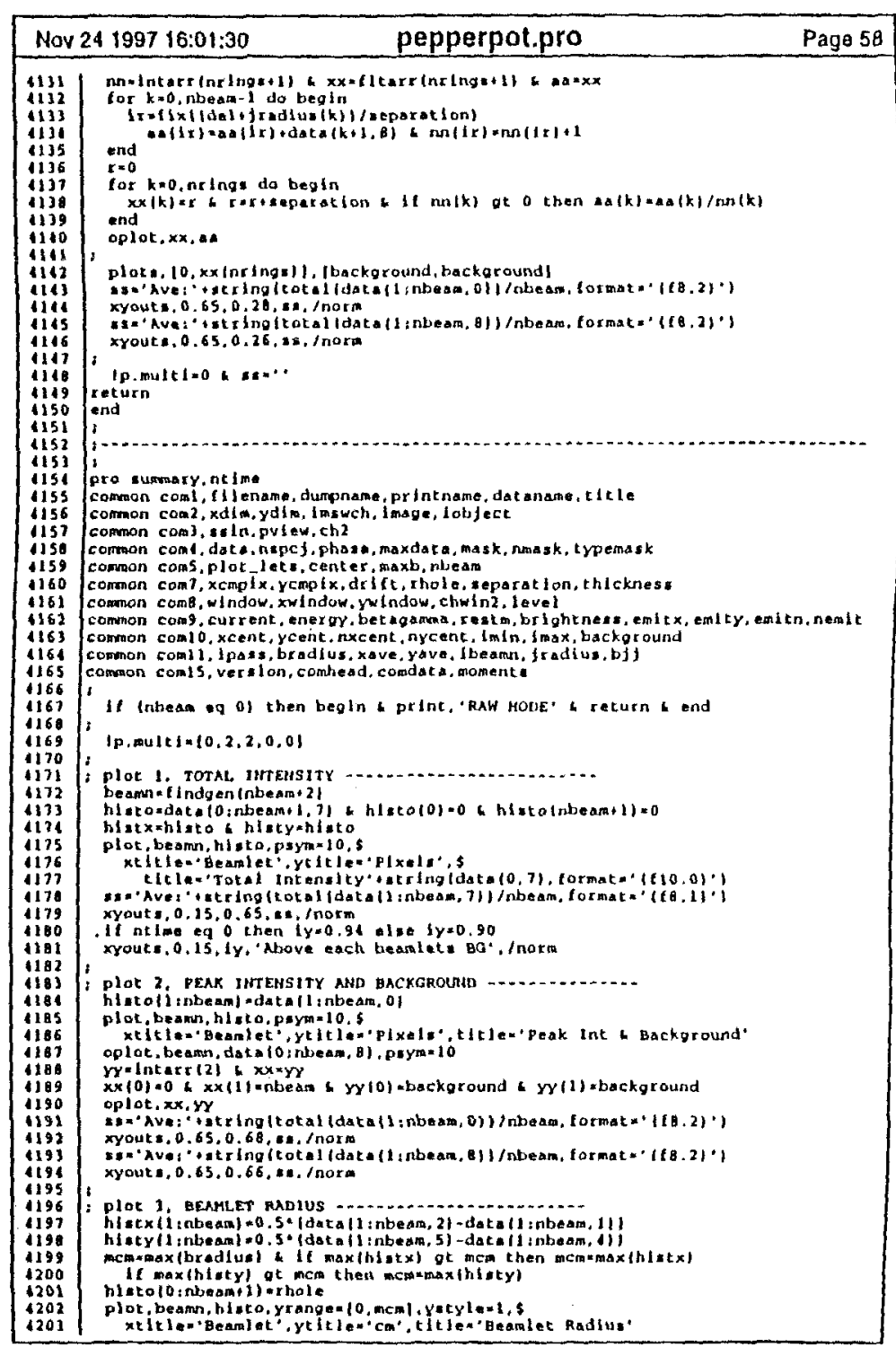




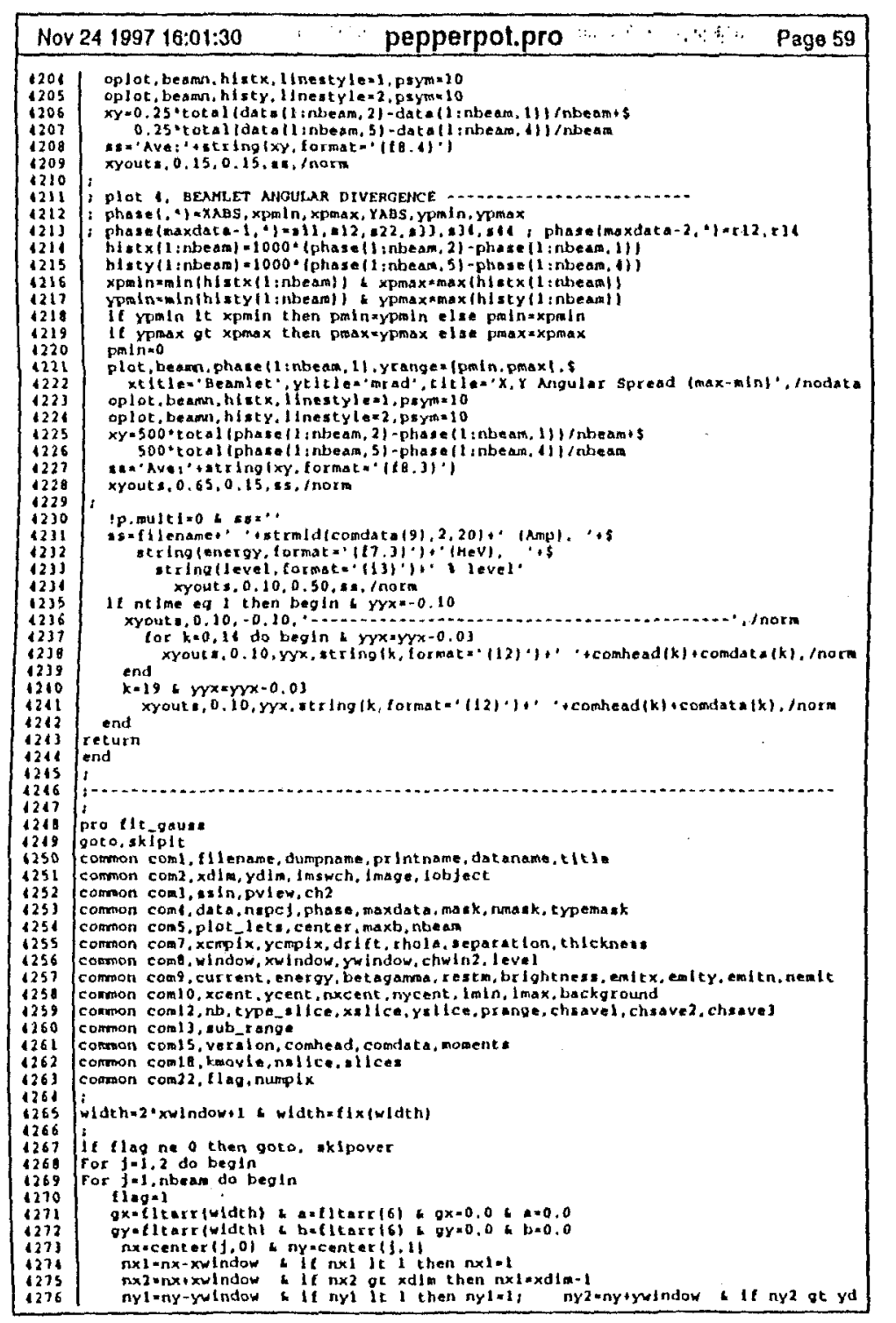

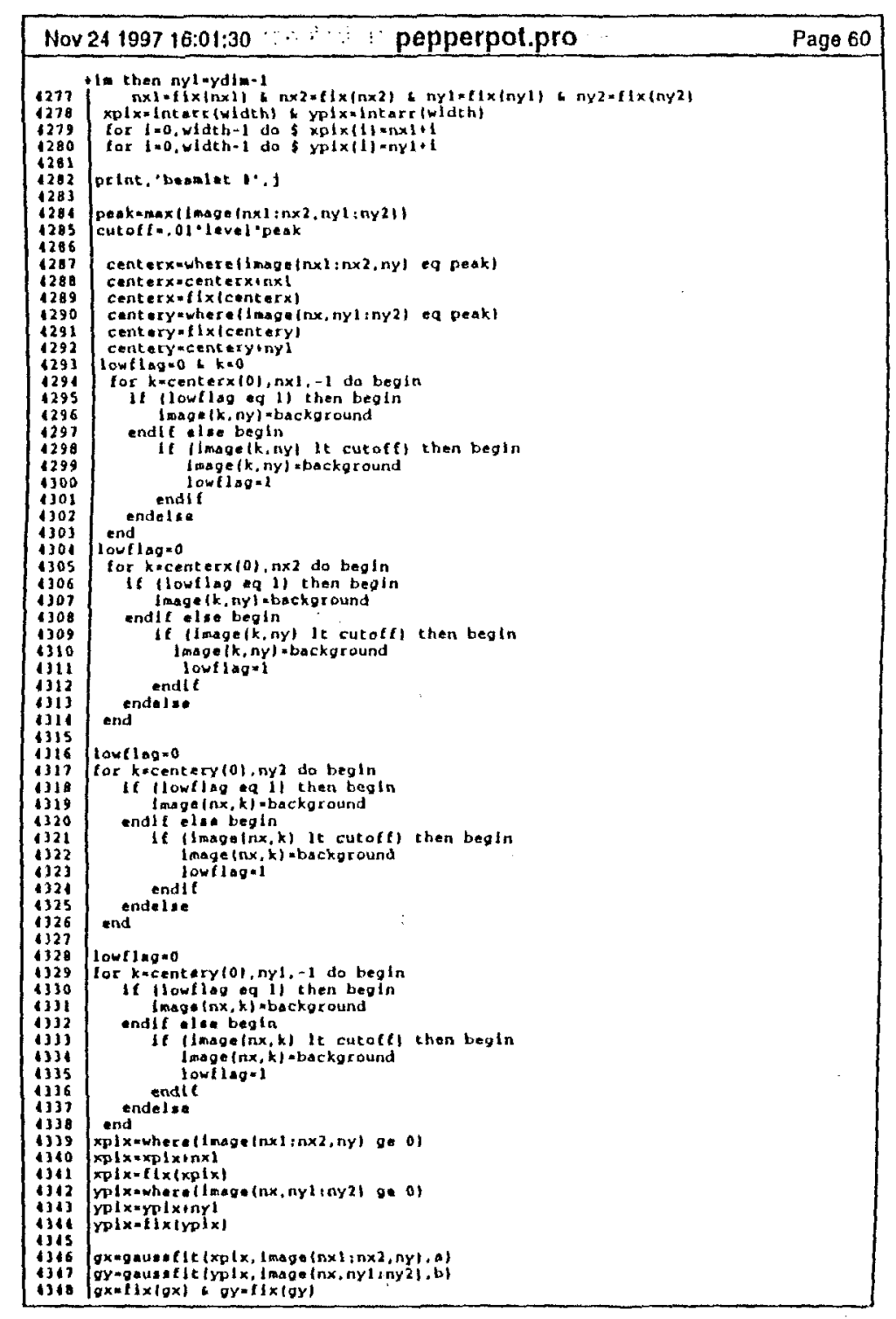




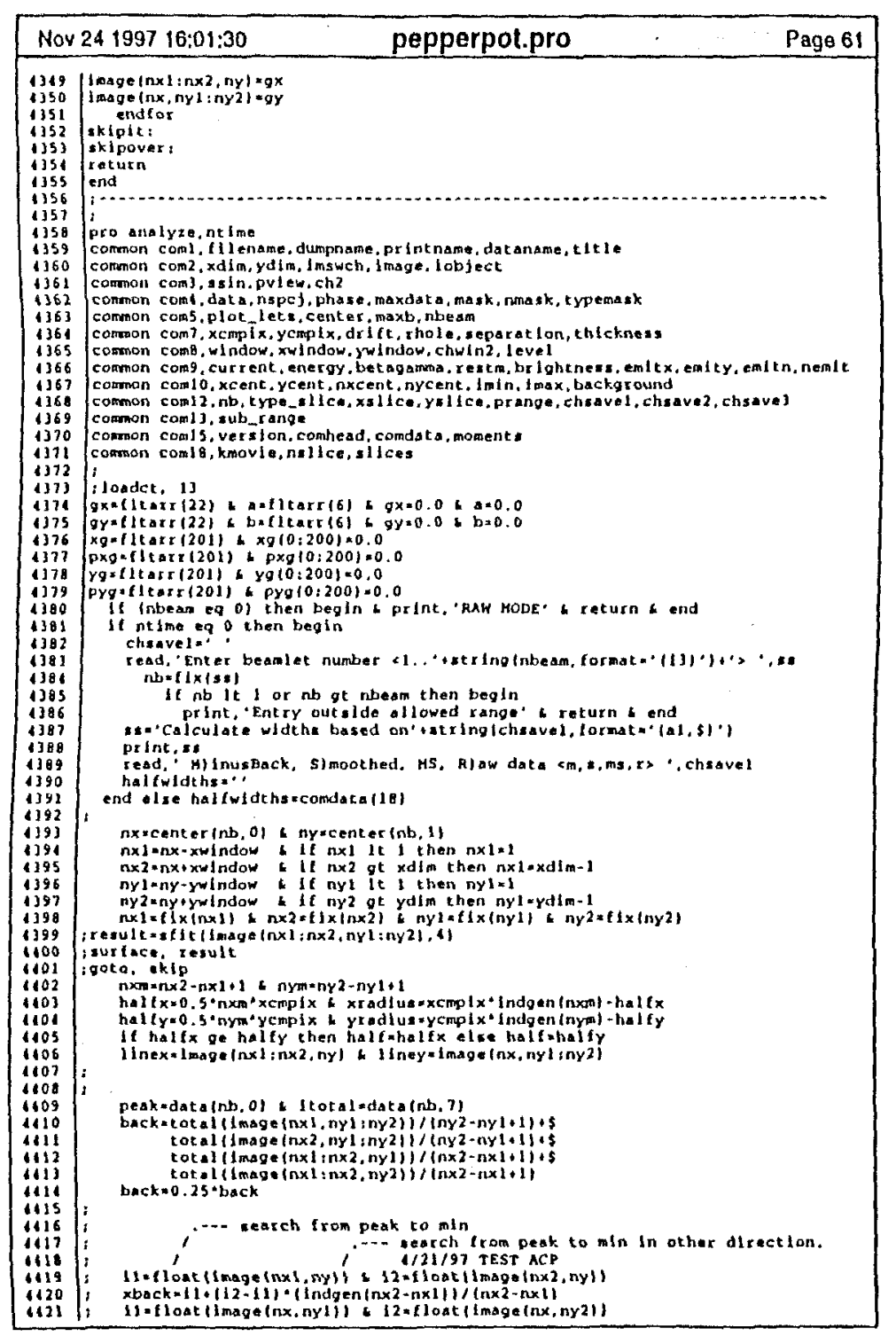

\begin{tabular}{|c|c|c|}
\hline Nov 2 & pepperpot.pro & Page 62 \\
\hline 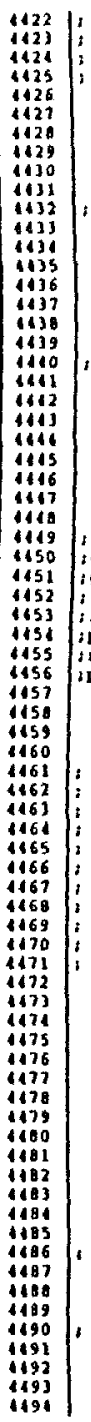 & 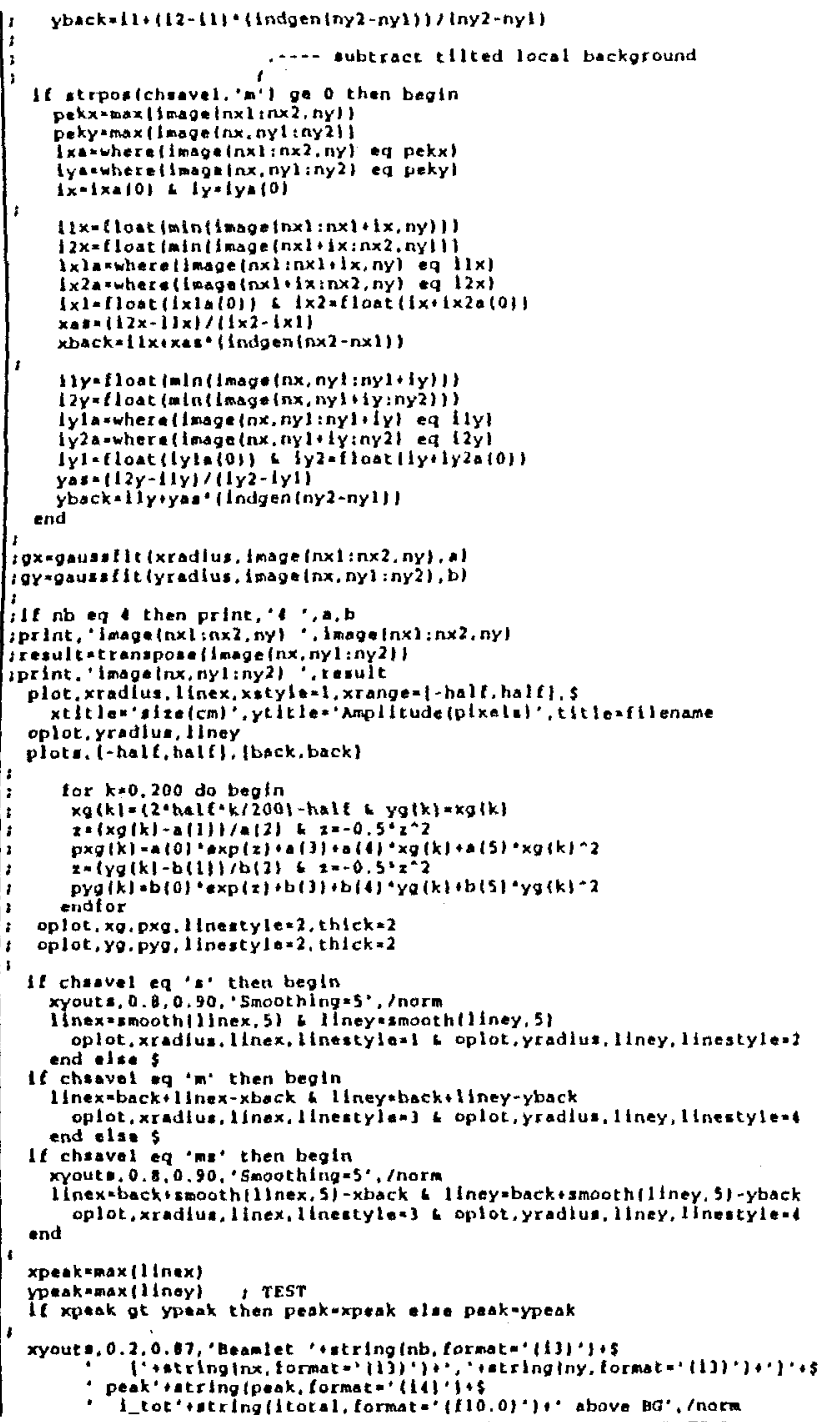 & \\
\hline
\end{tabular}



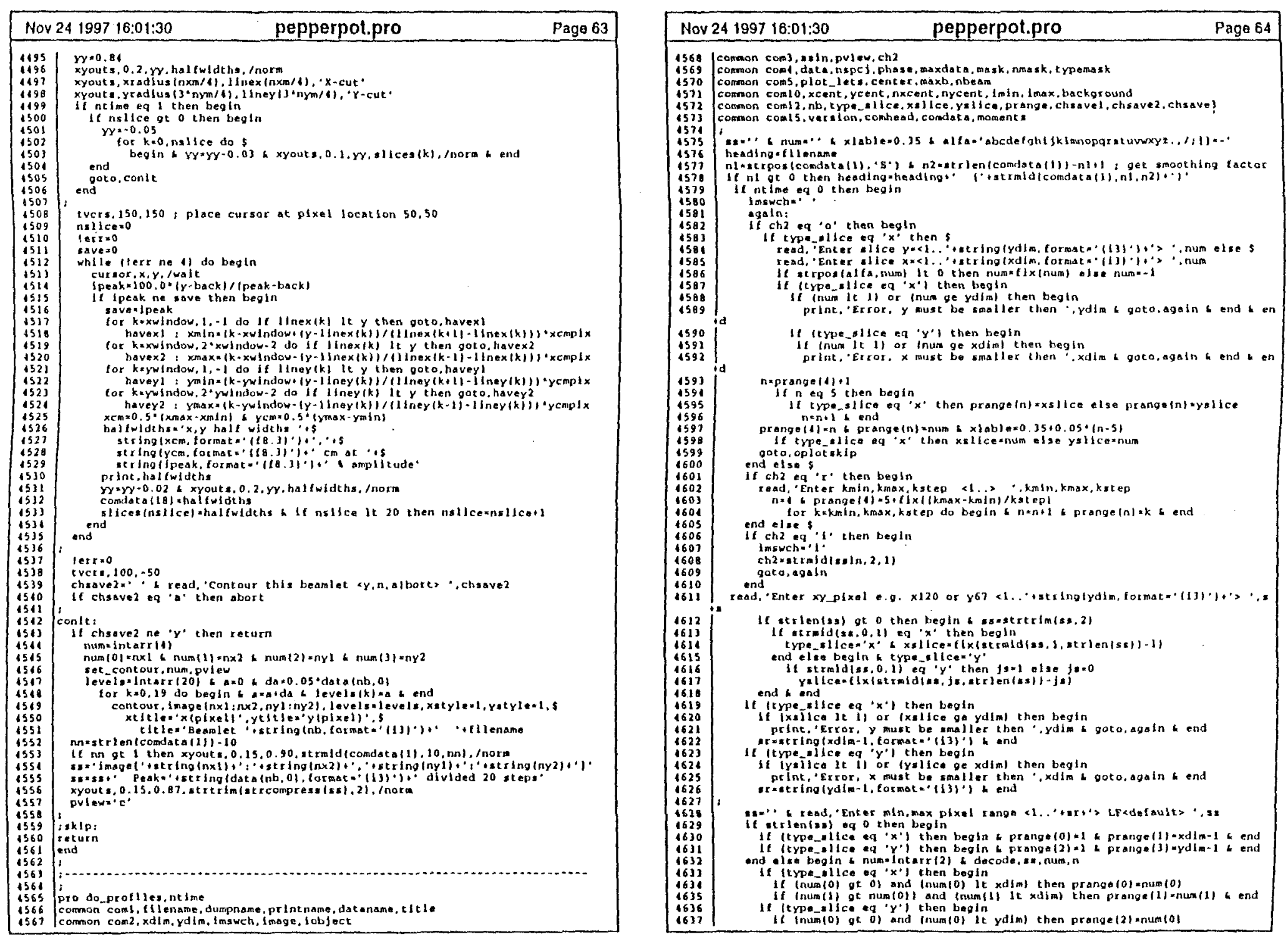

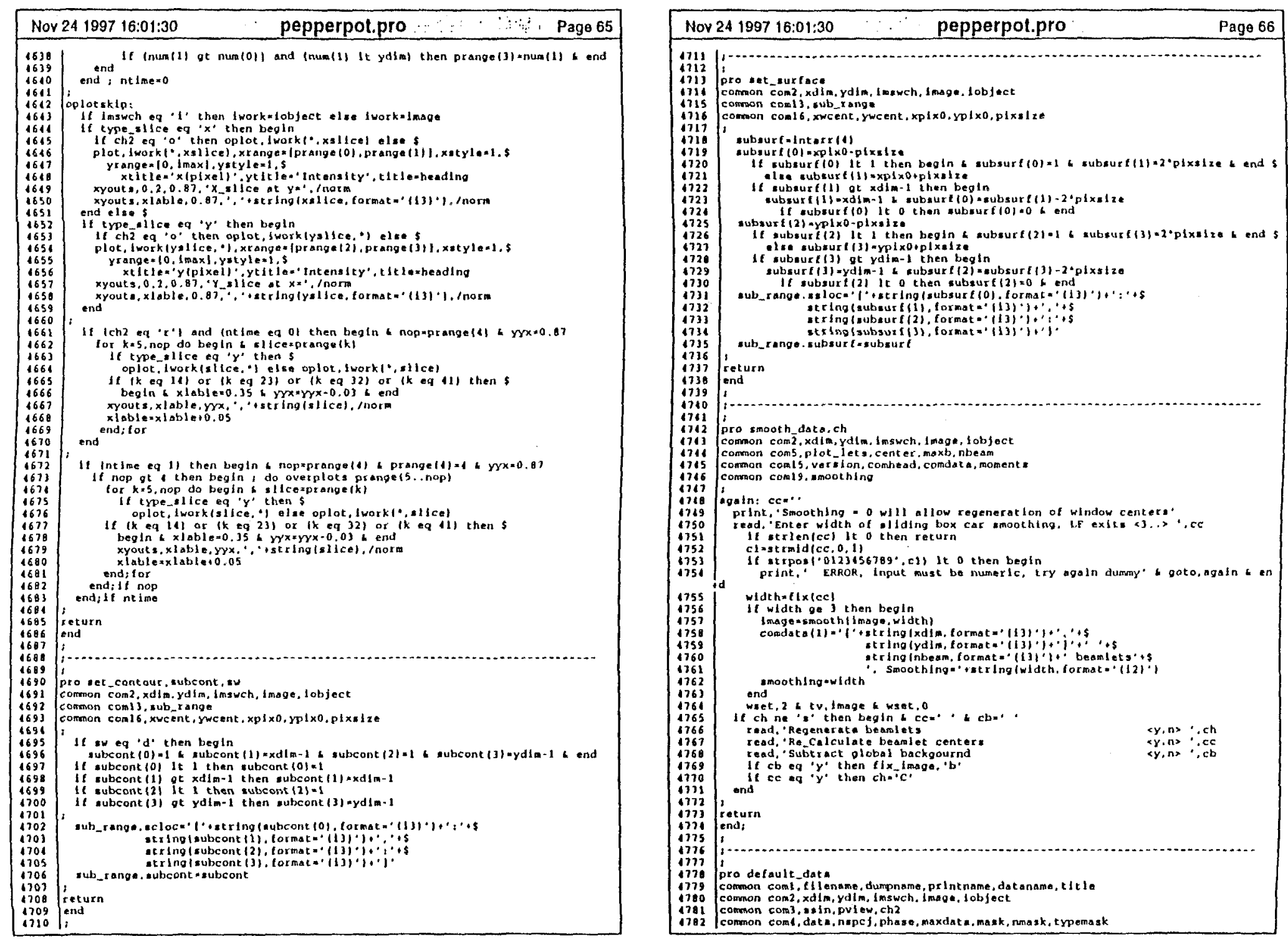

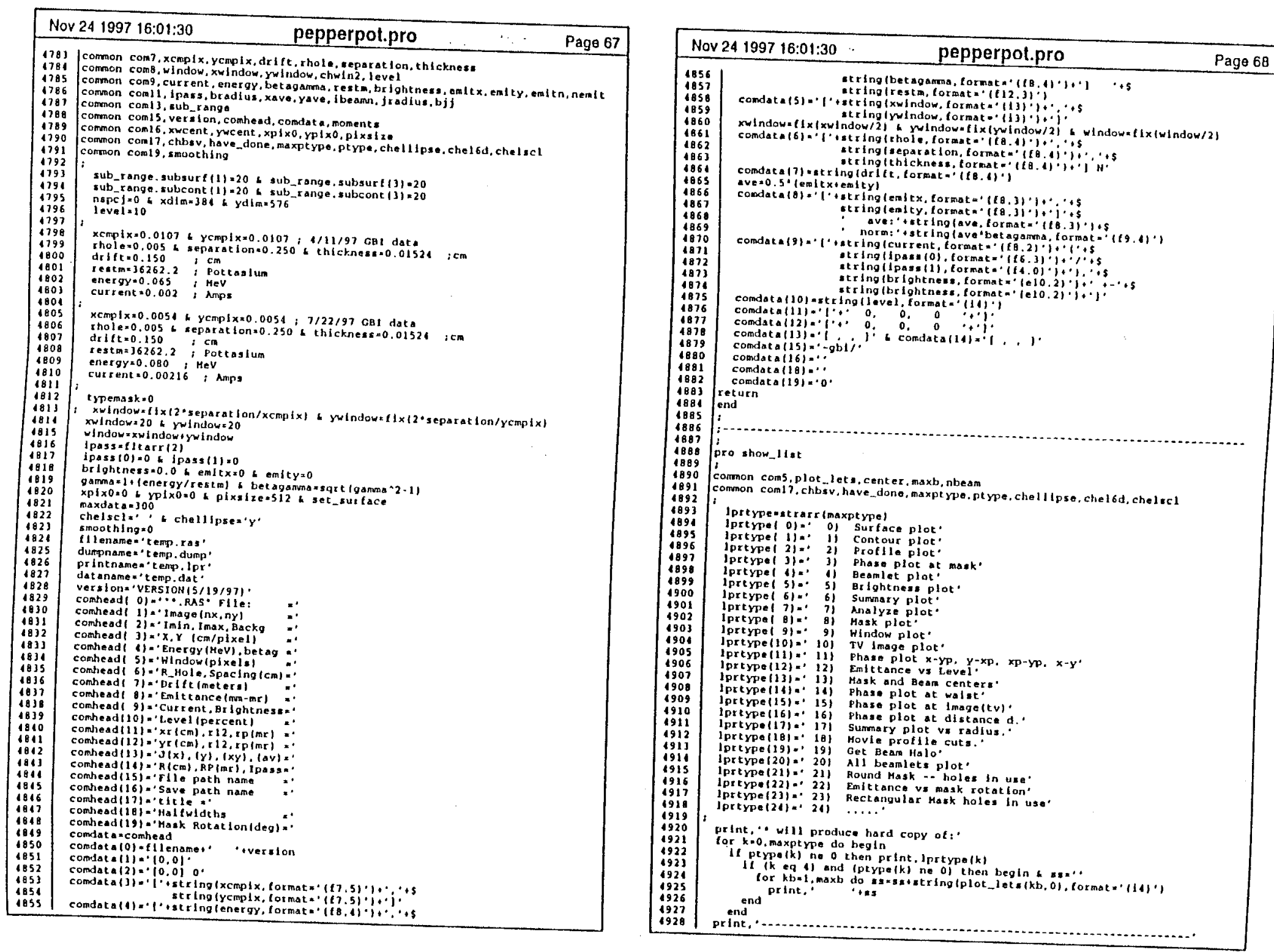

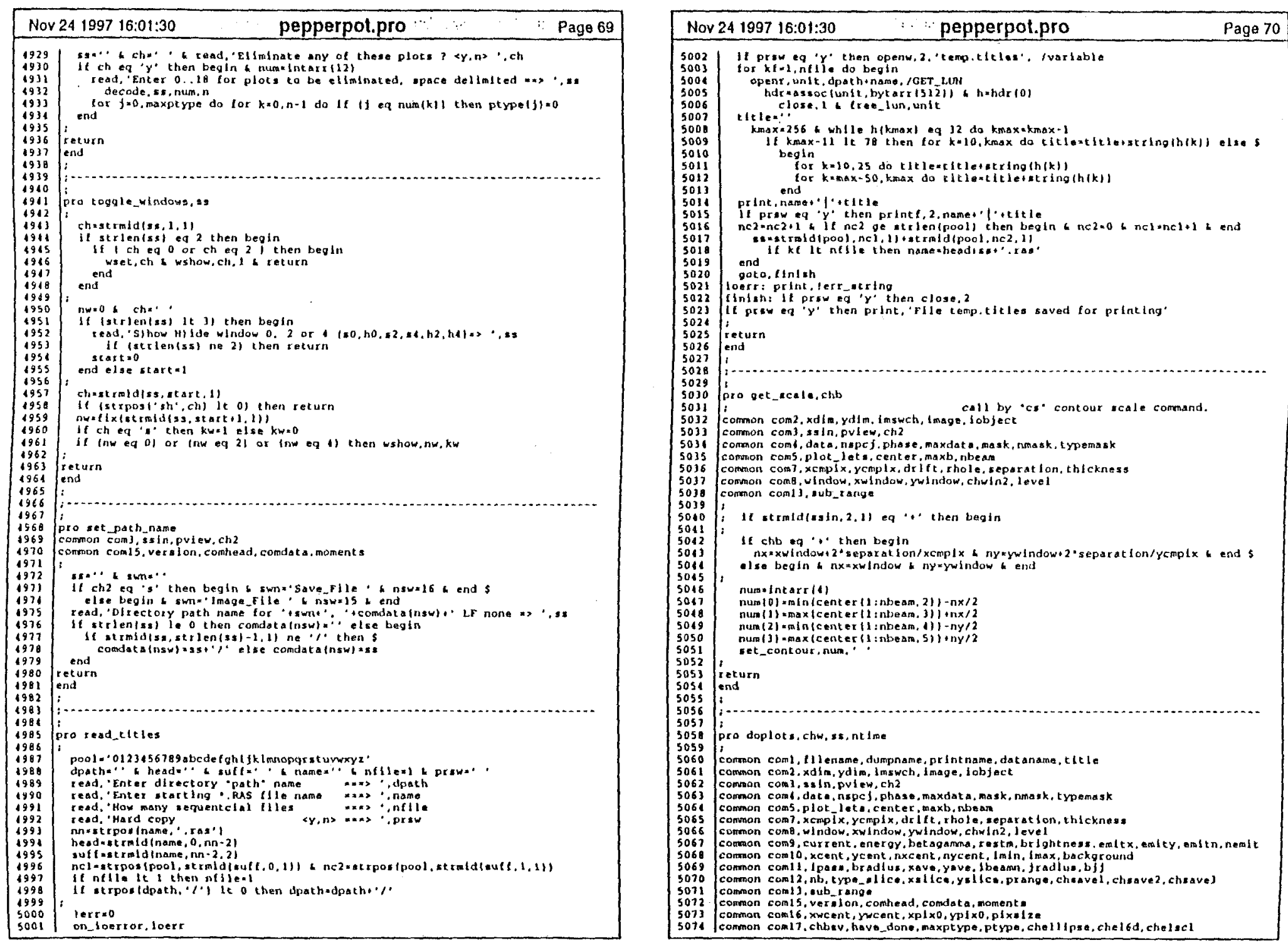

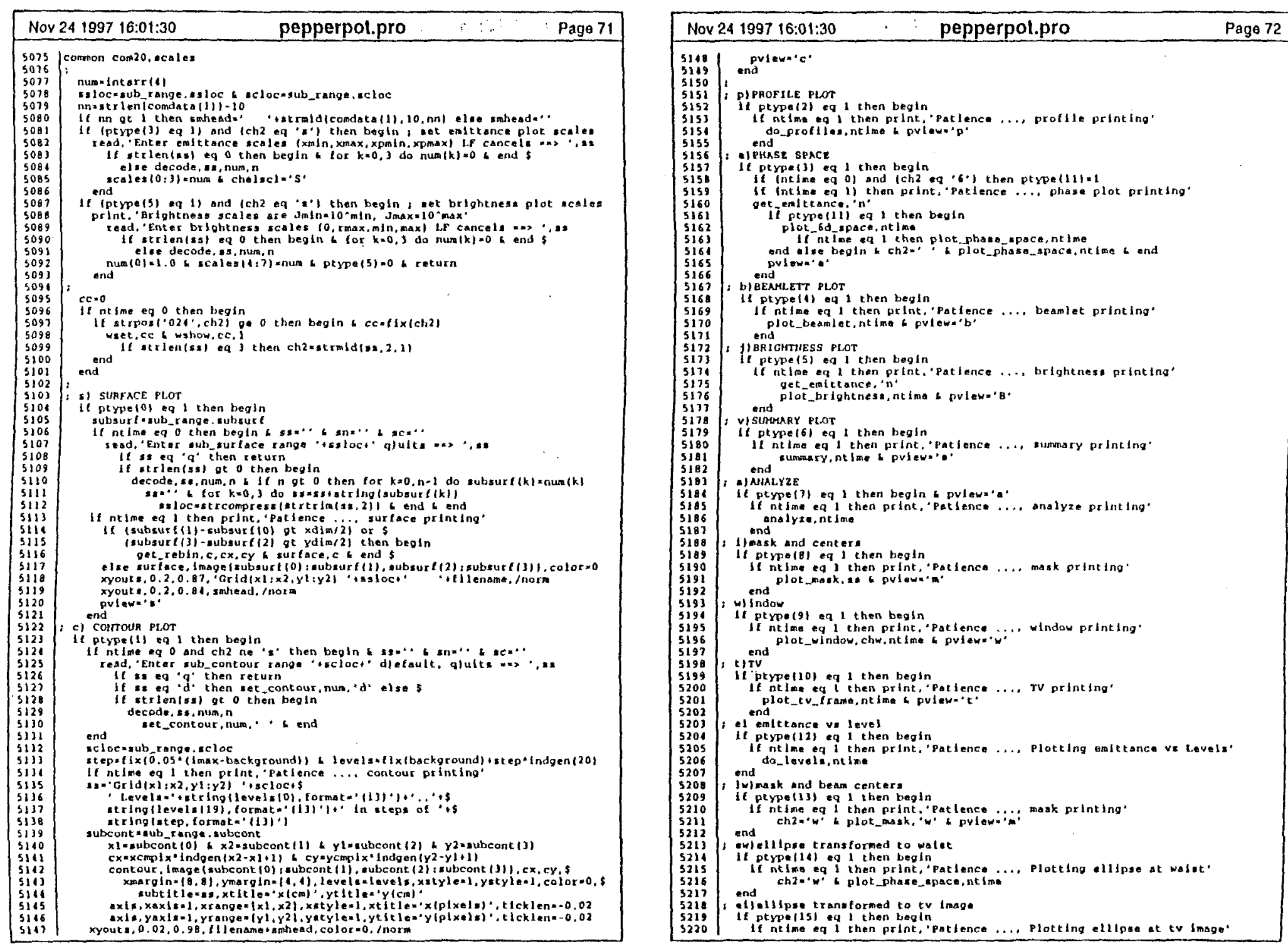

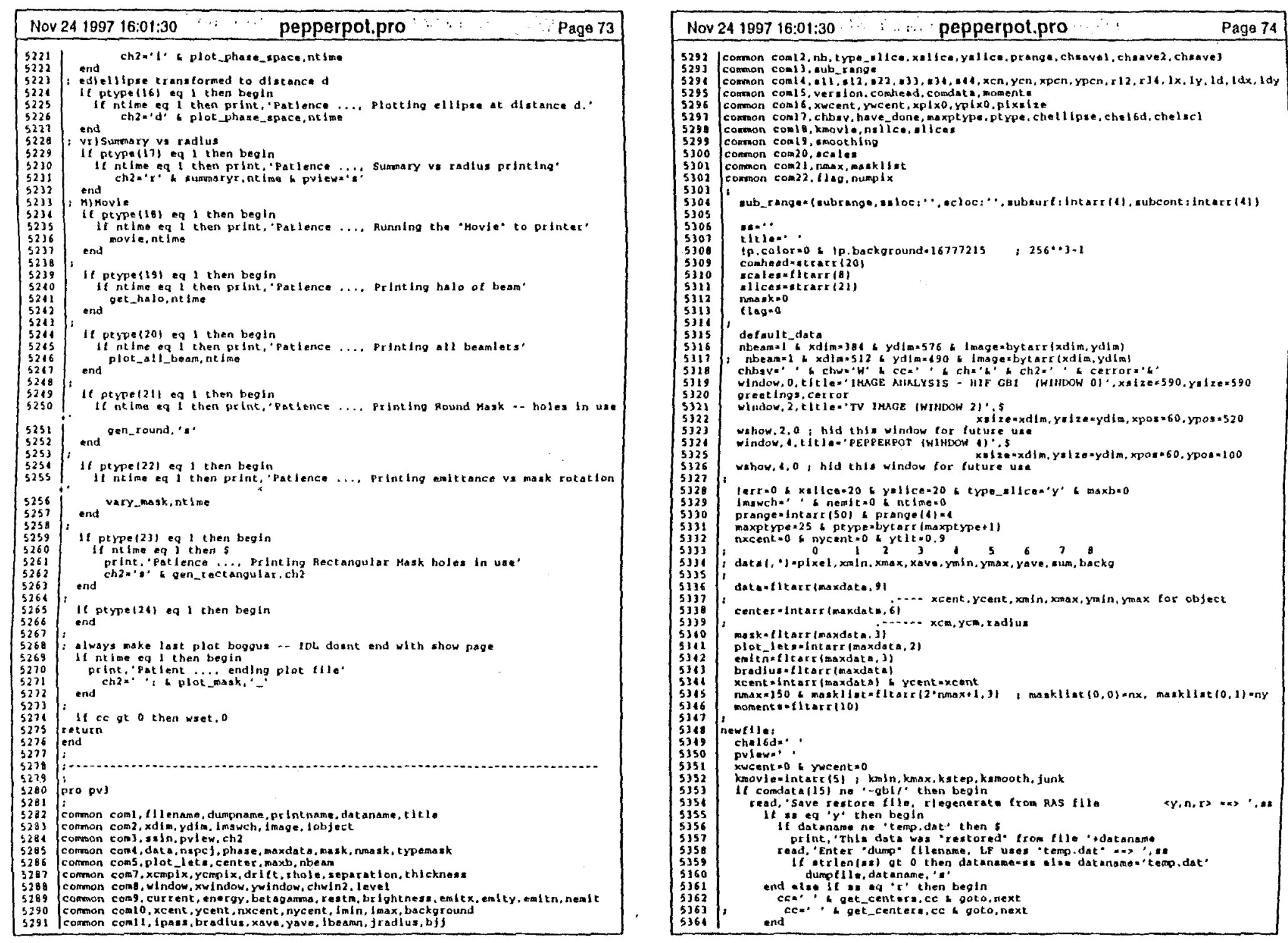

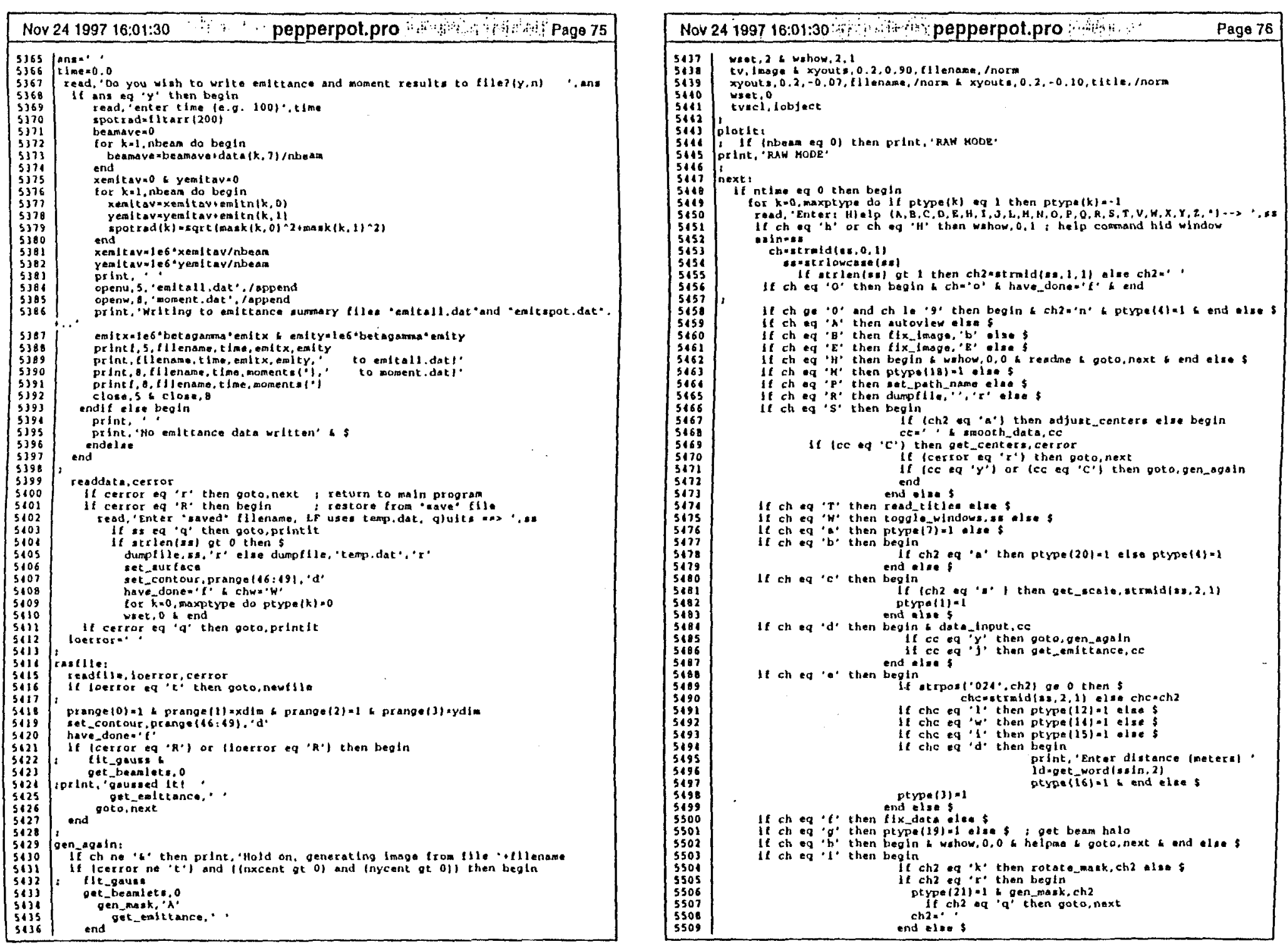

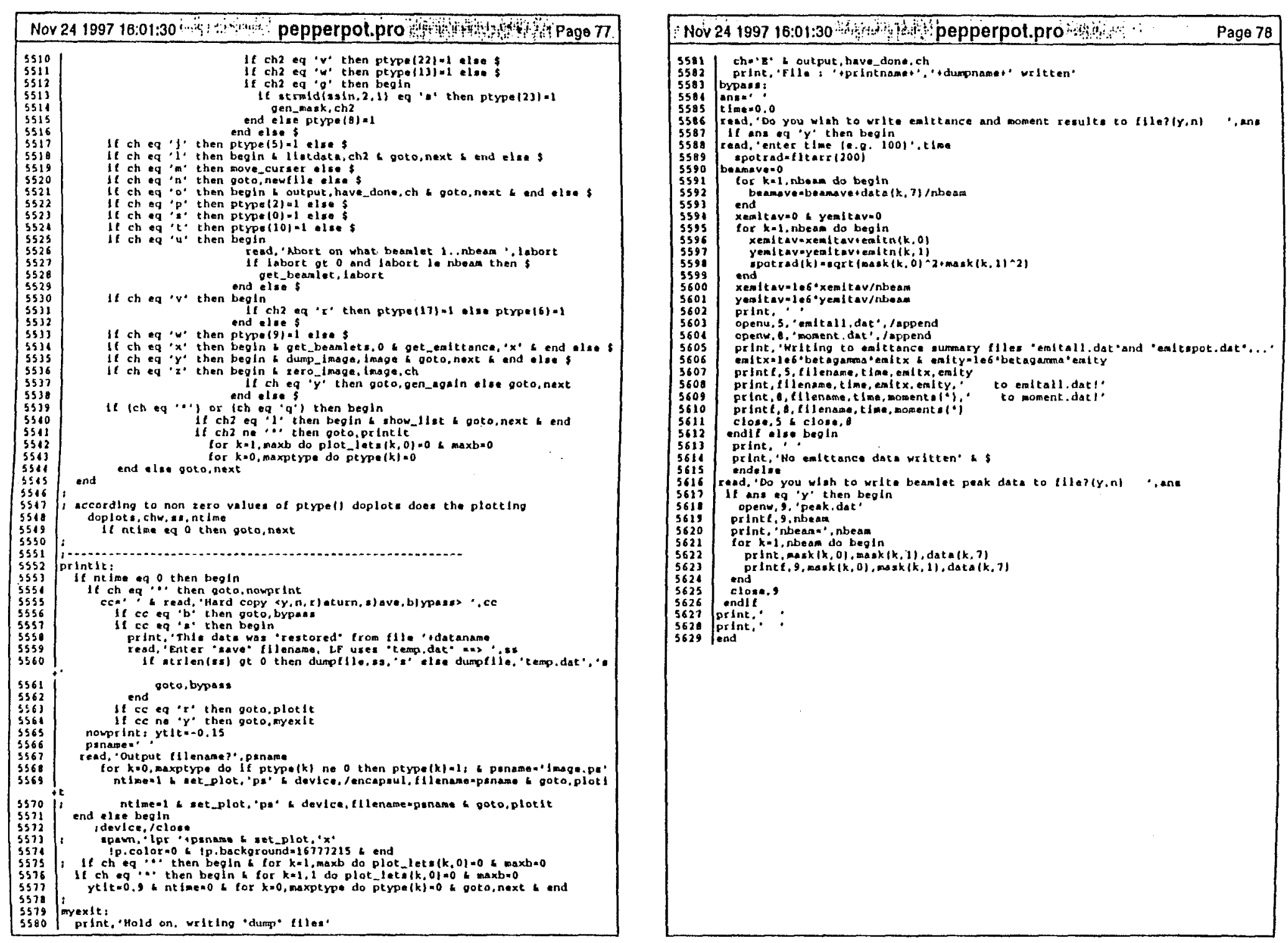


\section{References}

[1] Taubes, G. "News and Commentary" Science, Vol. 262, December, 1992.

[2] Friedman, A. et al, "Recirculating Induction Accelerators as Drivers for Heavy Ion Fusion" Phys. Fluids B: Plasma Physics 5, 2698 (1993)

[3] Sharp, W., "A Comparison of Driver Concepts for Heavy Ion Fusion" LLNL ICF Quarterly Report, Vol. 4, No. 2, March 1994

[4] Barnard J., et al, "Recirculating Induction Accelerators as Drivers for Heavy Ion Fusion" Phys. Fluids B5 (7), July 1993 2698-2706.

[5] Moir, R., "HYLIFE-II Inertial Confinement Fusion Power Plant Design" Particle Accelerators, 1992, Vols. 37-38, pp. 467-480

[6] Godlove, T., Keefe, D., Vaughan, D., "Heavy-Ion Accelerator Research for Inertial Fusion" LBL Publication, August, 1987.

[7] Friedman, A., Bangerter, R., Herrmannsfeldt, W., "Progress in Heavy Ion Drivers for Inertial Fusion" LLNL Report UCRL-JC-117332, December, 1994.

[8] Private communication with D. Callahan, October, 1997

[9] Cable, M., et al, "Heavy Ion Fusion Experiments at LLNL" Eigth ICFA Advanced Beam Dynamics Workshop on Space Charge Dominated Beams and Applications of High Brightness Beams, Bloomington, Indiana. October, 1995.

[10] Barnard J. J. et al, "Study of Recirculating Induction Accelerators as Drivers for Heavy Ion Fusion" LLNL Report UCRL-LR-108095, September 1991.

[11] Reiser, M. Theory and Design of Charged Particle Beams John Wiley and Sons, Inc., New York, 1994, p. 196.

[12] Kirby, H., et al, "Development of Solid-State Induction Modulators for High PRF Accelerators" LLNL Report UCRL-JC-119582, July, 1995. 
[13] Barnard, J., "Updated Recirculator Design Parameters", LLNL HIF Note No. 26, May, 1994.

[14] Barnard J., et al, "Physics Design and Scaling of Recirculating Induction Accelerators: From Benchtop Prototypes to Drivers" Fusion Engineering and Design 32-33 (1996) 247-258.

[15] Friedman, A., and Grote,D., "Three -Dimensional Particle Simulation of Heavy Ion Fusion Beams" Phys. Fluids B4 (7), July 1992

[16] " $\mathrm{K}^{+}$Source for the LLNL Recirculator Experiment", HIFAR Note 405, Lawrence Berkeley Laboratory, January, 1994.

[17] Wilson, M., et al, "Heavy Ion Fusion Impulse Injector Design, Construction, and Checkout" LLNL Report UCRL-ID-DRAFT, August, 1997.

[18] Karpenko, V., et al, "Mechanical Design of Recirculating Accelerator Experiments for Heavy Ion Fusion” LLNL Report UCRL-JC-119583, May, 1995.

[19] Reiser, M. Theory and Design of Charged Particle Beams John Wiley and Sons, Inc., New York, 1994

[20] Friedman, A., et al, "Construction of the First Recirculating Induction Accelerator" LLNL Proposal for a Director's Initiative LDRD Project, April, 1994.

[21] Reiser, M. Theory and Design of Charged Particle Beams John Wiley and Sons, Inc., New York, 1994, p. 363.

[22] Reiser, M. Theory and Design of Charged Particle Beams John Wiley and Sons, Inc., New York, 1994, p. 150.

[23] Reiser, M. Theory and Design of Charged Particle Beams John Wiley and Sons, Inc., New York, 1994, p. 360.

[24] Kapchinskij, I., and Vladmirskij, V. "Limitations of Proton Beam Current in a Strong Focusing Linear Accelerator Associated with Beam Space Charge", Proc. 
of the International Symposium on High Energy Accelerators, (CERN, Geneva, 1959), p. 274.

[25] Reiser, M. Theory and Design of Charged Particle Beams John Wiley and Sons, Inc., New York, 1994, Chapter 6.

[26] "Possible Emittance Increase Through Filamentation Due to Space Charge in Continuous Beams" IEEE Trans. Nucl. Sci.NS-18, No. 3, 1101, 1971.

[27] Sacherer, F., "RMS Envelope Equations with Space Charge" IEEE Trans. Nucl. Sci.NS-18, No. 3, 1105, 1971.

[28] Paul, A. et al, "Probing the Electron Distribution Inside the ATA Beam Pulse", Nucl. Instrum. Methods Phys. Rev. A300 (1991) 137-150.

[29] Barnard, J., "Emittance Growth from Rotated Quadrupoles in Heavy Ion Accelerators", 1995 Partcle Accelerator Conference, Dallas, TX.

[30] Lawson, J., Lapostelle, P., Gluckstern, R., "Emittance, Entropy, and Information" Particle Accelerators, 1973, Vol. 5, pp. 61-65

[31] Tiefenback, M., "Space-Charge Limits on the Transport of Ion Beams in a Long Alternating Gradient System" LBL Report 22465, 1986, p. 38.

[32] Signal Analytics Corporation, IPLab Spectrum ver. 3.1 Software User's Guide, 1996, p. 160.

[33] Research Systems, Inc., Interactive Data Language ver. 4.0 Software User's Guide, 1995.

[34] Li, C., Petrasso, R., "Charged-particle stopping powers in inertial confinement fusion plasma" Physical Review Letters Vol. 70, No. 20, May, 1993, pp. 3059-62.

[35] Knoll, G., Radiation Detection and Measurement John Wiley and Sons, Inc., New York, 1989 
[36] Ziegler, J., Biersack, J., Littmark, U., The Stopping and Range of Ions in Matter, Vol. 1 Pergamon Press, Inc., New York, 1985

[37] Galileo Electro-Optics Corporation Product Brochure, 1996

[38] Kaminsky, M., Atomic and Ionic Impact Phenomena on Metal Surfaces Academic Press, Inc., New York, 1965, p. 325.

[39] Princeton Instruments Corporation, PG-200 Programmable Pulse Generator Operating Manual, Ver. 2, rev. A, August 26, 1996.

[40] Princeton Instruments Corporation, ITE/CCD Detector Operating Manual, Ver. 1, rev. A, April 8, 1996.

[41] Princeton Instruments Corporation, ST-138 Camaera Controller Operating Manual, Ver. 3, rev. A, May 9, 1996, p. 25.

[42] Dragt, A., Neri, F., Rangarajan, G., General Moment Invariants for Linear Hamiltonian Systems, Phys. Rev. A., Vol. 45, No. 4, 1992, 2572-2585.

[43] Halbach, K., "Conceptual Design of a Permanent Quadrupole Magnet with Adjustable Strength", Nucl. Instrum. Methods Phys. Rev. A 206 (1983) 353-354.

[44] Spall, J., "Multivariate Stochastic Approximation Using a Simultaneous Perturbation Gradient Approximation", IEEE Trans. Automatic Control, vol. 37, pp. $332-341$.

[45] Spall, J., "A Model-Free Approach to Optimal Signal Light Timing for SystemWide Traffic Control", Proceedings of the 33rd Conference on Decision and Control, Lake Buena Vista, Fl., December 1994, pp. 1868-1875

[46] Spall, J., "A Neural Network Controller for Systems with Unmodeled Dynamics with Applications to Wastewater Treatment", IEEE Trans. Systems, Man, and Cybernetics, vol. 27, 1997. 
[47] Lund, S., et al, "Numerical Simulation of Intense Beam Experiments at LLNL and LBL" 12th International Symposium on Heavy Ion Inertial Fusion, Heidelberg, Germany, September 24-27, 1997.

[48] Friedman, A., et al, "Progress Toward a Prototype Recirculating Induction Accelerator for Heavy Ion Fusion", 1995 Partcle Accelerator Conference, Dallas, TX.

[49] Wolfram, S., Mathematica, A System for Doing Mathematics by Computer Addison-Wesley Publishing Company, Inc., Redwood City, Ca, 1988

[50] Barnard, J., et al, "Emittance Growth in Heavy Ion Recirculators", C. R. Hoffman (ed.), Proc Linear Accelerator Conf., Ottawa, August 1992, AECL 10728, 1992 , p. 229.

[51] Lee, E., Fessenden, T., Laslett, L., "Transportable Charge in a Periodic Alternating Gradient System" IEEE Trans. Nucl. Sci.NS-18, No. 32, 2489, 1985.

[52] BICRON Corporation Scintillator Product Brochure, 1990, p. B2.

[53] Spall, J., "Implementation of the Simultaneous Perturbation Algorithm for Stochastic Optimization", American Statistician, September, 1995.

[54] Private communication with S. Eylon, September, 1997

[55] Hopkins, H., LLNL HIF Note Number 96-5 "Physics design and Data Analysis for a Gata Beam Imager Diagnostic Instrument, July 2, 1996. 